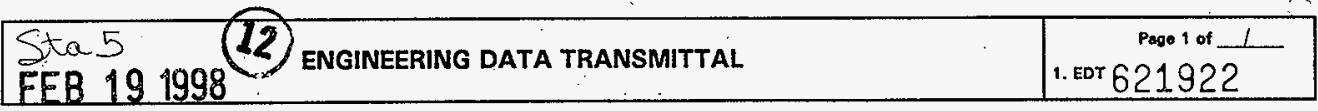

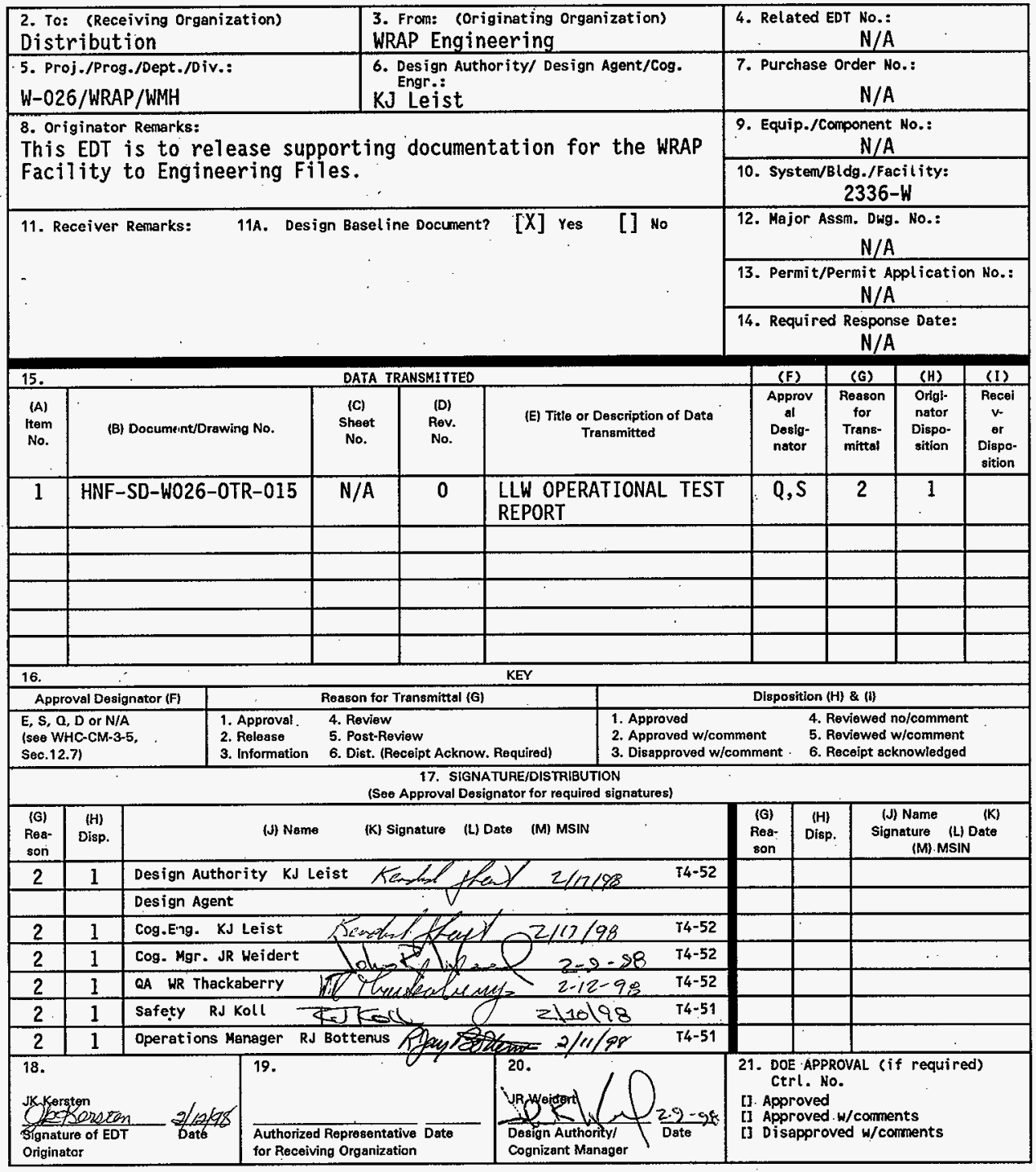

BD-7400-172-2 (05/96) GEF097 


\title{
WRAP LOW LEVEL WASTE (LLW) GLOVEBOX OPERATIONAL TEST REPORT
}

\author{
KJ Leist
}

Waste Management Federal Services of Hanford, Richland, WA 99352

U.S. Department of Energy Contract DE-AC06-96RL13200

\begin{tabular}{|c|c|c|}
\hline $\begin{array}{l}\text { EDT/ECN: } \\
\text { Org Code: } \\
\text { B\&R Code: }\end{array}$ & $\begin{array}{l}\text { EDT-621922 } \\
32620 \\
\text { EW3130020 }\end{array}$ & $\begin{array}{l}\text { UC: } 506 \\
\text { Charge Code: } \\
\text { Total Pages: }\end{array}$ \\
\hline
\end{tabular}

Key Words: W-026, ATR, Drum, Diversified Metal Products, DMS, PCS

Abstract: The purpose of this test was to verify the Plant Operating Procedures related to the LLW Process Gloveboxes are accurate so operations within the gloveboxes can be performed safely and desired results obtained. And to verify the Data Management System could properly interface with the Plant Control System.

* Drath \& Schrader is a registered trademark of Babcock Rohrleitungsbau, GMBH, Germany

* Nylon is a registered trademark of E.I. duPont de Nemours and Co., Wilmington, DE.

* Schilling Robotics System is a registered trademark of GEC ALSTHOM Schilling Robotics Systems, Davis, CA.

* TORR II D is a registered trademark of EG\&G Ortec, Oak Ridge, TN.

* Ziplock is a registered trademark of DowBrands, subsidiary of Dow Chemical Co., Indianapolis, IN.

TRADEMARK DISCLAIMER. Reference herein to any specific comercial product, process, or service by trade name, trademark, manufacturer, or otherwise, does not necessarily constitute or inply its endorsement, recommendation, or favoring by the United States Government or any agency thereof or its contractors or subcontractors.

Printed in the United States of America. To obtain copies of this document, contact: Document Control Services, P.0. Box 950, Mailstop H6-08, Richland WA 99352, Phone (509) 372-2420; Fax (509) 376-4989.
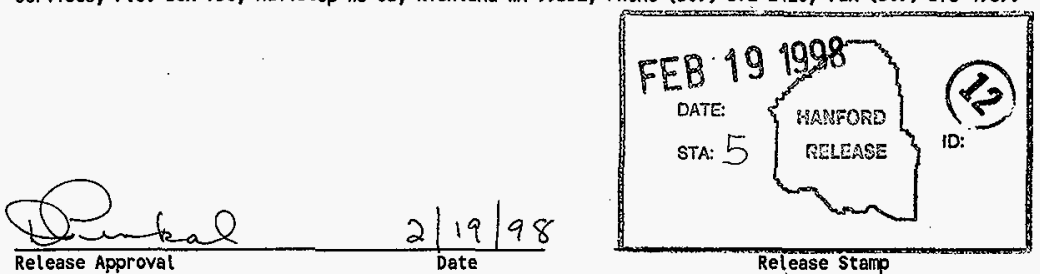

Approved for Public Release 


\title{
WASTE RECEIVING AND PROCESSING FACILITY (WRAP)
}

\author{
LOW LEVEL WASTE (LLW) PROCESS \\ GLOVEBOXES 101, 102, $104 \& 105$ \\ OPERATIONAL TEST REPORT
}

HNF-SD-W026-OTR-015 Rev. 0

WASTE MANAGEMENT HANFORD COMPANY - PROJECT W-026

January 28, 1998

SUM 1 of 9 
LLW GLOVEBOXES 101, 102, $104 \& 105$ OPERATIONAL TEST REPORT HNF-SD-W026-OTR-015 Rev. 0

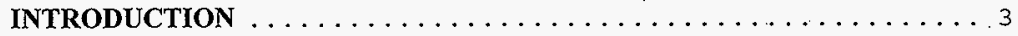

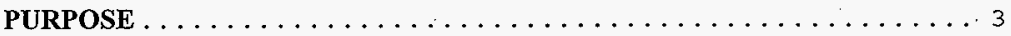

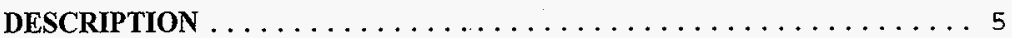

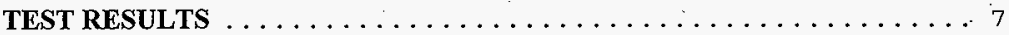

Exception Test Reports (ETR's) Summary . . . . . . . . . . . . 7

Additional Items. . . . . . . . . . . . . . . . . . . 7

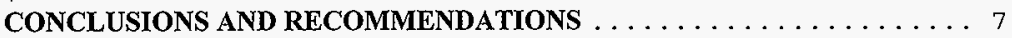

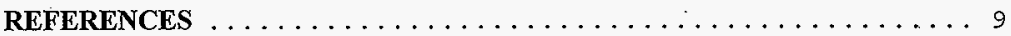

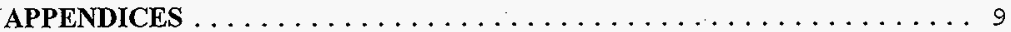

Appendix A - Exception Test Reports . . . . . . . . . . . . . . . . . . . . . . . .

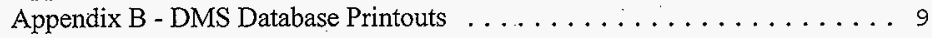

Appendix C - Plant Operating Procedures . . . . . . . . . . . . . 9 


\section{LLW GLOVEBOXES 101, 102, 104 \& 105 OPERATIONAL TEST REPORT HNF-SD-W026-OTR-015 Rev. 0}

\section{INTRODUCTION}

The Low Level Waste (LLW) Process Gloveboxes are designed to:

- Receive a 55 gallon drum in an 85 gallon overpack in the Entry glovebox (GB101).

- Open and sort the waste from the 55 gallon drum, place the waste back into drum and relid in the Sorting glovebox (GB102). In addition, waste which requires further examination is transferred to the LLW RWM Glovebox via the Drath \& Schraeder Bagless Transfer Port (DO-07-201) or sent to the Sample Transfer Port (STC).

- Crush the drum in the Supercompactor glovebox (GB104).

- Place the resulting puck (along with other pucks) into another 85 gallon overpack in the Exit glovebox (GB105).

The status of the waste items is tracked by the Data Management System (DMS) via the Plant Control System (PCS) barcode interface. As an item is moved from the entry glovebox to the exit glovebox, the Operator will track an items location using a barcode reader and enter any required data on the DMS console.

The Operational Test Procedure (OTP) will perform evolution's (described below) using the Plant Operating Procedures (POP) in order to verify that they are sufficient and accurate for controlled glovebox operation.

\section{PURPOSE}

The intent of this test was to satisfy two main objectives:

a. Verify that the Plant Operating Procedures related to the LLW Process Gloveboxes are accurate so that operations within the gloveboxes can be performed safely and desired results obtained.

During the performance of the OTP, the POP's were redlined to correct errors found during testing. These corrections were incorporated into updated revisions of the POP's. The original POP's from which this OTP was conducted are included with this (Operational Test Report) OTR so the evolution to the present state of the POP's can be traced. 


\section{LLW GLOVEBOXES 101, 102, 104 \& 105 OPERATIONAL TEST REPORT HNF-SD-W026-OTR-015 Rev. 0}

b. Verify that the Data Management System (DMS) could properly interface with the Plant Control System (PCS) to control and display the data obtained during drum, sample/packet or puck movement and treatment.

When both of the above objectives are met, the LLW Process Gloveboxes would be considered ready for operation. 


\section{LLW GLOVEBOXES 101, 102, 104 \& 105 OPERATIONAL TEST REPORT HNF-SD-W026-OTR-015 Rev. 0}

\section{DESCRIPTION}

The Operational Test Procedure (HNF-SD-W026-015) was started on August 8, 1997 and consisted of six main sections as described below. A complete description is included at the beginning of each Test Case under General Description.

a. LLW Glovebox Manual Operation

All three glovebox access ports (DO-101, DO-105 \& DO-106) are operated in manual mode. The respective lift table is placed in manual mode and the drum is mated to the port. Then by using manual mode for the Bagless Transfer port or the maintenance menus for the Entry/Exit ports, a manual open/closed cycle is completed.

The Transfer port (DO-102) and port seal will be cycled in manual mode.

The Transfer Car, Lidder/Delidder Fixture, Drum Grabber/Tilt Unit \& Sorting Table in the Sorting glovebox will be exercised in manual mode.

b. LLW Glovebox Automatic Test Case 1

Waste Processing Drum 1 will be loaded into the Entry glovebox and then sent to the Sorting glovebox via the Transfer port. The drum will be delidded, dumped on the sorting table and samples loaded in the Sample Transport Container (STC) for analysis. The remaining contents will be dumped back into the drum and relidded. The drum will be sent to the exit glovebox without being compacted and loaded into an overpack:

The Data Management System (DMS) will track the progress of the drum and samples by barcode throughout the evolution.

\section{c. LLW Glovebox Automatic Test Case 2}

Waste Processing Drum 2 will be loaded into the Entry glovebox and then sent to the Sorting glovebox via the Transfer port. The drum will be delidded, dumped on the sorting table and samples packets loaded on the transfer stand for transfer to the LLW/RWM glovebox. The remaining contents will be dumped back into the drum and relidded. The drum will be sent to the Compactor on the transfer car. After being compacted, the remaining puck is barcoded and placed in an overpack drum. 


\section{LLW GLOVEBOXES 101, 102, 104 \& 105 OPERATIONAL TEST REPORT HNF-SD-W026-OTR-015 Rev. 0}

The Data Management System (DMS) will track the progress of the drum and samples by barcode throughout the evolution.

d. LLW Glovebox Automatic Test Case 3

Waste Processing Drum 3 will be loaded into the Entry glovebox and then sent to the Sorting glovebox via the Transfer port. Instead of stopping at the delid position, the transfer car will take the drum to the Compactor without opening. After being compacted, the remaining puck is barcoded and placed in an overpack drum.

The Data Management System (DMS) will track the progress of the drum and samples by barcode throughout the evolution.

\section{e. LLW Glovebox Automatic Test Case 4}

Waste Processing Drum 4 will be loaded into the Entry glovebox and then sent to the Sorting glovebox via the Transfer port. The drum will be delidded, dumped on the sorting table and samples packets loaded on the transfer stand for transfer to the LLW/RWM glovebox. The remaining contents will be dumped back into the drum and relidded. The drum will be sent to the Compactor on the transfer car. After being compacted, the remaining puck is barcoded and placed in an overpack drum.

The Data Management System (DMS) will track the progress of the drum and samples by barcode throughout the evolution.

\section{f. LLW Glovebox Restart}

The Glovebox Emergency Stop button is pushed during a port opening sequence. Equipment status is recorded for recovery. 


\section{LLW GLOVEBOXES 101, 102, $104 \& 105$ OPERATIONAL TEST REPORT HNF-SD-W026-OTR-015 Rev. 0}

\section{TEST RESULTS}

\section{Exception Test Reports (ETR's) Summary}

35 ETR's were written during test performance, of which 10 are DMS related. As of December 17, 1997, all ETR's have been cleared.

\section{Additional Items}

WRP1-OP-0703 (Glovebox Lift Table Operation) was not referenced and therefore not verified in this OTP.

The "Glovebox Restart" portion of the OTP lacked enough depth to really verify that the glovebox can be successfully restarted for a selection of scenarios. Only one Emergency Stop pushbutton was checked. The balance of the section was merely a status check of the equipment in the glovebox. Operation Procedure OP0722 should be enhanced to included flow charts or the like to demonstrate a logical course of action after the status of the systems are known.

\section{CONCLUSIONS AND RECOMMENDATIONS}

a. The STC Port can be open when the STC has been removed; no mechanical interlock exists. This can result in a loss of glovebox differential pressure and possible contamination of the STC port seal.

b. The Bagless Transfer Port DO-07-105 operation should be incorporated into the existing PLC interface inștead of through a separate controller for the following reasons:

-The operator can only perform open and close functions when in automatic mode. Manual operation and recovery must be performed from the D\&S controller.

-There is no feed back to the operator in maintenance mode as to the position of the port components as there is for the entry/exit ports in this glovebox.

-The controller is programmed in German, requiring outside support for program changes.

-By eliminating the controller, the system is made simpler and therefore more reliable. 


\section{LLW GLOVEBOXES 101, 102, 104 \& 105 OPERATIONAL TEST REPORT HNF-SD-W026-OTR-015 Rev. 0}

c. Permanent bar code charts should be added to the glovebox. During the OTP barcodes were taped to a sheet of paper which in time will deteriorate and require replacement.

d. Since all ETR's have been cleared the Operational Test Procedure can be considered passed. However, modifications to the gloveboxes have been made since the completion of the OTP and will require verification prior to operation along with any other items that have been changed or reworked. 
LLW GLOVEBOXES 101, 102, $104 \& 105$ OPERATIONAL TEST REPORT HNF-SD-W026-OTR-015 Rev. 0

REFERENCES

Plant Operating Procedures.

WRP1-OP-0702 'Restart LLW Glovebox Operation

WRP1-OP-0706 Packet NDE Operation

WRP1-OP-0713 LLW Entry Glovebox Operation

WRP1-OP-0714 LLW Sorting Glovebox Operation

WRP1-OP-0715 LLW Supercompactor Operation

WRP1-OP-0716 LLW Exit Glovebox Operation

WRP1-OP-0719 LLW Gloveboxes Manual Operation

WRP1-OP-0722 Restart RWM Glovebox Operation

APPENDICES

Appendix A - Exception Test Reports

Appendix B - DMS Database Printouts

Appendix C - Plant Operating Procedures

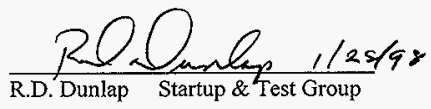

SUM 9 of 9 


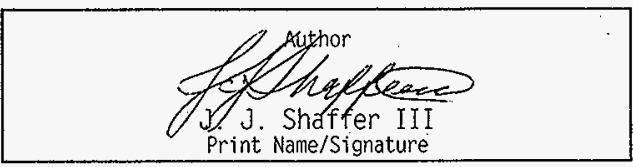

APPROVAL DESIGNATOR

PROCEDURE APPROVAL SIGNATURES

Ru Botterus WRAP 1 Startup Manager

Ru Bottehus Estoless WRAP 1 Lngineering Manager
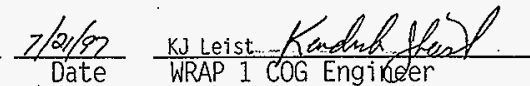

$\frac{7 / 17 / 97}{\text { Date }}$

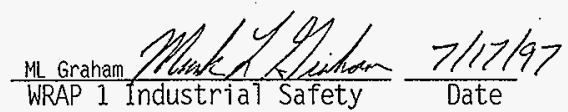

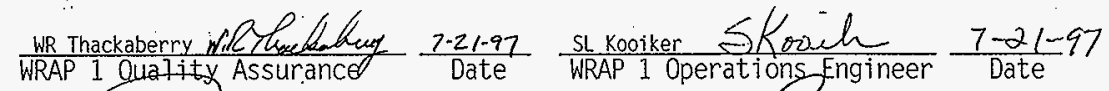
Plesydere beldele 秎A 1 Operation Manager $\frac{1 / 2 i / 97}{\text { Date }}$

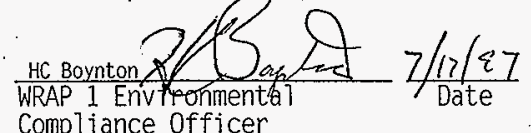
with WRAP 1 Records Management Specialist 
Waste Management Federal Services of Hanford, Inc.

Project $W-026$, WRAP I

operational Test - LLW Glovebox

\section{TABLE OF CONTENTS}

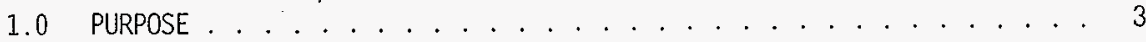

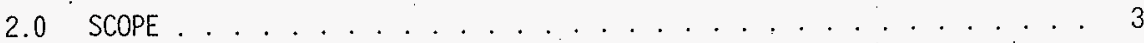

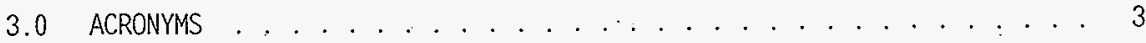

4.0 PRECAUTIONS AND LIMITATIONS . . . . . . . . . . . . . . . . . . . 4

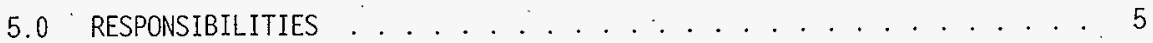

6.0 CHANGE CONTROL ........................ 6

7.0 REFERENCES .......................... 6

8.0 SAFETY .............................. 8

9.0 QuAlitY ASSURANCE . . . . . . . . . . . . . . . . 8

10.0 RECORDS . . . . . . . . . . . . . . . . . . . . 8

11.0 PREREQUISITES . . . . . . . . . . . . . . . . . . . . . . 9

12.0 PROCEDURE . . . . . . . . . . . . . . . . . 12

13.0 ACCEPTANCE CRITERIA . . . . . . . . . . . . . . . . . . . 13

ATTACHMENTS

ATTACHMENT 1 - TEST EXCEPTION LOG FORM . . . . . . . . . . . . . . 14

ATTACHMENT 2 - TEST EXCEPTION REPORT . . . . . . . . . . . . . 15

ATTACHMENT 3 - SIGNATURE RECORD . . . . . . . . . 16

ATTACHMENT 4 - TEST PLAN, LLW GLOVEBOX MANUAL OPERATION _... . . . . . 17

ATTACHMENT 5 - TEST PLAN, LLW GLOVEBOX AUTOMATIC TEST CASE $1 \ldots \ldots$. . . . . . . 27

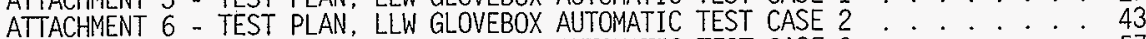

ATTACHMENT 7 - TEST PLAN, LLW GLOVEBOX AUTOMATIC TEST CASE $3 \ldots \ldots$. . . . . . 57

ATTACHMENT 8 - TEST PLAN, LLW GLOVEBOX AUTOMATIC TEST CASE $4 \ldots \ldots$. . . . . . 66

ATTACHMENT 9 - TEST PLAN, LLW GLOVEBOX RESTART . . . . . . . . . . 82

ATTACHMENT 10 - TEST ACCEPTANCE SIGNATURE RECORD . . . . . . . . . 91 


\subsection{PURPOSE}

1.1 This procedure has been prepared to verify that the Low Level Waste (LLW) Process Glovebox line operates in accordance with system design and specifications. This procedure will also validate portions of the WRAP-1 plant operating procedures listed in Section 7.2.

\subsection{SCOPE}

2.1 This Operating Test will demonstrate operability and waste processing functions for the following system components, collectively referred to as the "LLW GToveboxes":

LLW Entry Glovebox, (107-GB-07-101)

LLW Sorting Glovebox, (107-GB-07-102)

LLW Supercompactor GTovebox, (107-GB-07-104)

LLW Exit Glovebox, (107-GB-07-105)

2.2 Other interfacing systems outside the LLW Glovebox include the Internal Transport System, the Data Management System. the Plant Control System, and the Glovebox Barcode System.

2.3 When possible, this test wi 71 demonstrate al1 mechanical and control operations in manual and automatic modes. . Restart of the glovebox from an emergency shutdown will also be demonstrated.

\subsection{ACRONYMS}

3.1 AGV - Automatic Guided Vehicle.

3.2 DMS - Data Management System

3.3 HS - Hand Switch

3.4 OCS - Operating Control Station.

3.5 OIU - Operator Interface Unit

3.6 OP - Operating Procedure

3.7 OTP - Operational Test Procedure

3.8 PCS - Plant Control System

3.9 PLC - Programmable Logic Controller

3.10 RCT - Radiological Control Technician

3.11 TD - Test Director

3.12 TE - Test Engineer

3.13 MC - Maintenance Craft 
Waste Management Federal Services of Hanford, Inc.

Project $W-026$. WRAP I

Operational Test - LLW Glovebox

HNF-SD-W026-OTR-015

Rev: 0

Page 4 of 91

\subsection{PRECAUTIONS AND LIMITATIONS}

4.1 If during performance of this procedure, any of the following conditions are found:

- Any equipment malfunction which could prevent fulfillment of functional requirements.

- Personnel error or procedurat inadequacy which could prevent fulfillment of OTP procedural requirements.

THEN. IMMEDIATELY NOTIFY the TD and TE.

4.2 The TD and TE shall choose to stop work and place equipment in a safe condition based on the significance of a malfunction, error. or inadequacy.

4.3 Contact the TD and TE for additional instructions if changing plant conditions affect work or delays in work extending beyond (testing) shift.

4.4 If any waste is generated during performance of this OTP, THEN. consult Environmental Compliance Officer for specific instructions to ensure compliance with HNF and DOE environmenta? standards, , as «applikable, for correct disposal.

4.5 Comply with HNF and plant/facility specific lock and tag and over-tagging procedures, as applicable.

4.6 Measuring and Test Equipment (M\&TE), except timing devices used in the performance of this OTP, shall meet the following requirements:

- Be within its current calibration cycle as evidenced by an affixed calibration label.

- $\quad$ Be capable of the desired range.

- Have an accuracy (consistent with state-of-the-art limitations) equal to or greater than the accuracy specified in the OTP.

4.7 The equipment number and calibration information of $M$ \& TE used to perform this test shall recorded on the data sheets. 
Waste Management Federal Services of Hanford, Inc.

4.8 Timing measurements shall be made with commercially available timing devices.

4.9 A11 test data readings are to be taken and recorded for each location where the capability exists (i.e. local instrument. OIU. DMS termina1).

4.10 Al1 test data readings are to be taken and recorded for each location where the capability exists (i.e. Tocal observable instrument (LOI). operators screen (OS)).

\subsection{RESPONSIBILITIES}

5.1 Test Director:

- $\quad$ Schedules and reschedules operating tests as required.

- Notifies test participants before testing begins.

- Notifies personnel prior to testing of special safety precautions and conditions during testing.

- Schedules and conducts a daily pretest meeting with test participants.

- $\quad$ Record Data Exceptions and support OTP notes as required on the Attachment 1, TEST EXCEPTION LOG.

- Controls overall testing process and change record authorization for this OTP:

- Responsible for conducting the test, collecting data, and ensuring compliance with all OTP requirements.

- $\quad$ Ensures required changes to supporting operating procedures (OPS) are initiated upon completion of OTP testing.

5.2 Test Engineer:

- $\quad$ Provides technical support during testing.

- $\quad$ Provides programming support during testing.

- Participates in pre-test meetings, as necessary.

- $\quad$ Conducts a pre-test system wa7kdown, as necessary.

- Verifies that mechanical, electrical, and control systems are functioning correctly and can support testing.

5.3 Maintenance Craft:

- $\quad$ Provide assistance during OTP activities. 
Waste Management Federal Services of Hanford, .Inc. Project $W-026$, WRAP I

HNF-SD-W026-0TR-015

Operational Test - LLW Glovebox

Rev: 0

Page 6 of 91

5.4 Operations Personnel:

- Perform manipulations on Systems and Test Equipment.

- Start, stop and operate equipment related to OTP.

- Control and monitor parameters from Control Panels and Control Room.

- Inform Duty Operations Supervisor of conditions affecting plant stability or safety.

6.0 CHANGE CONTROL

6.1 OTP and supporting Operating Procedure (OP) administrative or editorial changes required during testing may be accommodated as redline exceptions in the test report, if these changes do not affect operating facility function, performance or safety that could compromise or influence data results. Changes to acceptance criteria, requirement changes, or changes to Cautions, Warnings, or any other safety items, and environmental instructions in the OTP or OP shall be approved by the signatories initially approving the OTP.

\subsection{REFERENCES}

7.1 The following documents were used in the preparation of, or are referenced in this procedure:

HSRCM-1. Hanford Site Radiological Control Manual

HNF-CM-5-34. Solid Waste Disposal Operations Administration

HNF-CM-5-36. SWD Internal Requirements

HNF-IP-0263-WRP1., Building Emergency P7an

HNF-CM-5-36. Chapter 1.10, Safety Manua 1

HNF-CM-5-36, Chapter 1.11. Industrial Hygiene Manual

HNF-CM-5-36, Chapter 3-5, Section 12.7

HNF-CM-5-36. Chapter 7.5. Environmental Comp 7iance Manual

HNF-CM-5-34, Section 3.1.4, Lock-out, Tag-out 
Waste Management Federal. Services of Hanford, Inc. Project. W-026. WRAP I Operational Test - LLW Glovebox

7.2 The following operating procedures (OPS) will be used to support test activities and objectives and will be validated during this 0TP:

Procedure \# Procedure Titile

WRP1-OP-0713, LLW Entry Glovebox Operation

WRP1-OP-0714, LLW Sorting Glovebox Operation

WRP1-OP-0715. LLW Supercompactor Operation

WRP1-0P-0716. LLW Exit G7ovebox Operation

WRP1-OP-0719. LLW Gloveboxes Manual Operation

WRP1-0P-0702, Restart LLW G1ovebox Operation

WRP1-0P-0706. Packet NDE Operation

NOTE

WRP1-0P-0701, WRP1-OP-0703, WRP1-OP-0704, WRP1-OP-0705 and WRP1-0P-0710 were validated in previous OTPs. They should still be redlined as necessary during this OTP to reflect any discrepancies specific to the LLW Gloveboxes.

WRP1-0P-0701, Glovebox Housekeeping

WRP1-0P-0703; Glovebox Lift Table Operation

WRP1-0P-0704, Glovebox Manipulator Operation

WRP1-0P-0705, Bagless Transfer Operation

WRP1-OP-0710, Glovebox Waste Sampling

A17 copies of the OPS used for testing shall be hand manket with the OTP number at the top of the first page and attached to the completed OTP test package.

Use of operating procedures to support the OTP wi11 provide validation of the procedures and minimize the amount of test paperwork generated.

7.3 The following procedures will be used for alarm response during OTP activities, as required:

WRP1-AR-0700, Process Area A7arm Response

WRP1-AR-1106; Process Area (Zone II). HVAC Alarm Response

WRP1-AR-1107, Glovebox HVAC Alarm Response. 
Waste Management Federal Services of Hanford, Inc. Project W-026. WRAP I

Operational Test - LLW Glovebox

HNF-SD-W026-0TR-015

Rev: 0

Page 8 of 91

7.4 The following administrative procedures control work performed in this OTP:

HSRCM-1, Hanford Site Radiological Control Manual

HNF-CM-5-34, Section 1.9, Quality Assurance Program Plan

HNF-CM-5-34, Section 3.1.4, Lock-out. Tag-out

HNF-CM-5-36, Chapter 1.10, Safety Manual

HNF-CM-5-36, Chapter 1.11, Industrial Hyqiene Manual

HNF-CM-5-36. Chapter 4-2, Quality Assurance Manual

HNF-CM-5-36, Chapter 7-5, Environmental Compliance Manual

HNF-IP-0263-WRP1. Building Emergency Plan

\subsection{SAFETY}

8.1 WARNING - In addition to construction and/or chemical, electrical, and oil contamination hazards. operators should be aware of the possibility of coming into contact with biological hazards.

8.2 The gloveboxes are equipped with dry chemical fire suppression systems which will discharge into the glovebox if the interior temperature at the top of the gloveboxes rises to $-190^{\circ} \mathrm{F}$.

8.3 Use care to minimize injury. Possible safety hazards include:

- Tripping / Slipping

- Injuries to the hands/head

- $\quad$ Automatic Guided Vehicle traffic.

8.4 RADIATION - CONTAMINATION CONTROL - WRAP 1 is a new facility, no radiation contamination concerns are expected during OTP activities.

\subsection{QUALITY ASSURANCE}

9.1 No Quality Assurance witness, hold points or verifications are required in this 0TP.

\subsection{RECORDS}

10.1 This LLW Glovebox OTP complete with attachments shall be filed as a permanent test record. 
Waste Management Federal Services of Hanford. Inc. Project W-026. WRAP I

Operational Test - LLW Glovebox

HNF-SD-W026-OTR-015

Rev: 0

Page 9 of 91

\subsection{PREREQUISITES}

11.1 VERIFY barcode Tabels attached to glovebox:

LOCATION COMMAND LABELS

LLW ENTRY END, CNCL PREV READ, CNCL OPERATION, YES, NO

LLW RWMPRT END. CNCL PREV READ, CNCL OPERATION, YES, NO

LLWEEXIT END, CNCL PREV READ, CNCL OPERATION, YES, NO

LLW'PP END, CNCL PREV READ, CNCL OPERATION, YES, NO

LLW'SORT END, CNCL PREV READ, CNCL OPERATION, YES, NO

LLW_STORE1 END, CNCL PREV READ, CNCL OPERATION, YES, NO

LLW_STORE2 END, CNCL PREV READ, CNCL OPERATION. YES, NO

11.2 VERIFY LLW process glovebox Acceptance Test Procedure is complete and a11 deficiencies or exceptions arising from the ATP have been resolved and closed out. or will not affect testing.

11.3 VERIFY al1 open (ATP/FTP) items will not effect OTP performance:

- Quality Assurance Nonconformance Reports (NCRs)

- Construction Punch Lists

- - Outstanding Engineering Change Notices (ECNS)

- Startup-originated Design Change Requests (CRs)

- Test Deficiency Reports

- Master System Punch List items 
Waste Management Federal Services of Hanford, Inc. Project W-026. WRAP I

\subsection{VERIFY materials are available as follows:}

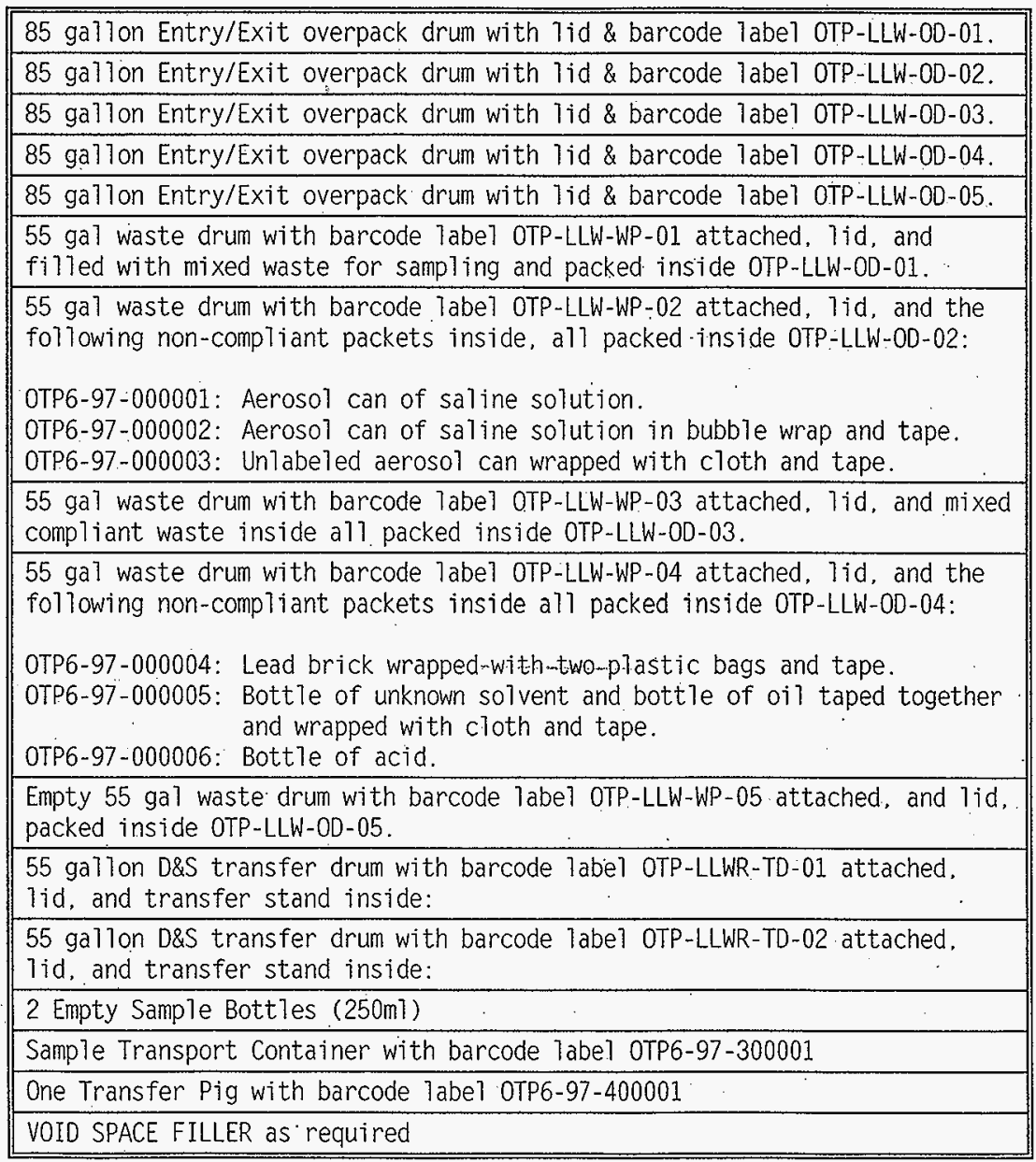

11.5 PREREQUISITES 11.1 through 11.4 complete:

TE Initials/Date:

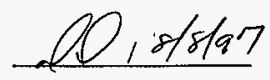


Waste Management Federal Services of Hanford, Inc.

Project W-026. WRAP I

Operational Test - LLW Glovebox

HNF-SD-W026-OTR-015

Rev: 0

Page 11 of 91

\section{NOTE \\ Steps 11.6 through 11.15 may be performed in any order.}

11.6 PERFORM a pretest briefing for all personnel involved in the OTP.

11.7 VERIFY two way portable radio communication (when necessary) between the testing and equipment locations.

11.8 VERIFY the official OTP document and all photocopies that will be used during testing are the latest approved revision.

11.9 DETERMINE appropriate interface support (personnel/equipment) from other departments and organizations and that support is available.

11.10 VERIFY personnel performing this procedure are qualified in accordance with HNF-CM-5-34, Section 1.8, Training Plan. HNF-IP-1242. WRAP 1 RGD Radiation Protection Program and on-the-job training.

11.11 VERIFY all personnel to be involved with this OTP have completed Attachment 3, SIGNATURE RECORD.

11.12 VERIFY al1 required LLW process glovebox components are installed and operational.

11.13 VERIFY a11 permanently installed and portable instrumentation listed in the applicable operating procedures and required for testing, is. within it's current calibration cycle.

11.14 VERIFY the DMS, PCS and OIUS for the LLW process glovebox are available and operational.

11.15 VERIFY process area HVAC and glovebox alarms are activated.

11.16 PREREQUISITES 11.6 through 11.15 complete:

To Initials/Date:

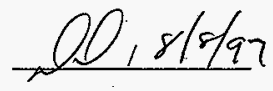


Waste Management Federal Services of Hanford, Inc.

Project $W-026$, WRAP I

Operational Test - LLW Glovebox

HNF-SD-W026-0TR-015

Rev: 0

Page 12 of 91

\subsection{PROCEDURE}

12.1 PERFORM Manual Operation test of the LLW Glovebox per Attachment 4 , TEST PLAN. LLW GLOVEBOX MANUAL OPERATION.

12.2 PERFORM Automatic Operation test of the LLW Glovebox for Test Case 1 per Attachment 5. TEST PLAN, LLW GLOVEBOX AUTOMATIC TEST CASE 1.

12.3 PERFORM Automatic Operation test of the LLW Glovebox for Test Case 2 per Attachment 6. TEST PLAN, LLW GLOVEBOX AUTOMATIC TEST CASE 2.

12.4 PERFORM Automatic Operation test of the LLW Glovebox for Test Case 3 per Attachment 7. TEST PLAN, LLW GLOVEBOX AUTOMATIC TEST CASE 3.

12.5 PERFORM Automatic Operation test of the LLW GTovebox for Test Case 4 per Attachment 8. TEST PLAN, LLW GLOVEBOX AUTOMATIC TEST CASE 4.

12.6 PERFORM Restart test of the LLW Glovebox per Attachment 9. TEST PLAN, LLW GLOVEBOX RESTART. 
Waste Management Federal Services of Hanford, Inc. Project $W-026$. WRAP I

\subsection{ACCEPTANCE CRITERIA}

13.1 Test results of each section will be considered acceptable if the following conditions are met:

13.1.1 The procedure test section was performed as written with expected results $\underline{O R}$ :

13.1.1.1 For editorial changes:

- The procedure was redined to reflect field conditions and the section in question performed as written with expected results.

- Redline and changes shall be in accordance with Section 6 . Change Control.

13.1.1.2 For changes other than editorial:

- Exceptions are documented on Attachment 2. Test Exception Report, and the Disposition Section is approved by the Test Engineer.

- Each Test. Exception Report is logged in Attachment 1. Test Exception Log.

- $\quad$ The Test Director sha11 review Attachment 1 . Test Exception Log, and ensure timely completion of all Disposition Actions and Retest Requirements. 
Waste Management Federa] Services of Hanford. Inc. Project $W-026$. WRAP I

Operational Test - LLW Glovebox

HNF-SD-W026-OTR-015

Rev: 0

Page 14 of 91

ATTACHMENT 1 - TEST EXCEPTION LOG FORM

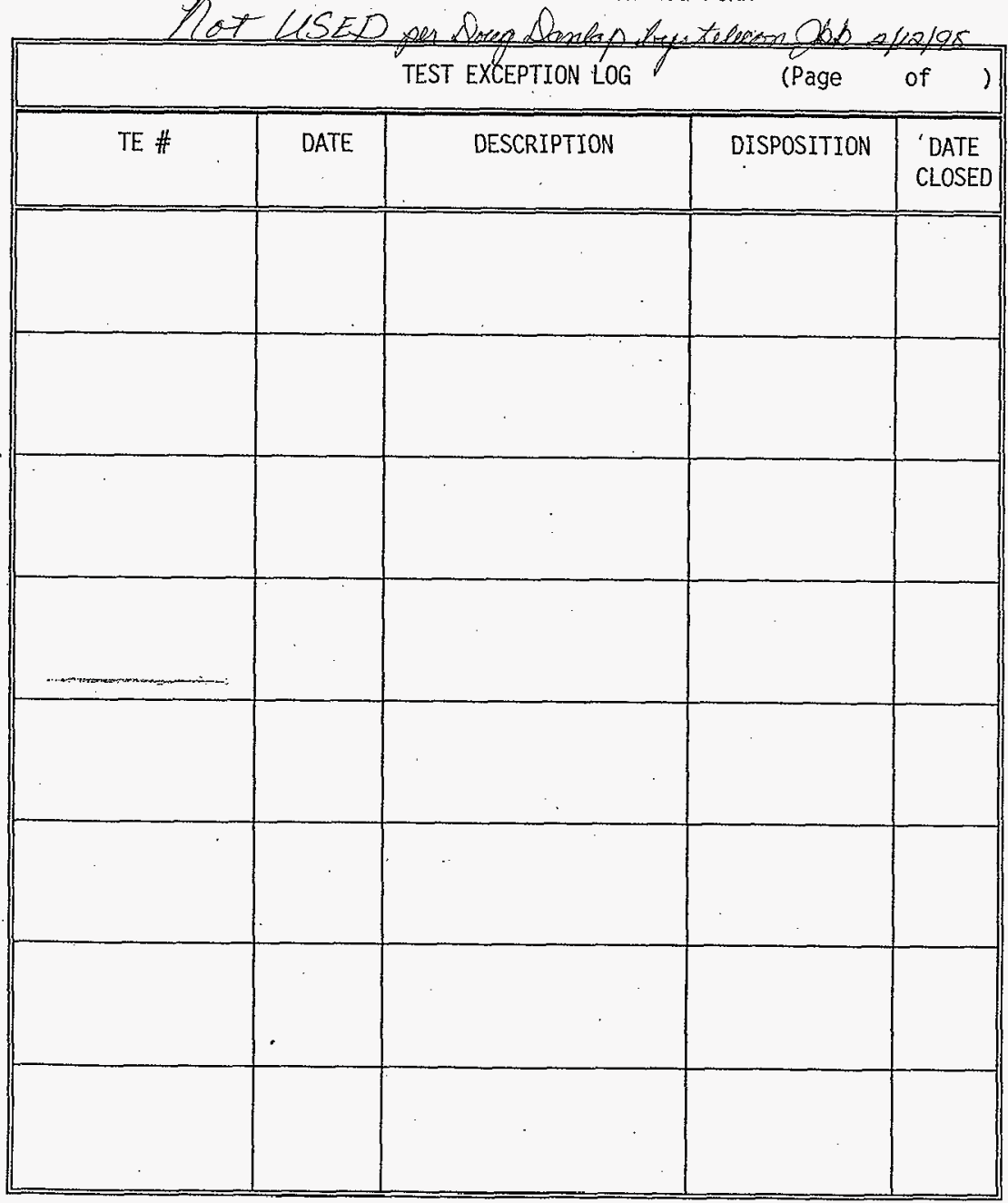



Waste Management Federal Services of Hanford, Inc. Project $W-026$, WRAP I

Operational Test - LLW Glovebox

HNF-SD-W026-0TR-015

Rev: 0

Page 16 of 91

\section{ATTACHMENT 3 - SIGNATURE RECORD}

\section{LOW LEVEL WASTE/RADWASTE MANAGEMENT GLOVEBOX OPERATIONAL TEST}

SIGNATURE RECORD: By signing below, I attest that I am aware of and understand my duties and responsibilities as described in the OTP and as assigned by the TO or TE.

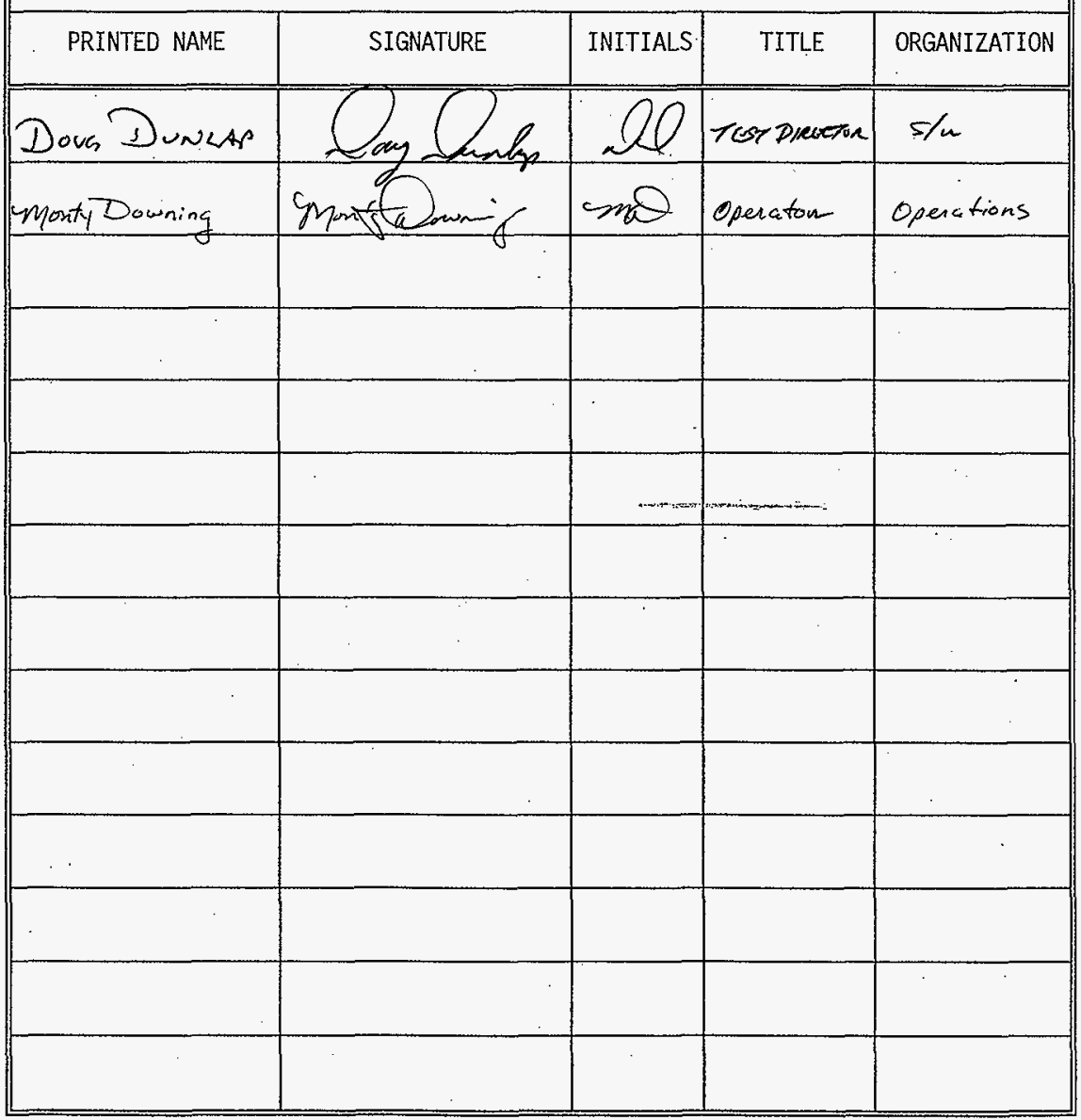


Waste Management Federai Services Of Hanford, Inc. Project $W-026$. WRAP I

ATTACHMENT 4 - TEST PLAN, LLW GLOVEBOX MANUAL OPERATION

\subsection{GENERAL DESCRIPTION}

1.1. This test plan will verify that each manual function of the LLW glovebox components may be individually operated. These operations wi 11 be tested using procedures WRP1-0P-0719. Low Level Waste G7ovebox Line Manual Operation and WRP1-OP-0715. LLW Supercompactor Operation. thereby also performing the required procedure validation for sections performed.

\subsection{SAFETY}

\subsection{Reference OTP Section 8. SAFETY.}

3.0 TOOLS, EQUIPMENT AND SUPPLIES

- Drum handling (manual) equipment or Pallet fork truck

- Spill Kit/Decontamination Equipment (oils/chemicals)

- Tape and rags

4.0. TEST PREREQUISITES

4.1 VERIFY OTP Section 11.0. PREREQUISITES are complete.

4.2 VERIFY PCS and DMS are ready to support testing.

4.3 TEST PREREQUISITES are complete and acceptable.

To Initials/Date:

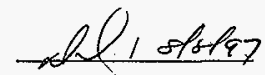


Waste Management Federal Services of Hanford, Inc.

Project $W-026$, WRAP I

Operational Test - LLW GTovebox

HNF-SD-W026-0TR-015

Rev: 0

Page 18 of 91

\section{ATTACHMENT 4 - TEST PLAN, LLW GLOVEBOX MANUAL OPERATION}

\subsection{PROCEDURE}

5.1 REFERENCE procedure WRP1-OP-0719, LOW LEVEL WASTE GLOVEBOX LINE MANUAL OPERATION:

\section{NOTE}

The following sections of this procedure will be tested at a later date:

1. VII.E "MATE SINGLE DRUM TO ENTRY PORT (107-DO-07-101)"

2. VII.G "OPEN ENTRY PORT (107-D0-07-101) (SINGLE DRUM)"

3. VII.I "CLOSE ENTRY PORT (107-D0-07-101) (SINGLE DRUM)"

\section{NOTE}

Sections VII.A thru VII.L of WRP1-OP-0719 test the manual functions of the LLW Entry Glovebox (107-GB-07-101).

5.1.1 PERFORM Section VII.A, "INITIALIZE ENTRY GLOVEBOX", of operating procedure as follows:

5.1.1.1 REDLINE procedure as needed during performance.

5.1.1.2 VERIFY results of performance are acceptable.

TD Initials/Date:

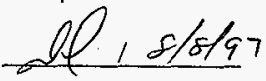

5.1.2 PERFORM Section VII.8. "ACCESS ENTRY GLOVEBOX MAINTENANCE MENUS", of operating procedure as follows:

5.1.2.1 REDLINE procedure as needed during performance.

5.1.2.2 VERIFY results of performance are acceptable.

TD Initials/Date:

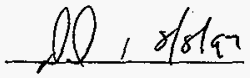


Waste Management Federal Services of Hanford, Inc. Project $W-026$, WRAP I Operational Test - LLW Glovebox
HNF-SD-W026-OTR-015

Rev: 0

Page 19 of 91

ATTACHMENT 4 - TEST PLAN, LLW GLOVEBOX MANUAL OPERATION

5.1.3 PERFORM Section VII.C, "OPERATE ENTRY GLOVEBOXX HOIST (107-CR-07-101A)". of operating procedure as follows:

5.1.3.1 REDLINE procedure as needed during performance.

5.1.3.2 VERIFY results of performance are acceptable.

TD Initials/Date:

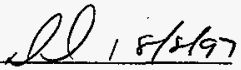

5.1.4 PERFORM Section VII.D. "MATE OVERPACK DRUM TO ENTRY PORT (107-D0-07-101)", of operating procedure as follows:

5.1.4.1 REDLINE.procedure as needed during performance.

5.1.4.2 VERIFY results of performance are acceptable.

TO Initials/Date:

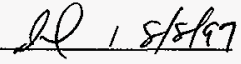

5.1.5 PERFORM Section VII.F, "OPEN ENTRY' PORT (107-00-07-101) (OVERPACK DRUM)", of operating procedure as follows:

5.1.5.1 REDLINE procedure as needed during performance.

5.1.5.2 VERIFY results of performance are acceptable.

TD Initials/Date:

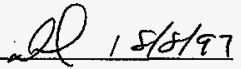

5.1.6 PERFORM Section VII.H, "CLOSE ENTRY PORT (107-D0-07-101) (OVERPACK DRUM)" , of operating procedure as follows:

5.1.6.1 REDLINE procedure as needed during performance.

5.1.6.2 VERIFY results of performance are acceptable.

TD Initials/Date:

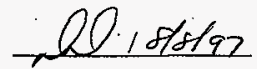


Waste Management Federal Services of Hanford, Inc. Project $W-026$. WRAP I

Operational Test - LLW Glovebox
HNF-SD-W026-OTR-015

Rev: 0

Page 20 of 91

ATTACHMENT 4 - TEST PLAN, LLW GLOVEBOX MANUAL OPERATION

5.1.7 PERFORM Section VII.J; "REMOVE OVERPACK DRUM FROM ENTRY PORT (107-D0-07-101)", of operating procedure as follows:

5.1.7.1 REDLINE procedure as needed during performance.

5.1.7.2 VERIFY results of performance are acceptable.

TO Initials/Date:

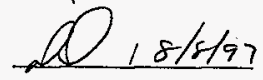

5.1.8 PERFORM Section VII.K, "OPEN TRANSFER PORT (107-D0-07-102A)", of operating procedure as follows:

5.1.8.1 REDLINE procedure as needed during performance.

5.1.8.2 VERIFY results of performance are acceptable.

TD Initials/Date:

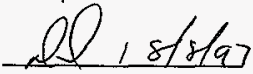

5.1.9 PERFORM Section VII.L, "CLOSE TRANSFER PORT (107-GB-07-102)", of operating procedure as follows:

5.1.9.1 REDLINE procedure as needed during performance.

5.1.9.2 VERIFY results of performance are acceptable.

TD Initials/Date:

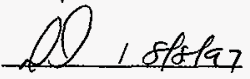

\section{NOTE}

Sections VIII.A thru VIII.O of WRP1-OP-0719 test the manual functions of the LLW Sorting Glovebox (107-GB-07-102).

5.1.10 PERFORM Section VIII.A. "INITIALIZE SORTING GLOVEBOX" of operating procedure as follows:

5.1.10.1 REDLINE procedure as needed during performance.

5.1.10.2 VERIFY results of performance are acceptable.

TD Initials/Date:

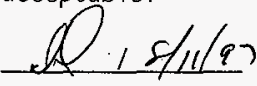




\section{ATTACHMENT 4 - TEST PLAN, LLW GLOVEBOX MANUAL OPERATION}

5.1.11 PERFORM Section VIII.B, "ACCESS SORTING GLOVEBOX MAINTENANCE MENUS", of operating procedure as follows:

5.1.11.1 REDLINE procedure as needed during performance.

5.1.11.2 VERIFY results of performance are acceptable.

TD Initials/Date:

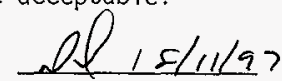

5.1.12 PERFORM Section VIII.C, "OPERATE SORTING GLOVEBOX HOIST (107-CR-07-101C)", of operating procedure as follows:

\subsubsection{REDLINE procedure as needed during performance.}

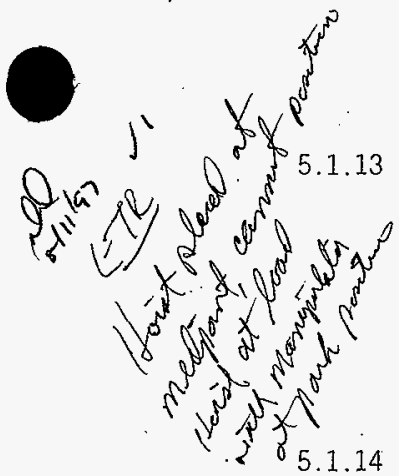

5.1.12.2 VERIFY results of performance are acceptable.

TD Initials/Date:

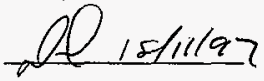

PERFORM Section VIII.D, "OPEN/CLOSE BAGLESS TRANSFER PORT (107-D0-07-105)", of operating procedure as follows:

5.1.13.1 REDLINE procedure âs needed during performance.

5.1.13.2 VERIFY results of performance are acceptable.

TD Initials/Date:

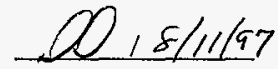

PERFORM Section VIII.E, "MOVE DRUM TRANSFER CAR (107-TC-07-101)", of operating procedure as follows:

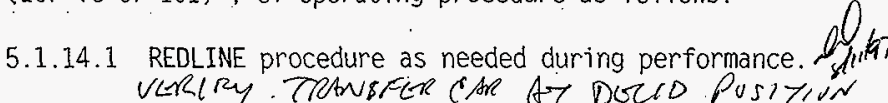

5.1.14.2 VERIFY results of performance are acceptable.

TD Initials/Date:

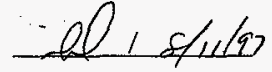


Waste Management Federal Services of Hanford, Inc.

\section{Project $W-026$. WRAP I}

Operational Test - LLW Glovebox

HNF-SD-W026-0TR-015

Rev: 0

Page 22 of 91

\section{pis $\hat{s}$ s ATTACHMENT 4 - TEST PLAN, LLW GLOVEBOX MANUAL OPERATION}

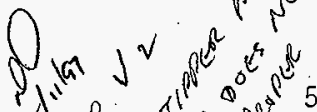

ANA

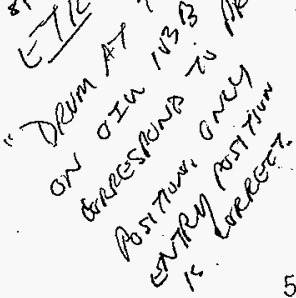

5.1.15 PERFORM Section VIII.F. "MOVE OR ROTATE DRLM LID/DELID FIXTURE (107-RT-07-101) of operating procedure as follows:

VIII.L - cower TIPPER Dl slilla]

5.1.15.1 REDLINE procedure as needed during performance.

5.1.15.2 VERIFY resu7ts of performance are acceptable.

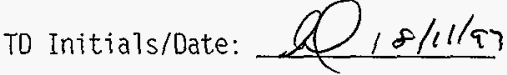

5.1.16 PERFORM Section VIII.G, "OPERATE LID/DELID SAW", of operating procedure as follows:

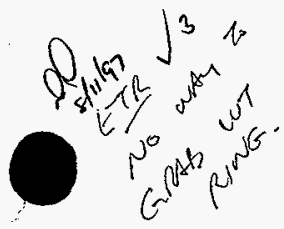

5.1.16.1 REDLINE procedure as needed during performance.

5.1.16.2 VERIFY results of performance are acceptable.

TD Initials/Date:

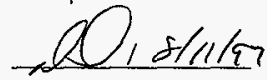

5.1.17 PERFORM Section VIII.H, "DEL ID WASTE DRUM", of operating procedure as follows:

5.1.17.1 REDLINE procedure as needed during performance.

5.1.17.2 VERIFY results of performance are acceptable.

TD Initials/Date:

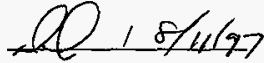

5.1.18 PERFORM Section VIII.I. "RELID WASTE DRUM", of operating procedure as follows:

5.1.18.1 REDLINE procedure as needed during performance.

5.1.18.2 VERIFY results of performance are acceptable:

TD Initials/Date:

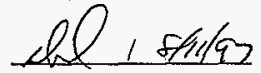


Waste Management Federal Services of Hanford. Inc. Project $W-026$. WRAP I operational Test - LLW Glovebox
HNF-SD-W026-0TR-015

Rev: 0

Page 23 of 91
Sin

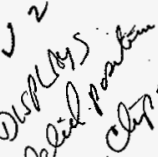

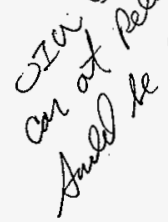

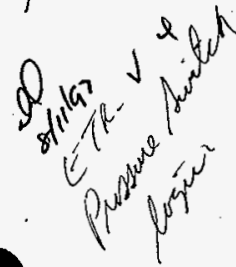

ATTACHMENT 4 - TEST PLAN, LLW GLOVEBOX MANUAL OPERATION

5.1.19 PERFORM Section VIII.J, "C-CLIP AND CRIMP LIO" , of operating procedure as follows:

5.1.19.1 REDLINE procedure as needed during performance.

5.1.19.2 VERIFY results of performance are acceptable.

TD Initials/Date:

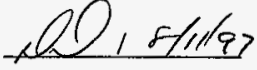

5.1.20 PERFORM Section VIII.K, "OPEN/CLOSE DRUM GRAB" of operating procedure as follows:

5.1.20.1 REDLINE procedure as needed during performance.

5.1.20.2 VERIFY results of performance are acceptable.

TD Initials/Date:

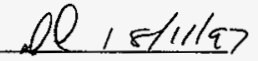

5.1.21 PERFORM Section VIII.L, "RAISE/LOWER/PARK DRUM TIPPER (107-DH-07-103)", of cperating procedure as follows:

5.1.21.1 REDLINE procedure as needed during performance.

5.1.21.2 VERIFY results of performance are acceptable.

TD Initials/Date:

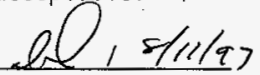

5.1.22 PERFORM Section VIII.M. "ROTATE DRUM TIPPER (107-DH-07-103)", of operating procedure as follows:

5.1.22.1 REDLINE procedure as needed during performance.

5.1.22.2 VERIFY results of performance are acceptable.

TD Initials/Date:

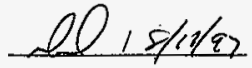


Waste Management Federa] Services of Hanford. Inc. Project W-026. WRAP I

Operational Test - LLW Glovebox

HNF-SD-W026-OTR-015

Rev: 0

Page 24 of 91

\section{ATTACHMENT 4 : TEST PLAN, LLW GLOVEBOX MANUAL OPERATION}

5.1.23 PERFORM Section VIII.N, "EXTEND/RETRACT SORTING TABLE (107-ST-07-101)", of operating procedure as follows:

5.1.23.1 REDLINE procedure as needed during performance.

5.1.23.2 VERIFY results of performance are acceptable.

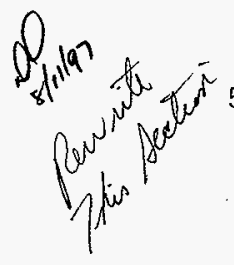

TD Initials/Date:

5.1.24 PERFORM Section VIII.0, "RAISE/LOWER SORTING TABLE

(107-ST-07-101)"., of operating procedure as follows:

5.1.24.1 REDLINE procedure as needed during performance.

5.1.24.2 VERIFY results of performance are acceptable.

TD Initials/Date: Dl , stilgr

\section{NOTE}

Sections IX.A thru IX.I of WRP1-OP-0719 test the manual functions of the LLW Exit Glovebox (107-GB-07-105).

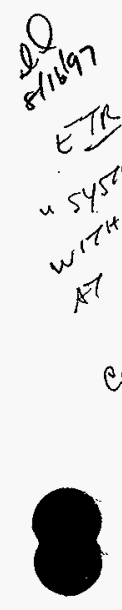

5.1.25 PERFORM Section IX.A. "INITIALIZE EXIT GLOVEBOX" of operating procedure as follows:

5.1.25.1 REDLINE procedure as needed during performance.

5.1.25.2 VERIFY results of performance are acceptable.

TD Initials/Date: $00,8 / 14 / 97$

PERFORM Section IX.B. "ACCESS EXIT GLOVEBOX MAINTENANCE MENUS", of operating procedure as follows:

5.1.26. I REDLINE procedure as needed during performance.

5.1.26.2 VERIFY results of performance are acceptable.

TO Initials/Date:

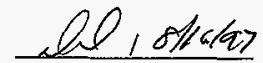


Waste Management Federal Services of Hanford. Inc. Project W-026. WRAP I operational Test - LLW Glovebox

HNF-SD-W026-0TR-015 Rev: 0

Page 25 of 91

\section{ATTACHMENT 4 - TEST PLAN, LLW GLOVEBOX MANUAL OPERATION}

5.1.27 PERFORM Section IX.C, "OPERATE SORTING GLOVEBOX HOIST (107-CR-07-1010)", of operating procedure as follows:

5.1.27.1 REDLINE procedure as needed during performance.

5.1.27.2 VERIFY results of performance are acceptable.

TD Initials/Date:

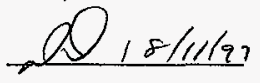

5.1.28 PERFORM Section IX.D, "MATE DRUM TO EXIT.PORT (107-D0-07-106)", of operating procedure as follows:

5.1.28.1 REDLINE procedure as needed during performance.

5.1.28.2 VERIFY results of performance are acceptable.

TD Initials/Date:

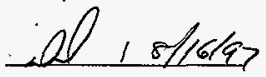

5.1.29 PERFORM Section IX.E. "OPEN EXIT PORT (107-D0-07-106)", of operating procedure as follows:

5.1:29.1 REDLINE procedure as needed during performance.

5.1.29.2 VERIFY results of performance are acceptable.

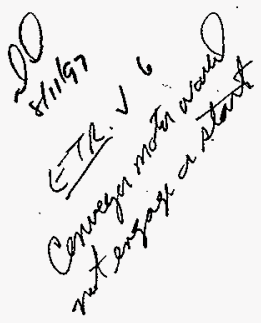

TO Initials/Date:

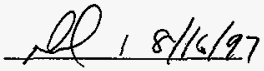

5.1.30 PERFORM Section IX.F, "OPERATE TRANSFER CAR CONVEYOR Of operating procedure as follows:

5.1.30.1 REDLINE procedure as needed during performance.

5.1.30.2 VERIFY results of performance are acceptable.

Tó Initials/Date:

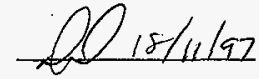


Waste Management Federal Services of Hanford, Inc. Project $W-026$. WRAP I

Operational Test - LLW GTovebox
HNF-SD-W026-0TR-015

Rev: 0

Page 26 of 91

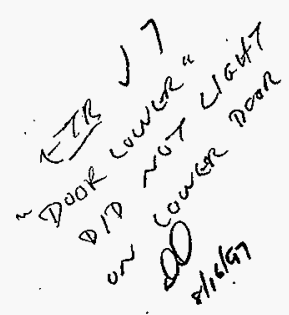

ATTACHMENT 4 - TEST PLAN, LLW GLOVEBOX MANUAL OPERATION

5.1.31 PERFORM Section IX.G. "RELID OVERPACK DRUM \& CLOSE EXIT PORT (107-D0-07-106)", of operating procedure as follows:

5.1.31.1 REDLINE procedure as needed during performance.

5.1.31.2 VERIFY results of performance are acceptable.

TD Initials/Date:

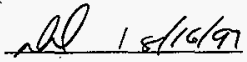

5.1.32 PERFORM Section IX.H, "REMOVE. OVERPACK DRUM FROM EXIT PORT (107-D0-07-106)", of operating procedure as follows:

5.1.32.1 REDLINE procedure as needed during performance.

5.1.32.2 VERIFY results of performance are acceptable.

TD Initials/Date:

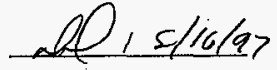

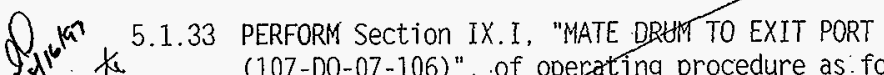

(107-D0-07-106)", of operating procedure as: follows:

5.1.33.1 REDLINE procedure as needed during performance.

5.1.33.2 VERIFY results of performance are acceptable. To Initials/Date:

5.2 REFERENCE procedure WRP1-OP-0715, LLW SUPERCOMPACTOR OPERATION:

5.2.1 PERFORM Section VII.E, "MANUAL OPERATION", of operating

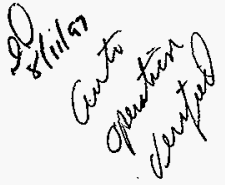
procedure as follows:

5.2.1.1 REDLINE procedure as needed during performance.

5.2.1.2 VERIFY results of performance are acceptable.

TO Initials/0ate:

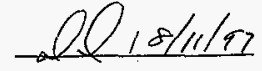


Waste Management Federa7 Services of Hanford, Inc. Project $W-026$. WRAP I Operational Test - LLW Glovebox

ATTACHMENT 5 - TEST PLAN, LLW GLOVEBOX AUTOMATIC TEST CASE 1

\subsection{GENERAL DESCRIPTION}

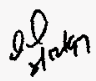

1.1 Test case one will sample LLW Test Drum 1 without compacting. These operations will be performed using procedures WRP1-OP-0710 "Glovebox Waste Sampling." WRP1-0P-0713 "LLW Entry Glovebox Operation," WRP1-OP-0714 "Low Level Waste (LLW) Sorting Glovebox Operation," WRP1-OP-0715 "LLW Supercompactor Operation" and WRP1-OP-0716 "LLW Exit Glovebox Operation," thereby also performing the required procedure validation for sections performed. The sequence of events is as follows:

\subsubsection{STC 0TP6-97-300001 is connected to the STC port.}

1.1.2 Transfer drum OTP-LLWR-TD-01 is connected to the LLW glovebox RWM bagless transfer port and the port is opened.

1.1.3 Transfer stand is moved to the sorting table.

1.1.4 85 gallon shipping overpack OTP-LLW-00-01, with 55 gallon LLW drum OTP-LLW-WP-01 inside, is connected to the LLW glovebox entry port and drum OTP-LLW-WP-01 is Tifted into the LLW Entry glovebox.

1.1.5 Drum OTP-LLW-WP-01 is checked for external contamination.

1.1.6 85 galion drum OTP-LLW-00-01 is closed and removed from the LLW Entry port and from lift table LT-09-202A.

1.1.7 Empty 85 gallon drum OTP-LLW-OD-01 (from entry port) is placed on lift table LT-09-202C (LLW Exit).

1.1.8 Drum OTP-LLW-OD-O1 is connected to the LLW glovebox exit port and the port is opened.

1.1.9 Drum OTP-LLW-WP-01 is lifted by the LLW entry glovebox hoist, lowered through transfer port, and placed on transfer car.

1.1.10 Drum OTP-LLW-WP-01 is moved to the drum tipper, the lid is removed, and the contents are dumped on the sorting table. 
Waste Management Federal Services of Hanford, Inc.

Project W-026. WRAP I

HNF-SD-W026-0TR-015

Rev: 0

Operational Test - LLW Glovebox

Page 28 of 91

\section{ATTACHMENT 5 - TEST PLAN, LLW GLOVEBOX AUTOMATIC TEST CASE 1}

1.1.11 A sample analysis request is prepared for drum OTP-LLW-WP-01. Sample 97-00001 is requested with analyses as directed by Sampling Representative. One bottle is required for each analysis.

1.1.12 Sample bottle barcode 7abe1s 97-00001-01L and 97-00001-02L are printed in the sample management office and the labels are placed on empty sample bottles.

1.1.13 Empty sample bott7es 97-00001-01L and 97-00001-02L are loaded into the LLW glovebox through the Consumable Materials port.

1.1.14 A soil sample is collected in sample bottles 97-00001-01L and 97-00001-02L.

1.1:15 Sample bottles 97-00001-01L and 97-00001-02L are placed in STC 0TP6-97-300001.

1.1.16 STC 0TP6-97-300001 is removed from the LLW STC port and placed in transfer pig 0TP6-97-400001.

1.1.17 The waste on the sorting table is loaded back into drum OTP-LLW-WP-01, the drum is placed on the transfer car, the drum is relidded, and the transfer car with the drum is moved to the compactor.

1.1.18 Drum OTP-LLW-WP-01 is moved to the exit glovebox without being compacted. "Puck" bar code label 0TP001 is placed on the drum and the barcode is read. The drum is then lifted by the hoist and the drum is weighed on the hoist scale. The ID and weight are sent to the DMS.

1.1.19 The waste drum is loaded into the overpack at the exit port.

1.1.20 Overpack drum OTP-LLW-OD-01 is (1) closed, (2) removed from the LLW glovebox exit port. (3) surveyed, and (4) removed from 7 ift table LT-09-202C.

\subsection{SAFETY}

\subsection{Reference OTP Section 8, SAFETY.}


Waste Management Federal Services of Hanford, Inc. Project $W-026$. WRAP I

Operational Test - LLW Glovebox

HNF-SD-W026-OTR-015

Rev: 0

Page 29 of 91

\section{ATTACHMENT 5 - TEST PLAN, LLW GLOVEBOX AUTOMATIC TEST CASE 1}

\subsection{TOOLS, EQUIPMENT AND SUPPLIES}

- Drum handling (manual) equipment or Pallet fork truck.

- $\quad$ Spil1 Kit/Decontamination Equipment (oils/chemicals)

- Tape and rags

- $\quad$ ther equipment, material or protective clothing (as required)

\subsection{TEST PREREQUISITES}

4.1 VERIFY OTP Section 11.0. PREREQUISITES are complete.

4.2 SET initial conditions as follows:

4.2.1 55 gallon LLW drum 0TP-LLW-WP-01, in shipping overpack OTP-LLW-00-01, is on lift table LT-09-202A.

4.2.2 The PCS man_op program has sent an AGVPCS AGVL message for the AGV located at AGVLLWENTR (LT-09-202A) with drum OTP-LLW-0D-01 (sets LCU conditions have been set for drum OTP-LLW-0D-01 on lift table LT-09-202A).

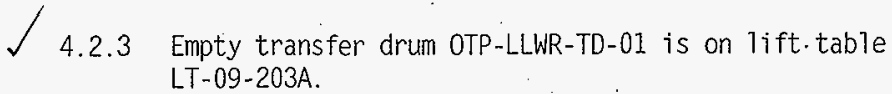

$\int$ 4.2.4 The PCS man op program has sent an AGVPCS AGVL message for the AGV located at AGVLLWRWX (LT-09-203A) with drum OTP-LLWR-TD-01 (sets LCU conditions for drum OTP-LLWR-TD-01 on lift table LT-09-203A).

\section{$\sqrt{4.2 .5}$ Empty sample transport container (STC) 0TP6-97-300001 is at} the $L L W$ glovebox ready to connect to the STC port.

$\longrightarrow$ 4.2.6 Transfer pig 0TP6-97-400001 is 1ocated near the LLW STC port. $\sqrt{4.2 .7}$ Sequentially numbered puck barcode labels are available in the exit glovebox.

4.2.8 Required sampling supplies are available in the glovebox..

4.2.9 Two empty unlabeled $250 \mathrm{ml}$ sample bottles are in the sample management office. 


\section{ATTACHMENT 5 - TEST PLAN, LLW GLOVEBOX AUTOMATIC TEST CASE 1}

$\sqrt{4.2 .10}$ A user with general operator (GENOP) privileges is logged on at DMS LLW Terminal (107-TE-12-104)

$\sqrt{4.2 .11}$ A user with chemist privileges is logged on at DMS Sample Management office Terminal (109-TE-12-107).

4:2.12 The sample-label barcode printer in the sample management office is loaded with blank labels and ready to print.

$\int 4.2 .13$ Test scripts have been run on the DMS server to:

$\checkmark$ 4.2.13.1 Initialize the DMS database.

$\checkmark$ 4.2.13.2 Load initial data for PINs:

PINS OTP-LLW-00-01, 0TP-LLW-00-02,

OTP-LLW-00-03, OTP-LLW-00-04,

OTP-LLW-WP-01, OTP-LLW-WP-02,

OTP-LLW-WP-03, OTP-LLW-WP-04,

OTP-LLWR-TD-01, OTP-LLWR-TD-02.

0TP6-97-300001, and OTP6-97-400001.

4.2.13.3 Reset. screen DMSS0504 sequential sample number to zero. (The first sample number assigned by screen DMSS0504 will be 0TP00001.)

4.2.13.4 Print report of DMS database: 
Waste Management Federal Services of Hanford, Inc.

Project $W-026$, WRAP I

Operational Test - LLW GTovebox

HNF-SD-W026-OTR-015

Rev: 0

Page 31 of 91

ATTACHMENT 5 - TEST PLAN, LLW GLOVEBOX AUTOMATIC TEST CASE 1

\subsubsection{VERIFY conditions:}

$\checkmark$ 4.2.14.1 Port 107-D0-07-101, CLOSED and LOCKED.

4.2.14.2 Port 107-D0-07-105, CLOSED and LOCKED.

$\checkmark$ 4.2.14.3 Lift Table 107-LT-09-202A at AGV height.

$\sqrt{ }$ 4.2.14.4 Lift Table 107-LT-09-202A. MAINTENANCE/OFF/NORMAL $(M / O / N)$ switch in NORMAL.

$\checkmark$ 4.2.14.5 Lift Table 107-LT-09-203A at AGV height.

4.2.14.6 Lift Table 107-LT-09-203A, MAINTENANCE/OFF/NORMAL (M/O/N) switch in NORMAL.

$\sqrt{ }$ 4.2.14.7 Manipulator 107-EM-07-101 PARKED.

4.3 UPDATE PCS Tracking Table as follows:

$\sqrt{4.3 .1}$ Drum OTP-LLW-WP-01 in shipping overpack OTP-LLW-0D-01 at LT- $09-202 \mathrm{~A}$.

$\sqrt{4.3 .2}$ Drum OTP-LLWR-TD-01 at LT-09-203A.

\begin{tabular}{|c|c|c|}
\hline \multicolumn{3}{|c|}{ PCS TRACKING TABLE FOR LLW LIFT TABLES } \\
\hline \multirow{2}{*}{ LIFT TABLE } & \multicolumn{2}{|c|}{ Registers } \\
\cline { 2 - 4 } & PIN & Drum Present $(=1)$ \\
\hline LT-09-202A & $-N 32: 35-41$ & N32: 46 bit 12 \\
\hline LT.09-203A & $-N 32: 47-53$ & N32: 58 bit 12 \\
\hline
\end{tabular}

4.4 TEST PREREQUISITES are comp Tete and acceptable.

TD Initials/Date: 
Waste Management Federal Services of Hanford, Inc.

Project $W-026$. WRAP I

operational Test - LLW Glovebox

HNF-SD-W026-OTR-015

Rev: 0

Page 32 of 91

ATTACHMENT 5 - TEST PLAN, LLW GLOVEBOX AUTOMATIC TEST CASE 1

\subsection{PROCEDURE}

5.1 REFERENCE procedure WRP1-OP-0710, GLOVEBOX WASTE SAMPLING:

5.1.1 PERFORM Section VII.D.1 "ATTACH STC TO GLOVEBOX", of operating procedure as required to attach empty STC-1 to gTovebox:

5.1.1.1 REDLINE procedure as needed during performance.

5.1.1.2 VERIFY results of performance are acceptable.

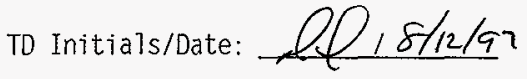

5.2 REFERENCE procedure WRP1-OP-0714, LOW LEVEL WASTE (LLW) SORTING

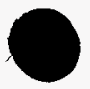

$\checkmark \quad \&$ S GLOVEBOX OPERATION:

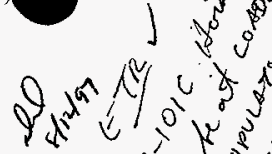

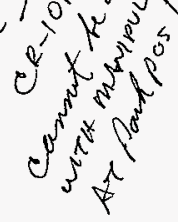

5.2 .1

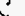

5.2.2 PERFORM Section VII.N. "MATE DRUM TO RWM TRANSFER PORT

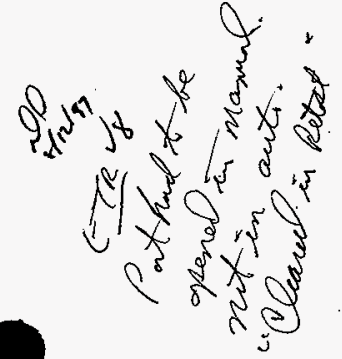

PERFORM Section VII.A, "INITIALIZE SYSTEM", of operating procedure as follows:

5.2.1.1 REDLINE procedure as needed during performance.

5.2.1.2 VERIFY results of performance are acceptable.

TD Initials/Date: 10 /8/12/a

(107-00-07-105)", of operating procedure as follows:

5.2.2.1 REDLINE procedure as needed during performance.

5.2.2.2 VERIFY drum OTP-LLWR-TD-01 connected to port 107-D0-07-105 and port OPEN.

5.2.2.3 VERIFY results of performance are acceptable.

To Initials/Date:

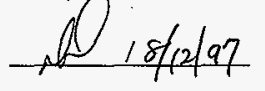


ATTACHMENT 5 - TEST PLAN, LLW GLOVEBOX AUTOMATIC TEST CASE 1

5.2.3 PERFORM Section VII.0, "REMOVE TRANSFER STAND FROM RWM TRANSFER DRUM", of operating procedure as follows:

5.2.3.1 REDLINE procedure aș needed during performance.

5.2.3.2 VERIFY transfer stand at. glovebox sorting table.

5.2.3.3 VERIFY results of performance are acceptable.

TD Initials/0ate:

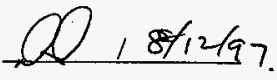

5.3 REFERENCE procedure WRP1-OP-0713, LLW ENTRY GLOVEBOX OPERATION:

5.3.1 PERFORM Section VII.A, "INITIALIZE SYSTEM", of operating procedure as follows:

5.3.1.1 REDLINE procedure as needed during performance.

5.3.1.2 VERIFY results of performance are acceptable.

TD Initials/Date:
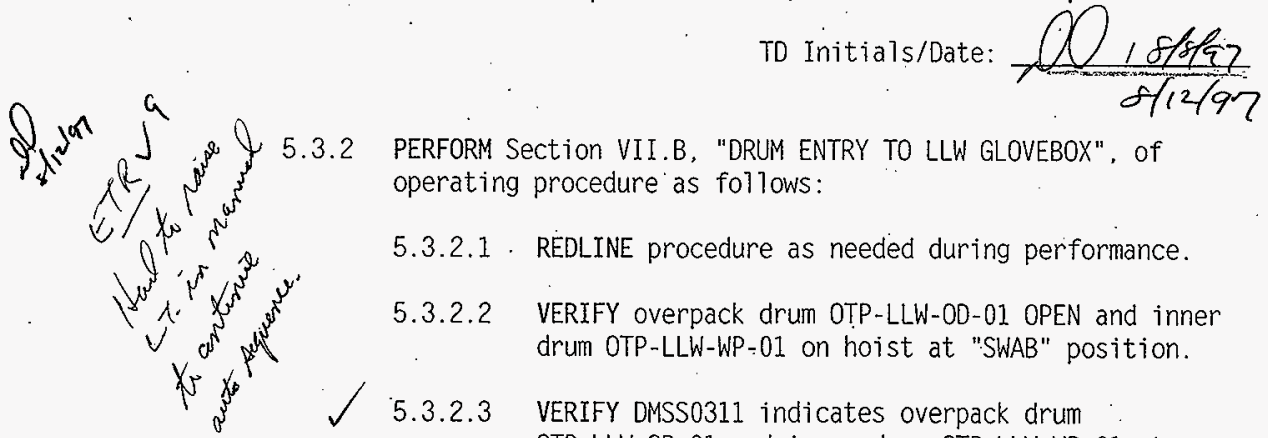

PERFORM Section VII.B, "DRUM ENTRY TO LLW GLOVEBOX", of operating procedure as follows:

5.3.2.1 REDLINE procedure as needed during performance.

5.3.2.2 VERIFY overpack drum OTP-LLW-00-01 OPEN and inner drum OTP-LLW-WP-01 on hoist at "SWAB" position.

5.3.2.3 VERIFY DMSS0311 indicates overpack drum OTP-LLW-0D-01 and inner drum. OTP-LLW-WP-01 at glovebox with message "Sorting Required".

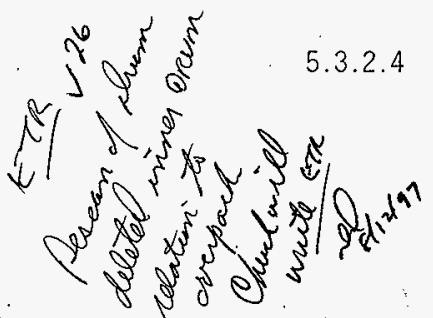

VERIFY results of performance are acceptable.

TD Initials/Date:

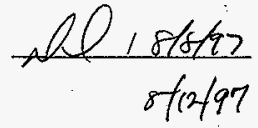




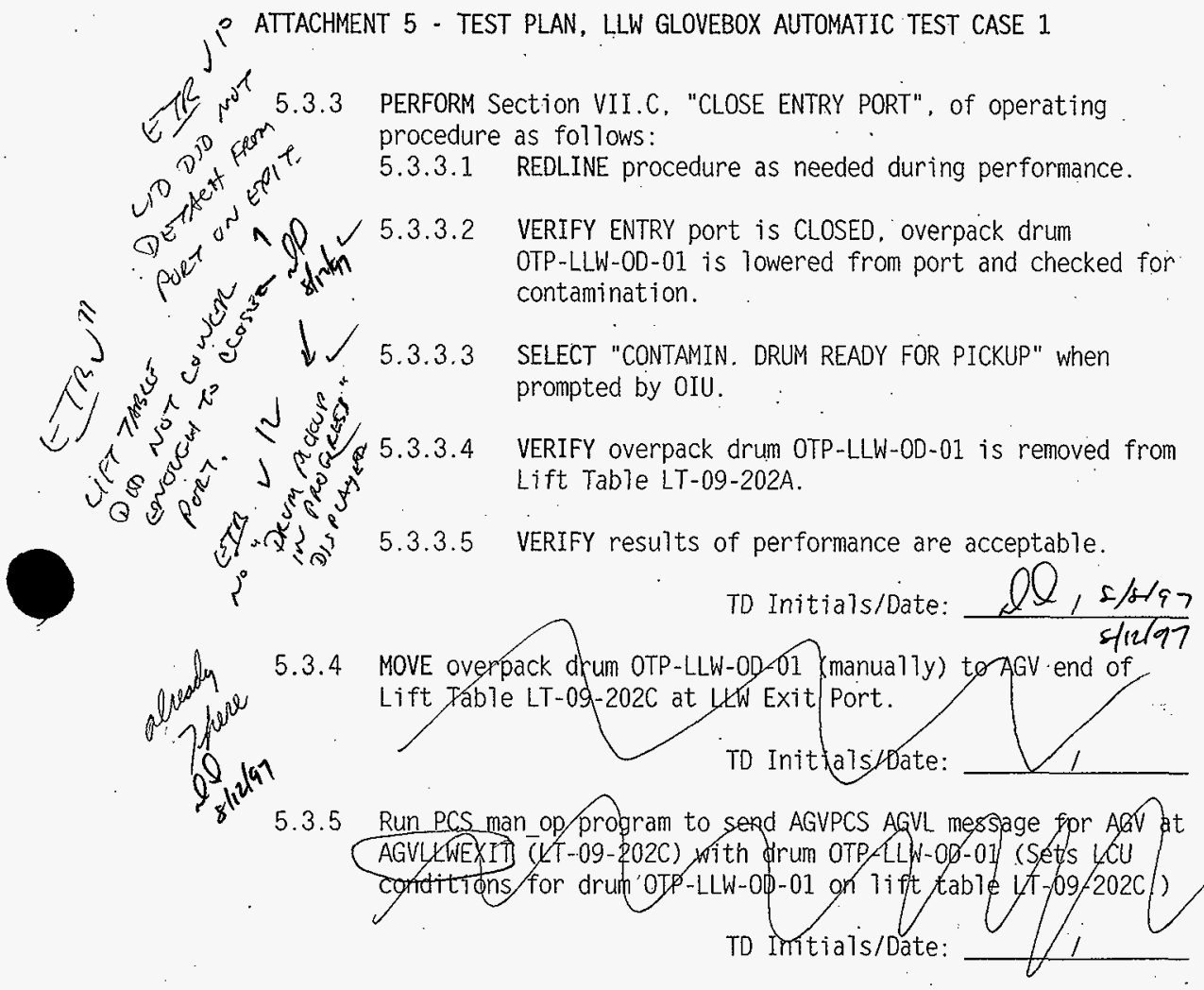

5.4 REFERENCE procedure WRP1-OP-0716. LLW EXIT GLOVEBOX OPERATION:

5.4.1 PERFORM Section VII.A. "INITIALIZE SYSTEM", of operating procedure as follows:

5.4.1.1 REDLINE procedure as needed during performance.

5.4.1.2 VERIFY results of performance are acceptable. 
Waste Management Federal Services of Hanford. Inc. Project $W-026$. WRAP I

Operational Test - LLW GTovebox

HNF-SD-W026-OTR-015

Rev: 0

Page 35 of 91

ATTACHMENT 5 - TEST PLAN, LLW GLOVEBOX AUTOMATIC TEST CASE 1

5.4.2 PERFORM Section VII.B, "EXIT DRUM ACCESS TO LLW GLOVEBOX", of operating procedure as follows:

5.4.2.1 REDLINE procedure as needed during performance.

5.4.2.2 VERIFY overpack drum OTP-LLW-OD-01 connected to LLW Exit Port and OPEN.

5.4.2.3 VERIFY results of performance are acceptable.

To Initials/Date:

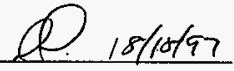

5.5 REFERENCE procedure WRP1-OP-0713, LLW ENTRY GLOVEBOX OPERATION:

5.5.1 PERFORM Section VII.D, "MOVE DRUM TO DRUM TRANSFER CAR". of operating procedure as follows:

5.5.1.1 REDLINE procedure as needed during performance.

$\sqrt{5.5 .1 .2}$ VERIFY drum OTP-LLW-WP-01 is positioned over transfer port 107-D0-07-102 and lowered onto drum transfer car.

5.5.1.3 VERIFY results of performance are acceptable.

TD Initials/Date:

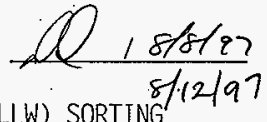

3 5.6 REFERENCE procedure WRP1-OP-0714. LOW LEVEL WASTE (LLW) SORTING GLOVEBOX OPERATION:

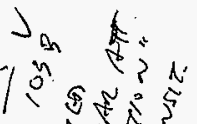

5.6.1 PERFORM Section VII.B, "TRANSFER WASTE DRUM TO DELID POSITION", of operating procedure as follows:

5.6.1.1 REDLINE procedure as needed during performance.

5.6.1.2 VERIFY drum OTP-LLW-WP-01 at "DELID" position.

5.6.1.3 VERIFY results of performance are acceptable.

TD Initials/Date:

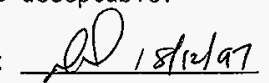




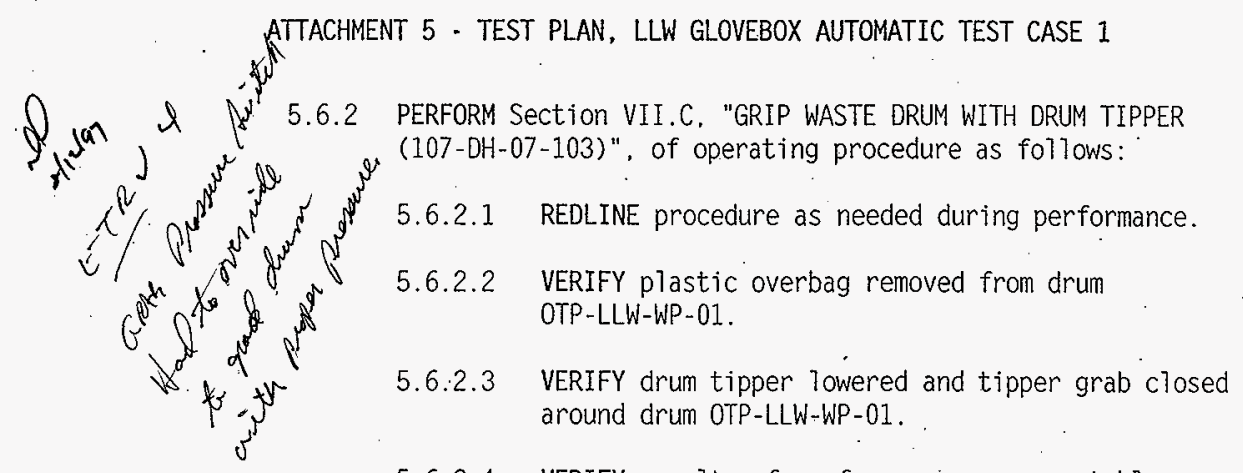

5.6.2.4 VERIFY results of performance are acceptable.
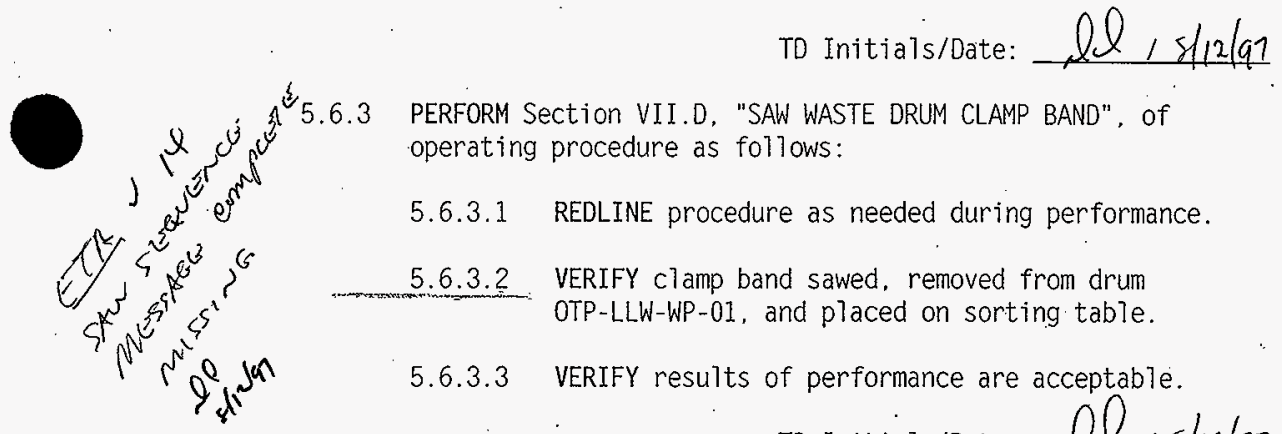

PERFORM Section VII.D. "SAW WASTE DRUM CLAMP BAND", of operating procedure as follows:

5.6.3.1 REDLINE procedure as needed during performance.

5.6.3.2 VERIFY clamp band sawed, removed from drum OTP-LLW-WP-01, and placed on sorting table.

5.6.3.3 VERIFY results of performance are acceptable.

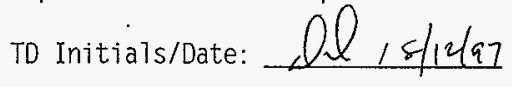

5.6.4 PERFORM Section VII.E, "DELID WASTE DRUM", of operating procedure as follows:

5.6.4.1 REDLINE procedure as needed during performance.

5.6.4.2 VERIFY delid fixture removes lid from drum OTP-LLW-WP-01, and returns to it's "raised/parked" position with the lid.

5.6.4.3 VERIFY results of performance are acceptable.

TD Initials/Date: $8 / 2 / 97$ 
Waste Management Federal Services of Hanford, Inc. Project $W-026$. WRAP I

Operational Test - LLW Glovebox

HNF-SO-W026-0TR-015

Rev: 0

Page 38 of 91

\section{ATTACHMENT 5 - TEST PLAN, LLW GLOVEBOX AUTOMATIC TEST CASE 1}

5.7 REFERENCE procedure WRP1-OP-0710, GLOVEBOX WASTE SAMPLING:

5.7.1 PERFORM Section VII.B. "PREPARING TO SAMPLE" of operating procedure as follows:

5.7.1.1 REDLINE procedure as needed during performance.

$\sqrt{5.7 .1 .2}$ VERIFY sample bottle labels OTP00001-01L and OTP00001-02L are printed using DMSS0508 and placed on sample bottles, when directed by the operating procedures.
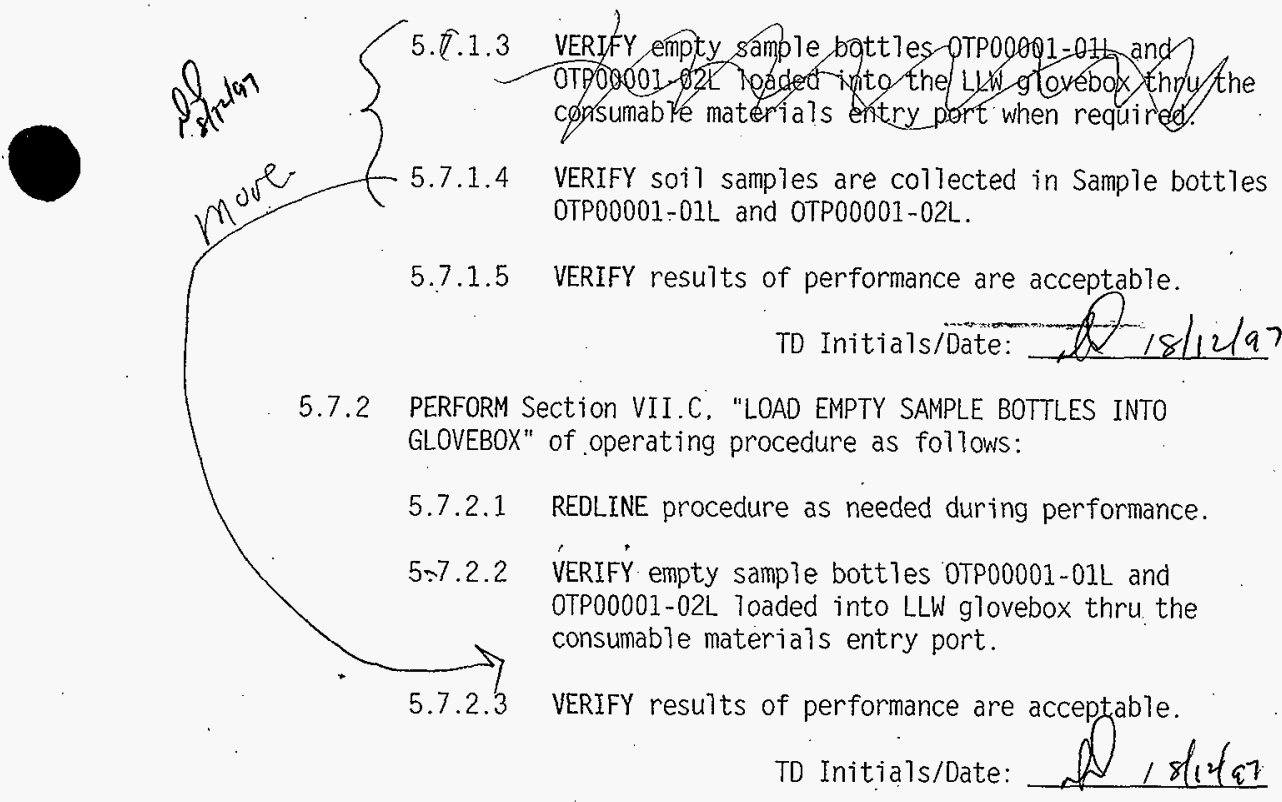
ATTACHMENT 5 - TEST PLAN, LLW GLOVEBOX AUTOMATIC TEST CASE 1

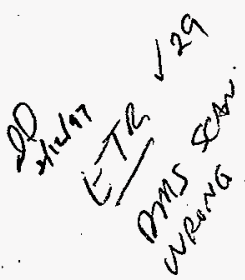

\section{F Oldivar}

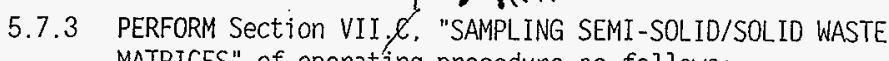
MATRICES" of operating procedure as follows:

5.7.3.1 REDLINE procedure as needed during performance.

5.7.3.2 VERIFY soil samples are collected in Sample bottles OTP00001-01L and OTP00001-02L.

5.7.3.3 VERIFY results of performance are acceptable.

To Initials/Date:

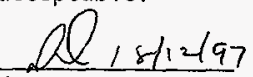

5.7.4 PERFORM Section VII.G. "FINAL SAMPLE PREPARATION AND LOADOUT", of operating procedure.

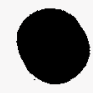

5.7.4.1 REDLINE procedure as needed during performance.

5.7.4.2 VERIFY sample bottles OTP00001-01L. OTP00001-02L are placed in STC-1.

5.7.4.3 VERIFY STC-1 removed from glovebox and placed in transfer pig 0TP5-97-400001.

5.7.4.4 VERIFY results of performance are acceptable.

TD Initials/Date:

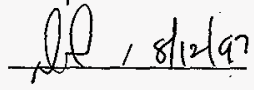

$\begin{array}{lll} & 5.8 & \text { REFERENCE procedure } \\ J & & \text { GLOVEBOX OPERATION: }\end{array}$

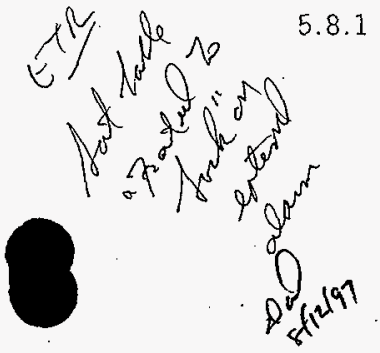

PERFORM Section VII:H, "RETURN COMPLIANT WASTE TO WASTE DRUM", of operating procedure as follows:

5.8.1.1 REDLINE procedure as needed during performance.

5.8.1.2 VERIFY results of performance are acceptable.

TD Initials/Date:

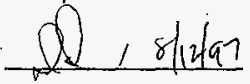




\section{ATTACHMENT 5 - TEST PLAN, LLW GLOVEBOX AUTOMATIC TEST CASE 1}
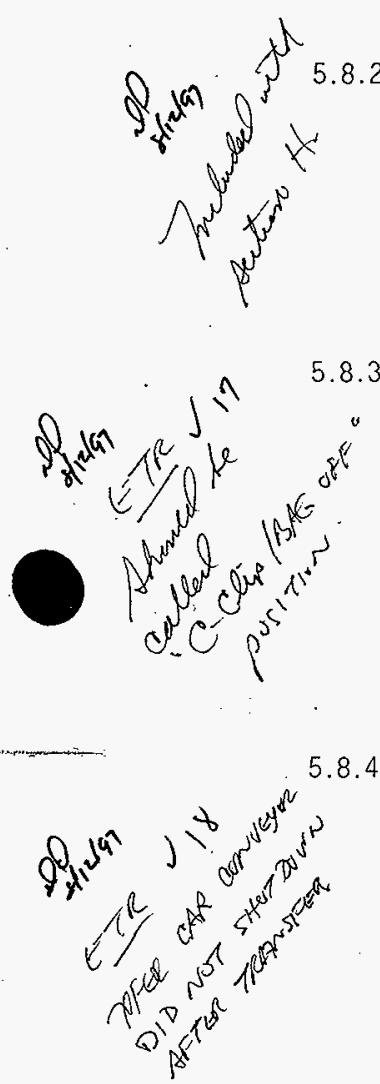

PERFORM Section VII.I. "MOVE WASTE ORUM TO RELID POSITION", of operating procedure as follows:

5.8.2.1 REDLINE procedure as needed during performance.

5.8:2.2 VERIEY results of performance are acceptable.

TD Initials/Date: 
Waste Management Federal Services of Hanford. Inc.

Project $W-026$. WRAP I

HNF-SD-W026-0TR-015

Operational Test - LLW Glovebox

Rev: 0

Page 41 of 91

ATTACHMENT 5 - TEST PLAN, LLW GLOVEBOX AUTOMATIC TEST CASE 1

5.9 REFERENCE procedure WRP1-OP-0716, LLW EXIT GLOVEBOX OPERATION:

5.9.1 PERFORM Section VII.C, "PUCK RECEIPT AND WEIGHT MEASUREMENT", of operating procedure as follows:

5.9.1.1 REDLINE procedure as needed during performance.

5.9.1.2 VERIFY puck barcode OTPO01 is placed on drum OTP-LLW-WP-01 and scanned.

5.9.1.3 VERIFY puck OTP001 is lifted and weighed on the Exit Glovebox hoist.

5.9.1.4 VERIFY results of performance are acceptable.

TD Initials/Date:

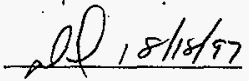

5.9.2 PERFORM Section VII.D. "MOVE PUCK FROM RECEIPT TO LOAD OUT", of operating procedure as follows:

5.9.2.1 REDLINE procedure as needed during performance.

5.9.2.2 VERIFY puck OTP001 is loaded into overpack drum OTP-LLW-OD-01 at Exit port.

5.9.2.3 VERIFY results of performance are acceptable.

To Initials/Date:

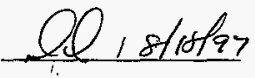




\section{ATTACHMENT 5 - TEST PLAN, LLW GLOVEBOX AUTOMATIC TEST CASE 1}

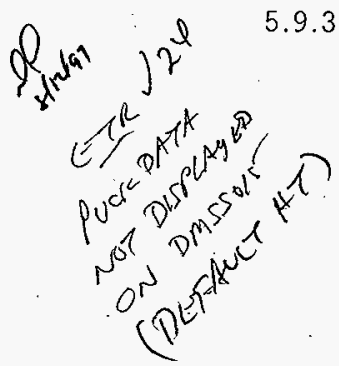

PERFORM Section VII.G, "RECORD COMPACT DRUM (PUCK) DATA", of operating procedure as follows:

5.9.3.1 REDLINE procedure as needed during performance.

5.9.3.2 ENTER on DMSS0315, the following data for puck OTP001 when directed by the operating procedure:

- WASTE DESCRIPTION:

- PUCK HEIGHT:

5.9.3.3. VERIFY results of performance are acceptable.

TD Initials/Date:

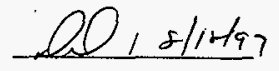

5.9.4 PERFORM Section VII.H, "REMOVE OVERPACK FROM GLOVEBOX", of operating procedure as follows:

5.9.4.1 REDLINE procedure as needed during performance.

5.9.4.2 VERIFY overpack drum OTP-LLW-0D-01 is:

1) CLOSED,

2) Removed from Exit port,

3) Surveyed,

4) Removed from Lift Table LT-09-202C.

5.9.4.3 VERIFY following data is entered on DMSS0315 when directed by operating procedure:

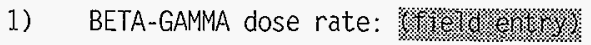

5.9.4.4 VERIFY results of performance are acceptable.

5.10 RUN DMS Report Script.

To Initials/Date: $01,8 / 18 / 57$

To Initials/Date: 01 , s/fla? 


\section{ATTACHMENT 6 - TEST PLAN, LLW GLOVEBOX AUTOMATIC TEST CASE 2}

\subsection{GENERAL DESCRIPTION}

1.1 Test case two will sample, compact and place in Exit Glovebox Storage LLW Test Drum 2. These operations will be performed using procedures WRP1-OP-0710 "Glovebox Waste Sampling," WRP1-OP-0713 "LLW Entry Glovebox Operation," WRP1-OP-0714 "Low Level Waste (LLW) Sorting G1ovebox Operation," WRP1-0P-0706 "Packet NDE Operation," WRP1-OP-0715 "LLW Supercompactor Operation" and WRP1-OP-0716 "LLW Exit Glovebox Operation, " thereby also performing the required procedure validation for sections performed. The sequence of events is as follows:

$\checkmark 1.1 .185$ gallon shipping overpack OTP-LLW-OD-02, with 55 gallon LLW drum OTP-LLW-WP-02 inside, is connected to the LLW glovebox entry port and drum OTP-LLW-WP-02 is lifted into the LLW Entry glovebox

$\sqrt{ }$ 1.1.2 Drum OTP-LLW-WP-02 is checked for external contamination.

1.1.3 85 gallon drum OTP-LLW-0D-02 is closed and removed from the LLW Entry port and from lift table LT-09-202A.

1.1.4 Empty 85 galion drum OTP-LLW-0D-02 (from entry port) is placed on lift table LT-09-202C (LLW Exit).

1.1.5 Drum OTP-LLW-00-02 is connected to the LLW glovebox exit port and the port is opened.

1.1.6 Drum OTP-LLW-WP-02 is lifted by the LLW entry g7ovebox hoist, lowered through transfer port, and placed on transfer car.

1.1.7 Drum OTP-LLW-WP-02 is moved to the drum tipper, the lid is removed, and the drum contents are dumped on the sorting table.

1.1.8 NDE hard copy, DMS NDE comments and Sorting Glovebox Packet NDE, are used to find and identify three non-compliant packets. 
Waste Management Federal Services of Hanford, Inc.

Project W-026. WRAP I

Operational Test - LLW Glovebox

HNF-SD-W026-0TR,-015

Rev: 0

Page 44 of 91

ATTACHMENT 6 - TEST PLAN, LLW GLOVEBOX AUTOMATIC TEST CASE 2

1.1.9 Packet bar code label OTP6-97-000001 is applied to the unwrapped aerosol can of saline solution, the bar code is read, the packet is placed on the transfer stand, and data is entered in the DMS.

1.1.10 Packet bar code label 0TP6-97-000002 is applied to the aerosol can of saline solution wrapped in bubble wrap and tape, the bar code is read, the packet is placed on the transfer stand, and data is entered in the DMS.

1.1.11 Packet bar code label 0TP6-97-000003 is applied to the unlabeled aerosol can wrapped in cloth material and secured with tape, the bar code is read, the packet is placed on the transfer stand, and data is entered in the DMS.

1.1.12 The waste data for the sorted drum is updated on the DMS.

1.1.13 The waste on the sorting table is loaded back into drum OTP-LLW-WP-02, the drum is placed on the transfer car, the drum is relidded, and the transfer car with the drum is moved to the compactor.

1.1.14 Drum OTP-LLW-WP-02 is compacted.

1.1.15 Compacted drum OTP-LLW-WP-02 is moved to the exit glovebox. Bar code label 0TP002 is placed on the puck and the barcode is read. The puck is then lifted by the hoist and the puck is weighed on the hoist scale. The ID and weight are sent to the DMS.

1.1.16 The puck is stored at Storage Location 1.

1.1.17 The transfer stand is placed in transfer drum OTP-LLWR-TD-01 and the drum is closed and removed from the transfer drum port and from iift table LT-09-201F.

\subsection{SAFETY}

2.1 Reference OTP Section 8, SAFETY. 
Waste Management Federai Services of Hanford. Inc.

Project W-026. WRAP I

HNF-SD-W026-0TR-015

Operational Test - LLW Glovebox

Rev: 0

Page 45 of 91

ATTACHMENT 6 - TEST PLAN, LLW GLOVEBOX AUTOMATIC TEST CASE 2

\subsection{TOOLS, EQUIPMENT AND SUPPLIES}

- Drum handling (manual) equipment or Pallet fork truck

- $\quad$ Spill Kit/Decontamination Equipment (oils/chemicals)

- Tape and rags

- Other equipment, material or protective clothing (as required)

\subsection{TEST PREREQUISITES}

4.1. VERIFY OTP Section 11.0, PREREQUISITES are complete.

4.2 SET initial conditions as follows:

$J_{4.2 .155}$ gallon LLW drum OTP-LLW-WP-02 in shipping overpack OTP-LLW-0D-02 is on lift table LT-09-202A.

$P \ln (x)$

4:2.2 The PCS man_op ppogram has sent an AGVPCS AGVL message for the AGV located at AGVLLWENTR (LT-09-202A) with drum OTP-LLW-00-02 (sets LCL Conditions for drum OTP-LLW-00-02 on lift table LT-09-202A).

Transfer drum OTP-LLWR-TO-01 is connected to the LLW glovebox RWM transfer port and the empty transfer stand is at the LLW glovebox sorting location.

4.2.4 Sequentially numbered packet barcode labels are available in the LLW sorting glovebox.

$\sqrt{4.2 .5}$ Sequentially numbered puck barcode labels are available in the LLW exit glovebox.

$\sqrt{\text { 4.2.6 A user with general operator (GENOP) privileges is logged on }}$ to the DMS LLW Termina] (107-TE-12-104)

4.2.7 DMS Database unchanged from Test Case 1.

$\int_{\text {4.2.8 PCS Database unchanged from Test Case } 1 .}$ 
Waste Management Federal Services of Hanford. Inc. Project $W$-026. WRAP I Operational Test - LLW Glovebox

HNF-SD-W026-0TR-015 Rev: 0

ATTACHMENT 6 - TEST PLAN, LLW GLOVEBOX AUTOMATIC TEST CASE 2

4.3 UPDATE PCS Tracking Table as follows:

4.3.1 Drum OTP-LLW-WP-02 in shipping overpack OTP-LLW-0D-02 at LT-09-202A.

\begin{tabular}{|c|c|c|}
\hline \multicolumn{3}{|c|}{ PCS TRACKING TABLE FOR LLW LIFT TABLES } \\
\hline \multirow{2}{*}{ LIFT TABLE } & \multicolumn{2}{|c|}{ Registers } \\
\hline & $\begin{array}{c}\text { PIN } \\
07 p-L C \omega-00-02\end{array}$ & Drum Present $(=1)$ \\
\hline LT-09-202A & N31: $35-41$ & N31: 46 bit 12 \\
\hline
\end{tabular}

4.4 TEST PREREQUISITES are complete and acceptable.

TD Initials/Date:

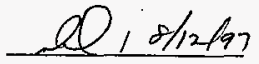


Waste Management Federal Services of Hanford. Inc.

Project $W-026$. WRAP I

operational Test - LLW Glovebox

HNF-SD-W026-OTR-015

Rev: 0

Page 47 of 91

\section{ATTACHMENT 6 - TEST PLAN, LLW GLOVEBOX AUTOMATIC TEST CASE 2}

\subsection{PROCEDURE}

5.1 REFERENCE procedure WRP1-OP-0713, LLW ENTRY GLOVEBOX OPERATION:

5.1.1 PERFORM Section VII:A, "INITIALIZE SYSTEM", of operating procedure as follows:

5.1.1.1 REDLINE procedure as needed during performance.

5.1.1.2 VERIFY results of performance are acceptable.

To Initials/Date:

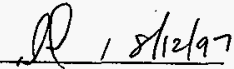

5.1.2 PERFORM Section VII.B, "DRUM ENTRY TO LLW GLOVEBOX", of
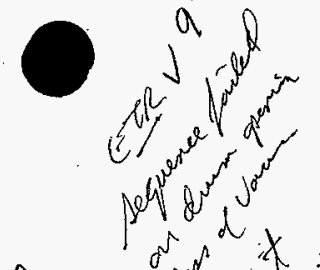

$p$

$P e^{2} x^{2 x}$

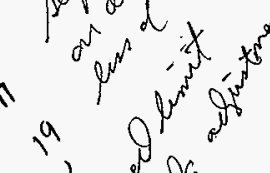

5.1.2.1 REDLINE procedure as needed during performance.

5.1.2.2 VERIFY overpack drum OTP-LLW-0D-02 OPEN and inner drum 0TP-LLW-WP-02 on hoist at "SWAB" position.

5.1.2.3 VERIFY DMSS0311 indicates overpack drum 0TP-LLW-00-02 and inner drum OTP-LLW-WP-02 at glovebox with message "Sorting Required".

5.1.2.4 VERIFY results of performance aré acceptable.

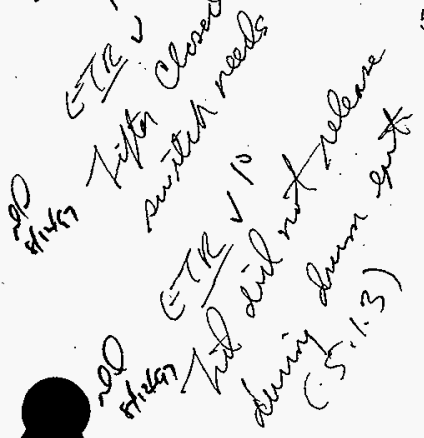

TD Initials/Date:

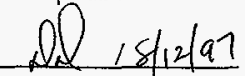


ATTACHMENT 6 - TEST PLAN, LLW GLOVEBOX AUTOMATIC TEST CASE 2

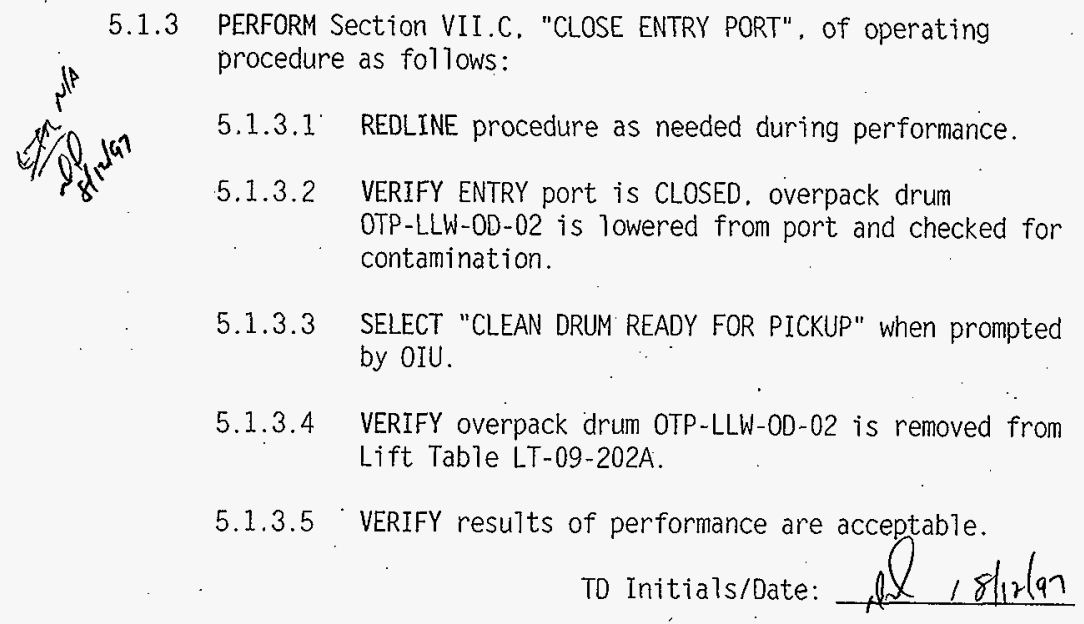

5.1.4 MOVE overpack drum OTP-LLW-0D-02 (manua71y) to AGV end of ${ }_{N / A}$ Lift Table LT-09-2026 at LtW Exit Port.

TD Initials/Date:

5.1.5 Run PCS man_op program to send AGVPCS AGVL message for AGV at AGVLLWEXIT (LT-09-202C) with-dru OTP-LLW-0D-02 (setS LCU

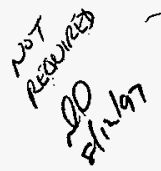
conditions for drum OTP-LLW-OD-02 on lift table LT-09-202C.)

TD Initials/Date: 1 
Waste Management Federal Services of Hanford, Inc. Project $W-026$. WRAP I

\section{ATTACHMENT 6 - TEST PLAN, LLW GLOVEBOX AUTOMATIC TEST CASE 2}

5.2 REFERENCE procedure WRP1-OP-0716, LLW EXIT GLOVEBOX. OPERATION:

5.2.1 PERFORM Section VII.A. "INITIALIZE SYSTEM", of operating procedure as follows:

5.2.1.1 REDLINE procedure as needed during performance.

5.2.1.2 VERIFY results of performance are acceptable.

TD Initia1s/Date:

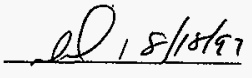

5.2.2 PERFORM Section VII.B. "EXIT DRUM ACCESS TO LLW GLOVEBOX", of operating procedure as follows:

5.2.2.1 REDLINE procedure as needed during performance.

5.2.2.2 VERIFY overpack drum OTP-LLW-00-02 connected to LLW Exit Port and OPEN.

5.2.2.3 VERIFY results of performance are acceptable

TD Initials/Date:

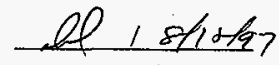

5.3 REFERENCE procedure WRP1-OP-0713, LLW ENTRY GLOVEBOX OPERATION:

5.3.1 PERFORM Section VII.D, "MOVE DRUM TO DRUM TRANSFER CAR". Of operating procedure as follows:

5.3.1.1 REDLINE procedure as needed during performance.

5.3.1.2 VERIFY drum OTP-LLW-WP-02 is positioned over transfer port 107-00-07-102 and lowered onto drum transfer car.

5.3.1.3 VERIFY results of performance are acceptable.

To Initials/Date:

5.4 REFERENCE procedure WRPI-OP-0714, LOW LEVEL WASTE (LLW) SORTING GLOVEBOX OPERATION: 

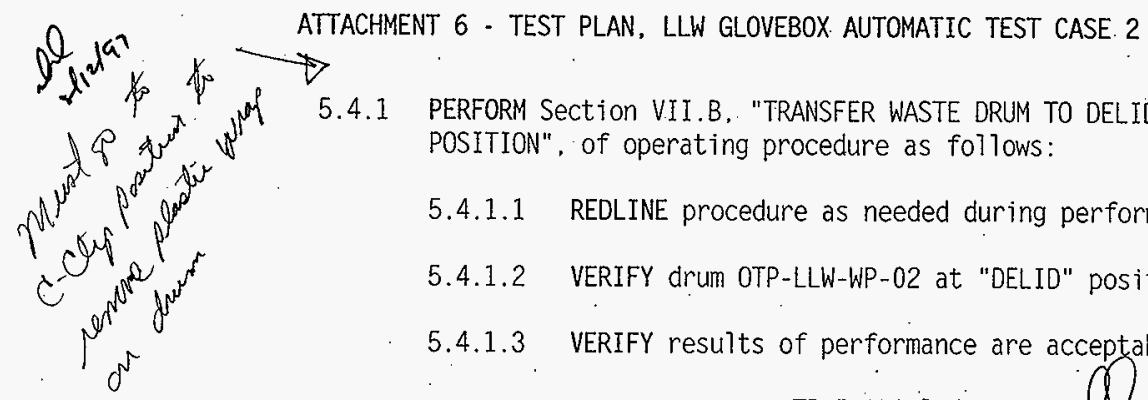

5.4.1 PERFORM Section VII.B. "TRANSFER WASTE DRUM TO DELID POSITION", of operating procedure as follows:

5.4.1.1 REDLINE procedure as needed during performance.

5.4.1.2 VERIFY drum OTP-LLW-WP-02 at "DELID" position.

5.4.1.3 VERIFY results of performance are acceptable.

TD Initials/Date:

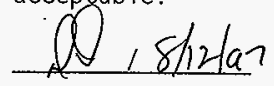

5.4.2 PERFORM Section VII.C. "GRIP WASTE DRUM WITH DRUM TIPPER

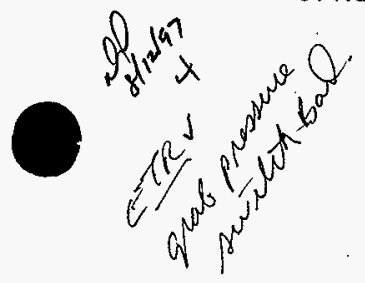
(107-OH-07-103)", of operating procedure as follows:

5.4.2.1 REDLINE procedure as needed during performance.

5.4.2.2 VERFFY plastic overbag removed from drum OTP-LLW-WP-O2.

5.4.2.3 VERIFY drum tipper lowered and tipper grab closed around drum OTP-LLW-WP-02.

5.4.2.4 VERIFY results of performance are acceptable.

TO Initials/Date:

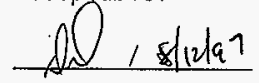

5.4.3 PERFORM Section VII.D. "SAW WASTE DRUM CLAMP BAND", of operating procedure as follows:

5.4.3.1 REDLINE procedure as needed during performance.

5.4.3.2 VERIFY clamp band sawed, removed from drum 0TP-LLW-WP-02, and placed on sorting table.

5.4.3.3 VERIFY results of performance are accepzoble.

TD Initials/Date:

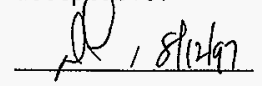

5.4.4 PERFORM Section VII.E, "DELID WASTE DRUM", of operating procedure as follows:

5.4.4.1 REDLINE procedure as needed during performance. 
Waste Management Federal Services of Hanford. Inc. Project $W-026$, WRAP I

Operational Test - LLW Glovebox

HNF-SD-W026-0TR-015

Rev: 0

Page 51 of 91

\section{ATTACHMENT 6 - TEST PLAN, LLW GLOVEBOX AUTOMATIC TEST CASE 2}

5.4.4.2 VERIFY delid fixture removes lid from drum 0TP-LLW-WP-02, and returns to it's "raised/parked" position with the lid.

5.4.4.3 VERIFY results of performance are acceptable. TD Initials/Date:

5:4.5 PERFORM Section VII.F, "EMPTY WASTE DRUM ONTO SORTING TABLE", of operating procedure as follows:

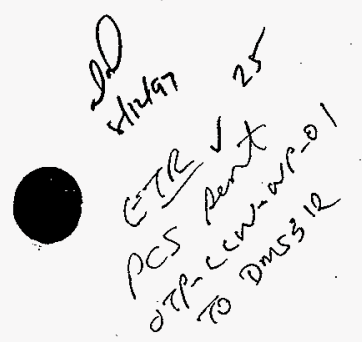

5.4.5.1 REDLINE procedure as needed during performance.

5.4.5.2 VERIFY tipper raises to dump contents of drum OTP-LLW-WP-02 onto sorting table, and returns to it's "lowered" position with the empty drum.

5.4.5.3 VERIFY DMSS0312 Parent PIN is OTP-LLW-WP-02, Sample $=$ NO and Compliant $?=$ NO.

5.4.5.4 VERIFY results of performance are acceptable.

TD Initials/Date:

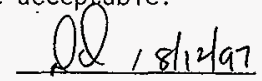


ATTACHMENT 6 - TEST PLAN, LLW GLOVEBOX AUTOMATIC TEST CASE 2

5.4.6 PERFORM Section VII.G, "REMOVE NON-COMPLIANT PACKETS FROM WASTE", of operating procedure as follows:

5.4.6.1 REDLINE procedure as needed during performance.

$\int$ 5.4.6.2 IDENTIFY three non-compliant packages using NDE hard copy from receiving. DMS NDE comments and Packet NDE:
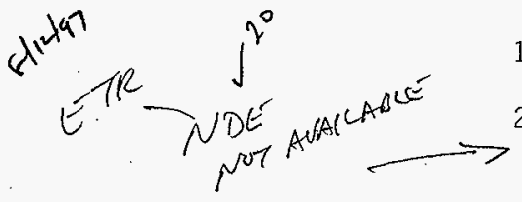

1) VIEW DMS NOE comments on DMSS0312
PERFORM WRP1-OP-0706, PACKET NDE OPERATION in it's entirety on at least one non-compliant package.

5.4.6.3 VERIFY Packet bar code 1abel OTP6-97-000001 is applied to the unwrapped aerosol can of saline solution, the bar code is read, and the packet is placed on the transfer stand.

1) DISPLAY DMSS0312 LLW Non-Compliant Screen after the barcodes are read.

2). ENTER the following for PIN 0TP6-97-000001:

Group: AC

Waste Description: AEROSOL CAN OF SALINE SOLUTION

5.4.6.4 VERIFY Packet bar code label OTP6-97-000002 is applied to the aerosol can of saline solution wrapped in bubble wrap and tape, the bar code is read, and packet is placed on the transfer stand.

1) REFRESH DMSS0312 LLW Non-Compliant Screen after the barcodes are read.

2) ENTER the following for PIN OTP6-97-000002:

Group: AC

Waste Description: AEROSOL CAN 


\section{ATTACHMENT 6 - TEST PLAN, LLW GLOVEBOX AUTOMATIC TEST CASE 2}

5.4.6.5 VERIFY Packet bar code label 0TP6-97-000003 is applied to the unlabeled aerosol can of saline solution wrapped in cloth and tape, bar code is read, and packet is placed on transfer stand.

1) REFRESH DMSS0312 LLW Non-Compliant Screen after the barcodes are read.

2) ENTER the following for.PIN 0TP6-97-000003:

Group: $A C$ Waste Description: AEROSOL CAN

5.4.6.6 UPDATE sorted drum data in DMS:

1) SELECT .DMSS0312 "Physcomp" screen.

- DELETE HAZARDOUS CONSTITUENTS record

- CHANGE CONWEB PADS volume percent to 98

- CHANGE WATER volume percent to 2.

2) SELECT DMSS0312 "Chemcomp" screen.

- DELETE the two records.

5.4.6.7 VERIFY results of performance are acceptable.

TD Initials/Date:

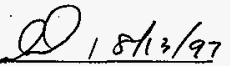

5.4.7 PERFORM Section VII.H, "RETURN COMPLIANT WASTE TO WASTE DRUM", of operating procedure as follows:

5.4.7.1 REDLINE procedure as needed during performance.

5.4.7.2 VERIFY results of performance are acceptable.

To Initials/Date:

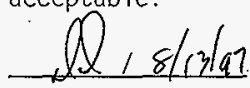




\section{ATTACHMENT 6 - TEST PLAN, LLW GLOVEBOX AUTOMATIC TEST CASE 2}

5.4.8 PERFORM Section VII.I. "MOVE WASTE DRUM TO RELID DOSITION",

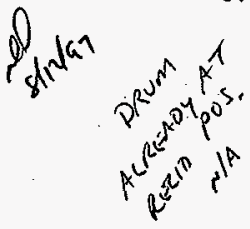
of operating procedure as follows:

5.4.8.1 REDLINE procedure as needed during performance.

5.4.8.2 VERIFY results of performance are acceptable.

TD Initials/Date:

5.4.9 PERFORM Section VII.J, "RELID. WASTE DRUM", of operating procedure as follows:

5.4.9.1 REDLINE procedure as needed during performance.

5.4.9.2 VERIFY drum OTP-LLW-WP-02 is re-lidded with c-clips attached.

5.4.9.3 VERIFY resuTts of performance are acceptable.

TD Initials/Date:

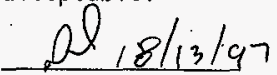

5.4.10 PERFORM Section VII.K, "TRANSFER WASTE DRUM TO SUPERCOMPACTOR", of operating procedure as follows:

5.4.10.1 REDLINE procedure as needed during performance.

5.4.10.2 PERFORM appropriate Sections of WPRI-0P-0715 to ensure Supercompactor is in Automatic and ready to receive drums.

5.4.10.3 VERIFY drum OTP-LLW-WP-02 is delivered to Supercompactor and automatically compacted.

5.4.10.4 VERIFY results of performance are acceptable.

TD Initials/Date:

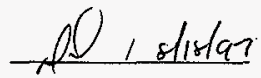


Waste Management Federal Services of Hanford. Inc. Project $W-026$, WRAP I Operational Test - LLW GTovebox

HNF-SD-W026-OTR-015

Rev: 0

Page 55 of 91

ATTACHMENT 6 - TEST PLAN, LLW GLOVEBOX AUTOMATIC TEST CASE 2

5.5 REFERENCE procedure WRP1-OP-0716, LLW EXIT GLOVEBOX OPERATION:

5.5.1 PERFORM Section VII.C, "PUCK RECEIPT AND WEIGHT MEASUREMENT", of operating procedure as follows:

5.5.1.1 REDLINE procedure as needed during performance.

$\sqrt{5.5 .1 .2}$ VERIFY puck barcode OTP002 is placed on drum OTP-LLW-WP-02 and scanned.

5.5.1.3 VERIFY puck OTP002 is 7 ifted and weighed on the Exit Glovebox hoist.

5.5.1.4 VERIFY results of performance are acceptable.

TD Initials/Date:

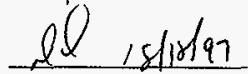

5.5.2 PERFORM Section VII.D. "MOVE PUCK FROM RECEIPT TO STORAGE", of operating procedure as follows:

$40_{8} 1291$

5.5.2.1 REDLINE procedure as needed during performance.

5.5.2.2 VERIFY drum OTP-LLW-WP-02 is moved to Storage Position 1.

5.5.2.3 VERIFY results of performance are acceptable.

TD Initials/Date:

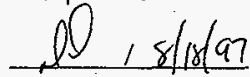


Waste Management Federal Services of Hanford, Inc.

Project $W-026$. WRAP I

Operational Test - LLW Glovebox

HNF-SD-W026-OTR-015

Rev: 0 .

Page 56 of 91

\section{ATTACHMENT 6 - TEST PLAN, LLW GLOVEBOX AUTOMATIC TEST CASE 2}

5.6 REFERENCE procedure WRP1-0P-0714, LOW LEVEL WASTE (LLW) SORTING GLOVEBOX OPERATION:

5.6.1 PERFORM Section VII.L, "PLACE TRANSFER STAND IN RWM TRANSFER DRUM", of operating procedure as follows:

5.6.1.1 REDLINE procedure as needed during performance.

5.6.1.2 VERIFY transfer stand is placed in transfer drum OTP-LLWR-TD-01.

5.6.1.3 VERIFY results of performance are acceptable.

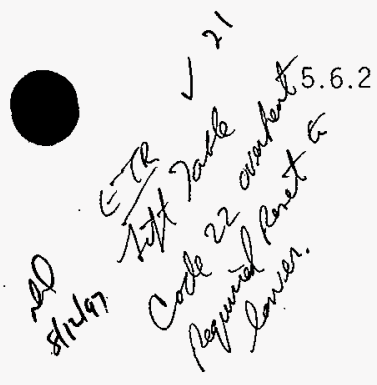

TD Initials/Date:

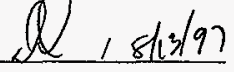

PERFORM Section VII.M, "REMOVE DRUM FROM RWM TRANSFER PORT (107-D0-07-105)" , of operating procedure as follows:

5.6.2.1 REDLINE procedure as needed during performance.

5.6.2.2 VERIFY transfer drum OTP-LLWR-TD-01 is closed and removed from port.

5.6.2.3 VERIFY results of performance are acceptable.

TD Initials/Date:

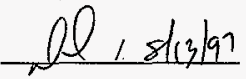

5.7 REMOVE transfer drum OTP-LLWR-TO-01 manual1y from Lift Table LT-09-201F.

5.8 RUN DMS Report Script.

TD Initiars/Date:

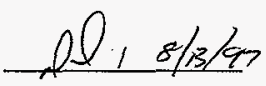


Waste Management Federal Services of Hanford. Inc. Project $W-026$, WRAP I

Operational Test - LLW Glovebox

HNF-SD-W026-0TR-015

Rev: 0

Page 57 of 91

\section{ATTACHMENT 7 - TEST PLAN, LLW GLOVEBOX AUTOMATIC TEST CASE 3}

\subsection{GENERAL DESCRIPTION}

1.1 Test case three wi11 compact and overpack LLW Test Drum 3 without sorting. These operations wi 71 be performed using procedures WRP1-OP-0713 "LLW Entry Glovebox Operation," WRP1-0P-0714 "Low Level Waste (LLW) Sorting Glovebox Operation," WRP1-OP-0715 "LLW Supercompactor Operation" and WRP1-0P-0716 "LLW Exit Glovebox operation." thereby also performing the required procedure validation for sections performed. The sequence of events is as follows:

1.1.1 85. gallon shipping overpack OTP-LLW-OD-03, with 55 gallon LLW drum OTP-LLW-WP-03 inside, is connected to the LLW glovebox entry port and drum OTP-LLW-WP-03 is lifted into the LLW Entry glovebox.

1.1.2 Drum OTP-LLW-WP-03 is checked for external contamination.

1.1.3 85 gallon drum OTP-LLW-OD-03 is closed and removed from the LLW Entry port and from lift table LT-09-202A.

1.1.4 Drum OTP-LLW-WP-03 is 7ifted by the LLW entry glovebox hojst. lowered through the transfer port, and placed on the transfer car.

1.1.5 Drum OTP-LLW-WP-03 is moved to the drum tipper and the lid. band clamp is removed.

1.1.6 Lid C clamps are put on drum OTP-LLW-WP-03 and the transfer car with the drum is moved to the compactor.

1.1.7 Drum OTP-LLW-WP-03 is compacted.

1.1.8 Compacted drum OTP-LLW-WP-03 is moved to the exit glovebox: Bar code label 0TP003 is placed on the puck and the barcode is read. The puck is then lifted by the hoist and the puck is weighed on the hoist scale. The ID and weight are sent to the DMS.

1.1.9 The puck is loaded into the overpack at the exit port. 
Waste Management Federal Services of Hanford. Inc.

Project $W-026$. WRAP I

Operationa] Test - LLW Glovebox

HNF-SD-W026-OTR-015

Rev: 0

Page 58 of 91

\section{ATTACHMENT 7 - TEST PLAN, LLW. GLOVEBOX AUTOMATIC TEST CASE 3}

\subsection{SAFETY}

2.1 Reference OTP Section 8. SAFETY.

$3.0 \cdot$ TOOLS, EQUIPMENT AND SUPPLIES

- Drum handling (manual) equipment or Pallet fork truck

- $\quad$ Spill Kit/Decontamination Equipment (oils/chemicals)

- Tape and rags

- Other equipment, material or protective clothing (as required)

\subsection{TEST PREREQUISITES}

4.1 VERIFY OTP Section 11.0, PREREQUISITES are complete.

4.2 SET initial conditions as follows:

4.2.1 55 gallon LLW drum OTP-LLW-WP-0.3 in shipping overpack

OTP-LLW-0D-03 is on lift table LT-09-202A.

4.2 .2 The PCS man op program has sent an AGVPCS AGVL messatge for
the AGV located at AGVLLWENTR (LT-09-202A) with drum OTP-LLW-DD-03 (sets LCU condritions for drum OTP-LLW-0D-03 on lift table LT-09-202A).

$\$$ 4.2.3. 85 gallon overpack drum 0TP-LLW-00-02 is connected to the LLW glovebox exit port.

4.2.4 Sequentially numbered puck barcode labels are available in the LLW exit glovebox.

$J_{4.2 .5}$ A user. with general operator (GENOP) privileges is logged on to the DMS LLW Terminal (107-TE-12-104)

$\int_{4.2 .6}$ DMS Database unchanged from Test Case 2.

$\sqrt{ }$ 4.2.7 PCS Database unchanged from Test Case 2. 
Waste Management Federal Services of Hanford, Inc.

Project $W-026$. WRAP I

Operationa 7 Test - LLW Glovebox

HNF-SD-W026-0TR-015

Rev: 0

Page 59 of 91

ATTACHMENT 7 - TEST PLAN, LLW GLOVEBOX AUTOMATIC TEST CASE 3

$\sqrt{4.3}$ UPDATE PCS Tracking Table as follows:

4.3.1 Drum OTP-LLW-WP-03 in shipping overpack OTP-LLW-0D-03 at LT-09-202A.

\begin{tabular}{|c|c|c|}
\hline \multicolumn{3}{|c|}{ PCS TRACKING TABLE FOR LLW LIFT TABLES } \\
\hline \multirow{2}{*}{ LIFT TABLE } & \multicolumn{2}{|c|}{ Registers } \\
\cline { 2 - 3 } & PIN & Drum Present $(=1)$ \\
\hline \hline LT.09-202A & N31: 35-41: & N31: 46 bit 12 \\
\hline
\end{tabular}

4.4 TEST PREREQUISITES are complete and acceptable.

TD Initials/Date:

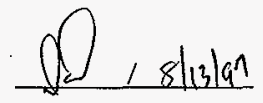


Waste Management Federal Services of Hanford, Inc.

Project W-026. WRAP I

Operational Test - LLW Glovebox

HNF-SD-W026-OTR-015

Rev: 0

Page 60 of 91

\section{ATTACHMENT 7 - TEST PLAN, LLW GLOVEBOX AUTOMATIC TEST CASE 3}

\subsection{PROCEDURE}

5.1 REFERENCE procedure WRP1-OP-0713. LLW ENTRY GLOVEBOX OPERATION:

5.1.1 PERFORM Section VII.A." "INITIALIZE SYSTEM". of operating procedure as follows:

5.1.1.1 REDLINE procedure as needed during performance.

5.1.1.2 VERIFY results of performance are acceptable.

TD Initials/Date:

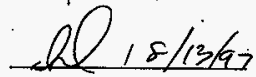

5.1.2 PERFORM Section VII.B, "DRUM ENTRY TO LLW GLOVEBOX", of operating procedure as follows:

5.1.2.1 REDLINE procedure as needed during performance.

5.1.2.2 VERIFY overpack drum OTP-LLW-0D-03 OPEN and inner drum OTP-LLW-WP-03 on hoist at "SWAB" position.

5.1.2.3-VERIFY DMSSO311 indicates overpack drum OTP-LLW-OD-03 and inner drum OTP-LLW-WP-03 at glovebox with message "Sorting Not Required".

5.1.2.4 VERIFY results of performance are acceptable.

TD Initials/Date:

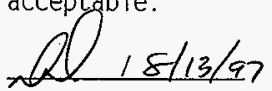


Waste Management Federa? Services of Hanford, Inc.

\section{ATTACHMENT 7 - TEST PLAN, LLW GLOVEBOX AUTOMATIC TEST CASE 3}

5.1.3 PERFORM Section VII.C, "CLOSE ENTRY PORT", of operating procedure as follows:

5.1.3.1 REDLINE procedure as needed during performance.

5.1.3.2 VERIFY ENTRY port is CLOSED, overpack drum OTP-LLW-0D-03 is lowered from port and checked for contamination.

5.1.3.3 SELECT "CLEAN DRUM READY FOR PICKUP" when prompted by OIU.

5.1.3.4 VERIFY overpack drum OTP-LLW-OD-03 is removed from Lift Table LT-09-202A.

5.1.3.5 VERIFY results of performance are acceptable.

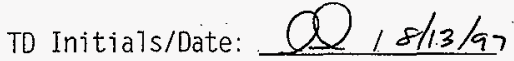

5.1.4 PERFORM Section VII.D, "MOVE DRUM TO DRUM TRANSFER CAR", of operating procedure as follows:

5.1.4.1 REDLINE procedure as needed during performance. transfer port 107-00-07-102 and lowered onto drum transfer car.

5.1.4.3 VERIFY results of performance are acceptable.

TD Initials/Date:

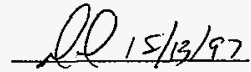


Waste Management Federal Services of Hanford. Inc. Project W-026. WRAP I

Operational Test - LLW Glovebox

HNF-SD-W026-0TR-015

Rev: 0

Page 62 of 91

\section{ATTACHMENT 7 - TEST PLAN, LLW GLOVEBOX AUTOMATIC TEST CASE 3}

5.2 REFERENCE procedure WRP1-OP-0714, LOW LEVEL WASTE (LLW) SORTING GLOVEBOX OPERATION:

5.2.1 PERFORM Section VII.B, "TRANSFER WASTE DRUM TO DELID POSITION", of operating procedure as follows:

5.2.1.1 REDLINE procedure as needed during performance.

5.2.1.2 VERIFY drum OTP-LLW-WP-03 at "DELID" position.

5.2.1.3 VERIFY results of performance are acceptable.

TD Initials/Date:
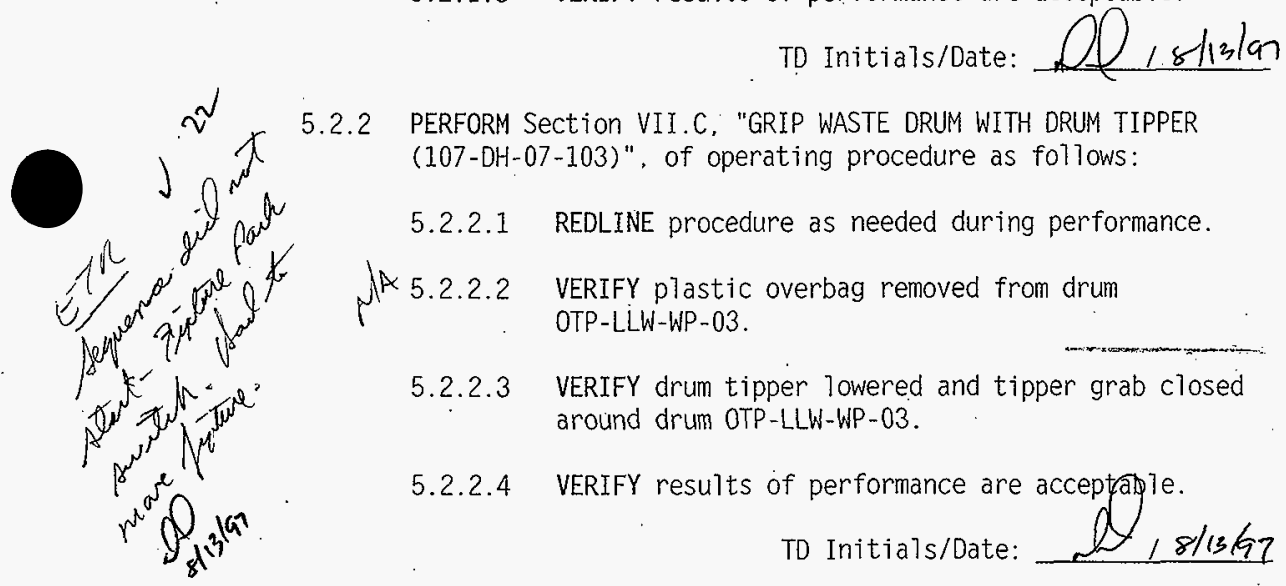

5.2.2 PERFORM Section VII.C, "GRIP WASTE DRUM WITH DRUM TIPPER (107-DH-07-103)", of operating procedure as follows:

5.2.2.1 REDLINE procedure as needed during performance.

A 5.2.2.2 VERIFY plastic overbag removed from drum OTP-LLW-WP-03.

5.2.2.3 VERIFY drum tipper lowered and tipper grab closed around drum OTP-LLW-WP-03.

5.2.2.4 VERIFY results of performance are acceptaple.

TD Initials/Date:

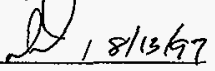

5.2.3 PERFORM Section VII.D, "SAW WASTE DRUM CLAMP BAND", of operating procedure as follows:

5.2.3.1 REDLINE procedure as needed during performance.

5.2.3.2 VERIFY clamp band sawed, removed from drum OTP-LLW-WP-03, and placed on sorting table.

5.2.3.3. VERIFY results of performance are acceptable.

TD Initials/Date: $8 / 1392$ 
Waste Management Federal Services of Hanford. Inc.

Project $W-026$, WRAP I

operational Test - LLW Glovebox

HNF-SD-W026-0TR-015

Rev: 0

Page 63 of 91

ATTACHMENT 7 - TEST PLAN, LLW GLOVEBOX AUTOMATIC TEST CASE 3

5.2.4 PERFORM Section VII.I, "MOVE WASTE DRUAHTO RELID POSITIOAN", of operating procedure as follows:

$\int_{8}^{8} \sin ^{4}$

5.2.4.1 REDLINE procedure as needed during-performance.

5.2.4.2 VERIFY results of performance are acceptable.

TD Initials/Date:

5.2.5 PERFORM Section VII.J, "RELIO WASTE DRUM", of operating procedure as follows:

5.2.5.1 REDLINE procedure as needed during performance.

5.2.5.2 VERIFY drum OTP-LLW-WP-03 is re-lidded with c-clips attached.

5.2.5.3 VERIFY results of performance are acceptable.

TD Initials/Date:

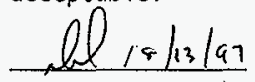

5.2.6 PERFORM Section VII.K, "TRANSFER WASTE DRUM TO

SUPERCOMPACTOR", of operating procedure as follows:

5.2.6.1 REDLINE procedure as needed during performance.

5:2.6:2 PERFORM appropriate Sections of WPR1-OP-0715 to ensure Supercompactor is in Automatic and ready to receive drums.

5.2.6.3 VERIFY drum OTP-LLW-WP-03 is delivered to Supercompactor and automatically compacted.

5.2.6.4 VERIFY results of performance are acceptable.

To Initiàls/Date:

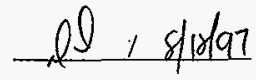


Waste Management Federal Services of Hanford. Inc. Project $W-026$. WRAP I

Operational Test - LLW Glovebox
HNF-SD-W026-OTR-015

Rev: 0

Page 64 of 91

\section{ATTACHMENT 7 - TEST PLAN, LLW GLOVEBOX AUTOMATIC TEST CASE 3}

5.3 REFERENCE procedure WRP1-OP-0716, LLW EXIT GLOVEBOX OPERATION:

5.3.1 PERFORM Section VII.C, "PUCK RECEIPT AND WEIGHT MEASUREMENT", of operating procedure as follows:

5.3.1.1 REDLINE procedure as needed during performance.

5.3.1.2 VERIFY puck barcode OTP003 is placed on drum OTP-LLW-WP-03 and scanned.

5.3.1.3 VERIFY puck OTP003 is lifted and weighed on the Exit Glovebox hoist.

5.3.1.4 VERIFY results of performance are acceptable.

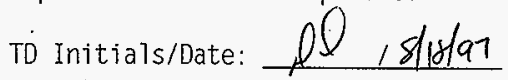

5:3.2 PERFORM Section VII.D, "MOVE PUCK FROM RECEIPT TO LOAD OUT", of operating procedure as follows:

5.3.2.1 REDLINE procedure as needed during performance.

5.3.2.2 VERIFY puck OTP003 is Toaded into overpack drum OTP-LLW-OD-02 at Exit port.

5.3.2.3 VERIFY results of performance are acceptable.

TD Initials/Date:

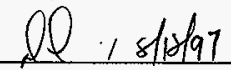


Waste Management Federal Services of Hanford. Inc. Project W-026. WRAP I

Operational Test - LLW GTovebox

HNF-SD-W026-0TR-015

Rev: 0

Page 65 of 91

ATTACHMENT 7 - TEST PLAN, LLW GLOVEBOX AUTOMATIC TEST CASE 3

5.3.3 PERFORM Section VII.G, "RECORD COMPACT DRUM (PUCK) DATA", of operating procedure as follows:

5.3.3.1 REDLINE procedure as needed during performance.

5.3.3.2 ENTER on DMSS0315, the following data for puck OTP003 when directed by the operating procedure:

- WASTE DESCRIPTION:

- PUCK HEIGHT:

5.3.3.3 VERIFY results of performance are acceptable.

TD Initials/Date:

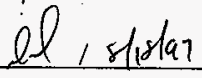

5.4. RUN DMS Report Script.

TD Initials/Date: _ol, $8 / 18 / 91$ 
Waste Management Federal Services of Hanford, Inc.

Project W-026, WRAP I

Operational Test - LLW Glovebox

HNF-SD-W026-OTR-015

Rev: 0

Page 66 of 91

\section{ATTACHMENT 8 - TEST PLAN, LLW GLOVEBOX AUTOMATIC TEST CASE 4}

\subsection{GENERAL. DESCRIPTION}

1.1 Test Case four will treat items in Transfer Drum 2 packets and samples in STC-2 which were taken from these items. These operations will be tested using procedures WRP1-OP-0710. Glovebox Waste Sampling. WRP1-OP-0728. LLW Glovebox Operation and WRP1-OP-0711. Waste Treatment, thereby also performing the required procedure validation for sections performed. The sequence of events is as follows:

1.1.1 Transfer drum OTP-LLWR-TD-02 is connected to the LLW glovebox RWM bagless transfer port and the port is opened.

1.1.2 Transfer stand is moved to the sorting table.

1.1.3 85 gallon shipping overpack OTP-LLW-00-04, with 55 gallon LLW drum OTP-LLW-WP-04 inside, is connected to the LLW glovebox entry port and drum OTP-LLW-WP-04 is lifted into the LLW Entry glovebox.

1.1.4 Drum OTP-LLW-WP-04 is checked for external contamination.

1.1.5 85 gallon drum OTP-LLW-00-04 is closed and removed from the LLW Entry port and from lift table LT-09-202A.

1.1.6 Drum 0TP-LLW-WP-04 is lifted by the LLW entry glovebox hoist. lowered through the transfer port, and placed on the transfer car.

1.1.7 Drum OTP-LLW-WP-04 is moved to the drum tipper, the Tid is removed. and the drum contents are dumped on the sorting table.

1.1.8 The Packet X-ray, NDE hard copy, and DMS NDE comments are used to find and identify three non-compliant packets.

1.1.9 Packet bar code label OTP6-97-000004 is applied to the lead brick in two plastic bags wrapped with tape, the bar code is read, the packet is placed on the transfer stand, and data is entered in the DMS. 


\section{ATTACHMENT 8 - TEST PLAN, LLW GLOVEBOX AUTOMATIC TEST CASE 4}

1.1.10 Packet bar code label 0TP6-97-000005 is applied to the bottle of solvent and bottle of oil wrapped together in cloth with tape, the bar code is read; the packet is placed on the transfer stand, and data is entered in the DMS.

1.1.11 Packet bar code label 0TP6-97-000006 is applied to the bottle of acid, the bar code is read, the packet is placed on the transfer stand, and data is entered in the DMS.

1.1.12 The waste data for the sorted drum is updated on the DMS.

1.1.13 The waste on the sorting table is loaded back into drum OTP-LLW-WP-04, the drum is placed on the transfer car, the drum is relidded, and the transfer car with the drum is moved to the compactor.

1.1.14 Drum OTP-LLW-WP-04 is compacted.

1.1.15 Compacted drum 0TP-LLW-WP-04 is moved to the exit glovebox. Bar code label 0TP004 is placed on the puck and the barcode is read. The puck is then lifted by the hoist and the puck is weighed on the hoist scale. The ID and weight-are sent to the DMS.

1.1.16 Puck P00004 is loaded into the overpack at the exit port.

1.1.17 Puck P00002 is lifted by the hoist, weighed, and placed in the overpack drum.

1.1.18 Filler is added to overpack drum OTP-LLW-00-01 and the drum is prepared for loadout.

1.1.19 Overpack drum OTP-LLW-OD-01 is (1) closed, (2) removed from the LLW glovebox exit port, (3) surveyed, and (4) removed from lift table LT-09-202C.

1.1.20 The transfer stand is placed in transfer drum OTP-LLWR-TD-02 and the drum is closed and removed from the transfer drum port and from Lift Table LT-09-201F. 
Waste Management Federal Services of Hanford. Inc.

Project $W-026$. WRAP I

Operational Test - LLW Glovebox

HNF-SD-W026-OTR-015

Rev: 0

Page 68 of 91

\section{ATTACHMENT $8^{\circ}-$ TEST PLAN, LLW GLOVEBOX AUTOMATIC TEST CASE 4}

\subsection{SAFETY}

2.1 Reference OTP Section 8, SAFETY.

3.0 TOOLS, EQUIPMENT AND SUPPLIES

- Drum handling (manual) equipment or Pallet fork truck

- Spill Kit/Decontamination Equipment (oils/chemicals)

- Tape and rags

- Other equipment. material or protective clothing (as required)

\subsection{TEST PREREQUISITES}

4.I VERIFY OTP Section 11.0. PREREQUISITES are complete.

4.2 SET initial conditions as follows:

$\sqrt{4.2 .155 \text { gallon LLW drum OTP-LLW-WP-04 in shipping overpack }}$ OTP-LLW-0D-04 is on lift table LT-09-202A.

Q

4.2.2 The PCS man_op program has sent an AGVPCS AGVL-message for the AGV located at AGVLLWRW (LT-09-202A) with drum OTP-LLW-WP-O4 (sets LCU conditions for drum OTP-LLW-WP-04 on lift table LT-09-202A.)

$\sqrt{4.2 .3}$ Empty transfer drum 0TP-LLWR-TD-02 is on lift table LT-09-203A.

4.2.4 The PCS man_op program has sent an AGVPCS AGVL message for the AGV located at AGVLLRWX (LT-09-203A) with drum
OTP-LLWR-TD-02 (sets LCU conditions for drum 0TP-LLWR-TD-02 on lift table (T-09-203A)

4.2.5 85 gallon overpack drum OTP-LLW-0D-02 is connected to the LLW glovebox exit port.

$\sqrt{4.2 .6}$ Sequentially numbered packet barcode labels are available in the LLW sorting glovebox.

$\int 4.2 .7$ Sequentially numbered puck barcode labels are available in the LLW exit glovebox. 
Waste Management Federal Services of Hanford. Inc. Project $W$-026. WRAP I

\section{ATTACHMENT 8 - TEST PLAN, LLW GLOVEBOX AUTOMATIC TEST CASE 4}

4.2.8 A user with general operator (GENOP) privileges is logged on to the DMS LLW Terminal (107-TE-12-104)

4.2.9 DMS Database unchanged from Test Case 3.

4.2.10 PCS Database unchanged from Test Case 3.

4.3 UPDATE PCS Tracking Table as follows:

4.3.1 Drum OTP-LLW-WP-04 in shipping overpack OTP-LLW-0D-04 at LT-09-202A.

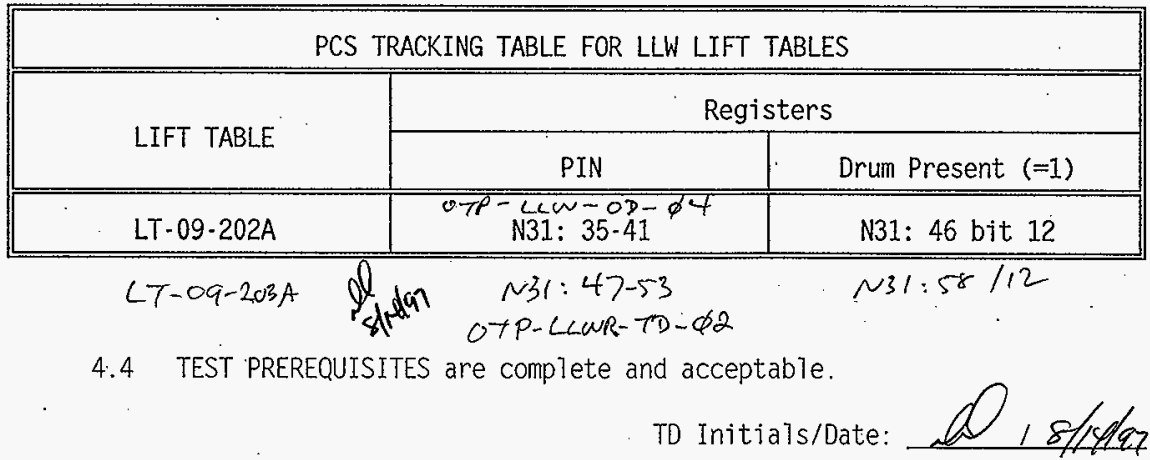


ATTACHMENT 8 - TEST PLAN, LLW GLOVEBOX AUTOMATIC TEST CASE 4

\subsection{PROCEDURE}

5.1 REFERENCE procedure WRP1-0P-0714, LOW LEVEL WASTE (LLW) SORTING GLOVEBOX OPERATION:

5.1.1 PERFORM Section VII.A, "INITIALIZE SYSTEM". of operating procedure as follows:

5.1.1.1 REDLINE procedure as needed during performance.

5.1.1.2 VERIFY results of performance are acceptable.

TD Initials/Date:

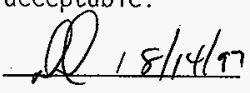

5.1.2 PERFORM Section VII.N. "MATE DRUM TO RWM TRANSFER PORT (107-D0-07-105)", of operating procedure as follows:

5.1.2.1 REDLINE procedure as needed during performance.

5.1.2.2 VERIFY drum OTP-LLWR-TD-02 connected to port 107-D0-07-105 and port OPEN.

5.1.2.3 VERIFY results of performance are acceptable.

TD Initials/Date:

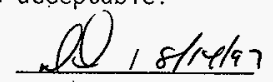

5.1.3 PERFORM Section VII.0, "REMOVE TRANSFER STAND FROM RWM TRANSFER DRUM", of operating procedure as follows:

5.1.3.1 REDLINE procedure as needed during performance.

5.1.3.2. VERIFY transfer stand at glovebox sorting table.

5.1.3.3 VERIFY results of performance are acceptable.

TD Initials/Date:

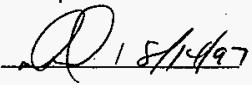


ATTACHMENT 8 - TEST PLAN, LLW GLOVEBOX AUTOMATIC TEST CASE 4

5.2 REFERENCE procedure WRP1-OP-0713, LLW ENTRY GLOVEBOX OPERATION:

5.2.1 PERFORM Section VII.A, "INITIALIZE SYSTEM", of operating procedure as follows:

5.2.1.1 REDLINE procedure as needed during performance.

5.2.1.2 VERIFY results of performance are acceptaple.

TO Initials/Date:

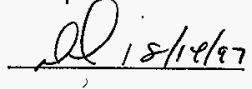

5.2.2 PERFORM Section VII.B, "DRUM ENTRY TO LLW GLOVEBOX", of operating procedure as follows:

5.2.2.1 REDLINE procedure as needed during performance.

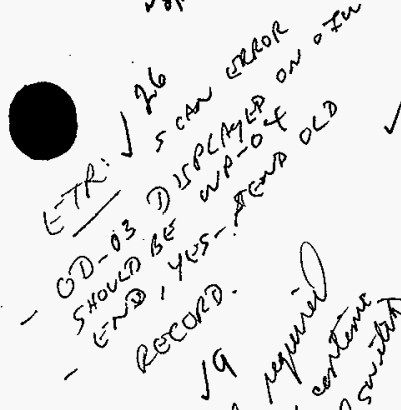

$\sqrt{5.2 .2 .2}$

5.2.2.3 VERIFY DMSS0311 indicates overpack drum OTP-LLW-0D-04 and inner drum OTP-LLW-WP-04 at glovebox with message "Sorting Required".

5.2.2.4 VERIFY results of performance are acceptable.

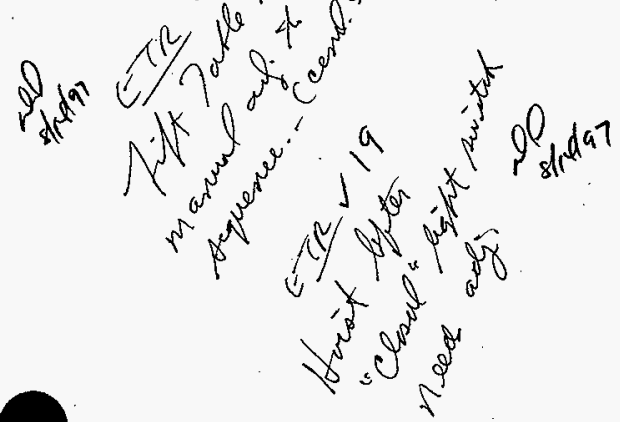

TD Initials/Date:

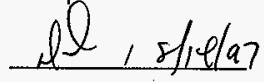


Waste Management Federal Services Of Hanford. Inc.

Project $W-026$, WRAP I

HNF-SD-W026-OTR-015

Operational Test - LLW Glovebox

Rev: 0

Page 72 of 91

\section{ATTACHMENT 8 - TEST PLAN, LLW GLOVEBOX AUTOMATIC TEST CASE 4}

5.2.3 PERFORM Section VII.C, "CLOSE ENTRY PORT", of operating procedure as follows:

5.2.3.1 REDLINE procedure as needed during performance.

5.2.3.2 VERIFY ENTRY port is CLOSED, overpack drum OTP-LLW-0D-04 is lowered from port and checked for contamination.

5.2.3.3 SELECT "CLEAN DRUM READY FOR PICKUP" when prompted by OIU.

5.2.3.4 VERIFY overpack drum OTP-LLW-OD-04 is removed from Lift Table LT-09-202A.

5.2.3.5 VERIFY results of performance are acceptable.

TD Initials/Date:

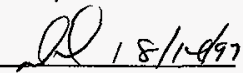

5.2.4 PERFORM Section VII.D, "MOVE DRUM TO DRUM TRANSFER CAR", of operating procedure as follows:

5.2.4.1 REDLINE procedure as needed during performance.

5.2.4.2. VERIFY drum OTP-LLW-WP-04 is positioned over transfer port 107-00-07-102 and lowered onto drum transfer car.

5.2.4.3 VERIFY results of performance are acceptable.

To Initials/Date:

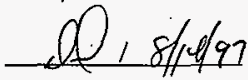




\section{ATTACHMENT 8 - TEST PLAN, LLW GLOVEBOX AUTOMATIC TEST CASE 4}

\subsection{REFERENCE procedure WRP1-OP-0714, LOW LEVEL WASTE (LLW) SORTING} GLOVEBOX OPERATION:

yier car to C.ccie rosition.3.1 To Remule pala

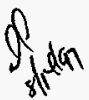

5.3.1.1 REDLINE procedure as needed during performance.

5.3.1.2 VERIFY drum OTP-LLW-WP-04 at "DELID" position.

5.3.1.3 VERIFY results of performance are acceptable.

TD Initials/Date:

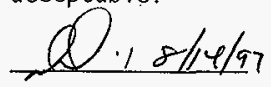

5.3.2. PERFORM Section VII.C, "GRIP WASTE DRUM WITH DRUM TIPPER (107-DH-07-103)", of operating procedure as follows:

5.3.2.1 REDLINE procedure as needed during performance.

Pele $n^{5} \varepsilon^{2} 3^{3}$
5.3.2.2 VERIFY plastic overbag removed from drum OTP-LLW-WP-04.

5.3.2.3 VERIFY drum tipper lowered and tipper grab closed around drum OTP-LLW-WP-04.

5.3.2.4 VERIFY results of performance are acceptable.

TO Initials/Date: $)$, s/plar

5.3.3 PERFORM Section VII.D, "SAW WASTE DRUM CLAMP BAND", of operating procedure as follows:

5.3.3.1 REDLINE procedure as needed during performance.

5.3.3.2 VERIFY clamp band sawed, removed from drum OTP-LLW-WP-04, and placed on sorting table.

5.3.3.3 VERIFY results of performance are acceptable.

TD Initials/Date:

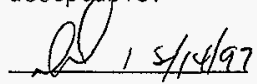


Waste Management Federal Services of Hanford, Inc. Project W-026. WRAP I Operational Test - LLW Glovebox

ATTACHMENT 8 - TEST PLAN, LLW GLOVEBOX AUTOMATIC TEST CASE 4

5.3.4 PERFORM Section VII.E, "DELIO WASTE DRUM", of operating procedure as follows:

5.3.4.1 REDLINE procedure as needed during performance.

$\int$ 5.3.4.2 VERIFY delid fixture removes lid from drum OTP-LLW-WP-04, and returns to it's "raised/parked" position with the lid.

5.3.4.3 VERIFY results of performance are acceptable.

TO Initials/Date:

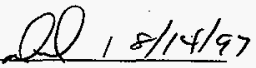

5.3.5. PERFORM Section VII.F, "EMPTY WASTE DRUM ONTO SORTING TABLE", of operating procedure as follows:

5.3.5.1 REDLINE procedure as needed during performance.

5.3.5.2 VERIFY tipper raises to dump contents of drum OTP-LLW-WP-04 onto sorting table, and returns to it's "lowered" position with the empty drum.

5.3.5.3 VERIFY DMSS0312 Parent PIN is OTP-LLW-WP-04, Sample $=$ NO and Compliant? $=$ NO.

5.3.5.4 VERIFY results of performance are acceptable.

TD Initials/Date:

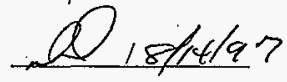


ATTACHMENT 8 - TEST PLAN, LLW GLOVEBOX AUTOMATIC TEST CASE 4

5.3.6 PERFORM Section VII.G, "REMOVE NON-COMPLIANT PACKETS FROM WASTE", of operating procedure as follows:

5.3.6.1 REDLINE procedure as needed during performance.

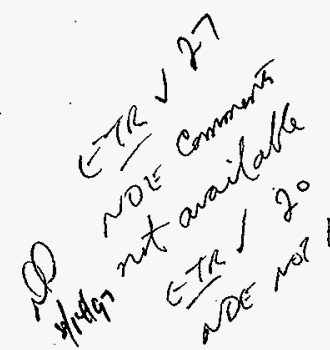

5.3.6.2 IDENTIFY three non-compliant packages using NDE hard copy from receiving, DMS NDE comments and Packet NDE:

1) VIEW DMS NDE comments on DMSS0312

2) PERFORM WRP1-OP-0706, PACKET NDE OPERATION in it's entirety on at least one non-compliant package.

5.3.6.3 VERIFY Packet bar code label 0TP6-97-000004 is applied to the lead brick in two plastic bags wrapped with tape, the bar code is read, and the packet is placed on the transfer stand:

1). DISPLAY DMSSO312 LLW Non-Compliant Screen after the barcodes are read.

2). ENTER the following for PIN OTP6-97-000004:

Group: PB

Waste Description: LEAD BRICK

5.3.6.4 VERIFY Packet bar code 7abel 0TP6-97-000005 is applied to the bottle of solvent and bottle of oil wrapped together in cloth and tape, the bar code is read, and packet is placed on the transfer stand.

1) REFRESH DMSS0312 LLW Non-Compliant Screen after the barcodes are read.

2) ENTER the following for PIN OTP6-97-000005:

Group: LQ Waste Description: BOTTLE OF SOLVENT AND BOTTLE OF OIL 
Waste Management Federal Services of Hanford, Inc. Project $W-026$. WRAP I

Operational Test - LLW Glovebox

HNF-SD-W026-OTR-015

Rev: 0

Page 76 of 91

ATTACHMENT 8 - TEST PLAN, LLW GLOVEBOX AUTOMATIC TEST CASE 4

5.3.6.5 VERIFY Packet bar code label 0TP6-97-000006 is applied to the bottle of acid, bar code is read. and packet is placed on transfer stand.

1) REFRESH DMSS0312 LLW Non-Compliant Screen after the barcodes are read.

2) ENTER the following for PIN 0TP6-97-000007:

Group: LQ

Waste Description: BOTTLE OF ACID

5.3.6.6 UPDATE sorted drum data in DMS:

1) SELECT DMSS0312 "Physcomp" screen.

- $\quad$ DELETE HAZARDOUS CONSTITUENTS record

- CHANGE PLASTIC/POLYURATHANE volume percent to 30

- CHANGE CLOTH/RAGS/NYLON volume percent tó 70 .

2) SELECT DMSSO312 "Chemcomp" screen.

- DELETE all records.

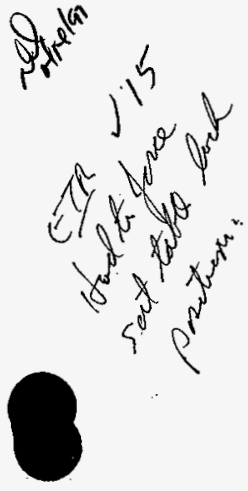

5.3.6.7 VERIFY results of performance are acceptable.

TD Initialș/Date:

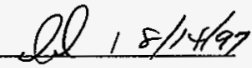

5.3.7 PERFORM Section VII.H. "RETURN COMPLIANT WASTE TO WASTE DRUM", of operating procedure as follows:

5.3.7.1 REDLINE procedure as needed during performance.

5.3.7.2 VERIFY results of performance are acceptable.

TO Initials/Date:

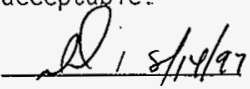




\section{ATTACHMENT 8 - TEST PLAN, LLW GLOVEBOX AUTOMATIC TEST CASE 4}

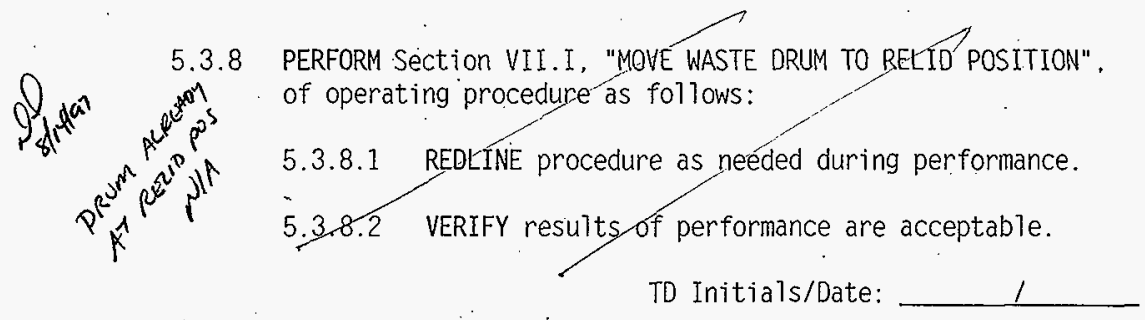

5.3.9 PERFORM Section VII.J, "RELID WASTE DRUM", of operating procedure as follows:

5.3.9.1 REDLINE procedure as needed during performance.

5.3.9.2 VERIFY drum OTP-LLW-WP-04 is re-7idded with c-clips attached.

5.3.9.3 VERIFY results of performance are acceptable.

TD Initials/Date:

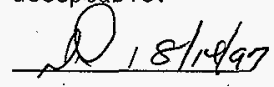

5.3.10 PERFORM Section VII.K, - TRANSFER-WASTE DRUM TO SUPERCOMPACTOR", of operating procedure as follows:

5.3.10.1 REDLINE procedure as needed during performance.

5.3.10.2 PERFORM appropriate Sections of WPRI-OP-0715 to ensure Supercompactor is in Automatic and ready to receive drums.

\subsubsection{VERIFY drum OTP-LLW-WP-04 is delivered to} Supercompactor and automatical1y compacted.

5.3.10.4 VERIFY results of performance are acceptable.

TO Initials/Date:

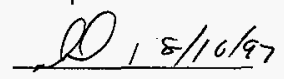



Waste Management Federa? Services of Hanford, Inc. Project $W-026$. WRAP I Operational Test - LLW Glovebox

HNF-SD-W026-OTR-015

Rev: 0

Page 79 of 91

ATTACHMENT 8 - TEST PLAN, LLW GLOVEBOX AUTOMATIC TEST CASE 4

5.4.3 PERFORM Section VII.G. "RECORD COMPACT DRUM (PUCK) DATA", Of operating procedure as follows:

5.4.3.1 REDLINE procedure as needed during performance.

5.4.3.2 ENTER on DMSSO315. the following data for puck OTP004 when directed by the operating procedure:

- WASTE DESCRIPTION:

- $\quad$ PUCK HEIGHT:

5.4.3.3 VERIFY results of performance are acceptable.

TD Initials/Date:

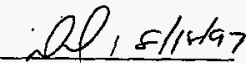

0

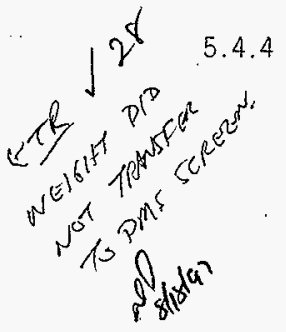

PERFORM SEction VII.D, "MOVE PUCK FROM STORAGE TO LOAD OUT",

of operating procedure as follows:

5.4.4.1 REDLINE procedure as needed during performance.

5.4.4.2 VERIFY puck OTP002 is Toaded into overpack drum OTP-LLW-0D-02 at Exit port.

5.4.4.3 VERIFY results of performance are acceptaple.

TD Initials/Date:

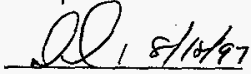

5.4.5 PERFORM Section VII.G, "RECORD COMPACT DRUM (PUCK) DATA", of operating procedure as follows:

5.4.5.1 REDLINE procedure as needed during performance.

5.4.5.2 ENTER on DMSS0315, the following data for puck OTP002 when directed by the operating procedure:

- WASTE DESCRIPTION:

- PUCK HEIGHT:

5.4.5.3 VERIFY results of performance are acceptable.

TD Initials/Date:

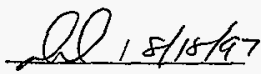


Waste Management Federal Services of Hanford, Inc. Project $W$-026. WRAP I

Operational Test - LLW Glovebox

HNF-SD-W026-OTR-015

Rev: 0

Page 80 of 91

\section{ATTACHMENT 8 - TEST PLAN, LLW GLOVEBOX AUTOMATIC TEST CASE 4}

5.4.6 PERFORM SEction VII.H, "REMOVE OVERPACK FROM GLOVEBOX", of operating procedure as follows:

5.4.6.1 REDLINE procedure as needed during performance.

5.4.6.2 ENTER on DMSS0315, the following data for puck OTP002 when directed by the operating procedure:

- wASTE DESCRIPTION: /

- filleR TYPE: \%

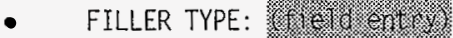

5.4:6.3 SELECT "SUM WASTE RECORDS" on DMSS0315 to sum the puck data.

5.4.6.4 VERIFY results of performance are acceptable

To Initials/Date:

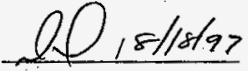

5.5 REFERENCE procedure WRP1-OP-0714, LOW LEVEL WASTE (LLW) SORTING GLOVEBOX OPERATION:

5.5.1 PERFORM Section VII.L, "PLACE TRANSFER STAND IN RWM TRANSFER DRUM", of operating procedure as fol7ows:

5.5.1.1 REDLINE procedure as needed during performance.

5.5.1.2. VERIFY transfer stand is placed in transfer drum OTP-LLWR-TD-021.

5.5.1.3 VERIFY results of performance are acceptable.

TD Initials/Date:

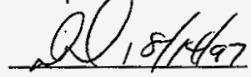


Waste Management Federal Services of Hanford. Inc. Project W-026. WRAP I

\section{ATTACHMENT 8 - TEST PLAN, LLW GLOVEBOX AUTOMATIC TEST CASE 4}

\subsubsection{PERFORM Section VII.M. "REMOVE DRUM FROM RWM TRANSFER PORT} (107-D0-07-105)", of operating procedure as follows:

5.5.2.1 REDLINE procedure as needed during performance.
5.5.2.2 VERIFY transfer drum OTP-LLWR-TD-02 is closed and removed from port.

5.5.2.3 VERIFY results of performance are acceptabie.

To Initials/Date:
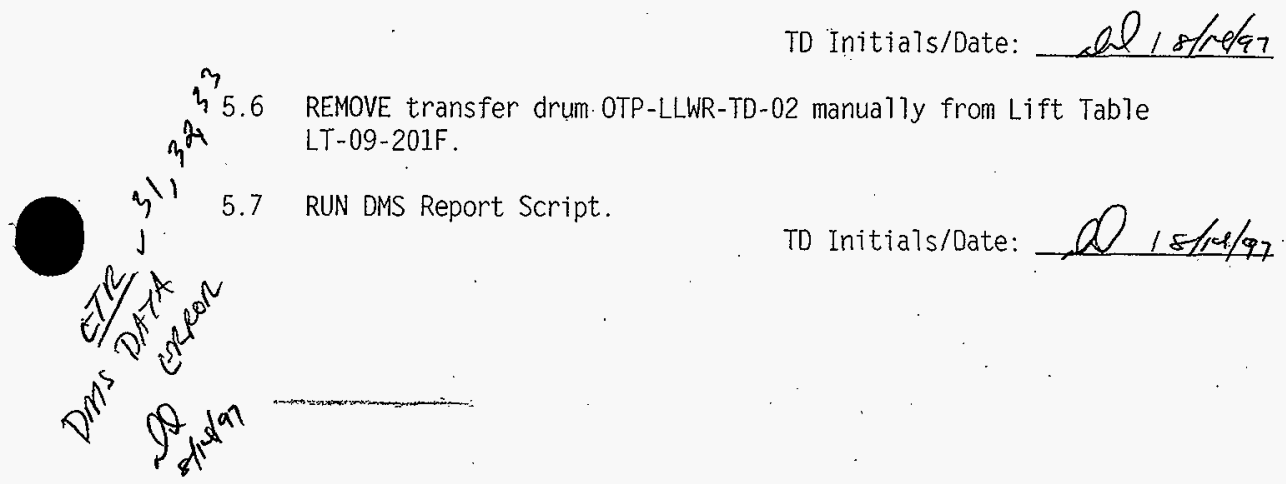


\section{ATTACHMENT 9 - TEST PLAN, LLW GLOVEBOX RESTART}

\subsection{GENERAL DESCRIPTION}

1.1. This test plan provides instructions for testing restart of the LLW Glovebox. These operations will be tested using procedures WRP1-0P-0702, Restart LLW Glovebox Operation, WRP1-OP-0713 "LLW Entry Glovebox Operation," and WRP1-OP-0714 "Low Level Waste (LLW) Sorting Glovebox Operation, thereby also performing the required procedure validation for sections performed. The sequence of events is as follows:

1.1.1 85 gallon shipping overpack 0TP-LLW-0D-05, with 55 gallon LLW drum OTP-LLW-WP-05 inside, is connected to the LLW giovebox entry port and drum OTP-LLW-WP-05 is lifted into the LLW Entry glovebox.

1.1.2 Drum 0TP-LLW-WP-05 is checked for external contamination.

1.1.3 85 gallon drum OTP-LLW-OD-05 is closed and removed from the LLW Entry port and from lift table LT-09-202A.

1.1.4 Empty 85 gallon drum OTP-LLW-OD-05 (from entry port) is placed on lift table LT-09-202C (LLW Exit).

1.1.5 Drum OTP-LLW-OD-05 is connected to the LLW glovebox exit port and the port is opened.

1.1.6 Drum 0TP-LLW-WP-05 is lifted by the LLW entry glovebox hoist. lowered through transfer port, and placed on transfer car.

1.1.7 Drum 0TP-LLW-WP-05 is moved to the delidder and sawing of the clam-band is begun.

1.1.8 Prior to completion of sawing the clamp band, the $L L W$ Emergency Stop is pressed.

1.1.9 The glovebox restart procedure is used to restore the glovebox components to operational status.

1.1:10 The test is terminated and all equipment removed from the gloveboxes. 
Waste Management Federal Services of Hanford. Inc.

HNF-SD-W026-0TR-015

Project $W-026$, WRAP I

Rev: 0

Operational Test - LLW Glovebox

Page 83 of 91

\section{ATTACHMENT 9 - TEST PLAN, LLW GLOVEBOX RESTART}

\subsection{SAFETY}

\subsection{Reference OTP Section 8. SAFETY.}

3.0 TOOLS, EQUIPMENT AND SUPPLIES

- Drum handling (manual) equipment or Pallet fork truck

- Spill Kit/Decontamination Equipment (oils/chemicals)

- Tape and rags

- Other equipment, material or protective clothing (as required)

\subsection{TEST PREREQUISITES}

4.I VERIFY OTP Section 11.0. PREREQUISITES are complete.

4.2 SET initial conditions as follows:

4.2.1 55 gallon LLW drum OTP-LLW-WP-05, in shipping overpack 0TP-LLW-0D-05, is on-7ift table LT-09-202A.

4.2.2 The PCS man op program has sent an AGVPCS AGVL message for the AGV located at AGVLLWENTR (LT-09-202A) with-drum 0TP-LLW-0D-05 (sets LCU conditions have been set for drum OTP-LLW-OD-05 on Tift table LT-09-202A).

4.2.3 Empty transfer drum OTP-LLWR-TD-05 is on lift table LT-09-203A.

4.2.4 The PCS man_op program has sent an AGVPCS AGVL message for the AGV located at AGVLLWRWX (LT-09-203A) with drum OTP-LLWR-TD-05 (sets LCU conditions for drum 0TP-LLWR-TD-05 on lift table (T-09-203A). 
Waste Management Federai Services of Hanford. Inc. Project $W-026$. WRAP I

\section{ATTACHMENT 9 - TEST PLAN, LLW GLOVEBOX RESTART}

4.3 UPDATE PCS Tracking Table as follows:

4.3.1 Drum 0TP-LLW-WP-05 in shipping overpack 0TP-LLW-00-05 at LT-09-202A.

4.3.2 Drum OTP-LLWR-TD-05 at $L T=09-203 A$.

\begin{tabular}{|c|c|c|}
\hline \multicolumn{3}{|c|}{ PCS TRACKING TABLE FOR LLW LIFT TABLES } \\
\hline \multirow{2}{*}{ LIFT TABLE } & PIN & Registers \\
\cline { 2 - 3 } & N32: $35-41$ & N32: 46 bit 12 \\
\hline LT-09-202A & N32: 47-53 & N32: 58 bit 12 \\
\hline LT-09-203A & Rresent (=1) \\
\hline
\end{tabular}

4.4 TEST PREREQUISITES are complete and acceptable.

To Initials/Date: 
Waste Management Federal Services of Hanford, Inc. Project $W-026$, WRAP I

operational Test - LLW Glovebox

HNF-SD-W026-OTR-015

Rev: 0

Page 85 of 91

\section{ATTACHMENT 9 - TEST PLAN, LLW GLOVEBOX RESTART}

\subsection{PROCEDURE}

5.1 REFERENCE procedure WRP1-OP-0713. LLW ENTRY GLOVEBOX OPERATION:

5.1.1 PERFORM Section VII.A, "INITIALIZE SYSTEM", of operating procedure as follows:

5.1.1.1 REDLINE procedure as needed during performance.

5.1.1.2 VERIFY results of performance are acceptable.

TD Initials/Date:

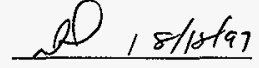

5.1.2 PERFORM Section VII.B, . "DRUM ENTRY TO LLW GLOVEBOX", of operating procedure as follows:

5.1.2.1 REDLINE procedure as needed during performance.

5.1.2.2 VERIFY overpack drum OTP-LLW-OD-05 OPEN and inner drum 0TP-LLW-WP-05 on hoist at "SWAB" position.

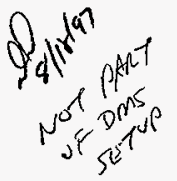

VERIFY DMSS0311 indicates overpack drum OTP-LLW-OD-05 and inner drum OTP-LLW-WP-05 at glovebox with message "Sorting Required".

5.1.2.4 VERIFY results of performance are acceptable.

TD Initials/Date:

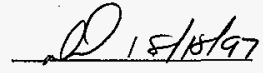


Waste Management Federal Services of Hanford, Inc. Project W-026. WRAP I

ATTACHMENT 9.- TEST PLAN, LLW GLOVEBOX RESTART

5.1.3 PERFORM Section VII.C. "CLOSE ENTRY PORT", of operating procedure as follows:

5.1.3.1. REDLINE procedure as needed during performance.

5.1.3.2 VERIFY ENTRY port is CLOSED. overpack drum OTP-LLW-OD-05 is lowered from port and checked for contamination.

5.1.3.3 SELECT "CONTAMIN. DRUM READY FOR PICKUP" when prompted by 0IU.

5.1.3.4 VERIFY overpack drum OTP-LLW-OD-05 is removed from Lift Table LT-09-202A.

5.1.3.5 VERIFY results of performance are acceptable.

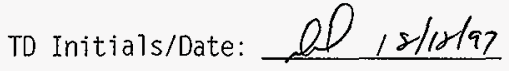

5.1.4 MOVE overpack drum OTP-LLW-OD-05 (manually) to AGV end of Lift Table LT-09-202C at LLW Exit Port.

TO Initials/Date:
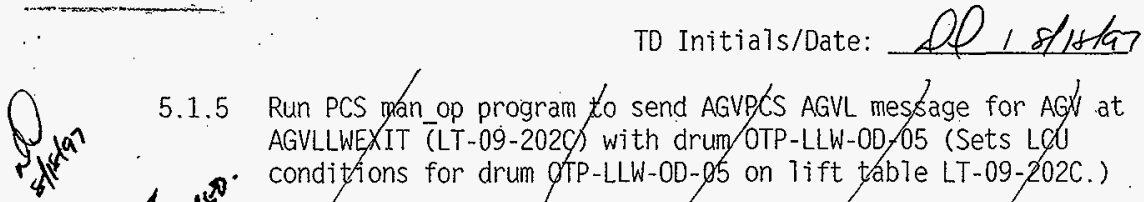

5.1 .5

Run PCS man_op program to send AGVPCS AGVL message for AGX at AGVLLWEXIT (LT-09-202C) with drum 0TP-LLW-00-05 (Sets LCU conditions for drum ofP-LLW-00-05 on iift table LT-09-202C.)
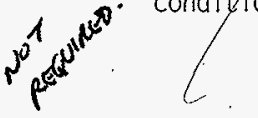

TD/Initials/Date:

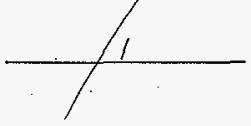




\section{ATTACHMENT 9 - TEST PLAN, LLW GLOVEBOX RESTART}

5.2 REFERENCE procedure WRP1-OP-0716, LLW EXIT GLOVEBOX OPERATION:

5.2.1 PERFORM Section VII.A. "INITIALIZE SYSTEM", of operating procedure as follows:

5.2.1.1 REDLINE procedure as needed during performance.

5.2.1.2 VERIFY results of performance are acceptable.

TD Initials/Date:

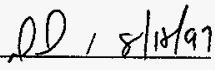

5.2.2 PERFORM Section VII.B, "EXIT DRUM ACCESS TO LLW GLOVEBOX", of operating procedure as follows:

5.2.2.1 REDLINE procedure as needed during performance.

5.2.2.2 VERIFY overpack drum OTP-LLW-OD-0\% conpected to LLW
Exit Port and OPEN.

5.2.2.3 VERIFY results of performance are acceptable.

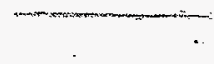

To Initials/Date: Ll 1 s/18/a?

5.3 REFERENCE procedure WRP1-OP-0713, LLW ENTRY GLOVEBOX OPERATION: $\begin{array}{lll}D_{\text {Jal }} & 5.3 .1 & \text { PERFORM Section VII.D, "MOVE DRUM TO DRUM TRANSFER CAR", of } \\ \text { operating procedure as follows: }\end{array}$

5.3.1.1 REDLINE procedure as needed during performance.

5.3.1.2 VERIFY drum OTP-LLW-WP-05 is positioned over transfer port 107-00-07-102 and lowered onto drum transfer car.

5.3.1.3 VERIFY results of performance are acceptable.

TD Initials/Date: $\mathrm{el} / \mathrm{s} / \mathrm{k} / \mathrm{a}$ 
Waste Management Federal Services of Hanford, Inc. Project $W$-026. WRAP I

\section{ATTACHMENT 9 - TEST PLAN, LLW GLOVEBOX RESTART}

5.4 REFERENCE procedure WRP1-OP-0714, LOW LEVEL WASTE (LLW) SORTING GLOVEBOX OPERATION:

5.4.1 PERFORM Section VII.B, "TRANSFER WASTE DRUM TO DELID POSITION", of operating procedure as follows:

5.4.1.1 REDLINE procedure as needed during performance.

5.4.1.2 VERIFY drum OTP-LLW-WP-05 at "DELID" position.

5.4.1.3 VERIFY results of performance are acceptable.

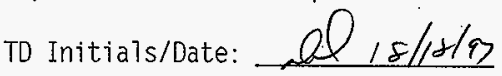

5.4.2 PERFORM Section VII.C, "GRIP WASTE DRUM WITH DRUM TIPPER (107-DH-07-103)". of operating procedure as follows:

5.4.2.1 REDLINE procedure as needed during performance.

$S_{1} j a ̂$

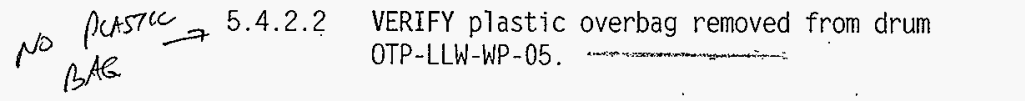

5.4.2.3 VERIFY drum tipper Towered and tipper grab closed around drum OTP-LLW-WP-05.

5.4.2.4 VERIFY results of performance are acceptable.

TD Initials/Date:

\section{NOTE}

The exact point at which EMERGENCY STOP is pressed is not critical, so long as the saw has not completed the cutting sequence. 
Waste Management Federal Services of Hanford, Inc.

\section{ATTACHMENT 9 - TEST PLAN, LLW GLOVEBOX RESTART}

5.4.3 PERFORM Section VII.D, "SAW WASTE DRUM CLAMP BAND", of operating procedure as follows:

5.4.3.1 REDLINE procedure as needed during performance.

5.4.3.2 WHEN the saw is cutting the clamp band.

- THEN, PRESS EMERGENCY STOP switch (107-HS-07-315) on DMS console (107-TE-12-103).

TD Initials/Date:

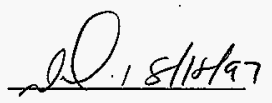

5.4.3.3 VERIFY LLW Glovebox operations STOP.

TD Initials/Date:

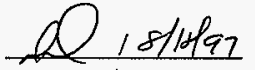

5.5 REFERENCE procedure WRP1-OP-0702, Restart LLW Glovebox Operation:

5.5.1 PERFORM Section VII.A. "DETERMINE GLOVEBOX EQUIPMENT STATUS", of operating procedure as follows:

5.5.1.1 REDLINE procedure as needed during performance.

5.5.1.2 VERIFY results of performance are acceptable.

TO Initials/Date: 1 , \&/1 $/ 97$

5.5.2 PERFORM Section VII.B. "RETURN ENTRY GLOVEBOX TO OPERATION", of operating procedure as follows:

5.5.2.1 REDLINE procedure as needed during performance.

5.5.2.2 VERIFY results of performance are acceptable.

To Initials/Date: 00, s/1s/a? 
Waste Management Federal Services of Hanford, Inc.

Project $W-026$, WRAP I

Operational Test - LLW Glovebox

HNF-SD-W026-0TR-015

Rev: 0

Page 90 of 91

\section{ATTACHMENT 9 - TEST PLAN, LLW GLOVEBOX RESTART}

5.5.3 PERFORM Section VII.C. "RETURN SORTING GLOVEBOX TO OPERATION", of operating procedure as follows:

5.5.3.1 REDLINE procedure as needed during performance.

5.5.3.2 VERIFY resu7ts of performance are acceptable.

To Initials/Date:

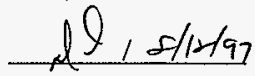

5.5.4 PERFORM Section VII.D, "RETURN EXIT GLOVEBOX TO OPERATION", of operating procedure as follows:

5.5.4.1 REDLINE procedure as needed during performance.

5.5.4.2 VERIFY results of performance are acceptable.

To Initials/Date:

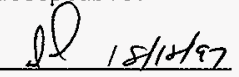

5.6 PERFORM sections of the following procdures as required to remove drums from glovebox and ports:

WRP1-OP-0713, LLW Entry Glovebox Operation WRP1-0P-0714. LLW Sorting Glovebox Operation WRP1-OP-0715, LLW Supercompactor Operation WRP1-OP-0716. LLW Exit GTovebox Operation WRP1-OP-0719, LLW Gloveboxes Manual Operation

5.6.1 VERIFY results of performance are acceptable.

TD Initials/Date:

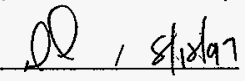

5.7 REMOVE a17 drums (manually) from LLW glovebox lift tables.

TD Initials/Date:

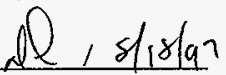

5.8 INFORM Operations Management testing is complete.

TO Initials/Date:

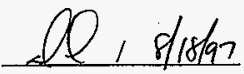




\section{Author}

J. J. Shaffer III

Print Mame/Signature

APPROVAL DESIGNATOR so

PROCEDURE APPROVAL SIGNATURES
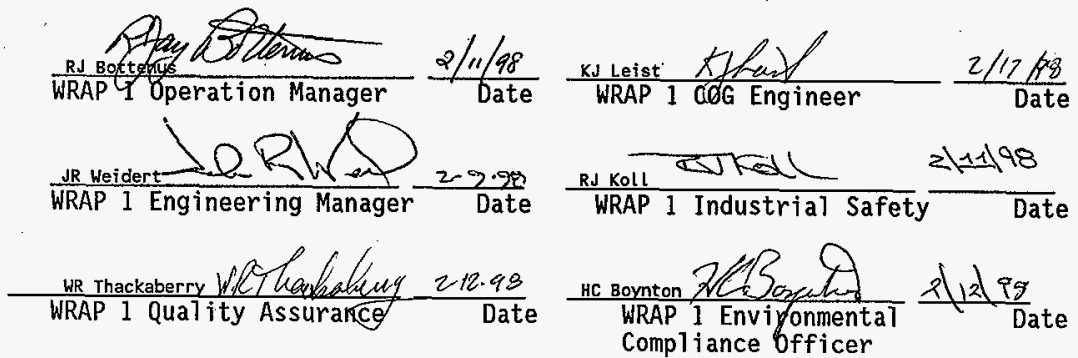
ATP_YN : N

Test : HNF-SD-W026-OTP-015

Step : A4,5.1,A5,5.2.1 Equipment Tag \#: CR-07-101C

Specification Section : 13462

Test Witness/Author :

Test Operator:

\section{Exception :}

Revision :

Date:

\begin{tabular}{ll} 
Responsible Company: & WMH \\
\hline Responsible Subcontractor: & \\
\hline Responsible Individual: & Leist \\
\hline
\end{tabular}

\section{Exception Description :}

Froist could not be parked at" "load" position due to mechanical interference with manipulator.

\section{Exception Disposition :}

\section{Exception Status :}

9-3-97 Duplicate of \#148. Closed.

Exception Closed By : Leist Fffun

\section{APPROVAL OF RESOLUTION}

Contractor Representative: (Only as needed)

WMH Representative:

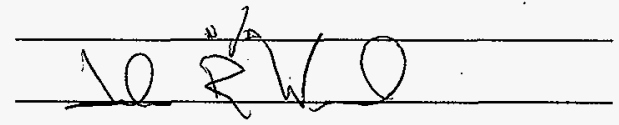

Additional Approval:

(Only as needed)
Date:

Date: $12-10-97$

Date:

Date: 
ATP_YN : N

SW

Test : HNF-SD-W026-OTP-015

Step : See Below Equipment Tag \#: TC-07-101

\section{Exception :}

Revision :

Date:

Specification Section: 13462

Test Witness/Author :

Dunlap

\section{Test Operator:}

Responsible Company: WMH

Responsible Subcontractor:

\section{Responsible Individual: Lane}

\section{Exception Description :}

Steps A4, 5.5.15, 5.1.19

Transfer car position indication on OIU-103B does not correspond to proper location of car except for "entry."

\section{Exception Disposition :}

\section{Exception Status :}

9-3-97 MP Lane to fix so that messaging triggered by location of car, retest. 9-25-97 - Code moditications completed, waiting on opportunity to test. 12-26.97: Retested 12-20-97. Sat is Factory.
Closed.

Exception Closed By: Tnlciver Pdow.

Date : $12-26-97$

\section{APPROVAL OF RESOLUTION}

Contractor Representative: (Only as needed)

WMH Representative:

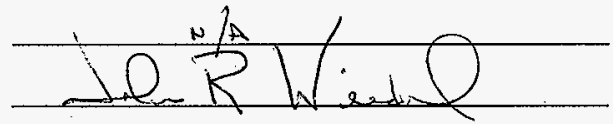

Additional Approval:

(Only as needed)
Date:

Date: $12 \cdot 26.97$

Date:

Date: 
ATP_YN : N

Test : HNF-SD-W026-OTP-015

Step : A4, 5.1.16 Equipment Tag \#: RT-07-101

\section{Exception :}

Revision :

Date:

Specification Section : 13462

Test Witness/Author :
Dunap

Test Operator:

$\begin{aligned} & \text { Responsible Company: } \\
& \text { Responsible Subcontractor : }\end{aligned}$
\begin{tabular}{l} 
Responsible Individual: \\
\hline
\end{tabular}

Exception Description:

After pertorming saw operation to remove drum lid, a tool is needed to grab the ring.

\section{Exception Disposition :}

\section{Exception Status :}

9-3-97Duplicate of 220. Closed.

Exception Closed By : Leist

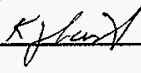

Date : $9 / 3 / 97$

\section{APPROVAL OF RESOLUTION}

Contractor Representative: (Only as needed)

WMH Representative:

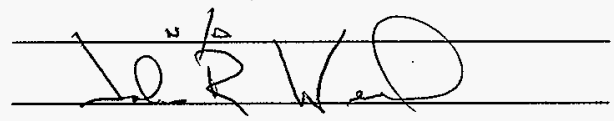

Additional Approval:

(Onlyas needed)
Date:

Date: $12-10-87$

Date:

Date:

FDNW Representative:

N

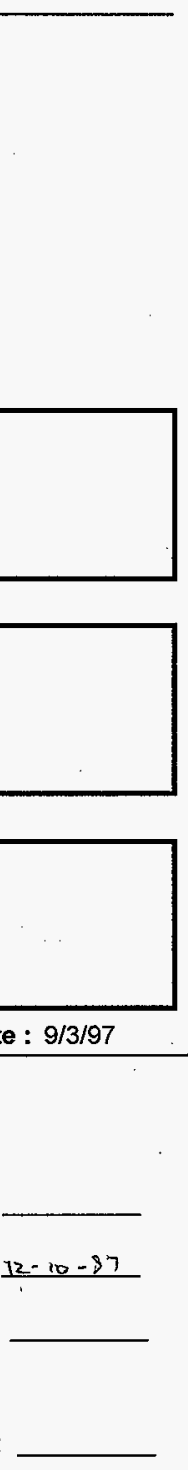


ATP_YN : N

Exception :

Test : HNF-SD-W026-OTP-015

Step : See Below Equipment Tag \#: DH-07-103

Revision :

Date:

Specification Section : 13462

Test Witness/Author:

Dunaap

\section{Test Operator:}

\begin{tabular}{ll} 
Responsible Company: & WMH \\
\hline Responsible Subcontractor: & \\
\hline Responsible Individual: & Warmenhoven \\
\hline
\end{tabular}

Exception Description :

Steps A4, 5.1.20, A5, 5.6.2, A6, 5.4.2

The pressure switch which indicates proper tipper grab pressure does not function. Drum is loose in grab arms.

\section{Exception Disposition :}

\section{Exception Status :}

9-3-97 Duplicate of 250 . Closed.

Exception Closed By : Leist

Date : $9 / 3 / 97$

\section{APPROVAL OF RESOLUTION}

Contractor Representative: (Only as needed)

WMH Representative:

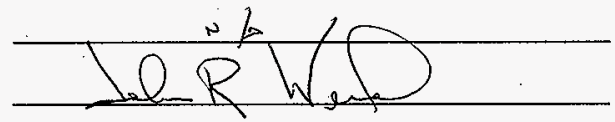

Additional Approval:

(Onily as needed)
Date:

Date: $12-10-97$

Date:

Date: 
ATP_YN : N

SW

Test : HNF-SD-W026-OTP-015

Step : A4, 5.1.25

Equipment Tag \#: CR-07-101D

\section{Exception :}

Revision :

Date:

\section{Specification Section : 13462}

Test Witness/Author :

$$
\text { Dunlap }
$$

\section{Test Operator:}

Responsible Company: $\quad$ WMH

Responsible Subcontractor:

Responsible Individual: Lane

\section{Exception Description :}

OIU-103C would indicate "system initialized" with the hoist not at the compact receipt position. The subscreen did indicate the hoist out of position (red).

\section{Exception Disposition :}

\section{Exception Status :}

9-3-97MP Lane to investigate, resolve. 9-20-97-Code examined. Hoist must be at compact receipt; with all three hoist position switches activated, before system is initialized. Once system is initialized, it remains so until switched back to maintenance mode. OIU also examined and OIU diagnostics screen accurately reflects hoist status. This exception is therefore closed.

\section{Exception Closed By: Lane Thieles Pare.}

Date : 9/20/97

\section{APPROVAL OF RESOLUTION}

Contractor Representative: (Only as needed)

WMH Representative:

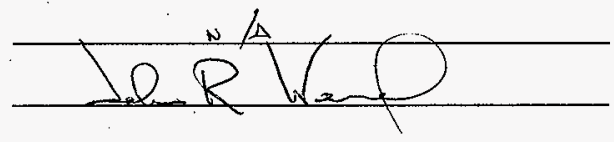

Additional Approval:

(Only as needed)
Date:

Date: $12-10-97$

Date:

Date: 
ATP YN : N

SW

Test : HNF-SD-W026-OTP-015

Step : A4, 5.1.30

Equipment Tag \#: TC-07-101/M1
Exception :

Revision :

Date:

Specification Section: 13462

Test Witness/Author :

Dunlap

Test Operator:

Responsible Company: WMH

Responsible Subcontractor:

Responsible Individual: Lane

Exception Description :

When attempting transfer of a drum to the compactor, the transfer car would not engage or start

\section{Exception Disposition :}

Exception Status :

9-3-97MP Lane to investigate, resolve. $11.24-97$ - pedoly to test. 12-26.97. Retested 12-23-97. Transfir effected in semi-auto pode. Test exceptian olused.

Exception 'Closed By :

Miciar Pdaes

Date : $12.26-97$

\section{APPROVAL OF RESOLUTION}

Contractor Representative: (Only as needed)

WMH Representative:

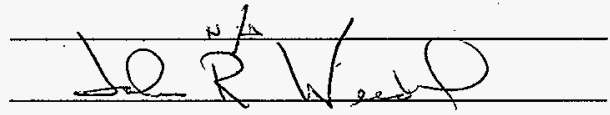

Additional Approval:

(Oniy as needed)
Date:

Date: $\underline{12-26-97}$

Date:

Date: 
ATP_YN : N

SW

Test : HNF-SD-W026-106

Step : A4, 5.1.31

Equipment Tag \#: DO-07-101
Exception :

Revision :

Date:

Specification Section : 13462

Test Witness/Author:

Dunlap

Test Operator:

Responsible Company: WMH

Responsible Subcontractor:

Responsible Individual: Lane

Exception Description:

The "close port" sequence did not complete. The lift table did not lower to close the port.

\section{Exception Disposition :}

\section{Exception Status :}

9-7-97 MPLane to investigate. $11.24-97$ - Ready to test $12-276-97-$ Retested Sat is Factory $12-20-97$. Closed.

Exception Closed By: Miciuel Pdor

Date : $12-20-97$

\section{APPROVAL OF RESOLUTION}

Contractor Representative: (Only as needed)

WMH Representative:

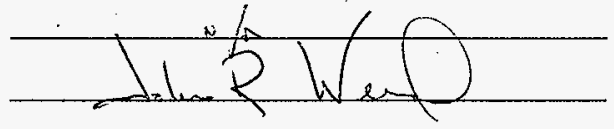

Additional Approval:

(Oniy as needed)
Date:

Date: $12-26 \cdot 97$

Date:

Date: 
ATP_YN : N

SW

Test : HNF-SD-W026-OTP-015

Step : A5, 5.2 .2

Equipment Tag \#: DO-07-105
Exception :

Revision :

Date:

Specification Section : 13462

Test Witness/Author :

Dunlap

Test Operator:

Responsible Company: WWH

Responsible Subcontractor:

Responsible Individual: Lane

Exception Description :

Port would not open in auto mode.

\section{Exception Disposition :}

Retested Port - operation sat. No reason determined for fall to open

\section{Exception Status :}

crosed

Exception Closed By: Lane ma Eles

Date : $9 / 3 / 97$

\section{APPROVAL OF RESOLUTION}

Contractor Representative: (Only as needed)

WMH Representative:

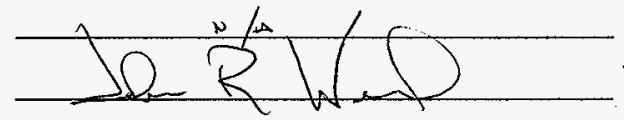

Additional Approval:

(Only as needed)
Date:

Date: $12-10-97$

Date:

Date: 
ATP_YN : N

SW

Test : HNF-SD-W026-015

Step : See Below

Equipment Tag \#: DO-07-101
Exception :

Revision :

Date:

Specification Section : 13462

Test Witness/Author :

Düntap

Test Operator:

Responsible Company: $\quad$ WMH

Responsible Subcontractor:

Responsible Individual: Lane

Exception Description :

Steps A5, 5.3.2, A6, 5.1.2, A8, 5.2.2

Lid in position sensor activated too soon and prevented lift table from opening port - sequence failed.

\section{Exception Disposition :}

\section{Exception Status :}

9-3-97 Duplicate of \#54. Closed.

Exception Closed By: Lane Micrees Pro

Date : $9 / 3 / 97$

\section{APPROVAL OF RESOLUTION}

Contractor Representative: (Only as needed)

WMH Representative:

Additional Approval:

(Oniy as needed)

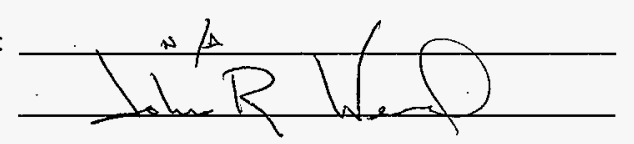

Date:

Date: $12-10-97$

Date:

Date: 
ATP_YN : N

Exception :

Test : HNF-SD-W026-OTP-015

Step : See Below Equipment Tag \#: DO-07-101

Revision :

Date:

Specification Section : 13462

Test Witness/Author :

Test Operator:

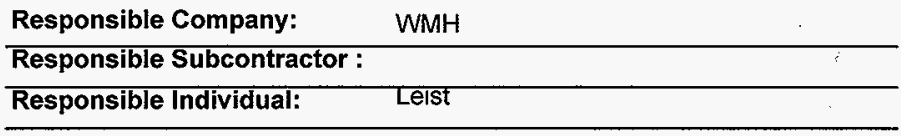

Exception Description :

Steps A5,5.3.3, A6, 5.1.2

The overpack drum lid did not detach from the port when the drum started the exit sequence.

\section{Exception Disposition :}

\section{Exception Status :}

$12 / 17 / 97$ New retention desigh ineplementud. Systam to be tesbed ad vern Foed.

Exception Closed By :

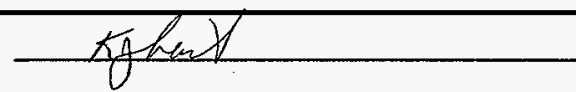

Date : $12 / 31 / 97$

\section{APPROVAL OF RESOLUTION}

Contractor Representative: (Only as needed)

WMH Representative:

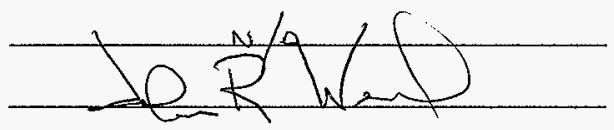

Additional Approval:

(Only as needed)

FDNW Representative:
Date:

Date: $12 m+9 n$

Date:

Date: 
ATP_YN : N

SW

Test : HNF-SD-W026-OTP-015

Step : A5, 5.3.3

Equipment Tag \#: DO-07-101

\section{Exception :}

Revision :

Date:

Specification Section : 13462

Test Witness/Author:

Test Operator:

$\begin{array}{ll}\text { Responsible Company: } & \text { WMH } \\ \text { Responsible Subcontractor: } & \\ \text { Responsible Individual: } & \text { Lane }\end{array}$

Exception Description :

The lift table did not lower enough to close the port-sequence falled.

\section{Exception Disposition :}

\section{Exception Status :}

9-3-97 Duplicate of \#257. Closed.

Exception Closed By : Lane noleves Pate : 9/5/97

\section{APPROVAL OF RESOLUTION}

Contractor Representative: (Only as needed)

WMH Representative:

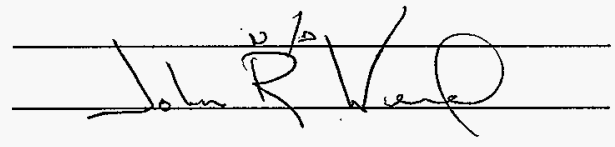

Additional Approval:

(Only as needed)
Date:

Date: $12 \cdot 10+97$

Date:

Date: 
ATP_YN : N

SW

Test : HNF-SD-W026-OTP-015

Step : A5, 5.3.3

Equipment Tag \#: DO-07-101
Exception :

Revision :

Specification Section : 13462

Test Witness/Author :

Date:

\section{Test Operator:}

Responsible Company: WMH

Responsible Subcontractor:

Responsible Individual: Lane

Exception Description :

After the exit sequence, message "drum pickup in progress" was not displayed.

\section{Exception Disposition :}

\section{Exception Status :}

9-3-97 MPLane to review, retest. 9-25-97-Code examined. Message code present. The following conditions; 1) Lift table at AGV height, 2) "Drum at End of Conveyor" switch activated and 3) Not "Notify AGV of Pickup" must be met before message will be displayed. Retested - message displayed for both clean, contaminated drums. Closed.

Exception Closed By: Lane Thicheses Pae

Date : $9 / 25 / 97$

\section{APPROVAL OF RESOLUTION}

Contractor Representative: (Only as needed)

WMH Representative:

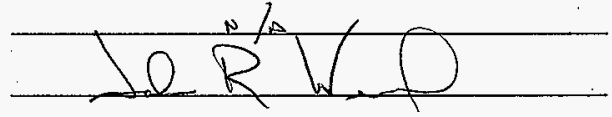

Additional Approval:

(Only as needed)

FDNW Representative:

$N \not$
Date:

Date: $12-10-57$

Date:

Date: 
ATP_YN : N

SW

Test : HNF-SD-W025-OTP-015

Step : A5, 5.6.1

Equipment Tag \#: TC-07-101
Exception :

Revision :

Date:

Specification Section : 13462

Test Witness/Author:

Dunlap

Test Operator:

Responsible Company: WMH

Responsible Subcontractor: SII

Responsible tnaividual: tane

\section{Exception Description :}

When the transfer car was sent to the delid position from the entry position, the display momentarily said "transfer can moving to delid position," but then said "transfer car at entry position" until the car reached the delid position.

\section{Exception Disposition :}

\section{Exception Status :}

$11.24-97$ pesdy to tost. hry, i2-26-97 Reteoted satistactory

12.20-97. C108-1.

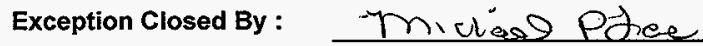

Date : $12-2<-97$

\section{APPROVAL OF RESOLUTION}

Contractor Representative: (Oniy as needed)

WMH Representative:

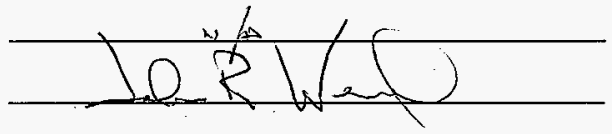

Additional Approval:

(Oniy as needed)
Date:

Date: $12-26-97$

Date:

Date:

FDNW Representative:

N

. 
ATP_YN : N

SW

Test : HNF-SD-W026-OTP-015

Step : A5,5.6.3

Equipment Tag \#: OIU-103B

\section{Exception :}

Revision :

Date:

Specification Section : 13462

Test Witness/Author:

Dunlap

Test Operator:

Responsible Company: WMH

Responsible Subcontractor: SII

Responsible Individual:_ Lame

Exception Description :

At completion of Saw sequence, message "saw sequence complete" missing.

Exception Disposition :

\section{Exception Status :}

9-3-97 Fixed. Closed.

Exception Closed By: Lane Mrentis Pore

Date : $9 / 3 / 97$

\section{APPROVAL OF RESOLUTION}

Contractor Representative: (Only as needed)

WMH Representative:

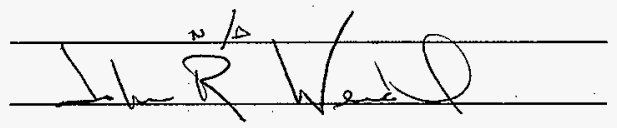

Additional Approval:

(Only as needed)
Date:

Date: $12-10-97$

Date:

Date:

N/ 
ATP_YN : N

Test : HNF-SD-W026-OTP-015

Step : See Below Equipment Tag \#: ST-07-101

\section{Exception :}

Revision :

Date:

Specification Section : 13462

Test Witness/Author:

Dunlap

\section{Test Operator:}

\begin{tabular}{ll} 
Responsible Company: & WMH \\
\hline Responsible Subcontractor : & \\
\hline Responsible Individual: & Warmenhoven \\
\hline
\end{tabular}

\section{Exception Description :}

Steps A5, 5.6.5, A8, 5.3.7

When extending sorting table, table stopped at tip poisition because tabnle locks position infor not available.

\section{Exception Disposition :}

\section{Exception Status :}

9-3-97 Duplicate of \#141. Closed.

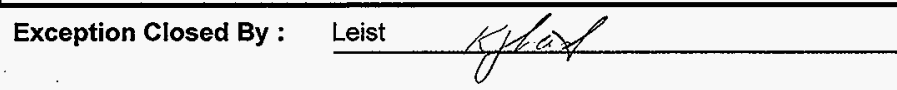

\section{APPROVAL OF RESOLUTION}

Contractor Representative: (Only as needed)

WMH Representative:

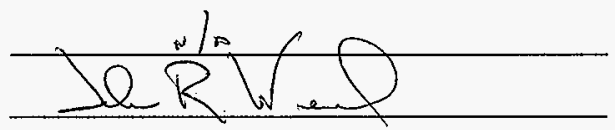

Date:

Date: $12-10.97$

Additional Approval:

Date:

(Only as needed)

FDNW Representative:

$s$

Date: 
ATP_YN : N

Test : HNF-SD-W026-OTP-015

Step : A5, 5.81

Equipment Tag \#: ST-07-101

\section{Exception :}

Revision :

Date:

Specification Section : 13462

Test Witness/Author:

Test Operator:

Responsible Company: WMH

Responsible Subcontractor :

Responsible Individual: Warmenhoven

Exception Description :

Sort table "failed to lock" on extend to tip command. Lock position info not avallable.

\section{Exception Disposition :}

\section{Exception Status :}

9-3-97 Duplicate of \# 141. Closed.

Exception Closed By : Leist

$12 / n / 97$

Date : $9 / 3 / 97$

\section{APPROVAL OF RESOLUTION}

Contractor Representative: (Only as needed)

WMH Representative:

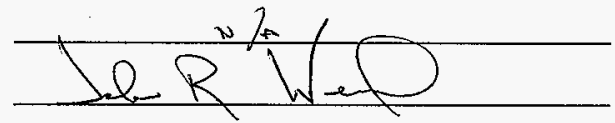

Additional Approval:

(Only as needed)
Date:

Date: $12-10-97$

Date:

Date: 


\section{WRAP ACCEPTANCE TEST PROCEDURE EXCEPTION LOG}

ATP_YN : N

SW

Test : HNF-SD-W026-OTP-015

Step : A5, 5.8.2

Equipment Tag \#: OIU-103B
Tracking \#: 267

Exception :

Revision :

Date:

Specification Section : 13462

Test Witness/Author : Dunlap

Test Operator:

\begin{tabular}{l} 
Responsible Company: $\quad$ WMH \\
Responsible Subcontractor: \\
Responsible Individual: Lane \\
\hline
\end{tabular}

\section{Exception Description :}

On the transfer car menu, the "relid" position should be renamed since relid not takes place at delid position. Possibly "c-clip/bar off."

\section{Exception Disposition :}

\section{Exception Status :}

9-3-97 Changed to "Debag/Crmp".

\section{Exception Closed By: Lane hn colas POrane}

\section{APPROVAL OF RESOLUTION}

Contractor Representative: (Only as needed)

WMH Representative:

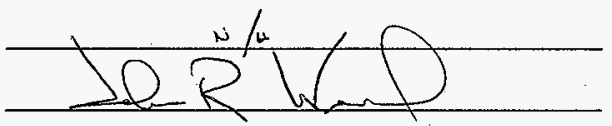

Additional Approval:

(Only as needed) it

Date: 
ATP_YN : N

SW

Test : HNF-SD_W026-OTP-015

Step : A5, 5.8 .4

Equipment Tag \#: TC-07-101

\section{Exception :}

Revision :

Date:

Specification Section : 13462

Test Witness/Author :

Dunlap

Test Operator:

Responsible Company:
Responsible Subcontractor:
Responsible Individual:

Exception Description :

After transfer to the super compactor, the transfer car conveyor motor did not stop or disengage.

\section{Exception Disposition :}

\section{Exception Status :}

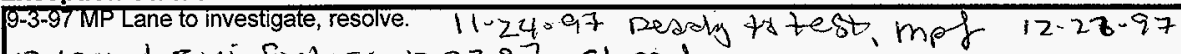
patested $5 u+$ is Fackir y 12-23-97. Closed.

\section{Exception Closed By: Thiciens Pdre}

Date: $12-26-97$

\section{APPROVAL OF RESOLUTION}

Contractor Representative: (Only as needed)

WMH Representative:

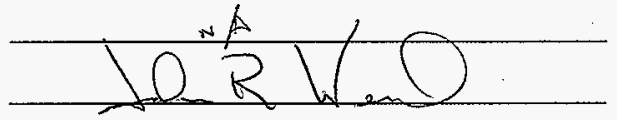

Date:

Date: $12-26 \cdot 97$

Additional Approvai:

(Only as needed)

Date:

FDNW Representative:

$\omega / 0$

Date: 


\section{WRAP ACCEPTANCE TEST PROCEDURE EXCEPTION LOG}

ATP_YN : N

Test : HNF-SD-W026-OTP-015

Step : See Below

Equipment Tag \#: DH-07-101

\section{Exception :}

Revision :

Date:

Specification Section : 13462

Test Witness/Author :

Duniap

\section{Test Operator:}

\begin{tabular}{ll} 
Responsible Company: & WMH \\
\hline Responsible Subcontractor: & \\
\hline Responsible Individual: & Lelst
\end{tabular}

\section{Exception Description :}

A6, 5.1.2, A8, 5.3.3

Drum lifter closed limit switch needs adjustment. The closed light on the control pendant did not illuminate.

\section{Exception Disposition :}

\section{Exception Status :}

WP-97-502 Item \#59. 12-26-97. patested 12-20-92 satis facterg. Cloged.

Exception Closed By : Th, etess p dree

Date: $12-26-97$

\section{APPROVAL OF RESOLUTION}

Contractor Representative: (Only as needed)

WMH Representative:

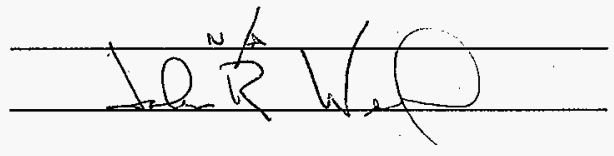

Date:

Date: $12-26-9)$

Additional Approval:

(Only as needed)

Date:

FDNW Representative:

$\therefore$ f

Date: 
ATP_YN : N

Test : HNF-SD-W025-OTP-015

Step : See Below Equipment Tag \#: ND-07-101

\section{Exception :}

Revision :

Specification Section : 13462

Test Witness/Author :

Duñap

Test Operator:

\section{Responsible Company: WMH}

\section{Responsible Subcontractor:}

Responsible Individual:

Humphrys

\section{Exception Description :}

A6, 5.4.6, A8, 3.6

It was not possible to perform the required portion of OP-706 (packet NDE operation) because the x-ray was unavailable (tray missing).

\section{Exception Disposition :}

\section{Exception Status :}

9-3-97 Install procured tray, test.

$$
\text { PACKET } X \text {-RAT ATP WAS PERFRMES } 11 / 26 / 96 .
$$

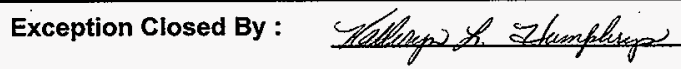

\section{APPROVAL OF RESOLUTION}

Contractor Representative: (Only as needed)

WMH Representative:

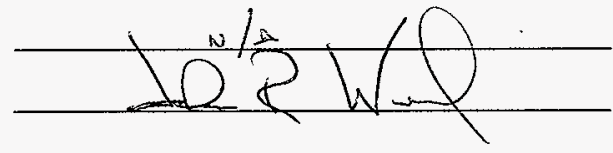

Additional Approval:

(Only as needed)
Date:

Date: $2-3 i-97$

Date:

Date: 
Test : HNF-SD-W026-OTP-015

Step : A6, 5.6.2

Equipment Tag \#: LT-09-203A

Revision :

Date:

Specification Section : 13462

Test Witness/Author:

Dunlap

Test Operator:

Responsible Company: WMH
Responsible Subcontractor:
Responsible Individual: $\quad$ Jordal

Exception Description :

The lift table falled to lower during the exit sequence of the RWV port. Cause was servo controller overheated and tripped out (code 22 indicater).

\section{Exception Disposition :}

\section{Exception Status :}

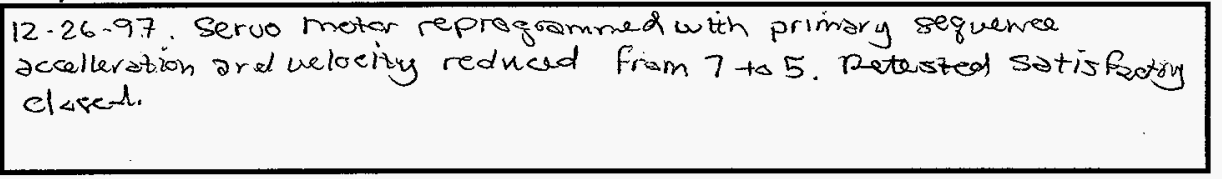

Exception Closed By : Micicos Pfre

Date : $12-26-97$

\section{APPROVAL OF RESOLUTION}

Contractor Representative: (Only as needed)

WMH Representative:

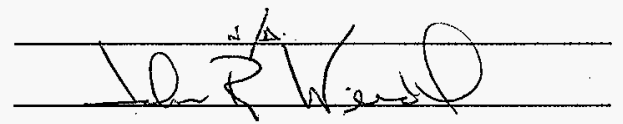

Additional Approval:

(Only as needed)
Date:

Date: $12-26-97$

Date:

Date: 
ATP_YN : N

Test : HNF-SD-W026-OTP-015

Step : A7, 5.2.2 Equipment Tag \#: RT-07-101

Specification Section : 13462

Test Witness/Author :

Test Operator:

\section{Exception :}

Revision :

Date:

\section{Responsible Company: WMH \\ Responsible Subcontractor : \\ Responsible Individual: Leist}

Exception Description :

$\mathrm{FAl}^{2} \mathrm{LQ⿻}^{\circ}$

The "lower with grab closed" sequencephecause the drum lid/delic fixture had moved approximately 6 " from the parked position.

\section{Exception Disposition :}

\section{Exception Status :}

9-3-97 Test error. Closed.

\section{Exception Closed By : Leist Kffuct $12 / 12 / 27$}

\section{APPROVAL OF RESOLUTION}

Contractor Representative: (Only as needed')

WMH Representative:

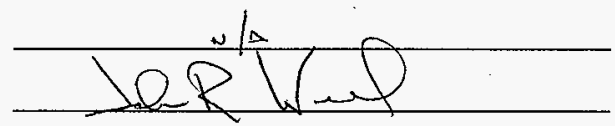

Additional Approval:

(Only as needed)
Date:

Date: $27-10-97$

Date:

Date: 
ATP_YN : N

SW

Test : HNF-SD-W026-OTP-015

Step : A9, 5.3.1

Equipment Tag \#: DO-07-101

\section{Exception :}

Revision :

Date:

\section{Specification Section : 13462 \\ Test Witness/Author: \\ Dunlap}

Test Operator:

$\begin{array}{ll}\text { Responsible Company: } & \text { WMH } \\ \text { Responsible Subcontractor: } & \text { StI } \\ \text { Responsible Indivauar: } & \text { Lame }\end{array}$

Exception Description :

When opening the drum transfer port, message "transfer port opening..." was displayed before the "open port" selection was pressed.

\section{Exception Disposition :}

\section{Exception Status :}

9-3-97 Test error - Leftover message. Closed.

\section{Exception Closed By: Lane Mrelal Potere Date: 9/3/97}

\section{APPROVAL OF RESOLUTION}

Contractor Representative: (Only as needed)

WMH Representative:

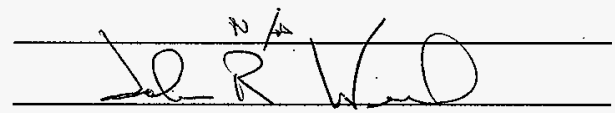

Additional Approval: (Only as needed)
Date:

Date: $12-10-97$

Date:

Date:

FDNW Representative:

$N / \mathrm{s}$ 
ATP_YN : N

DMS

Test : HNF-SD-W026-OTP-015

Step : Att.5, 5.93

Equipment Tag \#: DMS Software

\section{Exception :}

Revision :

Date:

Specification Section : DMS

Test Witness/Author:

Rosnick

Test Operator:

\begin{tabular}{ll} 
Responsible Company: & WMH \\
\hline Responsible subcontractor: & BDIV \\
Responsible Indivaual: & Weident
\end{tabular}

Exception Description :

Spec. is WHC-SD-W026-SD-001

Screen DMSS0315. The default puck height was not displayed. This was due to WRAP_FIELD_NAME being "DEFAULT_PCK_HGHT" in stead of "DEFAULT_PCK_HGT."

\section{Exception Disposition :}

Look-up table updated.

\section{Exception Status :}

9-18-97 Ready to test.

Look-up data corrected.

Exception Closed By :

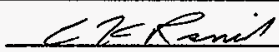

Date: $12 / 3, / 97$

\section{APPROVAL OF RESOLUTION}

Contractor Representative: (Only as needed)

WMH Representative:

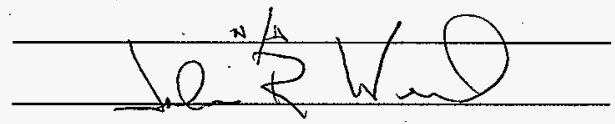

Date:

Date: $\underline{(2-3)-77}$

Additional Approval:

(Onily as needed)

Date:

FDNW Representative:<smiles>C1CC1C1CC1</smiles>

Date: 
ATP_YN : N

SW

Test : HNF-SD-W026-OTP-015

Step : A6, 5.4 .5

Equipment Tag \#: Sensor ZS-331

\section{Exception :}

Revision :

Date:

Specification Section : 13462

Test Witness/Author :

Dunlap

Test Operator:

$\begin{array}{ll}\text { Responsible Company: } & \text { WMH } \\ \text { Responsible Subcontractor: } & \text { Stl } \\ \text { Responsible Individual: } & \text { Lame }\end{array}$

Exception Description :

DMSS012 display pin OTP-LLW-WR-01 when OTP-CCW-WR-02 was actually on sort table. Senso did not register drum being loaded on transfer

\section{Exception Disposition :}

Software error was found and corrected.

\section{Exception Status :}

Retested SAT.

\section{Exception Closed By : Dunlap \\ APPROVAL OF RESOLUTION}

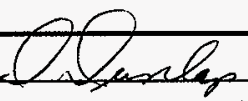

Contractor Representative: (Only as needed)

WMH Representative:

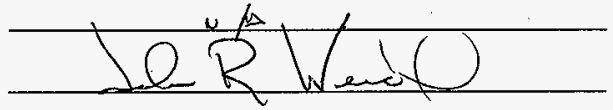

Date:

Date: $12-10-97$

Additional Approval:

(Only as needed)

Date:

FDNW Representative:

spo

Date: 
DMS

Exception : 26

Test : HNF-SD-W026-OTP-015

Step : See Below

Equipment Tag \#: DMS Software

Revision :

Date:

Specification Section: DMS

Test Witness/Author :

Rosnick

Test Operator:

\begin{tabular}{ll} 
Responsible Company: & WMH \\
\hline Responsible Subcontractor: & BDM \\
Responsible Individual: & Wetdert
\end{tabular}

Exception Description :

10-1-97 - Prior intormation incorrect, see\#233. Correct description below:

Step Att. 8, 5.2.2, 5, 5.3.2, Spec. WHC-SD-W026-SD-001.

DMSCOM:If multiple location messages are sent by the PCS with the same drum at LLW-Entry, the DMS dos not recognize the inner drum PIN, since the container relationship code has already been deleted.

Exception Disposition :

Writing sofware correction.

\section{Exception Status :}

9-18-97 Ready to test.

Exception Closed By :

Date : $10 / 2 / 9>$

\section{APPROVAL OF RESOLUTION}

Contractor Representative: (Only as needed)

WMH Representative:

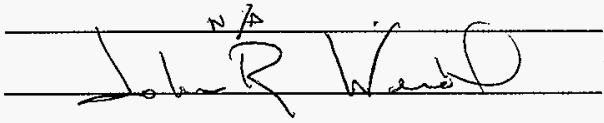

Additional Approval:

(Only as needed)
Date:

Date: $12-10 \cdot 97$

Date:

Date: 
ATP_YN : N

DMS

Test : See Below

Step : Att.8, 5.3.6

Equipment Tag \#: DMS Software
Exception : 27

Revision :

Specification Section: DMS

Test Witness/Author:

Rosnick

Test Operator:

\begin{tabular}{ll} 
! & WMH \\
\hline Responsible Subcontractor : & BDM \\
Responsible Individual: & Weddert
\end{tabular}

Exception Description :

Test-HINF-SD-W026-OTP-015(LLW)

Spec. - WHC-SD-WO26-SD-001

Screen DMSS0312. Pop-up "E532 No NDE comments records for this PIN" was displayed for OTP-LLW-WP-01

even though there were NDE records in the database.

\section{Exception Disposition :}

Waiting for software correction.

Exception Status :

9-18-97 Ready to test.

Exception Closed By: Srotenderon

Date : $12 / 51 / 97$

\section{APPROVAL OF RESOLUTION}

Contractor Representative: (Only as needed)

WMH Representative:

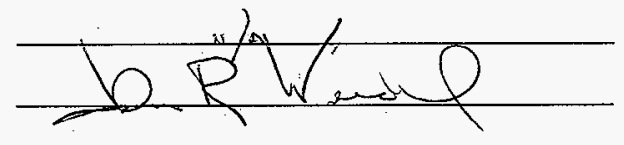

Additional Approval:

(Oniy as needed)
Date:

Date: 12.31 .97

Date:

Date:

FDNW Representative:<smiles>C1CC12CC2</smiles>

Date: 
ATP_YN : N

SW

Test : HNF-SD-W026-OTP-015

Step : A8, 8.4.4

Equipment Tag \#: PCS

\section{Exception :}

Revision :

Date:

Specification Section :

13462

Test Witness/Author :

Dunlap

Test Operator:

Responsible Company:
Responsible Subcontractor:
$\begin{array}{ll}\text { Responsible Individual: } & \text { Lane }\end{array}$

Exception Description :

Puck weight did not transfer to DMSS015 screen when puck moved to loadout position.

\section{Exception Disposition :}

\section{Exception Status :}

9-3-97 Fixed, retested satisfactory.

Exception Closed By: Lane Mr dree PQtex

Date : 9/3/97

\section{APPROVAL OF RESOLUTION}

Contractor Representative: (Only as needed)

WMH Representative:

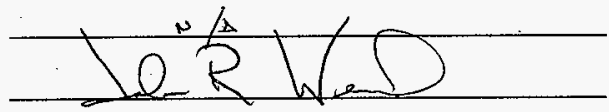

Additional Approval:

(Only as needed)
$N$

Date: 
ATP_YN : N

DMS

Test : See Below

Step : Att.5, 5.7 .3

Equipment Tag \#: DMS Software

Specification Section : DMS

Test Witness/Author:

Test Operator:
Exception : 29

Revision :

\section{Responsible Company: WMH \\ Responsible Subcontractor: BDM \\ Responsible Individual: Wetdert}

Exception Description :

Test - HNF-SD-W026-OTP-015 (LLW)

Spec. - WHC-SD-W026-SD-001

Screen DMSS0501. When the sample bottle is scanned and the wrong drum is at the sorting table, the error is reported, but it is not possible to process the next sample message until the erroneous data is committed.

\section{Exception Disposition :}

Waiting for software correction.

\section{Exception Status :}

9-18-97 Ready to test.

$$
\text { Tested seterfectory }
$$

Exception Closed By :

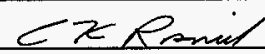

Date : $1 / 17 / 9>$

\section{APPROVAL OF RESOLUTION}

Contractor Representative: (Only as needed)

WMH Representative:

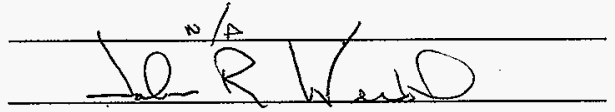

Additional Approval:

(Only as needed)
Date:

Date: $12-10-8 y$

Date:

Date: 
ATP YN : N

DMS

Test : See Below

Step : Att.5, 5.6 .5

Equipment Tag \#: DMS Software
Exception : 30

Revision: :

Date:

Specification Section: DMS

Test Witness/Author :

Rosnick

Test Operator:

\begin{tabular}{ll} 
Responsible Company: & WMH \\
\hline Responsible Subcontractor : & BDM \\
Responsible Individual: & Weidert \\
\hline
\end{tabular}

Exception Description :

Test - HNF-SD-W026-OTP-015 (LLW)

Spec. - WHC-SD-W026-001

Screen DMSS0311. Drum OTP-LLW-WP-01 was still shown on DMSS0311 as being at LLW Entry atter the drum was shown on DMSS0312 as being at LLW Sort.

\section{Exception Disposition :}

Waiting for software correction.

\section{Exception Status :}

9-18-97 Ready to test.

$$
\text { Tested ok }
$$

Exception Closed By :

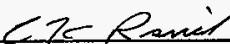

Date : $10 / 2 / 52$

\section{APPROVAL OF RESOLUTION}

Contractor Representative: (Only as needed)

WMH Representative:

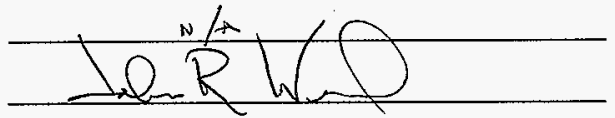

Date:

Date: $\quad(2-20-97$

Additional Approval:

Date:

(Only as needed)

Date: 
ATP_YN : N

DMS

Test : HNF-SD-W026-OTP

Step : Att.8, 5.7

Equipment Tag \#: DMS Software
Exception : 31

Revision :

Date:

Specification Section: DMS

Test Witness/Author :

Rosnick

Test Operator:

\begin{tabular}{ll} 
Responsible Company: & WMH \\
\hline Responsible Subcontractor: & BDM \\
Responsible Individual: & Wetdert
\end{tabular}

Exception Description :

Spec. - WHC-SD-W026-SD-007

DMS Screen DMSS0312. When a11 CHEMCOMP records for OTP-LLW-WP-02 were deleted the HDET record was not deleted and the CON PWTYP CD was not changed to "R." For OTP-LLW-WP-04, the HDET record was deleted but the CON_PWTYP_CD was not changed. See SDD, Appendix F, 3.1.3.1, UP2.

\section{Exception Disposition :}

Waiting for sofware correction.

\section{Exception Status :}

9-18-97 Ready to test.

$$
\text { Tested ok }
$$

Exception Closed By :

\section{APPROVAL OF RESOLUTION}

Contractor Representative: (Only as needed)

WMH Representative:

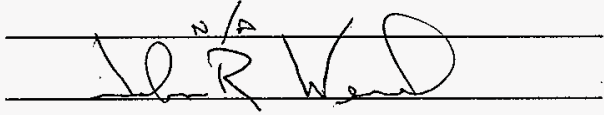

Date:

Date: $12-10-97$

Additional Approval:

Date:

(Only as needed)

ㄱ.

Date: 
Test : See Below

Step : Att. 8, $5.7 \quad$ Equipment Tag \#: DMS Software

\section{Revision :}

Date:

Specification Section: DMS

Test Witness/Author:

Rosnick

Test Operator:

\begin{tabular}{ll} 
Responsible Company: & WMH \\
\hline Responsible Subcontractor: & BDM \\
Responsible Individual: & Weldert
\end{tabular}

Exception Description :

Test - HNF-SD-W026-OTP-015 (LIWW)

Spec. - WHC-SD-W026-SD-001

DMSCOM. At LLW Entry the CONEXT_USE_CD for the waste (inner) drum is set to "WW." The use code should be left "WP."

\section{Exception Disposition :}

Waiting for software correction.

\section{Exception Status :}

9-18-97/Ready to test

$$
\text { Testat ok }
$$

Exception Closed By :

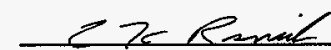

Date

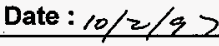

\section{APPROVAL OF RESOLUTION}

Contractor Representative: (Only as needed)

WMH Representative:

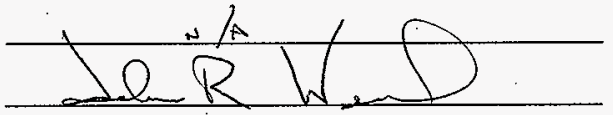

Additional Approval:

(Only as needed)
Date:

Date: $12-10-97$

Date:

Date: 
ATP_YN : N

DMS

Test : See Below

Step : Att.8, 5.7

Equipment Tag \#: DMS Software
Exception : 33

\section{Revision :}

Date:

Specification Section: DMS

Test Witness/Author :

Rosnick

Test Operator:

\begin{tabular}{ll} 
Responsible Company: & WMH \\
\hline Responsible Subcontractor: & BDM \\
\hline Responsible Individual: & Wetdert
\end{tabular}

Exception Description :

rest-HNF-SD-W026-OTP-015 (LLW)

Spec. - WHC-SD-W026-SD-001

DMS Screen DMSS0315. The "Sum Waste Records" did not create any NDAISO records.

\section{Exception Disposition :}

Walting for software correction.

\section{Exception Status :}

Software corract and tertid

Exception Closed By :

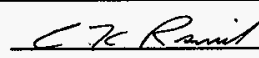

Date: $1 / 1>/ 9>$

\section{APPROVAL OF RESOLUTION}

Contractor Representative: (Only as needed)

WMH Representative:

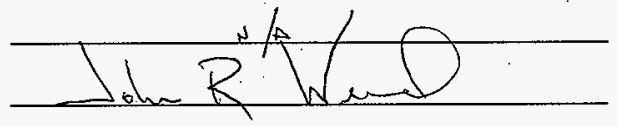

Additional Approval:

(Oniy as needed)
Date:

Date: $12-10-97$

Date:

Date:

N 
ATP_YN : N

DMS

Test : HNF-SD-W026-OTP-015

Step : Att.

Equipment Tag \#: DMS Software

\section{Exception :}

Revision :

Date:

Specification Section :

Test Witness/Author :

DMS

Rosnick

Test Operator:

\begin{tabular}{ll} 
Responsible Company: & WMH \\
Responsible Subcontractor : & BDM \\
Responsible Individual: & Weidert \\
\hline
\end{tabular}

Exception Description :

Spec. - WHC-SD-WO26-SD-001

DMSCOM. The CON_PKG_STATUS for the puck records is "T." The status should be the same as the original cirum, "R."

\section{Exception Disposition :}

Corrected prior to formal test and verified in dry run. DID NOT OCCUR DURING LLW-OTPTIESTING.

\section{Exception Status :}

Closed.

\section{APPROVAL OF RESOLUTION}

Contractor Representative: (Only as needed)

WMH Representative:

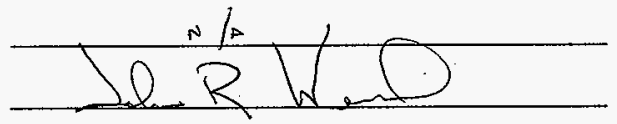

Date:

Date: $12-10-97$

Additional Approval:

(Only as needed)

Date: 
ATP_YN : N

DMS

Test: See Below

Step : Att. 5, 5.6.6

Equipment Tag \#: DMS Software

\section{Exception :}

Revision :

Date:

Specification Section: DMS

Test Witness/Author :

Rosnick

Test Operator:

$\begin{array}{ll}\text { Responsible Company: } & \text { WMH } \\ \text { Responsible Subcontractor : } & \text { BDHI } \\ \text { Responsible Individual: } & \text { Weidert }\end{array}$

Exception Description :

Test - HNF-SD-W026-OTP-015

DMS Screen DMSS0504, Analysis Request. If the [Add Sample] button is selected before the data for the current request is committed, the user is asked if they want to commit the data. If "no" or "cancel" is selected, the original data is still displayed but the sample number is incremented.

Exception Disposition :

Corrected prior to formal test and veritied in ary run. DID NOT OCCUR DURINGLLW-OTP iESTING.

\section{Exception Status :}

Closed.

Exception Closed By : Dunlap

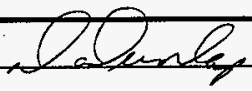

Date : $8 / 20 / 97$

\section{APPROVAL OF RESOLUTION}

Contractor Representative: (Only as needed)

WMH Representative:

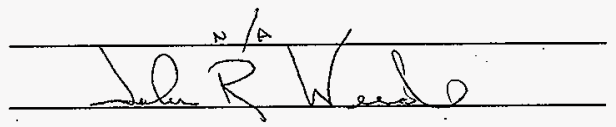

Additional Approval:

(Only as needed)
Date:

Date: $12+10 \cdot 97$

Date:

Date: 


\section{LLW GLOVEBOX OTP RETEST TO CLEAR DMS EXCEPTIONS $9 / 29 / 97$}

\section{ETRs To Be Cleared}

\#274 Default puck height is not set on LLW Exit (315) screen.

\#276 Rescanning of drum at LLW entry results in erroneous processing.

\#277 NDE comments not displayed on LLW Sort (312) pop-up.

\#279) Cannot process next sample bottle on 501 screen if wrong bottle is scanned. Popunp FRn-4065 7

\#280 Drum shown on LLW Entry (311) screen after drum is shown on LLW Sort (312) screen.

\#281 When a11 CHEMCOMP records are deleted on LLW Sort (312) CHEMCOMP pop-up, the HDET record should be deleted and CON PWTYP CD should be changed from ' $M$ ' to 'R'.

\#282 At LLW ENTRY DMSCOM should not change CONEXT_USE_CD for the innder drum from ' $\bar{R}^{\prime}$ to ' $W V$ '.

\#283 NDAISO records are not created for the puck when records are summed by the LLW Exit (315) screen.

\section{Test Steps}

1. Run WRESTORE to load database file BASE 970820.

2. Run script otp Tul.sql.

3. Run PCS Simulator with data file $17 w 1 . p c s$.

4. After 6 th message request sample 1 with 3 analysis.

5. After 8th message commit sample data for bottles 1 \& 2 .

6. Process remaining messages.

7. Run PCS Simulator with data file 11 w2.pcs.

8. After 4 th message request sample 2 with 1 analysis.

9. Print drum barcode label OTP-LLW-OD-03 and sample bottle labels 9700001-03L and 97-00002-01L.

10. Shut down simulator and start DMSCOM.

11. On LLW Exit (315) screen specify OTP-LLW-OD-01 and verify puck height is set to default (ETR \#274). Default. 21 . New value .5 entined

$X$ 12. On LLW Exit (315) screen Sum Waste Records and verify NDAISO records are created for the puck (ETR 283).

$\checkmark 13$. on LLW Sort (312) screen verify NDE comments are displayed on NDE pop-up screen for OTP-LLW-WP-02 (ETR \#277).

14. Scan bottle labels 97-00001-03L and 97-00002-01L and verify data can be entered on the Sample (501) screen for bottle 97-00002-01L after the

popan FRri-4065?

$\checkmark 15$. error message is cleared for bottle 97-00001-03

Delete the CHEMCOMP records on LLW Sort (312) screen CHEMCOMP pop-up screen and verify the HDET record has been deleted and CON PWTYP CD = ' $R$ ' for the puck (ETR \#281).

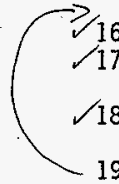

16 On the LLW Entry (311) screen verify there is no drum at entry (\#280). Perform 0TP-LLW-OD-03 barcode scan sequence twice at LLW_ENTRY and verify second message was not processed (ETR \#276).

18. Verify CONEXT USE CD for OTP-LLW-WP-03 was not changed when the barcode was scanned a $\bar{t}$ LLW ENTRY (ETR \#282).

19. Run query script ōpq_11w and print database 1isting. sompt run before scan int Luw-Enirey. 
Test LLw otp Rerun 9/29/9?

11W1.pCS PCS SIMULATOR DATA FILE FOR LLW GLOVEBOX OTP CASE 1

PCSDMS $\sim$ PPL LLW PP 0TP6-97-300001

PCSDMS $\sim C^{\sim} \sim L L W$ RWMPRT $\sim 1 \sim 0 T P-L L W R-T D-01 \sim$

PCSDMS $\sim \mathrm{CL}^{\sim} \sim \mathrm{LLW}^{-}$ENTRY $\sim 1 \sim 0 T P-L L W-0 D-01^{\sim}$

PCSDMS DCS $\sim L L W$ ENTRY OTP-LLLW-OD-01 $T^{\sim}$

PCSDMS CL $\sim$ LLW EXIT $\sim 1 \sim 0 T P-L L W-O D-01 \sim$ PCSDMS CL LLLW SORT 1 OTP-LLW-WP-01

4

5 PCSDMS SPDP $\sim$ LIW SORT 97-00001-01L OTP-LLW-WP-01

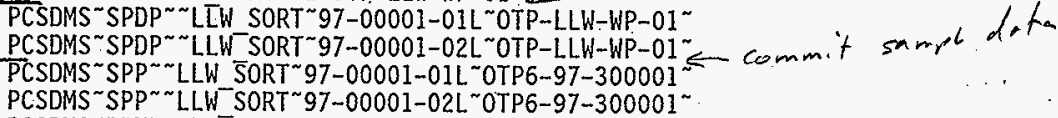

PCSDMS PPTP LLW PP $0 T P 6-97-300001$ 0TP6-97-400001

6 PCSDMS $\sim \mathrm{CL}^{\sim \sim} \sim L W$ CMPCT $\sim 1 \sim 0 T P-L L W-W P-01 \sim$

PCSDMS $\sim \mathrm{CL}^{\sim} \sim \mathrm{LLW}^{-} \mathrm{PUCK}^{\sim} \sim 1 \sim 0 T \mathrm{POOI}$

PCSDMS ${ }^{\sim} \mathrm{CLW}^{\sim} \sim \mathrm{LLW}$ PUCK ${ }^{\sim}$ OTPOO1 150

PCSDMS ${ }^{\sim}$ POPD. LLW_EXIT ${ }^{\sim} 0 T P 001 \sim 0 T P-L L W-O D-01 \sim$

\section{W2. pCS PCS SIMULATOR DATA FILE FOR LLW GLOVEBOX OTP CASE 2}

PCSDMS $\sim \mathrm{CL}^{\sim} \sim \mathrm{LLW}$ ENTRY 1 $^{\sim}$ OTP-LLW-OD-02

8 PCSDMS DCS $\sim$ LLW ENTRY OTP-LLW-OD-02 $\sim F^{\sim}$

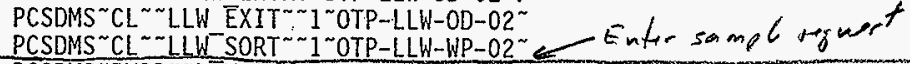

PCSDMS RWPP LLW SORT 0TP6-97-000001 0TP-LIW-WP-02"?

PCSDMS $\sim$ RWPT LLW'SORT 0TP6-97-000001 0TP-LLWR-TD-01

PCSDMS RWPP LLW'SORT 0TP6-97-000002 0TP-LLW-WP-02

PCSDMS ${ }^{2}$ RWPT $\sim$ LLW_SORT 0TP6-97-000002 0TP-LLWR-TD-01

PCSDMS RNDP LLW'SORT 0TP6-97-000003 0TP

PCSDMS RWPT LLW_SORT OTP6-97-000003 07P-LLWR-TD-01

PCSDMS ${ }^{\sim} \mathrm{CL}^{\sim}$ NLW CMPCT $\sim 10 T P-L L W-W P-027$

PCSDMS $\sim \mathrm{CL}^{\sim} \sim \mathrm{LLW}^{-} \mathrm{PUCK}^{\sim} \sim 1 \sim 0 T P 002^{\sim}$

PCSDMS CLW ${ }^{\sim}$ LLK PUCK $1^{\sim}$ OTPO02 $160 \%$

PCSDMS $\mathrm{CL}^{\sim} \sim \mathrm{LLW}$ SYORE1 $\sim 1 \sim 0 T P 002 \%$

11W3.pCS PCS SIMULATOR DATA FILE FOR LLW GLOVEBOX OTP. CASE 3

PCSDMS CL LLW ENTRY 1 OTP-LLW-OD-03

PCSDMS DCS LLW ENTRY \%TP- KLW-0D-03 $F^{\sim}$

PCSDMS $\sim \mathrm{CL}^{\sim} \sim \mathrm{LLW}$ CMPCT $\sim 1 \sim 0 T P$ - L LW-WP-03

PCSDMS CL $\sim$ LLW PUGK 1 0TPO03

PCSDMS $\sim C L \sim \sim L L W, P U C K \sim 1 \sim 0 T P O 03 \sim 150 \sim$

PCSDMS POPD LLW_EXIT OTPOO3 OTP-XLW-OD-02

11W4.pCS PCS SIMULATOR DATA FILE FOR LLW GLOVEBOX OTP CASE 4

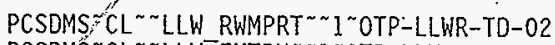

PCSDMS $\sim \mathrm{CL}^{\sim \sim L L W}{ }^{-}$ENTRY 1 OTP-LLW-0D-04

PCSBMS DCS LLW ENTRY OTP-LLW-OD-04 F

PCSDMS $\mathrm{CL}^{\sim} \sim \mathrm{LLW}$ SORT $\sim 1 \sim 0 T P-\mathrm{LLW}-\mathrm{WP}-04 \sim$

PCSDMS RWPP ${ }^{\sim}$ LEWSORT OTP6-97-000004 OTP-LLW-WP-04 


\section{0 rows selected. LLW otp Rerun 9/2\%/97}

SQL> msglog :

unknown command "msglog" - rest of line ignored.

Li> @msgiog

\&L> 1

Test

Step

Seq \# Date/Time EF Message

$124813 \quad 30-S E P-97 \mathrm{~N}$

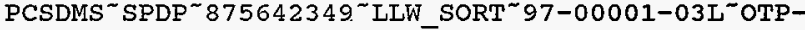
$\mathrm{LLW}-\mathrm{WP}-02^{\sim}$

14

$12481630-S E P-97 \mathrm{~N}$ PCSDMS $\sim \mathrm{SPDP}^{\sim} 875642373^{\sim} \mathrm{LLW}$ SORT $\sim 97-00002-01 \mathrm{~L}$ OTP$\mathrm{LLW}-\mathrm{WP}-02^{\sim}$

124865 30-SEP-97 N PCSDMS $\mathrm{CL}^{\sim} 875643465^{\sim}$ LLW ENTRY 1 OTP-LLW-OD-03 $12486630-\mathrm{SEP}-97 \mathrm{~N}$ $12488730-S E P-97 \mathrm{~N}$ DMSPCS ${ }^{-C L}$ - LLW ENTRY ${ }^{\sim}$ OTTP-LLW-WP-03 $124890 \quad 30-S E P-97 \mathrm{~N}$ PCSDMS $\sim \mathrm{CL}^{\sim} 875 \overline{6} 43923^{\sim}$ LLW ENTRY 1 OTP-LLW-OD 03PCSDMS $\sim \mathrm{CL}^{\sim} 875643946^{\sim}$ LLW_ENTRY $^{-1}$ 1 OTP-LLW-OD-03 $^{-}$

6 rows selected.

SQL $>$ 


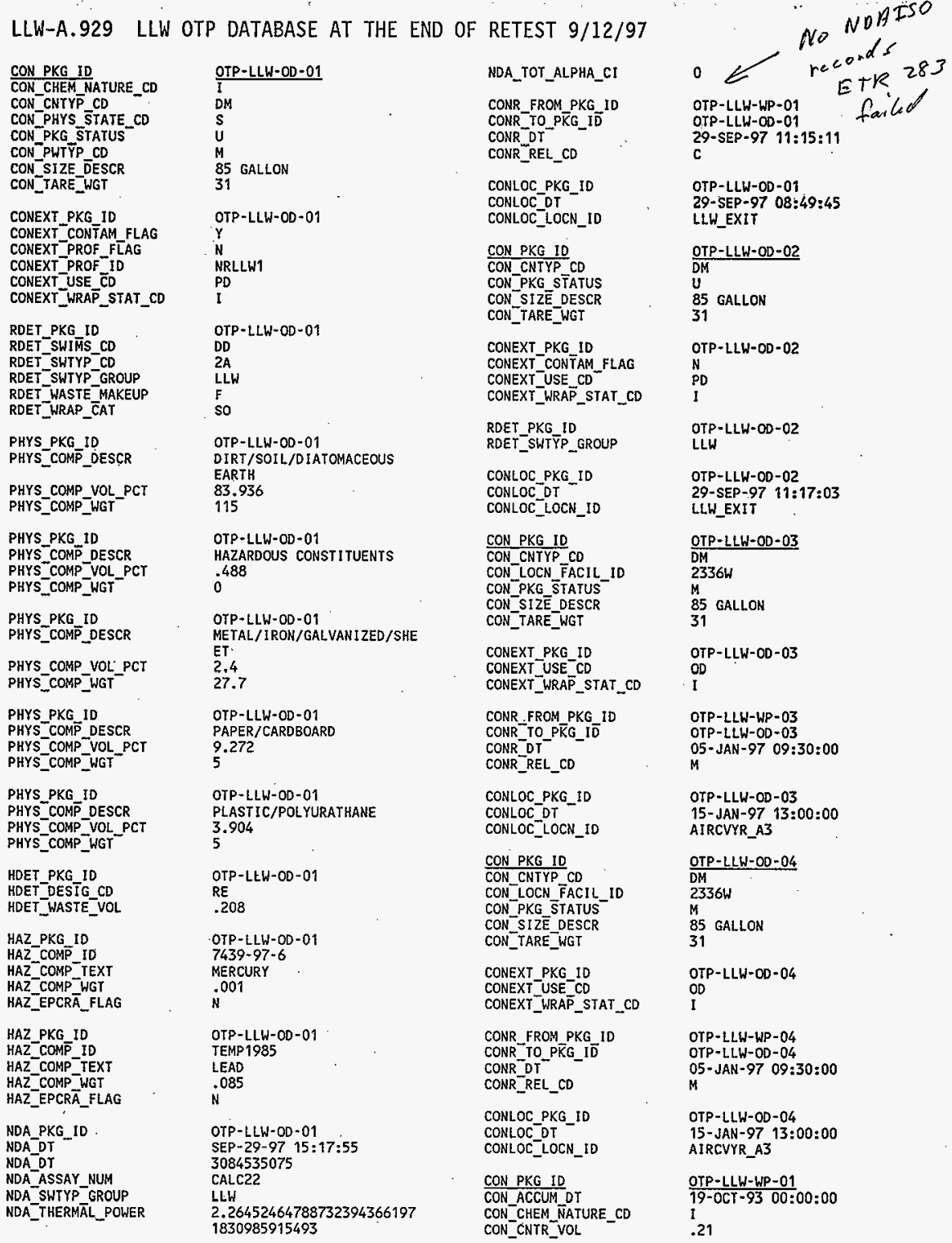




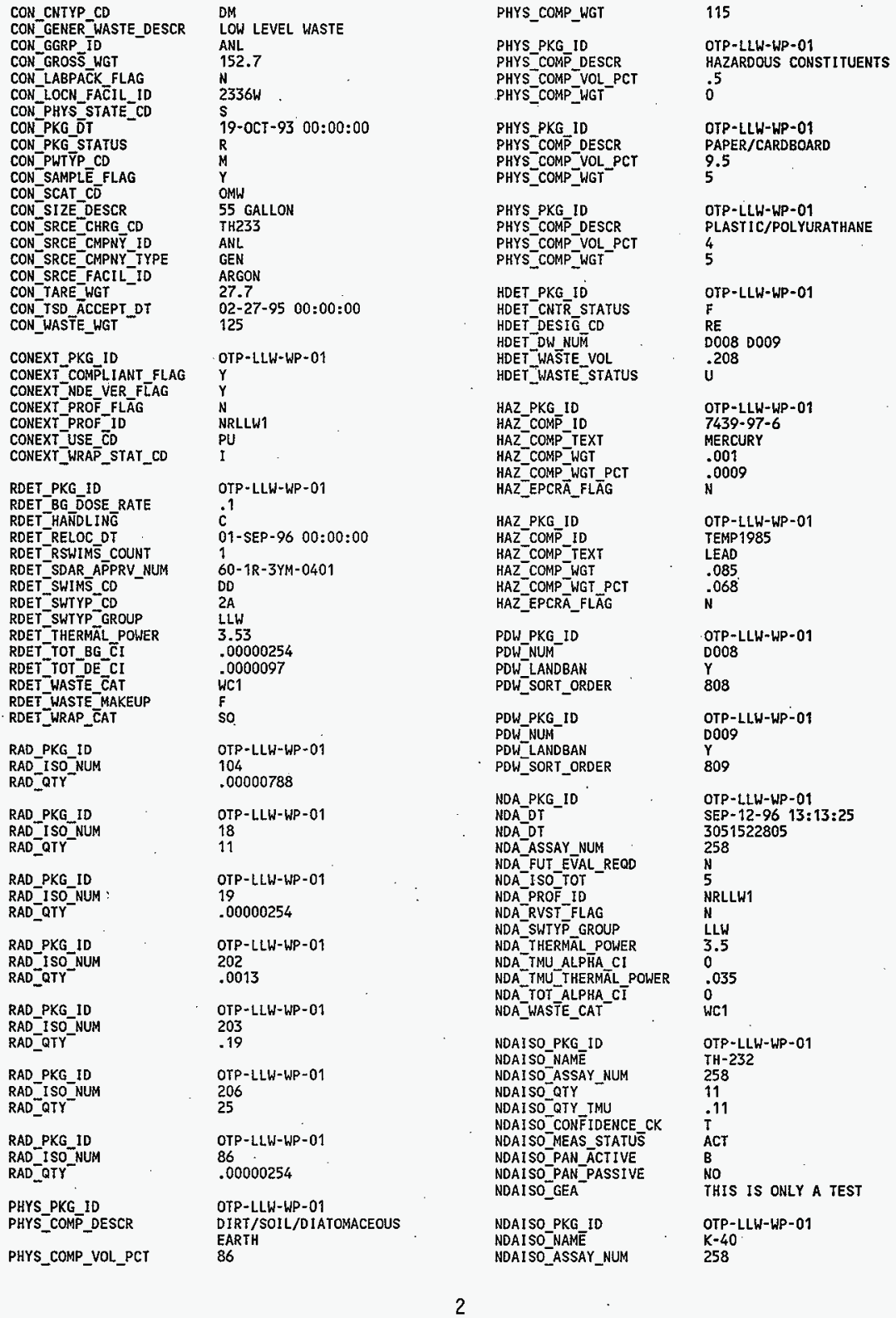




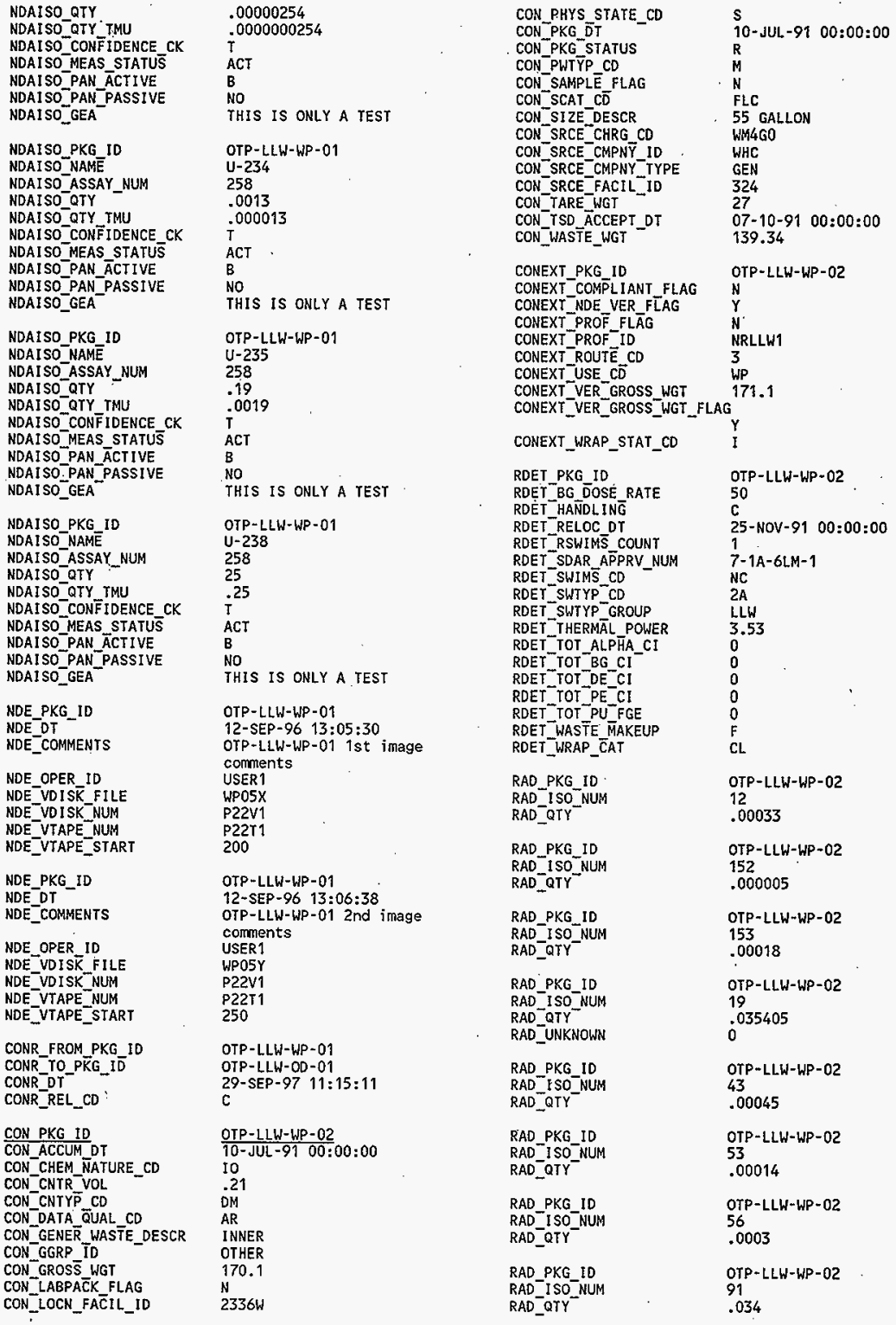




\begin{tabular}{|c|c|}
\hline $\begin{array}{l}\text { PHYS_PKG_ID } \\
\text { PHYS_COMP_DESCR } \\
\text { PHYS_COMP_VOL_PCT } \\
\text { PHYS_COMP_WGT }\end{array}$ & $\begin{array}{l}\text { OTP-LLW-WP- } 02 \\
\text { CONWEB PADS } \\
93 \\
127.5\end{array}$ \\
\hline $\begin{array}{l}\text { PHYS_PKG_ID } \\
\text { PHYS_COMP_OESCR } \\
\text { PHYSCOMP_VOL_PCT } \\
\text { PHYS_COMP_WGT }\end{array}$ & $\begin{array}{l}\text { OTP-LLW-WP- } 02 \\
\text { HAZARDOUS CONSTI IUENTS } \\
5 \\
.64\end{array}$ \\
\hline $\begin{array}{l}\text { PHYS_PKG_ID } \\
\text { PHYS_COMP_DESCR } \\
\text { PHYS_COMP_VOL_PCT } \\
\text { PHYS_COMP_WGT }\end{array}$ & $\begin{array}{l}\text { OTP-LLW-WP- } 02 \\
\text { WATER } \\
2 \\
3.9\end{array}$ \\
\hline $\begin{array}{l}\text { HDET_PKG_ID } \\
\text { HDET_DESIG_CD } \\
\text { HDET_DW_NUM } \\
\text { HDET_WASTE_VOL. }\end{array}$ & $\begin{array}{l}\text { OTP-LLW-WP-O2 } \\
\text { RD } \\
\text { D002 } \\
.21\end{array}$ \\
\hline $\begin{array}{l}\text { HAZ_PKG_ID } \\
\text { HAZ_COMPIID } \\
\text { HAZ_COMP_TEXT } \\
\text { HAZ_COMP_WGT }\end{array}$ & $\begin{array}{l}\text { OTP-LLLW-WP- } 02 \\
\text { TEMP2091 } \\
\text { LIQUID WASTE } \\
.6\end{array}$ \\
\hline $\begin{array}{l}\text { HAZ_PKG_ID } \\
\text { HAZ_COMP_ID } \\
\text { HAZ_COMP_TEXT } \\
\text { HAZ_COMP_WGT }\end{array}$ & $\begin{array}{l}\text { OTP-LLW-WP-02 } \\
\text { TEMP3 } 313 \\
\text { SODIUM CHLORIDE } \\
.04\end{array}$ \\
\hline 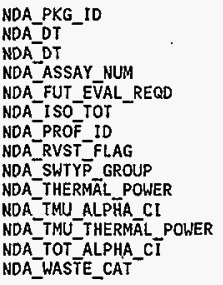 & $\begin{array}{l}\text { OIP-LLW-WP-02 } \\
\text { SEP-12-96 13:47:13 } \\
3051524833 \\
265 \\
N \\
1 \\
\text { NRLLW1 } \\
N \\
\text { LLW } \\
3.53 \\
0 \\
.0353 \\
0 \\
\text { GTWC3 }\end{array}$ \\
\hline $\begin{array}{l}\text { NDAISO_PKG_ID } \\
\text { NDAISO_NAME } \\
\text { NDAISO_ASSAY_NUM } \\
\text { NDAISO_OTY } \\
\text { NDAISO_QTY_TMU } \\
\text { NDAISO_CONFIDENCE_CK } \\
\text { NDAISO_MEAS_STATUS } \\
\text { NDAISO_PAN_ACTIVE } \\
\text { NDAISO_PAN_PASSIVE } \\
\text { NDAISO_GEA }\end{array}$ & $\begin{array}{l}\text { OTP-LLW-WP-02 } \\
\text { NA-22 } \\
265 \\
.0003 \\
.000003 \\
T \\
\text { ACT } \\
\text { A } \\
\text { NO } \\
\text { THS IS ONLY A TEST }\end{array}$ \\
\hline $\begin{array}{l}\text { NDE_OPER_IO } \\
\text { NDE_VDISK_FILE } \\
\text { NDE_VDISK_NUM } \\
\text { NDE_VIAPE_NUM } \\
\text { NDE_VTAPE_START }\end{array}$ & $\begin{array}{l}\text { OTP-LLW-WP-02 } \\
\text { 12-SEP-96 13:39:22 } \\
\text { OTP-LLW-WP-02 1st inage } \\
\text { comments } \\
\text { USER1 } \\
\text { WP17X } \\
\text { P22V1 } \\
\text { P22T1 } \\
200\end{array}$ \\
\hline $\begin{array}{l}\text { NDE_OPER_ID } \\
\text { NDE_VDISK_FILE } \\
\text { NDE_VOISK_NUM } \\
\text { NDE_VIAPE_NUM } \\
\text { NDE_VTAPE_START }\end{array}$ & $\begin{array}{l}\text { OTP-LLW-WP-02 } \\
12-\text { SEP-96 13:40:09 } \\
\text { OrP-LLW-WP-02 2nd image } \\
\text { comments } \\
\text { USER1 } \\
\text { WP11Y } \\
\text { P22V1 } \\
\text { P22I1 } \\
250\end{array}$ \\
\hline CONLOC_PKG_ID & OTP-LLW-WP-02 \\
\hline
\end{tabular}

$\begin{array}{ll}\text { CONLOC_DT } & \text { 29-SEP-97 } 11: 17: 06 \\ \text { CONLOC_LOCN_ID } & \text { LLW_SORT }\end{array}$

CON PKG ID OTP-LLW-WP-03

CON_ACCUM DT

CON_CNTR VOOL

CON CNTY $\bar{P}$ CD

CONDATA QUAL_CD

CON GENER WASTTE_DESCR

CON_GGRP ID

CON_GROSS WWT

CON LABPAC̄K FLAG

CON LOCN FAC IL ID

CONPHYS STATE_CD

CON PKG DT

CON PKG STATUS

CON PWTY P CD

CON_SAMPLE FLAG

CON SIZE DESCR

CON SRCE-CHRG CD

CON_SRCE_CMPNY ID

CON_SRCE CMPNY TYPE

CON_SRCE_FACIL_ID

CON_TARE WGT

CON TSD ACCEPT DT

CON_WASTTE_WGT

CONEXT PKG ID

CONEXT_COMPLIANT_FLAG

CONEXT NDE VER FLAG

CONEXT PRO $\bar{F}$ FLA $\bar{A} G$

CONEXT PROF ID

CONEXT USE $\overline{C D}$

CONEXT_WRAP_STAT_CD

RDET PKG ID

RDET_BG DOSE RATE

RDET HANDLING

RDET RELOC DT

RDET RSWIMS COUNT

RDET SOAR APPRV IUM

RDET_SWIMS CD

ROET SWTYP CD

ROET SWTYP GROUP

RDET THERMĀL POWER

RDET TOT BG Eे I

RDET TOT DE CI

RDET WAST̄E MAKEUP

RDET_WRAP_CAT

RAD_PKG_ID

RAD I SO NUM

RAD $Q T Y$

RAD UNKNOWN

RAD_PKG_ID

RAD ISO_NUM

RAD_QTY

RAD PKG ID

RAD ISO NUM

RAD_QTY

PHYS PKG ID

PHYS COMP DESCR

PHYS COMP VOL PCY

PHYS COMP WGT

PHYS PKG IO

PHYS_COMP DESCR

PHYS COMP VOL PCT

PHYS_COMP_WGT

PHYS PKG ID

PHYS COMI DESCR

PHYS COMP VOL PCT

PHYS_COMP_WGT
29-MAY-90 00:00:00

.21

DM

AR

INNER

OTHER

163.02

N

$2336 \mathrm{~W}$

$s$

29-MAY-90 00:00:00

$T$

$\mathrm{R}$

55 GALLON

B80545

PNL

GEN

324

27

05-29-90 00:00:00

136

OTP-LLW-WP-03

Y

N

NRLLW1

WP

I

OTP-LLW-WP-03

7

29-MAY-90 00:00:00

1

$2 A=1 A-1 A-0$

CE

2A

LLW

3.53

.01212

1

F

OTP-LLW-WP-03

19

.01212

.000324

OTP-LLW-WP-03

3

.00006

OTP-LLW-WP-03

.006

OTP-LLW-WP-03

CLOTH/RAGS/NYLON

10

14

OTP-LLW-WP-03

PAPER/CARDBOARD

30

41

OTP-LLW-WP-03

PLASTIC/POLYURATHANE

60

81 


\begin{tabular}{|c|c|}
\hline 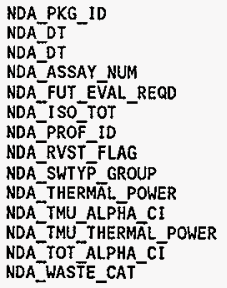 & $\begin{array}{l}\text { OTP-LLW-WP-03 } \\
\text { SEP-12-96 } 13: 34: 22 \\
3051524062 \\
262 \\
N \\
2 \\
\text { NRLLW1 } \\
N \\
\text { LLW } \\
5.45 \\
0 \\
.0545 \\
0 \\
\text { GTWC3 }\end{array}$ \\
\hline $\begin{array}{l}\text { NDAISO_PKG_ID } \\
\text { NDAISO_NAME } \\
\text { NDAISO_ASSAY_NUM } \\
\text { NDAISO_QTY - } \\
\text { NDAISO_QTY_TMU } \\
\text { NDAISO_CONFIDENCE_CK } \\
\text { NDAISO_MEAS_STATUS } \\
\text { NDAISO_PAN_ACTIVE } \\
\text { NDAISO_PAN_PASSIVE } \\
\text { NDAISO_GEA }\end{array}$ & $\begin{array}{l}\text { OTP-LLW-WP-03 } \\
\text { CS- } 137 \\
262 \\
.006 \\
.00006 \\
\vdots \\
\text { ACT } \\
\text { B } \\
\text { NO } \\
\text { THIS IS ONLY A TEST }\end{array}$ \\
\hline $\begin{array}{l}\text { NDAISO_PKG_ID } \\
\text { NDAISO_NAME } \\
\text { NDAISO_ASSAY_NUM } \\
\text { NDAISO_QTY } \\
\text { NDAISO_QTY_TMU } \\
\text { NDAISO_CONFIIDENCE_CK } \\
\text { NDAISO_MEAS_STATUS } \\
\text { NDAISO_PAN_ACTIVE } \\
\text { NDAISO_PAN_PASSIVE } \\
\text { NDAISO_GEA }\end{array}$ & $\begin{array}{l}\text { OTP-LLW-WP-03 } \\
\text { SR-90 } \\
262 \\
.00006 \\
.0000006 \\
\text { ACT } \\
\text { B } \\
\text { NO } \\
\text { THIS IS ONLY A TEST }\end{array}$ \\
\hline $\begin{array}{l}\text { NDE_PKG_ID } \\
\text { NDE_DT } \\
\text { NDE_COMMENTS }\end{array}$ & $\begin{array}{l}\text { OTP-LLW-WP-03 } \\
12-S E P-96 \quad 13: 31: 45 \\
\text { OTP-LLW-WP-03 1st image } \\
\text { comnents }\end{array}$ \\
\hline $\begin{array}{l}\text { NDE_OPER_ID } \\
\text { NDE_VDISK_FILE } \\
\text { NDE_VDISK_NUM } \\
\text { NDE_VTAPE_NUM } \\
\text { NDE_VIAPE_SIART, }\end{array}$ & $\begin{array}{l}\text { USER1 } \\
\text { WPO4X } \\
\text { P22V1 } \\
\text { P22T1 } \\
200\end{array}$ \\
\hline $\begin{array}{l}\text { NDE_PKG_ID } \\
\text { NDE_DT } \\
\text { NDE_COMMENTS }\end{array}$ & $\begin{array}{l}\text { OTP-LLW-WP-03 } \\
12-S E P-96 \quad 13: 33: 16 \\
\text { OTP-LLW-WP-03 2nd image } \\
\text { comments }\end{array}$ \\
\hline $\begin{array}{l}\text { NDE_OPER_ID } \\
\text { NDE_VDISK_FILE } \\
\text { NDE_VDISK_NUMH } \\
\text { NDE_VTAPE_NUM } \\
\text { NDE_VTAPE_START }\end{array}$ & $\begin{array}{l}\text { USER } 1 \\
\text { WPO4Y } \\
\text { P22V1 } \\
\text { P22T1 } \\
250\end{array}$ \\
\hline $\begin{array}{l}\text { CONR_FROM_PKG_ID } \\
\text { CONR_TO_PKG_ID } \\
\text { CONR_DT } \\
\text { CONR_REL_CD }\end{array}$ & $\begin{array}{l}\text { OTP-LLW-WP-03 } \\
\text { OTP-LLW-OD-03 } \\
05-J A N-9709: 30: 00 \\
M\end{array}$ \\
\hline 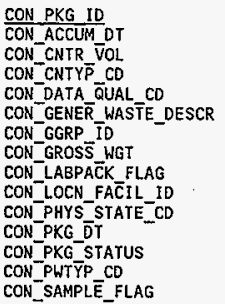 & $\begin{array}{l}\text { OTP-LLW-WP-04 } \\
05-J U L-90 \quad 00: 00: 00 \\
.21 \\
\text { OH } \\
\text { AR } \\
\text { INNER } \\
\text { OTHER } \\
358.34 \\
N \\
2336 \mathrm{~W} \\
S \\
05-J U L-90 \quad 00: 00: 00 \\
T \\
M \\
N\end{array}$ \\
\hline
\end{tabular}

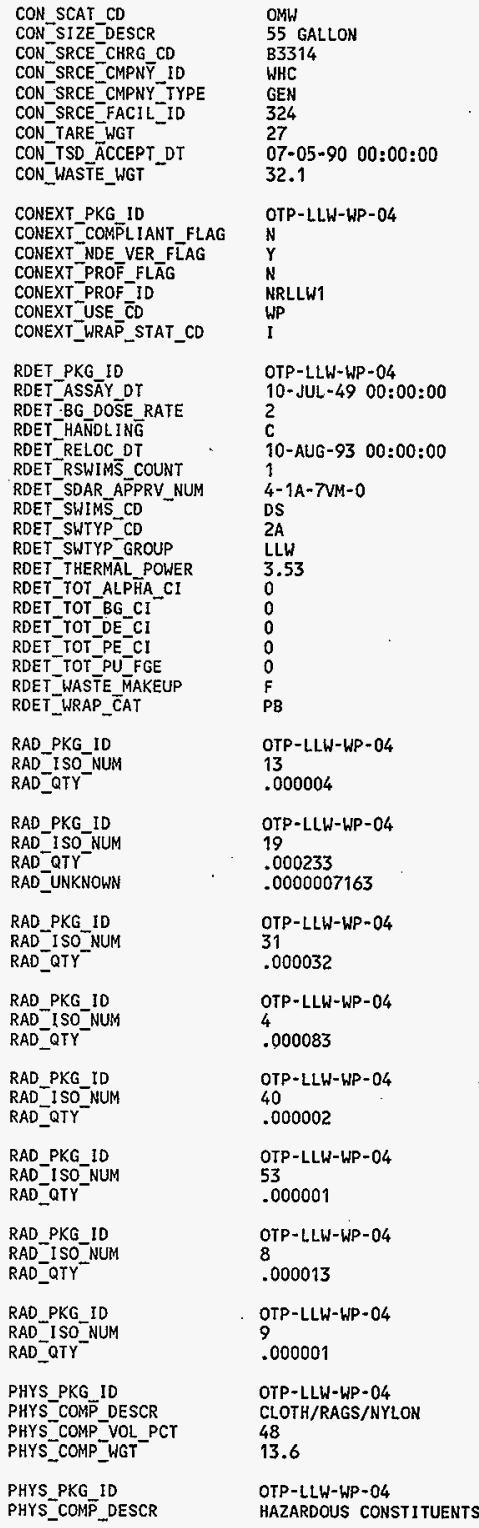




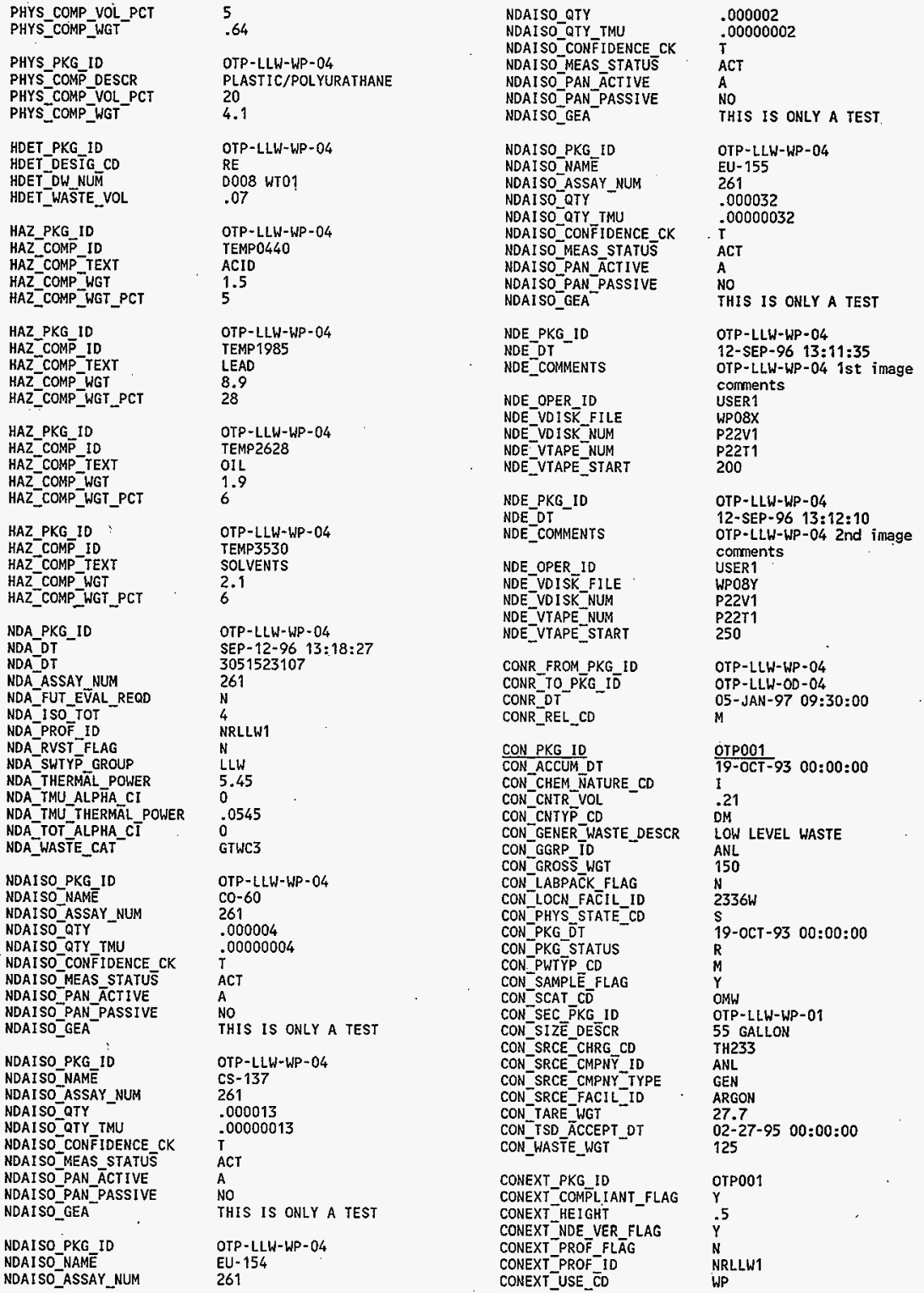




\begin{tabular}{|c|c|}
\hline CONEXT_WRAP_STAT_CD & I \\
\hline $\begin{array}{l}\text { RDET_PKG_ID } \\
\text { RDET_BG_DOSE_RATE } \\
\text { RDET_HANDLING } \\
\text { RDET_RELOC_DT } \\
\text { RDET_RSWIMS_COUNT } \\
\text { RDET_SSAR_APPRV_NUM } \\
\text { RDET_SWIMS_CD } \\
\text { RDET_SWTYP_CD } \\
\text { RDET_SWTYP_GROUP } \\
\text { RDET_THERMĀL_POWER } \\
\text { RDET_TOT_BG_CI } \\
\text { RDET_TOT_DE_CI } \\
\text { RDET_WASTE_CAT } \\
\text { RDET_WASTE_MAKEUP } \\
\text { RDET_WRAP_CAT }\end{array}$ & $\begin{array}{l}\text { OTP001 } \\
.1 \\
C \\
01-S E P-9600: 00: 00 \\
1 \\
60-1 R-3 Y M-0401 \\
\text { DD } \\
2 A \\
\text { LLW } \\
3.53 \\
.00000254 \\
.0000097 \\
\text { WC1 } \\
\text { S } \\
\text { SO }\end{array}$ \\
\hline $\begin{array}{l}\text { RAD_PKG_ID } \\
\text { RAD_ISO_NUM } \\
\text { RAD_QTY }\end{array}$ & $\begin{array}{l}\text { OTP001 } \\
104 \\
.00000788\end{array}$ \\
\hline $\begin{array}{l}\text { RAD_PKG_ID } \\
\text { RAD_ISO_NUM } \\
\text { RAD_QTY }\end{array}$ & $\begin{array}{l}\text { otP001 } \\
18 \\
11\end{array}$ \\
\hline $\begin{array}{l}\text { RAD_PKG_ID } \\
\text { RAD_ISO_NUN } \\
\text { RAD_QTY }\end{array}$ & $\begin{array}{l}\text { OTP001 } \\
19 \\
.00000254\end{array}$ \\
\hline $\begin{array}{l}\text { RAD_PKG_ID } \\
\text { RAD_ISO_NUM } \\
\text { RAD_QTY }\end{array}$ & $\begin{array}{l}\text { OTP001 } \\
202 \\
.0013\end{array}$ \\
\hline $\begin{array}{l}\text { RAD_PKG_ID } \\
\text { RAD_ISO_NUM } \\
\text { RAD_QTY }\end{array}$ & $\begin{array}{l}\text { OTP001 } \\
203 \\
.19\end{array}$ \\
\hline $\begin{array}{l}\text { RAD_PKG_ID } \\
\text { RAD_ISO_NUM } \\
\text { RAD_QTY }\end{array}$ & $\begin{array}{l}\text { OTP001 } \\
206 \\
25\end{array}$ \\
\hline $\begin{array}{l}\text { RAD_PKG_ID } \\
\text { RAD_ISO_NUM } \\
\text { RAD_QTY }\end{array}$ & $\begin{array}{l}\text { OTP001 } \\
86 \\
.00000254\end{array}$ \\
\hline $\begin{array}{l}\text { PHYS_PKG_ID } \\
\text { PHYS_COMP_DESCR } \\
\text { PHYS_COMP_VOL_PCT } \\
\text { PHYS_COMP_WGT }\end{array}$ & $\begin{array}{l}\text { OTP001 } \\
\text { DIRT/SOIL/DIATONACEOUS } \\
\text { EARTH } \\
86 \\
115\end{array}$ \\
\hline $\begin{array}{l}\text { PHYS_PKG_ID } \\
\text { PHYS_COMP_DESCR } \\
\text { PHYS_COMP_VOL_PCI } \\
\text { PHYS_COMP_WGI- }\end{array}$ & $\begin{array}{l}\text { OTP001 } \\
\text { HAZARDOUS CONSTITUENTS } \\
0^{5}\end{array}$ \\
\hline $\begin{array}{l}\text { PHYS_PKG_IO } \\
\text { PHYS_COMP_DESCR } \\
\text { PHYS_COMP_VOL_PCT } \\
\text { PHYS_COMP_WGT }\end{array}$ & $\begin{array}{l}\text { OTPQ01 } \\
\text { PAPER/CARDBOARD } \\
9.5 \\
5\end{array}$ \\
\hline $\begin{array}{l}\text { PHYS_PKG_ID } \\
\text { PHYS_COMP_DESCR } \\
\text { PHYS_COMP_VOL_PCT } \\
\text { PHYS_COMP_WGT }\end{array}$ & $\begin{array}{l}\text { OTP001 } \\
\text { PLASTIC/POLYURATHANE } \\
4 \\
5\end{array}$ \\
\hline $\begin{array}{l}\text { HDET_PKG_ID } \\
\text { HDET_CNT } \bar{R} \text { STATUS } \\
\text { HDET_DESIG_CD } \\
\text { HDET_DW_NUM } \\
\text { HDET_WASTE_VOL } \\
\text { HDET_WASTE_STATUS }\end{array}$ & $\begin{array}{l}\text { OTP001 } \\
F \\
\text { RE } \\
\text { D008 D009 } \\
i^{208}\end{array}$ \\
\hline $\begin{array}{l}\text { HAZ_PKG_ID } \\
\text { HAZ_COMP_ID } \\
\text { HAZ_COMP_TEXT }\end{array}$ & $\begin{array}{l}\text { OTPOO1 } \\
7439-97-6 \\
\text { MERCURY }\end{array}$ \\
\hline
\end{tabular}

\begin{tabular}{|c|c|}
\hline $\begin{array}{l}\text { HAZ_COMP_WGT } \\
\text { HAZ_COMP_WGT_PCT } \\
\text { HAZ_EPCRA_FLABG }\end{array}$ & $\begin{array}{l}.001 \\
\dot{N}^{0009}\end{array}$ \\
\hline $\begin{array}{l}\text { HAZ_PKG_ID } \\
\text { HAZ_COMP_ID } \\
\text { HAZ_COMP_TEXT } \\
\text { HAZ_COMP_WGT } \\
\text { HAZ_CONP_WGT_PCT } \\
\text { HAZ_EPCRA_FLA }\end{array}$ & $\begin{array}{l}\text { OTP001 } \\
\text { TEMP1985 } \\
\text { LEAD } \\
.085 \\
.068 \\
\text { N }^{.068}\end{array}$ \\
\hline $\begin{array}{l}\text { CON PKG ID } \\
\text { CON_CNTYP_CD } \\
\text { CON_LOCN_FACIL_ID } \\
\text { CON_PKG_STATUS } \\
\text { CON_SCAT_CO } \\
\text { CON_SIZE_DESCR } \\
\text { CON_TARE_WGT }\end{array}$ & $\begin{array}{l}\text { OTP-LLWR-TD-01 } \\
\text { DM } \\
2336 W \\
\text { G } \\
\text { ANY } \\
85 \text { GALLON } \\
31\end{array}$ \\
\hline $\begin{array}{l}\text { CONEXT_PKG_ID } \\
\text { CONEXT_CNT } \bar{R} \text { STATUS } \\
\text { CONEXT_USE_CD } \\
\text { CONEXT_WRA } \bar{P}_{-} \text {STAT_CD }\end{array}$ & $\begin{array}{l}\text { OTP-LLWR-TD-01 } \\
E \\
\text { ID } \\
\text { I }\end{array}$ \\
\hline $\begin{array}{l}\text { CONLOC_PKG_ID } \\
\text { CONLOC_OT } \\
\text { CONLOC_LOCN_ID }\end{array}$ & $\begin{array}{l}\text { OTP-LLWR-TD-01 } \\
\text { 29-SEP-97 08:49:34 } \\
\text { LLW_RWMPRT }\end{array}$ \\
\hline $\begin{array}{l}\text { CON PKG ID } \\
\text { CON_CNTYP_CD } \\
\text { CON_LOCN_FACIL_ID } \\
\text { CON_PKG_STATUS } \\
\text { CON_SCAT CD } \\
\text { CON_SIZE_DESCR } \\
\text { CON_TARE_WGT }\end{array}$ & $\begin{array}{l}\text { OTP-LLWR-TD }-02 \\
\text { DM } \\
2336 \mathrm{~W} \\
6 \\
\text { ANY } \\
85 \text { GALLON } \\
31\end{array}$ \\
\hline $\begin{array}{l}\text { CONEXT_PKG_ID } \\
\text { CONEXT_CNTR_STATUS } \\
\text { CONEXT_USE_CD } \\
\text { CONEXT_WRAP_STAT_CD }\end{array}$ & $\begin{array}{l}\text { OTP-LLWR-TD-02 } \\
\text { E } \\
\text { TD } \\
\text { I }\end{array}$ \\
\hline $\begin{array}{l}\text { CONLOC_PKG_10 } \\
\text { CONLOC_DT } \\
\text { CONLOC_LOCN_10 }\end{array}$ & $\begin{array}{l}\text { OTP-LLWR-TO-02 } \\
\text { 05-FEB-97 16:00:00 } \\
\text { RWM_CRSL. }\end{array}$ \\
\hline CON PKG ID & OTP6-97-300001 \\
\hline $\begin{array}{l}\text { CONEXT_PKG_ID } \\
\text { CONEXT_USE_CD }\end{array}$ & $\begin{array}{l}\text { OTP6-97-300001 } \\
\text { PP }\end{array}$ \\
\hline $\begin{array}{l}\text { CONR_FROM_PKG_ID } \\
\text { CONR_TO_PKG_ID } \\
\text { CONR_DT } \\
\text { CONR_REL_CD }\end{array}$ & $\begin{array}{l}\text { OTP6-97-300001 } \\
\text { OTP6-97-400001 } \\
29-\text { SEP-97 11:15:00 } \\
0\end{array}$ \\
\hline $\begin{array}{l}\text { CON PKG ID } \\
\text { CON_PKG_DT } \\
\text { CON_PKG_STATUS }\end{array}$ & $\frac{\text { OTP } 6-97-400001}{29-S E P-97 \quad 11: 15: 00}$ \\
\hline $\begin{array}{l}\text { CONEXT_PKG_ID } \\
\text { CONEXT_USE_CD }\end{array}$ & $\begin{array}{l}\text { OTP6-97-400001 } \\
\text { TP }\end{array}$ \\
\hline $\begin{array}{l}\text { CONR_FROM_PKG_ID } \\
\text { CONR_TO_PKG_ID } \\
\text { CONR_DT } \\
\text { CONR_REL_CD. }\end{array}$ & $\begin{array}{l}\text { OTP6-97-300001 } \\
\text { OTP6-97-400001 } \\
29-\text { SEP-97 } 11: 15=00 \\
0\end{array}$ \\
\hline $\begin{array}{l}\text { CONLOC_PKG_ID } \\
\text { CONLOC_DT } \\
\text { CONLOC_LOCN_10 }\end{array}$ & $\begin{array}{l}\text { OTP6-97-400001 } \\
29-S E P-97 \quad 11: 15: 00 \\
\text { LLW_PP. }\end{array}$ \\
\hline $\begin{array}{l}\text { CON PKG 1D } \\
\text { CON_ACCUMDT } \\
\text { CON_CHEM_NATURE_CD } \\
\text { CON_CNTR_VOL } \\
\text { CON_CNTY } \bar{P} \text { _CD } \\
\text { CON_DATA_QUAL_CD }\end{array}$ & $\begin{array}{l}\text { PUCK289 } \\
\frac{10-J U L-91}{10} 00: 00: 00 \\
\text { IO } \\
\text { DM } \\
\text { DM } \\
\text { AR }\end{array}$ \\
\hline
\end{tabular}




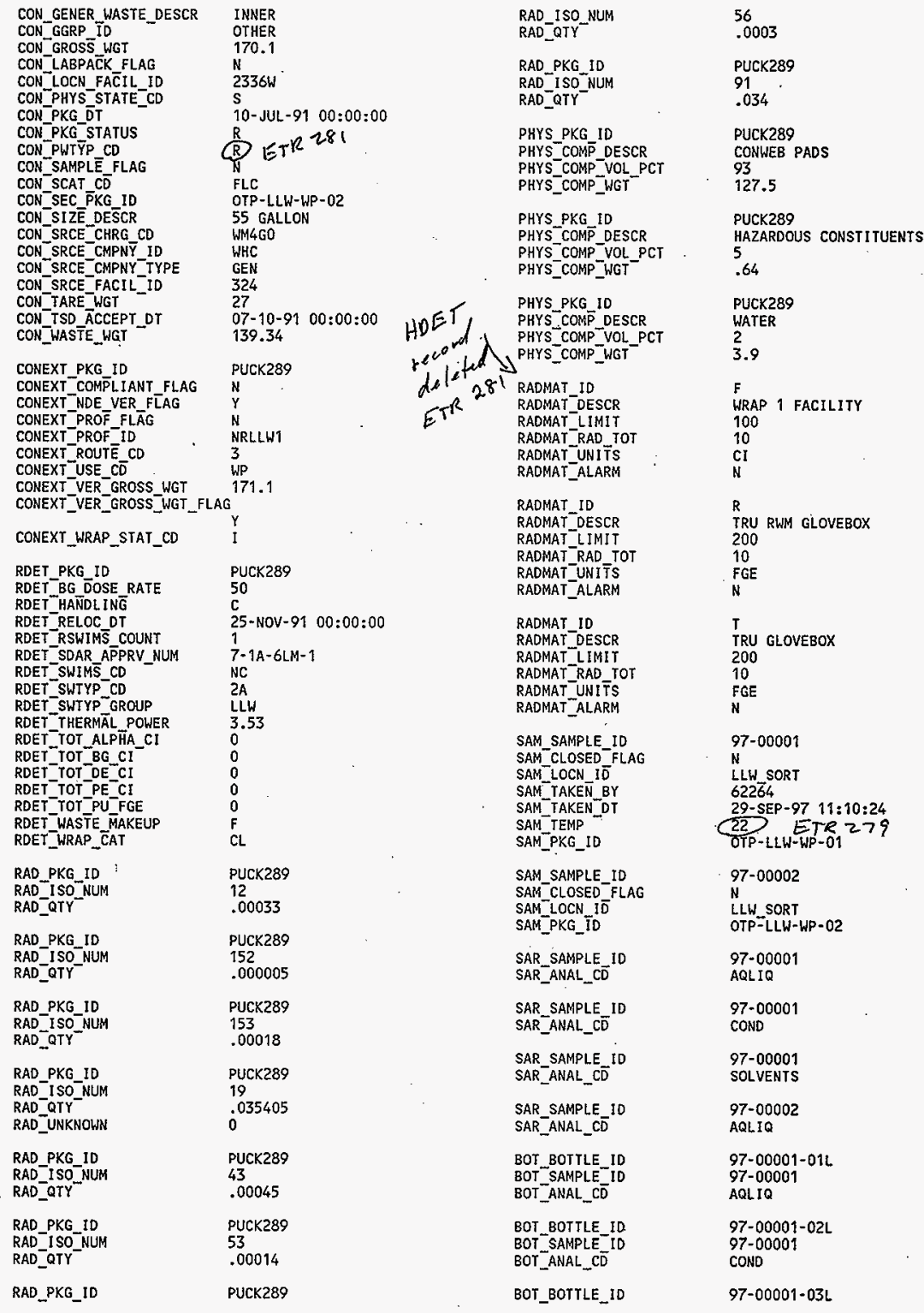




\begin{tabular}{|c|c|}
\hline $\begin{array}{l}\text { BOT_SAMPLE_ID } \\
\text { BOT_ANAL_CD }\end{array}$ & $\begin{array}{l}97-00001 \\
\text { SOLVENTS }\end{array}$ \\
\hline $\begin{array}{l}\text { BOT_BOTTLE_ID } \\
\text { BOT_SAMPLE_ID } \\
\text { BOT_ANAL_CD }\end{array}$ & $\begin{array}{l}97-00002-01 L \\
97-00002 \\
\text { AQLIQ }\end{array}$ \\
\hline $\begin{array}{l}\text { SAMREL_BOTTLE_ID } \\
\text { SAMREL_PKG_ID- } \\
\text { SAMREL_OT } \\
\text { SAMREL_USE_CD . }\end{array}$ & $\begin{array}{l}97-00001-01 L \\
\text { OTP-LLW-WP-01 } \\
29-\text { SEP-97 00:00:00 } \\
\text { WP }\end{array}$ \\
\hline $\begin{array}{l}\text { SAMREL_BOTTLE_ID } \\
\text { SAMREL_PKG_ID-ID } \\
\text { SAMREL_DT } \\
\text { SAMREL_USE_CD }\end{array}$ & $\begin{array}{l}97-00001-01 \mathrm{~L} \\
\text { OTP6-97-300001 } \\
29-\text { SEP-97 } 11: 14: 48 \\
\text { PP }\end{array}$ \\
\hline $\begin{array}{l}\text { SAMREL_BOTTLE_ID } \\
\text { SAMREL_PKG_ID- } \\
\text { SAMREL_DT } \\
\text { SAMREL_USE_CD }\end{array}$ & $\begin{array}{l}\text { 97-00001-02L } \\
\text { OTP-LLW-WP-01 } \\
\text { 29-SEP-97 00:00:00 } \\
\text { WP }\end{array}$ \\
\hline $\begin{array}{l}\text { SAMREL_BOTTLE_ID } \\
\text { SAMREL_PKG_ID- } \\
\text { SAMREL_DT } \\
\text { SAMREL_USE_CD }\end{array}$ & $\begin{array}{l}97-00001-02 L \\
\text { OTP6-97-300001 } \\
29-S E P-97 \quad 11: 14: 58 \\
\text { PP }\end{array}$ \\
\hline $\begin{array}{l}\text { MSGLOG_DT } \\
\text { MSGLOG_ERROR_FLAG } \\
\text { MSGLOG_GEN_TYPE } \\
\text { MSGLOG_SEQ_NUM } \\
\text { MSGLOG_SPEC_TYPE } \\
\text { MSGLOG_STRINGI }\end{array}$ & $\begin{array}{l}09-29-97 \quad 15: 23: 59 \\
N \\
\text { PCSDMS } \\
121819 \\
\text { SPDP } \\
\text { PCSDMS SPDP } 875571842^{\sim} L L W \\
\text { SORT } 97-00001-03 L^{\sim} \text { OTP-LL } \\
\text { W-WP-04 }\end{array}$ \\
\hline $\begin{array}{l}\text { MSGLOG_OT } \\
\text { MSGLOG_ERROR_FLAG } \\
\text { MSGLOG_GEN_TYPE } \\
\text { MSGLOG_SEQ_NUM } \\
\text { MSGLOG_SPEC̄_TYPE } \\
\text { MSGLOG_STRING }\end{array}$ & $\begin{array}{l}\text { O9-29-97 } 15: 24: 52 \\
N \\
\text { PCSOMS } \\
121822 \\
\text { SPDP } \\
\text { PCSDMS } \sim \text { SPDP 875571894 LLW } \\
\text { SORT } 97-00002-01 L^{\sim} \text { OTP-LL } \\
\text { W-WP-04 }\end{array}$ \\
\hline $\begin{array}{l}\text { MSGLOG_DT } \\
\text { MSGLOG_ERROR_FLAG } \\
\text { MSGLOG_GEN_TYYPE } \\
\text { MSGLOG_SEQ_NUM } \\
\text { MSGLOG_SPEC TYPE } \\
\text { MSGLOG_STRINTG1 }\end{array}$ & $\begin{array}{l}09-29-97 \quad 15: 26: 28 \\
N \\
\text { PCSDMS } \\
121829 \\
\text { SPDP } \\
\text { PCSDMS } \sim \text { SPDP } \sim 875571991 \sim L L W \\
\text { SORT } \% 7-00002-01 \text { WTP-LL }^{\sim} \text {-WP-04 }\end{array}$ \\
\hline
\end{tabular}


CON PKG ID

CON_CNTYP_CD

CON_LOCN_FACIL_ID

CON_PKG_STATUS

CON SIZE DESCR

CON_TARE_WGT

CON PKG ID

CON_CNTYP CD

CON L.OCN FACIL_ID

CON PKG STATUS

CON_SIZE DESCR

CON_TARE_WGT

CON PKG ID

CON CNTYP CD

CON_LOCN FACIL ID

CONPKG STATUS

CON_SIZE_DESCR

CON_TARE_WGT

CON PKG ID

CON CNTYP CD

CON_LOCN_FACIL_ID

CON PKG STATUS

CON $\$$ SIZE_DESCR

CON_TARE_WGT

CON PKG ID

CON ACCUM DT

CON_CHEM ÑATURE_CD

CON CNTR VOL

CON CNTYP CD

CON_GENER_WASTE_DESCR

CON_GGRP ID

CON GROSS̈ WGT

CON_LABPACK_FLAG

CON LOCN FACIL ID

CON_PHYS_STATE_CD

CONPKG DTT

CON PKG STATUS

CON PWTYYP CD

CON SAMPLE FLAG

CON SCAT CD

CON_SIZE-DESCR

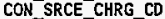

CON_SRCE_CMPNY ID

CON SRCE CMPNY TYPE

CON_SRCE_FACIL_ID

CON TARE WGT

CON TSD ACCEPT DT

CON_WASTE_WGT

CON PKG ID

CON_ACCUM DT

CON-CHEM_NATURE_CD

CON CNTR VOL

CON CNTYP CD

CONDATA QUAL CD

CON_GENER WASTE DESCR

CON GGRP ID

CON GROSS WGT

CON_LABPACK FLAG

CON LOCN FAC IL ID

CON_PHYS_STATE_CD

CON PKG $\bar{D} T$

CON PKG STATUS

CON_PWTYPPCD

CON SAMPLE FLAG

CON SCAT CD

CON_SIZE-DESCR

CON SRCE CHRG CD

CON_SRCE CMPNY ID

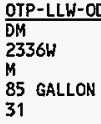

OTP-LLW-OD - 02

DM

$2336 \mathrm{~W}$

H

85 GALLON

31

OTP-LLW-OD-03

$2336 \mathrm{~W}$

$M$

85 GALLON

31

$O T P-L L W-O D-04$

DM

$2336 \mathrm{~W}$

$M$

85 GALLON

31

$\frac{\text { OTP-LLW-WP-01 }}{19-0 C T-93}$

.21

OM

LOW LEVEL WASTE

ANL

152.7

N

$2336 \mathrm{~W}$

19-OCT-93

T

Y

OMW

55 GALLON

TH233

ANL.

GEN

ARGON

27.7

27-FEB-95

125

OFP-LLW-WP-02

10-JUL-91

IO

.21

DH

AR

INNER

OTHER

170.1

$N$

$2336 \mathrm{~W}$

5

10-JUL -91

$T$

$M$

FLC

55 GALLON

WM4GO

WHC

$\begin{array}{ll}\text { CON_SRCE_CMPNY_TYPE } & \text { GEN } \\ \text { CON_SRCE_FACIL_ID } & 324 \\ \text { CON_TARE_WGT - } & 27 \\ \text { CON_TSD_ACCEPT_DT } & 10-\text { JUL-91 } \\ \text { CON_WASTE_WGT } & 139.34\end{array}$

CON PKG ID

CON ACCUM OT

CON_CNTR_VOL

CON CNTYP CD

CONDATA QUUALCD

CON_GENER_WASTE_DESCR

CON GGRP ID

CON_GROSS̄ WGT

CON LABPACK FLAG

CON_LOCN_EACIL_ID

CON PHYS STATE CD

CON PKG DT

CON PKG STATUS

CON PWTYYP CD

CON ${ }^{-}$SAMPLE ELAG

CON SIZE DESSR

CON-SRCE CHRG CD

CON_SRCE_CMPNŸ_ID

CON_SRCE_CMPNY TYPE

CON_SRCE_FACIL_ID

CON_TARE WGT

CON TSD A ACCEPT DT

CON_WASTE_WGT

OTP-LLW-WP-03

29-MAY-90

.21

DM

AR

INNER

OTHER

163.02

N

$2336 \mathrm{~W}$

S

29-MAY-90

T

$\mathrm{R}$

55 GALLON

B80545

PNL

GEN

324

27

29-MAY-90

136

CON PKG ID

CON_ACCUNDDT

CON CNTR VOL

CON CNTY $\vec{P}$ CD

CONDATA QUAL CD

CON GENER WASTE DESCR

CON GGRP ID

CON GROSS WGT

CON LABPAC̄K FLAG

CON_LOCN_FACIL_ID

CON PHYS STATE CD

CON PKG $\bar{D} T$

CON PKG STATUS

CON PWTYPP CD

CON_SAMPLE E FLAG

CON $S C A T C \bar{D}$

CON SIZEDESCR

CON SRCE CHRG CD

CON SRCE CMPNY ID

CON SRCE-CMPNY TYPE

CON SRCE FACILID

CON TARE WGT

CON_TSD ACCEPT DT

CON_WASTE_WGT

\section{CON PKG ID}

CON CNTYP CD

CON_LOCN_FACIL_ID

CON PKG STATUS

CON'SCAT_CD

CONSIZE DESCR

CON_TARE_WGT

\section{CON PKG ID}

CON_CNTYP_CD

CON_LOCN_FACIL_ID

CONPKG STATUS

CON SCAT CD

CON SIZE DESCR

CON_TARE_WGT

OTP-LLW-WP-O4

05-JUL-90

.21

DM

AR

INNER

OTHER

358.34

N

$2336 \mathrm{~W}$

05-JUL-90

T

OMW

55 GALLON

83314

WHC

GEN

324

27

05-JUL-90

32.1

OTP-LLWR-TD-01

DM

$2336 \mathrm{~W}$

G

ANY

85 GALLON

31

OTP-LLWR - TD - 02

DM

$2336 \mathrm{~W}$

ANY

85 GALLON

31

CON PKG ID

0TP6-97-300001 
CON PKG 10

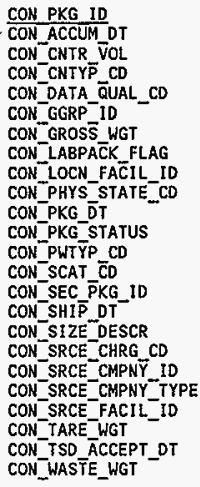

CON PKG ID

CON_ACCUM_DT

CON CNTR VOL

CON CNTYP CD

CON_DATA_QUAL_CO

CON GGRP ID

CON_GROS̄ $W G T$

CON LABPACKK FLAG

CON_LOCN_FAC̈IL_ID

CON PHYS STATE_CD

CON PKG DT

CONPKG STATUS

CON_PWTYP_CD

CON SCAT CD

CON_SEC PKG_1D

CON SHIP OT $^{-}$

CON_SIZE_DESCR

CON SRCE CHRG CD

CON SRCE CMPNY ID

CON_SRCE_CMPNY_TYPE

CON_SRCE_FACIL_ID

CON TARE WGT

CON_TSD ACCEPT DT

CON_WASTE_WGT

CONEXT PKG ID

CONEXT USE CD

CONEXT_WRAT̄_STAT_CD

CONEXT_PKG_ID

CONEXT USE CD

CONEXT_WRAP_STAT_CD

CONEXT_PKG_ID

CONEXT USE CD

CONEXT_WRAP_STAT_CD

CONEXT_PKG_ID

CONEXT USE CD

CONEXT_WRAP_STAT_CD

CONEXT PKG ID

CONEXT_COMPLIANT FLAG

CONEXT NDE VER FLAG

CONEXT PROF FLÄG

CONEXT_PROF_ID

CONEXT USE CD

CONEXT_WRAP_STAT_CD
OTP6-97-400001

\begin{tabular}{l} 
RHZ-101-A13568 \\
\hline $14-0 C T-85$ \\
.21 \\
DN \\
AR \\
PFP \\
72.98 \\
$N$ \\
$2336 \mathrm{~W}$ \\
S \\
$14-$ OCT -85 \\
$T$ \\
R \\
TRU \\
A13568 \\
$14-$ OCT -85 \\
55 GALLON \\
K6 \\
WHC \\
GEN \\
$2345 Z$ \\
27 \\
$14-$ OCT -85 \\
45.98
\end{tabular}

RHZ-103-A13561 14-0CT-85

.21

DN

AR

PFP

51.98

N

$2336 \mathrm{~W}$

5

14-OCT-85

$\mathrm{T}$

TRU

A13561

14-OCT-85

55 GALLON

K6

WHC

GEN

23452

27

14-oCT-85

24.98

OTP-LLW-OD-01

OD

OTP-LLW-OD-02

$O D$

I

OTP-L,LW-OD-03

OD

OTP-LLW-OD-04

OD

I

OTP-LLW-WP-01

$Y$
$y$

NRLLW1

WB

\begin{tabular}{|c|c|}
\hline $\begin{array}{l}\text { CONEXT_PKG_1D } \\
\text { CONEXT_COMPLIIANT_FLAG } \\
\text { CONEXT_NDE_VER_FLAG } \\
\text { CONEXT_PROF_FLAG } \\
\text { CONEXT_PROF_ID } \\
\text { CONEXI_ROUTE_CD } \\
\text { CONEXI_USE_CD } \\
\text { CONEXT_VER_GROSS_WGT } \\
\text { CONEXT_VER_GROSS_WGT_FLAO }\end{array}$ & $\begin{array}{l}\text { OTP-LLW-WP-02 } \\
\text { N } \\
Y \\
\text { W } \\
\text { WRLLW1 } \\
3 \\
\text { WP } \\
\text { I71.1 } \\
3\end{array}$ \\
\hline CONEXT_WRAP_STAT_CD & $\begin{array}{l}Y \\
\text { I }\end{array}$ \\
\hline $\begin{array}{l}\text { CONEXT_PKG_ID } \\
\text { CONEXT_COMPLIANT_FLAG } \\
\text { CONEXT_NDE_VER_FLAG } \\
\text { CONEXT_PROF_FLAG } \\
\text { CONEXT_PROF_ID } \\
\text { CONEXT_USE_CD } \\
\text { CONEXT_WRA } \bar{P} \text { STAT_CD }\end{array}$ & $\begin{array}{l}\text { OTP-LLW-WP-03 } \\
\text { Y } \\
Y \\
\text { N } \\
\text { NRLLW1 } \\
\text { WP } \\
\text { I }\end{array}$ \\
\hline $\begin{array}{l}\text { CONEXT_PKG_IO } \\
\text { CONEXT_COMPLIANT_FLAG } \\
\text { CONEXT_NDE VER_FLAG } \\
\text { CONEXT_PROF_FLAGG } \\
\text { CONEXT_PROF_ID } \\
\text { CONEXI_USE_CD } \\
\text { CONEXT_WRAP_STAT_CD }\end{array}$ & $\begin{array}{l}\text { OTP-LLW-WP-04 } \\
\text { W } \\
Y \\
\text { N } \\
\text { NRLLW1 } \\
\text { WP } \\
\text { I }\end{array}$ \\
\hline $\begin{array}{l}\text { CONEXI_PKG_ID } \\
\text { CONEXT_CNTR_STATUS } \\
\text { CONEXT_USE_CD } \\
\text { CONEXT_WRAP_STAT_CD }\end{array}$ & $\begin{array}{l}\text { OTP-L.LWR-TD-01 } \\
\text { E } \\
\text { TD } \\
\text { I }\end{array}$ \\
\hline $\begin{array}{l}\text { CONEXT_PKG_IO } \\
\text { CONEXT_CNTR_STATUS } \\
\text { CONEXT_USE_CD } \\
\text { CONEXT_WRAP_STAT_CD }\end{array}$ & $\begin{array}{l}\text { OTP-LLWR-TD-02 } \\
\text { E } \\
\text { TD } \\
1\end{array}$ \\
\hline $\begin{array}{l}\text { CONEXT_PKG_ID } \\
\text { CONEXT_USE_CD }\end{array}$ & $\begin{array}{l}\text { OTP6-97-300001 } \\
\text { PP }\end{array}$ \\
\hline $\begin{array}{l}\text { CONEXT_PKG_10 } \\
\text { CONEXT_USE_CD }\end{array}$ & $\begin{array}{l}\text { OTP6-97-400001 } \\
\text { TP }\end{array}$ \\
\hline $\begin{array}{l}\text { CONEXT_PKG_ID } \\
\text { CONEXT_NDA_DRUN_STATUS } \\
\text { CONEXT_PROF_ID } \\
\text { CONEXT_ROUTE_CD } \\
\text { CONEXT_USE_CD } \\
\text { CONEXT_VER_GROSS_WGT }\end{array}$ & $\begin{array}{l}\text { RHZ-101-A13568 } \\
\text { PROC } \\
\text { TEST } \\
1 \\
\text { WP } \\
0\end{array}$ \\
\hline CONEXI_VER_GROSS_WGT_FLAC & $\mathrm{H}_{\mathrm{H}}$ \\
\hline CONEXT_WRAP_STAT_CD & I \\
\hline $\begin{array}{l}\text { CONEXT_PKG_ID } \\
\text { CONEXT_NDA_DRUM_STATUS } \\
\text { CONEXI_PROF,ID } \\
\text { CONEXT_ROUTE_CD } \\
\text { CONEXT_USE_CD } \\
\text { CONEXI_VER_GROSS_WGT }\end{array}$ & $\begin{array}{l}\text { RHZ-103-A13561 } \\
\text { PROC } \\
\text { TEST } \\
1 \\
\text { WP } \\
48.35\end{array}$ \\
\hline CONEXI_VER_GROSS_WGT_FLAC & \\
\hline CONEXT_WRAP_STAT_CD & $i$ \\
\hline $\begin{array}{l}\text { ROET_PKG_ID } \\
\text { ROET_BG_DOSE_RATE } \\
\text { RDET_HANDLING } \\
\text { RDET_RELOC_DT } \\
\text { RDET_RSWIMS COUNT } \\
\text { RDET_SDAR_APPRV_NUM } \\
\text { ROET_SWIMS_CD } \\
\text { RDET_SWTYP_CD } \\
\text { RDET_SWTYP_GROUP } \\
\text { RDET_THERMAL_POWER } \\
\text { ROET_TOT_BG_Cl] } \\
\text { RDET_TOT_DE_CI }\end{array}$ & $\begin{array}{l}\text { OTP-LLW-WP-01 } \\
.1 \\
\text { C } \\
01-\text { SEP-96 } \\
1 \\
60-1 R-3 Y M-0401 \\
0 D \\
2 A \\
\text { LLW } \\
3.53 \\
.00000254 \\
.0000097\end{array}$ \\
\hline
\end{tabular}




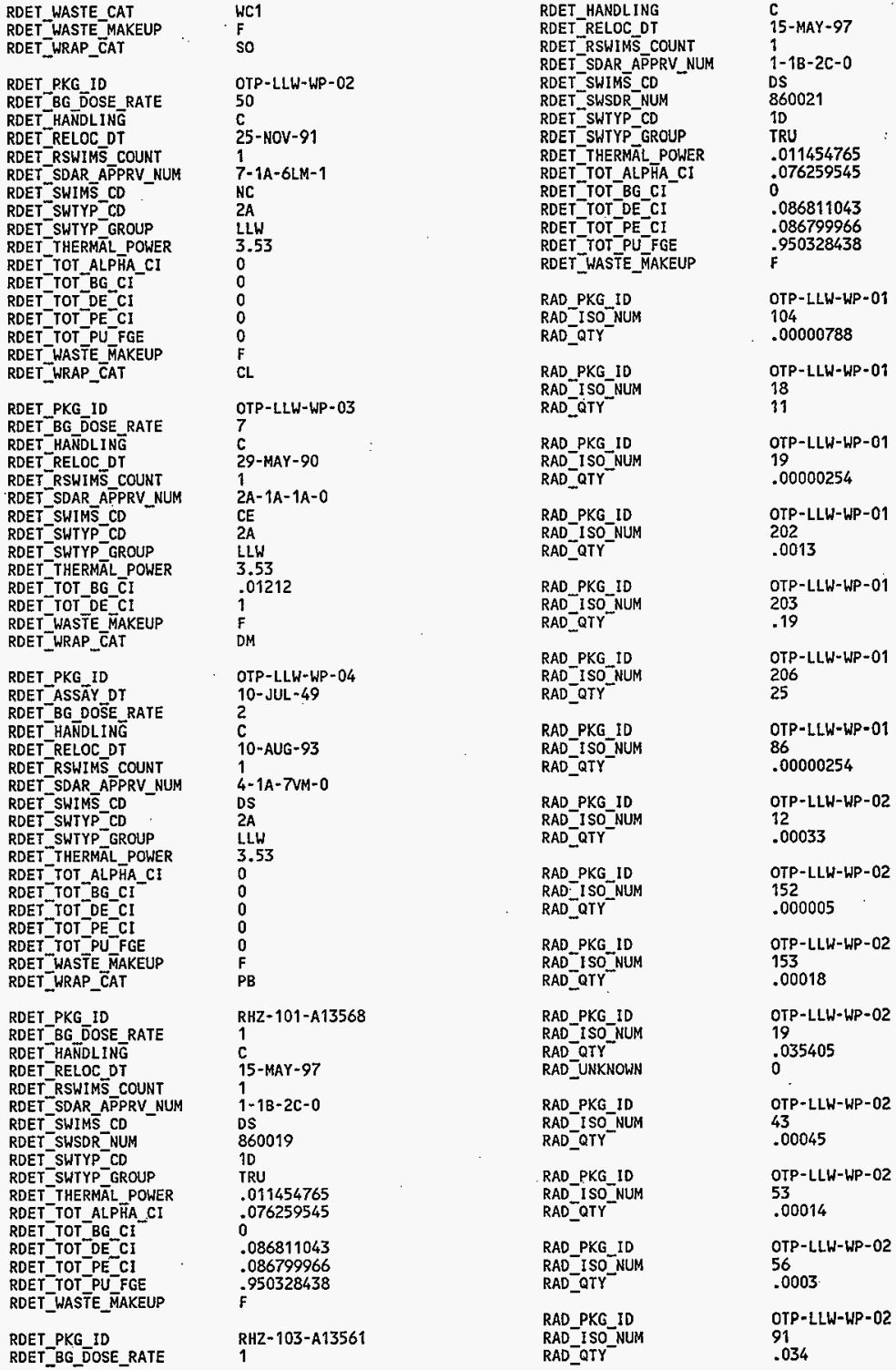




\begin{tabular}{|c|c|}
\hline $\begin{array}{l}\text { RAD_PKG_ID } \\
\text { RAD_ISO_NUM } \\
\text { RAD_QIY } \\
\text { RAD_UNKNOWN }\end{array}$ & $\begin{array}{l}\text { OrP-LLW-WP-03 } \\
19 \\
.01212 \\
.000324\end{array}$ \\
\hline $\begin{array}{l}\text { RAD_PKG_ID } \\
\text { RAD_ISO_NUM. . } \\
\text { RAD_QTY }\end{array}$ & $\begin{array}{l}\text { OTP-LLW-WP-03 } \\
3 \\
.00006\end{array}$ \\
\hline $\begin{array}{l}\text { RAD_PKG_ID } \\
\text { RAD_ISO_NUM } \\
\text { RAD_QTY }\end{array}$ & $\begin{array}{l}\text { OTP-LLW-WP- } 03 \\
8 \\
.006\end{array}$ \\
\hline $\begin{array}{l}\text { RAD_PKG_ID } \\
\text { RAD_ISO_NUM } \\
\text { RAD_QTY }\end{array}$ & $\begin{array}{l}\text { OTP-LLW-WP-04 } \\
13 \\
.000004\end{array}$ \\
\hline $\begin{array}{l}\text { RAD_PKG_ID } \\
\text { RAD_ISO_NUM } \\
\text { RAD_QTY } \\
\text { RAD_UNKNOWN }\end{array}$ & $\begin{array}{l}\text { OTP-LLW-WP-04 } \\
19 \\
.000233 \\
.0000007163\end{array}$ \\
\hline $\begin{array}{l}\text { RAD_PKG_ID } \\
\text { RAD_ISO_NUM } \\
\text { RAD_QTY }\end{array}$ & $\begin{array}{l}\text { OTP-LLW-WP-04 } \\
31 \\
.000032\end{array}$ \\
\hline $\begin{array}{l}\text { RAD_PKG_ID } \\
\text { RAD_ISO_NUM } \\
\text { RAD_QTY }\end{array}$ & $\begin{array}{l}\text { OTP-LLW-WP-04 } \\
4 \\
.000083\end{array}$ \\
\hline $\begin{array}{l}\text { RAD_PKG_ID } \\
\text { RAD_ISO_NUM } \\
\text { RAD_QTY }\end{array}$ & $\begin{array}{l}\text { OTP-LLW-WP- } 04 \\
40 \\
.000002\end{array}$ \\
\hline $\begin{array}{l}\text { RAD_PKG_ID } \\
\text { RAD_ISO_NUM } \\
\text { RAD_QTY }\end{array}$ & $\begin{array}{l}\text { OTP-LLW-WP-04 } \\
53 \\
.000001\end{array}$ \\
\hline $\begin{array}{l}\text { RAD_PKG_ID } \\
\text { RAD_ISO_NUM } \\
\text { RAD_QTY }\end{array}$ & $\begin{array}{l}\text { OTP-LLW-WP-04 } \\
8 \\
.000013\end{array}$ \\
\hline $\begin{array}{l}\text { RAD_PKG_ID } \\
\text { RAD_ISO_NUM } \\
\text { RAD_QTY }\end{array}$ & $\begin{array}{l}\text { OTP-LLW-WP- } 04 \\
9 \\
.000001\end{array}$ \\
\hline $\begin{array}{l}\text { RAD_PKG_ID } \\
\text { RAD_ISO_NUM } \\
\text { RAD_ALPHA_CI } \\
\text { RAD_PE_CI } \\
\text { RAD_PU_FGE } \\
\text { RAD_QTY }\end{array}$ & $\begin{array}{l}\text { RHZ-101-A13568 } \\
100 \\
.0580886376 \\
.05815044 \\
.9364 \\
.9364\end{array}$ \\
\hline $\begin{array}{l}\text { RAD_PKG_ID } \\
\text { RAD_ISO_NUM } \\
\text { RAD_QIY } \\
\text { RAD_UNKNOWN }\end{array}$ & $\begin{array}{l}\text { RHZ-101-A13568 } \\
19 \\
.05 \\
.05\end{array}$ \\
\hline $\begin{array}{l}\text { RAD_PKG_ID } \\
\text { RAD_ISO_NUM } \\
\text { RAD_ALPHA_CI } \\
\text { RAD_PE_CI } \\
\text { RAD_PU_FGE } \\
\text { RAD_QTY }\end{array}$ & $\begin{array}{l}\text { RHZ- } 101-\mathrm{A} 13568 \\
26 \\
.00030897 \\
.0003096 \\
.0000016875 \\
.00009\end{array}$ \\
\hline $\begin{array}{l}\text { RAD_PKG_ID } \\
\text { RAD_ISO_NUM } \\
\text { RAD_ALPHA_CI } \\
\text { RAD_PE_CI } \\
\text { RAD_PU_FGE } \\
\text { RAD_QYY }\end{array}$ & $\begin{array}{l}\text { RHZ- } 101-\mathrm{A} 13568 \\
41 \\
.00479304 \\
.00435272292 \\
.0000315 \\
.00028\end{array}$ \\
\hline $\begin{array}{l}\text { RAD_PKG_1D } \\
\text { RAD_ISO_NUM } \\
\text { RAD_ALPHA_CI } \\
\text { RAD_PE_CI }\end{array}$ & $\begin{array}{l}\text { RHZ- } 101-A 13568 \\
97 \\
.0130548 \\
.01311\end{array}$ \\
\hline
\end{tabular}

$\begin{array}{ll}\text { RAD_PU_FGE: } & .00129375 \\ \text { RAD_QTY } & .0575 \\ \text { RAD_PKG_ID } & \text { RHZ-101-A13568 } \\ \text { RAD_ISO_NUM } & 98 \\ \text { RAD_ALPŸA_CI } & .0000133112 \\ \text { RAD_PE_CI } & .010876488 \\ \text { RAD_PU_FGE } & .0126 \\ \text { RAD_QTY } & .0056\end{array}$

RAD PKG ID

RAD ISO NUM

RAD ALP $\overline{H A}$ CI

RAD_PE_CI

RAD PU FGE

RAD_QTYY

RAD PKG ID

RAD ISO NUM

RAD ALPHA CI

RAD PE CI

RAD PU FGE

RAD_QTY

RAD PKG ID

RAD_ISO_NUM

RAD_QTY

RAD_UNKNOWN

RAD PKG ID

RAD ISO NUM

RAD_ALPHA_C!

RAD_PE_CI

RAD PUFGE

RAD_QTY $\bar{Y}$

RAD_PKG_ID

RAD ISO NUM

RAD ALPHA CI

RAD_PE_CI

RAD PU FGE

RAD_QTY $\bar{Y}$

RAD PKG ID

RAD I SO NUM

RAD_ALPTHA_CI

RAD PE CI

RAD PU FGE

RAD_QTY

RAD PKG ID

RAD ISO NUM

RAD_ALPHA_CI

RAD PE CI

RAD PU FGE

RAD_QTY

RAD PKG_ID

RAD ISO NUM

RAD $^{-}$ALP ${ }^{-} A$ CI

RAD PECI

RAD PU FGE

RAD_QTY

PHYS PKG ID

PHYS_COMP_DESCR

PHYS COMP VOL PCT

PHYS_COMP-WGT

PHYS PKG ID

PHYS COMP DESCR

PHYS COMP VOL PCT

PHYS_COMP_WGT

PHYS_PKG_ID
RHZ-101-A13568

99

.0000007856

.00000071454474

.0000015

.0002

RHZ-103-A13561

100

.0580886376

.05815044

.9364

.9364

RHZ-103-A13561

19

.05

.05

RHZ - 103-A13561

26

.00030897

.0003096

.0000016875

.00009

RHZ-103-A13561

41

.00479304

.00435272292

.0000315

.00028

RHZ-103-A13561

97

.0130548

.01311

.00129375

.0575

RHZ-103-A13561

98

.0000133112

.010876488

.0126

.0056

RH2-103-A13561

99

.0000007856

.00000071454474

.0000015

.0002

OTP-LLW-WP-01

DIRT/SOIL/OLATOMACEOUS

EARTH

86

115

OTP-LLW-WP-01

HAZARDOUS CONSTITUENTS

.5

0

OTP-LLW-WP-01 
PHYS_COMP_DESCR

PHYS COMP VOL PCT

PHYS_COMP_WGT

PHYS PKG 10

PHYS_COMP DESCR

PHYS COMP VOL. PCT

PHYS_COMP_WGT

PHYS_PKG_ID

PHYS COMP DESCR

PHYS COMP VOL PCT

PHYSCOMP_WGT

PHYS PKG ID

PHYS_COMP DESCR

PHYS COMP VOL PCT

PHYS_COMP_WGT

PHYS PKG ID

PHYS COMP DESCR

PHYS COMP_VOL_PCT

PHYS_COMP_WGT

PHYS PKG ID

PHYS COMP DESCR

PHYS_COMP_VOL_PCT

PHYS_COMP_WGT

PHYS PKG ID

PHYS_COMP DESCR

PHYS COMP VOL PCT

PHYS_COMP_WGT

PHYS PKG ID

PHYS_COMP_DESCR

PHYS COMP VOL PCT

PHYS_COMP_WGT

PHYS PKG ID

PHYS_COMP_DESCR

PHYS_COMP_VOL_PCT

PHYS_COMP_WGT

PHYS PKG ID

PHYS_COMPDDESCR

PHYS COMP VOL PCT

PHYS_COMP_WGT

PHYS PKG ID

PHYS COMP DESCR

PHYS COMP VOL, PCT

PHYS_COMP_WGT

PHYS PKG 10

PHYS COM $\bar{P}$ OESCR

PHYS_COMP_VOL_PCT

PHYS PKG ID

PHYS COM $\bar{P}$ DESCR

PHYS_COMP_VOL_PCT

PHYS PKG 1D

PHYS COMP DESCR

PHYS_COMP_VOL_PCT

PHYS PKG ID

PHYS COMP DESCR

PHYS_COMP_VOL_PCT

PHYS PKG ID

PHYS_COMP_DESCR

PHYS_COMP_VOL_PCT

PHYS_PKG_10

PHYS COMP DESCR
PAPER/CAROBOARD

9.5

5

OTP-LLW-WP-01

PLASTIC/POLYURATHANE

4

OTP-LLW-WP-02

CONWEB PADS

93

127.5

OTP-LLW-WP-02

HAZARDOUS CONSTITUENTS

.64

OTP-LLW-WP-02

WATER

2

3.9

OTP-LLW-WP-O3

CLOTH/RAGS/NYLON

10

14

OTP-LLW-WP-03

PAPER/CARDBOARD

30

41

OTP-LLW-WP-03

PLASTIC/POLYURATHANE

60

81

OTP-LLW-WP- 04

CLOTH/RAGS/NYLON

48

13.6

OTP-LLW-WP-04

HAZARDOUS CONSTITUENTS

5

.64

OTP-LLW-WP-04

PLASTIC/POLYURATHANE

20

4.1

RHZ-109-A 13568

CLOTH/RAGS/NYLON

15

RHZ-101-A13568

PAPER/CARDBOARD

30

RHZ-101-A13568

PLASTIC/POLYURATHANE 40

RHZ-101-A13568

RUBBER

15

RHZ-103-A13561

CLOTH/RAGS/NYLON

15

RHZ-103-A13561

PAPER/CARDBOARD
PHYS_COMP_VOL_PCT

40

PHYS PKG ID

PHYS COMP DESCR

PHYS_COMP_VOL_PCT

PHYS PKG ID

PHYS COMP DESCR

PHYS_COMP_VOL_PCT

HDET PKG ID

HDET CNTR STATUS

HDET DESIG_CD

HDETDW NUM

HDET WAS̄TE VOL

HDET_WASTE_STATUS

HDET PKG ID

HDET DESĪG CD

HDET DW NUM

HDET_WASTTE_VOL

HDET PKG ID

HDET DESIGGCD

HDET DW NUM

HDET_WASTTE_VOL

HAZ_PKG_ID

HAZ $C O M \bar{P}$ ID

HAZ COMP TEXT

HAZ COMP WGT

HAZ COMP WGT PCT

HAZ_EPCRA_ELĀG

HAZ PKG ID

HAZ COMP ID

HAZ_COMP_TEXT

HAZ COMP WGT

HAZ COMP WGT_PCT

HAZ_EPCRA__FLA $\bar{A} G$

HAZ_PKG_ID

HAZ COMP ID

HAZ COMP_TEXT

HAZ_COMP_WGT

HAZ_PKG_ID

HAZ COMP ID

HAZ_COMP_TEXT

HAZ_COMP_WGT

HAZ_PKG_ID

HAZ COMP ID

HAZ_COMP_TEXT

HAZ COMP WGT

HAZ_COMP_WGT_PCT

HAZ PKG ID

HAZ COMP ID

HAZ COMP-IEXT

HAZ COMP WGT

HAZ_COMP_WGI_PCT

HAZ PKG ID

HAZ COMP ID

HAZ COMP-TEXT

HAZ COMP WGT

HAZ_COMP_WGT_PCT

HAZ PKG_ID

HAZ COMP ID

HAZ COMP TEXT

HAZ COMP WGT

HAZ_COMP_WGT_PCT

PDW_PKG_1D
RHZ-103-A13561

PLASTIC/POLYURATHANE

30

RHZ-103-A13561

RUBBER

15

OTP-LLW-WP-01

$F$

D008 D009

.208

U

OTP-LLW-WP-02

RD

D002

.21

OTP-LLW-WP-O4

RE

D008 WT01

.07

0TP-LLW-WP-01

7439-97-6

MERCURY

.001

.0009

N

OTP-LLW-WP-01

TEMP1985

LEAD

.085

.068

N

OTP-LLW-WP-02

TEMP2091

LIQUID WASTE

.6

OTP-LLW-WP-02

TEMP 3413

SODIUM CHLORIDE

.04

OTP-LLW-WP- 04

TEMPO 40

ACID

1.5

5

OTP-LL.W-WP-04

TEMP1985

LEAD

8.9

28

OTP-LLW-WP-04

TEMP2628

OIL

1.9

6

OTP-LLW-WP-04

TEMP3530

SOLVENTS

2.1

OTP-LLW-WP-01 


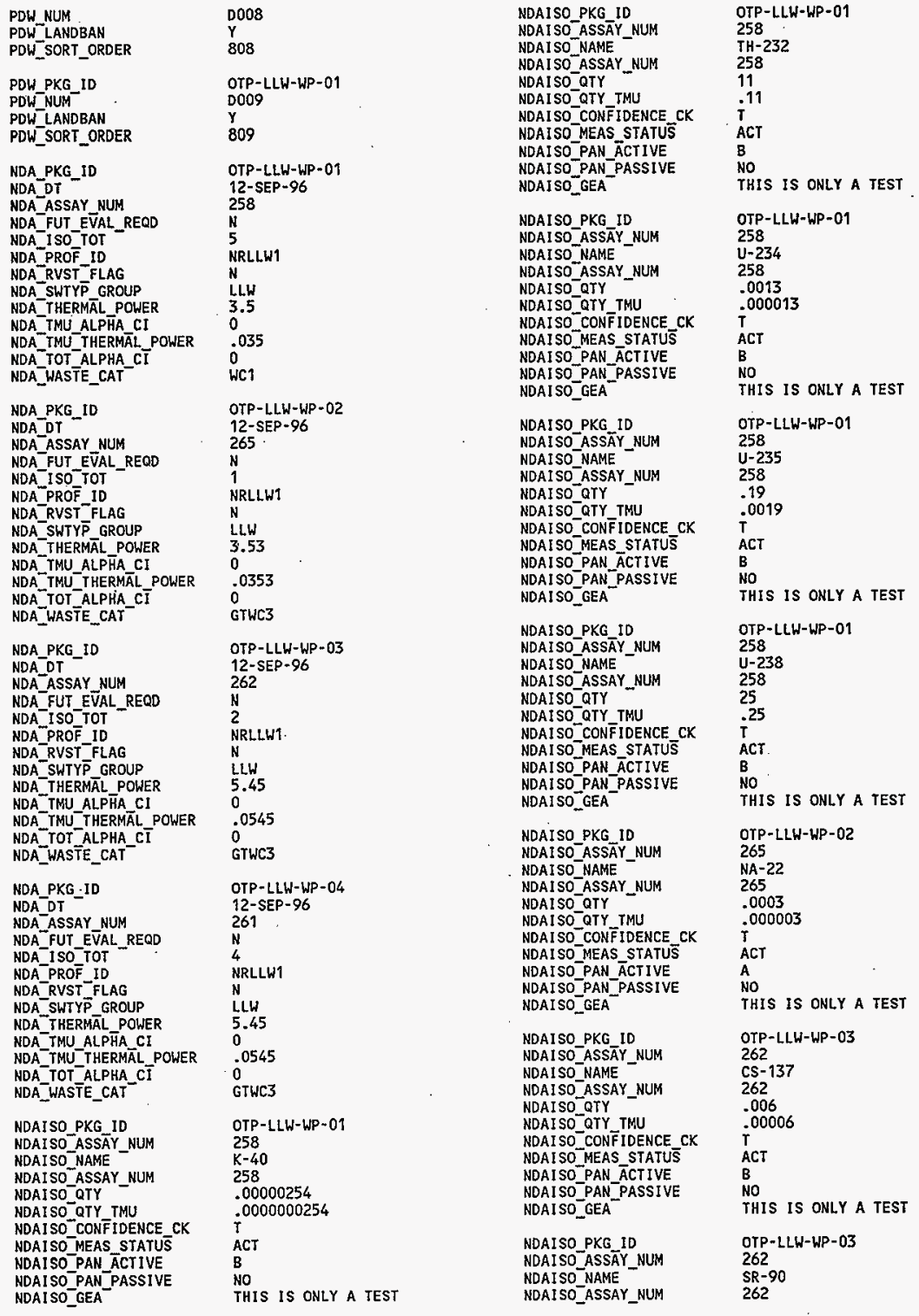




\begin{tabular}{|c|c|}
\hline $\begin{array}{l}\text { NDAISO_QTY } \\
\text { NDAISO_QTY_TMU } \\
\text { NDAISO_CONFIDENCE_CK } \\
\text { NDAISO_MEAS_STATUS } \\
\text { NDAISO_PAN_ACTIVE } \\
\text { NDAISO_PAN_PASSIVE } \\
\text { NDAISO_GEA }\end{array}$ & $\begin{array}{l}.00006 \\
.0000006 \\
\bar{T} \\
\text { ACT } \\
\text { B } \\
\text { NO } \\
\text { THIS IS ONLY A TEST }\end{array}$ \\
\hline $\begin{array}{l}\text { NDAISO_PKG_ID } \\
\text { NDAISO_ASSAY_NUM } \\
\text { NDAISO_NAME- } \\
\text { NDAISO_ASSAY_NUM } \\
\text { NDAISO_QTY } \\
\text { NDAISO_QTY_TMU } \\
\text { NDAISO_CONFIDENCE_CK } \\
\text { NDAISO_MEAS_STATUS } \\
\text { NDAISO_PAN_ACTIVE } \\
\text { NDAISO_PAN_PASSIVE } \\
\text { NDAISO_GEA- }\end{array}$ & $\begin{array}{l}\text { OTP-LLW-WP-04 } \\
261 \\
\text { CO-60 } \\
261 \\
.000004 \\
.00000004 \\
T \\
\text { ACT } \\
\text { A } \\
\text { NO } \\
\text { THIS IS ONLY A TEST }\end{array}$ \\
\hline $\begin{array}{l}\text { NDAISO_PKG_ID } \\
\text { NDAISO_ASSAY_NUM } \\
\text { NDAISO_NAME- } \\
\text { NDAISO_ASSAY_NUM } \\
\text { NDAISO_QTY } \\
\text { NDAISO_QTY_TMU } \\
\text { NDAISO_CONFIDENCE_CK } \\
\text { NDAISO_MEAS_STATUS } \\
\text { NDAISO_PAN_ACTIVE } \\
\text { NDAISO_PAN_PASSIVE } \\
\text { NDAISO_GEA }\end{array}$ & $\begin{array}{l}\text { OTP-LLW-WP-04 } \\
261 \\
\text { CS-137 } \\
261 \\
.000013 \\
.00000013 \\
T \\
\text { ACT } \\
\text { A } \\
\text { NO } \\
\text { THIS IS ONLY A TEST }\end{array}$ \\
\hline $\begin{array}{l}\text { NDAISO_PKG_ID } \\
\text { NDAISO_ASSAY_NUM } \\
\text { NDAISO_NAME } \\
\text { NDAISO_ASSAY_NUM } \\
\text { NDAISO_QTY_- } \\
\text { NDAISO_QTY_TMU } \\
\text { NDAISO_CONFIDENCE_CK } \\
\text { NDAISO_MEAS_STATUS } \\
\text { NDAISO_PAN_ACTIVE } \\
\text { NDAISO_PAN_PASSIVE } \\
\text { NDAISO_GEA }\end{array}$ & $\begin{array}{l}\text { OTP-LLW-WP-O4 } \\
261 \\
\text { EU-154 } \\
261 \\
.000002 \\
.00000002 \\
T \\
\text { ACT } \\
\text { A } \\
\text { NO } \\
\text { THIS IS ONLY A TEST }\end{array}$ \\
\hline $\begin{array}{l}\text { NDAISO_PKG_ID } \\
\text { NDAISO_ASSAY_NUM } \\
\text { NDAISO_NAME } \\
\text { MDAISO_ASSAY_NUM } \\
\text { NDAISO_QTY - } \\
\text { NDAISO_QTY_TMU } \\
\text { NDAISO_CONFIDENCE_CK } \\
\text { NDAISO_MEAS_STATUS } \\
\text { NDAISO_PAN_ACTIVE } \\
\text { NDAISO_PAN_PASSIVE } \\
\text { NDAISO_GEA }\end{array}$ & $\begin{array}{l}\text { OTP-LLW-WP-O4 } \\
261 \\
\text { EU-155 } \\
261 \\
.000032 \\
.00000032 \\
T \\
\text { ACT } \\
\text { A } \\
\text { NO } \\
\text { THIS IS ONLY A IEST }\end{array}$ \\
\hline $\begin{array}{l}\text { NDE_PKG_ID } \\
\text { NDE_DT } \\
\text { NDE_COMMENTS }\end{array}$ & $\begin{array}{l}\text { OTP-LLW-WP-01 } \\
12-S E P-96 \\
\text { OTP-LLW-WP-01 1ST IMAGE } \\
\text { COMMENTS }\end{array}$ \\
\hline $\begin{array}{l}\text { NDE_OPER_ID } \\
\text { NDE_VDISK_FILE } \\
\text { NDE_VDISK_NUM } \\
\text { NDE_VTAPE_NUM } \\
\text { NDE_VTAPE_START } \\
\text { N-_ }\end{array}$ & $\begin{array}{l}\text { USERT } \\
\text { WP05X } \\
\text { P22V1 } \\
\text { P22T1 } \\
200\end{array}$ \\
\hline $\begin{array}{l}\text { NDE_PKG_ID } \\
\text { NDE_DT } \\
\text { NDE_COMMENTS }\end{array}$ & $\begin{array}{l}\text { OTP-LLW-WP-01 } \\
\text { 12-SEP-96 } \\
\text { OTP-LLW-WP-01 2ND IMAGE } \\
\text { COMMENTS }\end{array}$ \\
\hline $\begin{array}{l}\text { NDE_OPER_IO } \\
\text { NDE_VDISK_FILE } \\
\text { NDE_VDISK_NUM } \\
\text { NDE_VTAPE_NUM } \\
\text { NDE_VTAPE-START }\end{array}$ & $\begin{array}{l}\text { USER1 } \\
\text { WP05Y } \\
\text { P22V1 } \\
\text { P22T1 } \\
250\end{array}$ \\
\hline
\end{tabular}

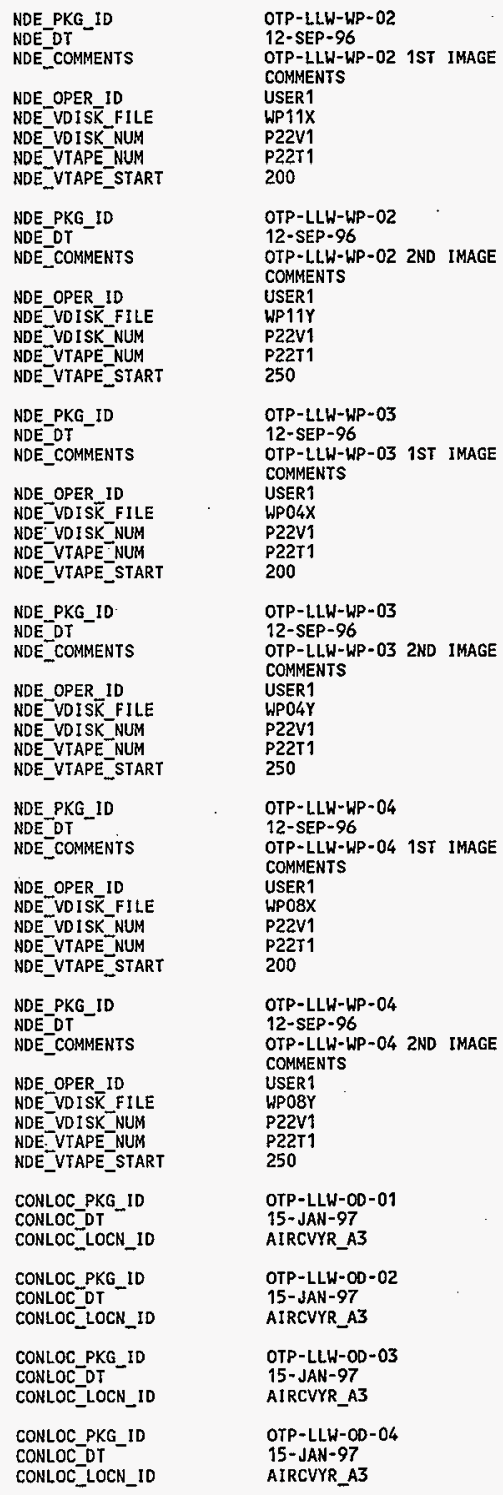




\begin{tabular}{|c|c|}
\hline $\begin{array}{l}\text { CONLOC_PKG_ID } \\
\text { CONLOC_DT } \\
\text { CONLOC_LOCN_ID }\end{array}$ & $\begin{array}{l}\text { OTP-LLWR-TD-01 } \\
\text { 05-FEB-97 } \\
\text { RWM_CRSL }\end{array}$ \\
\hline $\begin{array}{l}\text { CONLOC_PKG_ID } \\
\text { CONLOC_DT } \\
\text { CONLOC_LOCN_ID }\end{array}$ & $\begin{array}{l}\text { OTP-LLWR-TD-02 } \\
\text { O5-FEB-97 } \\
\text { RWM_CRSL }\end{array}$ \\
\hline $\begin{array}{l}\text { CONLOC_PKG_ID } \\
\text { CONLOC_DT } \\
\text { CONLOC_LOCN_ID }\end{array}$ & $\begin{array}{l}\text { OTP6-97-300001 } \\
\text { 04-FEB-97 } \\
\text { SAMPLE_MGT }\end{array}$ \\
\hline $\begin{array}{l}\text { CONLOC_PKG_ID } \\
\text { CONLOC_DT } \\
\text { CONLOC_LOCN_ID }\end{array}$ & $\begin{array}{l}\text { OTP6-97-400001 } \\
\text { O4-FEB-97 } \\
\text { SAMPLE_MGT }\end{array}$ \\
\hline $\begin{array}{l}\text { CONLOC_PKG_ID } \\
\text { CONLOC_DT } \\
\text { CONLOC_LOCN_ID }\end{array}$ & $\begin{array}{l}\text { RHZ-101-A13568 } \\
\text { 09-AUG-27 } \\
\text { AGVPANC_A }\end{array}$ \\
\hline $\begin{array}{l}\text { CONLOC_PKG_ID } \\
\text { CONLOC_DT } \\
\text { CONLOC_LOCN_ID }\end{array}$ & $\begin{array}{l}\text { RHZ-103-A13561 } \\
\text { O9-AUG-27 } \\
\text { INFDCVYRW }\end{array}$ \\
\hline $\begin{array}{l}\text { CONR_FROM_PKG_ID } \\
\text { CONR_TO_PKG_ID } \\
\text { CONR_DT } \\
\text { CONR_REL_CD }\end{array}$ & $\begin{array}{l}\text { OTP-LLW-WP-01 } \\
\text { OTP-LLW-OD-01 } \\
05-J A N-97 \\
M\end{array}$ \\
\hline $\begin{array}{l}\text { CONR_FROM_PKG_ID } \\
\text { CONR_TO_PKG_ID } \\
\text { CONR_DT- } \\
\text { CONR_REL_CD }\end{array}$ & $\begin{array}{l}\text { OTP-LLW-WP-02 } \\
\text { OTP- } L L W-0 D-02 \\
05-J A N-97 \\
M\end{array}$ \\
\hline $\begin{array}{l}\text { CONR_FROM_PKG_ID } \\
\text { CONR_TO_PKG_ID } \\
\text { CONR_DT } \\
\text { CONR_REL_CD }\end{array}$ & $\begin{array}{l}\text { OTP-LLW-WP-03 } \\
\text { OTP-LLW-OD-03 } \\
\text { O5-JAN-97 } \\
M\end{array}$ \\
\hline $\begin{array}{l}\text { CONR_FROM_PKG_ID } \\
\text { CONR_TO_PKG_ID } \\
\text { CONR_DT } \\
\text { CONR_REL_CD }\end{array}$ & $\begin{array}{l}\text { OTP-LLW-WP-04 } \\
\text { OTP-LLW-OD-04 } \\
\text { O5-JAN- } 97 \\
M\end{array}$ \\
\hline $\begin{array}{l}\text { RADMAT_ID } \\
\text { RADMAT_DESCR } \\
\text { RADMAT_LIMIT } \\
\text { RADMAT_RAD_TOT } \\
\text { RADMAT_UNITS } \\
\text { RADMAT_ALARM }\end{array}$ & $\begin{array}{l}\text { F } \\
\text { WRAP I FACILITY } \\
100 \\
10 \\
\text { CI } \\
\text { N }\end{array}$ \\
\hline $\begin{array}{l}\text { RADMAT_ID } \\
\text { RADMAT_DESCR } \\
\text { RADMAT_LIMIT } \\
\text { RADMAT_RAD_TOT } \\
\text { RADMAT_UNITS } \\
\text { RADMAT_ALARM }\end{array}$ & $\begin{array}{l}\text { R } \\
\text { TRU RWM GLOVEBOX } \\
200 \\
10 \\
\text { FGE } \\
\text { N }\end{array}$ \\
\hline $\begin{array}{l}\text { RADMAT_ID } \\
\text { RADMAT_DESCR } \\
\text { RADMAT_LIMIT } \\
\text { RADMAT_RAD_TOT } \\
\text { RADMAT_UNITS } \\
\text { RADMAT_ALARM }\end{array}$ & $\begin{array}{l}\text { T } \\
\text { TRU GLOVEBOX } \\
200 \\
10 \\
\text { FGE } \\
\text { N }\end{array}$ \\
\hline
\end{tabular}


CASEIA. 813

$8 / 13 / 9>$

\section{DMS DATABASE FOLLOWING THE LLW GLOVEBOX OTP SORTING OF OTP-LLW-WP-01 (CASE 1)}

\begin{tabular}{|c|c|}
\hline $\begin{array}{l}\text { CON PKG ID } \\
\text { CON_CNTYPCD } \\
\text { CON_PKG_STATUS } \\
\text { CON_SIZE_DESCR } \\
\text { CON_TARE_WGT }\end{array}$ & $\frac{O T P-L L W-O D-01}{\text { DM }}$ \\
\hline $\begin{array}{l}\text { CON PKG ID } \\
\text { CON_CNTYP_CD } \\
\text { CON_LOCN_FACIL_ID } \\
\text { CON_PKG_STATUS } \\
\text { CON_SIZE_DESCR } \\
\text { CON_TARE_WGT }\end{array}$ & $\begin{array}{l}\text { OTP-LLW-OD-02 } \\
\text { DM } \\
2336 \mathrm{~W} \\
M \\
85 \text { GALLON } \\
31\end{array}$ \\
\hline $\begin{array}{l}\text { CON_PKG 10 } \\
\text { CON_CNTYP_CD } \\
\text { CON_LOCN_FACIL_ID } \\
\text { CON_PKG_STATUS } \\
\text { CON_SIZE_DESCR } \\
\text { CON_TARE_WGT }\end{array}$ & 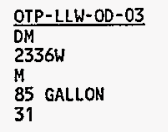 \\
\hline $\begin{array}{l}\text { CON_PKG ID } \\
\text { CON_CNTYP_CD } \\
\text { CON_LOCN_FACIL_ID } \\
\text { CON_PKG_STATUS } \\
\text { CON_SIZE_DESCR } \\
\text { CON_TARE_WGT }\end{array}$ & $\begin{array}{l}\frac{O T P-L L W-O D-04}{D H} \\
2336 \mathrm{~W} \\
M \\
85 \text { GALLON } \\
31\end{array}$ \\
\hline 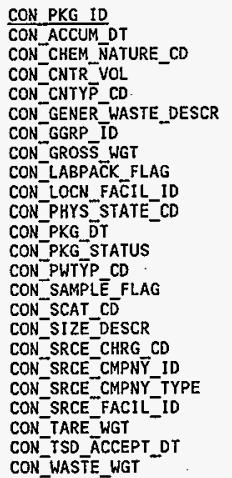 & $\begin{array}{l}\text { OTP-LLW-WP-01 } \\
19-0 C T-93 \\
1 \\
-21 \\
\text { DM } \\
\text { LOW LEVEL WASTE } \\
\text { ANL } \\
152.7 \\
\mathrm{~N} \\
2336 \mathrm{~W} \\
\mathrm{~S} \\
19-\text { OCT }-93 \\
\mathrm{R} \\
\mathrm{M} \\
Y \\
\text { OMW } \\
55 \text { GALLON } \\
\text { TH233 } \\
\text { ANL. } \\
\text { GEN } \\
\text { ARGON } \\
27.7 \\
27-\text { FEB-95 } \\
125\end{array}$ \\
\hline 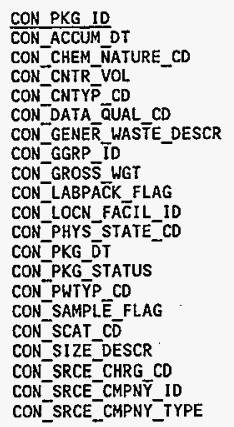 & $\begin{array}{l}\text { OTP-LLW-WP-02 } \\
10-J U L-91 \\
\text { IO } \\
-21 \\
\text { OM } \\
\text { AR } \\
\text { INNER } \\
\text { OTHER } \\
170.1 \\
N \\
2336 \mathrm{~W} \\
\text { S } \\
10-\text { JUL-91 } \\
\text { T } \\
\text { M } \\
\text { N } \\
\text { FLC } \\
55 \text { GALLON } \\
\text { WM4GO } \\
\text { WHC } \\
\text { GEN }\end{array}$ \\
\hline
\end{tabular}

$\begin{array}{ll}\text { CON_SRCE_FACIL_ID } & 324 \\ \text { CON_TARE_WGT } & 27 \\ \text { CON_TSD_ACCEPT_DT } & 10-J U L-91 \\ \text { CON_WASTE_WGT } & 139.34\end{array}$

CON_PKG ID
CON_ACCUM_DT
CON_CNTR_VO
CON_CNTYP_CD
CON_DATA_QUAL_CD
CON_GENER_WASTE_DESCR
CON_GGRP_ID
CON_GROSS WGT
CON_LABPACK_FLAG
CON_LOCN_FACIL_ID
CON_PHYS_STATE_CD
CON_PKG_DT
CON_PKG_STATUS
CON_PWTYP_CD
CON_SAMPLE_FLAG
CON_SIZE_DESCR
CON_SRCE_CHRG_CD
CON_SRCE_CMPNY ID
CON_SRCE_CMPNY_TYPE
CON_SRCE_FACIL_ID
CON_TARE_WGT
CON_TSD_ACCEPT_DT
CON_WASTE_WGT

OTP-LLW-WP-03

29-MAY $=90$

.21

DM

AR

INNER

OTHER

163.02

$2336 \mathrm{~W}$

$\mathrm{S}$

29-MAY-90

T

N

55 GALLON

B80545

PNL.

GEN

324

27

29-MAY-90

136

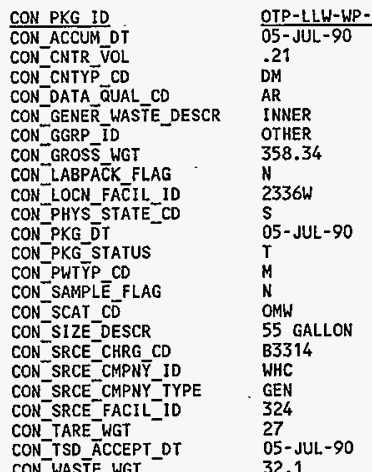

CON_WASTE_WGT

\section{CON PKG ID \\ CON_CNTYP_CD \\ CON_LOCN_FACIL.ID \\ CON-PKG_STATUS \\ CON_SCAT_CD \\ CONSIZE-DESCR \\ CON_TARE_WGT}

CON PKG ID

CON_CNTYP_CD

CON_LOCN FACIL_ID

CON PKG STATUS

CON SCAT CD

CON SIZE-DESCR

CON_TARE_WGT
OTP-LLWR-TD-01
DM
$2336 \mathrm{~W}$
G
ANY
85 GALLON
31

OTP-LLWR -TD-02

DM

$2336 \mathrm{~W}$

$G$

ANY

85 GALLON

31

CON PKG ID

0IP6-97-300001

CON PKG ID

OTP6-97-400001 


\begin{tabular}{|c|c|}
\hline $\begin{array}{l}\text { CON_PKG_DT } \\
\text { CON_PKG_STATUS }\end{array}$ & $\begin{array}{l}13-A U G-27 \\
G\end{array}$ \\
\hline $\begin{array}{l}\text { CON PKG ID } \\
\text { CON_ACCUM_DT } \\
\text { CON_CHEM_NATURE_CD } \\
\text { CON_CNTR_VOL } \\
\text { CON_CNIYP_CD } \\
\text { CON_GENER_WASTE_DESCR } \\
\text { CON_GGRP_ID } \\
\text { CON_GROSS_WGT } \\
\text { CON_LABPACK_FLAG } \\
\text { CON_LOCN_FACIL_ID } \\
\text { CON_PHYS_STATE_CD } \\
\text { CON_PKG_DT } \\
\text { CON_PKG_STATUS } \\
\text { CON_PHTYP_CD } \\
\text { CON_SAMPLE_FLAG } \\
\text { CON_SCAT_CD } \\
\text { CON_SEC_PKG_ID } \\
\text { CON_SIZE_DESCR } \\
\text { CON_SRCE_CHRG_CD } \\
\text { CON_SRCE_CHPNY_ID } \\
\text { CON_SRCE_CNPNY_TYPE } \\
\text { CON_SRCE_FACIL_ID } \\
\text { CON_TARE_WGT - } \\
\text { CON_TSD_ACCEPT_DT } \\
\text { CON_WASTE_WGT - }\end{array}$ & $\begin{array}{l}\frac{\text { PUCK278 }}{19-0 C T-93} \\
1 \\
.21 \\
\text { DM } \\
\text { LOW LEVEL WASTE } \\
\text { ANL } \\
152.7 \\
N \\
2336 \mathrm{~W} \\
S \\
19-\text { OCT-93 } \\
\text { R } \\
\text { M } \\
\text { Y } \\
\text { OMW } \\
\text { OTP-LLW-WP-01 } \\
55 \text { GALLON } \\
\text { TH233 } \\
\text { ANL } \\
\text { GEN } \\
\text { ARGON } \\
27.7 \\
27-\text { FEB-95 } \\
125\end{array}$ \\
\hline $\begin{array}{l}\text { CON PKG ID } \\
\text { CON_CNTYP CD } \\
\text { CON_PKG_STATUS } \\
\text { CON_SEC_PKG ID } \\
\text { CON_SIZE_DESCR } \\
\text { CON_TARE_WGT }\end{array}$ & $\begin{array}{l}\text { PUCK279 } \\
\text { DM } \\
R \\
\text { OTP-LLW-OD-01 } \\
85 \text { GALLON } \\
31\end{array}$ \\
\hline 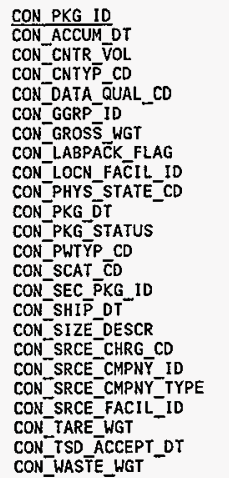 & $\begin{array}{l}\text { RHZ-101-A13568 } \\
\frac{14-O C T-85}{21} \\
.21 \\
\text { DM } \\
\text { AR } \\
\text { PFP } \\
72.98 \\
\text { N } \\
2336 \mathrm{~W} \\
\text { S } \\
14-\text { OCT }-85 \\
T \\
R \\
\text { TRU } \\
\text { A13568 } \\
14-0 \mathrm{CT}-85 \\
55 \text { GALLON } \\
\text { K6 } \\
\text { WHC } \\
\text { GEN } \\
23452 \\
27 \\
14-\text { OCT- } 85 \\
45.98\end{array}$ \\
\hline 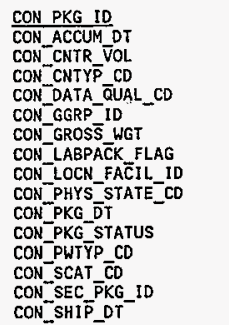 & $\begin{array}{l}\text { RHZ-103-A13561 } \\
14-0 C T-85 \\
.21 \\
\text { DM } \\
\text { AR } \\
\text { PFP } \\
51.98 \\
N \\
2336 \mathrm{~W} \\
\text { S } \\
14-\text { OCT }-85 \\
T \\
R \\
\text { TRU } \\
\text { A13561 } \\
14-0 C T-85\end{array}$ \\
\hline
\end{tabular}

$\begin{array}{ll}\text { CON_SIZE_DESCR } & 55 \text { GALLON } \\ \text { CON_SRCE_CHRGCCD } & \text { K6 } \\ \text { CON_SRCE_CMPNYYID } & \text { WHC } \\ \text { CON_SRCE_CMPNY_TYPE } & \text { GEN } \\ \text { CON_SRCE_FACIL_ID } & 23452 \\ \text { CON_TARE_WGT } & 27 \\ \text { CON_TSD_ACCEPT_DT } & 14-0 C T-85 \\ \text { CON_WASTE_WGT } & 24.98\end{array}$

CONEXT PKG ID

CONEXY USE CD

CONEXT_WRAP_STAT_CD I

CONEXT_PKG_ID OTP-LLW-OD-02

CONEXT_USECD $C D$

CONEXT_WRAP_STAT_CD

CONEXT_PKG_ID OTP-LLW-OD-03

CONEXT_USECD OD

CONEXT_WRAP_STAT_CD I

$\begin{array}{ll}\text { CONEXT_PKG_ID } & \text { OTP-LLW-OD-04 } \\ \text { CONEXT_USE_CD } & \text { OD }\end{array}$

CONEXT_WRAF_STAT_CD I

CONEXI_PKG_ID OTP-LLW-WP-01

CONEXT_COMPL,IANT_FLAG

CONEXT_NDE_VER_FLAG Y

CONEXT PROF FLA $\bar{A} G \quad N$

CONEXT_PROF_IO - NRLLWT

CONEXT_USE_CD WV

CONEXT_WRAP_STAT_CD I

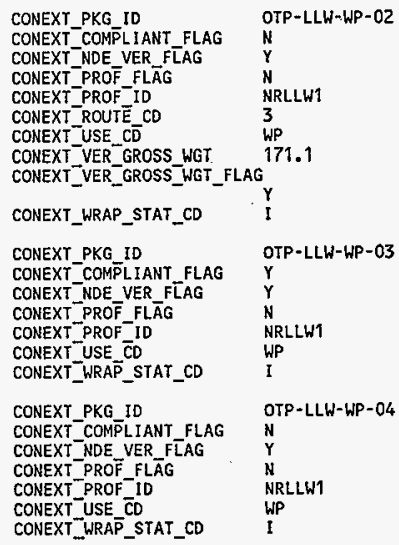

CONEXT PKG ID OTP-LLWR-TD-01

CONEXT_CNTR_STATUS E

$\begin{array}{ll}\text { CONEXT_USE_CD } & \text { TD } \\ \text { CONEXT_WRAP STAT CD I }\end{array}$

CONEXT_PKG_ID OTP-LLWR-TD-02

CONEXT CNTR STATUS E

$\begin{array}{ll}\text { CONEXT_USE_CD } & \text { TO } \\ \text { CONEXT_WRAP STAT_CD I } & \text { I }\end{array}$

CONEXI_PKG_ID OTP6-97-300001

CONEXI_USE_CD PP

CONEXT_PKG_ID OTP6-97-400001

CONEXT_USE-CD TP 


\begin{tabular}{|c|c|}
\hline 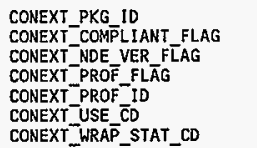 & $\begin{array}{l}\text { PUCK278 } \\
Y \\
\gamma \\
N \\
\text { NRLLW1 } \\
\text { WP } \\
\text { I }\end{array}$ \\
\hline $\begin{array}{l}\text { CONEXT_PKG_ID } \\
\text { CONEXT_USE_CD } \\
\text { CONEXT_WRAP_STAT_CD }\end{array}$ & $\begin{array}{l}\text { PUCK279 } \\
\text { EC } \\
1\end{array}$ \\
\hline $\begin{array}{l}\text { CONEXT_PKG_ID } \\
\text { CONEXT_NDA_DRUM_STATUS } \\
\text { CONEXT_PROF_ID } \\
\text { CONEXT_RADNAT_ID } \\
\text { CONEXT_ROUTE_CD } \\
\text { CONEXT_TO_PU, FGE } \\
\text { CONEXT_USE__CD } \\
\text { CONEXT_VER_GROSS_WGT } \\
\text { CONEXT_VER_GROSS_WGT_FLAC }\end{array}$ & $\begin{array}{l}\text { RHZ-101-A13568 } \\
\text { PROC } \\
\text { TEST } \\
\text { F } \\
1 \\
.141960683549683 \\
\text { WP } \\
66.4 \\
\end{array}$ \\
\hline CONEXT_WRAP_STAT_CD & 1 \\
\hline $\begin{array}{l}\text { CONEXT_PKG_ID } \\
\text { CONEXY_NDA_DRUM_STATUS } \\
\text { CONEXT_PROF_ID } \\
\text { CONEXT_RADMAT_ID } \\
\text { CONEXT_ROUTE_CD } \\
\text { CONEXT_TO_PU_FGE } \\
\text { CONEXT_USE_CD } \\
\text { CONEXT_VER_GROSS_WGT } \\
\text { CONEXT_VER_GROSS_WGT_FLAG }\end{array}$ & $\begin{array}{l}\text { RHZ-103-A } 13561 \\
\text { PROC } \\
\text { TEST } \\
\text { F } \\
1 \\
.84683609729712 \\
\text { WP } \\
48.35 \\
G\end{array}$ \\
\hline CONEXT_WRAP_STAT_CD & I \\
\hline $\begin{array}{l}\text { RDET_PKG_ID } \\
\text { RDET_BG_DOSE_RATE } \\
\text { RDET_HANDLING } \\
\text { RDET_RELOC_DT } \\
\text { RDET_RSWIMS_ COUNT } \\
\text { RDET_SDAR_APPRV_NUM } \\
\text { RDET_SWIMS_CD } \\
\text { RDET_SWTYP_CD } \\
\text { RDET_SWTYP_GROUP } \\
\text { RDET_THERMAL_POWER } \\
\text { RDET_TOT_BG_CI } \\
\text { RDET_TOT_DE_CI } \\
\text { RDET_WASTE_CAT } \\
\text { RDET_WASTE_MAKEUP } \\
\text { RDET_WRAP_CAT }\end{array}$ & $\begin{array}{l}\text { OTP-LLW-WP-01 } \\
-1 \\
\text { C } \\
01-\text { SEP-96 } \\
1 \\
60-1 R-3 Y M-0401 \\
\text { DD } \\
2 A \\
\text { LLW } \\
3.53 \\
.00000254 \\
.0000097 \\
\text { WC1 } \\
\text { F } \\
\text { SO }\end{array}$ \\
\hline $\begin{array}{l}\text { RDET_PKG_ID } \\
\text { RDET_BG_DOSE_RATE } \\
\text { RDET_HANDLING } \\
\text { ROET_RELOC_DT } \\
\text { RDET_RSWIMST_COUNT } \\
\text { RDET_SDAR_APPPR_NUM } \\
\text { RDET_SWIMS_CD } \\
\text { RDET_SWTYP_CD } \\
\text { RDET_SWTYP_GROUP } \\
\text { RDET_THERMAL_POWER } \\
\text { RDET_TOT_ALPHA_CI } \\
\text { RDET_TOT_BG_CI } \\
\text { RDET_TOT_DE_CI } \\
\text { RDET_TOT_PE_CI } \\
\text { RDET_TOT_PU_FGE } \\
\text { RDET_WASTE_MAKEUP } \\
\text { RDET_WRAP_CAT }\end{array}$ & $\begin{array}{l}\text { OTP-LLW-WP-02 } \\
50 \\
\text { C } \\
25-N O V-91 \\
1 \\
7-1 A-6 L M-1 \\
\text { NC } \\
2 A \\
\text { LLW } \\
3.53 \\
0 \\
0 \\
0 \\
0 \\
0 \\
F \\
C L\end{array}$ \\
\hline $\begin{array}{l}\text { RDET_PKG_ID } \\
\text { RDET_BG_DOSE_RATE } \\
\text { ROET_HANDLING } \\
\text { RDET_RELOC_DT } \\
\text { RDET_RSWIMS COUNT } \\
\text { RDET_SDAR_APPRV_NUM } \\
\text { RDET_SWIMS_CD }\end{array}$ & $\begin{array}{l}0 T P-1 L W-W P-03 \\
7 \\
C \\
29-M A Y-90 \\
1 \\
2 A-1 A-1 A-0 \\
C E\end{array}$ \\
\hline
\end{tabular}

$\begin{array}{ll}\text { ROET_SWTYP_CD } & \text { 2A } \\ \text { RDET_SWTYP_GROUP } & \text { LLW } \\ \text { RDET_THERMAL_POWER } & 3.53 \\ \text { RDET_TOT_BG_CI } & : 01212 \\ \text { RDET_TOT_DE_CI } & 1 \\ \text { RDET_WASTE_MAKEUP } & \text { F } \\ \text { RDET_WRAP_CAT } & \text { DH }\end{array}$

RDET_PKG_ID

RDET ASSAYY DT

RDET_BG_DOSEERATE

RDET HAÑDL ING

RDET RELOC DT

RDET_RSWIMS__COUNT

RDET SDAR APPRV NUM

RDET_SWIMS CD

RDET_SWTYP CD

RDET SWTYP GROUP

RDET THERMAL POWER

RDET TOT_ALPHA_CI

RDET TOT BG CI

RDET_TOT_DE_CI

RDET TOT PECI

RDET TOTPU PGE

RDET WASTTE MAKEUP

RDET_WRAP_CAT

RDET_PKG_ID

ROET_BG_DOSE RATE

RDET_HANDDLING

RDET RELOC DT

RDET_RSWIMS_COUNT

RDET_SDAR_APPRV_NUM

RDET SWIMS CD

RDET_SWTYP_CD

RDET SWTYP-GROUP

RDET_IHERMĀL_POWER

RDET_TOT_BG_ $\bar{C}$ I

RDET TOT DE CI

RDET_WASTE_CAT

RDET WASTE MAKEUP

RDET_WRAP_CAT

RDET_PKG_ID

RDEI BG DOSE RATE

RDET_HANDLING

RDET RELOC DT

ROET_RSWIMS_COUNT

RDET SDAR APPRV_NUM

RDET_SWIMS_CD

RDET_SWSOR_NUM

RDET SWTYP CD

RDET_SWTYP_GROUP

RDET_THERMAL POWER

RDET TOI ALPHA CI

ROET_TOT_BG_CI

RDET_TOT DE_CI

RDETTTOTPE-CI

RDET TOT PU FGE

RDET_WASTE_MAKEUP

RDET PKG ID

RDET BG DOSE RATE

RDET_HANNDLING

ROET RELOC DT

RDET_RSWIMS _COUNT

RDET_SDAR APPPRV_NUM

RDET'SWIMS̄ CD

RDET_SWSDR_NUM

RDET SWTYP CD

RDET SWTYP GROUP

RDET_THERMĀL_POWER

RDET-TOT ALPHA CI

RDET_TOT_BG_CI

RDET TOT DECI

RDET TOT PECI

RDET_TOT_PU_FGE

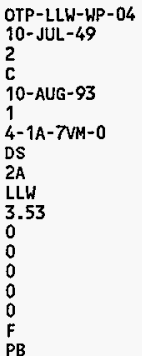

PUCK278

i

01-SEP-96

1

$60-1 R-3 Y M-0401$

DD

$2 A$

LLW

3.53

.00000254

.0000097

WC1

$\mathrm{F}$

so

RHZ-101-A13568

1

15-MAY-97

1

$1-1 \mathrm{~B}-2 \mathrm{C}-0$

DS

860019

10

TRU

.011454765

.076259545

0

.086811043

.086799966

.950328438

RHZ-103-A13561

1

15-MAY-97

1

$1-1 \mathrm{~B}-2 \mathrm{C}-0$

DS

860021

ID

TRU

.011454765

.076259545

.086811043

.086799966

.950328438 


\begin{tabular}{|c|c|}
\hline RDET_HASTE_MAKEUP & $\mathbf{F}$ \\
\hline $\begin{array}{l}\text { RAD_PKG_ID } \\
\text { RAD_ISO_NUM } \\
\text { RAD_QTY }\end{array}$ & $\begin{array}{l}\text { OTP-LLW-WP-01 } \\
104 \\
.00000788\end{array}$ \\
\hline $\begin{array}{l}\text { RAD_PKG_ID } \\
\text { RAD_ISO_NUM } \\
\text { RAD_QTY }\end{array}$ & $\begin{array}{l}\text { OTP-LLW-WP-01 } \\
18 \\
11\end{array}$ \\
\hline $\begin{array}{l}\text { RAD_PKG_ID } \\
\text { RAD_ISO_NUM } \\
\text { RAD_OTY }\end{array}$ & $\begin{array}{l}\text { OTP-LLW-WP-01 } \\
19 \\
.00000254\end{array}$ \\
\hline $\begin{array}{l}\text { RAD_PKG_ID } \\
\text { RAD_ISO_NUM } \\
\text { RAD_QTY }\end{array}$ & $\begin{array}{l}\text { OTP-LLW-WP-01 } \\
202 \\
.0013\end{array}$ \\
\hline $\begin{array}{l}\text { RAD_PKG_ID } \\
\text { RAD_ISO_NUM } \\
\text { RAD_QTY }\end{array}$ & $\begin{array}{l}\text { OTP-LLW-WP-01 } \\
203 \\
.19\end{array}$ \\
\hline $\begin{array}{l}\text { RAD_PKG_ID } \\
\text { RAD_ISO_NUM } \\
\text { RAD_QTY }\end{array}$ & $\begin{array}{l}\text { OTP-LLW-WP-01 } \\
206 \\
25\end{array}$ \\
\hline $\begin{array}{l}\text { RAD_PKG_ID } \\
\text { RAD_ISO_NUM } \\
\text { RAD_QYY }\end{array}$ & $\begin{array}{l}\text { OTP-LLW-WP-01 } \\
86 \\
.00000254\end{array}$ \\
\hline $\begin{array}{l}\text { RAD_PKG_ID } \\
\text { RAD_ISO_NUM } \\
\text { RAD_QTY }\end{array}$ & $\begin{array}{l}\text { OTP-LLW-WP-02 } \\
12 \\
.00033\end{array}$ \\
\hline $\begin{array}{l}\text { RAD_PKG_ID } \\
\text { RAD_ISO_NUM } \\
\text { RAD_QTY }\end{array}$ & $\begin{array}{l}\text { OTP-LLW-WP-02 } \\
152 \\
.000005\end{array}$ \\
\hline $\begin{array}{l}\text { RAD_PKG_ID } \\
\text { RAD_ISO_NUM } \\
\text { RAD_QTY }\end{array}$ & $\begin{array}{l}\text { OTP-LLW-WP-02 } \\
153 \\
.00018\end{array}$ \\
\hline $\begin{array}{l}\text { RAD_PKG_10 } \\
\text { RAD_ISO_NUM } \\
\text { RAD_QTY } \\
\text { RAD_UNKNOWN }\end{array}$ & $\begin{array}{l}\text { OTP-LLW-WP-02 } \\
19 \\
035405 \\
0\end{array}$ \\
\hline $\begin{array}{l}\text { RAD_PKG_ID } \\
\text { RAD_ISO_NUM } \\
\text { RAD_QTY }\end{array}$ & $\begin{array}{l}\text { OIP-LLH-WP-02 } \\
43 \\
.00045\end{array}$ \\
\hline $\begin{array}{l}\text { RAD_PKG_ID } \\
\text { RAD_ISO_NUM } \\
\text { RAD_QTY }\end{array}$ & $\begin{array}{l}\text { OTP-LLW-WP-02 } \\
53 \\
.00014\end{array}$ \\
\hline $\begin{array}{l}\text { RAD_PKG_ID } \\
\text { RAD_ISO_NUM } \\
\text { RAD_QTY }\end{array}$ & $\begin{array}{l}\text { OTP-LLW-WP-02 } \\
56 \\
.0003\end{array}$ \\
\hline $\begin{array}{l}\text { RAD_PKG_ID } \\
\text { RAD_ISO_NUM } \\
\text { RAD_QTY }\end{array}$ & $\begin{array}{l}\text { OTP-LLW-WP-02 } \\
91 \\
.034\end{array}$ \\
\hline $\begin{array}{l}\text { RAD_PKG_ID } \\
\text { RAD_ISO_NUM } \\
\text { RAD_QTY } \\
\text { RAD_UNKNOWN }\end{array}$ & $\begin{array}{l}\text { OTP-LLW-WP-03 } \\
19 \\
.01212 \\
.000324\end{array}$ \\
\hline $\begin{array}{l}\text { RAD_PKG_ID } \\
\text { RAD_ISO_NUM } \\
\text { RAD_QTY }\end{array}$ & $\begin{array}{l}\text { OTP-LLW-WP-03 } \\
3 \\
.00006\end{array}$ \\
\hline $\begin{array}{l}\text { RAD_PKG_ID } \\
\text { RAD_ISO_NUM } \\
\text { RAD_QTY }\end{array}$ & $\begin{array}{l}\text { OTP-LLW-WP-03 } \\
8 \\
.006\end{array}$ \\
\hline RAD_PKG_ID & OTP-LLW-WP-04 \\
\hline
\end{tabular}

\begin{tabular}{|c|c|}
\hline $\begin{array}{l}\text { RAD_ISO_NUM } \\
\text { RAD_QTY }\end{array}$ & $\begin{array}{l}13 \\
.000004\end{array}$ \\
\hline $\begin{array}{l}\text { RAD_PKG_ID } \\
\text { RAD_ISO_NUM } \\
\text { RAD_QTY } \\
\text { RAD_UNKNOWN }\end{array}$ & $\begin{array}{l}\text { OTP-LLW-WP-04 } \\
19 \\
.000233 \\
.0000007163\end{array}$ \\
\hline $\begin{array}{l}\text { RAD_PKG_ID } \\
\text { RAD_ISO_NUM } \\
\text { RAD_QTY }\end{array}$ & $\begin{array}{l}\text { OTP-LLW-WP-04 } \\
31 \\
.000032\end{array}$ \\
\hline $\begin{array}{l}\text { RAD_PKG_ID } \\
\text { RAD_ISO_NUM } \\
\text { RAD_QTY }\end{array}$ & $\begin{array}{l}\text { OTP-LLW-WP-04 } \\
4 \\
.000083\end{array}$ \\
\hline $\begin{array}{l}\text { RAD_PKG_ID } \\
\text { RAD_ISO_NUW } \\
\text { RAD_QTY }^{-}\end{array}$ & $\begin{array}{l}\text { OTP-LLW-WP-04 } \\
40 \\
.000002\end{array}$ \\
\hline $\begin{array}{l}\text { RAD_PKG_ID } \\
\text { RAD_ISO_NUM } \\
\text { RAD_QTY }\end{array}$ & $\begin{array}{l}\text { OTP-LLW-WP-04 } \\
53 \\
.000001\end{array}$ \\
\hline $\begin{array}{l}\text { RAD_PKG_ID } \\
\text { RAD_ISO-NUM } \\
\text { RAD_QTY }\end{array}$ & $\begin{array}{l}\text { OTP-LLW-WP-04 } \\
8 \\
.000013\end{array}$ \\
\hline $\begin{array}{l}\text { RAD_PKG_ID } \\
\text { RAD_ISO_NUM } \\
\text { RAD_QTY }\end{array}$ & $\begin{array}{l}\text { OTP-LLW-WP-04 } \\
9 \\
.000001\end{array}$ \\
\hline $\begin{array}{l}\text { RAD_PKG_ID } \\
\text { RAD_ISO_NUM } \\
\text { RAD_QTY }\end{array}$ & $\begin{array}{l}\text { PUCK278 } \\
104 \\
.00000788\end{array}$ \\
\hline $\begin{array}{l}\text { RAD_PKG_ID } \\
\text { RAD_ISO_NUM } \\
\text { RAD_QTY }\end{array}$ & $\begin{array}{l}\text { PUCK278 } \\
18 \\
11\end{array}$ \\
\hline $\begin{array}{l}\text { RAD_PKG_ID } \\
\text { RAD_ISO_NUM } \\
\text { RAD_QTY }\end{array}$ & $\begin{array}{l}\text { PUCK278 } \\
19 \\
.00000254\end{array}$ \\
\hline $\begin{array}{l}\text { RAD_PKG_ID } \\
\text { RAD_ISO_NUM } \\
\text { RAD_QTY }\end{array}$ & $\begin{array}{l}\text { PUCK278 } \\
202 \\
.0013\end{array}$ \\
\hline $\begin{array}{l}\text { RAD_PKG_ID } \\
\text { RAD_ISO_NUM } \\
\text { RAD_QTY }\end{array}$ & $\begin{array}{l}\text { PUCK278 } \\
203 \\
.19\end{array}$ \\
\hline $\begin{array}{l}\text { RAD_PKG_ID } \\
\text { RAD_ISO_NUM } \\
\text { RAD_QTY }\end{array}$ & $\begin{array}{l}\text { PUCK278 } \\
206 \\
25\end{array}$ \\
\hline $\begin{array}{l}\text { RAD_PKG_ID } \\
\text { RAD_ISO_NUM } \\
\text { RAD_QTY }\end{array}$ & $\begin{array}{l}\text { PUCK278 } \\
86 \\
.00000254\end{array}$ \\
\hline $\begin{array}{l}\text { RAD_PKG_ID } \\
\text { RAD_ISO_NUM } \\
\text { RAD_ALPHA_CI } \\
\text { RAD_PE_CI } \\
\text { RAD_PU_FGE } \\
\text { RAD_QTY }\end{array}$ & $\begin{array}{l}\text { RHZ-101-A13568 } \\
100 \\
.0580886376 \\
.05815044 \\
.9364 \\
.9364\end{array}$ \\
\hline $\begin{array}{l}\text { RAD_PKG_ID } \\
\text { RAD_ISO_NUM } \\
\text { RAD_QTY } \\
\text { RAD_UNKNOWN }\end{array}$ & $\begin{array}{l}\text { RHZ-101-A13568 } \\
19 \\
.05 \\
.05\end{array}$ \\
\hline $\begin{array}{l}\text { RAD_PKG_ID } \\
\text { RAD_ISO_NUM } \\
\text { RAD_ALPTHACI } \\
\text { RAD_PE_CI } \\
\text { RAD_PU_FGE }\end{array}$ & $\begin{array}{l}\text { RHZ-101-A13568 } \\
26 \\
.00030897 \\
.0003096 \\
.0000016875\end{array}$ \\
\hline
\end{tabular}




\begin{tabular}{|c|c|}
\hline RAD_QTY & .00009 \\
\hline $\begin{array}{l}\text { RAD_PKG_IO } \\
\text { RAD_ISO_NUM } \\
\text { RAD_ALPHA_CI } \\
\text { RAD_PE_CI } \\
\text { RAD_PU_FGE } \\
\text { RAD_QTY }\end{array}$ & $\begin{array}{l}\text { RHZ-101-A13568 } \\
41 \\
.00479304 \\
.00435272292 \\
.0000315 \\
.00028\end{array}$ \\
\hline $\begin{array}{l}\text { RAD_PKG_ID } \\
\text { RAD_ISO_NUM } \\
\text { RAD_ALPHA_CI } \\
\text { RAD_PE_CI } \\
\text { RAD_PU_FGE } \\
\text { RAD_QTY }\end{array}$ & $\begin{array}{l}\text { RHZ-101-A13568 } \\
97 \\
.0130548 \\
.01311 \\
.00129375 \\
.0575\end{array}$ \\
\hline $\begin{array}{l}\text { RAD_PKG_ID } \\
\text { RAD_ISO_NUM } \\
\text { RAD_ALP } \overline{H A C C I} \\
\text { RAD_PE_CI } \\
\text { RAD_PU_FGE } \\
\text { RAD_QIY }\end{array}$ & $\begin{array}{l}\text { RHZ-101-A13568 } \\
98 \\
.0000133112 \\
.010876488 \\
.0126 \\
.0056\end{array}$ \\
\hline $\begin{array}{l}\text { RAD_PKG_ID } \\
\text { RAD_ISO_NUM } \\
\text { RAD_ALPHA_CI } \\
\text { RAD_PE_CI } \\
\text { RAD_PU_FGE } \\
\text { RAD_QTY }\end{array}$ & $\begin{array}{l}\text { RHZ- } 101-A 13568 \\
99 \\
.0000007856 \\
.00000071454474 \\
.0000015 \\
.0002\end{array}$ \\
\hline $\begin{array}{l}\text { RAD_PKG_ID } \\
\text { RAD_ISO_NUM } \\
\text { RAD_ALPHA_CI } \\
\text { RAD_PE_CI } \\
\text { RAD_PU_FGE } \\
\text { RAD_QTY }\end{array}$ & $\begin{array}{l}\text { RHZ-103-A13561 } \\
100 \\
.0580886376 \\
.05815044 \\
.9364 \\
.9364\end{array}$ \\
\hline $\begin{array}{l}\text { RAD_PKG_ID } \\
\text { RAD_ISO_NUM } \\
\text { RAD_QTY- } \\
\text { RAD_UNKNOWW }\end{array}$ & $\begin{array}{l}\text { RHZ- 103-A13561 } \\
19 \\
.05 \\
.05\end{array}$ \\
\hline $\begin{array}{l}\text { RAD_PKG_ID } \\
\text { RAD_ISO_NUM } \\
\text { RAD_ALPHA_CI } \\
\text { RAD_PE_CI } \\
\text { RAD_PU_FGE } \\
\text { RAD_QTY }\end{array}$ & $\begin{array}{l}\text { RHZ-103-A13561 } \\
26 \\
.00030897 \\
.0003096 \\
.0000016875 \\
.00009\end{array}$ \\
\hline $\begin{array}{l}\text { RAD_PKG_ID } \\
\text { RAD_ISO_NUM } \\
\text { RAD_ALPHA_CI } \\
\text { RAD_PE_CI } \\
\text { RAD_PU_FGE } \\
\text { RAD_QTY }\end{array}$ & $\begin{array}{l}\text { RHZ-103-A13561 } \\
41 \\
.00479304 \\
.00435272292 \\
.0000315 \\
.00028\end{array}$ \\
\hline $\begin{array}{l}\text { RAD_PKG_IO } \\
\text { RAD_ISO_NUM } \\
\text { RAD_ALPHA_CI } \\
\text { RAD_PE_CI } \\
\text { RAD_PU_FGE } \\
\text { RAD_QTY }\end{array}$ & $\begin{array}{l}\text { RHZ-103-A13561 } \\
97 \\
.0130548 \\
.01311 \\
.00129375 \\
.0575\end{array}$ \\
\hline $\begin{array}{l}\text { RAD_PKG_ID } \\
\text { RAD_ISO_NUM } \\
\text { RAD_ALPHA_CI } \\
\text { RAD_PE_CI } \\
\text { RAD_PU_FGE } \\
\text { RAD_QTY }\end{array}$ & $\begin{array}{l}\text { RHZ-103-A13561 } \\
98 \\
.0000133112 \\
.010876488 \\
.0126 \\
.0056\end{array}$ \\
\hline $\begin{array}{l}\text { RAD_PKG_ID } \\
\text { RAD_ISO_NUM } \\
\text { RAD_ALPHA_CI } \\
\text { RAD_PE_CI } \\
\text { RAD_PU_FGE } \\
\text { RAD_QTY }\end{array}$ & $\begin{array}{l}\text { RHZ-103-A13561 } \\
99 \\
.0000007856 \\
.00000071454474 \\
.0000015 \\
.0002\end{array}$ \\
\hline
\end{tabular}

\begin{tabular}{|c|c|}
\hline $\begin{array}{l}\text { PHYS_PKG_ID } \\
\text { PHYS_COMP_DESCR } \\
\text { PHYS_COMP_VOL_PCT } \\
\text { PHYS_COMP_WGT }\end{array}$ & $\begin{array}{l}\text { OTP-LLW-WP- } 01 \\
\text { DIRT/SOIL/DIATOHACEOUS } \\
\text { EARTH } \\
86 \\
115\end{array}$ \\
\hline $\begin{array}{l}\text { PHYS_PKG_ID } \\
\text { PHYS_COMP_DESCR } \\
\text { PHYS_COMP_VOL_PCT } \\
\text { PHYS_COMP_WGT }\end{array}$ & $\begin{array}{l}\text { OTP-LLW-WP-01 } \\
\text { HAZARDOUS CONSTITUENTS } \\
.5 \\
0 .\end{array}$ \\
\hline $\begin{array}{l}\text { PHYS_PKG_ID } \\
\text { PHYS_COMP_DESCR } \\
\text { PHYS_COMP_VOL_PCT } \\
\text { PHYS_COMP_WGT }\end{array}$ & $\begin{array}{l}\text { OTP-LLW-WP-01 } \\
\text { PAPER/CARDBOARD } \\
9.5 \\
5\end{array}$ \\
\hline $\begin{array}{l}\text { PHYS_PKG_ID } \\
\text { PHYS_COMP_DESCR } \\
\text { PHYS_COMP_VOL_PCT } \\
\text { PHYS_COMP_WGT }\end{array}$ & $\begin{array}{l}\text { OTP-LLW-WP-01 } \\
\text { PLASTIC/POLYURATHANE } \\
4 \\
5\end{array}$ \\
\hline $\begin{array}{l}\text { PHYS_PKG_IO } \\
\text { PHYS_COMP_DESCR } \\
\text { PHYS_COMP_VOL_PCT } \\
\text { PHYS_COMP_WGT }\end{array}$ & $\begin{array}{l}\text { OTP-LLW-WP-02 } \\
\text { CONWEB PADS } \\
93 \\
127.5\end{array}$ \\
\hline $\begin{array}{l}\text { PHYS_PKG_ID } \\
\text { PHYS_COMP_DESCR } \\
\text { PHYS_COMP_VOL_PCT } \\
\text { PHYS_COMP_WGT }\end{array}$ & $\begin{array}{l}\text { OTP- } L L W-W P-02 \\
\text { HAZARDOUS CONSTITUENTS } \\
5 \\
.64\end{array}$ \\
\hline $\begin{array}{l}\text { PHYS_PKG_ID } \\
\text { PHYS_COMP_DESCR } \\
\text { PHYS_COMP_VOL_PCT } \\
\text { PHYS_COMP_WGT }\end{array}$ & $\begin{array}{l}\text { OTP-LLW-WP-02 } \\
\text { WATER } \\
2 \\
3.9\end{array}$ \\
\hline $\begin{array}{l}\text { PHYS_PKG_ID } \\
\text { PHYS_COMP_DESCR } \\
\text { PHYS_COMP_VOL_PCT } \\
\text { PHYS_COMP_WGT }\end{array}$ & $\begin{array}{l}\text { OTP-LLLW-WP-03 } \\
\text { CLOTH/RAGS/NYLON } \\
10 \\
14\end{array}$ \\
\hline $\begin{array}{l}\text { PHYS_PKG_IO } \\
\text { PHYS_COMP_DESCR } \\
\text { PHYS_COMP_VOL_PCT } \\
\text { PHYS_COMP_WGT }\end{array}$ & $\begin{array}{l}\text { OTP-LLW-WP-O3 } \\
\text { PAPER/CARDBOARD } \\
30 \\
41\end{array}$ \\
\hline $\begin{array}{l}\text { PHYS_PKG_ID } \\
\text { PHYS_COMP_DESCR } \\
\text { PHYS_COMP_VOL_PCT } \\
\text { PHYS_COMP_WGT }\end{array}$ & $\begin{array}{l}\text { OTP-LLW-WP-03 } \\
\text { PLASTIC/POLYURATHANE } \\
60 \\
81\end{array}$ \\
\hline $\begin{array}{l}\text { PHYS_PKG_ID } \\
\text { PHYS_COMP_DESCR } \\
\text { PHYS_COMP_VOL_PCT } \\
\text { PHYS_COMP_WGT }\end{array}$ & $\begin{array}{l}\text { OTP-LLW-WP-O4 } \\
\text { CLOTH/RAGS/NYLON } \\
48 \\
13.6\end{array}$ \\
\hline $\begin{array}{l}\text { PHYS_PKG_ID } \\
\text { PHYS_COMP_DESCR } \\
\text { PHYS_COMP_VOL_PCT } \\
\text { PHYS_COMP_WGT }\end{array}$ & $\begin{array}{l}\text { OTP-LLW-WP-O4 } \\
\text { HAZARDOUS CONSTITUENTS } \\
5 \\
.64\end{array}$ \\
\hline $\begin{array}{l}\text { PHYS_PKG_ID } \\
\text { PHYS_COMP_DESCR } \\
\text { PHYS_COMP_VOL_PCT } \\
\text { PHYS_COMP_WGT }\end{array}$ & $\begin{array}{l}\text { OTP-LLW-WP-04 } \\
\text { PLASTIC/POLYURATHANE } \\
20 \\
4.1\end{array}$ \\
\hline $\begin{array}{l}\text { PHYS_PKG_ID } \\
\text { PHYS_COMP_DESCR } \\
\text { PHYS_COMP_VOL_PCT } \\
\text { PHYS_COMP_WGT }\end{array}$ & $\begin{array}{l}\text { PUCK278 } \\
\text { DIRT/SOIL/OIATOMACEOUS } \\
\text { EARTH } \\
86 \\
115\end{array}$ \\
\hline $\begin{array}{l}\text { PHYS_PKG_ID } \\
\text { PHYS_COMP DESCR } \\
\text { PHYS_COMP_VOL_PCT } \\
\text { PHYSCOMP_WGT }\end{array}$ & $\begin{array}{l}\text { PUCK278 } \\
\text { HAZARDOUS CONSTITUENTS } \\
\dot{0}^{5}\end{array}$ \\
\hline
\end{tabular}




\begin{tabular}{|c|c|}
\hline $\begin{array}{l}\text { PHYS_PKG_ID } \\
\text { PHYS_COMP_DESCR } \\
\text { PHYS_COMP_VOL_PCT } \\
\text { PHYS_COMP_WGT. }\end{array}$ & $\begin{array}{l}\text { PUCK278 } \\
\text { PAPER/CARDBOARD } \\
9.5 \\
5\end{array}$ \\
\hline $\begin{array}{l}\text { PHYS_PKG_ID } \\
\text { PHYS_COMP_DESCR } \\
\text { PHYS_COMP_VOL_PCT } \\
\text { PHYS_COMP_WGT }\end{array}$ & $\begin{array}{l}\text { PUCK278 } \\
\text { PLASTIC/POLYURATHANE } \\
4 \\
5\end{array}$ \\
\hline $\begin{array}{l}\text { PHYS_PKG_10 } \\
\text { PHYS_COMP_DESCR } \\
\text { PHYS_COMP_VOL_PCT }\end{array}$ & $\begin{array}{l}\text { RHZ- } 101-A 13568 \\
\text { CLOTH/RAGS/NYLON } \\
15\end{array}$ \\
\hline $\begin{array}{l}\text { PHYS_PKG_1D } \\
\text { PHYS_COMP_DESCR } \\
\text { PHYS_COMP_VOL_PCT }\end{array}$ & $\begin{array}{l}\text { RHZ-101-A13568 } \\
\text { PAPER/CARDBOARO } \\
\text { 30 }\end{array}$ \\
\hline $\begin{array}{l}\text { PHYS_PKG_ID } \\
\text { PHYS_COMP_DESCR } \\
\text { PHYS_COMP_VOL_PCT }\end{array}$ & $\begin{array}{l}\text { RHZ- } 101-\text { A } 13568 \\
\text { PLASTIC/POLYURATHANE } \\
40\end{array}$ \\
\hline $\begin{array}{l}\text { PHYS_PKG_ID } \\
\text { PHYS_COMP_DESCR } \\
\text { PHYS_COMP_VOL_PCT }\end{array}$ & $\begin{array}{l}\text { RHZ-101-A13568 } \\
\text { RUBBER } \\
15\end{array}$ \\
\hline $\begin{array}{l}\text { PHYS_PKG_ID } \\
\text { PHYS_COMP_DESCR } \\
\text { PHYS_COMP_VOL_PCT }\end{array}$ & $\begin{array}{l}\text { RHZ- } 103-A 13561 \\
\text { CLOTH/RAGS/NYLON } \\
15\end{array}$ \\
\hline $\begin{array}{l}\text { PHYSPKG_ID } \\
\text { PHYS_COMP_DESCR } \\
\text { PHYS_COMP_VOL_PCT }\end{array}$ & $\begin{array}{l}\text { RHZ-103-A13561 } \\
\text { PAPER/CARDBOARD } \\
40\end{array}$ \\
\hline $\begin{array}{l}\text { PHYS_PKG_ID } \\
\text { PHYS_COMP_DESCR } \\
\text { PHYS_COMP_VOL_PCT }\end{array}$ & $\begin{array}{l}\text { RHZ-103-A13561 } \\
\text { PLASTIC/POLYURATHANE } \\
30\end{array}$ \\
\hline $\begin{array}{l}\text { PHYS_PKG_ID } \\
\text { PHYS_COMP_DESCR } \\
\text { PHYS_COMP_VOL_PCT }\end{array}$ & $\begin{array}{l}\text { RHZ-103-A13561 } \\
\text { RUBBER } \\
15\end{array}$ \\
\hline $\begin{array}{l}\text { HDET_PKG_ID } \\
\text { HDET_CNTR_STATUS } \\
\text { HDEI_DESI }{ }^{2} \text { CD } \\
\text { HDET_DW_NUM } \\
\text { HDET_WASTE_VOL } \\
\text { HDET_WASTE_STATUS }\end{array}$ & $\begin{array}{l}\text { OTP-LLW-WP-01 } \\
F \\
\text { RE } \\
\text { D008 } 0009 \\
\dot{u}^{208}\end{array}$ \\
\hline $\begin{array}{l}\text { HDET_PKG_ID } \\
\text { HDET_DESIG_CD } \\
\text { HDET_DW_NUM } \\
\text { HDET_WASTE_VOL }\end{array}$ & $\begin{array}{l}\text { OTP-LLW-WP-02 } \\
\text { RD } \\
0002 \\
.21\end{array}$ \\
\hline $\begin{array}{l}\text { HDET_PKG_ID } \\
\text { HDET_DESİG_CD } \\
\text { HDET_DW_NUM } \\
\text { HDET_WASTE_VOL }\end{array}$ & $\begin{array}{l}\text { OTP-LLW-WP- } 04 \\
\text { RE } \\
\text { D008 WT01 } \\
.07\end{array}$ \\
\hline $\begin{array}{l}\text { HDET_PKG_ID } \\
\text { HDET_CNTR STATUS } \\
\text { HDET_DESIG_CD } \\
\text { HDET_DW_NUM } \\
\text { HDET_WASTE_VOL } \\
\text { HDET_WASIE_STATUS }\end{array}$ & $\begin{array}{l}\text { PUCK278 } \\
\text { F } \\
\text { RE } \\
\text { D008 } 0009 \\
\dot{u}^{208}\end{array}$ \\
\hline $\begin{array}{l}\text { HAZ_PKG_ID } \\
\text { HAZ_COMP ID } \\
\text { HAZ_COMP_TEXT } \\
\text { HAZ_COMP_WGT } \\
\text { HAZ_COMP_WGT_PCT } \\
\text { HAZ_EPCRA_FLĀG }\end{array}$ & $\begin{array}{l}\text { OTP-LLW-WP-01 } \\
\text { 7439-97-6 } \\
\text { MERCURY } \\
.001 \\
.0009 \\
\hat{N}^{0009}\end{array}$ \\
\hline $\begin{array}{l}\text { HAZ_PKG_ID } \\
\text { HAZ_COMD_ID } \\
\text { HAZ_COMP_IEXT } \\
\text { HAZ_COMP_WGT }\end{array}$ & $\begin{array}{l}\text { OTP-LLW-WP }=01 \\
\text { TEMP1985 } \\
\text { LEAD } \\
.085\end{array}$ \\
\hline
\end{tabular}

\begin{tabular}{|c|c|}
\hline $\begin{array}{l}\text { HAZ_COMP_WGT_PCT } \\
\text { HAZ_EPCRA__FLÄG }\end{array}$ &.$_{\mathrm{N}}^{.068}$ \\
\hline $\begin{array}{l}\text { HAZ_PXE_ID } \\
\text { HAZ_COM } \bar{P} \text { ID } \\
\text { HAZ_COMP_TEXT } \\
\text { HAZ_COMP_WGT }\end{array}$ & $\begin{array}{l}\text { OTP-LLW-WP-02 } \\
\text { TEMP2091 } \\
\text { LIQUID WASTE } \\
.6\end{array}$ \\
\hline $\begin{array}{l}\text { HAZ_PKG_ID } \\
\text { HAZ_COMD_ID } \\
\text { HAZ_COMP_TEXT } \\
\text { HAZ_COMP_WGT }\end{array}$ & $\begin{array}{l}\text { OTP-LLW-WP-02 } \\
\text { TEMP3 } 413 \\
\text { SODIUN CHLORIDE } \\
.04\end{array}$ \\
\hline $\begin{array}{l}\text { HAZ_PKG_ID } \\
\text { HAZ_COMP_ID } \\
\text { HAZ_COMP_TEXT } \\
\text { HAZ_COMP_WGT } \\
\text { HAZ_COMP_WGT_PCT }\end{array}$ & $\begin{array}{l}\text { OTP-LLW-WP- } 04 \\
\text { TEMPO } 460 \\
\text { ACID } \\
1.5 \\
5\end{array}$ \\
\hline $\begin{array}{l}\text { HAZ_PKG_ID } \\
\text { HAZ_COMP_ID } \\
\text { HAZ_COMP_IEXT } \\
\text { HAZ_COMP_WGT } \\
\text { HAZ_COMP_WGT_PCT }\end{array}$ & $\begin{array}{l}\text { OTP-LLW-WP-04 } \\
\text { TEMP } 1985 \\
\text { LEAD } \\
8.9 \\
28\end{array}$ \\
\hline $\begin{array}{l}\text { HAZ_PKG_ID } \\
\text { HAZ_COMP_ID } \\
\text { HAZ_COMP_TEXT } \\
\text { HAZ_COMP_WGT } \\
\text { HAZ_COMP_WGT_PCT }\end{array}$ & $\begin{array}{l}\text { OTP-LLW-WP-04 } \\
\text { TEMP2628 } \\
\text { OIL } \\
1.9 \\
6\end{array}$ \\
\hline $\begin{array}{l}\text { HAZ_PKG_ID } \\
\text { HAZ_COMP_ID } \\
\text { HAZ_COMP_TEXT } \\
\text { HAZ_COMP_WGT } \\
\text { HAZ_COMP_WGT_PCT }\end{array}$ & $\begin{array}{l}\text { OTP-LLW-WP-04 } \\
\text { TEMP } 3530 \\
\text { SOLVENTS } \\
2.1 \\
6\end{array}$ \\
\hline $\begin{array}{l}\text { HAZ_PKG_ID } \\
\text { HAZ_COMP_ID } \\
\text { HAZ_COMP_TEXT } \\
\text { HAZ_COMP_WGT } \\
\text { HAZ_COMP_WGT_PCT } \\
\text { HAZ_EPCRA_FLAG }\end{array}$ & $\begin{array}{l}\text { PUCK278 } \\
7439-97-6 \\
\text { MERCURY } \\
.001 \\
.0009 \\
\text { N }\end{array}$ \\
\hline $\begin{array}{l}\text { HAZ_PKG_ID } \\
\text { HAZ_COMP_ID } \\
\text { HAZ_COMP_TEXT } \\
\text { HAZ_COMP_WGT } \\
\text { HAZ_COMP_WGT_PCT } \\
\text { HAZ_EPCRA_FLAG }\end{array}$ & $\begin{array}{l}\text { PUCK278 } \\
\text { TEMP1985 } \\
\text { LEAD } \\
.085 \\
.068 \\
\mathrm{~N}\end{array}$ \\
\hline $\begin{array}{l}\text { PDW_PKG_ID } \\
\text { PDW_NUM } \\
\text { PDW_LANDBAN } \\
\text { POW_SORT_ORDER }\end{array}$ & $\begin{array}{l}\text { OTP-LLW-WP-01 } \\
\text { D008 } \\
Y \\
808\end{array}$ \\
\hline $\begin{array}{l}\text { PDW_PKG_ID } \\
\text { POW_NUM } \\
\text { PDW_LANDBAN } \\
\text { PDW_SORT_ORDER }\end{array}$ & $\begin{array}{l}\text { OTP-LLW-WP-01 } \\
0009 \\
Y \\
809\end{array}$ \\
\hline $\begin{array}{l}\text { NDA_PKG_ID } \\
\text { NDA_DT } \\
\text { NDA_DT } \\
\text { NDA_ASSAY_NUM } \\
\text { NDA_FUT_EVAL_REQD } \\
\text { NDA_ISO_TOT } \\
\text { NDA_PROF_ID } \\
\text { NDA_RVST_FLAG } \\
\text { NDA_SWTYP_GROUP } \\
\text { NDA_THERMAL_POWER } \\
\text { NDA_TMU_ALPBA_CI } \\
\text { NDA_TMU_THERMA L_POWER } \\
\text { NDA_TOT_ALPHA_CI } \\
\text { NDA_WASTE_CAT }\end{array}$ & $\begin{array}{l}\text { OIP-LLW-WP-01 } \\
\text { SEP-12-96 13:13:25 } \\
3051522805 \\
258 \\
N \\
5 \\
\text { NRLLW1 } \\
N \\
\text { LLW } \\
3.5 \\
0 \\
.035 \\
0 \\
\text { WC1 }\end{array}$ \\
\hline NDA PKG_ID & OTP-LLW-WP-02 \\
\hline
\end{tabular}




\begin{tabular}{|c|c|}
\hline $\begin{array}{l}\text { NDA_DT } \\
\text { NDA_DT } \\
\text { NDA_ASSAY_NUM } \\
\text { NDA_FUT_EVAL_REQD } \\
\text { NDA_ISO_TOT } \\
\text { NDA_PROF_ID } \\
\text { NDA_RVST_FLAG } \\
\text { NDA_SWTYP_GROUP } \\
\text { NDA_THERMAL_POWER } \\
\text { NDA_TMU_ALPHA_CI } \\
\text { NDA_TMU_THERMAL_POWER } \\
\text { NDA_TOT_ALPHA_CI } \\
\text { NDA_WASTE_CAT- }\end{array}$ & $\begin{array}{l}\text { SEP-12-96 13:47:13 } \\
3051524833 \\
265 \\
N \\
1 \\
\text { NRLLW1 } \\
N \\
\text { LLW } \\
3.53 \\
0 \\
.0353 \\
0 \\
\text { GIWC3 }\end{array}$ \\
\hline 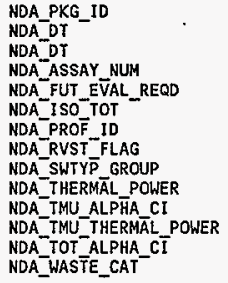 & $\begin{array}{l}\text { OTP-LLW-WP-O3 } \\
\text { SEP-12-96 13:34:22 } \\
3051524062 \\
262 \\
N \\
2 \\
\text { NRLLW1 } \\
N \\
\text { LLW } \\
5.45 \\
0 \\
-0545 \\
0 \\
\text { GTWC3 }\end{array}$ \\
\hline 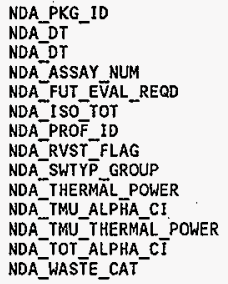 & $\begin{array}{l}\text { OTP-LLW-WP-04 } \\
\text { SEP-12-96 } 13: 18: 27 \\
3051523107 \\
261 \\
\text { N } \\
4 \\
\text { NRLLW1 } \\
N \\
\text { LLW } \\
5.45 \\
0 \\
.0545 \\
0 \\
\text { GTWC3 }\end{array}$ \\
\hline $\begin{array}{l}\text { NDA_PKG_ID } \\
\text { NDA_DT } \\
\text { NDA_DT } \\
\text { NDA_ASSAY_NUM } \\
\text { NDA_FUT_EVAL_REQD } \\
\text { NDA_ISO_TOT } \\
\text { NDA_PROF_ID } \\
\text { NDA_RVST_FLAG } \\
\text { NDA_TOT_ALPHA_CI } \\
\text { NDA_TOT_ALPHA_CI_TMU }\end{array}$ & $\begin{array}{l}\text { RHZ-101-A } 13568 \\
\text { AUG-08-97 18:42:20 } \\
3080054540 \\
1 \\
\text { Y } \\
60 \\
\text { TEST } \\
\text { N } \\
.000663124491192445 \\
.000027472306898023\end{array}$ \\
\hline $\begin{array}{l}\text { NDA_PKG_ID } \\
\text { NDA_DT } \\
\text { NDA_DT } \\
\text { NDA_ASSAY_NUM } \\
\text { NDA_FUT_EVAL_REQQD } \\
\text { NDA_ISO_TOT } \\
\text { NDA_PROF__ID } \\
\text { NDA_RVST_FLAG } \\
\text { NDA_TOT_ALPHA_CI } \\
\text { NDA_TOT_ALPHA_CI_TMU }\end{array}$ & $\begin{array}{l}\text { RHZ-101-A13568 } \\
\text { AUG }-08-9722: 48: 00 \\
3080069280 \\
4 \\
Y \\
60 \\
\text { TEST } \\
\text { N } \\
.00307933704290451 \\
.000132092725764085\end{array}$ \\
\hline $\begin{array}{l}\text { NDA_PKG_ID } \\
\text { NDA_DT. } \\
\text { NDA_DT } \\
\text { NDA_ASSAY_NUM } \\
\text { NDA_FUT_EVAL_REQD } \\
\text { NDA_ISO_TOT } \\
\text { NOA_PROF_ID } \\
\text { NDA_RVST_FLAG } \\
\text { NDA_TOT_ALPHA_CI } \\
\text { NDA_TOT_ALPHA_CI_IMU }\end{array}$ & $\begin{array}{l}\text { RHZ-103-A13561 } \\
\text { AUG-08-97 18:44:21 } \\
3080054661 \\
2 \\
Y \\
60 \\
\text { TEST } \\
\text { N } \\
.0734832895599637 \\
.00278999978807915\end{array}$ \\
\hline
\end{tabular}

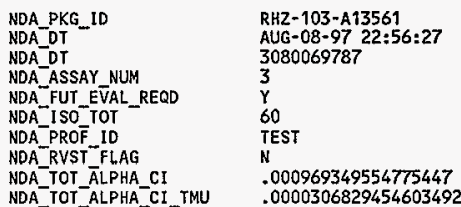

NOA_PKG_ID RHZ-103-A13561

NOA-DT

NDA_DT

NDA ASSAY NUM

NDA FUT EVAL REQD

NDA_ISO TOT

NDA-PROF ID

NDA RVST FLAG

NDA_TOT_ALPHA_CI

NDA_TOT_ALPHA_CI_TMU

NDAISO PKG ID

NDAISO ${ }^{-}$ASSĀY NUM

MDAISO NAME

NDAISO ASSAY NUM

NDAISO QTY

NDAISO-QTY TMU

NDAISO CONF IDENCE CK

NDAISO_MEAS_STATUS

NDAISO PAN ACTIVE

NDAISO-PAN_PASSIVE

NDAISO GEA

AUG-09-97 01:14:00

3080078040

5

60

TEST

.0711790870036994 .00283427410788774

NDAISO PKG ID

NDAISO ASSATY NUM

NDAI SO NAME

NDAISO_ASSAY NUM

NDAISO QTY

NDAISO QTY TMU

NDAISO CONF IDENCE CK

NDAISO MEAS STATUS

NDAISO PAN ÄCTIVE

NDAISO PAN PASSIVE

NDAISO_GEA

NDAISO PKG ID

NOAISO ASSĀY NUM

NDA1 SO NAME

NDAISO_ASSAY_NUM

NDA1SO QTY

NDAISO QTY TMU

NDAISO CONFIDENCE CK

NDAISO MEAS STATUS $\bar{S}$

NDAISO PAN ACTIVE

NDAI SO PAN PASSIVE

NDAISO_GEA

NDAISO_PKG ID

NDAISO ASSĀY NUM

NDAISO NAME

NDAISO ASSAY NUM

NDAISO QTY

NDAISO-QTY TMU

NDAISO CONFIDENCE CK

NDAISO MEAS STATUS

NDAISO_PAN_ACTIVE

NDAISO PAN PASSIVE

NDAISO_GEA

OTP-LLW-WP-01
258
K-40
258
.00000254
.0000000254
TCT
B
NO

THIS IS ONLY A TEST

OTP-LL.W-WP-01

258

TH-232

258

11

.11

$T$

ACT

$B$

NO

YHIS IS ONLY A TEST

OTP-LLW่-WP-01

258

$\mathrm{U}-234$

258

.0013

.000013

$\mathrm{T}$

ACT

$B$

NO

THIS IS ONLY A TEST

OTP-LLW-WP-01

258

U-235

258

.19

.0019

$T$

ACT

B

No

THIS IS ONLY A TEST

NDAISO PKG ID

NDAISO_ASSĀY_NUM

NDAISO NAME

NDAISO ASSAY NUM

NDAISO QTY

NDAISO QTY TMU

NDAISO CONFIDENCE CK.
OTP-LLW-WP-01

258

U-238

258

25

.25 


\begin{tabular}{|c|c|}
\hline $\begin{array}{l}\text { NDA1 SO_MEAS_STATUS } \\
\text { NDAI SO_PAN_ACTIVE } \\
\text { NDA1SO_PAN_PASSIVE } \\
\text { NDAISO_GEA- }\end{array}$ & $\begin{array}{l}\text { ACT } \\
\text { B } \\
\text { NO } \\
\text { THIS IS ONLY A TEST }\end{array}$ \\
\hline $\begin{array}{l}\text { NDAISO_PKG_ID } \\
\text { NDAISO_ASSAY_NUM } \\
\text { NDAISO_NAME } \\
\text { NDAISO_ASSAY_NUM } \\
\text { NDAISO_QTY } \\
\text { NDAISO_QTY_TMU } \\
\text { NDAISO_CONFIDENCE_CK } \\
\text { NDAISO_MEAS_STATUS } \\
\text { NDAISO_PAN_ACTIVE } \\
\text { NDAISO_PAN_PASSIVE } \\
\text { NDAISO_GEA_- }\end{array}$ & $\begin{array}{l}\text { OTP-LLW-WP-02 } \\
265 \\
\text { NA-22 } \\
265 \\
.0003 \\
.000003 \\
\text { ACT } \\
\text { A } \\
\text { NO } \\
\text { THIS IS ONLY A TEST }\end{array}$ \\
\hline $\begin{array}{l}\text { NDAISO_PKG_10 } \\
\text { NDAI SO_ASSAY_NUM } \\
\text { NDAI SO_NAME } \\
\text { NDAISO_ASSAY_NUN } \\
\text { NDAISO_QTY } \\
\text { NDAISO_QTY_TMU } \\
\text { NDAISO_CONFIDENCE_CK } \\
\text { NDAISO_MEAS_STATUS } \\
\text { NDAISO_PAN__CTIVE } \\
\text { NDAISO_PAN_PASSIVE } \\
\text { NDAISO_GEA . }\end{array}$ & $\begin{array}{l}\text { OTP-LLW-WP-03 } \\
262 \\
\text { CS-137 } \\
262 \\
.006 \\
.00006 \\
\text { ACT } \\
\text { B } \\
\text { NO } \\
\text { THIS IS ONLY A TEST }\end{array}$ \\
\hline $\begin{array}{l}\text { NDAISO_PKG_ID } \\
\text { NDAISO_ASSAY_NUM } \\
\text { NDAISO_NAME } \\
\text { NDAISO_ASSAY_NUM } \\
\text { NDAISO_QIY } \\
\text { NDAISO_QIY_TMU } \\
\text { NDAISO_CONFIDENCE_CK } \\
\text { NDAISO_MEAS_STATUS } \\
\text { NDAISO_PAN_ACTIVE } \\
\text { NDAISO_PAN_PASSIVE } \\
\text { NDAISO_GEA }\end{array}$ & $\begin{array}{l}\text { OTP-LLW-WP-03 } \\
262 \\
\text { SR-90 } \\
262 \\
.00006 \\
.0000006 \\
T \\
\text { ACT } \\
\text { B } \\
\text { NO } \\
\text { THIS IS ONLY A TEST }\end{array}$ \\
\hline $\begin{array}{l}\text { NDAISO_PKG_1D } \\
\text { NDAISO_ASSĀY_NUM } \\
\text { NDAISO_NAME } \\
\text { NDAISO_ASSAY_NUM } \\
\text { NDAISO_QIY - } \\
\text { NDAISO_QTY_TMU } \\
\text { NDAISO_CONFIDENCE_CK } \\
\text { NDAISO_MEAS_STATUS } \\
\text { NDAISO_PAN_ACTIVE } \\
\text { NDAISO_PAN_PASSIVE } \\
\text { NDAISO_GEA }\end{array}$ & $\begin{array}{l}\text { OTP-LLW-WP-04 } \\
261 \\
\text { CO-60 } \\
261 \\
.000004 \\
.00000004 \\
\text { T } \\
\text { ACT } \\
\text { A } \\
\text { NO } \\
\text { THIS IS ONLY A TEST }\end{array}$ \\
\hline $\begin{array}{l}\text { NDAISO_PKG_IO } \\
\text { NDAISO_ASSAYY_NUM } \\
\text { NDAISO_NAME } \\
\text { NDAISO_ASSAY_NUM } \\
\text { NDAISO_QTY } \\
\text { NDAISO_QTY_IMU } \\
\text { NDAISO_CONFFIDENCE_CK } \\
\text { NDAISO_MEAS_SIATUS } \\
\text { NDAISO_PAN_ACTIVE } \\
\text { NDAISO_PAN_PASSIVE } \\
\text { NDAISO_GEA- }\end{array}$ & $\begin{array}{l}\text { OTP-LLW-WP-04 } \\
261 \\
\text { CS-137 } \\
261 \\
-000013 \\
.00000013 \\
T \\
\text { ACT } \\
\text { A } \\
\text { NO } \\
\text { THIS IS ONLY A TEST }\end{array}$ \\
\hline $\begin{array}{l}\text { NDAISO_PKG_ID } \\
\text { NDAISO_ASSĀY_NUM } \\
\text { NDAISO_NAME_- } \\
\text { NDAISO_ASSAY_NUM } \\
\text { NDAISO_QTY } \\
\text { NDAISO_QTY_TMU } \\
\text { NDAISO_CONFIIDENCE_CK } \\
\text { NDAISO_MEAS_SIATUS } \\
\text { NDAISO_PAN_ACIIVE } \\
\text { NDAISO_PAN_PASSIVE } \\
\text { NDAISO_GEA }\end{array}$ & $\begin{array}{l}\text { OTP-LLW-WP-04 } \\
261 \\
\text { EU- } 154 \\
261 \\
.000002 \\
.00000002 \\
\text { T } \\
\text { ACT } \\
\text { A } \\
\text { NO } \\
\text { THIS IS ONLY A TEST }\end{array}$ \\
\hline
\end{tabular}

OTP-LLW-WP- 04

261

EU- 155

261

.000032

.00000032

$T$

ACT

A

THIS IS ONLY A TEST

RHZ-101-A13568

1

BE-10

1

0

NA

NP

NO

NONE

RHZ- 10 ? -A13568

c-14

0

NA

NP

NONE

RHZ-101-A13568

CD $-113 \mathrm{M}$

NDAISO NAME

NDAISO-ASSAY_NUM

NDAISO QTY

NDAISO OTY IMU

NDAI SO CONFIDENCE CK

NDAISO MEAS STATUS

NDAISO PAN A ACTIVE

NDAISO PAN PASSIVE

NDAISO_GEA

NDAISO PKG ID

NDAISO_ASSĀY_NUM

NDAI SO NAME

NDAISO_ASSAY_NUM

NDAISO QTY

NDAISO QTY TMU

NDAISO_CONFIDENCE_CK

NDAISO MEAS STATUS

NDAISO PAN ACT IVE

NDAISO_PAN_PASSIVE

NDAISO_GEA

NDAISO PKG ID

NDAISO-ASSĀY NUM

NDAISO NAME

NDAISO ASSAY NUM

NDAISO QTY

NDAISO_QTY TMU

NDAISO CONFIDENCE CK

NDAISO MEAS_STATUS

NDAI SO PAN ĀCTIVE

NDAISO PAN PASSIVE

NDAISO_GEA

NDAISO PKG ID

NOAISO ASSÄY NUM

NDAISO NAME

NOAISO ASSAY NUM

NDAISO-QTY
1
0
0
$N A$
NP
NO
NO

NA

$N P$

NO

NONE

RHZ-101-A13568

1

CL. 36

1

0

NA

NP

NONE

RHZ-101-A13568
1
CO-60
1
-0000000608653537929058
-00000000191544050421885
NA
ACT
NO
NO
LOD
RHZ-101-A13568
1
$H-3$
1
0




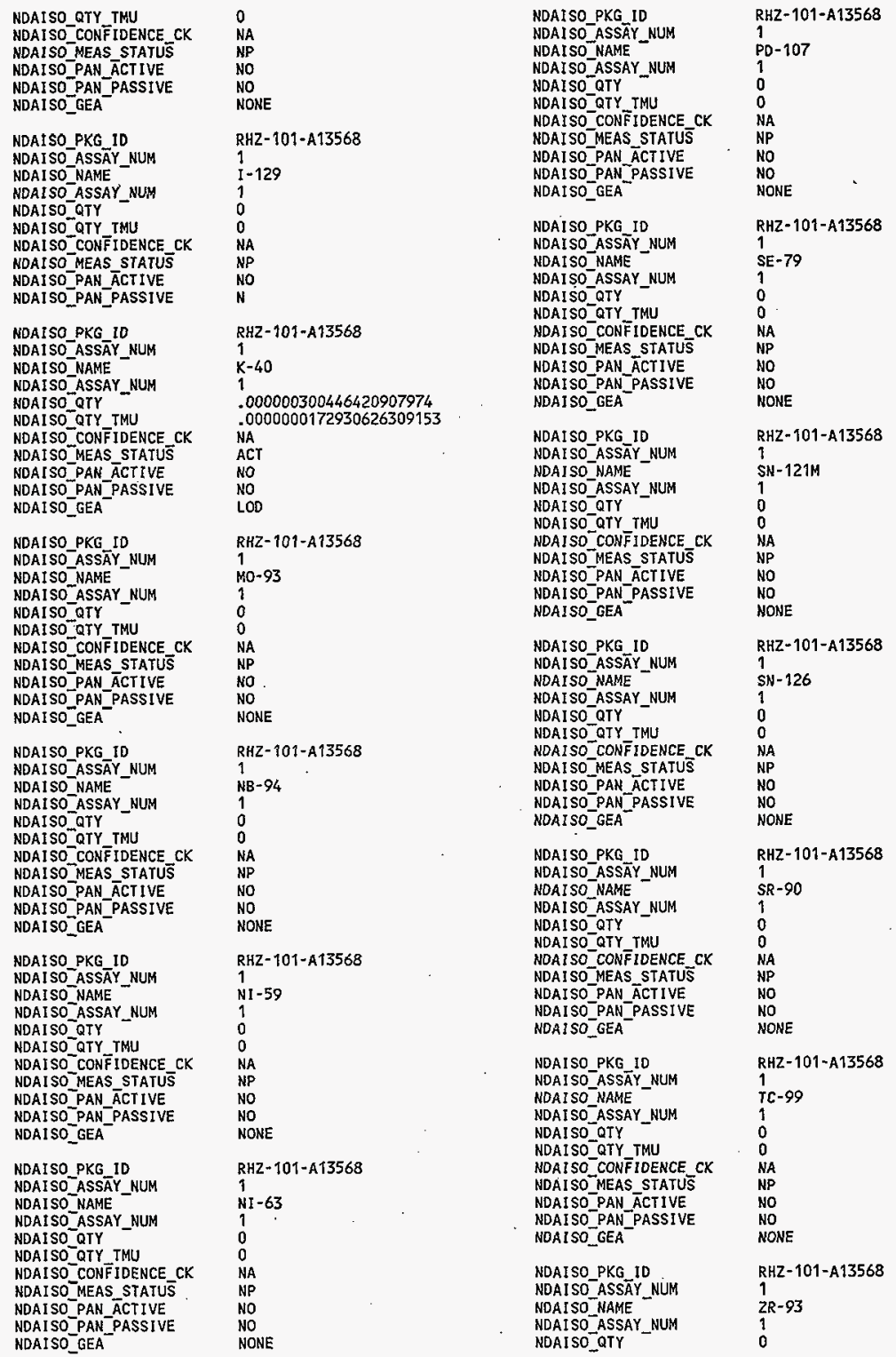




\begin{tabular}{|c|c|}
\hline $\begin{array}{l}\text { NDAISO_QTY_TMU } \\
\text { NDAISO_CONFIDENCE_CK } \\
\text { NDAISO_MEAS_STAIUS } \\
\text { NDAISO_PAN_ACTIVE } \\
\text { NDAISO_PAN_PASSIVE } \\
\text { NDAISO_GEA }\end{array}$ & $\begin{array}{l}0 \\
\text { NA } \\
\text { NP } \\
\text { NO } \\
\text { NO } \\
\text { NONE }\end{array}$ \\
\hline $\begin{array}{l}\text { NDAISO_PKG_ID } \\
\text { NDAISO_ASSAY_NUM } \\
\text { NDAISO_NAME } \\
\text { NDAISO_ASSAY_NUM } \\
\text { NDAISO_OTY } \\
\text { NDAISO_QTY_TMU } \\
\text { NDAISO_CONE IDENCE_CK } \\
\text { NDAISO_MEAS_STATUS } \\
\text { NDAISO_PAN_ACTIVE } \\
\text { NDAISO_PAN_PASSIVE } \\
\text { NDAISO_GEA }\end{array}$ & $\begin{array}{l}\text { RHZ-101-A13568 } \\
4 \\
\text { BE-10 } \\
4 \\
0 \\
0 \\
\text { NA } \\
\text { NP } \\
\text { NO } \\
\text { NO } \\
\text { NONE }\end{array}$ \\
\hline $\begin{array}{l}\text { NDAISO_PKG_ID } \\
\text { NDAISO_ASSAY_NUM } \\
\text { NDAISO_NAME } \\
\text { NDAISO_ASSAY_NUM } \\
\text { NDAISO_QTY } \\
\text { NDAISO_QTY_TMU } \\
\text { NDAISO_CONFIDENCE_CX } \\
\text { NDAISO_MEAS_STATUS } \\
\text { NDAISO_PAN ACTIVE } \\
\text { NDAISO_PAN_PASSIVE } \\
\text { NDAISO_GEA- }\end{array}$ & $\begin{array}{l}\text { RHZ-101-A13568 } \\
4 \\
\text { C- } 14 \\
4 \\
0 \\
0 \\
\text { NA } \\
\text { NP } \\
\text { NO } \\
\text { NO } \\
\text { NONE }\end{array}$ \\
\hline $\begin{array}{l}\text { NDAISO_PKG_ID } \\
\text { NDAISO_ASSAY_NUM } \\
\text { NDAISO_NAME- } \\
\text { NDAISO_ASSAY_NUM } \\
\text { NDAISO_QTY } \\
\text { NDAISO_QTY_TMU } \\
\text { NDAISO_CONFIDENCE_CK } \\
\text { NDAISO_MEAS_STATUS } \\
\text { NDAISO_PAN_ACTIVE } \\
\text { NDAISO_PAN_PASSIVE } \\
\text { NDAISO_GEA }\end{array}$ & $\begin{array}{l}\text { RHZ-101-A13568 } \\
4 \\
\text { CD-113M } \\
4 \\
0 \\
0 \\
\text { NA } \\
\text { NP } \\
\text { NO } \\
\text { NO } \\
\text { NONE }\end{array}$ \\
\hline $\begin{array}{l}\text { NDAISO_PKG_ID } \\
\text { NDAISO_ASSAYY_NUM } \\
\text { NDAISO_NAME } \\
\text { NDAISO_ASSAY_NUM } \\
\text { NOAISO_QTY - } \\
\text { NDAISO_QTY_TMU } \\
\text { NDAISO_CONFIDENCE_CK } \\
\text { NDAISO_MEAS_SIATUS } \\
\text { NDAISO_PAN_ACTIVE } \\
\text { NDAISO_PAN_PASSIVE } \\
\text { NDAISO_GEA- }\end{array}$ & $\begin{array}{l}\text { RHZ-101-A13568 } \\
4 \\
C L-36 \\
4 \\
0 \\
0 \\
\text { NA } \\
\text { NP } \\
\text { NO } \\
\text { NO } \\
\text { NONE }\end{array}$ \\
\hline $\begin{array}{l}\text { NDAISO_PKG_ID } \\
\text { NDAISO_ASSAY_NUM } \\
\text { NDAISO_NAME } \\
\text { NDAISO_ASSAY_NUM } \\
\text { NDAISO_QTY _._ } \\
\text { NDAISO_QTY_TMU } \\
\text { NDAISO_CONFIDENCE_CK } \\
\text { NDAISO_MEAS_STATUS } \\
\text { NDAISO_PAN_ACTIVE } \\
\text { NDAISO_PAN_PASSIVE } \\
\text { NDAISO_GEA }\end{array}$ & $\begin{array}{l}\text { RHZ- } 101-A 13568 \\
4 \\
\text { CO-60 } \\
4 \\
.0000000646429136395454 \\
\text { N00000000150498808764362 } \\
\text { NA } \\
\text { ACT } \\
\text { NO } \\
\text { NO } \\
\text { LOD }\end{array}$ \\
\hline $\begin{array}{l}\text { NDAISO_PKG_ID } \\
\text { NDAISO_ASSAY_NUM } \\
\text { NDAISO_NAME - } \\
\text { NDAISO_ASSAY_NUM } \\
\text { NDAISO_QTY } \\
\text { NDAISO_QTY_TMU } \\
\text { NDAISO_CONFIDENCE_CK } \\
\text { NDAISO_MEAS_STATUS } \\
\text { WDAISO_PAN_ACTIVE } \\
\text { NDAISO_PAN-PASSIVE }\end{array}$ & $\begin{array}{l}\text { RHZ-101-A13568 } \\
4 \\
H-3 \\
4 \\
0 \\
0 \\
\text { NA } \\
\text { HP } \\
\text { NO } \\
\text { NO }\end{array}$ \\
\hline
\end{tabular}

\begin{tabular}{|c|c|}
\hline NDAISO_GEA & NONE \\
\hline $\begin{array}{l}\text { NDAISO_PKG_ID } \\
\text { NDAISO_ASSĀY_NUM } \\
\text { NDAISO_NAME- } \\
\text { NDAISO_ASSAY_NUN } \\
\text { NDAISO_QTY - } \\
\text { NDAISO_QTY_TMU } \\
\text { NDAISO_CONFIDENCE_CK } \\
\text { NDAISO_MEAS_STATUS } \\
\text { NDAISO_PAN_ACTIVE } \\
\text { NDAISO_PAN_PASSIVE }\end{array}$ & $\begin{array}{l}\text { RHZ-101-A13568 } \\
4 \\
\text { I-129 } \\
4 \\
0 \\
0 \\
\text { NA } \\
\text { NP } \\
\text { NO } \\
\text { N }\end{array}$ \\
\hline $\begin{array}{l}\text { NDAISO_PKG_ID } \\
\text { NDAISO_ASSAY_NUM } \\
\text { NDAISO_NAME- } \\
\text { NDAISO_ASSAY_NUM } \\
\text { NDAISO_QTY _- } \\
\text { NDAISO_QTY_TMU } \\
\text { NDAISO_CONFIDENCE_CK } \\
\text { NDAISO_MEAS_STATUS } \\
\text { NDAISO_PAN_ACTIVE } \\
\text { NDAISO_PAN_PASSIVE } \\
\text { NDAISO-GEA- }\end{array}$ & $\begin{array}{l}\text { RHZ-101-A13568 } \\
4 \\
\text { K-40 } \\
4 \\
.000000516251444816589 \\
.0000000218220007845962 \\
\text { NA } \\
\text { ACT } \\
\text { NO } \\
\text { NO } \\
\text { LOD }\end{array}$ \\
\hline
\end{tabular}

NDAISO_PKG_ID .

NDAISO ASSATY NUM NOAISO"NAME

NDAISO ASSAY NUM

NDAISO QTY

NDAISO OTY TMU

NDAI SO CON F IDENCE CK

NDAISO MEAS STATUS

NDAISO PAN ACTIVE

NDAISO PAN PASSIVE

NDAISO_GEA

NDAISO PKG ID

NDAISO ASSĀY_NUM

NDAISO NAME

NDAISO ASSAY NUM

NDAISO-QTY

NDAISO-QTY TMU

NDAISO ${ }^{-}$CONF IDENCE CK

NDAISO MEAS STATUS

NDAISO PAN ACTIVE

NDAISO PAN_PASSIVE

NDAISO_GEA

NDAISO_PKG_ID

NDAISO_ASSÄY_NUM

NDAISO NAME

NDAISO_ASSAY_NUM

NDAISO ${ }^{-}$QTY

NDAISO QTY TMU

NDAISO_CONFIDENCE_CK

NDAISO MEAS STATUS

NDAISO PAN_ACT IVE

NDAISO PAN PASSIVE

NDAISO GEA

NDAISO PKG ID

NDAISO ASSĀY NUM

NDAISO NAME

NDAISO ASSAY NUM

NDAISO QTY

NDAISO QTY TMU

NDAISO CONF FIDENCE CK

NDAISO MEAS STATUS

NDAI SO PAN ACTIVE

NDAISO PAN PASSIVE

NDAISO-GEA

RHZ-101-A13568

4

MO-93

4

0

NA

NA

NO

NO

NONE

RHZ-101-A13568

4

NB-94

4

0

NA

NP

NO

NO

NONE

RHZ - 101-A13568

4

NI -59

4

0

NA

NP

NO

NO

NONE

RHZ-101-A13568

4

$\mathrm{NI}-63$

4

NA

NP

NO

NO

NONE

NDAISO PKG IO

NDAISO ASSĀY NUM

NDAISO NAME

NDAISO_ASSAY_NUM
RHZ-101-A13568

4

PD- 107 


\begin{tabular}{|c|c|}
\hline $\begin{array}{l}\text { NDAISO_QTY } \\
\text { NDAISO_QTY_TMU } \\
\text { NDAISO_CONFIDENCE_CK } \\
\text { NDAISO_MEAS_STATUS } \\
\text { NDAISO_PAN_ACTIVE } \\
\text { NDAISO_PAN_PASSIVE } \\
\text { NDAISO_GEA }\end{array}$ & $\begin{array}{l}0 \\
0 \\
\text { NA } \\
\text { NP } \\
\text { NO } \\
\text { NO } \\
\text { NONE }\end{array}$ \\
\hline $\begin{array}{l}\text { NDAISO_PKG_ID } \\
\text { NDAISO_ASSAY_NUM } \\
\text { NDAISO_NANE } \\
\text { NDAISO_ASSAY_NUM } \\
\text { NDAISO_QTY } \\
\text { NDAISO_QTY_TMU } \\
\text { NDAISO_CONFIOENCE_CK } \\
\text { NDAISO_MEAS_STATUS } \\
\text { NDAISO_PAN_ACTIVE } \\
\text { NDAISO_PAN_PASSIVE } \\
\text { NDAISO_GEA }\end{array}$ & $\begin{array}{l}\text { RHZ-101-A13568 } \\
4 \\
\text { SE-79 } \\
4 \\
0 \\
0 \\
\text { NA } \\
\text { NP } \\
\text { NO } \\
\text { NO } \\
\text { NONE }\end{array}$ \\
\hline $\begin{array}{l}\text { NDAISO_PKG_ID } \\
\text { NDAISO_ASSĀY_NUM } \\
\text { NDAISO_NAME - } \\
\text { NDAISO_ASSAY_NUM } \\
\text { NDAISO_QTY } \\
\text { NDAISO_QTY_TMU } \\
\text { NDAISO_CONFIDENCE_CK } \\
\text { NDAISO_MEAS_STATUS } \\
\text { NDAISO_PAN_ACTIVE } \\
\text { NDAISO_PAN_PASSIVE } \\
\text { NDAISO_GEA }\end{array}$ & $\begin{array}{l}\text { RHZ-101-A13568 } \\
4 \\
\text { SN-121M } \\
4 \\
0 \\
0 \\
\text { NA } \\
\text { UP } \\
\text { NO } \\
\text { NO } \\
\text { NONE }\end{array}$ \\
\hline $\begin{array}{l}\text { NDAISO_PKG_ID } \\
\text { NDAISO_ASSAY_NUM } \\
\text { NDAISO_NAME } \\
\text { NDAISO-ASSAY_NUM } \\
\text { NDAISO_QTY } \\
\text { NDAISO_QTY_TMU } \\
\text { NDAISO_CONFIDENCE_CK } \\
\text { NDAISO_MEAS_STATUS } \\
\text { NDAISO_PAN_ACTIVE } \\
\text { NDAISO_PAN_PASSIVE } \\
\text { NDAISO_GEA- }\end{array}$ & $\begin{array}{l}\text { RHZ-101-A13568 } \\
4 \\
\text { SN-126 } \\
4 \\
0 \\
0 \\
\text { NA } \\
\text { NP } \\
\text { NO } \\
\text { NO } \\
\text { NONE }\end{array}$ \\
\hline $\begin{array}{l}\text { NDAISO_PKG_ID } \\
\text { NDAISO_ASSAY_NUM } \\
\text { NDAISO_NAME } \\
\text { NDAISO-ASSAY_NUM } \\
\text { NDAISO_QTY - } \\
\text { NDAISO_QTY_TWU } \\
\text { NDAISO_CONFIDENCE_CK } \\
\text { NDAISO_MEAS_STATUS } \\
\text { NDAISO_PAN_ACTIVE } \\
\text { NDAISO_PAN_PASSIVE } \\
\text { NDAISO_GEA }\end{array}$ & $\begin{array}{l}\text { RHZ- 101-A13568 } \\
4 \\
\text { SR-90 } \\
4 \\
0 \\
0 \\
\text { NA } \\
\text { NP } \\
\text { NO } \\
\text { NO } \\
\text { NONE }\end{array}$ \\
\hline $\begin{array}{l}\text { NDAISO_PKG_ID } \\
\text { NDAISO_ASSAY_NUM } \\
\text { NDAISO_NAME } \\
\text { NDAISO_ASSAY_NUM } \\
\text { NDAISO_QTY } \\
\text { NDAISO_QTY_TMU } \\
\text { NDAISO_CONFIDENCE_CK } \\
\text { NDAISO_MEAS_STATUS } \\
\text { NDAISO_PAN_ACTIVE } \\
\text { NDAISO_PAN_PASSIVE } \\
\text { NDAISO_GEA }\end{array}$ & $\begin{array}{l}\text { RHZ-101-A13568 } \\
4 \\
\text { TC-99 } \\
4 \\
0 \\
0 \\
\text { NA } \\
\text { NP } \\
\text { NO } \\
\text { NO } \\
\text { NONE }\end{array}$ \\
\hline $\begin{array}{l}\text { NDAISO_PKG_ID } \\
\text { NDAISO-ASSAY_NUM } \\
\text { NDAISO_NAME } \\
\text { NDAISO_ASSAY_NUM } \\
\text { NDAISO_QTY } \\
\text { NDAISO_QTY_TMU } \\
\text { NDAISO_CONFIDENCE_CK } \\
\text { NDAISO_MEAS_STATUS } \\
\text { NDAISO_PAN_ACTIVE }\end{array}$ & $\begin{array}{l}\text { RHZ- 101-A13568 } \\
4 \\
\text { ZR-93. } \\
4 \\
0 \\
0 \\
\text { NA } \\
\text { NP } \\
\text { NO }\end{array}$ \\
\hline
\end{tabular}

$\begin{array}{ll}\text { NDAISO_PAN_PASSIVE } & \text { NO } \\ \text { NDAISO_GEA- } & \text { NONE } \\ \text { NDAISO_PKG_ID } & \text { RHZ-103-A13561 } \\ \text { NDAISO_ASSAY_NUM } & 2 \\ \text { NDAISO_NAME } & \text { BE-10 } \\ \text { NDAISO_ASSAY_NUM } & 2 \\ \text { NDAISO_QTY } & 0 \\ \text { NDAISO_QTY_TMU } & 0 \\ \text { NDAISO_CONFIDENCE_CK } & \text { NA } \\ \text { NDAISO_MEAS_STATUS } & \text { NP } \\ \text { NDAISO_PAN_ACTIVE } & \text { NO } \\ \text { NDAISO_PAN_PASSIVE } & \text { NO } \\ \text { NDAISO_GEA } & \text { NONE }\end{array}$

MDAISO PKG ID

NDAISO ASSĀY NUM

NDAISO NAME - C-

NDAISO_ASSAY_NUM 2

NDAISO QTY -

NDAISO_QTY TMU

NDAISO CONFIDENCE CK NA

NDAISO MEAS STATUS NP

NDAISO_PAN_ $\bar{A} C T I V E$ NO

NDAISO PAN PASSIVE NO

NDAISO_GEA NONE

NDAISO PKG_IO

NDAISO ASSATY NUM

NDAISO NAME

NDAISO_ASSAY_NUM

NDAISO-QTY

NDAISO QTY TMU

NDAI SO_CONFIDENCE CK

NDAISO MEAS STATUS

NDAISO PAN ÄCTIVE

NDAISO_PAN_PASSIVE

NDAISO GEA

MDAISO_PKG_ID

NDAISO ASSĀY_NUM

NDAISO NAME

NDAISO_ASSAY_MUM

NDAISO QTY

NDAISO-OTY TMU

NDAI SO CONFIDENCE CK.

NDAISO MEAS_STATUS

NDAISO PAN ACTIVE

NDAISO_PAN_PASSIVE

NDA ISO GEA

RHZ-103-A13561

2

CD $-113 \mathrm{M}$

2

0
0

NA

NO

No

NONE

RHZ-103-A13561

2

CL-36

2

0

NA

NP

NO

NO

NONE

NDAISO PKG ID

NDAISO_ASSAYY_NUM

NDAISO NAME

NDAISO_ASSAY_NUM

NDAISO QTY

NDAISO QTY IMU

NDAISO_CONFIDENCE_CK

NDAISO MEAS SIATUS

NDAISO_PAN_ACTIVE

NDAISO PAN_PASSIVE

NDAISO_GEA

NDAI SO_PKG_10

NDAISO_ASSĀY_NUM

NDAISO_NAME

MDAISO ASSAY_NUM

NDAISO_QTY

NDAISO QTY TMU

NDAISO CONF̈IDENCE CK

NDAISO MEAS STATUS

NDAISO PAN ÄCT IVE

NDAISO PAN PASSIVE

NDAISO GEA

RHZ-103-A13561

2

Co-60

2

.0000000665036588907242

.0000000017466502985813

NA

ACT

NO

No

LOD

RHZ-103-A13561

2

H-3

2

NA

NA
NO

NO

NONE

NDAISO_PKG_ID

NDAISO_ASSĀY_NUM
RHZ - 103-A13561

2 


\begin{tabular}{|c|c|}
\hline $\begin{array}{l}\text { NDAISO_NAME } \\
\text { NDAISO_ASSAY_NUM } \\
\text { NDAISO_QTY } \\
\text { NDAISO_QTY_TMU } \\
\text { NDAISO_CONFIDENCE_CK } \\
\text { NDAISO_MEAS_STATUS } \\
\text { NDAISO_PAN_ACTIVE } \\
\text { NDAISO_PAN_PASSIVE }\end{array}$ & $\begin{array}{l}I-129 \\
2 \\
0 \\
0 \\
\text { NA } \\
\text { NP } \\
\text { NO } \\
\text { N }\end{array}$ \\
\hline $\begin{array}{l}\text { NDAISO_PKG_ID } \\
\text { NDAISO_ASSAY_NUM } \\
\text { NDAISO_NAME- } \\
\text { NDAISO_ASSAY_NUM } \\
\text { NDAISO_QIY - } \\
\text { NDAISO_QTY_IMU } \\
\text { NDAISO_CONFIDENCE_CK } \\
\text { NDAISO_MEAS_STATUS } \\
\text { NDAISO_PAN_ACTIVE } \\
\text { NDAISO_PAN_PASSIVE } \\
\text { NDAISO_GEA }\end{array}$ & $\begin{array}{l}\text { RHZ-103-A13561 } \\
2 \\
\text { K-40 } \\
2 \\
.000000627851963043213 \\
\text {.0000000299940485083089 } \\
\text { NA } \\
\text { ACT } \\
\text { NO } \\
\text { NO } \\
\text { LOD }\end{array}$ \\
\hline $\begin{array}{l}\text { NDAISO_PKG_ID } \\
\text { NDAISO_ASSAY_NUM } \\
\text { NDAISO_NAME - } \\
\text { NDAISO_ASSAY_NUN } \\
\text { NDAISO_QTY _- } \\
\text { NDAISO_QTY_TMU } \\
\text { NDAISO_CONFIDENCE_CK } \\
\text { NDAISO_MEAS_STATUS } \\
\text { NDAISO_PAN_ACTIVE } \\
\text { NDAISO_PAN_PASSIVE } \\
\text { NDAISO_GEA }\end{array}$ & $\begin{array}{l}\text { RHZ-103-A13561 } \\
2 \\
\text { MO-93 } \\
2 \\
0 \\
0 \\
\text { NA } \\
\text { NP } \\
\text { NO } \\
\text { NO } \\
\text { NONE }\end{array}$ \\
\hline $\begin{array}{l}\text { NDAISO_PKG_ID } \\
\text { NDAISO_ASSAY_NUM } \\
\text { NDAISO_NAME } \\
\text { NDAISO_ASSAY_NUM } \\
\text { NDAISO_QTY } \\
\text { NDAISO_OTY_TMU } \\
\text { NDAISO_CONFIDENCE_CK } \\
\text { NDAISO_MEAS_SIAIUS } \\
\text { NDAISO_PAN_ACTIVE } \\
\text { NDAISO_PAN_PASSIVE } \\
\text { NDAISO_GEA }\end{array}$ & $\begin{array}{l}\text { RHZ-103-A13561 } \\
2 \\
\text { NB-94 } \\
2 \\
0 \\
0 \\
\text { NA } \\
\text { NP } \\
\text { NO } \\
\text { NO } \\
\text { NONE }\end{array}$ \\
\hline $\begin{array}{l}\text { NDAISO_PKG_IO } \\
\text { NDAISO_ASSAY_NUM } \\
\text { NDAISO_NAME } \\
\text { NDAISO_ASSAY_NUM } \\
\text { NDAISO_QTY _ } \\
\text { NDAISO_QTY_TMU } \\
\text { NDAISO_CONFIDENCE_CK } \\
\text { NDAISO_MEAS_STATUS } \\
\text { NDAISO_PAN_ACTIVE } \\
\text { NDAISO_PAN_PASSIVE } \\
\text { NDAISO_GEA }\end{array}$ & $\begin{array}{l}\text { RHZ-103-A13561 } \\
2 \\
\text { N1-59 } \\
2 \\
0 \\
0 \\
\text { NA } \\
\text { NP } \\
\text { NO } \\
\text { NO } \\
\text { NONE }\end{array}$ \\
\hline $\begin{array}{l}\text { NDAI SO_PKG_ID } \\
\text { NDAISO_ASSAY_NUM } \\
\text { NDAISO_NAME } \\
\text { NDAISO_ASSAY_NUM } \\
\text { NDAISO_OTY } \\
\text { NDAISO_OTY_TMU } \\
\text { NDAISO_CONFIDENCE_CK } \\
\text { NDAISO_MEAS_STATUS } \\
\text { NDAISO_PAN_ACTIVE } \\
\text { NDAISO_PAN_PASSIVE } \\
\text { NDAISO_GEA- }\end{array}$ & $\begin{array}{l}\text { RHZ-103-A13561 } \\
2 \\
\text { NI }-63 \\
2 \\
0 \\
0 \\
\text { NA } \\
\text { NP } \\
\text { NO } \\
\text { NO } \\
\text { NONE }\end{array}$ \\
\hline $\begin{array}{l}\text { NDAISO_PKG_ID } \\
\text { NDAISO_ASSAY_NUM } \\
\text { NDAISO_NAME } \\
\text { NDAISO_ASSAY_NUM } \\
\text { NDAISO_QIY } \\
\text { NDAISO_QTY_TMU } \\
\text { NDAISO_CONFIDENCE_CK } \\
\text { NDAISO_MEAS_STATUS }\end{array}$ & $\begin{array}{l}\text { RHZ-103-A13561 } \\
2 \\
\text { PD-107 } \\
2 \\
0 \\
0 \\
\text { NA } \\
\text { NP }\end{array}$ \\
\hline
\end{tabular}

$\begin{array}{ll}\text { NDAISO_PAN_ACTIVE } & \text { NO } \\ \text { NDAISO_PAN_PASSIVE } & \text { NO } \\ \text { NDAISO_GEA } & \text { NONE } \\ \text { NDAISO_PKG_ID } & \text { RHZ-103-A13561 } \\ \text { NDAISO_ASSAY_NUM } & 2 \\ \text { NDAISO_NAME_ } & \text { SE-79 } \\ \text { NDAISO_ASSAY_NUM } & 2 \\ \text { NDAISO_QTY } & 0 \\ \text { NDAISO_QTY_TMU } & 0 \\ \text { NDAISO_CONFIDENCE_CK } & \text { NA } \\ \text { NDAISO_MEAS_STATUS } & \text { NP } \\ \text { NDAISO_PAN_ACTIVE } & \text { NO } \\ \text { NDAISO_PAN_PASSIVE } & \text { NO } \\ \text { NDAISO_GEA } & \text { NONE }\end{array}$

NDAISO PKG ID

NDAISO_ASSĀY_MUM

NDAI SO NAME

NDAISO_ASSAY_NUM

NDAISO QTY

NDAISO QTY TMU

NDAISO CONFIDENCE CK

NDAISO MEAS_STATUS

NDAISO PAN ÁCTIVE

NDAISO_PAN_PASSIVE

NDAISO-GEA

NDAISO_PKG_ID

NDAISO-ASSĀY NUM

NDAISO_NAME

NDAISO-ASSAY_NUM

NDAISO QTY

NDAISO_QTY_TMU

NDAISO CONFIDENCE CK

NDAISO MEAS SIATUS

NDAI SO PAN_ACTIVE

NDAISO PAN PASSIVE

NDAISO_GEA

NDAISO PKG ID

NDAISO_ASSATY_NUM

NDAISO NAME

NDAISO_ASSAY_MUM

NDAISO-QTY

NDAISO QTY TMU

NDAISO CONFIDENCE CK

NDAISO MEAS STATUS

NDAISO PAN ACTIVE

NDAISO_PAN_PASSIVE

NDAISO_GEA

NDAISO_PKG_ID

NDAISOAASSĀY_NUM

NDAISO NAME

NDAISO_ASSAY_NUM

NDAISO QTY

NDAISO_QTY_TMU

NDAISO- CONFIDENCE CK

NDAISO MEAS SIATUS

NDAISO_PAN_ACTIVE

NDAISO PAN PASSIVE

NDAISO_GEA

NDAISO_PKG_ID

NDAISO_ASSĀY_NUM

NDAISO NAME

NDAISO_ASSAY_NUM

NDAISO QTY

MDAISO QTY TMU

NDAISO CONFIDENCE_CK

NDAISO MEAS STATUS

NDAISO_PAN ACCTIVE

MDAISO_PAN_PASSIVE

NDAISO_GEA

NDAISO_PKG_IO
RHZ-103-A13561

SN-121M

2

0

NA

NO

NO

NONE

RH2-103-A13561

2

SN-126

2

0

NA

NP

NO

NO

NONE

RHZ-103-A1356?

2

SR -90

2

0

NA

NP

NO

NO

NONE

RHZ-103-A13561

2

TC-99

2

NA

NO

NO

NONE

RHZ-103-A13561

2

ZR-93

0

NA

NP

NO

NO

NONE

RHZ-103-A13561 


\begin{tabular}{|c|c|}
\hline $\begin{array}{l}\text { NDAISO_ASSAY_NUM } \\
\text { NDAISO_NAME } \\
\text { NDAISO_ASSAY_NUM } \\
\text { NDAISO_QTY } \\
\text { NDAISO_QTY_TMU } \\
\text { NDAISO_CONFIDENCE_CK } \\
\text { NDAISO_MEAS_STATUS } \\
\text { NDAISO_PAN_ACTIVE } \\
\text { NDAISO_PAN_PASSIVE } \\
\text { NDAISO_GEA }\end{array}$ & $\begin{array}{l}3 \\
\text { BE-10 } 10 \\
3 \\
0 \\
0 \\
\text { NA } \\
\text { NP } \\
\text { NO } \\
\text { NO } \\
\text { NONE }\end{array}$ \\
\hline $\begin{array}{l}\text { NDAISO_PKG_ID } \\
\text { NDAISO_ASSAY_NUM } \\
\text { NDAISO_NAME_- } \\
\text { NDAISO_ASSAY_NUM } \\
\text { NDAISO_QTY - } \\
\text { NDAISO_QTY_TMU } \\
\text { NDAISO_CONFIDENCE_CK } \\
\text { NDAISO_MEAS_STATUS } \\
\text { NDAISO_PAN_ACTIVE } \\
\text { NDAISO_PAN_PASSIVE } \\
\text { NDAISO_GEA }\end{array}$ & $\begin{array}{l}\text { RHZ-103-A13561 } \\
3 \\
\text { C-14 } \\
3 \\
0 \\
0 \\
\text { NA } \\
\text { NP } \\
\text { NO } \\
\text { NO } \\
\text { NONE }\end{array}$ \\
\hline $\begin{array}{l}\text { NDAISO_PKG_ID } \\
\text { NDAISO_ASSAY_NUM } \\
\text { NDAISO_NAME } \\
\text { NOAISO_ASSAY_NUM } \\
\text { NDAISO_QTY _- } \\
\text { NDAISO_QTY_TMU } \\
\text { NDAISO_CONFIDENCE_CK } \\
\text { NDAISO_MEAS_STATUS } \\
\text { NDAISO_PAN_ACTIVE } \\
\text { NDAISO_PAN_PASSIVE } \\
\text { NDAISO_GEA }\end{array}$ & $\begin{array}{l}\text { RHZ-103-A13561 } \\
3 \\
\text { CD- } 113 \mathrm{M} \\
3 \\
0 \\
0 \\
\text { NA } \\
\text { NP } \\
\text { NO } \\
\text { NO } \\
\text { NONE }\end{array}$ \\
\hline $\begin{array}{l}\text { NDAISO_PKG_ID } \\
\text { NDAISO_ASSAY_NUM } \\
\text { NDAISO_NAME_- } \\
\text { NDAISO_ASSAY_NUM } \\
\text { NDAISO_QTY _- } \\
\text { NDAISO_GTY_TMU } \\
\text { NDAISO_CONFFIDENCE_CK } \\
\text { NDAISO_MEAS_STATUS } \\
\text { NDAISO_PAN_ACTIVE } \\
\text { NDAISO_PAN_PASSIVE } \\
\text { NDAISO_GEA- }\end{array}$ & $\begin{array}{l}\text { RHZ-103-A13561 } \\
3 \\
\text { CL-36 } \\
3 \\
0 \\
0 \\
\text { NA } \\
\text { NP } \\
\text { NO } \\
\text { NO } \\
\text { NONE }\end{array}$ \\
\hline $\begin{array}{l}\text { NDAISO_PKG_1D } \\
\text { NDAISO_ASSAY_NUM } \\
\text { NDAISO_NAME_- } \\
\text { NDAISO_ASSAY_NUM } \\
\text { NOAISO_QTY _- } \\
\text { NDAISO_QTY_TMU } \\
\text { NDAISO_CONFIDENCE_CK } \\
\text { NDAISO_MEAS_STATUS } \\
\text { NDAISO_PAN_ACTIVE } \\
\text { NDAISO_PAN_PASSIVE } \\
\text { NDAISO_GEA- }\end{array}$ & $\begin{array}{l}\text { RHZ-103-A13561 } \\
3 \\
\text { CO-60 } \\
3 \\
.0000000583489090204239 \\
.00000000154896691983814 \\
\text { NA } \\
\text { ACT } \\
\text { NO } \\
\text { NO } \\
\text { LOD }\end{array}$ \\
\hline $\begin{array}{l}\text { NDAISO_PKG_IO } \\
\text { NDAISO_ASSAY_NUM } \\
\text { NDAISO_NAME_- } \\
\text { NDAISO_ASSAY_NUM } \\
\text { NDAISO_QTY _- } \\
\text { NDAISO_QTY_TMU } \\
\text { NDAISO_CONFIDENCE_CK } \\
\text { NDAISO_MEAS_STATUS } \\
\text { NDAISO_PAN_ACTIVE } \\
\text { NDAISO_PAN_PASSIVE } \\
\text { NDAISO_GEA }\end{array}$ & $\begin{array}{l}\text { RHZ-103-A1356! } \\
3 \\
\mathrm{H}-3 \\
3 \\
0 \\
0 \\
\text { MA } \\
\text { NP } \\
\text { NO } \\
\text { NO } \\
\text { NONE }\end{array}$ \\
\hline $\begin{array}{l}\text { NDAISO_PKG_ID } \\
\text { NDAISO_ASSAY_NUM } \\
\text { NDAISO_NAME } \\
\text { NDAISO_ASSAY_NUM } \\
\text { NDAISO_QTY } \\
\text { NDAISO_QTY_TMU }\end{array}$ & $\begin{array}{l}\text { RHZ-103-A13561 } \\
3 \\
1-129 \\
3 \\
0 \\
0\end{array}$ \\
\hline
\end{tabular}

$\begin{array}{ll}\text { NDAISO_CONFIDENCE_CK } & \text { NA } \\ \text { NDAISO_MEAS_SIATUS } & \text { NP } \\ \text { NDAISO_PAN_ACTIVE } & \text { NO } \\ \text { NDAISO_PAN_PASSIVE } & \text { N }\end{array}$

$\begin{array}{ll}\text { NDAISO_PKG_ID } & \text { RHZ-103-A13561 } \\ \text { NDAISO_ASSAY_NUM } & 3 \\ \text { NDAISO_NAME } & \mathrm{K}-40 \\ \text { NDAISO_ASSAY_NUM } & 3\end{array}$

NDAISO_ASSAY_NUM $\quad 3.00000396498471498489$

NDAISO_QTY TMU

NDAISO CONFIDENCE_CK

NDAISO MEAS_STATUS

NDAISO PAN ACTIVE

NDAISO PAN PASSIVE

NDAISO_GEA

NDAISO PKG 10

NDAISO-ASSAYYNUM

NDAISO NAME

NDAISO_ASSAY_NUM

NDAISO_QTY

NDAISO QTY TMU

NDAISO_CONFIDENCE_CK

NDAISO MEAS STATUS

NDAISO PAN ACTIVE

NDAISO PAN PASSIVE

NDAISO_GEA

NDAISO_PKG 10

NDAISO-ASSÄY_NUM

NDAISO_NAME

NDAISO-ASSAY NUM

NDAISO-QTY

NDAISO-QTY TMU

NDAISO CONFIDENCE CK

NDAISO MEAS STATUS

NDAISO PAN ACTIVE

NDAISO PAN PASSIVE

NDAISO GEA

.0000000187943022787267

NA

NA

NO

NO

LOD

RHZ-103-A13561

3

MO-93

3

0

NA

NA

NO

NO

NONE

RHZ-103-A13561

3

NB- 94

3

NA

NA

NO

NONE

NDAISO PKG ID

NDAISO ASSAY NUM •

NDAISO NAME

NDAISO_ASSAY_NUM

NDAISO-QTY

NDAISO QTY TMU

NDAISO CONF IDENCE CK

NDAISO MEAS STATUS

NDAISO PAN ACTIVE

NDAISO PAN PASSIVE

MOAISO_GEA

\section{NDAISO PKG ID}

NDAISO_ASSĀY_NUM

NDAISO-NAME

NDAI SO ASSAY NUM

NDAISO QTY

NDAISO QTY TMU

NDAISO CONFFIDENCE CK

NDAISO MEAS STATUS

NDAISO_PAN_ACTIVE

NDAI SO PAN PASSIVE

NDAISO_GEA

NDAISO PKG ID

NDAISO ASSAYY_NUM

NDAISO NAME

NDAISO_ASSAY_NUM

NDAISO-QTY

NDAISO QTY TMU

NDAISO_CONFIDENCE_CK

NDAI SO MEAS STATUS

NDAISO_PAN_ÄCTIVE

NDAISO PAN PASSIVE

NDAISO_GEA
3

NI -59

3

0

NA

NP

NO

NO

NONE

RHZ-103-A13561

3

NI -63

3

0

NA

NP

NO

NO

NONE

RHZ-103-A13561

3

PD -107

3

0

NA

NO

NO

NONE
RHZ-103-A13561 


\begin{tabular}{|c|c|}
\hline NDAISO_PKG_ID & RHZ-103-A13561 \\
\hline NDAISO ASSĀY NUM & 3 \\
\hline NDAISO_NAME & SE-79 \\
\hline NDAISO- ASSAY NUM & 3 \\
\hline NDAISO_QTY - & 0 \\
\hline NDAISO_QTY_TMU & 0 \\
\hline NDAISO_CONFIIDENCE_CK & NA \\
\hline NDAISO_MEAS_STATUS & NP \\
\hline NDAISO_PAN_ACTIVE & NO \\
\hline NDAISO PAN PASSIVE & NO \\
\hline NDAISO_GEA & NONE \\
\hline NDAISO_PKG_ID & RHZ-103-A13561 \\
\hline NDAISO_ASSĀY_NUM & 3 \\
\hline NDAISO_NAME - & $S N-121 M$ \\
\hline NDAISO_ASSAY_NUM & 3 \\
\hline NDAISO_QTY - & 0 \\
\hline NDAISO_QTY_TMU & 0 \\
\hline NDAISO_CONFIDENCE_CK & NA \\
\hline NDAISO_MEAS_STATUS & NP \\
\hline NDAISO_PAN_ACTIVE & NO \\
\hline NDAISO_PAN_PASSIVE & NO \\
\hline NDAISO_GEA & NONE \\
\hline NDAISO_PKG_ID & RHZ-103-A13561 \\
\hline NDAISO_ASSĀY_NUM & 3 \\
\hline NDAISO_NAME - & $S N-126$ \\
\hline NDAISO_ASSAY_NUM & 3 \\
\hline NDAISO_QTY - & 0 \\
\hline NDAISO_QTY_TMU & 0 \\
\hline NDAISO_CONF IDENCE_CK & NA \\
\hline NOAISO_MEAS_STATUS & NP \\
\hline NDAISO_PAN_ACTIVE & NO \\
\hline NDAISO_PAN_PASSIVE & NO \\
\hline NDAISO_GEA & NONE \\
\hline NDAISO_PKG_ID & $R H Z-103-A 13561$ \\
\hline NDAISO_ASSĀY_NUM & 3 \\
\hline NDAISO NAME - & $S R-90$ \\
\hline NDAISO_ASSAY_NUM & 3 \\
\hline NDAISO_QTY - & 0 \\
\hline NDAISO_QTY_TMU & 0 \\
\hline NDAISO_CONF IDENCE_CK & NA \\
\hline NDAISO_MEAS_STATUS & NP \\
\hline NDAISO_PAN_ĀCTIVE & NO \\
\hline NDAISO_PAN_PASSIVE & NO \\
\hline NDAISO_GEA & NONE \\
\hline NDAISO_PKG_ID & RHZ-103-A13561 \\
\hline NOAISO-ASSAYYNUM & 3 \\
\hline NDAISO_NAME - & TC-99 \\
\hline NDAISO_ASSAY_NUM & 3 \\
\hline NOAISO_QTY & 0 \\
\hline NDAISO_OTY_TMU & 0 \\
\hline NDAI\$O_CONFIDENCE_CK & NA \\
\hline NDAISO_MEAS_STATUS & NP \\
\hline NDAISO_PAN_ $\bar{A} C T$ IVE & NO \\
\hline NDAISO_PAN_PASSIVE & NO \\
\hline NDAISO_GEA- & NONE \\
\hline NDAISO_PKG_ID & RHZ-103-A13561 \\
\hline NDAISO_ASSÄY_NUM & 3 \\
\hline NDAISO_NAME - & $28-93$ \\
\hline NDAISO_ASSAY_NUM & 3 \\
\hline NDAISO_QTY. & 0 \\
\hline NDAISO_QTY_TMU & 0 \\
\hline NDAISO-CONF-IDENCE_CK & NA \\
\hline NDAISO_MEAS_STATUS & NP \\
\hline NDAISO_PAN_ACTIVE & NO \\
\hline NDAISO_PAN_PASSIVE & NO \\
\hline NDAISO_GEA & NONE \\
\hline NDAISO_PKG_ID & RHZ-103-A13561 \\
\hline NDAYSO_ASSĀY_NUM & 5 \\
\hline NDAISO_NAME - & $B E-10$ \\
\hline NDAISO_ASSAY_NUM & 5 \\
\hline NDAISO_QTY & 0 \\
\hline
\end{tabular}

\begin{tabular}{|c|c|}
\hline $\begin{array}{l}\text { NDAISO_QTY_TMU } \\
\text { NDAISO_CONFIDENCE_CK } \\
\text { NDAISO_MEAS_STATUS } \\
\text { NDAISO_PAN_ACTIVE } \\
\text { NDAISO_PAN_PASSIVE } \\
\text { NDAISO_GEA }\end{array}$ & $\begin{array}{l}0 \\
\text { NA } \\
\text { NP } \\
\text { NO } \\
\text { NO } \\
\text { NONE }\end{array}$ \\
\hline $\begin{array}{l}\text { NDAISO_PKG_ID } \\
\text { NDAISO_ASSAY_NUM } \\
\text { NDAISO_NAME } \\
\text { NDAISO_ASSAY;NUM } \\
\text { NDAISO_QTY } \\
\text { NDAISO_QTY_TMU } \\
\text { NDAISO_CONFIDENCE_CK } \\
\text { NDAISO_MEAS_STATUS } \\
\text { NDAISO_PAN_ACTIVE } \\
\text { NDAISO_PAN_PASSIVE } \\
\text { NDAISO_GEA }\end{array}$ & $\begin{array}{l}\text { RHZ-103-A13561 } \\
5 \\
\text { C- } 14 \\
5 \\
0 \\
0 \\
\text { NA } \\
\text { NP } \\
\text { NO } \\
\text { NO } \\
\text { NONE }\end{array}$ \\
\hline $\begin{array}{l}\text { NDAISO_PKG_ID } \\
\text { NDAISO_ASSAY_NUM } \\
\text { NDAISO_NAME } \\
\text { NDAISO_ASSAY_NUM } \\
\text { NDAISO_QTY } \\
\text { NDAISO_QTY_IMU } \\
\text { NDAISO_CONF_IDENCE_CK } \\
\text { NDAISO_MEAS_STATUS } \\
\text { NDAISO_PAN_ACTIVE } \\
\text { NDAISO_PAN_PASSIVE } \\
\text { NDAISO_GEA }\end{array}$ & $\begin{array}{l}\text { RHZ-103-A13561 } \\
5 \\
\mathrm{CD}-113 \mathrm{M} \\
5 \\
0 \\
0 \\
\text { NA } \\
\text { NP } \\
\text { NO } \\
\text { NO } \\
\text { NONE }\end{array}$ \\
\hline $\begin{array}{l}\text { NDAISO_PKG_ID } \\
\text { NDAISO_ASSAY_NUM } \\
\text { NDAISO_NAME } \\
\text { NDAISO_ASSAY_NUM } \\
\text { NDAISO_QTY } \\
\text { NDAISO_QTY_TNU } \\
\text { NDAISO_CONFIDENCE_CK } \\
\text { NDAISO_MEAS_STATUS } \\
\text { NDAISO_PAN_ACTIVE } \\
\text { NDAISO_PAN_PASSIVE } \\
\text { NDAISO_GEA- }\end{array}$ & $\begin{array}{l}\text { RHZ-103-A13561 } \\
5 \\
\text { CL-36 } \\
5 \\
0 \\
0 \\
\text { NA } \\
\text { NP } \\
\text { NO } \\
\text { NO } \\
\text { NONE }\end{array}$ \\
\hline $\begin{array}{l}\text { NDAISO_PKG_ID } \\
\text { NDAISO_ASSAY_NUM } \\
\text { NDAISO_NAME } \\
\text { NDAISO_ASSAY_NUM } \\
\text { NDAISO_QTY } \\
\text { NDAISO_QTY_TMU } \\
\text { NDAISO_CONFIDENCE_CK } \\
\text { NDAISO_MEAS_STATUS } \\
\text { NDAISO_PAN_ACTIVE } \\
\text { NDAISO_PAN_PASSIVE } \\
\text { NDAISO_GEA }\end{array}$ & $\begin{array}{l}\text { RHZ-103-A13561 } \\
5 \\
\text { CO-60 } \\
5 \\
.0000000616424940526485 \\
.0000000016189767151073 \\
\text { NA } \\
\text { ACT } \\
\text { NO } \\
\text { NO } \\
\text { LOD }\end{array}$ \\
\hline $\begin{array}{l}\text { NDAISO_PKG_ID } \\
\text { NDAISO_ASSAY_MUM } \\
\text { NDAISO_NAME } \\
\text { NDAISO_ASSAY_NUM } \\
\text { NDAISO_QTY } \\
\text { NDAISO_QTY_TMU } \\
\text { NDAISO_CONFIDENCE_CK } \\
\text { NDAISO_MEAS_STATUS } \\
\text { NDAISO_PAN_ACTIVE } \\
\text { NDAISO_PAN_PASSIVE } \\
\text { NDAISO_GEA- }\end{array}$ & $\begin{array}{l}\text { RHZ-103-A } 13561 \\
5 \\
\text { H-3 } \\
5 \\
0 \\
0 \\
\text { NA } \\
\text { NP } \\
\text { NO } \\
\text { NO } \\
\text { NONE }\end{array}$ \\
\hline $\begin{array}{l}\text { NDAISO_PKG_ID } \\
\text { NDAISO_ASSAY_NUM } \\
\text { NDAISO_NAME - } \\
\text { NDAISO_ASSAY_NUM } \\
\text { NDAISO_QTY } \\
\text { NDAISO_QTY_TMU } \\
\text { NDAISO_CONFIDENCE_CK } \\
\text { NDAISO_MEAS_STATUS } \\
\text { NDAISO_PAN_ACTIVE } \\
\text { NDAISO_PAN_PASSIVE }\end{array}$ & $\begin{array}{l}\text { RHZ-103-A13561 } \\
5 \\
i-129 \\
5 \\
0 \\
0 \\
\text { NA } \\
\text { NP } \\
\text { NO } \\
\text { N }\end{array}$ \\
\hline
\end{tabular}




\begin{tabular}{|c|c|}
\hline $\begin{array}{l}\text { NDAISO_PKG_ID } \\
\text { NDAISO_ASSAY_NUM } \\
\text { NDAISO_NAME } \\
\text { NDAISO_ASSAY_NUM } \\
\text { NDAISO_QTY } \\
\text { NDAISO_OTY_TMU } \\
\text { NDAISO_CONFIDENCE_CK } \\
\text { NDAISO_MEAS_STATUS } \\
\text { NDAISO_PAN_ACTIVE } \\
\text { NDAISO_PAN_PASSIVE } \\
\text { NDAISO_GEA- }\end{array}$ & $\begin{array}{l}\text { RHZ-103-A13561 } \\
5 \\
\text { K- } 40 \\
5 \\
.000000627062678337097 \\
\text { NA } \\
\text { ACT } \\
\text { NO } \\
\text { NO } \\
\text { LOD }\end{array}$ \\
\hline $\begin{array}{l}\text { NDAISO_PKG_ID } \\
\text { NDAISO_ASSAY_NUM } \\
\text { NDAISO_NAME - } \\
\text { NDAISO_ASSAY_NUM } \\
\text { NDAISO_QTY - } \\
\text { NDAISO_QTY_TMU } \\
\text { NDAISO_CONFIDENCE_CK } \\
\text { NDAISO_MEAS_STATUS } \\
\text { NDAISO_PAN_ACTIVE } \\
\text { NDAISO_PAN_PASSIVE } \\
\text { NDAISO_GEA }\end{array}$ & $\begin{array}{l}\text { RHZ-103-A13561 } \\
5 \\
\text { MO-93 } \\
5 \\
0 \\
0 \\
\text { NA } \\
\text { NP } \\
\text { NO } \\
\text { NO } \\
\text { NONE }\end{array}$ \\
\hline $\begin{array}{l}\text { NDAISO_PKG_ID } \\
\text { NDAISO_ASSAY_NUM } \\
\text { NDAISO_NAME_- } \\
\text { NDAISO_ASSAY_NUM } \\
\text { NDAISO_QTY _ } \\
\text { NDAISO_QTY_TMU } \\
\text { NDAISO_CONFIDENCE_CK } \\
\text { NDAISO_MEAS_STATUS } \\
\text { NDAISO_PAN_ACTIVE } \\
\text { NDAISO_PAN_PASSIVE } \\
\text { NDAISO_GEA }\end{array}$ & $\begin{array}{l}\text { RHZ-103-A13561 } \\
5 \\
\text { NB-94 } \\
5 \\
0 \\
0 \\
\text { NA } \\
\text { NP } \\
\text { NO } \\
\text { NO } \\
\text { NONE }\end{array}$ \\
\hline $\begin{array}{l}\text { NDAISO_PKG_ID } \\
\text { NDAISO_ASSĀY_NUM } \\
\text { NDAISO_NAME_- } \\
\text { NDAISO_ASSAY_NUM } \\
\text { NDAISO_QTY _- } \\
\text { NDAISO_QTY_IMU } \\
\text { NDAISO_CONFIDENCE_CK } \\
\text { NDAISO_MEAS_STATUS } \\
\text { NDAISO_PAN_ACTIVE } \\
\text { NDAISO_PAN_PASSIVE } \\
\text { NDAISO_GEA }\end{array}$ & $\begin{array}{l}\text { RHZ-103-A13561 } \\
5 \\
\text { NI }-59 \\
5 \\
0 \\
0 \\
\text { NA } \\
\text { NP } \\
\text { NO } \\
\text { NO } \\
\text { NONE }\end{array}$ \\
\hline $\begin{array}{l}\text { NDAISO_PKG_ID } \\
\text { NDAISO_ASSĀY_NUM } \\
\text { NDAISO_NAME } \\
\text { NDAISO_ASSAY_NUM } \\
\text { NDAISO_QTY _ } \\
\text { NDAISO_QTY_TMU } \\
\text { NDAISO_CONFEIDENCE_CK } \\
\text { NDAISO_MEAS_STATUS } \\
\text { NDAISO_PAN_ACTIVE } \\
\text { NDAISO_PAN_PASSIVE } \\
\text { NDAISO_GEA }\end{array}$ & $\begin{array}{l}\text { RHZ-103-A13561 } \\
5 \\
\text { NI }-63 \\
5 \\
0 \\
0 \\
\text { NA } \\
\text { NP } \\
\text { NO } \\
\text { NO } \\
\text { NONE }\end{array}$ \\
\hline $\begin{array}{l}\text { NDAISO_PKG_ID } \\
\text { NDAISO_ASSAY_NUM } \\
\text { NDAISO_NAME_- } \\
\text { NDAISO_ASSAY_NUM } \\
\text { NDAISO_QTY _- } \\
\text { NDAISO_QTY_TMU } \\
\text { NDAISO_CONFIDENCE_CK } \\
\text { NDAISO_MEAS_STATUS } \\
\text { NDAISO_PAN_ACTIVE } \\
\text { NDAISO_PAN_PASSIVE } \\
\text { NDAISO_GEA }\end{array}$ & $\begin{array}{l}\text { RHZ-103-A13561 } \\
5 \\
\text { PD-107 } \\
5 \\
0 \\
0 \\
\text { NA } \\
\text { NP } \\
\text { NO } \\
\text { NO } \\
\text { NONE }\end{array}$ \\
\hline $\begin{array}{l}\text { NDAISO_PKG_ID } \\
\text { NDAISO_ASSAY_NUM } \\
\text { NDAISO_NAME- } \\
\text { NDAISO_ASSAY_NUM } \\
\text { NDAISO_QTY }\end{array}$ & $\begin{array}{l}\text { RHZ-103-A13561 } \\
5 \\
\text { SE-79 } \\
5 \\
0\end{array}$ \\
\hline
\end{tabular}

\begin{tabular}{|c|c|c|}
\hline $\begin{array}{l}\text { NDAISO_QTY_TMU } \\
\text { NDAISO_CONFF IDENCE_CK } \\
\text { NDAISO_MEAS_STATUS } \\
\text { NDAISO_PAN_ACTIVE } \\
\text { NDAISO_PAN_PASSIVE } \\
\text { NDAISO_GEA }\end{array}$ & $\begin{array}{l}0 \\
\text { NA } \\
\text { NP } \\
\text { NO } \\
\text { NO } \\
\text { NONE }\end{array}$ & \\
\hline $\begin{array}{l}\text { NDAISO_PKG_ID } \\
\text { NDAISO_ASSAY_NUM } \\
\text { NDAISO_NAME_- } \\
\text { NDAISO_ASSAY_NUM } \\
\text { NDAISO_QTY _- } \\
\text { NDAISO_QTY_IMU } \\
\text { NDAISO_CONFIDENCE_CK } \\
\text { NDAISO_MEAS_STATUS } \\
\text { NDAISO_PAN_ACTIVE } \\
\text { NDAISO_PAN_PASSIVE } \\
\text { NDAISO_GEA }\end{array}$ & $\begin{array}{l}\text { RHZ-103-A13561 } \\
5 \\
\text { SN-121M } \\
5 \\
0 \\
0 \\
\text { NA } \\
\text { NP } \\
\text { NO } \\
\text { NO } \\
\text { NONE }\end{array}$ & \\
\hline $\begin{array}{l}\text { NDAISO_PKG_ID } \\
\text { NDAISO_ASSAY_NUM } \\
\text { NDAISO_NAME_- } \\
\text { NDAISO_ASSAY_NUM } \\
\text { NDAISO_QTY } \\
\text { NDAISO_QTY_TMU } \\
\text { NDAISO_CONFIDENCE_CK } \\
\text { NDAISO_MEAS_STATUS } \\
\text { NDAISO_PAN_ACTIVE } \\
\text { NDAISO_PAN_PASSIVE } \\
\text { NDAISO_GEA- }\end{array}$ & $\begin{array}{l}\text { RHZ-103-A13561 } \\
5 \\
\text { SN-126 } \\
5 \\
0 \\
0 \\
\text { NA } \\
\text { NP } \\
\text { NO } \\
\text { NO } \\
\text { NONE }\end{array}$ & . \\
\hline $\begin{array}{l}\text { NDAISO_PKG_ID } \\
\text { NDAISO_ASSAY_NUM } \\
\text { NDAISO_NAME } \\
\text { NDAISO_ASSAY_NUM } \\
\text { MDAISO_QTY } \\
\text { NDAISO_QTY_IMU } \\
\text { NDAISO_CONFIDENCE_CK } \\
\text { NDAISO_MEAS_STATUS } \\
\text { NDAISO_PAN_ACTIVE } \\
\text { NDAISO_PAN_PASSIVE } \\
\text { NDAISO_GEA }\end{array}$ & $\begin{array}{l}\text { RHZ-103-A13561 } \\
5 \\
\text { SR-90 } \\
5 \\
0 \\
0 \\
\text { NA } \\
\text { NP } \\
\text { NO } \\
\text { NO } \\
\text { NONE }\end{array}$ & \\
\hline $\begin{array}{l}\text { NDAISO_PKG_ID } \\
\text { NDAISO_ASSĀY_NUM } \\
\text { NDAISO_NAME } \\
\text { NDAISO_ASSAY_NUM } \\
\text { NDAISO_QTY } \\
\text { NDAISO_QTY_TMU } \\
\text { NDAISO_CONFIDENCE_CK } \\
\text { NDAISO_MEAS_STATUS } \\
\text { NDAISO_PAN_ACTIVE } \\
\text { NDAISO_PAN_PASSIVE } \\
\text { NDAISO_GEA }\end{array}$ & $\begin{array}{l}\text { RHZ-103-A13561 } \\
5 \\
\text { TC-99 } \\
5 \\
0 \\
0 \\
\text { NA } \\
\text { NP } \\
\text { NO } \\
\text { NO } \\
\text { NONE }\end{array}$ & \\
\hline $\begin{array}{l}\text { NDAISO_PKG_ID } \\
\text { NDAISO_ASSAY_NUM } \\
\text { NDAISO_NAME } \\
\text { NDAISO_ASSAY_NUM } \\
\text { NDAISO_QTY - } \\
\text { NDAISO_QTY_TMU } \\
\text { NDAISO_CONFIDENCE_CK } \\
\text { NDAISO_MEAS_STATUS } \\
\text { NDAISO_PAN_ACTIVE } \\
\text { NDAISO_PAN_PASSIVE } \\
\text { NDAISO_GEA }\end{array}$ & $\begin{array}{l}\text { RHZ-103-A13561 } \\
5 \\
\text { ZR-93 } \\
5 \\
0 \\
0 \\
\text { NA } \\
\text { NP } \\
\text { NO } \\
\text { NO } \\
\text { NONE }\end{array}$ & \\
\hline $\begin{array}{l}\text { NDE_PKG_ID } \\
\text { NDE_DT - } \\
\text { NDE_COMMENTS }\end{array}$ & $\begin{array}{l}\text { OTP-LLW-WP-01 } \\
12-S E P-96 \\
\text { OTP-LLW-WP-01 1ST } \\
\text { COMMENTS }\end{array}$ & T IMAGE \\
\hline $\begin{array}{l}\text { NDE_OPER_ID } \\
\text { NDE_VDISK_FILE } \\
\text { NDE_VDISK_NUM } \\
\text { NDE_VTAPE_NUM } \\
\text { NDE_VTAPE_START }\end{array}$ & $\begin{array}{l}\text { USER1 } \\
\text { WP05X } \\
\text { P22V1 } \\
\text { P22T1 } \\
200\end{array}$ & \\
\hline
\end{tabular}




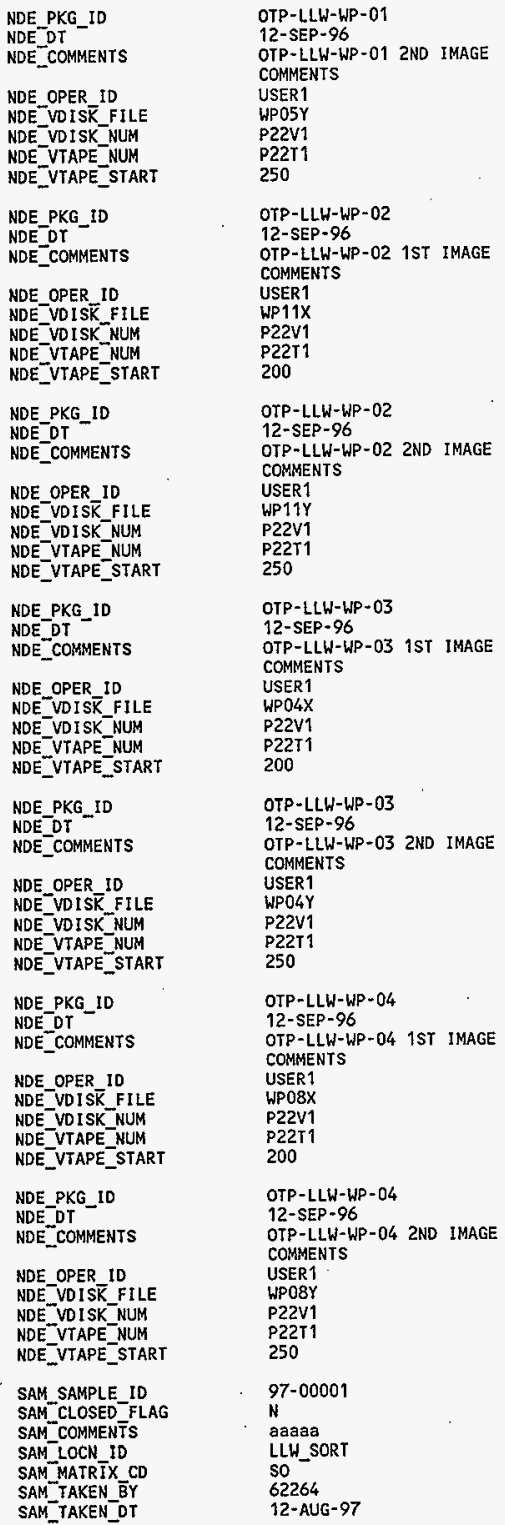

\begin{tabular}{|c|c|}
\hline $\begin{array}{l}\text { SAM_TEMP } \\
\text { SAM_PKG_ID }\end{array}$ & $\begin{array}{l}22 \\
\text { OTP-LLW-WP-01 }\end{array}$ \\
\hline $\begin{array}{l}\text { SAR_SAMPLE_ID } \\
\text { SAR_ANAL_CD }\end{array}$ & $\begin{array}{l}97-00001 \\
\text { solis }\end{array}$ \\
\hline $\begin{array}{l}\text { SAR_SAMPLE ID } \\
\text { SAR_ANAL_CD }\end{array}$ & $\begin{array}{l}97-00001 \\
\text { TCLP }\end{array}$ \\
\hline $\begin{array}{l}\text { BOT_BOTTLE_ID } \\
\text { BOT_SAMPLE_ID } \\
\text { BOT_ANAL_CD }\end{array}$ & $\begin{array}{l}97-00001-01 \mathrm{~L} \\
97-00001 \\
\text { TCLP }\end{array}$ \\
\hline $\begin{array}{l}\text { BOT_BOTTLE_ID } \\
\text { BOT_SAMPLE_ID } \\
\text { BOT_ANAL_CD }\end{array}$ & $\begin{array}{l}97-00001-02 L \\
97-00001 \\
\text { SOILS }\end{array}$ \\
\hline $\begin{array}{l}\text { SAMREL_BOTTLE_ID } \\
\text { SAMREL_PKG_ID } \\
\text { SAMREL_DT } \\
\text { SAMREL_USE_CD }\end{array}$ & $\begin{array}{l}97-00001-01 L \\
\text { OIP-LLW-WP-01 } \\
12-A U G-97 \\
\text { WV }\end{array}$ \\
\hline $\begin{array}{l}\text { SAMREL_BOITLE_ID } \\
\text { SAMREL_PKG_ID- } \\
\text { SAMREL_DI } \\
\text { SAMREL_USE_CD }\end{array}$ & $\begin{array}{l}97-00001-01 L \\
\text { OTP6-97-300001 } \\
13-A U G-27 \\
\text { PP }\end{array}$ \\
\hline $\begin{array}{l}\text { SAMREL_BOTTLE_ID } \\
\text { SAMREL_PKG_ID- } \\
\text { SAMREL_DT } \\
\text { SAMREL_USE_CD }\end{array}$ & $\begin{array}{l}97-00001-02 L \\
\text { OTP-LLW-WP-01 } \\
12-A \cup G-97 \\
W N\end{array}$ \\
\hline $\begin{array}{l}\text { SAMREL_BOTTLE_ID } \\
\text { SAMREL_PKG_ID- } \\
\text { SAMREL_DT } \\
\text { SAMREL_USE_CD }\end{array}$ & $\begin{array}{l}97-00001-02 \mathrm{~L} \\
0 \mathrm{TP} 6-97-300001 \\
13-A U G-27 \\
\text { PP }\end{array}$ \\
\hline $\begin{array}{l}\text { CONLOC_PKG_10 } \\
\text { CONLOC_DT } \\
\text { CONLOC_LOCN_ID }\end{array}$ & $\begin{array}{l}\text { OTP-LLW-OD-01 } \\
13-A U G-27 \\
\text { LLW_ENTRY }\end{array}$ \\
\hline $\begin{array}{l}\text { CONLOC_PKG_ID } \\
\text { CONLOC_DT } \\
\text { CONLOC_LOCN_ID }\end{array}$ & $\begin{array}{l}\text { OTP-LLW-OD-02 } \\
15-J A N-97 \\
\text { AIRCVYR_A3 }\end{array}$ \\
\hline $\begin{array}{l}\text { CONLOC_PKG_ID } \\
\text { CONLOC_DT } \\
\text { CONLOC_LOCN_ID }\end{array}$ & $\begin{array}{l}\text { OTP-LLW-OD-03 } \\
15-J A N-97 \\
\text { AIRCVYR_A3 }\end{array}$ \\
\hline $\begin{array}{l}\text { CONLOC_PKG_ID } \\
\text { CONLOC_DT } \\
\text { CONLOC_LOCN_ID }\end{array}$ & $\begin{array}{l}\text { OTP-LLW-OD-04 } \\
\text { 15-JAN-97 } \\
\text { AIRCVYR_A3 }\end{array}$ \\
\hline $\begin{array}{l}\text { CONLOC_PKG_ID } \\
\text { CONLOC_DT } \\
\text { CONLOC_LOCN_ID }\end{array}$ & $\begin{array}{l}\text { OTP-LLW-WP-01 } \\
13-A U G-27 \\
\text { LLW_SORT }\end{array}$ \\
\hline $\begin{array}{l}\text { CONLOC_PKG_ID } \\
\text { CONLOC_DY } \\
\text { CONLOC_LOCN_ID }\end{array}$ & $\begin{array}{l}\text { OTP-LLWR-TD-01 } \\
13-A U G-27 \\
\text { LLW_RWMPRT }\end{array}$ \\
\hline $\begin{array}{l}\text { CONLOC_PKG_ID } \\
\text { CONLOC_DT } \\
\text { CONLOC_LOCN_ID }\end{array}$ & $\begin{array}{l}\text { OTP-LLWR-TD-02 } \\
\text { O5-FEB- } 97 \\
\text { RWM_CRSL }\end{array}$ \\
\hline $\begin{array}{l}\text { CONLOC_PKG_ID } \\
\text { CONLOC_DT } \\
\text { CONLOC_LOCN_10 }\end{array}$ & $\begin{array}{l}\text { OTP6-97-400001 } \\
13-A U G-27 \\
\text { LLW_PP }\end{array}$ \\
\hline $\begin{array}{l}\text { CONLOC_PKG_ID } \\
\text { CONLOC_DT } \\
\text { CONLOC_LOCN_ID }\end{array}$ & $\begin{array}{l}\text { RHZ-101-A13568 } \\
10 \text {-AUG-27 } \\
\text { INFDCVYRW }\end{array}$ \\
\hline $\begin{array}{l}\text { CONLOC_PKG_ID } \\
\text { CONLOC_DT } \\
\text { CONLOC_LOCN_ID }\end{array}$ & $\begin{array}{l}\text { RHZ-103-A13561 } \\
\text { 10-AUG-27 } \\
\text { AGVPANC_A }\end{array}$ \\
\hline
\end{tabular}




\begin{tabular}{|c|c|}
\hline $\begin{array}{l}\text { CONR_FROM_PKG_ID } \\
\text { CONR_TO_PKG_ID } \\
\text { CONR_DT- } \\
\text { CONR_REL_CD }\end{array}$ & $\begin{array}{l}\text { OTP-LLW-WP-01 } \\
\text { OTP-LLW-OD-01 } \\
12-A U G-97 \\
M\end{array}$ \\
\hline $\begin{array}{l}\text { CONR_FROM_PKG_ID } \\
\text { CONR_TO_PKG_ID } \\
\text { CONR_DT } \\
\text { CONR_REL_CD }\end{array}$ & $\begin{array}{l}\text { OTP-LLW-WP-02 } \\
\text { OTP- LLW-OD-02 } \\
\text { O5-JAN- } 97 \\
M\end{array}$ \\
\hline $\begin{array}{l}\text { CONR_FROM_PKG_ID } \\
\text { CONR_TO_PKG_ID } \\
\text { CONR_DT } \\
\text { CONR_REL_CD }\end{array}$ & $\begin{array}{l}\text { OTP-LLW-WP-03 } \\
\text { OTP-LLH-OD-03 } \\
\text { O5-JAN-97 } \\
M\end{array}$ \\
\hline $\begin{array}{l}\text { CONR_FROM_PKG_ID } \\
\text { CONR_TO_PKG_ID } \\
\text { CONR_DT_ } \\
\text { CONR_REL_CD }\end{array}$ & $\begin{array}{l}\text { OTP-LLW-WP-04 } \\
\text { OTP-LLW-OD-04 } \\
\text { O5-JAN-97 } \\
\text { M }\end{array}$ \\
\hline $\begin{array}{l}\text { CONR_FROM_PKG_ID } \\
\text { CONR_IO_PKG_ID } \\
\text { CONR_DT } \\
\text { CONR_REL_CD }\end{array}$ & $\begin{array}{l}\text { OTP6-97-300001 } \\
\text { OTP6-97-400001 } \\
\text { 13-AUG-27 } \\
\text { o }\end{array}$ \\
\hline $\begin{array}{l}\text { RADMAT_ID } \\
\text { RADMAT_DESCR. } \\
\text { RADMAT_LIMIT } \\
\text { RADMAT_RAD_TOT } \\
\text { RADMAT_UN1TS } \\
\text { RADMAT_ALARM }\end{array}$ & $\begin{array}{l}\text { F } \\
\text { WRAP } 1 \text { FACILITY } \\
100 \\
10 \\
\text { CI } \\
\text { N }\end{array}$ \\
\hline $\begin{array}{l}\text { RADMAT_ID } \\
\text { RADMAT_DESCR } \\
\text { RADMAT_LIMIT } \\
\text { RADMAT_RAD_TOT } \\
\text { RADMAT_UNITS } \\
\text { RADMAT_ALARM }\end{array}$ & $\begin{array}{l}\text { R } \\
\text { TRU RWM GLOVEBOX } \\
200 \\
10 \\
\text { FGE } \\
\text { N }\end{array}$ \\
\hline $\begin{array}{l}\text { RADMAT_ID } \\
\text { RADMAT_DESCR } \\
\text { RADMAT_LIMIT } \\
\text { RADMAT_RAD_TOT } \\
\text { RADMAT_UNITS } \\
\text { RADMAT_ALARM }\end{array}$ & $\begin{array}{l}\text { T } \\
\text { TRU GLOVEBOX } \\
200 \\
10 \\
\text { FGE } \\
N\end{array}$ \\
\hline $\begin{array}{l}\text { MSGLOG_DT } \\
\text { MSGLOG_ERROR FLAG } \\
\text { MSGLOG_GEN_TYPE } \\
\text { MSGLOG_SEQ_NUM } \\
\text { MSGLOG_SPEC_TYPE } \\
\text { MSGLOG_STRING1 }\end{array}$ & 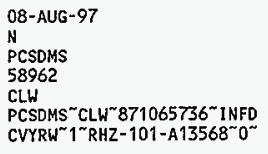 \\
\hline $\begin{array}{l}\text { MSGLOG_DT } \\
\text { MSGLOG_ERROR_FLAG } \\
\text { MSGLOG_GEN_TYYPE } \\
\text { MSGLOG_SEQ_NUM } \\
\text { MSGLOG_SPEC_TYPE } \\
\text { MSGLOG_STRINGI }\end{array}$ & 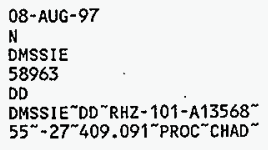 \\
\hline $\begin{array}{l}\text { MSGLOG_DT } \\
\text { MSGLOG_ERROR_FLAG } \\
\text { MSGLOG_GEN_TYPE } \\
\text { MSGLOG_SEQ_NUM } \\
\text { MSGLOG_SPECTIYPE } \\
\text { MSGLOG_STRING1 }\end{array}$ & $\begin{array}{l}\text { 08-AUG-97 } \\
\text { N } \\
\text { PCSDMS } \\
58970 \\
\text { CLW } \\
\text { PCSDMS CLW } 871065860 \sim 1 \text { HFD } \\
\text { CVYRW 1 RHZ-103-A13561 } 48\end{array}$ \\
\hline $\begin{array}{l}\text { MSGLOG_DT } \\
\text { MSGLOG_ERROR_FLAG } \\
\text { MSGLOG_GEN_TYPE } \\
\text { MSGLOG_SEQ_NUM }\end{array}$ & $\begin{array}{l}\text { O8-AUG-97 } \\
N \\
\text { DMSS!E } \\
5897 !\end{array}$ \\
\hline
\end{tabular}

MSGLOG_SPEC_TYPE MSGLOG_STRINTG

MSGLOG_DT

MSGLOG ERROR FLAG

MSGLOG_GEN_TYYPE

MSGLOG SEQ NUM

MSGLOG SPEC TYPE

MSGLOG_STRIN̄G1

MSGLOG DT

MSGLOG ERROR FLAG

MSGLOG_GEN_TȲPE

MSGLOG SEQ NUM

MSGLOG SPEC TYPE

MSGLOG_STRINGI

MSGLOG OT

MSGLOG ERROR FLAG

MSGLOG_GEN_TYYPE

MSGLOG SEQ NUM

MSGLOG SPE $\bar{C}$ TYPE

MSGLOG_STRINGTI

MSGLOG DT

MSGLOG_ERROR FLAG

MSGLOG GEN TYYPE

MSGLOG SEQ NUM

MSGLOG_SPE $\bar{C}$ TYPE

MSGLOG_STRIN̄GI

MSGLOG DT

MSGLOG ERROR FLAG

MSGLOG_GEN_TYYPE

MSGLOG SEQ NUM

MSGLOG SPEC TYPE

MSGLOG_STRIN̄G1

MSGLOG DT

MSGLOG ERROR FLAG

MSGLOG GEN TYPE

MSGLOG SEQ NUM

MSGLOG SPEC TYPE

MSGLOG STRINTE1

\section{MSGLOG DT}

MSGLOG_ERROR FLAG

MSGLOG_GEN_TȲPE

MSGLOG SEQ NUM

MSGLOG_SPEC TYPE

MSGLOG_SIRING 1

MSGLOG OT

MSGLOG ERROR FLAG

MSGLOG_GEN_TYYPE

MSGLOG SEQ NUM

MSGLOG SPE $\bar{C}$ TYPE

MSGLOGSTRING!

MSGLOG DT

MSGLOG ERROR FLAG MSGLOG GEN TYYPE MSGLOG SEQ NUM MSGLOG_SPEC MSGLOG STRING 1
DD

DMSSIE DD RHZ-103-A13561 $55^{\sim} 21.35^{\sim} 409.091^{\sim}{ }^{\sim P R O C}{ }^{\sim} \mathrm{CHA}^{\circ}$

08-AUG-97

$Y$

PCSDMS

59112

$\mathrm{CL}$

PCSDMS ${ }^{\sim} \mathrm{CL} \sim 871069005$ NDE_B $\sim 1 \sim$ COLDRUN-BACK 1 1

08-AUG-97

$\mathrm{N}$

PCSDMS

59208

$\mathrm{CL}$

PCSDMS $\%$ CL 871071150 AGVPA NC $A^{\sim \sim} 1^{\sim}$ RHZ - 101-A13568

08-AUG-97

$N$

DMSRSIE

59209

SDP

DMSRSIE SOP RHZ-101-A1356

$8^{-1} A^{*}$

08-AUG-97

N

PCSDMS

59228

CL

PCSDMS CL 871071546 AGVPA NC $A^{\sim} 1 \sim R H Z-101-A 13568^{\sim}$

08-AUG-97

N

DMSRSIE

59229

SDP

DMSRSIE SDP RHZ-101-A1356

$8^{\sim} A^{2}$

08-AUG-97

N

PCSDMS

59282

$\mathrm{Cl}$.

PCSDMS ${ }^{\sim} \mathrm{CL}^{\sim} 871072652^{\sim}$ AGVPA NC_A $A^{\sim} 1 \sim R H Z-101-A 13568 \sim$

08-AUG-97

$N$

DMSRSIE

59283

SDP

DMSRSIE SDP RHZ- 101-A1356

$8^{2} A^{2}$

08*AUG-97

$N$

PCSDNS

59318

CL

PCSDMS CL 871073307 AGVPA NC $A^{\sim \sim 1 \sim R H Z-101-A 13568 ~}$

08-AUG-97

N

DMSRSIE

59319

SDP

DMSRSIE SDP RHZ-101-A1356

$8^{\sim} A^{*}$ 


\begin{tabular}{|c|c|}
\hline $\begin{array}{l}\text { MSGLOG_DT } \\
\text { MSGLOG_ERROR_FLAG } \\
\text { MSGLOG_GEN_TYPE } \\
\text { MSGLOG_SEQ_NUM } \\
\text { HSGLOG_SPEC_TYPE } \\
\text { MSGLOG_STRINGT }\end{array}$ & 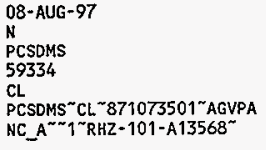 \\
\hline $\begin{array}{l}\text { MSGLOG_DT } \\
\text { MSGLOG_ERROR_FLAG } \\
\text { MSGLOG_GEN_TYPE } \\
\text { MSGLOG_SEQ_NUM } \\
\text { MSGLOG_SPE } \bar{C} \text { TYPE } \\
\text { MSGLOG_SIRINGGT }\end{array}$ & $\begin{array}{l}\text { 08-AUG- } 97 \\
\text { N } \\
\text { OMSRSIE } \\
59335 \\
\text { SDP } \\
\text { DMSRSIE }{ }^{\sim} \text { SDP RHZ-101-A1356 }^{\sim} \text { 8 }^{\sim}\end{array}$ \\
\hline $\begin{array}{l}\text { MSGLOG_OT } \\
\text { MSGLOG_ERROR_FLAG } \\
\text { MSGLOG_GEN_TYYPE } \\
\text { MSGLOG_SEQ_NUM } \\
\text { MSGLOG_SPEC TYPE } \\
\text { MSGLOG_STRING }\end{array}$ & 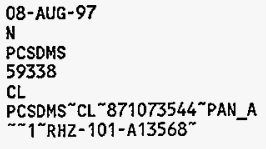 \\
\hline $\begin{array}{l}\text { MSGLOG_DT } \\
\text { MSGLOG_ERROR_FLAG } \\
\text { MSGLOG_GEN_TYYPE } \\
\text { MSGLOG_SEQ_NUM } \\
\text { MSGLOG_SPEC̄_TYPE } \\
\text { MSGLOG_STRINGG1 }\end{array}$ & 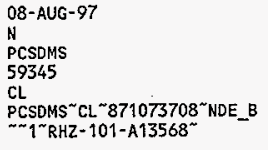 \\
\hline $\begin{array}{l}\text { MSGLOG_DT } \\
\text { MSGLOG_ERROR_FLAG } \\
\text { MSGLOG_GEN_TYPE } \\
\text { MSGLOG_SEQ_NUM } \\
\text { MSGLOG_SPEC.TYPE } \\
\text { MSGLOG_STRING1. }\end{array}$ & $\begin{array}{l}\text { 08-AUG-97 } \\
\text { N } \\
\text { PCSDMS } \\
59392 \\
\text { CL } \\
\text { PCSOMS CL } \sim 871074731 \sim L L W E \\
\text { NTRY } \sim 1 \sim O T P-L L W-O D-01 \sim\end{array}$ \\
\hline $\begin{array}{l}\text { MSGLOG_DT } \\
\text { MSGLOG_ERROR_FLAG } \\
\text { MSGLOG_GEN_TYPE } \\
\text { MSGLOG_SEQ_NUM } \\
\text { MSGLOG_SPEC̄_TYPE } \\
\text { MSGLOG_STRING }\end{array}$ & $\begin{array}{l}08-A U G-97 \\
N \\
\text { DMSPCS } \\
59393 \\
\text { CL. } \\
\text { DMSPCS } \sim C^{\sim} \sim \text { LL_ENTRY } 1 \sim 0 T P \\
\text {-LLW-WP-01 }\end{array}$ \\
\hline $\begin{array}{l}\text { MSGLOG_DT } \\
\text { MSGLOG_ERROR_FLAG } \\
\text { MSGLOG_GEN_TYPE } \\
\text { MSGLOG_SEQ_NUM } \\
\text { MSGLOG_SPEC TYPE } \\
\text { MSGLOG_STRINGI }\end{array}$ & $\begin{array}{l}08-A U G-97 \\
N \\
\text { PCSOMS } \\
59400 \\
C L \\
\text { PCSDMS } \\
\text { AC_B } \text { B }^{\sim \sim} 1^{\sim} \text { RHZ- } 871074842^{\sim} \text { AGVGE }\end{array}$ \\
\hline $\begin{array}{l}\text { MSGLOG_DT } \\
\text { MSGLOG_ERROR_FLAG } \\
\text { MSGLOG_GEN_TYYPE } \\
\text { MSGLOG_SEQ_NUM } \\
\text { MSGLOG_SPEC_TYPE } \\
\text { MSGLOG_STRINGI }\end{array}$ & $\begin{array}{l}\text { 08-AUG-97 } \\
\text { N } \\
\text { DMSRSIE } \\
59401 \\
\text { SDG } \\
\text { DMSRSIE-SDG RHZ-101-A1356 } \\
8^{\sim} B^{\sim}\end{array}$ \\
\hline $\begin{array}{l}\text { MSGLOG_DT } \\
\text { MSGLOG_ERROR_FLAG } \\
\text { MSGLOG_GEN_TYPE } \\
\text { MSGLOG_SEQ_NUM } \\
\text { MSGLOG_SPEC̄TYPE } \\
\text { MSGLOG_STRINTG1 }\end{array}$ & 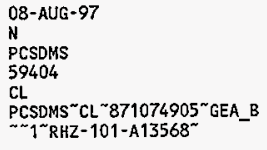 \\
\hline $\begin{array}{l}\text { MSGLOG_DT } \\
\text { MSGLOG_ERROR_FLAG } \\
\text { MSGLOG_GEN_TYPE } \\
\text { MSGLOG_SEQ_NUM } \\
\text { MSGLOG_SPEC_TYPE }\end{array}$ & $\begin{array}{l}\text { O8-AUG }-97 \\
Y \\
\text { PCSDMS } \\
59413 \\
\text { CL }\end{array}$ \\
\hline
\end{tabular}

\section{MSGLOG_STRING1 \\ MSGLOG DT MSGLOG ERROR FLAG MSGLOG_GEN TYYPE MSGLOG_SEQ NUM MSGLOG_SPEC_TYPE MSGLOG_STRING 1}

MSGLÓG DT

MSGLOG_ERROR_FLAG MSGLOG_GEN_TYTPE

MSGLOG SEQ NUM MSGLOG_SPEC _TYPE MSGLOG_STRIN̄G1

MSGLOG DT

MSGLOG_ERROR_FLAG

MSGLOG GEN TYYPE

MSGLOG SEQ NUM

MSGLOG_SPEE TYPE

MSGLOG_STRIN̄GI

MSGLOG DT

MSGLOG_ERROR_FLAG

MSGLOGGEN TYYPE

MSGLOG SEQ NUM

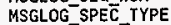

MSGLOG_STRINGI

MSGLOG DT

MSGLOG_ERROR_FLAG

MSGLOG_GEN_TYPE

MSGLOG SEQ NUM

MSGLOG SPEC TYPE

MSGLOG_STRINGGI

MSGLOG DT

MSGLOG ERROR FLAG

MSGLOG_GEN_TYPPE

MSGLOG SEQ NUM

MSGLOG SPEC TYPE

MSGLOG_STRIN̄G1

MSGLOG DT

MSGLOG ERROR FLAG

MSGLOG_SEQ NÜM

MSGLOG_STRINNGI

MSGLOG DT

MSGLOG ERROR FLAG

MSGLOG GEN TYPE

MSGLOG_SEQ NUM

MSGLOG SPEC TYPE

MSGLOG_STRING 1

MSGLOG DT

MSGLOG ERROR FLAG

MSGLOG GEN TYPE

MSGLOG SEQ NUM

MSGLOG SPEC TYPE

MSGLLOG_STRINTG1

MSGLOG_DT
PCSDMS CL $\% 871075070$ AS_RS “435 1 EMPTY101

08-AUG-97

$Y$

PCSDMS

59421

$\mathrm{CL}$

PCSOMS ${ }^{2}$ CL $871075242 \% A S$ RS “935 1 1"EMPTYO99"

08-AUG-97

$\mathrm{N}$

PCSDMS

59427

CL

PCSDMS $\approx C L$ \%871075316 AGVPA NC_A $\sim \sim 1 \sim R H Z-103-A 13561 \%$

08-AUG-97

$\mathrm{N}$

DMSRSIE

59428

SDP

DMSRSIE SDP $\sim R H Z-103-A 1356$

$1 \sim A^{*}$

08-AUG-97

N

PCSDMS

59431

CL

PCSOMS"CL $871075371^{\sim P A N}$ _A

$\sim \sim$ 1 RHZ-103-A13561

08-AUG-97

$Y$

SIEDMS

59444

RESS

SIEOMS ${ }^{R E S S}$ "RHZ-101-A 1356

$8^{\sim} T 3080054540^{\sim} T^{\sim} L L W^{\sim \sim} 0^{-8} 8$.

08-AUG-97

$Y$

DMSPCS

59445

SDMS

DMSPCS ${ }^{2}$ SOMS $00400000 \% 1012$

ERROR ORA-1400 SEQH $=5944$

08-AUG-97

$Y$

59446

O LONG RAW DATA ELEHENT

(msglog_string2) HAS

ENTIR

08-ALIG-97

N

PCSOMS

59488

CL

PCSDHS CL 871076500 AGVGE AC_A $1 \sim R H Z-103-A 13561^{\sim}$

08-AUG-97

N

DMSRSIE

59489

SDG

DMSRSIE* SDG ${ }^{\sim}$ RHZ - 103-A1356

$1 \sim A^{*}$

08-AUG-97 
CASE $4 X$

$H W W-4 * .814$ LLW GLOVEBOX OTP DMS DATABASE PRIOR TO THE FOUR FEED DRUMS GOING TO THE SUPERCOMPACTOR.

\begin{tabular}{|c|c|}
\hline $\begin{array}{l}\text { CON PKG ID } \\
\text { CON_CNTYP_CD } \\
\text { CON_PKG_STATUS } \\
\text { CON_SIZE_DESCR } \\
\text { CON_TARE_WGT }\end{array}$ & $\begin{array}{l}\frac{O T P-L L W-O D-01}{D M} \\
\$ 85 \text { GALLON } \\
31\end{array}$ \\
\hline $\begin{array}{l}\text { CONEXT_PKG_ID } \\
\text { CONEXT_USE_CD } \\
\text { CONEXT_WRAP_STAT_CD }\end{array}$ & $\frac{O T P}{\frac{C W V}{I}}$ \\
\hline $\begin{array}{l}\text { CONR_FROM_PKG_ID } \\
\text { CONR_TO_PKG_ID } \\
\text { CONR_DT } \\
\text { CONR_REL_CD }\end{array}$ & $\begin{array}{l}\text { OTP-LLW-WP-01 } \\
\text { OTP-LLW-OD-01 } \\
08-12-97 \quad 10: 08=07 \\
\mathrm{M}\end{array}$ \\
\hline $\begin{array}{l}\text { CON_PKG_ID } \\
\text { CON_CNTYP_CD } \\
\text { CON_PKG_STATUS } \\
\text { CON_SIZE_DESCR } \\
\text { CON_TARE_WGT }\end{array}$ & $\begin{array}{l}\frac{O T P-L L W-O D-02}{D M} \\
0 \\
85 \text { GALLON } \\
31\end{array}$ \\
\hline $\begin{array}{l}\text { CONEXT_PKG_ID } \\
\text { CONEXT_CONTAM_FLAG } \\
\text { CONEXT_USE_CD } \\
\text { CONEXT_WRAP_STAT_CD }\end{array}$ & $\frac{\text { OTP-LLW-00-02 }}{\frac{Y}{\mathrm{I}}}$ \\
\hline $\begin{array}{l}\text { CON PKG ID } \\
\text { CON_CNTYP_CD } \\
\text { CON_PKG_STATUS } \\
\text { CON_SIZE_DESCR } \\
\text { CON_TARE_WGT. }\end{array}$ & $\begin{array}{l}\text { OIP-LLW-OD-03 } \\
08 \\
85 \text { GALLON } \\
31\end{array}$ \\
\hline $\begin{array}{l}\text { CONEXT_PKG_ID } \\
\text { CONEXT_USE_CD } \\
\text { CONEXT_WRAP_STAT_CD }\end{array}$ & ${ }_{\text {WIP }}^{\text {OTPLW-OD-03 }}$ \\
\hline $\begin{array}{l}\text { CON PKG ID } \\
\text { CON_CNTYP_CO } \\
\text { CON_PKG_STATUS } \\
\text { CON_SIZE_DESCR } \\
\text { CON_TARE_WGT }\end{array}$ & $\begin{array}{l}\frac{O T P+L L W-O D-04}{D M} \\
\frac{R}{85} \text { GALLON } \\
31\end{array}$ \\
\hline $\begin{array}{l}\text { CONEXT_PKG_ID } \\
\text { CONEXT_USE_CD } \\
\text { CONEXT_WRAP_STAT_CD }\end{array}$ & $\begin{array}{l}\text { OIP-LLW-OD-04 } \\
\text { (WV) }\end{array}$ \\
\hline $\begin{array}{l}\text { CONR_FROM_PKG ID } \\
\text { CONR_TO_PKG_IJ } \\
\text { CONR_DT } \\
\text { CONR_REL_CD }\end{array}$ & $\begin{array}{l}\text { OTP-LLW-WP-04 } \\
\text { OTP-LLW-OD-04 } \\
08-14-97 \quad 09: 07: 47 \\
M\end{array}$ \\
\hline $\begin{array}{l}\text { NDE_PKG_ID } \\
\text { NDE_DT } \\
\text { NDE_COMMENTS } \\
\text { NDE_OPER_ID } \\
\text { NDE_VDISK_FILE } \\
\text { NDE_VDISK_NUM } \\
\text { NDE_VTAPE_NUM } \\
\text { NDE_VTAPE_START }\end{array}$ & $\begin{array}{l}\text { OTP-LLW-0D-04 } \\
09-12-96 \quad 13: 11: 35 \\
\text { OTP-LLH-WP-04 } 1 \text { ST IMAGE } \\
\text { COMMENTS } \\
\text { USER1 } \\
\text { WP08X } \\
\text { P22V1 } \\
\text { P22T1 } \\
200\end{array}$ \\
\hline $\begin{array}{l}\text { NDE_PKG_ID } \\
\text { NDE_DT } \\
\text { NDE_COMMENTS } \\
\text { NDE_OPER_ID } \\
\text { NDE_VDISK_FILE } \\
\text { NDE_VDISK_NUM } \\
\text { NDE_VTAPE_NUM } \\
\text { NDE_VTAPE_START }\end{array}$ & $\begin{array}{l}\text { OTP-LLW-00-04 } \\
09-12-96 \quad 13: 12: 10 \\
\text { OTP-LLW-WP-04 2ND IMAGE } \\
\text { COMMENTS } \\
\text { USER1 } \\
\text { WP08Y } \\
\text { P22V1 } \\
\text { P22T1 } \\
250\end{array}$ \\
\hline
\end{tabular}

CON_PKG ID
CON_ACCUM_DT
CON_CHEM_NATURE_CD
CON_CNTR_VOL
CON_CNTYP_CD
CON_GENER_WASTE_DESCR
CON_GGRP_ID
CON_GROSS_WGT
CON_LABPACK_FLAG
CON_LOCN_FACIL_ID
CON_PHYS_STATE_CD
CON_PKG_DT
CON_PKG_STATUS
CON_PWTYP_CD
CON_SAMPLE_FLAG
CON_SCAT_CD
CON_SIZE_DESCR
CON_SRCE_CHR_CD
CON_SRCE_CMPNY_ID
CON_SRCE_CMPNY_TYPE
CON_SRCE_FACIL_ID
CON_TARE_WGT _
CON_TSD_ACCEPT_DT
CON_WASTE_WGT -
CONEXT_PKG ID

CONEXT_COMPLIANT_FLAG

CONEXT NDE VER FLAG

CONEXT PROF FLA $\bar{F}$

CONEXT_PROF_ID

CONEXT USE CD

CONEXT_WRAP्P_STAT_CD

HDET_PKG_ID
HDET_CNTR_STATUS
HDET_DESI $\bar{G}$ CD
HDET_DW NUM
HDET_WASTE_VOL.
HDET_WASTE_STATUS
RDET PKG ID

RDET BG DOSE RATE

RDET HAÑDLING

RDET RELOC DT

RDET RSWIMS COUNT

RDET SDAR APPRV NUM

RDET SWIMS CD

RDET_SWTYP_CD

RDET SWTYP-GROUP

RDET THERMÄL POWER

RDET_TOT_BG_CI

RDET TOT DE CI

RDET_WAST̄E_CAT

RDET WASTE MAKEUP

RDET_WRAP_CAT

HAZ_PKG_ID
HAZ_COMP_ID
HAZ_COMP_TEXT
HAZ_COMP_HGT
HAZ_COMP_WGT_PCT
HAZ_EPCRA_FLAAG
HAZ_PKG_ID
HAZ_COMP__ID
HAZ_COMP_TEXT
HAZ_COMP_WGT
HAZ_COMP_WGT_PCT
HAZ_EPCRA_FLAG
PHYS_PKG_ID

OTP-LLW-WP- 01

10-19-93 00:00:00

1

.21

DM

LOW LEVEL WASTE

ANL

152.7

2336W

5

10-19-93 00:00:00

$\$$

$Y$

55 GALLON

TH233

ANL

GEN

ARGON

27.7

02-27-95 00:00:00

125

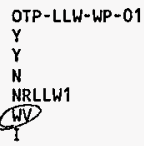

OTP-LLW-WP-01

RE

D008 D009

.208

u

OTP-LLW-WP-01

c 1

09-01-96 00:00:00

1

$60-1 R-3 Y M-0401$

DD

$2 A$

LLW

.00000254

.0000097

WC1

$F$

So

OTP-LLW-WP-01

7439-97-6

MERCURY

.001

.0009

N

OTP-LLW-WP-01

TEMP 1985

LEAD

.085

.068

N

OTP-LLW-WP-01 


\begin{tabular}{|c|c|}
\hline $\begin{array}{l}\text { PHYS_COMP_DESCR } \\
\text { PHYS_COMP_VOL_PCT } \\
\text { PHYS_COMP_WGT }\end{array}$ & $\begin{array}{l}\text { DIRT/SOIL/DIATOMACEOUS } \\
\text { EARTH } \\
86 \\
115\end{array}$ \\
\hline $\begin{array}{l}\text { PHYS_PKG_ID } \\
\text { PHYS_COMP_DESCR } \\
\text { PHYS_COMP_VOL_PCT } \\
\text { PHYS_CONP_WGT }\end{array}$ & $\begin{array}{l}\text { OTP-LLW-WP-01 } \\
\text { HAZARDOUS CONSTITUENTS } \\
: 5 \\
0\end{array}$ \\
\hline $\begin{array}{l}\text { PHYS_PKG_ID } \\
\text { PHYS_COMP_DESCR } \\
\text { PHY_COMP_VOL_PCT } \\
\text { PHYS_COMP_WGT }\end{array}$ & $\begin{array}{l}\text { OTP-LLW-WP-01 } \\
\text { PAPER/CARDBOARD } \\
9.5 \\
5\end{array}$ \\
\hline $\begin{array}{l}\text { PHYS_PKG_ID } \\
\text { PHYS_COMP_DESCR } \\
\text { PHYS_CONP_VOL_PCT } \\
\text { PHYS_CONP_WGT }\end{array}$ & $\begin{array}{l}\text { OTP-LLW-WP-01 } \\
\text { PLASTIC/POLYURATHANE } \\
4 \\
5\end{array}$ \\
\hline $\begin{array}{l}\text { POW_PKG_ID } \\
\text { PDW_NUN } \\
\text { PDW_LANDBAN } \\
\text { PDW_SORT_ORDER }\end{array}$ & $\begin{array}{l}\text { OTP-LLW-WP-01 } \\
\text { D008 } \\
Y \\
808\end{array}$ \\
\hline $\begin{array}{l}\text { POW_PKG_ID } \\
\text { POW_NUM } \\
\text { POW_LANDBAN } \\
\text { POW_SORT_ORDER }\end{array}$ & $\begin{array}{l}\text { OTP-LLLW-WP-01 } \\
\text { D009 } \\
\text { Y } \\
809\end{array}$ \\
\hline $\begin{array}{l}\text { RAD_PKG_ID } \\
\text { RAD_ISO_NUM } \\
\text { RAD_QTY }\end{array}$ & $\begin{array}{l}\text { OTP-LLW-WP-01 } \\
104 \\
.00000788\end{array}$ \\
\hline $\begin{array}{l}\text { RAD_PKG_ID } \\
\text { RAD_ISO_NUM } \\
\text { RAD_QTY }\end{array}$ & $\begin{array}{l}\text { OTP-LLW-WP-01 } \\
18 \\
11\end{array}$ \\
\hline $\begin{array}{l}\text { RAD_PKG_ID } \\
\text { RAD_ISO_NUM } \\
\text { RAD_QTY }\end{array}$ & $\begin{array}{l}\text { OTP-LLW-WP-01 } \\
19 \\
.00000254\end{array}$ \\
\hline $\begin{array}{l}\text { RAD_PKG_ID } \\
\text { RAD_ISO_NUM } \\
\text { RAD_QTY }\end{array}$ & $\begin{array}{l}\text { OTP-LLW-WP-01 } \\
202 \\
.0013\end{array}$ \\
\hline $\begin{array}{l}\text { RAD_PKG_ID } \\
\text { RAD_ISO_NUM } \\
\text { RAD_QTY }\end{array}$ & $\begin{array}{l}\text { OTP-LLW-WP-01 } \\
203 \\
.19\end{array}$ \\
\hline $\begin{array}{l}\text { RAD_PKG_ID } \\
\text { RAD_ISO_NUM } \\
\text { RAD_QTY }\end{array}$ & $\begin{array}{l}\text { OTP-LLW-WP-01 } \\
206 \\
25\end{array}$ \\
\hline $\begin{array}{l}\text { RAD_PKG_ID } \\
\text { RAD_ISO_NUM } \\
\text { RAD_QTY }\end{array}$ & $\begin{array}{l}\text { OTP-LLW-WP- } 01 \\
86 \\
.00000254\end{array}$ \\
\hline $\begin{array}{l}\text { CONR_FROM_PKG_ID } \\
\text { CONR_TO_PKG_ID } \\
\text { CONR_DT } \\
\text { CONR_REL_CD }\end{array}$ & $\begin{array}{l}\text { OTP-LLW-WP-01 } \\
\text { OTP-LLW-OD-01 } \\
08-12-97 \text { 10:08:07 } \\
M\end{array}$ \\
\hline 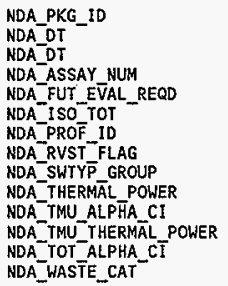 & $\begin{array}{l}\text { OTP-LLW-WP-01 } \\
\text { SEP-12-96 13:13:25 } \\
3051522805 \\
258 \\
\text { N } \\
5 \\
\text { NRLLWT } \\
\text { W } \\
\text { LLW } \\
3.5 \\
0 \\
.035 \\
0 \\
\text { WC1 }\end{array}$ \\
\hline
\end{tabular}

\begin{tabular}{|c|c|}
\hline $\begin{array}{l}\text { NDAISO_PKG_ID } \\
\text { NDAISO_DT } \\
\text { NDAISO_NAME } \\
\text { NDAISO_ASSAY_NUH } \\
\text { NDAISO_QTY } \\
\text { NDAISO_QTY_TMU } \\
\text { NDAISO_CONFIDENCE_CK } \\
\text { NDAISO_MEAS_STATUS } \\
\text { NDAISO_PAN_ACTIVE } \\
\text { NDAISO_PAN_PASSIVE } \\
\text { NDAISO_GEA }\end{array}$ & $\begin{array}{l}\text { K-40 } \\
258 \\
.00000254 \\
0000000254 \\
T \\
\text { ACT } \\
\text { B } \\
\text { NO } \\
\text { THIS IS ONLY A TEST }\end{array}$ \\
\hline $\begin{array}{l}\text { NDAISO_PKG_ID } \\
\text { NDAISO_DT } \\
\text { NDAISO_NAME } \\
\text { NDAISO_ASSAY_NUM } \\
\text { NDAISO_QTY } \\
\text { NDAISO_OTY_TMU } \\
\text { NDAISO_CONFIDENCE_CK } \\
\text { NDAISO_MEAS_STATUS } \\
\text { NDAISO_PAN_ACTIVE } \\
\text { NDAISO_PAN_PASSIVE } \\
\text { NDAISO_GEA }\end{array}$ & $\begin{array}{l}\text { TH-232 } \\
258 \\
11 \\
-11 \\
\text { J } \\
\text { ACT } \\
\text { B } \\
\text { NO } \\
\text { THIS IS ONLY A TEST }\end{array}$ \\
\hline $\begin{array}{l}\text { NDAISO_PKG_ID } \\
\text { NDAISO_DT } \\
\text { NDAISO_NAME } \\
\text { NDAISO_ASSAY_NUM } \\
\text { NDAISO_QTY } \\
\text { NDAISO_QTY_IMU } \\
\text { NDAISO_CONFIDENCE_CK } \\
\text { NDAISO_MEAS_STATUS } \\
\text { NDAISO_PAN_ACTIVE } \\
\text { NDAISO_PAN_PASSIVE } \\
\text { NDAISO_GEA }\end{array}$ & $\begin{array}{l}\text { OTP-LLW-WP-01 } \\
\text { U-234 } \\
258 \\
.0013 \\
.000013 \\
T \\
\text { ACT } \\
\text { B } \\
\text { NO } \\
\text { THIS IS ONEY A TEST }\end{array}$ \\
\hline $\begin{array}{l}\text { NDAISO_PKG_ID } \\
\text { NDAISO_DI } \\
\text { NDAISO_NAME } \\
\text { NDAISO_ASSAY_NUM } \\
\text { NDAISO_QTY } \\
\text { NDAISO_QIY_IMU } \\
\text { NDAISO_CONFIDENCE_CK } \\
\text { NDAI SO_MEAS_STATUS } \\
\text { NDAISO_PAN_ACYIVE } \\
\text { NDAISO_PAN_PASSIVE } \\
\text { NDAISO_GEA }\end{array}$ & $\begin{array}{l}\text { OTP-LLW-WP-01 } \\
\text { U-235 } \\
258 \\
.19 \\
.0019 \\
\text { ACT } \\
\text { B } \\
\text { NO } \\
\text { THIS IS ONLY A TEST }\end{array}$ \\
\hline $\begin{array}{l}\text { NDAISO_PKG_ID } \\
\text { NDAISO_DI } \\
\text { NDAISO_NAME } \\
\text { NDAISO_ASSAY_NUM } \\
\text { NDAISO_QTY } \\
\text { NDAISO_OTY_TMU } \\
\text { NDAISO_CONFIDENCE_CK } \\
\text { NDAISO_MEAS_STATUS } \\
\text { NDAISO_PAN_ACTIVE } \\
\text { NDAISO_PAN_PASSIVE } \\
\text { NDAISO_GEA }\end{array}$ & $\begin{array}{l}\text { OTP-LLW-WP-01 } \\
\text { U-238 } \\
258 \\
25 \\
: 25 \\
\text { ACT } \\
\text { B } \\
\text { NO } \\
\text { THIS IS ONLY A TEST }\end{array}$ \\
\hline $\begin{array}{l}\text { NDE_PKG_ID } \\
\text { NDE_DT } \\
\text { NDE_COMMENIS }\end{array}$ & $\begin{array}{l}\text { OTP-LLW-WP-01 } \\
09-12-96 \text { 13:05:30 } \\
\text { OIP-LLW-WP-01 1ST IMAGE }\end{array}$ \\
\hline $\begin{array}{l}\text { NDE_OPER_ID } \\
\text { NDE_VDISK_FILE } \\
\text { NDE_VDISK_NUM } \\
\text { NDE_VTAPE_NUM } \\
\text { NDE_VTAPE_START }\end{array}$ & $\begin{array}{l}\text { USER1 } \\
\text { WP05X } \\
\text { P22V1 } \\
\text { P22J1 } \\
200\end{array}$ \\
\hline $\begin{array}{l}\text { NDE_PKG_ID } \\
\text { NDE_DI } \\
\text { NDE_COMMENIS }\end{array}$ & $\begin{array}{l}\text { OTP-LLW-WP-01 } \\
\text { 09-12-96 13:06:38 } \\
\text { OTP-LLW-WP-01 2ND IMAGE } \\
\text { COMHENTS }\end{array}$ \\
\hline $\begin{array}{l}\text { NDE_OPER_ID } \\
\text { NDE_VDIS }{ }^{-} \text {_FILE } \\
\text { NDE_VDISK_NUM }\end{array}$ & $\begin{array}{l}\text { USER1 } \\
\text { WP05Y } \\
\text { P22V1 }\end{array}$ \\
\hline
\end{tabular}




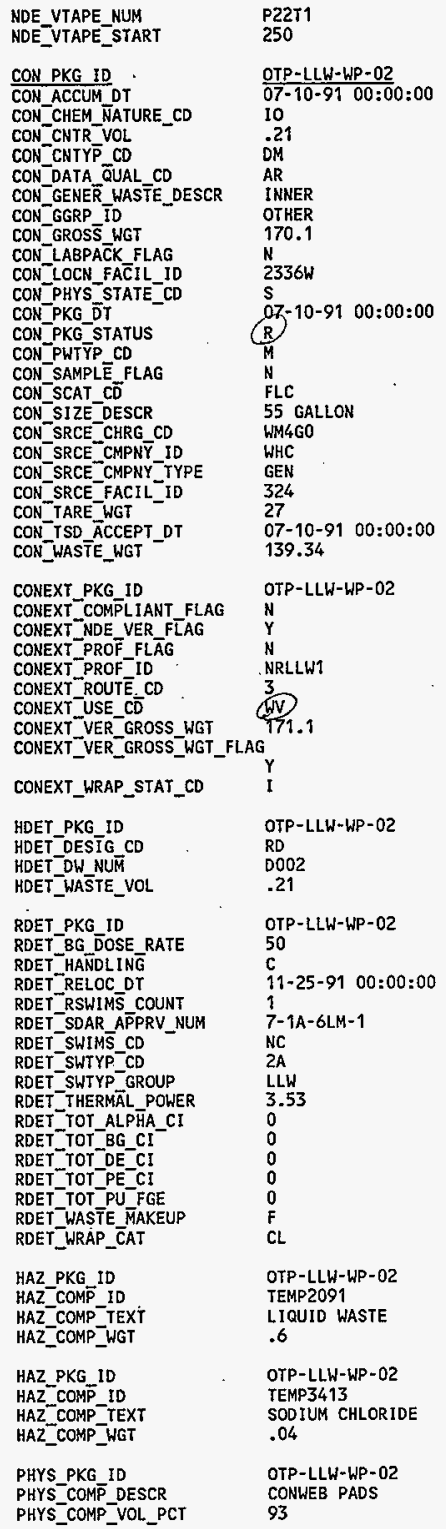

\begin{tabular}{|c|c|}
\hline PHYS_COMP_WGT & 127.5 \\
\hline $\begin{array}{l}\text { PHYS_PKG_ID } \\
\text { PHYS_COMP_DESCR } \\
\text { PHYS_COMP_VOL_PCT } \\
\text { PHYS_COMP_WGT }\end{array}$ & $\begin{array}{l}\text { OTP-LLW-WP-02 } \\
\text { HAZARDOUS CONSTITUENTS } \\
5 \\
.64\end{array}$ \\
\hline $\begin{array}{l}\text { PHYS_PKG_ID } \\
\text { PHYS_COMP_DESCR } \\
\text { PHYS_COMP_VOL_PCT } \\
\text { PHYS_COMP_WGT }\end{array}$ & $\begin{array}{l}\text { OTP-LLW-WP-02 } \\
\text { WATER } \\
2 \\
3.9\end{array}$ \\
\hline $\begin{array}{l}\text { RAD_PKG_ID } \\
\text { RAD_ISO_NUM } \\
\text { RAD_QTY }\end{array}$ & $\begin{array}{l}\text { OTP-LLW-WP-02 } \\
12 \\
.00033\end{array}$ \\
\hline $\begin{array}{l}\text { RAD_PKG_ID } \\
\text { RAD_ISO_NUM } \\
\text { RAD_QTY }\end{array}$ & $\begin{array}{l}\text { OTP-LLW-WP-02 } \\
152 \\
.000005\end{array}$ \\
\hline $\begin{array}{l}\text { RAD_PKG_ID } \\
\text { RAD_ISO_NUM } \\
\text { RAD_OTY }^{-}\end{array}$ & $\begin{array}{l}\text { OTP-LLW-WP-02 } \\
153 \\
.00018\end{array}$ \\
\hline $\begin{array}{l}\text { RAD_PKG_ID } \\
\text { RAD_ISO_NUM } \\
\text { RAD_QTY } \\
\text { RAD_UNKNOWN }\end{array}$ & $\begin{array}{l}\text { OTP-LLW-WP-02 } \\
19 \\
035405 \\
0\end{array}$ \\
\hline $\begin{array}{l}\text { RAD_PKG_ID } \\
\text { RAD_ISO_NUM } \\
\text { RAD_QTY }\end{array}$ & $\begin{array}{l}\text { OTP-LLW-WP-02 } \\
43 \\
.00045\end{array}$ \\
\hline $\begin{array}{l}\text { RAD_PKG_10 } \\
\text { RAD_ISO_NUM } \\
\text { RAD_QTY }\end{array}$ & $\begin{array}{l}\text { OTP-LLW-WP-02 } \\
53 \\
.00014\end{array}$ \\
\hline $\begin{array}{l}\text { RAD_PKG_ID } \\
\text { RAD_ISO_NUM } \\
\text { RAD_QTY }\end{array}$ & $\begin{array}{l}\text { OTP-LLW-WP-02 } \\
56 \\
.0003\end{array}$ \\
\hline $\begin{array}{l}\text { RAD_PKG_ID } \\
\text { RAD_ISO_NUM } \\
\text { RAD_QTY }^{-}\end{array}$ & $\begin{array}{l}\text { OTP-LLW-WP- } 02 \\
91 \\
.034\end{array}$ \\
\hline $\begin{array}{l}\text { CONR_FROM_PKG_ID } \\
\text { CONR_TO_PKG_ID } \\
\text { CONR_DT- } \\
\text { CONR_REL_CD }\end{array}$ & $\begin{array}{l}\text { OTP-LLW-WP-02 } \\
\text { OTP6-97-000001 } \\
08-14-27 \quad 17: 26: 29 \\
\mathbf{s}\end{array}$ \\
\hline $\begin{array}{l}\text { CONR_FROM_PKG_ID } \\
\text { CONR_TO_PKG_ID } \\
\text { CONR_DT } \\
\text { CONR_REL_CD }\end{array}$ & $\begin{array}{l}\text { OTP-LLW-WP-02 } \\
\text { OTP6-97-000002 } \\
08-14-27 \quad 17: 26: 00 \\
\text { S }\end{array}$ \\
\hline $\begin{array}{l}\text { CONR_FROM_PKG_ID } \\
\text { CONR_TO_PKG_ID } \\
\text { CONR_DT } \\
\text { CONR_REL_CD }\end{array}$ & $\begin{array}{l}\text { OTP-LLW-WP-02 } \\
\text { OTP6-97-000003 } \\
\text { 08-14-27 17:27:06 } \\
\text { s }\end{array}$ \\
\hline $\begin{array}{l}\text { NDA_PKG_ID } \\
\text { NDA_DT } \\
\text { NDA_DT } \\
\text { NDA_ASSAY_NUM } \\
\text { NDA_FUT_EVAL_REQD } \\
\text { NDA_ISO_TOT - } \\
\text { NDA_PROF_ID } \\
\text { NDA_RVST_FLAG } \\
\text { NDA_SWTYP_GROUP } \\
\text { NDA_THERMAL_POWER } \\
\text { NDA_TWU_ALPHA_CI } \\
\text { NDA_TMU_THERMAL_POWER } \\
\text { NDA_TOT_ALPHA_CI } \\
\text { NDA_WASTE_CAT }\end{array}$ & $\begin{array}{l}\text { OTP-LLW-WP-02 } \\
\text { SEP-12-96 13:47:13 } \\
3051524833 \\
265 \\
N \\
1 \\
\text { NRLLW1 } \\
N \\
\text { LLW } \\
3.53 \\
0 \\
\text { O0353 }_{0} \\
\text { GTWC3 }\end{array}$ \\
\hline
\end{tabular}

OTP-LLW-WP-02 HAZARDOUS CONSTITUENTS .64 NDAISO_DT 


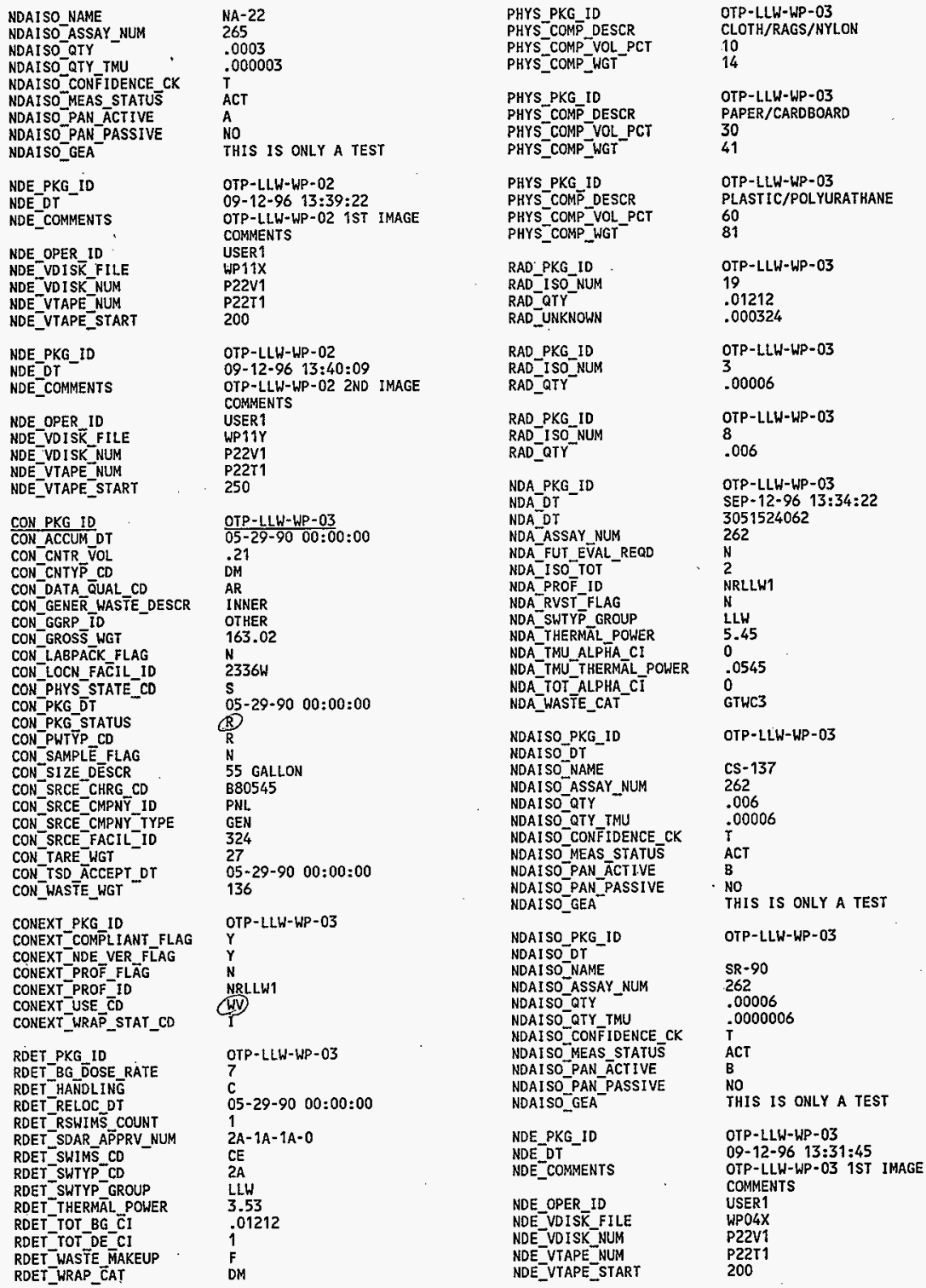




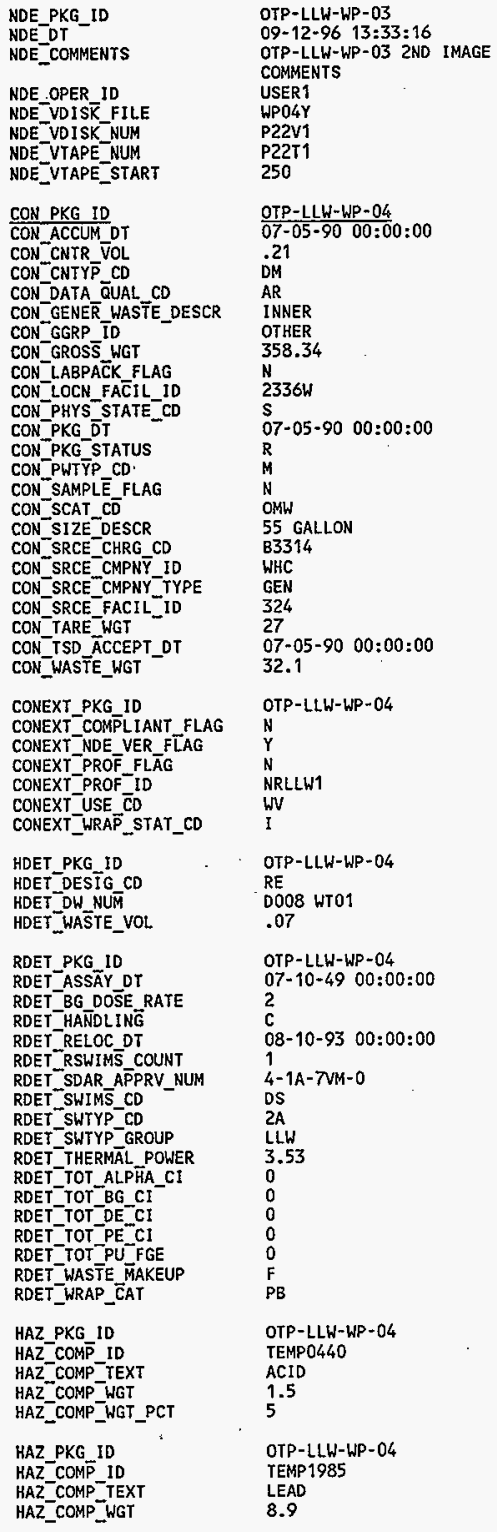

$\begin{array}{ll}\text { HAZ_COMP_WGT_PCT } & 28 \\ \text { HAZ_PKG_ID } & \text { OTP-LLW-WP-04 } \\ \text { HAZ_COMP_ID } & \text { TEMP2628 } \\ \text { HAZ_COMP_TEXT } & \text { OIL } \\ \text { HAZ_COMP_WGT } & 1.9 \\ \text { HAZ_COMP_WGT_PCT } & 6 \\ \text { HAZ_PKG_ID } & \\ \text { HAZ_COMP_ID } & \text { OTP-LLW-WP-04 } \\ \text { HAZ_COMP_TEXT } & \text { TEMP3530 } \\ \text { HAZ_COMP_WGT } & \text { SOLVENTS } \\ \text { HAZ_COMP_WGT_PCT } & 2.1 \\ \end{array}$

PHYS PKG ID OTP-LLW-WP-04

PHYS_COMP_DESCR CLOTH/RAGS/NYLON PHYS COMP VOL PCT PHYS_COMP_WGT

PHYS PKG ID PHYS COMP DESCR PHYS_COMP_VOL_PCT PHYS_COMP_WGT

PHYS PKG ID PHYS_COMP DESCR PHYS_COMP_VOL_PCT PHYS_COMP_WGT

RAD_PKG_ID
RAD_ISO_NUM
RAD_QTY

RAD_PKG_ID

RAD ISO NUM

RAD QTY

RAD_UNKNOWN

RAD_PKG_ID

RAD ISO NUM

RAD QIY

RAD_PKG ID

RAD_ISO-NUM

RAD_QTY

RAD_PKG_ID

RAD_ISO_NUM

RAD_QTY
RAD PKG ID
RAD_ISO_NUM
RAD_QTY

RAD_PKG_ID

RAD 150 NUM

RAD_QTY

RAD_PKG_ID
RAD_ISO_NUM
RAD_QTY

CONR_FROM PKG ID CONR TOPKKG_I $\vec{D}$

CONR_OT

CONR_REL_CD

CONR_FROM_PKG_ID CONR TO PKG ID

CONR DT

CONR_REL_CD

CONR_FROM_PKG_ID CONR TO PKG_ID CONR_DT CONR_REL_CD
48

13.6

OTP-LLW-WP-04 HAZARDOUS CONSTITUENTS 5

.64

OTP-LLH-UP-04 PLASTIC/POLYURATHANE 20

4.1

OTP-LLW-WP-04

13

.000004

OTP-LLW-WP-04

19

.000233

.0000007163

OTP-LLW-WP-04

31

.000032

OTP-LLW-WP-04

4

.000083

OTP-LLW-WP-04

40

.000002

OTP-LLW-WP- 04

53

.000001

OTP-LLW-WP-04

8

.000013

OTP-LLW-WP-04

9

.000001

OTP-LLW-WP-04

OTP-LLW-OD-04

08-14-97 09:07:47

M

OTP-LLW-WP-04 OTP6-97-000004 08-15-27 17:56:20 S

OTP-LLW-WP- 04 OTP6-97-000005 08-15-27 17:57:08 $\mathrm{s}$ 


\begin{tabular}{|c|c|}
\hline $\begin{array}{l}\text { CONR_FROM_PKG_ID } \\
\text { CONR_TO_PKG_ID } \\
\text { CONR_DT } \\
\text { CONR_REL_CD }\end{array}$ & $\begin{array}{l}\text { OTP-LLW-WP-04 } \\
\text { OTP6-97-000006 } \\
\text { O8-15-27 } 17: 57: 37 \\
\text { S }\end{array}$ \\
\hline $\begin{array}{l}\text { NDA_PKG_ID } \\
\text { NDA_DT } \\
\text { NDA_DT } \\
\text { NDA_ASSAY_NUM } \\
\text { NDA_FUT_EVAL_REQD } \\
\text { NDA_ISO_TOT - } \\
\text { NDA_PROF_ID } \\
\text { NDA_RVST_FLAG } \\
\text { NDA_SWTYP GROUP } \\
\text { NDA_THERMAL_POWER } \\
\text { NDA_TMU_ALPFA_CI } \\
\text { NDA_TMU_THERMAL_POWER } \\
\text { NDA_TOT_ALPHA_CI I TOT } \\
\text { NDA_WASTE_CAT }\end{array}$ & $\begin{array}{l}\text { OTP-LLW-WP-04 } \\
\text { SEP-12-96 13:18:27 } \\
3051523107 \\
261 \\
N \\
4 \\
\text { NRLLW1 } \\
N \\
\text { LLW } \\
5.45 \\
0 \\
\text {.0545 } \\
0 \\
\text { GTWC3 }\end{array}$ \\
\hline $\begin{array}{l}\text { NDAISO_PKG_ID } \\
\text { NDAISO_DT } \\
\text { NDAISO_NAME } \\
\text { NDAISO_ASSAY_NUM } \\
\text { NDAISO_QTY } \\
\text { NDAISO_QTY_THU } \\
\text { NDAISO_CONFIDENCE_CK } \\
\text { NDAISO_MEAS_STATUS } \\
\text { NDAISO_PAN_ACTIVE } \\
\text { NDAISO_PAN_PASSIVE } \\
\text { NDAISO_GEA }\end{array}$ & $\begin{array}{l}\text { CO-60 } \\
261 \\
.000004 \\
.00000004 \\
\dot{Y} \\
\text { ACT } \\
\text { A } \\
\text { NO } \\
\text { THIS IS ONLY A TEST }\end{array}$ \\
\hline $\begin{array}{l}\text { NDAISO_PKG_ID } \\
\text { NDAISO_DT } \\
\text { NDAISO_NAME } \\
\text { NDAISO_ASSAY_NUM } \\
\text { NDAISO_QTY } \\
\text { NDAISO_QTY_IMU } \\
\text { NDAISO_CONFIDENCE_CK } \\
\text { NDAISO_MEAS_SIAIUS } \\
\text { NDAISO_PAN_ACIIVE } \\
\text { NDAISO_PAN_PASSIVE } \\
\text { NDAISO_GEA }\end{array}$ & $\begin{array}{l}\text { OTP-LLW-WP-04 } \\
C S-137 \\
261 \\
=000013 \\
-00000013 \\
\text { ACT } \\
\text { A } \\
\text { NO } \\
\text { THIS IS ONLY A TEST }\end{array}$ \\
\hline $\begin{array}{l}\text { NDAISO_PKG_ID } \\
\text { NDAISO_DT } \\
\text { NDAISO_NAME } \\
\text { NDAISO_ASSAY_NUM } \\
\text { NDAISO_QTY } \\
\text { NDAISO_QTY_TMU } \\
\text { NDAISO_CONFIDENCE_CK } \\
\text { NDAISO_MEAS_STATUS } \\
\text { NDAISO_PAN_ACTIVE } \\
\text { NDAISO_PAN_PASSIVE } \\
\text { NDAISO_GEA- }\end{array}$ & $\begin{array}{l}\text { OTP-LLW-WP-04 } \\
\text { EU- } 154 \\
261 \\
.000002 \\
: 00000002 \\
\text { ACT } \\
\text { A } \\
\text { NO } \\
\text { THIS IS ONLY A TEST }\end{array}$ \\
\hline $\begin{array}{l}\text { NDAISO_PKG_ID } \\
\text { NDAISO_DT } \\
\text { NDAISO_NAME } \\
\text { NDAISO_ASSAY_NUM } \\
\text { NDAISO_QTY } \\
\text { NDAISO_QTY_TMU } \\
\text { NDAISO_CONFIDENCE_CK } \\
\text { NDAISO_MEAS_STATUS } \\
\text { NDAISO_PAN_ACTIVE } \\
\text { NDAISO_PAN_PASSIVE } \\
\text { NDAISO_GEA }\end{array}$ & $\begin{array}{l}\text { OTP-LLW-WP-04 } \\
\text { EU-155 } \\
261 \\
-000032 \\
=00000032 \\
T \\
\text { ACT } \\
\text { A } \\
\text { NO } \\
\text { THIS IS ONLY A TEST }\end{array}$ \\
\hline $\begin{array}{l}\text { CON_PKG ID } \\
\text { CON_CNTYP_CD } \\
\text { CON_LOCN_FACIL_ID } \\
\text { CON_PKG_STATUS } \\
\text { CON_SCAT_CD } \\
\text { CON_SIZE_DESCR } \\
\text { CON_TARE_WGT }\end{array}$ & $\begin{array}{l}\text { OTP-LLWR-TD-01. } \\
\text { DM } \\
2336 \mathrm{~W} \\
\mathbf{G} \\
\text { ANY } \\
85 \text { GALLON } \\
31\end{array}$ \\
\hline CONEXT_PKG_ID & OTP-LLWR-TD-01 \\
\hline
\end{tabular}

\begin{tabular}{|c|c|}
\hline $\begin{array}{l}\text { CONEXT_CNTR_STATUS } \\
\text { CONEXT_USE_CD } \\
\text { CONEXT_WRAP_STAT_CD }\end{array}$ & $\begin{array}{l}\text { P } \\
\text { TO } \\
I\end{array}$ \\
\hline $\begin{array}{l}\text { CONR_FROM_PKG_ID } \\
\text { CONR_TO_PKG_ID } \\
\text { CONR_DT } \\
\text { CONR_REL_CD }\end{array}$ & $\begin{array}{l}\text { OTP6-97-000001 } \\
\text { OTP-LLWR-TD-01 } \\
08-14-27 \quad 17: 26: 29 \\
0\end{array}$ \\
\hline $\begin{array}{l}\text { CONR_FROM_PKG_ID } \\
\text { CONR_TO_PKG_ID } \\
\text { CONR_DT } \\
\text { CONR_REL_CD }\end{array}$ & $\begin{array}{l}\text { OTP6-97-000002 } \\
\text { OTP-LLWR-TD-01 } \\
08-14-27 \quad 17: 26: 00 \\
0\end{array}$ \\
\hline $\begin{array}{l}\text { CONR_FROM_PKG_ID } \\
\text { CONR_TO_PKG_ID } \\
\text { CONR_DT } \\
\text { CONR_REL_CD }\end{array}$ & $\begin{array}{l}\text { OTP6-97-000003 } \\
\text { OTP-LLWR-TD-01 } \\
08-14-27 \quad 17: 27: 07 \\
0\end{array}$ \\
\hline $\begin{array}{l}\text { CON PKG 1D } \\
\text { CON_CNTYP_CD } \\
\text { CON_LOCN_FACIL_10 } \\
\text { CON_PKG_STATUS } \\
\text { CON_SCAT_CD } \\
\text { CON_SIZE_DESCR } \\
\text { CON_TARE_WGT }\end{array}$ & $\begin{array}{l}\text { OTP-LLWR-TD-02 } \\
\text { DM } \\
2336 \mathrm{~W} \\
\text { G } \\
\text { ANY } \\
85 \text { GALLON } \\
31\end{array}$ \\
\hline $\begin{array}{l}\text { CONEXT_PKG_ID } \\
\text { CONEXT_CNTR STATUS } \\
\text { CONEXT_USE_CD } \\
\text { CONEXT_WRAP_STAT_CD }\end{array}$ & $\begin{array}{l}\text { OTP-LLWR-TD-02 } \\
\text { P } \\
\text { TD } \\
1\end{array}$ \\
\hline $\begin{array}{l}\text { CONR_FROM_PKG_ID } \\
\text { CONR_TO_PKG_ID } \\
\text { CONR_OT } \\
\text { CONR_REL_CD }\end{array}$ & $\begin{array}{l}\text { OTP6-97-000004 } \\
\text { OTP-L.LWR-TD-02 } \\
08-15-27 \quad 17: 56: 21 \\
0\end{array}$ \\
\hline $\begin{array}{l}\text { CONR_FROM_PKG_ID } \\
\text { CONR_TO_PKG_ID } \\
\text { CONR_DT } \\
\text { CONR_REL_CD }\end{array}$ & $\begin{array}{l}\text { OTP6-97-000005 } \\
\text { OTP-LLWR-TD-02 } \\
08-15-27 \quad 17: 57: 08 \\
0\end{array}$ \\
\hline $\begin{array}{l}\text { CONR_FROM PKG_ID } \\
\text { CONR_TO_PKG_ID } \\
\text { CONR_DT } \\
\text { CONR_REL_CD }\end{array}$ & $\begin{array}{l}\text { OTP6-97-000006 } \\
\text { OTP-LLWR-TD-02 } \\
08-15-27 \quad 17: 57: 38 \\
0\end{array}$ \\
\hline$\frac{\text { CON PKG ID }}{\text { CON_GENER_WASTE_DESCR }}$ & $\begin{array}{l}\text { OTP6-97-000001 } \\
\text { AEROSOL CAN OF SALINE } \\
\text { SOLUTION }\end{array}$ \\
\hline $\begin{array}{l}\text { CONEXT_PKG_ID } \\
\text { CONEXT_MAT_GRP_CD } \\
\text { CONEXT_USE_CD }\end{array}$ & $\begin{array}{l}\text { OTP6-97-000001 } \\
\text { AC } \\
\text { PK }\end{array}$ \\
\hline $\begin{array}{l}\text { RDET_PKG_ID } \\
\text { RDET_SWTYP_CD } \\
\text { RDET_SWTYP_GROUP }\end{array}$ & $\begin{array}{l}\text { orP6-97-000001 } \\
\text { 2A } \\
\text { LLW }\end{array}$ \\
\hline $\begin{array}{l}\text { CONR_FROM_PKG_ID } \\
\text { CONR_TO_PKG_ID } \\
\text { CONR_DT } \\
\text { CONR_REL_CD }\end{array}$ & $\begin{array}{l}\text { OTP-LLW-WP-02 } \\
\text { OTP6-97-000001 } \\
08-14-27 \quad 17: 26: 29 \\
\text { S }\end{array}$ \\
\hline $\begin{array}{l}\text { CONR_FROM_PKG_ID } \\
\text { CONR_TO_PKG_ID } \\
\text { CONR_DI } \\
\text { CONR_REL_CD }\end{array}$ & $\begin{array}{l}\text { OTP6-97-000001 } \\
\text { OTP-LLWR-TD-01 } \\
08-14-27 \quad 17: 26: 29 \\
0\end{array}$ \\
\hline$\frac{\text { CON PKG ID }}{\text { CON_GENER_WASTE_DESCR }}$ & $\begin{array}{l}\text { OIP6-97-000002 } \\
\text { AEROSOL CAN OF SALINE } \\
\text { SOLUTION }\end{array}$ \\
\hline $\begin{array}{l}\text { CONEXT_PKG_ID } \\
\text { CONEXT_MAT_GRP_CO } \\
\text { CONEXT_USE_CD - }\end{array}$ & $\begin{array}{l}\text { OTP6-97-000002 } \\
\text { AC } \\
\text { PK }\end{array}$ \\
\hline
\end{tabular}




\begin{tabular}{|c|c|}
\hline $\begin{array}{l}\text { RDET_PKG_tD } \\
\text { RDET_SWTYP_CD } \\
\text { RDET_SWTYP_GROUP }\end{array}$ & $\begin{array}{l}\text { OTP6-97-000002 } \\
\text { 2A } \\
\text { LLW }\end{array}$ \\
\hline $\begin{array}{l}\text { CONR_FROM_PKG_ID } \\
\text { CONR_TO_PKG_ID } \\
\text { CONR_DT } \\
\text { CONR_REL_CD }\end{array}$ & $\begin{array}{l}\text { OTP-LLW-WP-02 } \\
\text { OTP6-97-000002 } \\
08-14-27 \quad 17=26=00 \\
\text { s }\end{array}$ \\
\hline $\begin{array}{l}\text { CONR_FROM_PKG_ID } \\
\text { CONR_TO_PKG_ID } \\
\text { CONR_DT } \\
\text { CONR_REL_CD }\end{array}$ & $\begin{array}{l}\text { OTP6-97-000002 } \\
\text { OTP-1LWR-TD-01 } \\
08-14-27 \quad 17: 26: 00 \\
0\end{array}$ \\
\hline$\frac{\text { CON PKG ID }}{\text { CON_GENER_WASTE_DESCR }}$ & $\frac{\text { OTP6-97-000003 }}{\text { AEROSOL CAN OF UNKNOWN }}$ \\
\hline $\begin{array}{l}\text { CONEXT_PKG_ID } \\
\text { CONEXT_MAT_GRP_CD } \\
\text { CONEXT_USE_CD }\end{array}$ & $\begin{array}{l}\text { OTP6-97-000003 } \\
\text { AC } \\
\text { PK }\end{array}$ \\
\hline $\begin{array}{l}\text { RDET_PKG_ID } \\
\text { RDET_SWTYP_CD } \\
\text { RDET_SWTYP_GROUP }\end{array}$ & $\begin{array}{l}\text { OTP6-97-000003 } \\
\text { 2A } \\
\text { LLW }\end{array}$ \\
\hline $\begin{array}{l}\text { CONR_FROM_PKG_ID } \\
\text { CONR_TO_PKG_ID } \\
\text { CONR_OT } \\
\text { CONR_REL_CD }\end{array}$ & $\begin{array}{l}\text { OTP-ILW-WP-02 } \\
\text { OTPS-97-000003 } \\
\text { 08-14-27 17:27:06 } \\
\text { S }\end{array}$ \\
\hline $\begin{array}{l}\text { CONR_FROM_PKG_ID } \\
\text { CONR_TO_PKG_ID } \\
\text { CONR_DT } \\
\text { CONR_REL_CD }\end{array}$ & $\begin{array}{l}\text { OTP6-97-000003 } \\
\text { OTP-LLWR-TD-01 } \\
08-14-27 \quad 17: 27: 07 \\
0\end{array}$ \\
\hline$\frac{\text { CON PKG ID }}{\text { CON_GENER_WASTE_DESCR }}$ & $\frac{\text { OTPG-97-000004 }}{\text { LEAD BRICK }}$ \\
\hline $\begin{array}{l}\text { CONEXT_PKG_ID } \\
\text { CONEXT_MAT_GRP_CD } \\
\text { CONEXT_USE_CD }\end{array}$ & $\begin{array}{l}\text { OTP6-97-000004 } \\
\text { PB } \\
\text { PK }\end{array}$ \\
\hline $\begin{array}{l}\text { RDET_PKG_ID } \\
\text { RDET_SWTYP_CD } \\
\text { RDET_SWTYP_GROUP }\end{array}$ & $\begin{array}{l}\text { OTP6-97-000004 } \\
2 A \\
\text { LLW }\end{array}$ \\
\hline $\begin{array}{l}\text { CONR_FROM_PKG_ID } \\
\text { CONR_TO_PKG_ID } \\
\text { CONR_DT } \\
\text { CONR_REL_CD }\end{array}$ & $\begin{array}{l}\text { OTP-LLW-WP-04 } \\
\text { OTP6-97-000004 } \\
08-15-27 \quad 17: 56: 20 \\
\text { s }\end{array}$ \\
\hline $\begin{array}{l}\text { CONR_FROM_PKG_ID } \\
\text { CONR_TO_PKG_ID } \\
\text { CONR_DT_- } \\
\text { CONR_REL_CD }\end{array}$ & $\begin{array}{l}\text { OTPG-97-000004 } \\
\text { OTP-LLWR-TD-02 } \\
08-15-27 \quad 17: 56: 21 \\
0\end{array}$ \\
\hline$\frac{\text { CON_PKG ID }}{\text { CON_GENER_WASTE_DESCR }}$ & $\begin{array}{l}\text { OTP6-97-000005 } \\
\text { BOTTLE OF OIL \& BOTTLE OF } \\
\text { SOLVENT }\end{array}$ \\
\hline $\begin{array}{l}\text { CONEXT_PKG_ID } \\
\text { CONEXT_MAT_GRP_CD } \\
\text { CONEXT_USE_CD }\end{array}$ & $\begin{array}{l}\text { OTP6-97-000005 } \\
\text { LQ } \\
\text { PK }\end{array}$ \\
\hline $\begin{array}{l}\text { RDET_PKG_IO } \\
\text { RDET_SWTYP_CD } \\
\text { RDET_SWTYP_GROUP }\end{array}$ & $\begin{array}{l}\text { OTP6-97-000005 } \\
2 A \\
\text { LLW }\end{array}$ \\
\hline $\begin{array}{l}\text { CONR_FROM_PKG_ID } \\
\text { CONR_TO_PKG_1D } \\
\text { CONR_DT } \\
\text { CONR_REL_CD }\end{array}$ & $\begin{array}{l}\text { OTP-LLW-WP-04 } \\
\text { OTP6-97-000005 } \\
08-15-27 \quad 17: 57: 08 \\
\text { s }\end{array}$ \\
\hline $\begin{array}{l}\text { CONR_FROM_PKG_ID } \\
\text { CONR_TO_PKG_ID } \\
\text { CONR_DT- } \\
\text { CONR_REL_CD }\end{array}$ & $\begin{array}{l}\text { OTP6-97-000005 } \\
\text { OTP-LLWR-TD-02 } \\
08-15-27 \quad 17: 57: 08 \\
0\end{array}$ \\
\hline
\end{tabular}

\begin{tabular}{|c|c|}
\hline$\frac{\text { CON PKG ID }}{\text { CON_GENER_WASTE_DESCR }}$ & $\frac{\text { OTP6-97-000006 }}{\text { BOTTLE OF ACID }}$ \\
\hline $\begin{array}{l}\text { CONEXT_PKG_ID } \\
\text { CONEXT_MAT_GRP_CD } \\
\text { CONEXT_USE_CD }\end{array}$ & $\begin{array}{l}\text { OTP6-97-000006 } \\
\text { LQ } \\
\text { PK }\end{array}$ \\
\hline $\begin{array}{l}\text { RDET_PKG_ID } \\
\text { RDET_SWTYYP_CD } \\
\text { RDET_SWTYP_GROUP }\end{array}$ & $\begin{array}{l}\text { OTP6-97-000006 } \\
\text { 2A } \\
\text { LLW }\end{array}$ \\
\hline $\begin{array}{l}\text { CONR_FROM_PKG_ID } \\
\text { CONR_TO_PKG_ID } \\
\text { CONR_DT } \\
\text { CONR_REL_CD }\end{array}$ & $\begin{array}{l}\text { OTP-LLW-WP-04 } \\
\text { OTP6-97-000006 } \\
\text { 08-15-27 } \quad 17: 57: 37 \\
\text { s }\end{array}$ \\
\hline $\begin{array}{l}\text { CONR_FROH_PKG_ID } \\
\text { CONR_TO_PKG_ID } \\
\text { CONR_DT } \\
\text { CONR_REL_CD }\end{array}$ & $\begin{array}{l}\text { OTPG-97-000006 } \\
\text { OTP-LLLR - TD-02 } \\
\text { O8-15-27 } \quad 17: 57: 38 \\
0\end{array}$ \\
\hline CON PKG_ID & 0TP6-97-300001 \\
\hline $\begin{array}{l}\text { CONEXT_PKG_ID } \\
\text { CONEXT_USE_CD }\end{array}$ & $\begin{array}{l}\text { OTP6-97-300001 } \\
\text { PP }\end{array}$ \\
\hline $\begin{array}{l}\text { CONR_FROM_PKG_ID } \\
\text { CONR_TO_PKG_ID } \\
\text { CONR_DT } \\
\text { CONR_REL_CD }\end{array}$ & $\begin{array}{l}\text { OTP6-97-300001 } \\
\text { OTP6-97-400001 } \\
08-13-27 \quad 21: 57: 45 \\
0\end{array}$ \\
\hline $\begin{array}{l}\text { CON PKG ID } \\
\text { CON_PKG_DT } \\
\text { CON_PKG_STATUS }\end{array}$ & $\frac{\text { OTP6-97-400001 }}{08-13-2721: 57: 45}$ \\
\hline $\begin{array}{l}\text { CONEXT_PKG_ID } \\
\text { CONEXT_USE_CD }\end{array}$ & $\begin{array}{l}\text { OTP6-97-400001 } \\
\text { TP }\end{array}$ \\
\hline $\begin{array}{l}\text { CONR_FROM_PKG_ID } \\
\text { CONR_TO,PKG_ID } \\
\text { CONR_DT } \\
\text { CONR_REL_CD }\end{array}$ & $\begin{array}{l}\text { OTP6-97-300001 } \\
\text { OTP6-97-400001 } \\
\text { 08-13-27 21:57:45 } \\
0\end{array}$ \\
\hline $\begin{array}{l}\text { CON_PKG ID } \\
\text { CON_ACCUM_OT } \\
\text { CON_CHEM_NATURE_CD } \\
\text { CON_CNTR_VOL } \\
\text { CON_CNTYP_CD } \\
\text { CON_GENER_WASTE_DESCR } \\
\text { CON_GGRP_ID } \\
\text { CON_GROSS_WGT } \\
\text { CON_LABPACK_FLAG } \\
\text { CON_LOCN_FACIL_ID } \\
\text { CON_PHYS_STATE_CD } \\
\text { CON_PKG_DT } \\
\text { CON_PKG_STATUS } \\
\text { CON_PWTYP_CD } \\
\text { CON_SAMPLE_FLAG } \\
\text { CON_SCAT_CD } \\
\text { CON_SEC_PKG_ID } \\
\text { CON_SIZE_DESCR } \\
\text { CON_SRCE_CHRG_CD } \\
\text { CON_SRCE_CMPNY IID } \\
\text { CON_SRCE_CMPNY_IYPE } \\
\text { CON_SRCE_FACIL_ID } \\
\text { CON_TARE_WGI } \\
\text { CON_TSD_ACCEPT_OT } \\
\text { CON_WASTE_WGT - }\end{array}$ & $\begin{array}{l}\text { PUCK278 } \\
10-19-93 \quad 00: 00: 00 \\
\text { I } \\
\text { DM } \\
\text { DM } \\
\text { LOW LEVEL WASTE } \\
\text { ANL } \\
152.7 \\
N \\
2336 W \\
S \\
10-19-93 \quad 00: 00: 00 \\
R \\
\text { M } \\
\text { Y } \\
\text { OMW } \\
\text { OTP-LLW-WP-01 } \\
55 \text { GRLLON } \\
\text { TH233 } \\
\text { ANL } \\
\text { GEN } \\
\text { ARGON } \\
27.7 \\
02-27-95 \quad 00: 00: 00 \\
125\end{array}$ \\
\hline $\begin{array}{l}\text { CON PKG ID } \\
\text { CON_CNTYP CD } \\
\text { CON_PKG_STATUS } \\
\text { CON_SEC_PKG_ID } \\
\text { CON_SIZE_DESCR } \\
\text { CON_TARE_WGT }\end{array}$ & $\begin{array}{l}\text { PUCK279 } \\
\text { DM } \\
R \\
\text { OTP-LLW-OD-01 } \\
85 \text { GALLON } \\
31\end{array}$ \\
\hline CON PKG ID & PUCK281 \\
\hline
\end{tabular}




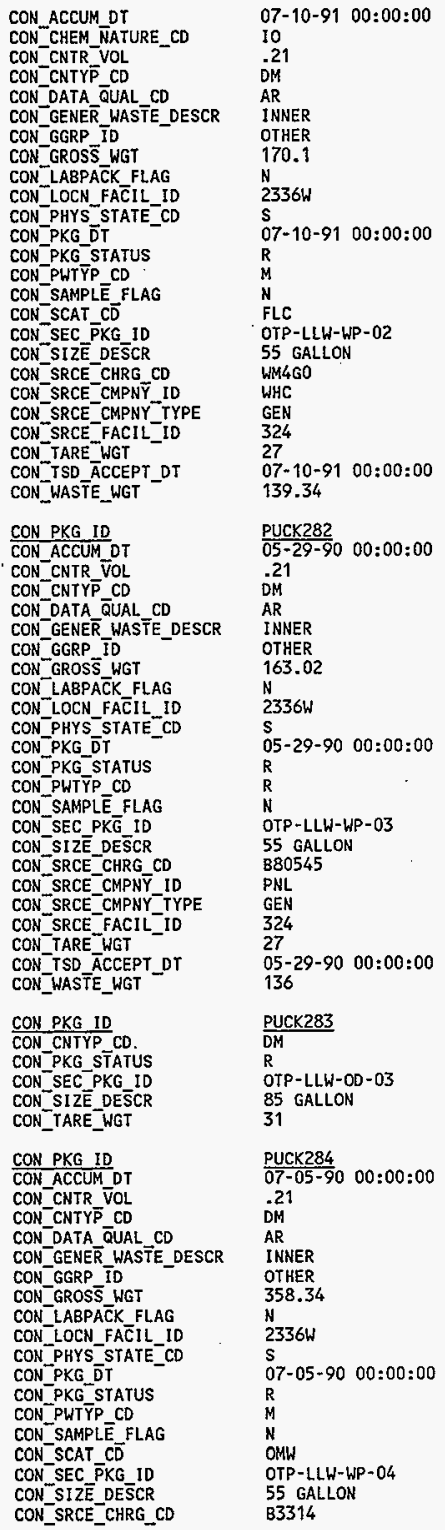

\begin{tabular}{|c|c|}
\hline $\begin{array}{l}\text { CON_SRCE_CMPNY_ID } \\
\text { CON_SRCE_CMPNY_TYPE } \\
\text { CON_SRCE_FACIL_ID } \\
\text { CON_TARE_WGT } \\
\text { CON_TSD_ACCEPT_DT } \\
\text { CON_WASTE_WGT }\end{array}$ & $\begin{array}{l}\text { WHC } \\
\text { GEN } \\
324 \\
27 \\
07-05-90 \quad 00: 00: 00 \\
32.1\end{array}$ \\
\hline $\begin{array}{l}\text { CON PKG ID } \\
\text { CON_CNTYP_CD } \\
\text { CON_PKG_STATUS } \\
\text { CON_SEC_PKG_ID } \\
\text { CON_SIZE_DESCR } \\
\text { CON_TARE_WGT }\end{array}$ & $\begin{array}{l}\text { PUCK285 } \\
\text { DM } \\
R \\
\text { OTP-LLW-OD-04 } \\
85 \text { GALLON } \\
31\end{array}$ \\
\hline $\begin{array}{l}\text { CONEXT_PKG_ID } \\
\text { CONEXT_COMPLIANT_FLAG } \\
\text { CONEXT_NDE_VER_FLAG } \\
\text { CONEXT_PROF_FLAG } \\
\text { CONEXT_PROF_ID } \\
\text { CONEXT_USE_CO } \\
\text { CONEXT_WRAP_STAT_CD }\end{array}$ & $\begin{array}{l}\text { PUCK278 } \\
Y \\
Y \\
\text { N } \\
\text { NRLLW1 } \\
\text { WP } \\
\text { I }\end{array}$ \\
\hline $\begin{array}{l}\text { CONEXT_PKG_ID } \\
\text { CONEXT_USE_CD } \\
\text { CONEXT_WRAP_STAT_CD }\end{array}$ & $\begin{array}{l}\text { PUCK279 } \\
\text { EC } \\
1\end{array}$ \\
\hline $\begin{array}{l}\text { CONEXT_PKG_ID } \\
\text { CONEXT_COMPLIANT_FLAG } \\
\text { CONEXT_NDE_VER_FLAG } \\
\text { CONEXT_PROF_FLĀG } \\
\text { CONEXT_PROF-ID } \\
\text { CONEXT_ROUTE_CD } \\
\text { CONEXT_USE_CD } \\
\text { CONEXT_VER_GROSS_WGT }\end{array}$ & $\begin{array}{l}\text { PUCK281 } \\
N \\
Y \\
N \\
\text { NRLLW1 } \\
3 \\
\text { WP } \\
T 71.1\end{array}$ \\
\hline CONEXT_VER_GROSS_WGT_FLAG & \\
\hline CONEXT_WRAP_STAT_CD & I \\
\hline $\begin{array}{l}\text { CONEXT_PKG_ID } \\
\text { CONEXT_COMPLIANT_FLAG } \\
\text { CONEXT_NDE_VER_F'AAG } \\
\text { CONEXT_PROF_FLAG } \\
\text { CONEXT_PROF_ID } \\
\text { CONEXT_USE_CD . } \\
\text { CONEXT_WRAP_STAT_CD }\end{array}$ & $\begin{array}{l}\text { PUCK282 } \\
Y \\
Y \\
N \\
\text { NRLLWT } \\
\text { WP } \\
\text { I }\end{array}$ \\
\hline $\begin{array}{l}\text { CONEXT_PKG_ID } \\
\text { CONEXT_USE_CD } \\
\text { CONEXT_WRAP_STAT_CD }\end{array}$ & $\begin{array}{l}\text { PUCK283 } \\
\text { EC } \\
\text { I }\end{array}$ \\
\hline $\begin{array}{l}\text { CONEXT_PKG_ID } \\
\text { CONEXT_CONPLIIANT_FLAG } \\
\text { CONEXT_NDE_VER FLAG } \\
\text { CONEXT_PROF_FLAG } \\
\text { CONEXT_PROF_ID } \\
\text { CONEXT_USE_CD } \\
\text { CONEXT_WRAP_STAT_CD }\end{array}$ & $\begin{array}{l}\text { PUCK284 } \\
\text { N } \\
\text { Y } \\
\text { N } \\
\text { NRLLW1 } \\
\text { WP } \\
\text { I }\end{array}$ \\
\hline $\begin{array}{l}\text { CONEXT_PKG_ID } \\
\text { CONEXT_USE_CD } \\
\text { CONEXT_WRAP_STAT_CD }\end{array}$ & $\begin{array}{l}\text { PUCK285 } \\
\text { EC } \\
\text { I }\end{array}$ \\
\hline $\begin{array}{l}\text { HDET_PKG_ID } \\
\text { HDET_CNTR_STATUS } \\
\text { HDET_DESIG_CD } \\
\text { HDET_DW_NUM } \\
\text { HDET_WASTE_VOL } \\
\text { HDET_WASTE_STATUS }\end{array}$ & $\begin{array}{l}\text { PUCK278 } \\
\text { F } \\
\text { RE } \\
\text { D008 D009 } \\
i^{208} .\end{array}$ \\
\hline $\begin{array}{l}\text { HDET_PKG_ID } \\
\text { HDET_DESIG_CD } \\
\text { HDET_DW_NUM } \\
\text { HDET_WASTE_VOL }\end{array}$ & $\begin{array}{l}\text { PUCK281 } \\
\text { RD } \\
\text { D002 } \\
.21\end{array}$ \\
\hline $\begin{array}{l}\text { HDET_PKG_ID } \\
\text { HDET_DESIG_CD } \\
\text { HDET_DW_NUM }\end{array}$ & $\begin{array}{l}\text { PUCK284 } \\
\text { RE } \\
\text { D008 WT01 }\end{array}$ \\
\hline
\end{tabular}




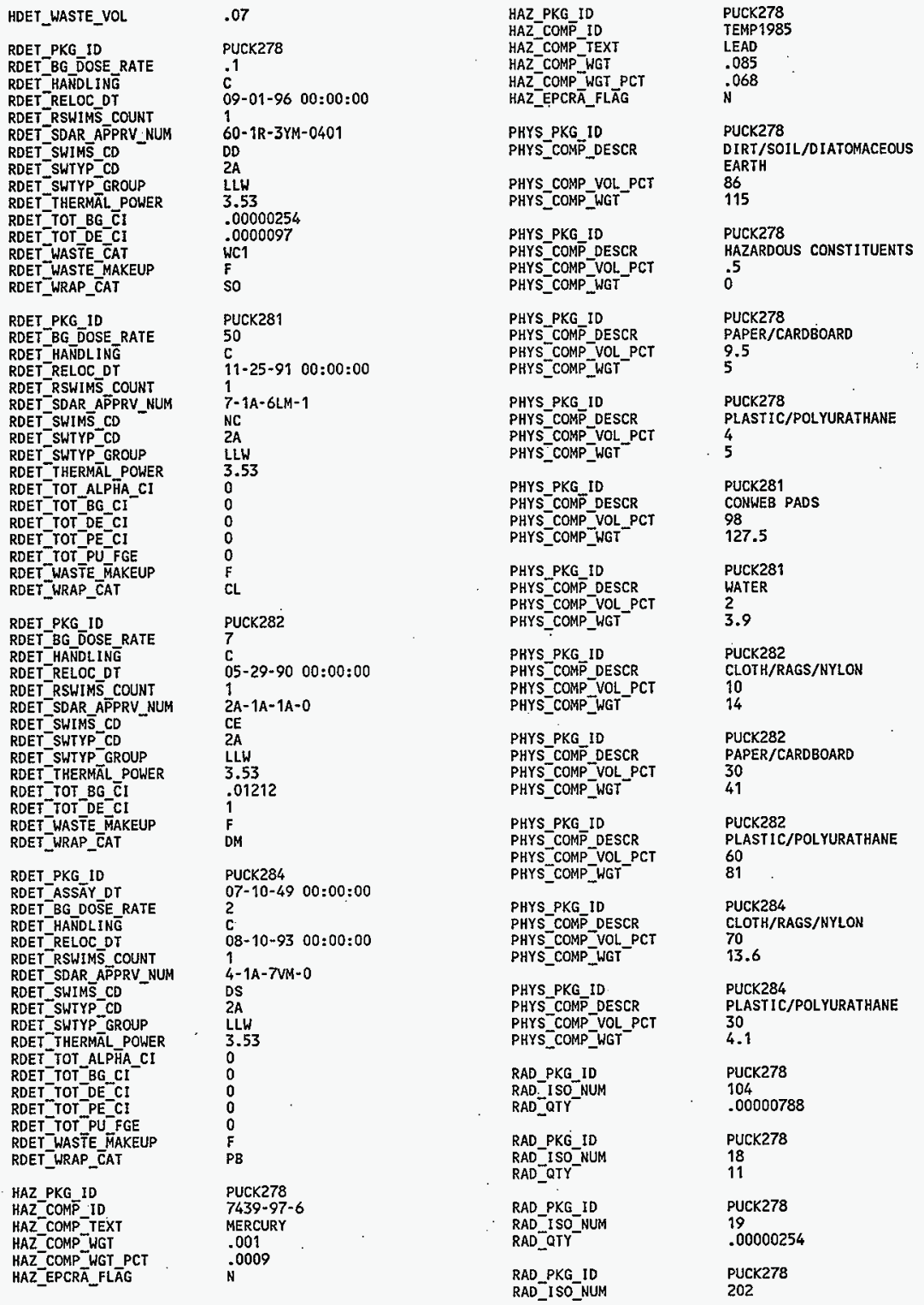




\begin{tabular}{|c|c|}
\hline RAD_QTY & .0013 \\
\hline $\begin{array}{l}\text { RAD_PKG_ID } \\
\text { RAD_ISO_NUM } \\
\text { RAD_QTY }\end{array}$ & $\begin{array}{l}\text { PUCK278 } \\
203 \\
.19\end{array}$ \\
\hline $\begin{array}{l}\text { RAD_PKG_ID } \\
\text { RAD_ISO_NUM } \\
\text { RAD_QTY }\end{array}$ & $\begin{array}{l}\text { PUCK278 } \\
206 \\
25\end{array}$ \\
\hline $\begin{array}{l}\text { RAD_PKG_ID } \\
\text { RAD_ISO_NUM } \\
\text { RAD_QTY }^{-{ }^{2}}\end{array}$ & $\begin{array}{l}\text { PUCK278 } \\
86 \\
.00000254\end{array}$ \\
\hline $\begin{array}{l}\text { RAD_PKG_ID } \\
\text { RAD_ISO_NUM } \\
\text { RAD_OTY }\end{array}$ & $\begin{array}{l}\text { PUCK281 } \\
12 \\
.00033\end{array}$ \\
\hline $\begin{array}{l}\text { RAD_PKG_ID } \\
\text { RAD_ISO_NUM } \\
\text { RAD_QTY }\end{array}$ & $\begin{array}{l}\text { PUCK281 } \\
152 \\
.000005\end{array}$ \\
\hline $\begin{array}{l}\text { RAD_PKG_ID } \\
\text { RAD_ISO_NUM } \\
\text { RAD_QTY }\end{array}$ & $\begin{array}{l}\text { PUCK281 } \\
153 \\
.00018\end{array}$ \\
\hline $\begin{array}{l}\text { RAD_PKG_ID } \\
\text { RAD_ISO_NUM } \\
\text { RAD_QTY } \\
\text { RAD_UNKNOWN }\end{array}$ & $\begin{array}{l}\text { PUCK281 } \\
19 \\
0^{-035405}\end{array}$ \\
\hline $\begin{array}{l}\text { RAD_PKG_ID } \\
\text { RAD_ISO_NUM } \\
\text { RAD_QTY }^{-}\end{array}$ & $\begin{array}{l}\text { PUCK281 } \\
43 \\
.00045\end{array}$ \\
\hline $\begin{array}{l}\text { RAD_PKG_ID } \\
\text { RAD_ISO_NUM } \\
\text { RAD_QTY }\end{array}$ & $\begin{array}{l}\text { PUCK281 } \\
53 \\
.00014\end{array}$ \\
\hline $\begin{array}{l}\text { RAD_PKG_ID } \\
\text { RAD_ISO_NUM } \\
\text { RAD_QTY }\end{array}$ & $\begin{array}{l}\text { PUCK281 } \\
56 \\
.0003\end{array}$ \\
\hline $\begin{array}{l}\text { RAD_PKG_ID } \\
\text { RAD_ISO_NUM } \\
\text { RAD_QTY }\end{array}$ & $\begin{array}{l}\text { PUCK281 } \\
91 \\
.034\end{array}$ \\
\hline $\begin{array}{l}\text { RAD_PKG_ID } \\
\text { RAD_ISO_NUM } \\
\text { RAD_QTY- } \\
\text { RAD_UNKNOWN }\end{array}$ & $\begin{array}{l}\text { PUCK282 } \\
19 \\
.01212 \\
.000324\end{array}$ \\
\hline $\begin{array}{l}\text { RAD_PKG_ID } \\
\text { RAD_ISO_NUM } \\
\text { RAD_QTY- }\end{array}$ & $\begin{array}{l}\text { PUCK282 } \\
3 \\
.00006\end{array}$ \\
\hline $\begin{array}{l}\text { RAD_PKG_ID } \\
\text { RAO_ISO_NUH } \\
\text { RAD_QTY }\end{array}$ & $\begin{array}{l}\text { PUCK282 } \\
8 \\
.006\end{array}$ \\
\hline $\begin{array}{l}\text { RAD_PKG_ID } \\
\text { RAD_ISO_NUM } \\
\text { RAD_QTY }\end{array}$ & $\begin{array}{l}\text { PuCK284 } \\
13 \\
.000004\end{array}$ \\
\hline $\begin{array}{l}\text { RAD_PKG_ID } \\
\text { RAD_ISO_NUM } \\
\text { RAD_QTY- } \\
\text { RAD_UNKNOWN }\end{array}$ & $\begin{array}{l}\text { PUCK284 } \\
19 \\
.000233 \\
.0000007163\end{array}$ \\
\hline $\begin{array}{l}\text { RAD_PKG_ID } \\
\text { RAD_ISO_EUM } \\
\text { RAD_QTY }\end{array}$ & $\begin{array}{l}\text { PuCK284 } \\
31 \\
.000032\end{array}$ \\
\hline $\begin{array}{l}\text { RAD_PKG_ID } \\
\text { RAD_ISO_NUN } \\
\text { RAD_QTY }\end{array}$ & $\begin{array}{l}\text { PUCK284 } \\
4 \\
.000083\end{array}$ \\
\hline
\end{tabular}

\begin{tabular}{|c|c|}
\hline $\begin{array}{l}\text { RAD_PKG_ID } \\
\text { RAD_ISO_NUM } \\
\text { RAD_QTY }\end{array}$ & $\begin{array}{l}\text { PUCK284 } \\
40 \\
.000002\end{array}$ \\
\hline $\begin{array}{l}\text { RAD_PKG_ID } \\
\text { RAD_ISO_NUM } \\
\text { RAD_QTY }\end{array}$ & $\begin{array}{l}\text { PUCK284 } \\
53 \\
.000001\end{array}$ \\
\hline $\begin{array}{l}\text { RAD_PKG_ID } \\
\text { RAD_ISO_NUM } \\
\text { RAD_QTY }\end{array}$ & $\begin{array}{l}\text { PUCK284 } \\
8 \\
.000013\end{array}$ \\
\hline $\begin{array}{l}\text { RAD_PKG_ID } \\
\text { RAD_ISO_NUM } \\
\text { RAD_QTY }\end{array}$ & $\begin{array}{l}\text { PUCK284 } \\
9 \\
.000001\end{array}$ \\
\hline $\begin{array}{l}\text { CONLOC_PKG_ID } \\
\text { CONLOC_OT } \\
\text { CONLOC_LOCN_ID }\end{array}$ & $\begin{array}{l}\text { OTP-LLW-0D-01 } \\
08-13-27 \quad 17: 01: 00 \\
\text { LLW_ENTRY }\end{array}$ \\
\hline $\begin{array}{l}\text { CONLOC_PKG_ID } \\
\text { CONLOC_DT } \\
\text { CONLOC_LOCN_ID }\end{array}$ & $\begin{array}{l}\text { OTP-LLW-OD-02 } \\
\text { O8-13-27 23:50:10 } \\
\text { LLW_ENTRY }\end{array}$ \\
\hline $\begin{array}{l}\text { CONLOC_PKG_IO } \\
\text { CONLOC_DT } \\
\text { CONLOC_LOCN_ID }\end{array}$ & $\begin{array}{l}\text { OTP-LLW-00-03 } \\
\text { 08-15-27 15:38:27 } \\
\text { LLW_ENTRY }\end{array}$ \\
\hline $\begin{array}{l}\text { CONLOC_PKG_ID } \\
\text { CONLOC_DT } \\
\text { CONLOC_LOCN_ID }\end{array}$ & $\begin{array}{l}\text { OTP-LLW-OD-04 } \\
08-15-27 \text { 16:06:40 } \\
\text { L.LW_ENTRY }\end{array}$ \\
\hline $\begin{array}{l}\text { CONLOC_PKG_ID } \\
\text { CONLOC_DT } \\
\text { CONLOC_LOCN_ID }\end{array}$ & $\begin{array}{l}\text { OTP-LLW-WP-01 } \\
08-14-27 \text { 01:42:44 } \\
\text { LLW_SORT }\end{array}$ \\
\hline $\begin{array}{l}\text { CONLOC_PKG_ID } \\
\text { CONLOC_DT } \\
\text { CONLOC_LOCN_ID }\end{array}$ & $\begin{array}{l}\text { OTP-LLW-WP-02 } \\
\text { 08-14-27 01:55:59 } \\
\text { LLW_SORT }\end{array}$ \\
\hline $\begin{array}{l}\text { CONLOC_PKG_ID } \\
\text { CONLOC_DT } \\
\text { CONLOC_LOCN_1D }\end{array}$ & $\begin{array}{l}\text { OTP-LLW-WP-03 } \\
\text { 08-13-97 13:25:13 } \\
\text { LLW_ENTRY }\end{array}$ \\
\hline $\begin{array}{l}\text { CONLOC_PKG_10 } \\
\text { CONLOC_DT } \\
\text { CONLOC_LOCN_10 }\end{array}$ & $\begin{array}{l}\text { OTP-LLW-WP-O4 } \\
08-15-2722: 10: 48 \\
\text { LLW_CMPCT. }\end{array}$ \\
\hline $\begin{array}{l}\text { CONLOC_PKG_ID } \\
\text { CONLOC_OT } \\
\text { CONLOC_LOCN_ID }\end{array}$ & $\begin{array}{l}\text { OTP-LLWR-TD-01 } \\
\text { O8-13-27 16:29:26 } \\
\text { LLW_RWMPRT }\end{array}$ \\
\hline $\begin{array}{l}\text { CONLOC_PKG_ID } \\
\text { CONLOC_DT } \\
\text { CONLOC_LOCN_ID }\end{array}$ & $\begin{array}{l}\text { OTP-LLWR-TD-02 } \\
\text { O8-15-27 15:38:57 } \\
\text { LLW_RWMPRT }\end{array}$ \\
\hline $\begin{array}{l}\text { CONLOC_PKG_ID } \\
\text { CONLOC_DT } \\
\text { CONLOC_LOCN_ID }\end{array}$ & $\begin{array}{l}\text { OTP6-97-400001 } \\
\text { 08-13-27 21:57:45 } \\
\text { LLW_PP }\end{array}$ \\
\hline $\begin{array}{l}\text { CONLOC_PKG_10 } \\
\text { CONLOC_OT } \\
\text { CONLOC_LOCN_ID }\end{array}$ & $\begin{array}{l}\text { RHZ-101-A } 13568 \\
08-10-2701: 55: 56 \\
\text { INFDCVYRW }\end{array}$ \\
\hline $\begin{array}{l}\text { CONLOC_PKG_ID } \\
\text { CONLOC_DT } \\
\text { CONLOC_LOCN_I0 }\end{array}$ & $\begin{array}{l}\text { RHZ-103-A13561 } \\
08-10-27 \quad 02: 02: 46 \\
\text { AGVPANC_A }\end{array}$ \\
\hline $\begin{array}{l}\text { RADMAT_ID } \\
\text { RADMAT_OESCR } \\
\text { RADMAT_LIMIT } \\
\text { RADMAT_RAD_TOT } \\
\text { RADMAT_UNITS } \\
\text { RADMAT_ALARM }\end{array}$ & $\begin{array}{l}\text { F } \\
\text { WRAP I FACILITY } \\
1433 \\
20 \\
\text { CI } \\
N\end{array}$ \\
\hline $\begin{array}{l}\text { RADMAT_ID } \\
\text { RADMAT_DESCR }\end{array}$ & $\begin{array}{l}\text { R } \\
\text { TRU RWM GLOVEBOX }\end{array}$ \\
\hline
\end{tabular}


RADMAT_LIMIT

RADMAT RAD TO

RADMAT_UNITS

RADMAT_ALARM

RADMAT ID

RADMAT DESCR

RADMATLIMIT

RADMAT_RAD_TOT

RADMAT UNITS

RADMAT_ALARM

MSGLOG DT

MSGLOG ERROR FLAG

MSGLOG GEN TYPE

MSGLOG_SEQ_NUM

MSGLOG SPE $\bar{C}$ TYPE

MSGLOG_STRIN̄G1

MSGLOG DT

MSGLOG ERROR FLAG

MSGLOG GEN TYYPE

MSGLOG_SEQ_NUM

MSGLOG SPE $\bar{C}$ TYPE

MSGLOG_STRINTGI

MSGLOG DT

MSGLOG ERROR FLAG

MSGLOG_GEN_TYPE

MSGLOG_SEQ NUM

MSGLOG SPE $\bar{C}$ TYPE

MSGLOG_STRINAGI

MSGLOG DT

MSGLOG ERROR FLAG

MSGLOG_GEN TYYPE

MSGLOG SEQ NUM

MSGLOG SPEC TYPE

MSGLOG_STRING 1

MSGLOG DT

MSGLOG ERROR FLAG

MSGLOG GEN TYYPE

MSGLOG SEQ NUM

MSGLOG SPEC TYPE

MSGLOG_STRIÑGI

MSGLOG DT

MSGLOG ERROR FLAG

MSGLOG GEN TYPE

MSGLOG SEQ NUM

MSGLOG SPE $\bar{C}$ TYPE

MSGLOG_STRINGG1

MSGLOG DT

MSGLOG ERROR FLAG

MSGLOG GEN TYPE

MSGLOG SEQ NUM

MSGLOG SPE $\bar{C}$ TYPE

MSGLOG_STRINGI

MSGLOG DT

MSGLOG ERROR FLAG

MSGLOG_GEN_TYPE

MSGLOG SEQ NUM

MSGLOG SPEC TYPE

MSGLOG_STRIN̄G!

MSGLOG_DT

177
10
FGE
N
T
TRU GLOVEBOX
177
10
FGE
N

08-11-97 16:16:34

$Y$

PCSDMS

62523

CL

PCSDMS“CL“871340844"LLW_E

XIT $\sim 1$ TEST DRUM *

08-11-97 16:53:56

$Y$

PCSDMS

62625

PCSDMS“CL“871343085“LLW_S

TORE1 $\sim 1$ TEST PIN \#2

08-12-97 08:35:29

$\mathrm{Y}$

PCSOMS

63927

CL

PCSDMS $\approx 2$ 871399578 LLW_S

TORE2 $\sim 1 \sim$ BTST PIN \#3

08-12-97 08:37:48

$Y$

PCSDMS

63935

CLW

PCSDMS CLW 871399716 L1W PUCK 1 BIST PIN \#3 ${ }_{503}$

08-12-97 08:38:55

$Y$

PCSOMS

63941

$\mathrm{Cl}$

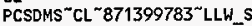

TORE2 $\sim 1 \sim$ BTST PIN \#3

08-12-97 08:39:13

Y

PCSDMS

63943

CL.

PCSDMS“CL 871399801 LLW_S

TORE2 ${ }^{\sim \sim} 1$ "BTST PIN \#3

08-12-97 08:43:46

$Y$

PCSDNS

63957

CI

PCSDMS CL"871400075 LLW_S

TORE2 ${ }^{\sim-1}$ BTST PIN \#3

08-12-97 08:54:34

$Y$

PCSDMS

63989

CL

PCSDNS CL 871400723\%LLW_S

TORE2 $1 \sim$ BTST PIN \#3 -

08-12-97 08:59:05
MSGLOG_ERROR_FLAG

MSGLOG GEN TYPE

MSGLOG SEQ NUM

MSGLOG_SPE $\bar{C}$ TYPE

MSGLOG STRIÑGI

MSGLOG DT

MSGLOG_ERROR FLAG

MSGLOG GEN TYYPE

MSGLOG SEQ NUM

MSGLOG_SPE $\bar{C}$ TYPE

MSGLOG_STRINTGI

MSGLOG_DT

MSGLOG ERROR FLAG

MSGLOG GEN TYPE

MSGLOG_SEQ NUM

MSGLOG_SPEC TYPE

MSGLOG_SIRIN̄G1

MSGLOG_DT

MSGLOG ERROR FLAG

MSGLQG GEN T $\bar{Y} P E$

MSGLOE_SEQ NUM

MSGLOG SPEC TYPE

MSGLOG_STRINTG1

MSGLOG_DT

MSGLOG ERROR FLAG

MSGLOG GEN_TYPPE

MSGLOG SEQ NUM

MSGLOG SPE $\bar{C}$ TYPE

MSGLOG_STRINGG

MSGLOG_DT

MSGLOG ERROR FLAG

MSGLOG_GEN TYYPE

MSGLOG SEQ NUM

MSGLOG SPE $\bar{C}$ TYPE

MSGLOG_STRIN̄G1

MSGLOG_DT

MSGLOG ERROR FLAG

MSGLOG_GEN_TYYPE

MSGLOG SEQ NUM

MSGLOG SPEC TYPE

MSGLOG_STRINTG1

MSGLOG DT

MSGLOG ERROR FLAG

MSGLOG GEN TYPPE

MSGLOG SEQ NUM

MSGLOG SPEC̄ TYPE

MSGLOG_STRINGG1

MSGLOG DT

MSGLOG ERROR FLAG

MSGLOG GEN TYPE

MSGLOG SEQ NUM

MSGLOG SPEC TYPE

MSGLOG_SIRINTG1

MSGLOG_DT

MSGLOG ERROR FLAG

MSGLOG GEN TYYPE.

MSGLOG SEQ NUM

MSGLOG_SPEC__TYPE
PCSDMS

64003

PPL

PCSDMS $\% P L$ \%71400993 LLW. $P P^{\sim} 0 T P 6-97-300001^{\sim}$

$08-12-97 \quad 09: 00: 50$

$\mathrm{N}$

PCSDMS

64008

$\mathrm{PPL}$

PCSDMS PPL $871401099^{2}$ LLW_ PP OTP6-97-300001

08-12-97 09:06:37

$\mathrm{N}$

PCSOMS

64025

POPD

PCSDMS POPD $871401446 \sim \mathrm{LLW}$

EXII $\sim$ BIST PIN \#3 TEST

08-12-97 09:09:53

$N$

PCSDMS

64034

$\mathrm{CL}$

PCSOMS CL $\% 871401641 \%$ LLW R WMPRT $\sim 1 \sim$ OTP-LLWR-TD-01

08-12-97 09:38:38

$\mathrm{N}$

PCSDMS

64113

ct.

PCSDMS $\mathrm{CL}^{2} 871403366$ LLW WMPRT ${ }^{m-1} 1^{\prime \prime}$ OTP-LLWR-TD-01"

08-12-97 10:01:19

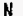

PCSOMS

64174

$\mathrm{CL}$

PCSOMS “CL 871404727 "LLW_E NTRY ${ }^{\sim}$ OTP-LLW-OD-01

08-12-97 10:01:31

N

DMSPCS

64175

CL

DMSPCS“CL“LLW_ENTRY 1 OTP -LLW-OD-01

$08-12-97$ 10:10:12

$Y$

PCSDMS

64200

CL

PCSDMS"CL"871405260"LLW_E NTRY $\sim$ T OTP-LLW-OD-01

$08-12-97 \quad 10: 10: 18$

N

DMSPCS

64202

$\mathrm{CL}$

DMSPCS“CL“LLW_ENTRY 1 10TP - LLW-WP-01

08-12-97 10:20:06

$Y$

PCSDMS

64229

$\mathrm{CL}$ 
MSGLOG_STRING1

MSGLOG DT

MSGLOG ERROR FLAG

MSGLOG_GEN_TYPPE

MSGLOG SEQ NUM

MSGLOG_SPE $\vec{C}$ TYPE

MSGLOG_STRINTG1

MSGLOG DT

MSGLOG ERROR FLAG

MSGLOG GEN TYPE

MSGLOG SEQ NUM

MSGLOG SPE $\bar{C}$ TYPE

MSGLOG_STRINGI

MSGLOG DT

MSGLOG_ERROR FLAG

MSGLOG GEN TYPE

MSGLOG_SEO-NUM

MSGLOG SPEC TYPE

HSGLOG_STRIN̄GI

MSGLOG DT

MSGLOG ERROR FLAG

MSGLOG_GEN_TYYPE

MSGLOG SEQ NUM

MSGLOG_SPEC $\bar{C}$ TYPE

MSGLOG_STRIN̄G1

MSGLOG DT

MSGLOG_ERROR_FLAG

MSGLOG GEN TYYPE

MSGLOG SEQ NUM

MSGLOG SPE $\bar{C}$ TYPE

MSGLOG_STRINGG1

MSGLOG DT

MSGLOG ERROR FLAG

MSGLOG_GEN_TYPE

MSGLOG SEQ NUM

MSGLOG SPEC TYPE

MSGLOG_STRING1

MSGLOG DT

MSGLOG ERROR FLAG

MSGLOG GEN TYYPE

MSGLOG_SEQ_NUM

MSGLOG SPEC TYPE

MSGLOG STRINTGI

MSGLOG_DT

MSGLOG ERROR FLAG

MSGLOG GEN TYPPE

MSGLOG SEQ NUM

MSGLOG SPEC TYPE

MSGLOG_STRINGI

MSGLOG DT

MSGLOG_ERROR FLAG

MSGLOG_GEN TYYPE
PCSDMS CL"871405855\%LLWE T ${ }^{\sim}$ TEST DRUM -

08-12-97 13:34:05

N

PCSDMS

64747

CL

PCSDMS CL"871417493\%LWS

ORT 1 OTP-LLW-WP-01

08-12-97 14:20:40

N

PCSDMS

64874

SPDP

PCSDMS ${ }^{2}$ SPDP $871420287 \%$ LLW

SORT $97-00001$-01L“OTP1-0

08-12-97 14:21:15

N

PCSOMS

64875

SPDP

PCSDMS ${ }^{\prime}$ SPDP ${ }^{2} 871420323 \sim \mathrm{LLW}$

SORT $97-00001-02 L=0 T P 1-0$

08-12-97 14:35:51

PCSDMS

64916

SPDP

PCSDMS“SPDP $871421198 \sim \mathrm{LLW}$

SORT ${ }^{297-00001-02 L “ O T P 1-0 ~}$

08-12-97 14:38:35

$\mathrm{N}$

PCSDMS

64923

SPDP

PCSDMS N SPDP $871421363^{\sim L L W}$

SORT $\% 97-00001-02 L \sim 0 T P$-LL

08-12-97 14:44:25

$N$

PCSDMS

64940

SPOP

PCSOMS $\sim$ SPDP $871421713 \sim 1 L W$

_SORT $297-00001-01 L^{\sim} 0 T P-L L$

08-12-97 14:45:19

$N$

PCSDNS

64943

SPDP

PCSOMS ${ }^{\sim}$ SPDP ${ }^{\sim} 871421767^{\sim 2 L L W}$

_SORT $\sim 97-00001-02 L \sim 0 T P-L L$

08-12-97 14:52:42

N

PCSDMS

64964

SPP

PCSDMS ${ }^{2 P P} 871422210 \% \mathrm{LLW}$ SORT. 97-00001-02L * OTP6-97

$08-12-97$ 14:53:02

N

PCSDMS
MSGLOG_SEQ_NUM

MSGLOG SPE $\vec{C}$ TYPE

MSGLOG_STRINGI

MSGLOG DT

MSGLOG ERROR FLAG

MSGLOG_GEN_IYPE

$M S G L O G$ SEQ NUM

MSGLOG SPEC TYPE

MSGLOG_SIRIÑGI

MSGLOG DT

MSGLOGERROR FLAG

MSGLOG GEN TYYPE

MSGLOG SEQ NUM

MSGLOG SPEC TYPE

MSGLOG_STRINGI

MSGLOG DT

MSGLOG_ERROR FLAG

MSGLOG GEN TYYPE

MSGLOG SEO NUM

MSGLOG_SPE $\bar{C}$ TYPE

MSGLOG_STRIN̄GI

MSGLOG DT

MSGLOG ERROR FLAC

MSGLOG GEN TYYPE

MSGLOG SEQ NUM

MSGLOG SPE $\bar{C}$ IYPE

MSGLOG_STRIM̄G1

MSGLOG DT

MSGLOG ERROR FLAG

MSGLOG_GEN_TȲPE

MSGLOG SEQ NUM

MSGLOG SPEC TYPE

MSGLOG_STRIN̄G?

MSGLOG DT

MSGLOG ERROR FLAG

MSGLOG GEN TYPE

MSGLOG SEQ NUM

MSGLOG SPEC TYPE

MSGLOG_STRINGG1

MSGLOG_DT

MSGLOG ERROR FLAG

MSGLOG GEN TYPE

MSGLOG SEQ NUM

MSGLOG SPEC TYPE

MSGLOG_STRIN̄G1

MSGLOG_DT

MSGLOG ERROR FLAG

MSGLOG GEN TYYPE

MSGLOG SEQ NUM

MSGLOG SPEC TYPE

MSGLOG_STRIN̄GI

MSGLOG_DT

MSGLOG ERROR FLAG

MSGLOG GEN TYPE

MSGLOG SEQ NUM

MSGLOG_SPEC_TYPE
64967

SPP

PCSDMS SPP 871422230 LLW SORT $27-00001-01 L^{\sim 0 T P 6-97}$

08-12-97 14:58:48

N

PCSOMS

64982

PPL

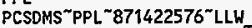
PP“OTP6-97-300001

08-12-97 15:02:47

$\mathrm{N}$

PCSDMS

64995

PPL

PCSDMS PPL 871422815 LLH PP $\approx T P 6-97-300001^{2}$

08-12-97 15:06:57

$\mathrm{N}$

PCSDMS

65006

PPTP

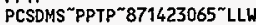

_PP־TP6-97-300001 OTP6-9

08-12-97 15:56:19

$Y$

PCSDMS

65141

CL

PCSOMS"Cl. "871426026"LLW_E XIT $\sim 1 \% T E S T$ DRUN

08-12-97 16:01:58

$Y$

PCSDMS

65159

CI

PCSDMS"CL" 871426365 LLLEE

XIT $1 \sim$ TEST DRUM

08-12-97 16:06:10

$Y$

PCSDMS

65171

CL

PCSDNS“CL $\% 871426617 \% L L W E$ XIT $\sim$ १ TEST DRUM

08-12-97 16:06:12

$Y$

PCSDMS

65173

$\mathrm{CL}$

PCSDHS“CL"871426618\%LLWE XIT $\sim 1 \sim T E S T$ DRUM

$08-12-97 \quad 16: 06: 14$

$Y$

PCSDMS

65175

$\mathrm{CL}$

PCSDMS“CL 871426618 นLW_E XIT $\sim 1 \sim T E S T$ DRUH

08-12-97 16:06:16

$Y$

PCSDMS

65177

CL 


\begin{tabular}{|c|c|}
\hline MSGLOG_STRING1 & $\begin{array}{l}\text { PCSDMS CL } 871426623 \sim 3^{\sim} \text { LLWE } \\
T^{\sim} \text { 1 }^{\sim} \text { TEST DRUM }\end{array}$ \\
\hline $\begin{array}{l}\text { MSGLOG_DT } \\
\text { MSGLOG_ERROR_FLAG } \\
\text { MSGLOG_GEN_TYPE } \\
\text { MSGLOG_SEQ_NUH } \\
\text { MSGLOG_SPEC TYYPE } \\
\text { MSGLOG_STRINGT }\end{array}$ & 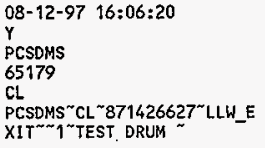 \\
\hline $\begin{array}{l}\text { MSGLOG_DT } \\
\text { MSGLOG_ERROR_FLAG } \\
\text { MSGLOG_GEN_TYPE } \\
\text { MSGLOG_SEQ_NUM } \\
\text { MSGLOG_SPEC TYPE } \\
\text { MSGLOG_STRING1 }\end{array}$ & 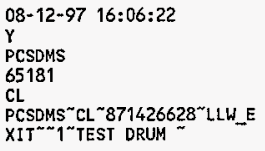 \\
\hline $\begin{array}{l}\text { MSGLOG_DT } \\
\text { MSGLOG_ERROR_FLAG } \\
\text { MSGLOG_GEN_TYPE } \\
\text { MSGLOG_SEQ_NUM } \\
\text { MSGLOG_SPEC̄_TYPE } \\
\text { MSGLOG_STRING1 }\end{array}$ & 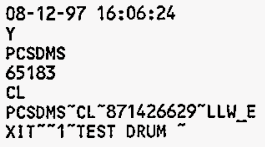 \\
\hline $\begin{array}{l}\text { MSGLOG_DT } \\
\text { MSGLOG_ERROR_FLAG } \\
\text { MSGLOG_GEN_TYPE } \\
\text { MSGLOG_SEQ_NUM } \\
\text { MSGLOG_SPEC CTYPE } \\
\text { MSGLOG_STRING1 }\end{array}$ & 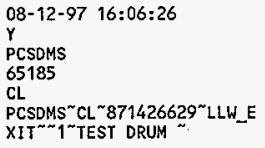 \\
\hline $\begin{array}{l}\text { MSGLOG_DT } \\
\text { MSGLOG_ERROR_FLAG } \\
\text { MSGLOG_GEN_TYPE } \\
\text { MSGLOG_SEQ_NUM } \\
\text { MSGLOG_SPEC_TYPE } \\
\text { MSGLOG_STRING1 }\end{array}$ & $\begin{array}{l}08-12-97 \quad 16: 06: 28 \\
Y \\
\text { PCSDMS } \\
65187 \\
C L \\
\text { PCSDMS } \\
\text { XIT } \sim 1^{\sim} \text { TEST DRUM } 871426631 \sim L L H E\end{array}$ \\
\hline $\begin{array}{l}\text { MSGLOG_DT } \\
\text { MSGLOG_ERROR_FLAG } \\
\text { MSGLOG_GEN_TYPE } \\
\text { MSGLOG_SEQ_NUM } \\
\text { MSGLOG_SPEC_TYPE } \\
\text { MSGLOG_STRING1 }\end{array}$ & $\begin{array}{l}08-12-97 \quad 16: 06: 30 \\
Y \\
\text { PCSDMS } \\
65189 \\
C L \\
\text { PCSDMS CL } 871426631 \sim \text { LLW_E } \\
\text { XIT } \sim 1 \sim T E S T \text { DRUM } \sim\end{array}$ \\
\hline $\begin{array}{l}\text { MSGLOG_DT } \\
\text { MSGLOG_ERROR_FLAG } \\
\text { MSGLOG_GEN_TYPE } \\
\text { MSGLOG_SEQ_NUM } \\
\text { MSGLOG_SPEC_TYPE } \\
\text { MSGLOG_SIRINTGT }\end{array}$ & 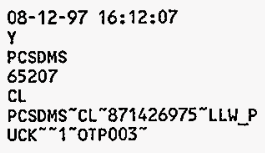 \\
\hline $\begin{array}{l}\text { MSGLOG_DT } \\
\text { MSGLOG_ERROR_FLAG } \\
\text { MSGLOG_GEN_TYPE } \\
\text { MSGLOG_SEQ_NUM } \\
\text { MSGLOG_SPEC_TYPE } \\
\text { MSGLOG_STRING1 }\end{array}$ & $\begin{array}{l}08-12-97 \quad 16: 59: 22 \\
N \\
\text { PCSDMS } \\
65335 \\
\text { CL } \\
\text { PCSOMS } \sim \text { CLL }^{\sim} 871429810 \sim \text { LLW_E } \\
\text { NTRY } \sim 1^{\sim} \text { OTP-LLW-0D-02\% }\end{array}$ \\
\hline $\begin{array}{l}\text { MSGLOG_DT } \\
\text { MSGLOG_ERROR_FLAG } \\
\text { MSGLOG_GEN_TYPE } \\
\text { MSGLOG_SEQ_NUM } \\
\text { MSGLOG_SPEC TYPE } \\
\text { MSGLOG_STRING1 }\end{array}$ & 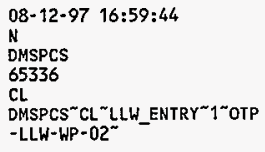 \\
\hline $\begin{array}{l}\text { MSGLOG_DT } \\
\text { MSGLOG_ERROR_FLAG }\end{array}$ & $\begin{array}{l}08-12-97 \quad 17: 27: 29 \\
Y\end{array}$ \\
\hline
\end{tabular}

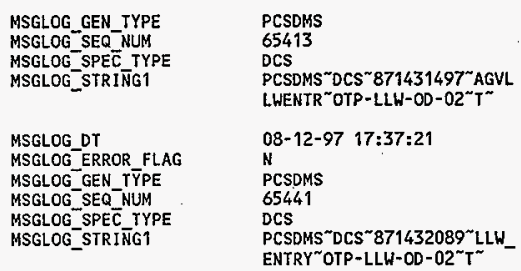

MSGLOG_DT

MSGLOG ERROR FLAG

MSGLOG_GEN_TYPE

MSGLOG SEQ NUM

MSGLOG SPE $\bar{C}$ TYPE

MSGLOG_STRINGI

MSGLOG DT

MSGLOGERROR FLAG

MSGLOG_GEN_TȲPE

MSGLOG SEQ NUM

MSGLOG_SPE $\bar{C}$ TYPE

MSGLOG_STRIN̄G1

MSGLOG DT

MSGLOG ERROR FLAG

MSGLOG_GEN_IYPE

MSGLOG SEQ NUM

MSGLOG_SPEC TYPE

MSGLOG_STRIN̄G1

MSGLOG DT

MSGLOG_ERROR_FLAG

MSGLOG_GEN_TYYPE

MSGLOG SEQ NUM

MSGLOG_SPEC_TYPE

MSGLOG_STRINTG1

MSGLOG DT

MSGLOG_ERROR_FLAG

MSGLOG GEN TYYPE

MSGLOG_SEQ NUM

MSGLOG_SPEC _TYPE

MSGLOG_STRING1

MSGLOG DT

MSGLOG_ERROR_FLAG

MSGLOG GEN TYYPE

MSGLOG SEQ NUM

MSGLOG_SPEC TYPE

MSGLOG_STRING!

MSGLOG DT

MSGLOG_ERROR_FLAG

MSGLOG GEN TYPE

MSGLOG_SEQ_NUM

MSGLOG_SPEC TYPE

MSGLOG_STRIÑGI

MSGLOG DT

MSGLOG_ERROR_FLAG MSGLOG GEN TYPE MSGLOG_SEQ_NUM MSGLOG_SPEC TYPE MSGLOG_STRIN̄GI
08-12-97 18:05:22

$Y$

PCSOMS

65516

$\mathrm{CL}$

PCSDMS“CL 871433770 "LLW_P UCK 1 " OTPOO1

08-12-97 18:05:32

$Y$

PCSDMS

65518

$\mathrm{CL}$

PCSDMS CL 2871433779 LLW_P UCK ${ }^{\sim}$ 1 OTP010

08-12-97 18:05:39

$Y$

PCSDMS

65520

CL

PCSOMS CL 871433786 LLW P UCK $\sim$ ฯ OTPOO1

08-12-97 18:11:40

$Y$

PCSDMS

65538

CL.

PCSDMS"CL 871434147"LLH_P UCK ${ }^{* * 1 \% O T P 011 " ~}$

08-12-97 $18: 16: 37$

$Y$

PCSDMS

65554

CL

PCSDMS“CL 871434445 LLW_P UCK $\sim$ ๆ"OTPOO1

08-12-97 18:38:43

$Y$ PCSDMS

65616

CL.

PCSDMS“CL “871435768 LLW_S TORE $1^{\sim} 1_{1}$ TEST PIN \#2

08-12-97 18:39:39

$Y$

PCSDMS

65620

CL.

PCSDMS“CL $\approx 871435827 \sim$ LLW S TORE1 1 TEST PIN \#2 -

$08-12-97 \quad 18: 39: 46$

$Y$

PCSDMS

65622

CL

PCSDMS CL $871435833^{\sim}$ LLW_S TORE1 1 TEST PIN \#2 -- 
MSGLOG_DT

MSGLOG_ERROR FLAG

MSGLOG GEN_TYPE

MSGLOG SEQ NUM

MSGLOG SPE $\bar{C}$ TYPE

MSGLOG_STRINTG1

MSGLOG DT

MSGLOG ERROR FLAG

MSGLOG GEN TYPE

MSGLOG SEQ NUM

MSGLOG SPEC TYPE

HSGLOG_STRIN̄G1

MSGLOG DT

MSGLOG ERROR FLAG

MSGLOG-GEN TYYPE

MSGLOG SEQ NUM

MSGLOG SPE $\bar{C}$ TYPE

MSGLOG_STRIN̄GI

MSGLOG DT

MSGLOG ERROR FLAG

MSGLOG_GEN_TYYPE

MSGLOG SEQ NUM

MSGLOG SPEC TYPE

MSGLOG STRIN̈G1

MSGLOG DT

MSGLOG ERROR FLAG

MSGLOG_GEN TYYPE

MSGLOG SEQ NUM

MSGLOG SPEC TYPE

MSGLOG_STRIÑGI

MSGLOG DT

MSGLOG ERROR FLAG

MSGLOG GEN TYYPE

MSGLOG SEQ NUM

MSGLOG_SPE $\bar{C}$ TYPE

MSGLOG_STRINTG1

\section{MSGLOG DT}

MSGLOG ERROR,FLAG

MSGLOG_GEN TYYPE

MSGLOG SEQ NUM

MSGLOG SPEC TYPE

MSGLOG SIRIN̄GI

MSGLOG DT

MSELOG ERROR FLAG

MSGLOG GEN TŸPE

MSGLOG SEQ NUM

MSGLOG'SPEC $\bar{C}$ TYPE

MSGLOG_SIRIÑG1

MSGLOG DT

MSGLOG_ERROR_FLAG

MSGLOG_GEN_TYYPE

MSGLOG SEQ NUM

MSGLOG SPEC̄ TYPE

MSGLOG_STRINGG1

MSGLOG DT

MSGLOGERROR_FLAG

MSGLOG GEN TYPE

MSGLOGSSEONUM

MSGLOG_SPEC_TYPE
08-12-97 18:44:00

$Y$

PCSDMS

65636

C.

PCSDMS CL. 871436088"LLW_P UCK ${ }^{\sim} 1 \sim$ OTP010

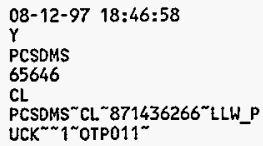

PCSOMS“CL_ 871436266 LLW_P UCK ${ }^{*} 1$ "OTP011

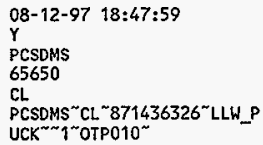

CL

PCSDMS“CL_871436564“LLW_S ORT ${ }^{\sim} \sim$ OTP-LLW-WP-01

08-12-97 19:03:15

$Y$

PCSDMS

65693

$\mathrm{CL}$

PCSDMS $\approx$ CL 871437242 LLW_S TORE2 $\sim 1 \%$ OTP010

08-12-97 19:03:33

$\mathrm{Y}$

PCSDMS

65697

$\mathrm{CL}$.

PCSDMS ${ }^{2} \approx 871437260 \sim$ LLW_S TORE2 1 OTP010

08-12-97 19:03:58

$Y$

PCSDMS

65699 MSGLOG DT

$\mathrm{CL}$

PCSDMS CL $2871437285^{\sim}$ LLWS TORE2 $\sim 1 \sim$ OTPO10

08-12-97 19:05:11

N

PCSDMS

65705

CL

PCSOMS CL-871437359 LLW_S ORT $\sim 1 \sim O T P-L L W-W P-02^{\sim}$

08-12-97 19:07:19

$Y$

PCSDNS

65712

$\mathrm{CL}$

PCSDMS CL“871437487 LLW_S TORE2 ${ }^{\sim \sim} 1$ OTPO10

08-12-97 19:07:22

Y

PCSDMS

65714

CL.

MSGLOG DT
MSGLOG_STRINGI

MSGLOGERROR FLAG

MSGLOG GEN TYYPE

MSGLOG_SEQ_NUM

MSGLOG SPEC TYPE

HSGLOG_STRINGT

MSGLOG_DI

MSGLOG ERROR FLAG

MSGLOG_GEN_TŸPE

MSGLOG_SEQ NUM

MSGLOG SPEC TYPE

MSGLOG_STRINGI

MSGLOG_DT

MSGLOG ERROR FLAG

MSGLOG GEN_TYYPE

MSGLOG SEQ NUM

MSGLOG SPE $\bar{C}$ TYPE

MSGLOG_STRINGGI

MSGLOG DT

MSGLOG ERROR FLAG

MSGLOG_GEN_TYYPE

MSGLOG SEQ NUM

MSGLOG_SPEC̄ TYPE

MSGLOG_STRIN̄G1

MSGLOG OT

MSGLOG ERROR FLAG

MSGLOG_GEN_TYYPE

MSGLOG SEQ NUM

MSGLOG SPE $\vec{C}$ TYPE

MSGLOG_STRINGI

MSGLOG DT

MSGLOG ERROR FLAG

MSGLOG_GEN_TYPE

MSGLOG SEQ MUM

MSGLOG_SPEC $\bar{C}$ TYPE

MSGLOGSTRIN̄G1

MSGLOG ERROR FLAE

MSGLOG_GEN_TYPE

MSGLOG SEQ NUM

MSGLOG_SPE $\bar{C}$ TYPE

MSGLOG STRIN̄G?

MSGLOG DT

MSGLOG ERROR FLAG

MSGLOG_GEN_TYPE

MSGLOG SEQ NUM

MSGLOG_SPEC _TYPE

MSGLOG STRIN̈G!

MSGLOG DT

MSGLOG ERROR FLAG

MSGLOG_GEN_TYPE

MSGLOG SEQ NUM

MSGLOG_SPEC

MSGLOG STRINTG1

MSGLOG DT

MSGLOG_ERROR_FLAG

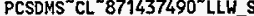
IORE2 $^{\sim}$ 1 OTP010 $^{\sim}$

08-12-97 19:07:27

$Y$

PCSDMS

65716

$\mathrm{CL}$

PCSDMS $\sim 2$ 871437495 LLW_S TORE2 $\sim 1 \sim 0 T P 010 \sim$

08-12-97 19:07:42

$Y$

PCSOMS

65718

PCSOMS"CL 781437510 LLW_S TORE2 $\sim 1$ \%TP010"

08-12-97 19:07:49

$Y$

PCSDMS

65720

$\mathrm{CL}$

PCSDMS ${ }^{\sim}$ CL871437516 LLW_S TORE2 1 OTP010

08-12-97 19:08:07

$Y$

PCSDMS

65724

$\mathrm{CL}$

PCSOMS CL 879437534 LLWS TORE2 $\sim 1 \sim$ OTP010

08-12-97 19:08:23

$Y$

PCSDMS

65726

$\mathrm{CL}$

PCSDMS $\mathrm{CL}^{\sim} 871437550^{\sim}$ LLHS TORE2 $2^{\sim 1} 1$ "OTP010

08-12-97 19:08:53

$Y$

PCSDHS

65730

$\mathrm{CL}$

PCSDMS“CL 871437581“LLW_S TORE2 $\sim 1 \sim O T P 010^{\sim}$

08-12-97 19:09:47

PCSDNS

65734

$\mathrm{CL}$

PCSDMS CL 271437635 LLW_S TORE2 $\sim 1 \% 0 T P 010 \%$

08-12-97 19:09:57

Y

PCSDNS

65736

CL.

PCSDHS CL 871437644 LLW S TORE2 $\sim 1 \sim 0 T P 010 \sim$

08-12-97 19:11:44

$Y$

PCSDMS

65744

CL

PCSDMS $\sim 2 L 871437751 \sim L L H S$

TORE2 " 1“OTP010

08-12-97 19:13:28

$Y$ 
MSGLOG_GEN_TYPE

MSGLOGSSEQ NUM

MSGLOG_SPEC̈ TYPE

MSGLOG_STRINGG1

MSGLOG DT

MSGLOG_ERROR FLAG

MSGLOG GEN_TYPE

MSGLOG SEQ NUM

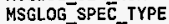

MSGLOG_STRIN̄G1

MSGLOG DT

MSGLOG_ERROR_FLAG

MSGLOG GEN TYPE

MSGLOG SEQ NUM

MSGLOG_SPE $\bar{C}$ TYPE

MSGLOGSTRIN̄GI

MSGLOG DT

MSGLOG_ERROR FLAG

MSGLOG GEN TŸPE

MSGLOG_SEQ NUM

MSGLOG SPE $\vec{C}$ TYPE

MSGLOG_STRIN̄G1

MSGLOG_DT

MSGLOG ERROR FLAG

MSGLOG GEN TYYPE

MSGLOG SEQ NUM

MSGLOG SPE $\bar{C}$ TYPE

MSGLLOG_STRIN̄G1

MSGLOG DT

MSGLOG_ERROR_FLAG

MSGLOG GEN TYYPE

MSGLOG SEQ NUM

MSGLOG_SPEC TYPE

MSGLOG SIRIN̄G1

HSGLOG DT

MSGLOG ERROR FLAG

MSGLOG GEN TYYPE

MSGLOG_SEQ-NUM

MSGLOG SPEC TYPE

MSGLOG_STRIN̄G1

MSGLOG DT

MSGLOG ERROR FLAG

MSGLOG_GEN_TYPE

MSGLOG SEQ NUM

MSGLOG-SPEC TYPE

MSGLOG_STRINGG1

MSGLOG DT

MSGLOGERROR FLAG

MSGLOG GEN TYYPE

MSGLOG SEQ NUM

MSGLOG SPEC TYPE

MSGLOG_STRING1

MSGLOG_DT
PCSDMS

65750

C..

PCSDMS $\approx$ CL"871437855 LLW_S TORE2 $\sim 1$ "OTP010

08-12-97 19:13:58

$Y$

PCSDMS

65754

CL

PCSDMS“CL"871437884"LLW_S

TORE2 $\sim 1$ OTPO10

08-12-97 19:27:21

$Y$

PCSOMS

65790

CL.

PCSDMS ${ }^{2 L} \approx 871438689^{\sim L L W}$ LP

UCK 1 OTP011

08-13-97 10:35:14

$\mathrm{N}$

PCSDMS

67080

RWPP

PCSDMS RWPP 871493160 LLW

SORT OTP6-97-000002 OTP-

$.08-13-97 \quad 10: 35: 19$

N

PCSDMS

67081

RWPI

PCSDMS ${ }^{\text {RWPT }}$ \%871493160 $\mathrm{LLW}$ SORT OTP6-97-000002 OTP-

08-13-97 10:35:43

$N$

PCSDMS

67082

RWPP

PCSDMS RWPP 871493189 LLW SORT OTPG-97-000001 OTP-

08-13-97 10:35:44

$N$

PCSDMS

67083

RWPT

PCSDMS"RWPT 871493189 LLW SORT OTP6-97-000001 OTP-

08-13-97 10:36:20

PCSOMS

67086

RWPP

PCSDMS RWPP $871493226 \sim$ LLW SORT OTP6-97-000003“OTP-

08-13-97 10:36:21

N

PCSDMS

67087

RWPT

PCSDMS RWPT 871493227 LLW _SORT"OTP6-97-000003 OTP-

08-13-97 12:45:05
MSGLOG_ERROR_FLAG

MSGLOG_GEN_TȲPE

MSGLOG SEQ NUM

MSGLOG_SPEC TYPE

MSGLOG_STRINTG1

MSGLOG DT

MSGLOG_ERROR_FLAG

MSGLOG GEN TYYPE

MSGLOG SEQ NUM

MSGLOG SPE $\vec{C}$ TYPE

MSGLOG_STRING1

MSGLOG DT

MSGLOG_ERROR FLAG

MSGL OG GEN TYYPE

MSGLOG_SEQ_NUM

MSGLOG_SPEC_TYPE

MSGLOG_STRIN̄GI

MSGLOG DT

MSGLOG_ERROR FLAG

MSGLOG GEN TYYPE

MSGLOG_SEQ_NUM

MSGLOG SPEC TYPE

MSGLOG_STRING1

\section{MSGLOG DT}

MSGLOG_ERROR_FLAG

MSGLOG GEN TYPE

MSGLOG_SEQ_NUM

MSGLOG SPEC TYPE

MSGLOG_STRIN̄G?

MSGLOG DT

MSGLOGERROR FLAG

MSGLOG GEN TYPE

MSGLOG_SEQ_NUM

MSGLOG SPE $\bar{C}$ TYPE

MSGLOG_STRIN̄G1

MSGLOG

MSGLOG DT

MSGLOG ERROR FLAG

MSGLOG GEN TYYPE

MSGLOG SEQ NUM

MSGLOG SPEC TYPE

MSGLOG_STRINGG

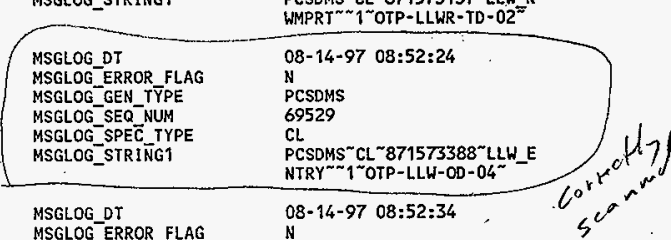

MSGLOG ERROR FLAG

MSGLOG_GEN_TȲPE

MSGLOG SEQ NUM

MSGLOG SPE $\bar{C}$ TYPE

MSGLOG_STRIN̄G1

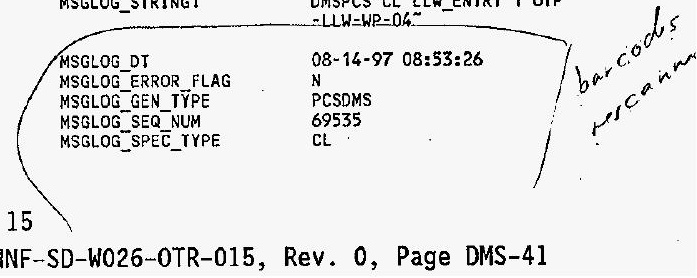

$Y$

PCSDMS

67432

$\mathrm{CL}$

PCSDMS $\sim C^{\sim}$ 871500950 LLWE XII $\sim \mathcal{I}^{\sim}$ TEST DRUM

08-13-97 13:24:56

$N$

PCSDMS

67540

CL

PCSOMS“CL "871503342"LLW_E

NTRY $\sim 1 \sim O T P-L L W-O D-03 \approx-$

08-13-97 13:25:13

$\mathrm{N}$

DMSPCS

67541

$\mathrm{CL}$

DMSPCS CL LLW_ENTRY"1“OTP

-LLW-WP-03

08-13-97 13:51:03

$Y$

PCSONS

67612

CL.

PCSDMS “CL -871504908 LLH_E XIT $T^{\sim}$ TEST DRUM *

08-14-97 08:47:43

N

PCSDMS

69514

$\mathrm{CL}$

PCSDMS CL 871573107“LLH_E NTRY 1 OTP-LLW-OD-03

$08-14-9708: 48: 01$

N

DMSPCS

69515

$\mathrm{CL}$

DHSPCS“CL“LLH_ENTRY 1 \%TP

-LLW-OD-03

08-14-97 08:48:12

PCSDMS

69518

08-14-97 08:52:24

PCSDUS

69529

N

DMSPCS

69530

DMSPCS $\sim 2 L \sim L H E N T R \sim 1 \% O T P$

08-14-97 08:53:26

69535

MSGLOG GEN TYYPE

MSGLOG SEQ NUM

MSGLOG_SPEC_TYPE

Rev. 0, Page DMS-41 


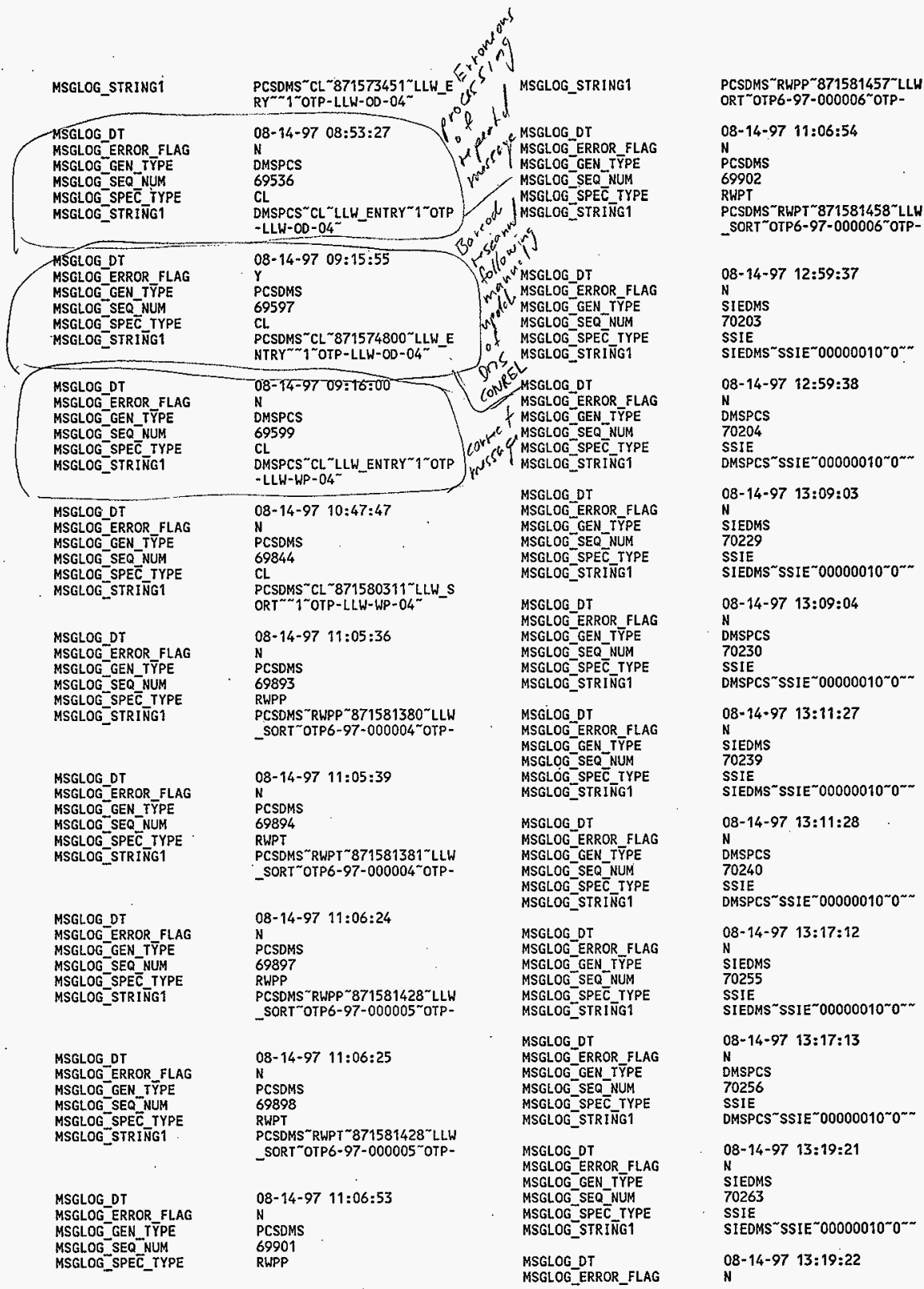




\begin{tabular}{|c|c|}
\hline $\begin{array}{l}\text { MSGLOG_GEN_TYPE } \\
\text { MSGLOG_SEQ_NUM } \\
\text { MSGLOG_SPEC̄TYPE } \\
\text { MSGLOG_STRINGG1 }\end{array}$ & $\begin{array}{l}\text { DMSPCS } \\
70264 \\
\text { SSIE } \\
\text { DHSPCS } \\
\text { SSIE } 00000010^{\sim} 0^{\sim \sim}\end{array}$ \\
\hline $\begin{array}{l}\text { MSGLOG_DT } \\
\text { MSGLOG_ERROR_FLAG } \\
\text { MSGLOG_GEN_TYPE } \\
\text { MSGLOG_SEQ_NUM } \\
\text { MSGLOG_SPEC_TYPE } \\
\text { MSGLOG_STRINGG1 }\end{array}$ & $\begin{array}{l}08-14-97 \quad 13: 28: 12 \\
\text { N } \\
\text { SIEDMS } \\
70289 \\
\text { SSIE } \\
\text { SIEDMS SSIE } 00400022 \sim 1040 \\
\text { 5\%Failed to connect to a }\end{array}$ \\
\hline $\begin{array}{l}\text { MSGLOG_DT } \\
\text { MSGLOG_ERROR_FLAG } \\
\text { MSGLOG_GEN_TYPE } \\
\text { MSGLOG_SEQ_NUM } \\
\text { MSGLOG_SPEC CTYPE } \\
\text { MSGLOG_STRING1 }\end{array}$ & $\begin{array}{l}\text { 08-14-97 } 13: 28: 13 \\
\text { N } \\
\text { DMSPCS } \\
70290 \\
\text { SSIE } \\
\text { DMSPCS SSIE 00400022 1040 } \\
5 \% \text { Failed to connect to a }\end{array}$ \\
\hline $\begin{array}{l}\text { MSGLOG_DT } \\
\text { MSGLOG_ERROR_FLAG } \\
\text { MSGLOG_GEN_TYPE } \\
\text { MSGLOG_SEQ_NUM } \\
\text { MSGLOG_SPEC_TYPE } \\
\text { MSGLOG_STRING1 }\end{array}$ & $\begin{array}{l}08-14-97 \quad 13: 32: 41 \\
N \\
\text { SIEDMS } \\
70303 \\
\text { SSIE } \\
\text { SIEDMS }{ }^{\sim} S_{1 E} 00000010^{\sim} 0^{\sim-}\end{array}$ \\
\hline $\begin{array}{l}\text { MSGLOG_DT } \\
\text { MSGLOG_ERROR_FLAG } \\
\text { MSGLOG_GEN_TYPE } \\
\text { MSGLOG_SEQ_NUM } \\
\text { MSGLOG_SPEC_TYPE } \\
\text { MSGLOG_STRING1 }\end{array}$ & $\begin{array}{l}\text { 08-14-97 13:32:42 } \\
\text { N } \\
\text { DMSPCS } \\
70304 \\
\text { SSIE } \\
\text { DMSPCS SSIE } 00000010^{\sim} 0^{\sim}\end{array}$ \\
\hline $\begin{array}{l}\text { MSGLOG_DT } \\
\text { MSGLOG_ERROR_FLAG } \\
\text { MSGLOG_GEN_TYPE } \\
\text { MSGLOG_SEQ_NUM } \\
\text { MSGLOG_SPEC_TYPE } \\
\text { MSGLOG_STRING1 }\end{array}$ & $\begin{array}{l}08-14-97 \quad 13: 32: 43 \\
N \\
\text { SIEDMS } \\
\text { 70305 } \\
\text { SSIE } \\
\text { SIEDMS SSIE } 00400022^{\sim} 1041 \\
\text { O Failed to connect to a }\end{array}$ \\
\hline $\begin{array}{l}\text { MSGLOG_DT } \\
\text { MSGLOG_ERROR_FLAG } \\
\text { MSGLOG_GEN_TYPE } \\
\text { MSGLOG_SEQ_NUM } \\
\text { MSGLOG_SPEC TYPE } \\
\text { MSGLOG_STRING1 }\end{array}$ & $\begin{array}{l}08-14-97 \quad 13: 32: 44 \\
\text { N } \\
\text { DMSPCS } \\
70306 \\
\text { SSIE } \\
\text { DMSPCS SSIE } 00400022 \sim 1041 \\
0^{\sim} \text { Failed to connect to a }\end{array}$ \\
\hline $\begin{array}{l}\text { MSGLOG_DT } \\
\text { MSGLOG_ERROR_FLAG } \\
\text { MSGLOG_GEN_TYPE } \\
\text { MSGLOG_SEQ_NUM } \\
\text { MSGLOG_SPEC̄_TYPE } \\
\text { MSGLOG_STRING] }\end{array}$ & $\begin{array}{l}08-14-97 \quad 13: 32: 50 \\
\text { N } \\
\text { SIEDMS } \\
70307 \\
\text { SSIE } \\
\text { SIEDMS SSIE } 00000010^{\sim} 0^{\sim \sim}\end{array}$ \\
\hline $\begin{array}{l}\text { MSGLOG_DT } \\
\text { MSGLOG_ERROR_FLAG } \\
\text { MSGLOG_GEN_TYPE } \\
\text { MSGLOG_SEQ_NUM } \\
\text { MSGLOG_SPEC_TYPE } \\
\text { MSGLOG_STRINGG }\end{array}$ & $\begin{array}{l}\text { 08-14-97 13:32:51 } \\
\text { N } \\
\text { DMSPCS } \\
70308 \\
\text { SSIE } \\
\text { DMSPCS } \sim S S 1 E \sim 00000010^{\sim} 0^{\sim}\end{array}$ \\
\hline $\begin{array}{l}\text { MSGLOG_DT } \\
\text { MSGLOG_ERROR_FLAG } \\
\text { MSGLOG_GEN_TYPE } \\
\text { MSGLOG_SEQ_NUM } \\
\text { MSGLOG_SPEC_TYPE } \\
\text { MSGLOG_STRINTG1 }\end{array}$ & $\begin{array}{l}08-14-9713: 32: 52 \\
N \\
\text { SIEOMS } \\
70309 \\
\text { SSIE } \\
\text { SIEDMS SSIE } 00400022 \sim 1041 \\
\text { 0Failed to connect to a }\end{array}$ \\
\hline $\begin{array}{l}\text { MSGLOG_DT } \\
\text { MSGLOG_ERROR_FLAG } \\
\text { MSGLOG_GEN_TYYPE } \\
\text { MSGLOG_SEQ_NUM }\end{array}$ & $\begin{array}{l}08-14-9713: 32: 53 \\
\text { N } \\
\text { DMSPCS } \\
70310\end{array}$ \\
\hline
\end{tabular}

MSGLOG_SPEC TYPE
MSGLOG_STRINGG1

MSGLOG DT MSGLOG ERROR FLAG MSGLOG_GEN_TȲPE MSGLOG SEQ NUM MSGLOG_SPEC $\bar{C}$ TYPE MSGLOG_STRIN̄GI

MSGLOG_DT MSGLOG ERROR FLAG MSGLOG_GEN_TYYPE MSGLOG_SEQ_NUM MSGLOG SPEC TYPE MSGLOG_STRINGI

MSGLOG DT

MSGLOG_ERROR FLAG

MSGLOG GEN TY YPE

MSGLOG_SEQ_NUM

MSGLOG SPEC TYPE

MSGLOG_STRINTG1

MSGLOG DT

MSGLOG_ERROR_FLAG

MSGLOG GEN_TYPE

MSGLOG_SEQ NUM

MSGLOG SPEE TYPE

MSGLOG_STRINGG1

MSGLOG DT

MSGLOG ERROR FLAG

MSGLOG_GEN TYYPE

MSGLOG_SEQNNUM

MSGLOG SPEC TYPE

MSGLOG_STRINGI

MSGLOG DT

MSGLOG_ERROR' FLAG

MSGLOG GEN TYYPE

MSGLOG SEQ MUM

MSGLOG SPEC TYPE

MSGLOG_STRINTG1

MSGLOG DT

MSGLOGERROR FLAG

MSGLOG GEN TYPPE

MSGLOG_SEQ NUM

MSGLOG_SPEC

MSGLOG_STRIN̄G1

MSGLOG DT

MSGLOG_ERROR FLAG

MSGLOG_GEN_TYPPE

MSGLOG_SEQ_NUM

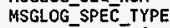

MSGLOG_STRINTGI

\section{MSGLOG DT}

MSGLOG_ERROR_FLAG

MSGLOG GEN TYYPE

MSGLOG_SEQ NUM

MSGLOG_SPEC

MSGLOG_STRING 1

MSGLOG_DT

MSGLOG ERROR FLAG

MSGLOG_GEN_TYYPE

MSGLOG SEQ NUM

MSGLOG_SPEC CTYPE

MSGLOG_STRINTG1
SSIE

DMSPCS“SSIE 00400022 1041

0 \%Failed to connect to a

08-14-97 13:32:59

N

SIEDMS

70311

SSIE

SIEDMS ${ }^{\sim} S S I E \sim 00000010^{\sim} 0^{\sim}$

08-14-97 13:33:00

N

DMSPCS

70312

SSIE

DMSPCS $\sim S[E \sim 00000010 \sim 0 \sim$

08-14-97 13:33:01

N

SIEDMS

70313

SSIE

SIEDMS SSIE $00400022 \sim 1041$

$0 \sim$ Failed to connect to a

08-14-97 13:33:02

N

DMSPCS

70314

SSIE

DMSPCS SSIE $00400022 \sim 1041$

$0 \sim$ Failed to connect to a

08-14-97 13:33:04

N

SIEDMS

70315

SSIE

SIEDMS $\sim S S^{\sim} \sim 0000010 \sim 0 \sim$

08-14-97 13:33:05

N

DMSPCS

70316

SSIE

DMSPCS SSIE $00000010^{\sim} 0^{\sim}$

$08-14-97 \quad 13: 33: 08$

N

SIEDMS

70319

SSIE

SIEDMS ${ }^{\sim}$ SSIE $~ 00400022^{\sim} 1041$

$0^{-}$Failed to connect to a

08-14-97 13:33:09

N

DMSPCS

70320

SSIE

DMSPCS SSIE $\sim 00400022 \sim 1041$

O Failed to connect to a

08-14-97 13:33:11

N

SIEDHS

70321

SSIE

SIEDMS SSIE $00000010^{\sim} 0^{--}$

08-14-97 13:33:12

N

DMSPCS

70322

SSIE

DMSPCS SSIE $00000010^{\sim} 0^{\sim \sim}$ 
MSGLOG_DT

MSGLOG ERROR FLAG

MSGLOG GEN TYPE

MSGLOG_SEQ NUM

MSGLOG SPE $\bar{C}$ TYPE

MSGLOG_STRINTG1

MSGLOG DT

MSGLOG ERROR FLAG

MSGLOG_GEN_TYPE

MSGLOG SEQ NUM

MSGLOG SPEC TYPE

MSGLOG_STRIN̄G1

MSGLOG DT

MSGLOG ERROR FLAG

MSGLOG_GEN_TYPE

MSGLOG_SEQ NUM

MSGLOG SPEC TYPE

MSGLOG_STRIN̄G!

MSGLOG DT

MSGLOG ERROR FLAG

MSGLOG_GEN_TȲPE

MSGLOG SEQ NUM

MSGLOG SPEC TYPE

MSGLOG STRINGG1

MSGLOG DT

MSGLOG_ERROR FLAG

MSGLOG GEN TYYPE

MSGLOG SEQ NUM

MSGLOG SPEC TYPE

MSGLOG_STRINGTI

MSGLOG DT

MSGLOG ERROR FLAG

MSGLOG GEN TYYPE

MSGLOG SEQ NUM

MSGLOG SPEC TYPE

MSGLOG_STRINGG1.

MSGLOG DT

MSGLOG ERROR FLAG

MSGLOG GEN TYYPE

MSGLOG_SEQ NUM

MSGLOG SPEC TYPE

MSGLOG SIRINTG

MSGLOG OT

MSGLOG ERROR FLAG

MSGLOG GEN TYPE

MSGLOG SEQ NUM

MSGLOG SPEC TYPE

MSGLOG STRING?

MSGLOG DT

MSGLOG ERROR FLAG

MSGLOG GEN TŸPE

MSGLOG SEQ NUM

MSGLOG SPEC TYPE

MSGLOG_STRINGG1

MSGLOG DT

MSGLOG ERROR FLAG

MSGLOG GEN TYPE

MSGLOG SEQ NUM

MSGLOG SPE $\bar{C}$ TYPE

MSGLOG_STRINTG1

MSGLOG_DT
08-14-97 13:33:13

N

SIEDMS

70323

SSIE

SIEDMS ${ }^{\sim} S$ SIE $^{\sim} 00400022 \sim 1041$

o Failed to connect to a

08-14-97 13:33:14

N

DMSPCS

70324

SSIE

DMSPCS“SSIE"00400022 1041

0 \% Failed to connect to a

08-14-97 13:33:46

N

SIEDMS

70325

SSIE

SIEDMS SSIE 00000010"0

$08-14-97 \quad 13: 33: 47$

$\mathrm{N}$

DMSPCS

70326

SSIE

DMSPCS ${ }^{2 S H E} 00000010^{\sim 0} 0^{\sim}$

08-14-97 13:33:48

N

SIEDMS

70327

SSIE

SIEDMS ${ }^{\sim}$ SSIE $00400022 \sim 1041$

$0 \sim$ Failed to connect to a

08-14-97 13:33:49

$\mathrm{N}$

DMSPCS

70328

SSIE

DMSPCS SSIE $00400022 \sim 1041$

$0 \sim$ Failed to connect to a

08-14-97 13:33:53

$\mathrm{N}$

SIEDMS

70331

SSIE

SIEDMS SSIE $00000010 \sim 0 \sim$

08-14-97 13:33:54

$N$

DMSPCS

70332

SSIE

DMSPCS SSIE $00000010 \sim 0 \sim$

08-14-97 13:33:55

4

SIEDMS

70333

SSIE

SIEDMS"SSIE" 00400022 1041

0 Failed to connect to a

08-14-97 13:33:56

N

DMSPCS

70334

SSIE

DMSPCS"SSIE“00400022"1041

0 Failed to connect to a

08-14-97 13:36:57
MSGLOG_ERROR_FLAG

MSGLOG_GEN_IYYPE

MSGLOG SEQ NUM

MSGLOG_SPE $\bar{C}$ TYPE

MSGLOG_STRIN̄G1

MSGLOG DT

MSGLOG ERROR FLAG

MSGLOG_GEN_TȲPE

MSGLOG SEQ NUM

MSGLOG SPEC TYPE

MSGLOG_STRINTGI

MSGLOGDT

MSGLOG ERROR FLAG

MSGLOG GEN TȲPE

MSGLOG_SEQ NUM

MSGLOG SPEC TYPE

MSGLOG_SIRIN̄G1

MSGLOG DT

MSGLOG ERROR FLAG

MSGLOG_GEN_TYYPE

MSGLOG SEQ NUM

MSGLOG SPEC TYPE

MSGLOG_STRINTG1

MSGLOGDT

MSGLOG ERROR FLAG

MSGLOG_GEN_TYPE

MSGLOG SEQ NUM

MSGLOG SPE $\bar{C}$ TYPE

MSGLOG_STRING1

MSGLOG_DT

MSGLOG ERROR FLAG

MSGLOG_GEN_TYPE

MSGLOG SEQ NUM

MSGLOG SPE $\bar{C}$ TYPE

MSGLOG_STRINTG1

MSGLOG DT

MSGLOG ERROR FLAG

MSGLOG_GEN_TYPE

MSGLOG SEQ NUM

MSGLOG SPE $\bar{C}$ TYPE

MSGLOG_STRINGI

MSGLOG DT

MSGLOG ERROR FLAG

MSGLOG GEN TYPE

MSGLOG SEQ MUM

MSGLOG SPE $\bar{C}$ TYPE

MSGLOG_STRINTG1

MSGLOG_DT

MSGLOG ERROR FLAG

MSGLOG_GEN_TYYPE

MSGLOG SEQ NUM

MSGLOG SPE $\bar{C}$ TYPE

MSGLOG STRINGI

MSGLOG DT

MSGLOG ERROR FLAG

MSGLOG GEN TYYPE

MSGLOG SEQ NUM

MSGLOG_SPEC TYPE

MSGLOG SIRINGG1
$N$

SIEDMS

70343

SSIE

SIEDMS SSIE $00000010^{\sim} 0^{\sim}$

08-14-97 13:36:58

N

DNSPCS

70344

SSIE

DMSPCS SSIE $00000010^{\sim} 0^{\sim \sim}$

08-14-97 13:36:59

$\mathrm{N}$

SIEDMS

70345

SSIE

SIEDMS SSIE"00400022 1041

$0^{\sim}$ Fajted to connect to a

08-14-97 13:37:00

$N$

DMSPCS

70346

SSIE

DMSPCS"SSIE"00400022 1041

0 Failed to connect to a

08-14-97 15:19:20

N

DMSPCS

70423

FCL

DMSPCS $\sim F C L \approx 2.000000 E+01 \% F$

08-14-97 15:20:04

N

PCSDMS

70426

CL

PCSDMS"CL"871596648 LLW_C MPCT \%1 OTP-LLW-WP-04

$08-11-97 \quad 16: 16: 34$

$Y$

PCSDNS

62523

$\mathrm{CL}$

PCSDMS"CL"871340844"LLHE XIT $\sim$ ๆTEST DRUM

08-11-97 16:53:56

$Y$

PCSDMS

62625

$\mathrm{CL}$

PCSDMS “CL 871343085 LLH_S

TORE1 $\sim 1$ TEST PIN \#2

08-12-97 08:35:29

$Y$

PCSDMS

63927

$\mathrm{CL}$

PCSDMS“CL“871399578 LLW_S TORE2 $\sim 1 \sim$ BTST PIN \#3

08-12-97 08:37:48

$Y$

PCSDMS

63935

CLW

PCSOMS ${ }^{\circ L W} 871399716$ LLW. PUCK $1 \sim$ BIST PIN \#3 503 
MSGLOG_OT

MSGLOG_ERROR_FLAG

MSGLOG_GEN_TYPE

MSGLOG SEQ NUM

MSGLOG SPE $\bar{C}$ TYPE

MSGLOG_STRIÑG1

MSGLOG DT

MSGLOG_ERROR FLAG

MSGLOG_GEN_TYPE

MSGLOG SEQ NUM

MSGLOG_SPE $\bar{C}$ TYPE

MSGLOG_STRINTG1

\section{MSGLOG DT}

MSGLOG_ERROR_FLAG

MSGLOG GEN TYYPE

MSGLOG_SEQ NUM

MSGLOG_SPEC $\bar{C}$ TYPE

MSGLOG_STRINGI

MSGLOG DT

MSGLOG_ERROR FLAG

MSGLOG_GEN_TYPE

MSGLOG_SEQ_NUM

MSGLOG_SPE $\bar{C}$ TYPE

MSGLOG_STRIN̄G1

MSGLOG DT

MSGLOG_ERROR_FLAG

MSGLOG GEN TYYPE

MSGLOG_SEQ_NUM

MSGLOG_SPEC̈.TYPE

MSGLOG_STRIN̄GI

MSGLOG DT

NSGLOG_ERROR FLAG

MSGLOG GEN TYYPE

MSGLOG SEQ NUM

MSGLOG_SPEC TYPE

MSGLOG_STRINGI

MSGLOG DT

MSGLOG_ERROR_FLAG

MSGLOG GEN TYYPE

MSGLOG_SEQ_NUM

MSGLOG SPE $\bar{C}$ TYPE

NSGLOG_STRINGG1

MSGLOG DT

MSGLOG_ERROR_FLAG

MSGLOG GEN TYPE

MSGLOG_SEQ_NUM

MSGLOG_SPEC TYPE

MSGLOG_STRIN̈G1

MSGLOG DT

MSGLOGERROR FLAG

MSGLOG GEN TYPE

MSGLOG_SEQ NUN

MSGLOG_SPEC TYPE

MSGLOG_STRINTG1

MSGLOG DT

MSGLOG_ERROR_FLAG

MSGLOG GEN TYYPE

MSGLOG_SEQ_NUM

MSGLOG_SPEC_TYPE
08-12-97 08:38:55

$\mathrm{Y}$

PCSDMS

63941

CL

PCSOMS CL“871399783“LLW_S

TORE2 ${ }^{-m} \eta^{-1} B T S T$ PIN \#3 -

08-12-97 08:39:13

$Y$

PCSDMS

63943

CL

PCSDMS $\%$ CL 871399801“LLW_S

TORE2 $\sim 1 \sim$ BTST PIN \#3 $\sim$

08-12-97 08:43:46

$Y$

PCSDMS

63957

CL.

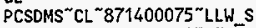

TORE2 $\sim 1 \sim B T S T$ PIN \#3

08-12-97 08:54:34

$\mathrm{Y}$

PCSDMS

63989

CL.

PCSOMS ${ }^{\sim}$ CL 871400723\%LLW_S

TORE2 ${ }^{\sim} 1$ BIST PIN \#3 $\sim$

$08-12-97 \quad 10: 10: 12$

$\mathrm{Y}$

PCSDMS

64200

C.

PCSDMS “CL $\approx 71405260$ LLW_E

NTRY $\sim 1 \sim O T P-L L W-O D-01^{\sim}$

08-12-97 10:20:06

$y$

PCSOMS

64229

$\mathrm{CL}$

PCSDMS ${ }^{2}$ CL 871405855 LLW_E

XIT $\sim 1^{\sim T E S T ~ D R U M ~}$

08-12-97 15:56:19

$\mathrm{Y}$

PCSDMS

65141

CL

PCSDMS“CL“871426026"LLH_E XIT $\sim 1 \sim$ TEST DRUM

08-12-97 16:01:58

$Y$

PCSDMS

65159

CL

PCSDMS“CL 871426365 LLW_E XIT T" 1 "TEST DRUM

08-12-97 16:06:10

$Y$

PCSDNS

65171

CL

PCSDMS ${ }^{2}$ × 871426617 1LW_E

XIT $\sim 1 \sim T E S T$ DRUM

08-12-97 16:06:12

$\mathrm{Y}$

PCSDMS

65173

CL
MSGLOG_STRING1

MSGLOG DI

MSGLOG ERROR FLAG

MSGLOG_GEN_TY $\bar{Y} P E$

MSGLOG SEQ NUM

MSGLOG_SPEC TYPE

MSELOG_STRINGI

MSGLOG DT

MSGLOGERROR_FLAG

MSGLOG_GEN_TYPPE

MSGLOG SEQ NUM

MSGLOG_SPEC__TYPE

MSGLOG_SIRINGI

MSGLOG DT

MSGLOG_ERROR_FLAG

MSGLOG GEN TYYPE

MSGLOG SEQ NUM

MSGLOG_SPE $\vec{C}$ IYPE

MSGLOG STRIN $\bar{N}$ 1

MSGLOG DT

MSGLOG_ERROR FLAG

MSGLOG_GEN_TŸPE

MSGLOG SEQ HUM

MSGLOG_SPEC _TYPE

NSGLOG_STRINTG1

MSGLOG OT

MSGLOG_ERROR_FLAG

MSGLOG GEN T YPPE

MSGLOG SEQ NUM

MSGLOG_SPEC TYPE

MSGLOG_STRINTG1

MSGLOG DT

MSGLOG_ERROR_FLAG

MSGLOG GEN. TYPE

MSGLOG_SEQ NUM

MSGLOG_SPE $\bar{C}$ TYPE

MSGLOG_STRINTG1

MSGLOG DT

MSGLOG_ERROR FLAG

MSGLOG GEN TYPE

MSGLOG_SEQ MUM

MSGLOG SPE $\bar{C}$ TYPE

MSGLOG STRINGI

MSGLOG DT

MSGLOG_ERROR FLAG

MSGLOG GEN TYYPE

MSGLOG SEQ NUM

USGLOG_SPEC TYPE

MSGLOG_SIRINTG1

MSGLOG_OT

MSGLOG_ERROR_FLAG

MSGLOG GEN TYPE

MSGLOG SEQ NUM

MSGLOG SPEC TYPE

MSGLOG_STRIN̄G1.

MSGLOG DT

MSGLOG_ERROR_FLAG
PCSDMS ${ }^{\sim}$ CL $871426618 \sim$ LL_E XIT $\sim 1^{\sim T E S T ~ D R U M ~ * ~}$

08-12-97 16:06:14

PCSDMS

65175

CL.

PCSDMS $\sim$ CL $871426618 \% L L H E$ XIT $\sim 1$ TEST DRUM *

08-12-97 16:06:16

$\gamma$

PCSDMS

65177

$\mathrm{CL}$.

PCSDMS ${ }^{-1} 871426623$ LL.WE XIT $\sim$ TEST DRUM -

08-12-97 16:06:20

$Y$

PCSDNS

65179

CL.

PCSOMS“CL. 871426627 LLW_E

XIT ${ }^{\sim \sim} 1^{\sim}$ TEST DRUM

08-12-97 96:06:22

$Y$

PCSDMS

65181

CL

PCSDMS“CL"871426628 LLHE

XIT $\sim$ TEST DRUM -

08-12-97 16:06:24

$Y$

PCSDMS

65183

CL

PCSDHS CL 871426629"LLWE

XIT $\sim$ १TEST DRUM -

08-12-97 16:06:26

$Y$

PCSDMS *

65185

$\mathrm{CL}$

PCSDMS“CL“871426629 LLW_E XIT $\sim 1$ TEST DRUM

08-12-97 16:06:28

$Y$

PCSOMS

65187

CL.

PCSDMS ${ }^{\circ L}$ \%871426631 LLW_E $X I T^{\sim \sim 1}$ TEST DRUM

08-12-97 16:06:30

$\gamma$

PCSDMS

65189

$\mathrm{CL}$

PCSDMS ${ }^{2 L}$ \%871426631 LLH_E XIT $\sim 1^{\sim T E S T ~ D R U M ~}$

08-12-97 16:12:07

$Y$

PCSDMS

65207

CL

PCSOMS CL 871426975 LLH_P UCK $^{\mu \sim}$ 1 OTP003

08-12-97 17:27:29

Y 


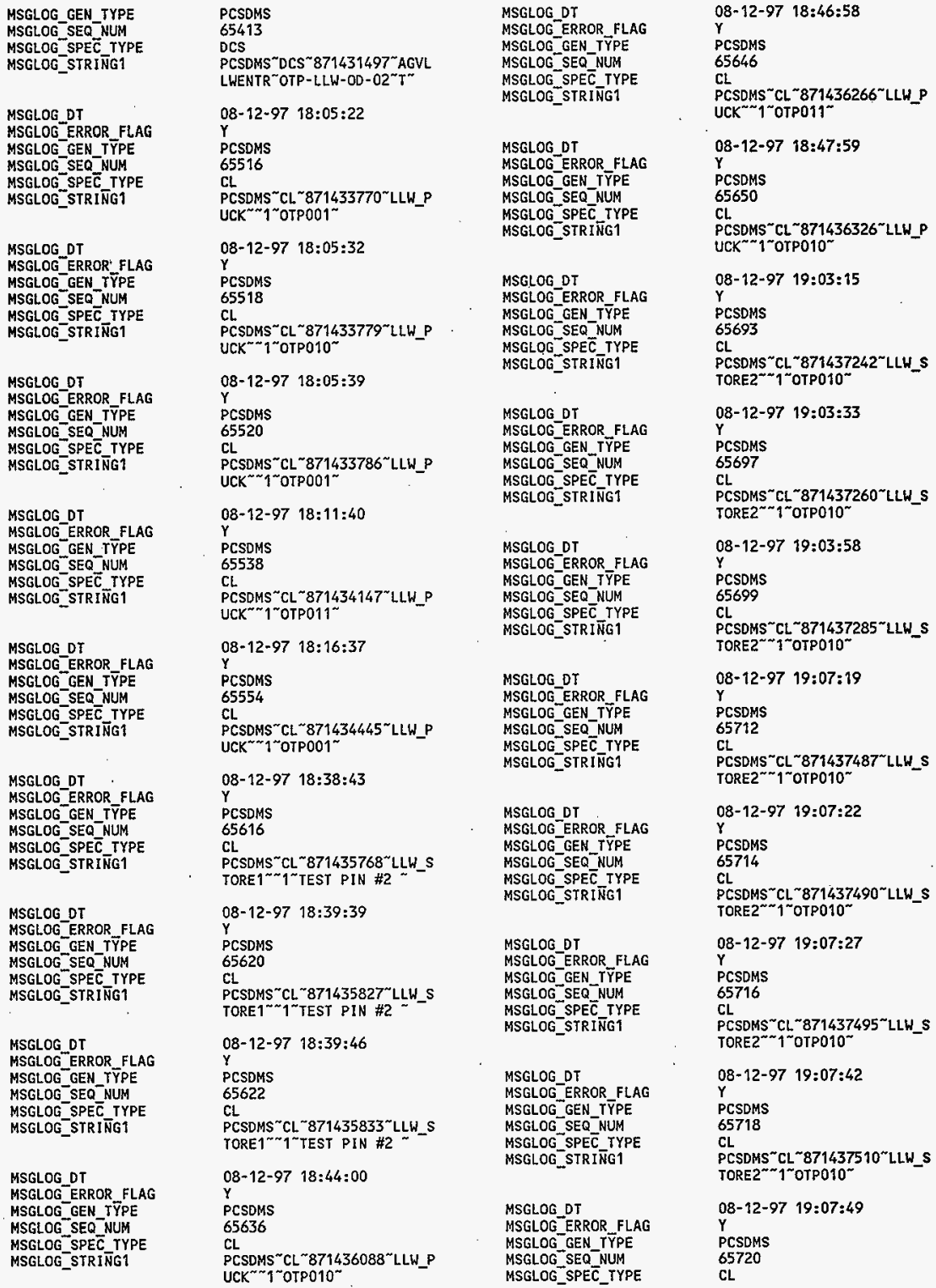


MSGLOG_STRING1

MSGLOG DT

MSGLOG ERROR FLAG

MSGLOG_GEN_TYYPE

MSGLOG SEO MUM

MSGLOG SPE $\bar{C}$ TYPE

MSGLOG_STRINGGI

\section{MSGLOG DT}

MSGLOG ERROR FLAG

MSGLOG_GEN_TYYPE

MSGLOG SEQ NUM

MSGLOG_SPEC

MSGLOG_STRIN̄GI

\section{MSGLOG DT}

MSGLOG_ERROR_FLAG

MSGLOG GEN TYYPE

MSGLOG SEQ NUM

MSGLOG_SPEC TYPE

MSGLOG_STRINGG1

MSGLOG DT

MSGLOG_ERROR_FLAG

MSGLOG GEN T TYPE

MSGLOG_SEQ_NUM

MSGLOG_SPEC TYPE

MSGLOG_STRINGG1

MSGLOG DT

MSGLOG_ERROR FLAG

MSGLOG_GEN TYYPE

MSGLOG SEQ_NUM

MSGLOG SPEC TYPE

MSGLOG_STRINGI

MSGLOG OT

MSGLOG_ERROR_FLAG

MSGLOG GEN TYPPE

MSGLOG SEQ NUM

MSGLOG_SPEC TYPE

MSGLOG_STRIN̄G1

MSGLOG DT

MSGLOGERROR FLAG

MSGLOG GEN T TYPE

MSGLOG_SEQ MUM

MSGLOG SPEC TYPE

MSGLOG_STRINGI

MSGLOG OT

MSGLOG_ERROR_FLAG

MSGLOG GEN TYPE

MSGLOG_SEQ_NUM

MSGLOG SPEC TYPE

MSGLOG_STRINTG1

MSGLOG DT

MSGLOG_ERROR FLAG

MSGLOG GEN TYYPE

MSGLOG SEQ NUM

MSGLOG SPE $\bar{C}$ TYPE

MSGLOG_STRINTG1

MSGLOG_DT

MSGLOG_ERROR_FLAG
PCSDMS“CL“871437516“LLW_S TORE2 $\sim 1 \sim$ OTP010"

08-12-97 19:08:07

$Y$

PCSDMS

65724

$\mathrm{CL}$

PCSDMS“CL 7871437534 "LLW_S

TORE2 $\sim 1$ "OTPO10"

08-12-97 19:08:23

$Y$

PCSDMS

65726

CL.

PCSDMS ${ }^{\sim} \mathrm{CL}^{-871437550}$ \%LL_S

TORE2 $~ 1 \%$ OTP010

08-12-97 19:08:53

PCSDMS

65730

$\mathrm{CL}$

PCSDMS ${ }^{\sim}$ \% $871437581 \sim \mathrm{LLWS}$

TORE2 $\sim 1 \sim$ OTP010

08-12-97 19:09:47

$\mathrm{Y}$

PCSDMS

65734

CL

PCSDMS“CL "871437635 LLW_S

TORE2 $\sim \sim 1 \sim$ OTPO10

08-12-97 19:09:57

$y$

PCSDMS

65736

CL

PCSDMS ${ }^{\sim}$ CL $871437644^{\sim}$ LLW_S

TORE2 $\sim 1$ \%TPO10

08-12-97 19:11:44

$\mathrm{Y}$

PCSDMS

65744

Cl.

PCSDMS "CL“871437751“LLW_S TORE2 $2^{N-1} 1^{\text {"OTP010" }}$

08-12-97 19:13:28

$Y$

PCSDMS

65750

CL

PCSDMS“CL ${ }^{2871437855 \% L L W ~ S ~}$ TORE2 ${ }^{\sim}{ }^{\sim}$ OTP010

08-12-97 19:13:58

$Y$

PCSDMS

65754

CL

PCSDMS“CL“871437884 "LLW_S TORE2 $\sim 1 \sim$ OTP010"

08-12-97 19:27:21

$Y$

PCSDMS

65790

$\mathrm{CL}$

PCSDMS“CL "871438689 LLW_P

UCK $\sim 1$ 1"OTP011

08-13-97 12:45:05

$Y$ $\begin{array}{ll}\text { MSGLOG_GEN_TYPE } & \text { PCSDMS } \\ \text { MSGLOG SEQ-NUM } & 67432\end{array}$

MSGLOG SPEC TYPE

MSGLOG STRINTGI

MSGLOG DT

MSGLOG ERROR FLAG

MSGLOG_GEN_T TPE

MSGLOG SEQ NUM

MSGLOG SPECे TYPE

MSGLOG_STRIÑG1

MSGLOG DT

MSGLOG_ERROR_FLAG

MSGLOG GEN TYPE

MSGLOG SEQ NUM

MSGLOG SPEC TYPE

MSGLOG_STRINGG!
PCSDMS ${ }^{\circ} \mathrm{CL}^{\sim} 871500950^{\circ} \mathrm{LLH} E$ XIT ${ }^{\sim} \sim$ TEST DRUM

$08-13-97 \quad 13: 51: 03$

$Y$

PCSDMS

67612

$\mathrm{CL}$

PCSDMS $\sim \mathrm{CL}^{\sim 871504908 \sim L L W ~ E ~}$ $\mathrm{XIT}^{\sim} \mathrm{T}^{\sim}$ TEST DRUM

$08-14-97 \quad 09: 15: 55$

$Y$

PCSDNS

69597

ct.

PCSDMS־CL $871574800 \%$ LLHE NTRY ${ }^{\sim-1}$-OTP-LLW-OD-04 
CHSEYA 14 LLW GLOVEBOX OTP DMS DATABASE FOLLOWING CASE 4 8/18/97

CON PKG ID

CON CNTYP $C D^{\circ}$

CONPKG ST̃ATUS

CON'SIZE_DESCR

CON_TARE_WGT

CONEXT PKG ID

CONEXT USE-CD

CONEXT_WRAP_STAT_CD

RDET PKG ID

RDET_SWTYP_GROUP

CONR FROM PKG ID

CONR_TOP $\bar{K} \mathrm{CB}_{-} \mathrm{ID}$

CONR_DT

CONR_REL_CD

CON PKG 10

CON ACCUM DT

CON_CHEM_NATURE_CD

$\mathrm{CON}^{-} \mathrm{CNTYP}$ CD

CONGENER_WASTE_DESCR $Y Y \bar{Y}$

CON_PHYS_STATE_CD

CON PKG DT

CON-PKG STATUS

CON_PWTYP CD

CON SIZE DESCR

CON_TARE_WGT

CONEXT PKG ID

CONEXT_CONTAM_FLAG

CONEXT FILLER WGT

CONEXT PROF FLAG

CONEXT_PROF_ID

CONEXT USE $\overline{C D}$

CONEXT_WRAP_STAT_CD

HDET PKG ID

HDET-DESIG_CD

HDET_WASTE_VOL

RDET PKG ID

RDET_BG DOSE RATE

RDET_SWIMS_CD

RDET SWTYP-CD

RDET_SWTYP GROUP

RDET_VOID_CD

RDET WASTE MAKEUP

RDET_WRAP_CAT

PHYS PKG ID

PHYS_COMP DESCR

PHYS COMP VOL PCT

PHYS_COMP_WGT

PHYS PKG_ID

PHYS COMP DESCR

PHYS_COMP VOL PCT

PHYS_COMP_WGT

PHYS_PKG_ID

PHYS_COMP_DESCR

PHYS_COMP_VOL_PCT PHYS_COMP_WGT

PHYS PKG ID

PHYS_COMP_DESCR

PHYS COMP VOL PCT

PHYS_COMP_WGT
OTP-LLW-OD-01
DM fo possing PHYSPKG_ID

B5 GALLON of rescens Entry PHYSCOMP-VOLPCT

31 (ETR)

OTP-LLW-OD-01.

$\mathrm{PD}$

I

OTP-LLW-OD-01

LLW

OTP-LLW-WP-01

OTP-LLW-OD-01

08-12-97 10:08:07

$\mathrm{M}$

PHYS PKG ID

PHYS_COMP_DESCR

PHYS COMP VOL PCT

PHYS_COMP_WGT

CONR FROM PKG ID

CONR_TO P $\overline{K G}$. $\bar{D}$

CONR DT

CONR_REL_CD

CONR_FROM PKG ID

CONR_TO_PKG_ID

CONRDT

OTP- $L L W-O D-02$

$\frac{018-18-97 \cdot 15: 56: 25}{10}$

I0.

DN?

CONR_REL_CD

CONR FROM PKG ID

CONR_TO_PKG_ID

CONR DT

CONR_REL_CD

s

08-18-97 15:56:25

(d.) Rest ant airlocic

is

85 GaLLON

31.

OTP-LLW-OD-02

$Y$.

10

NRLLW1 -

(PD) $O K$

IS

OIP-1LW-OD-02

$\mathrm{RE}^{\prime}$

.28

OTP-LLW-OD-02

2

$\mathrm{NC}$

2A

GRAVEL

( $\mathrm{F}$ C

OTP-LLW-OD-02

CLOTH/RAGS/NYLON

28.444\%

$27.6^{2}$

OTP-LLW-OD-02

CONWEB PADS -

23.956

127.5

OTP-LLW-OD-02

METAL/IRON/GALVANIZED/SHE

ET.

$4.444^{\prime}$

OTP-LLW-OD-02

PAPER/CARDBOARD

10.667

41
NDA THERMALL POWER
CONEXT PKG ID

CONEXT USE CD

CON PKG ID

CON CNTYP CD

CON PKG STATUS

CON SIZE DESCR

CON_TARE_WGT

CONEXT PKG ID

CONEXT USE CD

CONR_EROM_PKG_ID

CONR TO PKG ID

CONRDT

CONR_REL_CD

NDE_PKG_ID

NDE DT

NDE_COMMENTS

NDE_OPER_ID

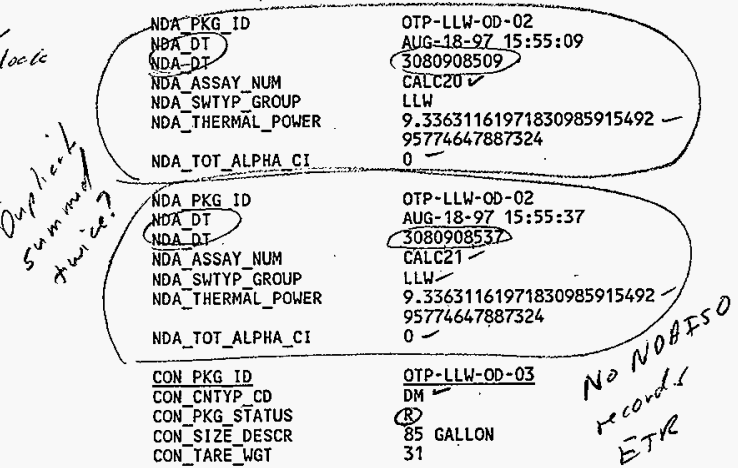

OTP- $11 W-00-02$

PLASTIC/POLYURATHANE

32 -

85.1

OTP-LLW-OD-02

WATER

.489

$3.9^{-}$

OTP-LLW-WP-02

OTP-LLW-OD-02

08-19-27 22:35:12

C -

OTP-LLW-WP-03

OTP-LLW-CD-02

08-19-27 18:59:10

$\mathrm{c}-$

OTP-LLW'WP-O4',

OTP-LLW-OD-02

$08-19-27 \quad 17: 02: 51$

C

CONEXT WRA $\bar{P}$ STAT CD

OTP-LLW-OD-03

from resean 5,00

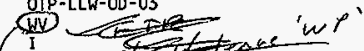
CONEXT WRAP STAT CD

DM

(B)

85 GALLON

31

OIP-LLW-0D-04

GV

OTP-LLW-WP-O4

$\left.\begin{array}{l}\text { OTP-LLW-WP-04 } \\ \text { OTP-LLW-OD-04 } \\ 08-14-9709: 07: 47\end{array}\right\}$ to to anuallow to

$\mathrm{M}$

OTP-LLW-OD-04

09-12-96 13:11:35

OTP-LLW-WP-04 1ST IMAGE

COMMENTS

USER 1 


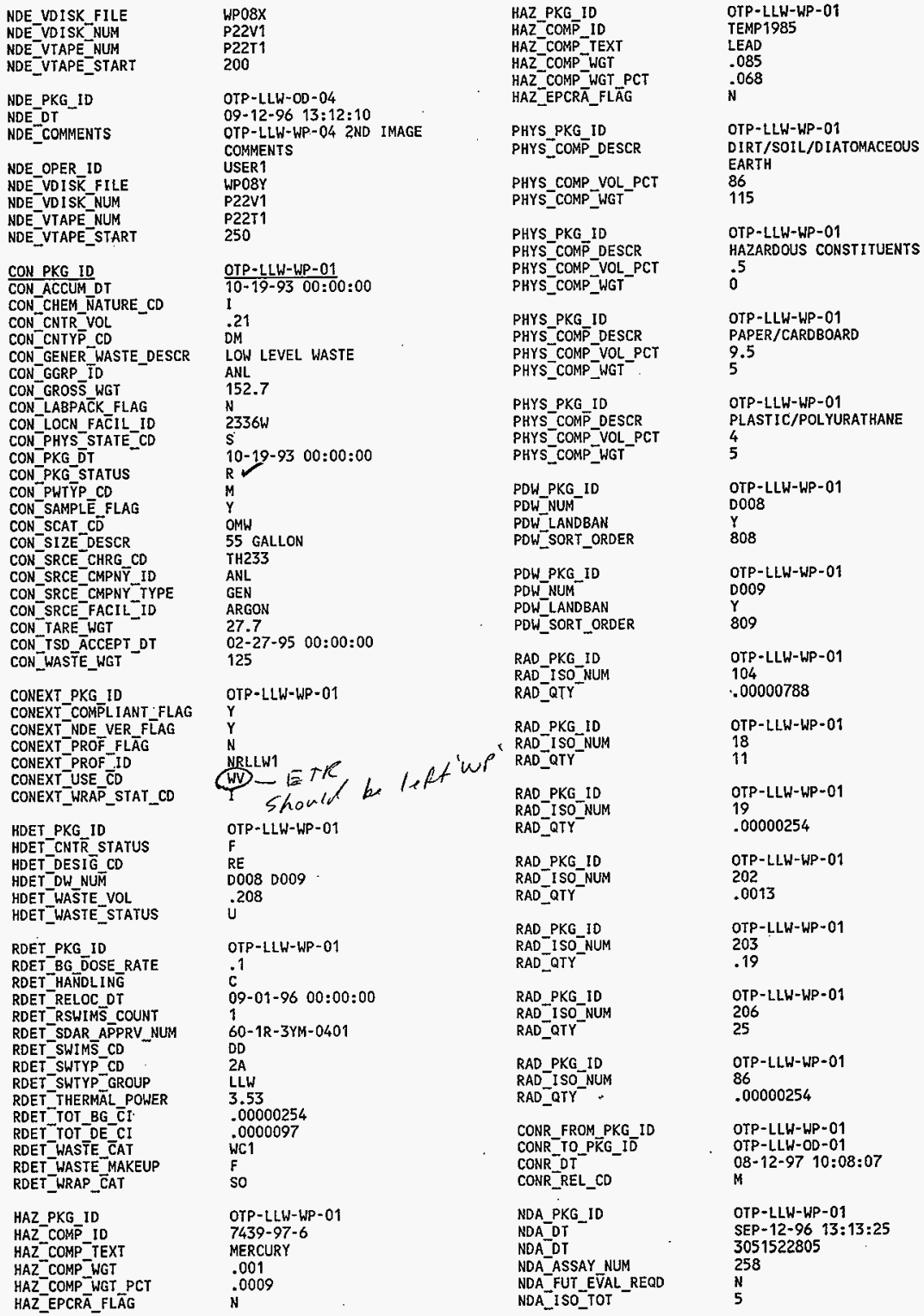




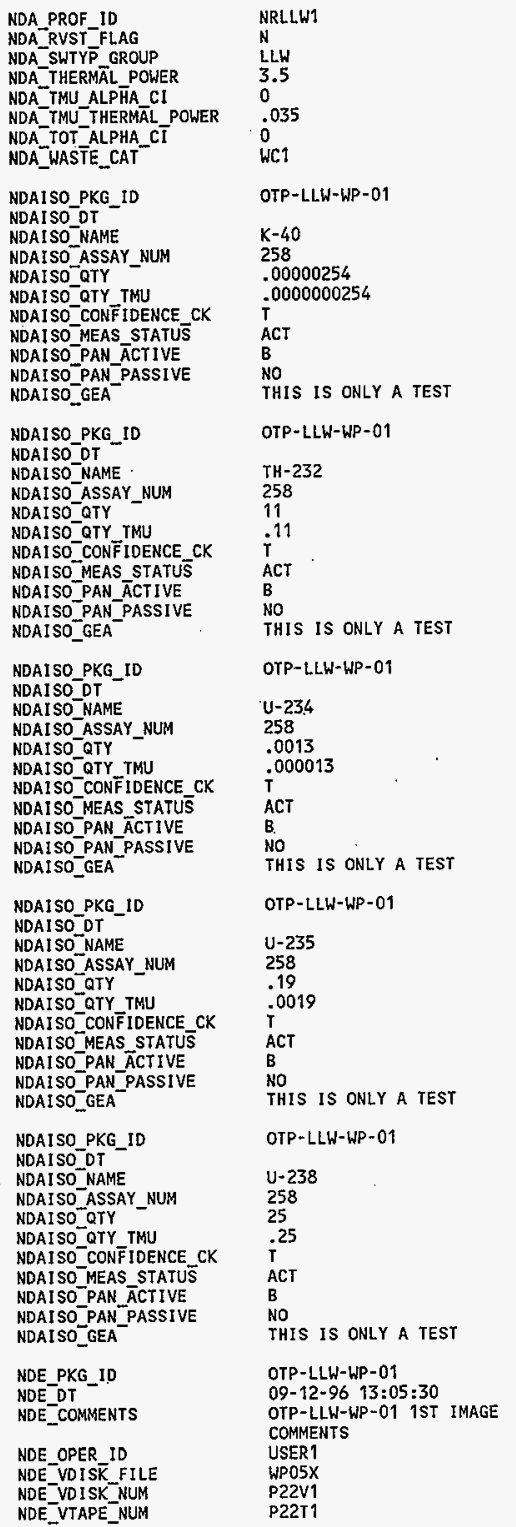

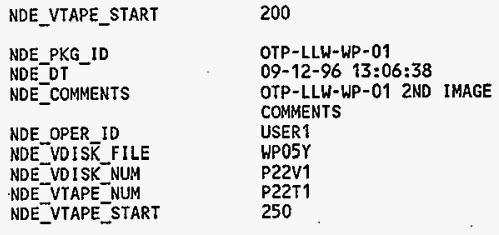

1

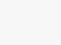




\begin{tabular}{|c|c|}
\hline $\begin{array}{l}\text { HAZ_PKG_ID } \\
\text { HAZ_COMP_ID } \\
\text { HAZ_COMP_TEXT } \\
\text { HAZ_COMP_WGT }\end{array}$ & $\begin{array}{l}\text { OTP-LLW-WP-02 } \\
\text { TEMP } 3413 \\
\text { SODIUM CHLOR IDE } \\
.04\end{array}$ \\
\hline $\begin{array}{l}\text { PHYS_PKG_ID } \\
\text { PHYS_COMP_DESCR } \\
\text { PHYS_COMP_VOL_PCT } \\
\text { PHYS_COMP_WGT- }\end{array}$ & $\begin{array}{l}\text { OTP-LLW-WP-O2 } \\
\text { CONWEB PADS } \\
93 \\
127.5\end{array}$ \\
\hline $\begin{array}{l}\text { PHYS_PKG_ID } \\
\text { PHYS_COMP_DESCR } \\
\text { PHYS_COMP_VOL_PCT } \\
\text { PHYS_COMP_WGT }\end{array}$ & $\begin{array}{l}\text { OTP-LLW-WP-02 } \\
\text { HAZARDOUS CONSTITUENTS } \\
5 \\
.64\end{array}$ \\
\hline $\begin{array}{l}\text { PHYS_PKG_ID } \\
\text { PHYS_COMP_DESCR } \\
\text { PHYS_COMP_VOL_PCT } \\
\text { PHYS_COMP_WGT }\end{array}$ & $\begin{array}{l}\text { OTP-LLW-WP-02 } \\
\text { WATER } \\
2 \\
3.9\end{array}$ \\
\hline $\begin{array}{l}\text { RAD_PKG_1D } \\
\text { RAD_ISO_NUM } \\
\text { RAD_QTY }\end{array}$ & $\begin{array}{l}\text { OTP-LLW-WP-02 } \\
12 \\
.00033\end{array}$ \\
\hline $\begin{array}{l}\text { RAD_PKG_ID } \\
\text { RAD_ISO_NUM } \\
\text { RAD_QTY }\end{array}$ & $\begin{array}{l}\text { OrP-LLW-WP-02 } \\
152 \\
.000005\end{array}$ \\
\hline $\begin{array}{l}\text { RAD_PKG_ID } \\
\text { RAD_ISO_NUM } \\
\text { RAD_QTY }\end{array}$ & $\begin{array}{l}\text { OTP-LLW-WP-02 } \\
153 \\
.00018\end{array}$ \\
\hline $\begin{array}{l}\text { RAD_PKG_ID } \\
\text { RAD_ISO_NUN } \\
\text { RAD_QTY } \\
\text { RAD_UNKNOWN }\end{array}$ & $\begin{array}{l}\text { OTP-LLW-WP-02 } \\
19 \\
035405\end{array}$ \\
\hline $\begin{array}{l}\text { RAD_PKG_ID } \\
\text { RAD_ISO_MUM } \\
\text { RAD_QTY }\end{array}$ & $\begin{array}{l}\text { OTP-LLW-WP-02 } \\
43 \\
.00045\end{array}$ \\
\hline $\begin{array}{l}\text { RAD_PKG_ID } \\
\text { RAD_ISO_NUM } \\
\text { RAD_QTY }\end{array}$ & $\begin{array}{l}\text { OTP-LLW-WP-02 } \\
53 \\
.00014\end{array}$ \\
\hline $\begin{array}{l}\text { RAD_PKG_ID } \\
\text { RAD_ISO_NUM } \\
\text { RAD_QTY }\end{array}$ & $\begin{array}{l}\text { OTP-LLW-WP-02 } \\
56 \\
.0003\end{array}$ \\
\hline $\begin{array}{l}\text { RAD_PKG_ID } \\
\text { RAD_ISO_NUM } \\
\text { RAD_QTY }\end{array}$ & $\begin{array}{l}\text { OTP-LLW-WP-02 } \\
91 \\
.034\end{array}$ \\
\hline $\begin{array}{l}\text { CONR_FROM_PKG_ID } \\
\text { CONR_TO_PKG_ID } \\
\text { CONR_DT } \\
\text { CONR_REL_CD }\end{array}$ & $\begin{array}{l}\text { OTP-LLW-WP-02 } \\
\text { OTP-LLW-OD-02 } \\
08-19-27 \quad 22: 35: 12 \\
\text { C }\end{array}$ \\
\hline $\begin{array}{l}\text { CONR_FROM_PKG_ID } \\
\text { CONR_TO_PKG_ID } \\
\text { CONR_DT } \\
\text { CONR_REL_CD }\end{array}$ & $\begin{array}{l}\text { OTP-LLW-WP-02 } \\
\text { OTP6-97-000001 } \\
08-14-27 \quad 17: 26: 29 \\
s\end{array}$ \\
\hline $\begin{array}{l}\text { CONR_FROM_PKG_ID } \\
\text { CONR_TO_PKG_ID } \\
\text { CONR_DT } \\
\text { CONR_REL_CD }\end{array}$ & $\begin{array}{l}\text { OTP-LLW-WP-02 } \\
\text { OTP6-97-000002 } \\
08-14-27 \quad 17=26=00 \\
S\end{array}$ \\
\hline $\begin{array}{l}\text { CONR_FROM_PKG_ID } \\
\text { CONR_TO_PKG_ID } \\
\text { CONR_DT } \\
\text { CONR_REL_CD }\end{array}$ & $\begin{array}{l}\text { OrP-LLW-WP-02 } \\
\text { OTP6-97-000003 } \\
08-14-27 \quad 17: 27: 06 \\
\text { s }\end{array}$ \\
\hline $\begin{array}{l}\text { NDA_PKG_10 } \\
\text { NDA_DT } \\
\text { NDADT } \\
\text { NDA_ASSAY_NUN }\end{array}$ & $\begin{array}{l}\text { OTP-LLW-WP-02 } \\
\text { SEP- } 12-9613: 47: 13 \\
3051524833 \\
265\end{array}$ \\
\hline
\end{tabular}

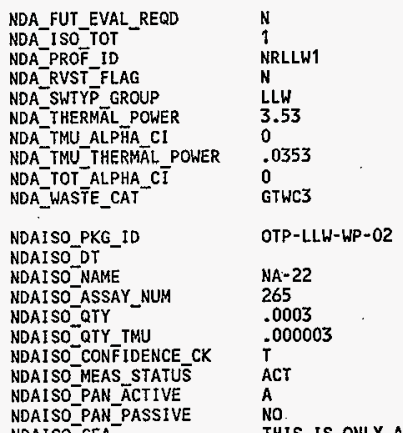

NDAISO_GEA

NDE. PKG_ID

NDE DT

NOE_COMMENTS

NDE OPER ID

NDE_VDISK FILE

NDE_VDISK_NUM

NDE VTAPE NUM

NDE_VTAPE_START

NDE_PKG_ID
NDE_DT

NDE_COMMENTS

NDE_OPER ID

NDE VDISK FILE

NDE VDISK NUM

NDE_VTAPE_NUM

NDE_VIAPE_START

CON PKG ID
CON_ACCUM DT
CON_CNTR_VOL

CON CNTYP CD

CON DATA QUAL CD

CON GENER WASTE_DESCR

CON_GGRP ID

CON_GROSS WGT

CON LABPACK FLAG

CON_LOCN_FACIL ID

CON_PHYS_STATE_CD

CON PKG $\bar{D} T$

CON PKG_STATUS

CON PWTYY CD

CON_SAMPLE FLAG

CONSSIZE_DESCR

CON SRCE_CHRG CO

CON_SRCE_CNPNY

CON SRCE_CMPNY TYPE

CON SRCE FACIL ID

CON_TARE_WGT

CON_TSO ACCEPT_DT

CON_WASTEE_WGT

CONEXT_PKG_ID
CONEXT_COMPLIANT_FLAG
CONEXT_NDE_VER_FLAG
CONEXT_PROF_FLAG
CONEXT_PROF_ID
CONEXT_USE_CD
CONEXT_WRAP_STAT_CD
RDET_PKG_ID
RDET_BG_DOSE_RATE

OTP-LLW-WP-02

$09-12-96 \quad 13: 39: 22$

OTP-LLW-WP-02 IST IMAGE

COMMENTS

USER 1

WP11X

P22V 1

P22T 1

200

OTP-LLW-WP-02

09-12-96 13:40:09

OTP-1LW-WP-02 2ND IMAGE COMMENTS

USER 1

WP11Y

P22V1

P22T1

250

OTP-LLW-WP-03

05-29-90 00:00:00

.21

DM

AR

INNER

OTHER

163.02 .

N

2336W

$\mathrm{s}$

05-29-90 00:00:00

R

R

N

55 GALLON

B80545

PNL

GEN

324

27

$05-29-90 \quad 00: 00: 00$

136

OTP-LLW-WP-03

$Y$
$Y$

Y

NRLLW1

PU

I

OTP-LLW-WP-03

7 


\begin{tabular}{|c|c|}
\hline 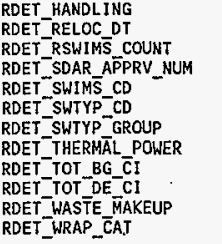 & $\begin{array}{l}C \\
05-29-90 \quad 00: 00: 00 \\
1 \\
2 A-1 A-1 A-0 \\
C E \\
2 A \\
L L W \\
3.53 \\
.01212 \\
1 \\
F \\
D M\end{array}$ \\
\hline $\begin{array}{l}\text { PHYS_PKG_ID } \\
\text { PHYS_COMP_DESCR } \\
\text { PHYS_COMP_VOL_PCT } \\
\text { PHYS_COMP_WGT }\end{array}$ & $\begin{array}{l}\text { OTP-LLW-WP-03 } \\
\text { CLOTH/RAGS/NYLON } \\
10 \\
14\end{array}$ \\
\hline $\begin{array}{l}\text { PHYS_PKG_ID } \\
\text { PHYS_COMP_DESCR } \\
\text { PHYS_COMP_VOL_PCT } \\
\text { PHYS_COMP_WGT- }\end{array}$ & $\begin{array}{l}\text { OTP-LLW-WP-03 } \\
\text { PAPER/CARDBOARD } \\
30 \\
41\end{array}$ \\
\hline $\begin{array}{l}\text { PHYS_PKG_ID } \\
\text { PHYS_COMP_DESCR } \\
\text { PHYS_COMP_VOL_PCT } \\
\text { PHYS_COMP_WGT }\end{array}$ & $\begin{array}{l}\text { OTP-LLW-WP-03 } \\
\text { PLAST IC/POLYURATHANE } \\
60 \\
81\end{array}$ \\
\hline $\begin{array}{l}\text { RAD_PKG_ID } \\
\text { RAD_ISO_NUM } \\
\text { RAD_QTY } \\
\text { RAD_UNKNOWN }\end{array}$ & $\begin{array}{l}\text { OIP-LLW-WP-03 } \\
19 \\
.01212 \\
.000324\end{array}$ \\
\hline $\begin{array}{l}\text { RAD_PKG_ID } \\
\text { RAD_ISO_NUM } \\
\text { RAD_QTY }\end{array}$ & $\begin{array}{l}\text { OTP-LLW-WP-03 } \\
3 \\
.00006\end{array}$ \\
\hline $\begin{array}{l}\text { RAD_PKG_ID } \\
\text { RAD_ISO_NUM } \\
\text { RAD_QTY }\end{array}$ & $\begin{array}{l}\text { OTP-LLW-WP-03 } \\
8 \\
.006\end{array}$ \\
\hline $\begin{array}{l}\text { CONR_FROM_PKG_ID } \\
\text { CONR_TO_PKG_ID } \\
\text { CONR_DT-_ } \\
\text { CONR_REL_CD }\end{array}$ & $\begin{array}{l}\text { OTP-LLW-WP-03 } \\
\text { OTP-LLW-OD-02 } \\
08-19-27 \quad 18: 59=10 \\
\text { C }\end{array}$ \\
\hline 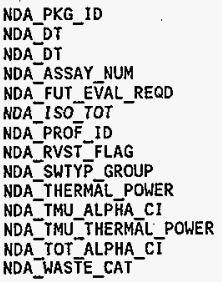 & $\begin{array}{l}\text { OTP-LLW-WP-03 } \\
\text { SEP- } 12-96 \quad 13: 34: 22 \\
3051524062 \\
262 \\
N \\
2 \\
\text { NRLLW1 } \\
N \\
\text { LLW } \\
5.45 \\
0 \\
.0545 \\
0 \\
\text { GTWCS }\end{array}$ \\
\hline $\begin{array}{l}\text { NDAISO_PKG_ID } \\
\text { NDAISO_DT } \\
\text { NDAISO_NAME } \\
\text { NDAISO_ASSAY_NUM } \\
\text { NDAISO_GTY - } \\
\text { NDAISO_OTY_IMU } \\
\text { NDAISO_CONFIDENCE_CK } \\
\text { NDAISO_MEAS_STAYUS } \\
\text { NDAISO_PAN_ACTIVE } \\
\text { NDAISO_PAN_PASSIVE } \\
\text { NDAISO_GEA }\end{array}$ & $\begin{array}{l}\text { OTP-LLW-WP-O3 } \\
C S-137 \\
262 \\
-006 \\
.00006 \\
\text { T } \\
\text { ACT } \\
\text { B } \\
\text { NO } \\
\text { THIS IS ONLY A TEST }\end{array}$ \\
\hline $\begin{array}{l}\text { NOAISO_PKG_ID } \\
\text { NOAISO_DT } \\
\text { NDAISO_NAME } \\
\text { NDAISO_ASSAY_NUM }\end{array}$ & $\begin{array}{l}\text { OTP-LLW-WP-03 } \\
\text { SR-90 }\end{array}$ \\
\hline
\end{tabular}

$\begin{array}{ll}\text { NDAISO_QTY } & .00006 \\ \text { NDAISO_QTY_TMU } & .0000006 \\ \text { NDAISO_CONFIDENCE_CK } & \text { T } \\ \text { NDAISO_MEAS_STATUS } & \text { ACT } \\ \text { NDAISO_PAN_ACTIVE } & \text { B } \\ \text { NDAISO_PAN_PASSIVE } & \text { NO }\end{array}$

NORISO_GEA THIS IS ONLY A TEST

NDE_PKG_ID

NDEDT

NDE_COMMENTS

NDE OPER ID

NDE VDISK_FILE

NDE VOISK NUM

NDE_VTAPE_NUM

NDE_VIAPE_START

NDE_PKG_1D

NDE-DT

NDE_COMMENTS

NDE_OPER_ID

NDE VDISK FILE

NDE VDISK NUM

NDE_VTAPE_NUM

NDE-VIAPE_START

OTP-LLW-WP-03

09-12-96 13:31:45

OTP-LLW-WP-03 1ST IMAGE COMMENTS

USER 1

WP0 $4 X$

P22V1

P22T1

200

OTP-LLW-WP-03

09-12-96 13:33:16

OTP-LLW-WP-03 2ND IMAGE COMMENTS

USER1

WP04Y

P22V1

P22T1

250

CON PKG ID
CON_ACCUM DT
CON_CNTR VOL
CON_CNTYP CD
CON_DATA QDUL_CD
CON_GENER WASTE_DESCR
CON_GGRP DD
CON_GROSS WGT

OTP-LLW-LP-O4

07-05-90 00:00:00

.21

DH

AR

INWER

OTHER

358.34

CON LABPACK FLAG

CON_LOCN FACIL_ID

CON PHYS STATE-CD

CON_PKG_DT

CONPKG STATUS

CON PWTYYP_CD

CON_SAMPLE FLAG

CON'SCAT CD D

CON_SIZE_DESCR

CON SRCE-CHRG CD

CON ${ }^{-}$SRCE CMPNY $\vec{Y}$ ID

CON_SRCE_CMPNY_TYPE

CON SRCE FACIL ID

CON TARE WGT

CON TSD ACCEPT DT

CON_WASTE_WGT

CONEXT_PKG_ID

CONEXT COMPLLIANT FLAG

CONEXT_NDE_VER_FIAG

CONEXT-PROF FLAG

CONEXT PROF ID

CONEXT USE $\bar{C} D$

CONEXT_WRAP्PSSTAT_CO

HDET_PKG_ID

HDETDESĪG CD

HDET_DW_NUM

HDET_WASTE_VOL

RDET_PKG_ID

RDET_ASSAY DT

RDET BG DOSEE RATE

RDET_HAÑDLING

RDET RELOC DT

RDET_RSWIMS_COUNT

RDET SDAR APPRV NUM

RDET'SWIMS CD

RDET_SWTYP_CD

RDET SWTYP GROUP

RDET_THERMÁ_POWER
A

2336W

07-05-90 00:00:00

R

N

OMW

55 GALLON

B3314

WHC

GEN

324

07-05-90 00:00:00

32.1

OTP-LLW-WP-04
Y
N
NRLLW1
PU
I

OTP-LLW-WP-04

RE

D008 WT01

.07

OTP-L.LW-WP-04

07-10-49 00:00:00

C

08-10-93 00:00:00

1

4- $1 A-7 V M-0$

DS

$2 A$

LLW

3.53 


\begin{tabular}{|c|c|}
\hline $\begin{array}{l}\text { RDET_TOT_ALPHA_CI } \\
\text { RDET_TOT_BG_CI } \\
\text { RDET_TOT_DE_CI } \\
\text { RDET_TOT_PE_CI } \\
\text { RDET_TOT_PU_FGE } \\
\text { RDET_WASTE_MAKEUP } \\
\text { RDET_WRAP_CAT }\end{array}$ & $\begin{array}{l}0 \\
0 \\
0 \\
0 \\
0 \\
F \\
P B\end{array}$ \\
\hline $\begin{array}{l}\text { HAZ_PKG_ID } \\
\text { HAZ_COMP_ID } \\
\text { HAZ_COMP_TEXT } \\
\text { HAZ_COMP_WGI } \\
\text { HAZ_COMP_WGI_PCr }\end{array}$ & $\begin{array}{l}\text { OTP-LLW-WP-04 } \\
\text { TEMP0440 } \\
\text { ACID } \\
1.5 \\
5\end{array}$ \\
\hline $\begin{array}{l}\text { HAZ_PKG_ID } \\
\text { HAZ_COMP_ID } \\
\text { HAZ_COMP_JEXT } \\
\text { HAZ_COMP_WGT } \\
\text { HAZ_COMP_WGT_PCT }\end{array}$ & $\begin{array}{l}\text { OTP-LLW-WP- } 04 \\
\text { TEMP } 1985 \\
\text { LEAD } \\
8.9 \\
28\end{array}$ \\
\hline $\begin{array}{l}\text { HAZ_PKG_ID } \\
\text { HAZ_COMP_ID } \\
\text { HAZ_COMP_TEXT } \\
\text { HAZ_COMP_WGT } \\
\text { HAZ_COMP_WGT_PCT }\end{array}$ & $\begin{array}{l}\text { OTP-LLW-WP- } 04 \\
\text { TEMP } 2628 \\
\text { OIL } \\
1.9 \\
6\end{array}$ \\
\hline $\begin{array}{l}\text { HAZ_PKG_ID } \\
\text { HAZ_COMP_ID } \\
\text { HAZ_COMP_TEXT } \\
\text { HAZ_COMP_WGT } \\
\text { HAZ_COMP_WGT_PCT }\end{array}$ & $\begin{array}{l}\text { OTP-LLW-WP-04 } \\
\text { TENP3530 } \\
\text { SOLVENTS } \\
2.1 \\
6\end{array}$ \\
\hline $\begin{array}{l}\text { PHYS_PKG_ID } \\
\text { PHYS_COMP_DESCR } \\
\text { PHYS_COMP_VOL_PCT } \\
\text { PHYS_COMP_WGT }\end{array}$ & $\begin{array}{l}\text { OTP-LLW-WP-04 } \\
\text { CLOTH/RAGS/NYLON } \\
48 \\
13.6\end{array}$ \\
\hline $\begin{array}{l}\text { PHYS_PKG_ID } \\
\text { PHYS_COMP_DESCR } \\
\text { PHYS_COMP_VOL_PCT } \\
\text { PHYS_COMP_WGT }\end{array}$ & $\begin{array}{l}\text { OTP-LLW-WP- } 04 \\
\text { HAZARDOUS CONSTITUENTS } \\
5 \\
.64\end{array}$ \\
\hline $\begin{array}{l}\text { PHYS_PKG_ID } \\
\text { PHYS_COMP_DESCR } \\
\text { PHYS_COMP_VOL_PCT } \\
\text { PHYS_COMP_WGT }\end{array}$ & $\begin{array}{l}\text { OTP-LLW-WP-04 } \\
\text { PLASTIC/POLYURATHANE } \\
20 \\
4.1\end{array}$ \\
\hline $\begin{array}{l}\text { RAD_PKG_ID } \\
\text { RAD_ISO_NUM } \\
\text { RAD_QTY }\end{array}$ & $\begin{array}{l}\text { OTP-LLW-WP-04 } \\
13 \\
.000004\end{array}$ \\
\hline $\begin{array}{l}\text { RAD_PKG_ID } \\
\text { RAD_ISO_NUM } \\
\text { RAD_QTY } \\
\text { RAD_UNKNOWN }\end{array}$ & $\begin{array}{l}\text { OTP-LLW-WP-O4 } \\
19 \\
.000233 \\
.0000007163\end{array}$ \\
\hline $\begin{array}{l}\text { RAD_PKG_ID } \\
\text { RAD_ISO_NUM } \\
\text { RAD_QTY }\end{array}$ & $\begin{array}{l}\text { OTP-LLW-WP-04 } \\
31 \\
.000032\end{array}$ \\
\hline $\begin{array}{l}\text { RAD_PKG_ID } \\
\text { RAD_ISO_NUM } \\
\text { RAD_QTY }\end{array}$ & $\begin{array}{l}\text { OTP-LLW-WP-04 } \\
4 \\
.000083\end{array}$ \\
\hline $\begin{array}{l}\text { RAD_PKG_ID } \\
\text { RAD_ISO_NUM } \\
\text { RAD_QTY }\end{array}$ & $\begin{array}{l}\text { OTP-LLW-WP-04 } \\
40 \\
.000002\end{array}$ \\
\hline $\begin{array}{l}\text { RAD_PKG_ID } \\
\text { RAD_1SO_NUM } \\
\text { RAD_QTY }\end{array}$ & $\begin{array}{l}\text { OTP-LLW-WP-04 } \\
53 \\
.000001\end{array}$ \\
\hline $\begin{array}{l}\text { RAD_PKG_ID } \\
\text { RAD_ISO-NUM } \\
\text { RAD_QIY- }\end{array}$ & $\begin{array}{l}\text { OTP-LLW-WP- } 04 \\
8 \\
.000013\end{array}$ \\
\hline RAD_PKG & OTP-LLW-WP- 04 \\
\hline
\end{tabular}

RAD_ISO_NUM 9

RAD_QTY $\quad .000001$

CONR_FROM_PKG $10 \quad$ OTP-LLW-WP-04

CONR TO_PKG_ID OTP-LLW-OD-02

CONR_DT ${ }^{-}$08-19-27 $\$ 7: 02: 51$

CONR_REL_CD

CONR FROM PKG $10 \quad$ OTP-LLW-WP-04

CONR TOP PKG_ID OTP-LLW-OD-04

CONR DT

CONR_REL_CD

CONR FROM PKG ID

CONR_TO_PKG_ID

CONR DT.

CONR_REL_CD

CONR_FROM_PKG_ID

CONR_TO_PKG_I $\bar{D}$

CONR_DT

CONR_REL_CD

CONR_FROM_PKG_ID

CONR TO PKGGID

CONR_DT

CONR_REL_CD

NDA_PKG_ID

NDA-OT

NDA-DT

NDA-ASSAY NUM

NDA_FUT_EVAL_REQD

NDA_ISO-TOT

NDA PROF ID

NDA_RVST FLAG

NDA SWTY $\bar{P}$ GROUP

NDA THERMĀL POWER

NDA_TMU_ALPHA_CI

NDA TMU THERMÁL POWER

NOA_TOT_ALPHA_CI

NDA_WASTE_CAT

NDAISO_PKG_ID

NDAISO DT

NOAISO NAME

NDAISO_ASSAY_NUM

NDAISO-OTY

NDAISO-QTY TMU

NDAISO-CONFIDENCE CK

NDAISO MEAS STATUS

NDAISO_PAN_ $\bar{A} C T I V E$

NDAISO PAN PASSIVE

NDAISO_GEA

NDAISO_PKG_ID

NDAISO DT

NDA1 SO NAME

NDAISO ASSAY NUM

NDAISO-QTY

NDAISO-OTY TMU

NDAISO CONFIDENCE CK

NDAISO_MEAS STATUS

NDAISO PAN ÄCTIVE

NDAISO_PAN_PASSIVE

NDAISO_GEA

NDAISO_PKG_ID

MDAISO DT

NDAISO NAME

NDAISO ASSAY NUM

NDAISO QTY

NDAI SO QTY TMU

NDAISO CONFIDENCE CK

NDAISO MEAS STATUS

NDAISO PAN ACTIVE

NDAISO PAN PASSIVE
08-14-97 09:07:47

M

OTP-LLW-WP-04

OTP6-97-000004

08-15-27 17:56:20

$\mathrm{s}$

OTP-LLW-WP- 04

OTP6-97-000005

08-15-27 17:57:08

s

OTP-LLW-WP-04

OTP6-97-000006

08-15-27 17:57:37

$\mathrm{s}$

OTP-LLW-WP-04

SEP-12-96 13:18:27

उ051523107

261

4

NRLLW1

N

LLW

5.45

0

0

GIWC3

OTP-LLW-WP-04

Co- 60

261

.000004

.00000004

$T$

ACT

A

THIS IS ONLY A TEST

OTP-LLW-WP-04

Cs -137

261

.000013

.00000013

T

ACT

A

THIS IS ONLY A TEST

OTP-LLW-WP-04

EU-154

261

.000002

.00000002

T

ACT

A

NO 


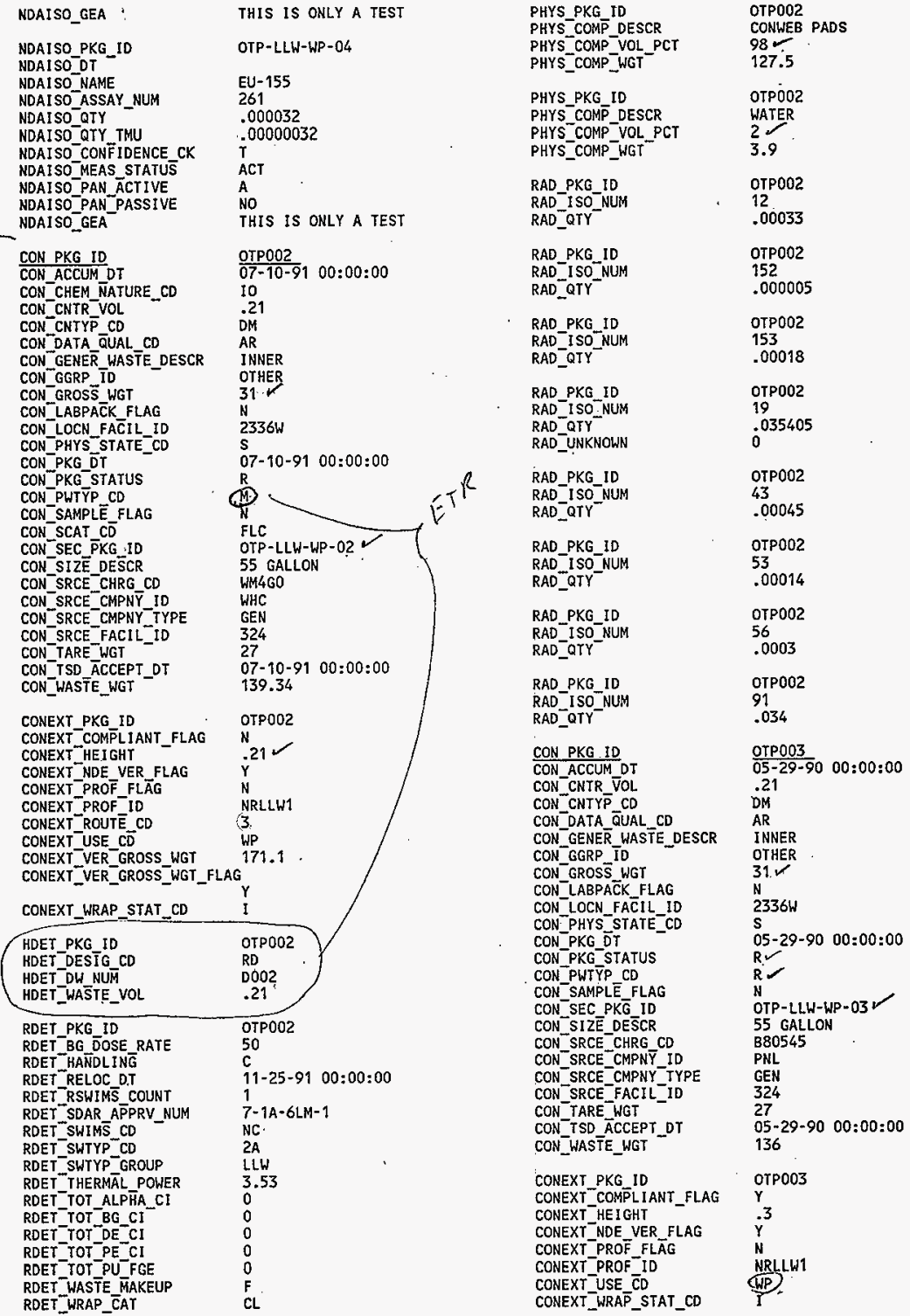




\begin{tabular}{|c|c|}
\hline $\begin{array}{l}\text { RDET_PKG_ID } \\
\text { RDET_BG_DOSE_RATE } \\
\text { RDET_HANDLING } \\
\text { RDET_RELOC_DT } \\
\text { RDET_RSWIMS__COUNT } \\
\text { RDET_SDAR_APPRV_NUM } \\
\text { RDEI_SWIMS_CD } \\
\text { RDET_SWTYP_CD } \\
\text { RDET_SWTYP_GROUP } \\
\text { RDET_THERMAL_POWER } \\
\text { RDET_TOT_BG_CI } \\
\text { RDET_TOT_DE_CI } \\
\text { RDET_WASTE_MAKEUP } \\
\text { RDET_WRAP_CAT }\end{array}$ & $\begin{array}{l}\text { OrP003 } \\
7 \\
C \\
05-29-90 \quad 00: 00: 00 \\
1 \\
2 A-1 A-1 A-0 \\
C E \\
2 A \\
\text { LLW } \\
3.53 \\
; \\
i \\
\text { F } \\
\text { DH }\end{array}$ \\
\hline $\begin{array}{l}\text { PHYS_PKG_IO } \\
\text { PHYS_COMP_DESCR } \\
\text { PHYS_COMP_VOL_PCT } \\
\text { PHYS_COMP_WGT }\end{array}$ & $\begin{array}{l}\text { OTPQ03 } \\
\text { CLOTH/RAGS/NYLON } \\
10 \\
14\end{array}$ \\
\hline $\begin{array}{l}\text { PHYS_PKG_ID } \\
\text { PHYS_COMP_DESCR } \\
\text { PHY_COMP_VOL_PCT } \\
\text { PHYS_COMP_WGT }\end{array}$ & $\begin{array}{l}\text { OTP003 } \\
\text { PAPER/CAROBOARD } \\
30 \\
41\end{array}$ \\
\hline $\begin{array}{l}\text { PHYS_PKG_1D } \\
\text { PHYS_COMP_DESCR } \\
\text { PHYS_COMP_VOL_PCT } \\
\text { PHYS_CONP_WGT }\end{array}$ & $\begin{array}{l}\text { OTP003 } \\
\text { PLASTIC/POLYURATHANE } \\
60 \\
81\end{array}$ \\
\hline $\begin{array}{l}\text { RAD_PKG_ID } \\
\text { RAD_ISO_NUM } \\
\text { RAD_OTY } \\
\text { RAD_UNKNOWN }\end{array}$ & $\begin{array}{l}\text { 0TP003 } \\
19 \\
.01212 \\
.000324\end{array}$ \\
\hline $\begin{array}{l}\text { RAD_PKG_ID } \\
\text { RAD_ISO_NUH } \\
\text { RAD_QTY }\end{array}$ & $\begin{array}{l}\text { OTP003 } \\
3 \\
.00006\end{array}$ \\
\hline $\begin{array}{l}\text { RAD_PKG_ID } \\
\text { RAD_ISO_NUM } \\
\text { RAD_QTY }^{-} .\end{array}$ & $\begin{array}{l}0 T P 003 \\
8 \\
.006\end{array}$ \\
\hline 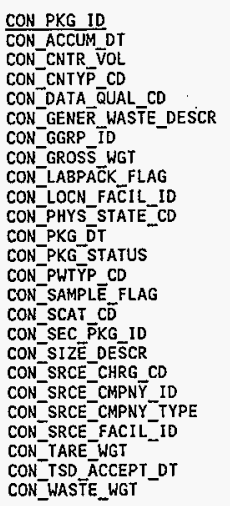 & $\begin{array}{l}\text { OTP004 } \\
07-05-90 \quad 00: 00: 00 \\
\text { OM } \\
\text { OM } \\
\text { AR } \\
\text { INNER } \\
\text { OTHER } \\
32 \\
\text { N } \\
2336 \mathrm{~W} \\
\text { S } \\
\text { O7-05-90 00:00:00 } \\
\text { R } \\
\text { M. ET ER } \\
\text { OMW } \\
\text { OMW-LLW-WP-04 } \\
\text { OTP-LLW } \\
55 \text { GALLON } \\
\text { B3314 } \\
\text { WHC } \\
\text { GEN } \\
324 \\
27 \\
\text { O7-05-90 } 00: 00: 00 \\
32.1\end{array}$ \\
\hline $\begin{array}{l}\text { CONEXI_PKG_ID } \\
\text { CONEXI_COMPLIANT_FLAG } \\
\text { CONEXI_HEIGHT } \\
\text { CONEXT_NDE_VER_FLAG } \\
\text { CONEXT_PROF_FLĀG } \\
\text { CONEXT_PROF_ID } \\
\text { CONEXT_USE_CD } \\
\text { CONEXT_WRA } \bar{P} \text { STAT_CD }\end{array}$ & $\begin{array}{l}\text { OTP004 } \\
N \\
\dot{Y}^{3} \\
N \\
\text { NRLLW1 } \\
\text { WP } \\
\text { I }\end{array}$ \\
\hline
\end{tabular}

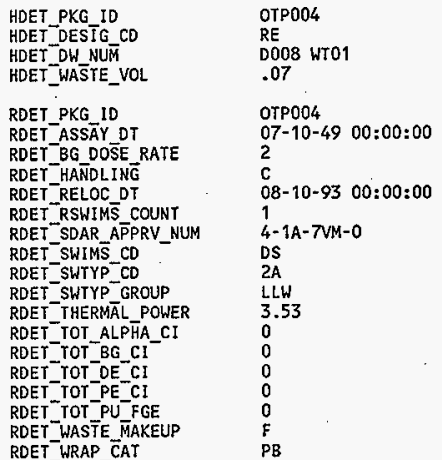

PHYS PKG_ID OTP004

PHYS COM $\bar{P}$ OESCR CLOTH/RAGS/NYLON

PHYS COMP VOL PCT $70{ }^{2}$

PHYSCONP_WGT

PHYS_PKG $10 \quad$ OTP004

PHYS COMP DESCR PLASTIC/POLYURATHANE

PHYS_COMP_VOL_PCT

PHYS_COMP_WGT

RAD_PKG_ID

RAD_ISO-NUM

RAD_QTY

RAD_PKG_ID

RAD_1SO NUM

RAD QTY

RAD_UNKNOWN

RAD_PKG_ID
RAD_ISO_NUM
RAD_QTY

RAD_PKG_10

RAD_ISO_NUM

RAD_QTY

RAD_PKG ID

RAD ISO_NUM

RAD_QTY

RAD_PKG_ID
RAD_ISO_NUM

RAD_QTY

RAD_PKG_ID

RAD ISO NUM

RAD_QTY

RAD:PKG_ID

RAD ISO NUM

RAD QTY

PLASTIC/POLYURATHANE
40.1
OTP004
13
.000004
OTP004
19
.000233
.0000007163
OTPO04
31
.000032
OTP004
4
.000083
OTP004
40
.000002
OTP004
53
.000001
OTP004
8
.000013
OTP004
9
.000001
OTP-LLWR-TO-01
DM
$2336 \mathrm{~W}$
ANY
85 GALLON
$31-$
$0 T P-L L W R-T D-01$
P 
CONEXT USE CD CONEXT_WRAP_STAT_CD

CONR FROM PKG ID CONR TO PKG_ID

CONR DT

CONR_REL_CD

CONR FROM PKG ID

CONR_TO PKG I $\bar{D}$

CONRDT

CONR_REL_CD

CONR_FROM_PKG_ID

CONR_TO_PKG_ID

CONR_DT

CONR_REL_CD

CON PKG ID

CON CNTYP CD

CON_LOCN FACIL_ID

CON PKG STATUS

CON SCAT CD

CON SIZE DESCR

CON_TARE_WGT

CONEXT PKG ID

CONEXT CNTR STATUS

CONEXT USE $\overline{C D}$

CONEXT_WRAP _STAT_CD

CONR_FROM PKG ID

CONR_TO_PKG_ID

CONR DT

CONR_REL_CD

CONR_FROM PKG ID

CONR_TO_PKG_ID

CONR-DT

CONR_REL_CD

CONR_FROM_PKG_ID

CONR TO P $\bar{K} G$ ID

CONR-DT

CONR_REL_CO

CON PKG ID

CON_GENER_WASTE_DESCR

CONEXT_PKG_ID
CONEXT_MAT_GRP_CD
CONEXT_USE_CD
RDET_PKG_ID
RDET_SWTYP_CD
RDET_SWTYP_GROUP
CONR_FROM_PKG_ID.
CONR_TO_PKG_ID
CONR_OT
CONR_REL_CD
CONR_FROM_PKG_ID
CONR_TO_PKG_ID
CONR_DT
CONR_REL_CD
CONPKG ID
CON_GENER_WASTE_DESCR

CONEXT_PKG_ID

CONEXT_MAT_GRP_CD

CONEXT_USE_CD

RDET_PKG_ID
TD

OTP6-97-000001

OTP-LLWR-TD-01 -

$08-14-27$ 17:26:29

0 -

0TP6-97-000002

OTP-LLWR-TD-01

08-14-27 17:26:00

0 -

0TP6-97-000003

OTP-LLWR-TD-01

$08-14-27 \quad 17: 27: 07$

0

OTP-LLWR - TD - 02

DM -

$2336 \mathrm{w}-$

G -

ANY -

85 GALLON

31

OTP-LLWR-TD-O2"

P -

TD

Ir

OTP6-97-000004 OTP-LLWR-TD-02 -

08-15-27 17:56:21

$0-$

OTP6-97-000005 -

OTP-LLWR-TD-02

08-15-27 17:57:08

0 -

OTP6-97-000006-

OTP-LLWR-TD-02 -

08-15-27 17:57:38

0 -

0TP6-97-000001

AEROSOL CAN OF SALINE -

SOLUTION

OTP6-97-000001

$A C$ -

PK -

0IP6-97-000001 -

2A

LLWT-

OTP-LLW-WP-02.

0TP6-97-000001

08-14-27 17:26:29

OTP6-97-000001,

OTP-LLWR-TD-01

08-14-27 17:26:29

$0<$

0TP6-97-000002

AEROSOL CAN OF SALINE SOLUTION

OTP6-97-000002

$A C \div$

PK -

0TP6-97-000002
RDET_SWTYP_CD $2 A$

RDET_SWTYP_GROUP LLW -

CONR_FROM PKG ID OTP-LLW-WP-02-

CONR TO P $\bar{K} G$ ID $\overline{\text { OTPG-97-000002 }}$

CONR_DT 08-14-27 17:26:00

CONR_REL_CD S -

CONR_FRON PKG ID

CONR TO PKG_ID

CONR_DT

CONR_REL_CD

CON PKG ID

CON_GENER_WASTE_DESCR

CONEXT_PKG_ID

CONEXT MAT GRP CD

CONEXT_USE_CD -

RDET PKG ID

RDET SWTYP CD

RDET_SWTYP_GROUP

CONR FROM PKG ID

CONR_TO_PKG_I $\bar{D}$

CONR DT

CONR_REL_CD

CONR_FROM_PKG_ID

CONR TO PKG I $\bar{D}$

CONR_DT

CONR_REL_CD

CON PKG ID

CON_GENER_WASTE_DESCR

CONEXT_PKG_ID

CONEXT_MAT_GRP_CD

CONEXT_USE_CD -

RDET_PKG ID

RDET SWT YP CD

RDET_SWTYP_GROUP

CONR FROM PKG ID

CONR_TO_PKG_ID

CONR DT

CONR_REL_CD

CONR FROM PKG. ID

CONR_TO_PKG_ID

CONRDT

CONR_REL_CD

CON PKG ID

CON_GENER_WASTE_DESCR

CONEXT_PKG_ID

CONEXT_MAT_GRP_CD

CONEXT_USE_CD -

RDET_PKG_ID

RDET SWTYYP CD

RDET_SWTYP_GROUP

CONR FROM PKG ID

CONR_TOPEG_ID

CONR DT

CONR_REL_CD.

CONR FROM PKG ID

CONR_TO_PKG_ID

CONR DT-

CONR_REL_CO

OTP6-97-000003 -
OTP6-97-000002 -

OTP-LLWR-TD-01-

08-14-27 17:26:00

0 -

OTP6-97-000003

AEROSOL CAN OF UNKNOWN -

0TP6-97-000003 -

$A C=$

PK

OTP6-97-000003 -

2A -

LLW-

OTP-LLW-WP-02 OTP6-97-000003 -

08-14-27 17:27:06

S -

OTP-LLWR-TD-01

$08-14-27 \quad 17: 27: 07$

0 -

OTP6-97-000004

LEAD BRICK

OTP6-97-000004

PB -

PK-

OTP6-97-000004

$2 A-$

LLW -

OTP-LLW-WP- 04 OTP6-97-000004 -

08-15-27 17:56:20

S -

OTP6-97-000004 -

OTP-LLWR-TD-02

08-15-27 17:56:21

0 -

0TP6-97-000005 -

BOTTLE OF OIL \& BOTTLE OF SOLVENT

OTP6-97-000005 -

LQ -

PK -

OTP6-97-000005 -

$2 A$

LLW -

OTP-LLWW-WP-04 OTP6-97-000005 $08-15-27 \quad 17: 57: 08$

$s$.

OTP6-97-000005 -

OTP-LLWR-TD=02 $08-15-27 \quad 17: 57: 08$ 0 - 


\begin{tabular}{|c|c|}
\hline$\frac{\text { CON PKG ID }}{\text { CON_GENER_WASTE_DESCR }}$ & $\frac{\text { OTP6-97-000006 }}{\text { BOTTLE OF ACID - }}$ \\
\hline $\begin{array}{l}\text { CONEXT_PKG_ID } \\
\text { CONEXT_MAT_GRP_CD } \\
\text { CONEXT_USE_CD - }\end{array}$ & $\begin{array}{l}\text { OTP6-97-000006 } \\
\text { LQ - } \\
\text { PK- }\end{array}$ \\
\hline $\begin{array}{l}\text { RDET_PKG_ID } \\
\text { RDET_SWTYP_CD } \\
\text { RDET_SWTYP_GROUP }\end{array}$ & $\begin{array}{l}\text { OIP6-97-000006 } \\
\text { 2A - } \\
\text { LLW - }\end{array}$ \\
\hline $\begin{array}{l}\text { CONR_FROM_PKG_ID } \\
\text { CONR_TO_PKG_ID } \\
\text { CONR_DT } \\
\text { CONR_REL_CD }\end{array}$ & $\begin{array}{l}\text { OTP-LLW-WP-04, } \\
\text { OTP6-97-000006 } \\
08-15-27 \quad 17: 57: 37 \\
S\end{array}$ \\
\hline $\begin{array}{l}\text { CONR_FROM_PKG_ID } \\
\text { CONR_TO_PKG_ID } \\
\text { CONR_DT_ } \\
\text { CONR_REL_CO }\end{array}$ & $\begin{array}{l}\text { OTP6-97-000006 - } \\
\text { OTP-LLWR-TD-02 - } \\
08-15-27 \quad 17: 57: 38 \\
0\end{array}$ \\
\hline CON PKG ID & OTP6-97-300001 \\
\hline $\begin{array}{l}\text { CONEXI_PKG_ID } \\
\text { CONEXI_USE_CD }\end{array}$ & $\begin{array}{l}\text { OTPG-97-300001 } \\
\text { PP }\end{array}$ \\
\hline $\begin{array}{l}\text { CONR_FROM_PKG_ID } \\
\text { CONR_TO_PKG_ID } \\
\text { CONR_DT } \\
\text { CONR_REL_CD. }\end{array}$ & $\begin{array}{l}\text { OTP6-97-300001: } \\
\text { 0TP6-97-400001, } \\
08-13-27 \quad 21: 57: 45 \\
0\end{array}$ \\
\hline $\begin{array}{l}\text { CONPKG ID } \\
\text { CON_PKG_DT } \\
\text { CON_PKG_STATUS }\end{array}$ & $\begin{array}{l}\frac{0 T P 6-97-400001}{08-13-2721: 57: 45} \\
G\end{array}$ \\
\hline $\begin{array}{l}\text { CONEXI_PKG_ID } \\
\text { CONEXI_USE_CD }\end{array}$ & $\begin{array}{l}\text { OIPG-97-400001 } \\
\text { TP }\end{array}$ \\
\hline $\begin{array}{l}\text { CONR_FROM_PKG_10 } \\
\text { CONR_TO_PKG_ID } \\
\text { CONR_DT } \\
\text { CONR_REL_CD }\end{array}$ & $\begin{array}{l}\text { OTP6-97-300001 } \\
\text { OTP6-97-400001 } \\
08-13=27 \quad 21: 57: 45 \\
0\end{array}$ \\
\hline $\begin{array}{l}\text { CON PKG ID } \\
\text { CON_ACCUM_DT } \\
\text { CON_CHEM_NATURE_CD } \\
\text { CON_CNTR_VOL } \\
\text { CON_CNTYP_CD } \\
\text { CON_GENER_WASTE_DESCR } \\
\text { CON_GGRP_ID } \\
\text { CON_GROSS_WGT } \\
\text { CON_LABPACK_FLAG } \\
\text { CON_LOCN_FACIL_ID } \\
\text { CON_PHYS_STATE_CD } \\
\text { CON_PKG_DT } \\
\text { CON_PKG_STATUS } \\
\text { CON_PWTYP_CD } \\
\text { CON_SAMPL_FLAG } \\
\text { CON_SCAT_CD } \\
\text { CON_SEC_PKG_ID } \\
\text { CON_SIZE_DESCR } \\
\text { CON_SRCE_CHRG_CD } \\
\text { CON_SRCE_CMPNY _ID } \\
\text { CON_SRCE_CMPNY_TYPE } \\
\text { CON_SRCE_FACIL_ID } \\
\text { CON_TARE_WGT _ } \\
\text { CON_TSD_ACCEPT_DT } \\
\text { CON_WASTE_WGT - }\end{array}$ & $\begin{array}{l}\text { PUCK278 } \\
10=19-93 \quad 00: 00: 00 \\
.21 \\
\text { DM } \\
\text { LOW LEVEL. WASTE } \\
\text { ANL } \\
152.7 \\
N \\
2336 W \\
\text { S } \\
10-19-93 \quad 00: 00: 00 \\
\text { R } \\
\text { H } \\
\text { Y } \\
\text { ONW } \\
\text { OTP-LLW-WP-01 } \\
\text { 55 GALTON } \\
\text { TH233 } \\
\text { ANL } \\
\text { GEN } \\
\text { ARGON } \\
27.7 \\
\text { O2-27-95 00:00:00 } \\
125\end{array}$ \\
\hline $\begin{array}{l}\text { CON PKG ID } \\
\text { CON_CNTYP_CD } \\
\text { CON_PKG_STATUS } \\
\text { CON_SEC_PKG_ID } \\
\text { CON_SIZE__DESCR } \\
\text { CON_TARE_WGT }\end{array}$ & $\begin{array}{l}\text { PUCK279 } \\
\text { DK } \\
R \\
\text { OTP-LLW-OD-01> } \\
\frac{85 \text { GALLON }}{31}\end{array}$ \\
\hline CON PKG ID & PUCK283 \\
\hline
\end{tabular}

\begin{tabular}{|c|c|}
\hline $\begin{array}{l}\text { CON_CNTYP_CD } \\
\text { CON_PKG_STATUS } \\
\text { CON_SEC_PKG_ID } \\
\text { CON_SIZE_DESCR } \\
\text { CON_TARE_WGT }\end{array}$ & $\begin{array}{l}D M \\
R \\
\text { OTP-LLW-OD-03 } \\
85 \text { GALLON } \\
31\end{array}$ \\
\hline $\begin{array}{l}\text { CON_PKG 1D } \\
\text { CON_CNTYP CD } \\
\text { CON_PKG_STATUS } \\
\text { CON_SEC_PKG_ID } \\
\text { CON_SIZE_DESCR } \\
\text { CON_TARE_WGT }\end{array}$ & $\begin{array}{l}\text { PUCK285 } \\
\text { DM- } \\
R \\
\text { OTP-LLW-0D-04 } \\
=85=-G A L L O N \\
31\end{array}$ \\
\hline $\begin{array}{l}\text { CONEXT_PKG_ID } \\
\text { CONEXT_CONPLIANT_FLAG } \\
\text { CONEXT_NDE_VER_FLAG } \\
\text { CONEXT_PROF_FLAG } \\
\text { CONEXT_PROF_ID } \\
\text { CONEXT_USE_CD } \\
\text { CONEXT_WRAP_STAT_CD }\end{array}$ & $\begin{array}{l}\text { PUCK278 } \\
\text { Y } \\
\text { Y } \\
\text { N } \\
\text { NRLEWT } \\
\text { WP } \\
1\end{array}$ \\
\hline $\begin{array}{l}\text { CONEXT_PKG_ID } \\
\text { CONEXT_USE_CD } \\
\text { CONEXT_WRAP_STAT_CD }\end{array}$ & $\begin{array}{l}\text { PUCK279 } \\
\text { EC } \\
\text { I }\end{array}$ \\
\hline $\begin{array}{l}\text { CONEXT_PKG_ID } \\
\text { CONEXT_USE-CD } \\
\text { CONEXT_WRAP_STAT_CD }\end{array}$ & $\begin{array}{l}\text { PUCK283 } \\
\text { EC } \\
\text { I }\end{array}$ \\
\hline $\begin{array}{l}\text { CONEXT_PKG_ID } \\
\text { CONEXT_USE_CD } \\
\text { CONEXT_WRAP_STAT_CD }\end{array}$ & $\begin{array}{l}\text { PUCK285 } \\
\text { EC } \\
\text { I }\end{array}$ \\
\hline $\begin{array}{l}\text { HDET_PKG_ID } \\
\text { HDET_CNT }{ }^{\circ} \text { STATUS } \\
\text { HDET_DESI } \bar{G} \text { CD } \\
\text { HDET_DW_HUM } \\
\text { HDET_WASTE_VOL } \\
\text { HDET_WASTE_STATUS }\end{array}$ & $\begin{array}{l}\text { PUCK278 } \\
\text { F } \\
\text { RE } \\
\text { D008 D009 } \\
\dot{U}^{208}\end{array}$ \\
\hline $\begin{array}{l}\text { RDET_PKG_ID } \\
\text { RDET_BG_DOSE_RATE } \\
\text { RDET_HANDLING } \\
\text { RDET_RELOC_DT } \\
\text { RDET_RSWIMS_COUNT } \\
\text { RDET_SDAR_APPRV_MUM } \\
\text { RDET_SWIMS_CD } \\
\text { RDET_SWTYP_CD } \\
\text { RDET_SWTYP_GROUP } \\
\text { ROET_THERMAL_POWER } \\
\text { RDET_TOT_BG_CI } \\
\text { RDET_TOT_DE_CI } \\
\text { RDET_WASTE_CAT } \\
\text { RDET_WASTE_MAKEUP } \\
\text { RDET_WRAP_CAT }\end{array}$ & $\begin{array}{l}\text { PUCK278 } \\
.1 \\
c \\
09-01-96 \quad 00: 00: 00 \\
1 \\
60-1 R-3 Y M-0401 \\
D D \\
2 A \\
\text { LLW } \\
3.53 \\
.00000254 \\
.0000097 \\
\text { WC1 } \\
\text { F } \\
\text { So }\end{array}$ \\
\hline $\begin{array}{l}\text { HAZ_PKG_ID } \\
\text { HAZ_COMP_ID } \\
\text { HAZ_COMP_TEXT } \\
\text { HAZ_COMP_WGT } \\
\text { HAZ_COMP_WGT_PCT } \\
\text { HAZ_EPCRA_FLAG }\end{array}$ & $\begin{array}{l}\text { PUCK278 } \\
7439-97-6 \\
\text { MERCURY } \\
.001 \\
\dot{N}^{0009}\end{array}$ \\
\hline $\begin{array}{l}\text { HAZ_PKG_ID } \\
\text { HAZ_COMP_ID } \\
\text { HAZ_COMP_IEXT } \\
\text { HAZ_COMP_WGT } \\
\text { HAZ_COMP_WGT_PCT } \\
\text { HAZ_EPCRA_FLAG }\end{array}$ & $\begin{array}{l}\text { PUCK278 } \\
\text { TEMP1985 } \\
\text { LEAD } \\
.085 \\
.068 \\
\text { N }\end{array}$ \\
\hline $\begin{array}{l}\text { PHYS_PKG_ID } \\
\text { PHYS_COMP_DESCR } \\
\text { PHYS_COMP_VOL_PCT } \\
\text { PHYS_COMP_WGT }\end{array}$ & $\begin{array}{l}\text { PUCK278 } \\
\text { DIRT/SOIL/DIATOMACEOUS } \\
\text { EARTH } \\
86 \\
115\end{array}$ \\
\hline PHYS_PKG_ID & PUCK278 \\
\hline
\end{tabular}


PHYS COMP_DESCR

PHYS COMP VOL PCT

PHYS_COMP_WGT

PHYS_PKG_10

PHYS_COMP _DESCR

PHYS_COMP_VOL_PCT

PHYS_COMP_WGT

PHYS PKG 10

PHYS_COMP DESCR

PHYSCOMP_VOL PCT

PHYS_COMP_WGT

RAD_PKG_ID
RAD_ISO_NUM
RAD_QTY

RAD PKG ID

RAD_ISO_NUM

RAD_QTY

RAD_PKG_ID

RAD_ISO_NUM

RAD_QTY

RAD_PKG_ID

RAD_ISO_NUM

$R A D_{-}^{-} \mathrm{QTY}^{-}$

RAD_PKG_ID

RAD_ISO_NUM

RAD_QTY

RAD PKG ID

RAD_ISO_NUM

RAD_QTY-

RAD_PKG_ID

RADIISO_NUM.

RAD QTY

CONLOC PKG ID

CONLOCDT

CONLOC_LOCN_10

CONLOC_PKG_ID

CONLOCDT

CONLOC_LOCN_IO

CONLOC PKG ID

CONLOCDO

CONLOC_LOCN_ID

CONLOC_PKG_ID

CONLOCDT

CONLOC_LOCN_ID

CONLOC_PKG_ID

CONLOC-DT

CONLOC_LOCN_ID

CONLOC_PKG ID

CONLOCDT

CONLOC_LOCN_ID

CONLOC PKG ID

CONLOCDT

CONLOC_LOCN_ID

CONLOC_PKG_ID

CONLOCDI

CONLOC_LOCN_ID

CONLOC_PKG_IO

CONLOCDT

CONLOC_LOCN_ID
HAZARDOUS CONSTITUENTS

$i^{5}$

PUCK278

PAPER/CARDBOARD

9.5

5

PUCK278

PLASTIC/POLYURATHANE

4

5

PUCK278

104

.00000788

PUCK278

18

11

PUCK278

19

.00000254

PUCK278

202

.0013

PUCK278

203

203
.19

PUCK278

206

25

PUCK278

86

.00000254

OTP-LLW-0D-01

08-20-27 01:07:46

LLW_EXIT

OTP-LLW-0D-02

08-19-27 22:30:25

LLW_EXIT -

OTP-LLW-OD-03

08-15-27 15:38:27

LLW_ENTRY

OTP-LLW-OD-04

08-15-27 16:06:40

LLW_ENTRY

OTP-LLH-OD-05

08-19-27 20:58:52

LLW_ENTRY -

OTP-LLW-WP-01

$08-14=27 \quad 01: 42: 44$

LLW SORT

OTP-LLWR-TD-01

08-13-27 16:29:26

LLH_RWMPRT -

OTP-LLWR-TD-02 08-15-27 15:38:57.

LLW_RWMPRT -

OTP6-97-400001 -

08-13-27 21:57:45

LLW_PP
CONLOC_PKG_IO

CONLOC DT

CONLOC_LOCN_ID

CONLOC_PKG_ID

CONLOCDT

CONLOC_LOCN_ID

RADMAT_ID

RADMAT DESCR

RADMAT_LIMIT

RADMAT_RAD_TOT

RADMAT UNITSS

RADMAT_ALARM

RADMAT_ID

RADMAT-DESCR

RADMAT LIMIT

RADMAT RAD IOT

RADMAT UNITS .

RADMAT_ALARM

RADMAT ID

RADMAT DESCR

RADMAT LIMIT

RADMAT_RAD TOT

RADMAT UNIT̄S

RADMAT_ALARM

MSGLOG DT

MSGLOG_ERROR_FLAG

MSGLOG_GEN_TYYPE

MSGLOG SEQ NUM

MSGLOG SPE $\bar{C}$ TYPE

MSGLOG_STRIN̄G1

MSGLOG DT

MSGLOG_ERROR_FLAG

MSGLOG_GEN_TYYPE

MSGLOG_SEQ NUM

MSGLOG_SPE $\overline{\mathrm{C}}$ TYPE

MSGLOG_STRINTGI

MSGLOG OT

MSGLOG_ERROR_FLAG

MSGLOG GEN TYYPE

MSGLOG SEQ NUM

MSGLOG_SPE $\bar{C}$ TYPE

MSGLOG_STRE $\overline{N G 1}$.

MSGLOG DT

MSGLOG_ERROR_FLAG

MSGLOG GEN TYPPE

MSGLOG_SEQ NUM

MSGLOG SPE $\bar{C}$ TYPE

MSGLOG_STRINTG1

MSGLOG_DT

MSGLOG_ERROR FLAG

MSGLOG GEN TŸPE

MSGLOGSSEQNUM

MSGLOG SPEC TYPE

MSGLOG_STRIÑNG1

MSGLOG DT

MSGLOG_ERROR FLAG

MSGLOG GEN TYYPE

MSGLOG_SEQ NUM

MSGLOG SPEC TYPE

MSGLOG_STRIÑGI
RHZ-101-A13568

$08-99-27$ 16:2\%:20

DISCHCVR

RHZ-103-A13561

08-10-27 02:02:46

Non
terting

$F$

WRAP 1 FACILITY

1433

123.456789

CI

N

$R$

IRU RWM GLOVEBOX

177

123.456789

FGE

N

T

TRU GLOVEBOX

177

123.456789

FGE

$\mathrm{N}$

08-15-97 08:34:21

Y

PCSDMS

70534

$\mathrm{CL}$

PCSDMS CL $\% 871658704 \% T R U$ S

ORT $\sim 1 \sim 202 A$-TEST-CASE

$08-15-97 \quad 09: 43: 25$

$Y$

PCSDMS

70720

$\mathrm{CL}$

PCSDMS ${ }^{2}$ "871662848"LLW_S

TORE $1 \sim \sim 1 \sim$ OTP010

$08-15-97 \quad 09: 44: 10$

$\mathrm{N}$

PCSDMS

70724

$\mathrm{CL}$

PCSDMS“CL $\approx 871662893$ "LLW_C MPCT $^{\sim} \sim$ OTP-LLW-WP-04

08-15-97 09:53:52

$N$

PCSDMS

70751

$\mathrm{CL}$

PCSDMS ${ }^{2}$ C 871663474 ¿LW_C MPCr $\sim$ 1 OTP-LLW-WP-04

08-15-97 10:58:33

$\mathrm{N}$

PCSDMS

70924

$\mathrm{Cl}$

PCSDMS CL 871667355 LLW_P

UCK $^{\sim \sim 1} 1^{\sim}$ OTPO99

08-15-97 11:22:14

$\mathrm{Y}$

PCSDMS

70989

$\mathrm{CL}$

PCSDMS $\approx C L \approx 871668776$ "LLH_P

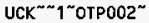


MSGLOG DT

MSGLOG ERROR FLAG

MSGLOG GEN TYPE

MSGLOG-SEQ NUM

MSGLOG SPE $\bar{C}$ TYPE

MSGLOG_STRINGG1

MSGLOG DT

MSGLOG ERROR FLAG

MSGLOG_GEN_TYYPE

MSGLOG SEQ NUM

MSGLOG SPEC TYPE

MSGLOG_STRINTG1

MSGLOG DT

MSGLOG ERROR FLAG

MSGLOG GEN TYYPE

MSGLOG SEQ NUM

MSGLOG SPEC TYPE

MSGLOG_STRIN̂G1

MSGLOG DT

MSGLOG ERROR FLAG

MSGLOG_GEN_TYYPE

MSGLOG SEQ NUM

MSGLOG SPE $\bar{C}$ TYPE

MSGLOG STRIN̄GI

MSGLOG DT

MSGLOG ERROR FLAG

MSGLOG GEN TYPE

MSGLOG SEQ NUM

MSGLOG SPE $\bar{C}$ TYPE

MSGLOG STRINTG1

MSGLOG OT

MSGLOG ERROR FLAG

MSGLOG GEN TYPE

MSGLOG SEQ NUM

MSGLOG-SPE $\bar{C}$ TYPE

MSGLOG STRIN̄G1

MSGLOG OT

MSGLOG ERROR, FLAG

MSGLOG GEN TYPE

MSGLOG SEQ NUM

MSGLOG SPEC TYPE

MSGLOG STRINTG1

MSGLOG DI

MSGLOG_ERROR_FLAG

MSGLOG_GEN TYYPE

MSGLOG SEQ NUM

MSGLOG_SPE $\bar{C}$ TYPE

MSGLOG SIRINGI

MSGLOG DT

MSGLOG_ERROR FLAG

MSGLOG GEN TYYPE

MSGLOG SEQ NUM

MSGLOG SPEC TYPE

MSGLOG_STRING

MSGLOG OT

MSGLOG_ERROR_FLAG

MSGLOG GEN TYYPE

MSGLOG SEQ NUM

MSGLOG_SPEC TYPE
Y

PCSDMS

71057

CL.

PCSDMS“CL“871670253“LLW_P UCK $\sim 1 " O T P 001$

08-15-97 12:29:53

$N$

DMSPCS

71171

ECL

DMSPCS FCL $\% 5.678000 E-05 \% F$

08-15-97 12:39:35

N

DMSPCS

71198

FCL

DMSPCS ${ }^{\sim} C_{C L} \sim 1.234500 E-03^{\sim} F$

08-15-97 12:40:28

DMSPCS

71201

FCL

DMSPCS ${ }^{\sim} \mathrm{FCL}^{\sim} 1.234568 \mathrm{E}+03^{\sim} \mathrm{F}$

08-15-97 12:49:18

DMSPCS

71226

FCL

DMSPCS FCL $1.234568 E+02 \% F$

08-15-97 12:50:54

DMSPCS

71231

FML

DMSPCS ${ }^{\sim}$ FML“AREA_3 ${ }^{\sim 1.23456}$

$8 \mathrm{E}+02^{\prime \prime} \mathrm{F}^{\prime \prime}$

08-15-97 12:51:09

$N$

DMSPCS

71232

FML

DMSPCS FML“TRU_GLVEBX 1.2

$34568 \mathrm{E}+02 \sim \mathrm{F}$

08-15-97 12:51:16

$Y$

PCSDMS

71233

$\mathrm{CL}$

PCSDMS“CL"871674118 LLW_P

UCK $\sim 1 \% O T P 001 \sim$

08-15-97 12:51:55

$Y$

PCSOMS

71237

$\mathrm{CL}$

PCSDMS “CL 871674156 "LLW_P

UCK 1 1 OTP001"

08-15-97 12:52:13

$\mathrm{Y}$

PCSDMS

71239

$\mathrm{CL}$
MSGLOG_SIRINGI

MSGLOG_DT

MSGLOG ERROR FLAG

MSGLOG GEN TYYPE

MSGLOG_SEQ NUM

MSGLOG SPEC TYPE

MSGLOG_STRING 1

MSGLOG DT

MSGLOG ERROR FLAG

MSGLOG GEN TYYPE

MSGLOG SEQ NUM

MSGLOG SPEC IYPE

MSGLOGSSTRINGG1

MSGLOG DT

MSGLOG ERROR FLAG

MSGLOG_GEN_TYYPE

MSGLOG SEQ NUM

MSGLOG SPEC TYPE

MSGLOG_STRIN̈GI

MSGLOG DT

MSGLOG ERROR FLAG

MSGLOG_GEN_TY YPE

MSGLOG SEQ NUM

MSGLOG SPE $\bar{C}$ TYPE

MSGLOG STRINGI

\section{MSGLOG DT}

MSGLOG ERROR FLAG

MSGLOG_GEN_TYYPE

MSGLOG SEQ NUM

MSGLOG_SPE $\bar{C}_{\text {TYPE }}$

MSGLOG STRIN̄GI

MSGLOG OT

MSGLOG_ERROR FLAG

MSGLOG GEN TYYPE

MSGLOG SEQ NUM

MSGLOG_SPEC TYPE

MSGLOG_STRIN̄GI

MSGLOG DT

MSGLOG_ERROR FLAG

MSGLOG GEN IYYPE

MSGLOG SEQ NUM

MSGLOG SPEC TYPE

MSGLOG_STRIÑG1

MSGLOG OY

MSGLOG_ERROR_FLAG

MSGLOG GEN TYYPE

MSGLOG SEQ NUM

MSGLOG SPEC TYPE

MSGLOG_STRINGG1

MSGLOGDT

MSGLOG ERROR FLAG

MSGLOG GEN TYYPE

MSGLOG SEQ NUM

MSGLOG SPEC TYPE

MSGLOG_SIRIN̄G1

MSGLOG DT

MSGLOG_ERROR_FLAG
PCSDMS“CL 271674175 "LLW_P UCK" ${ }^{N 1}$ "OTP001

08-15-97 12:54:08

$Y$

PCSDMS

71247

$\mathrm{CL}$

PCSDMS"CL "871674289"LLW_P $U^{U} \mathrm{CK}^{\sim \sim 1}$ 1 OTPOO1

08-15-97 13:01:54

$Y$

PCSOMS

71269

$\mathrm{CL}$

PCSONS“CL "871674756"LLS TORE $1 \sim 1 \sim$ OTPOO ${ }^{\sim}$

08-15-97 13:04:44

$Y$

PCSDMS

71277 .

$\mathrm{CL}$

PCSDMS “ CL"871674926“LLW_S TORE $1^{\sim-1}$ 1"OTPOO1"

08-15-97 13:09:55

$Y$

PCSDMS

71293

$\mathrm{CL}$

PCSDMS ${ }^{\circ}$ 871675237 LLW_S

TORE1 ${ }^{\sim 1} 1$ OTPOO1

08-15-97 13:09:57

$Y$

PCSDMS

71295

$\mathrm{CL}$

PCSDMS“CL “871675239 LLW_S

TORE $1 \sim \sim 1^{\sim}$ OTPOO $1^{\sim}$

08-15-97 13:10:02

$Y$

PCSDMS

71297

$\mathrm{CL}$.

PCSDMS CL 871675244 LLW_S TORE1 " 1"OTPOO1"

08-15-97 $13: 10: 14$

$Y$

PCSOMS

71301

$\mathrm{CL}$

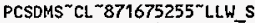
TORE1 $"$ " 1"OTPOO1"

08-15-97 13:10:32

$Y$

PCSDMS

71303

CL

PCSOMS“CL" "871675274"LLW_S TORE1" ${ }^{\sim} \sim$ "OTPOO1"

$08-15-97 \quad 13: 11: 36$

$\mathrm{Y}$

PCSDMS

71309

$\mathrm{CL}$

PCSDMS“CL“871675336“LLWS

TORE $1 \sim 1$ OTPOOT

$08-15-97 \quad 13: 12: 30$

Y 
MSGLOG_GEN_TYPE

MSGLOG_SEQ_NUM

MSGLOG SPE $\bar{C}$ TYPE

MSGLOG_STRIN̄G1

MSGLOG OT

MSGLOG_ERROR_FLAG

MSGLOG_GEN_TYPPE

MSGLOG SEQ NUM

MSGLOG SPE $\bar{C}$ TYPE

NSGLOG_STRIN̄GI

MSGLOG DT

MSGLOG_ERROR_FLAG

MSGLOG GEN TYPE

MSGLOG SEQ NUM

MSGLOG_SPEC TYPE

MSGLOG_STRINGG1

MSGLOG DT

MSGLOG_ERROR_FLAG

MSGLOG GEN TYPE

MSGLOG SEQ NUM

MSGLOG_SPEC _TYPE

MSGLOG_SIRINGA1

MSGLOG DT

MSGLOG_ERROR FLAG

MSGLOG_GEN_TŸPE

MSGLOG_SEQ_NUM

MSGLOG SPED TYPE

MSGLOG_STRIÑG1

MSGLOG DT

MSGLOG ERROR FLAG

MSGLOG GEN TŸPE

MSGLOG SEQ NUM

MSGLOG SPE C TYPE

MSGLOG_STRIN̄G?

MSGLOG DT

MSGLOG_ERROR_FLAG

MSGLOG GEN TYYPE

MSGLOGSSEQ_NUM

MSGLOG SPED TYPE

MSGLOG_STRINNGI

MSGLOG DT

MSGLOG_ERROR_FLAG

MSGLOG GEN TYYPE

MSGLOG_SEQ_NUM

MSGLOG SPE $\bar{C}$ TYPE

MSGLOG_STRIÑGT

MSGLOG.DT

MSGLOG ERROR FLAG

MSGLOG GEN TYYPE

MSGLOG SEQ NUM

MSGLOG SPEC TYPE

MSGLOG_STRIN̄G1

MSGLOG DT

MSGLOG ERROR FLAG

MSGLOG GEN TYPE

MSGLOG SEQ NUM

MSGLOG SPE $\bar{C}$ TYPE

MSGLOG_STRINTG?
PCSDMS

71313

$\mathrm{CL}$

PCSDHS “CL $871675392^{\sim}$ LLW_S

TORE1 $~ 1$ "OTPOO1

08-15-97 13:26:58

$Y$

PCSDMS

71353

CL

PCSDMS $\%$ CL871676260 LLW_P UCK $\sim 1 \% 0 T P 002 \sim$

$\begin{array}{ll}08-15-9713: 27: 00 & \\ Y & \text { MSGLOG_DT } \\ \text { PCSDMS } & \text { MSGLOG_ERROR_FLAG } \\ 71355 & \text { MSGLOG_GEN_TYPE } \\ \text { CL } & \text { MSGLOG_SEQ_NUM } \\ \text { PCSDMS"CL_871676261 LLWP } & \text { MSGLOG_SPEC TYPE } \\ \text { UCK"1\%OTP002 } & \text { MSGLOG_STRING1 }\end{array}$

08-15-97 13:28:04

$Y$

PCSDMS

71358

$\mathrm{CL}$.

PCSDMS “CL 871676326 LLW_P

UCK $\sim 1^{\sim \cdots}$

08-15-97 13:32:31

$\mathrm{Y}$

PCSDMS

71372

CL

PCSDMS $\approx C L ~ 871676593 \% L L W P P$ UCK $\sim 1 \sim$

08-15-97 13:35:44

$Y$

PCSDMS

71384

$\mathrm{CL}$

PCSDNS"CL"871676786"LLW_P UCK ${ }^{\sim-1 " O T P 002 " ~}$

08-15-97 13:35:46

$Y$

PCSDMS

71386

CL

PCSOMS $\approx C L \approx 871676787 \%$ LLW_P UCK ${ }^{m \sim 1}$ "OTP002

08-15-97 13:35:47

$Y$

PCSDMS

71387

CL.

PCSDMS CL 871676788 LLW_P UCK ${ }^{\sim}$ 1 OTPO02 $^{\sim}$

08-15-97 13:37:32

$Y$

PCSDMS

71392

$\mathrm{CL}$

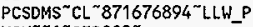
UCK $^{\sim}$ ? ${ }^{\sim}$ OTPO02

08-15-97 13:37:36

$Y$

PCSDMS

71394

CL

PCSDMS ${ }^{\circ}$ \%871676897“LLW_P UCK $\sim 1 \sim 0 T P 002 \sim$
MSGLOG DT

MSGLOG ERROR FLAG

MSGLOG GEN TYPE

MSGLOG SEQ NUM

MSGLOG_SPE $\vec{C}$ TYPE

MSGLOG_STRIN̄G1

MSGLOG DT

MSGLOG ERROR FLAG

MSGLOG_GEN_TYPE

MSGLOG SEQ NUM

MSGLOG SPEC TYPE

MSGLOG SIRING 1

MSGLOG DI

MSGLOG ERROR FLAG

MSGLOG_STRINNG1

MSGLOG ERROR FLAG

MSGLOG_GEN_TYPE

MSGLOG SEQ NUM

MSGLOG SPE $\bar{C}$ TYPE

MSGLOG_STRIN̄G1

MSGLOG DT

MSGLOG ERROR FLAG

MSGLOG_GEN_TYYPE

MSGLOG SEQ NUM

MSGLOG_SPE $\bar{C}$ TYPE

MSGLOG_STRINGG1

MSGLOG DT

MSGLOG_ERROR FLAG

MSGLOG GEN TYYPE

MSGLOG SEQ NUM

MSGLOG_SPEC̄_TYPE

MSGLOG STRINGG1

MSGLOG DT

MSGLOG_ERROR_FLAG

MSGLOG GEN TYYPE

MSGLOG SEQ NUM

MSGLOG_SPEC TYPE

USGLOG STRINGI

MSGLOG DT

MSGLOG_ERROR_FLAG

MSGLOG GEN TYYPE

MSGLOG SEQ NUM

MSGLOG SPEC TYPE

MSGLOG_SIRIN̄G1

MSGLOG DT

MSGLOG_ERROR_FLAG

MSGLOG GEN TY'PE

MSGLOG SEQ NUM

MSGLOG SPE $\bar{C}$ TYPE

MSGLOG_SIRINTG1

MSGLOG DI

MSGLOG_ERROR_FLAG

MSGLOG GEN TYYPE

MSGLOG SEQ NUM

MSGLOG_SPEC IYPE
MSGLOG DT
08-15-97 14:26:51

$\mathrm{N}$

PCSDMS

71527

$\mathrm{CL}$

PCSDMS“CL"871679853"LLW_P

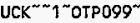

08-15-97 14:34:55

$\mathrm{N}$

PCSDMS

71550

$\mathrm{CL}$

PCSDMS $\approx C L ~ 871680337$ LLW_P UCK 1 \% OTP099

08-15-97 14:35:14

$Y$

PCSDHS

71551

CL

PCSDMS CL $871680356 \% T R U$ C OMPCT $^{\sim 1} 1^{\approx} 96000001$

08-15-97 $94: 35: 16$

$Y$

PCSDMS

71553

$\mathrm{CL}$

PCSDMS ${ }^{\circ}{ }^{\sim} 871680356$ TRU C OMPCT $^{\sim} \%, 6000001$

08-15-97 14:35:18

PCSDMS

71555

$\mathrm{CL}$

PCSDMS $\approx C L \approx 871680357 \%$ TRU_C OMPCT $^{\sim 1} 1 \sim 96000001$

08-15-97 14:35:20

$Y$

PCSDMS

71557

$\mathrm{CL}$

PCSOMS $\sim \mathrm{CL}^{\sim} 871680357$ TRU C OMPCI $\sim 1 \sim 96000001$

08-15-97 14:37:20

$\mathrm{Y}$

PCSDMS

71565

$\mathrm{CL}$

PCSDMS CL 871680481 TRU_C OMPCT $\sim 1 \sim 96000001^{\sim}$

08-15-97 14:37:22

$Y$

PCSDMS

71567

$\mathrm{Cl}$.

PCSDHS"CL 2871680482 TRU_C OMPCT $\sim 1 \sim 96000001^{2}$

08-15-97 14:37:24

$Y$

PCSDMS

71569

$\mathrm{CL}$

PCSDMS"CL "871680482"TRU_C OMPCI $\sim 1 \% 96000001$

08-15-97 14:37:55

$Y$

PCSDMS

71573

$\mathrm{Cl}$. 
MSGLOG_STRINGI

MSGLOG_DT

MSGLOG ERROR FLAG

MSGLOG GEN TYYPE.

MSGLOG SEQ NUM

MSGLOG SPEC TYPE

MSGLOG_STRIN̄GI

MSGLOG DT

MSGLOG ERROR FLAG

MSGLOG_GEN_TYTPE

MSGLOG SEQ NUM

MSGLOG SPEC TYPE

MSGLOG_SIRIN̄O1

MSGLOG DT

MSGLOG ERROR FLAG

USGLOG_GEN_TYYPE

MSGLOG_SEQ_NUM

MSGLOG SPE $\bar{C}$ TYPE

MSGLOG_STRIN̄G1

MSGLOG_DT

MSGLOG ERROR FLAG

MSGLOG_GEN_TYYPE

MSGLOG SEQ NUM

MSGLOG SPEC TYPE

MSGLOG_STRIN̄GI

MSGLOG DT

MSGLOG ERROR FLAG

MSGLOG_GEN_TŶPE

MSGLOG SEQ NUM

MSGLOG SPEC TYPE

MSGLOG_STRIN̄GI

MSGLOG DT

MSGLOG ERROR FLAG

MSGLOG GEN TYYPE

MSGLOG SEQ NUM

MSGLOG_SPEC TYPE

MSGLOG_STRINGG1

MSGLOG DT

MSGLOGERROR FLAG

MSGLOG GEN TYPE

MSGLOG SEQ NUM

MSGLOG_SPEC TYPE

MSGLOG_STRI NGG1

MSGLOG DT

MSGLOG ERROR FLAG

MSGLOG GEN TYPE

MSGLOG SEQ NUM

MSGLOG SPEC TYPE

MSGLOG_STRING 1

MSGLOG_DT

MSGLOG_ERROR, FLAG

MSGLOG GEN TȲPE

MSGLOG_SEQ_NUM

MSGLOG SPE $\bar{C}$ TYPE

MSGLOG_STRIN̄G1

MSGLOG_DT
PCSDMS“CL"871680517“TRU_C OMPCT ${ }^{\sim 1} 96000001$

08-15-97 14:37:57

$Y$

PCSDMS

71575

CL

PCSDMS $\mathrm{CL}^{\sim} 871680517$ TRU C OMPCT $^{\sim} \sim 96000001$

08-15-97 14:37:59

$Y$

PCSDMS

71577

$\mathrm{Cl}$

PCSDMS $\sim 2 L ~ 871680518 \sim T R U \_C$ OMPCT $^{\sim} 1^{\sim} 96000001$

08-15-97 14:38:01

$Y$

PCSDMS

71579

PCSDMS “CL"871680518“TRU_C OMPCT $^{\sim 1} 1 \mp 9600001$

08-15-97 14:38:03

$Y$

PCSOMS

71581

CL.

PCSDMS CL 871680518 TRU_C OMPCT $\sim 1 \sim 9600001$

08-15-97 15:19:03

$Y$

PCSDNS

71691

CL

PCSDMS ${ }^{2} \mathrm{CL}^{\sim} 871682985^{\sim}$ LLW_E

XII $\sim 1^{\sim}$ TEST DRUM

$08-15-97 \quad 15: 21: 11$

N
PCSOMS
71699
PAMR
PCSDMS ${ }^{\sim}$ PAMR $871683112^{\sim} \sim \mathrm{LLH}$
ENIRY 12345678901234 1.2

08-15-97 15:36:16

N

PCSDMS

71740

CL.

PCSDMS"CL“871684017 "LLW_P UCK 1"OTP099

08-15-97 15:48:44

$N$

PCSOMS

71775

$\mathrm{CL}$

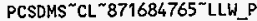
UCK 1 OTP099

08-15-97 15:48:48

$N$

PCSOMS

71776

$\mathrm{CL}$

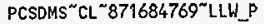

UCK $\sim$ 1 OTP099"

08-15-97 15:48:50
MSGLOG ERROR FLAG

MSGLOG GEN IYYPE

MSGLOG SEQ NUM

MSGLOG SPEC̈ TYPE

MSGLOG_STRING

MSGLOG DT

MSGLOG ERROR FLAG

MSGLOG_GEN_TYYPE

MSGLOG SEQ NUM

MSGLOG SPEC TYPE

MSGLOG_STRIN̄G1

MSGLOG DT

MSGLOG ERROR FLAG

MSGLOG_GEN_TYPE

MSGLOG SEQ NUM

MSGLOG SPE $\bar{C}$ TYPE

MSGLOG_STRINTG1

MSGLOG DT

MSGLOG ERROR FLAG

MSGLOG_GEN_TYYPE

MSGLOE SEQ NUM

MSGLOG SPEC $\bar{C}$ TYPE

MSGLOG STRINGI

MSGLOG_DT

MSGLOGERROR FLAG

MSGLOG_GEN_TŸPE

MSGLOG SEQ NUM

MSGLOG_SPEC̄ TYPE

MSGLOG STRINGI

MSGLOG_DT

MSGLOG_ERROR FLAG

MSGLOG_GEN_TYYPE

MSGLOG SEQ NUM

MSGLOG_SPE $\bar{C}_{\text {TYPE }}$

MSGLOG_STRINGA

MSGLOG DT

MSGLOG_ERROR_FLAG

MSGLOG_GEN TYYPE

MSGLOG SEQ NUM

MSGLOG_SPEC TYPE

MSGLOG STRIN̈G1

MSGLOG DT

MSGLOG_ERROR FLAG

MSGLOG GEN TYYPE

MSGLOG SEQ NUM

MSGLOG_SPEC_TYPE

MSGLOG SIRINNGI

MSGLOG DT

MSGLÓG_ERROR_FLAG

MSGLOG GEN TYYPE

MSGLOG SEQ NUM

MSGLOG_SPE $\vec{C}$ TYPE

MSGLOG_STRING'

MSGLOG DT

MSGLOG_ERROR_FLAG

MSGLOG GEN TYYPE

MSGLOG SEQ NUM

MSGLOG SPE $\bar{C}$ TYPE
$\mathrm{N}$

PCSDMS

71777

$\mathrm{CL}$

PCSDNS“CL 871684772\%LLW_P

$\mathrm{K}^{\sim \sim 1 \sim 0 T P 099 \sim}$

08-15-97 15:48:53

N

PCSDMS

71778

CL

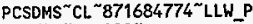
UCK $\sim 1$ OTP099

08-15-97 15:49:04

N

PCSDMS

71779

$\mathrm{CL}$

PCSDMS CL 871684785 LLW_P UCK $\sim$ ๆ OTP099

08-15-97 15:56:02

$Y$

PCSDMS

71800

CL

PCSDMS CL 871685204“LLW_E

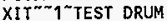

08-15-97 17:20:39

$Y$

PCSDMS

72026

$\mathrm{CL}$.

PCSDMS $\sim 2 L 871690281$ "LLH_E XIT $\sim 1 \sim T$ TEST DRUM

08-15-97 17:31:50

N

PCSDMS

72058

$\mathrm{CL}$

PCSDMS"CL 871690952 LLW_P UCK 1 ОTP099

08-15-97 17:31:52

N

PCSDMS

72059

$\mathrm{CL}$

PCSDMS“CL 871690953 LLW_P UCK $^{\sim 1 \text { 1 OTPO99 }}$

08-15-97 17:31:55

N

PCSDMS

72060

CL

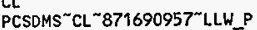
UCK" 1 OTP099

08-15-97 17:31:58

$N$

PCSOMS

72061

Cl.

PCSDMS ${ }^{2}$-871690959 LLH_P UCK 1 1 OTP099

08-15-97 17:32:01

N

PCSDMS

72064

CL 
MSGLOG_STRING1

MSGLOG DT

MSGLOG_ERROR FLAG

MSGLOG_GEN_TYYPE

MSGLOG SEQ NUM

MSGLOG_SPE $\bar{C}$ TYPE

MSGLOG_STRINGG1

MSGLOG DT

MSGLOG_ERROR FLAG

MSGLOG_GEN TYPPE

MSGLOG SEQ NUM

MSGLOG_SPE $\bar{c}$ TYPE

HSGLOG_STRING

MSGLOG DT

MSGLOG ERROR FLAG

MSGLOG_GEN TYYPE

MSGLOG SEQ NUM

MSGLOG_SPEC TYPE

MSGLOG_STRIN̄G1

\section{MSGLOG DT}

MSGLOG_ERROR - FLAG

HSGLOG GEN TYPE

MSGLOG SEO NUM

MSGLOG_SPEC TYPE

MSGLOG_STRIN̄G1

\section{MSGLOG DT}

MSGLOG_ERROR_FLAG

MSGLOG GEN TYPE

MSGLOG SEQ NUM

MSGLOG_SPEC TYPE

MSGLOG_STRINGGI

MSGLOG DT

MSGLOG_ERROR FLAG

MSGLOG_GEN TYPE

MSGLOG SEQ NUM

MSGLOG_SPEC̄ TYPE

MSGLOG_STRIN̈G1

\section{MSGLOG DT}

MSGLOG_ERROR_FLAG

MSGLOG_GEN TYYPE

MSGLOG SEQ NUM

MSGLOG_SPEC TYPE

MSGLOG_STRIN̄G1

MSGLOG DT

MSGLOG ERROR FLAG

MSGLOG_GEN TYPE

MSGLOG SEQ MUM

MSGLOG SPE $\bar{C}$ TYPE

MSGLOG_STRINGI

MSGLOG DT

MSGLOG_ERROR FLAG

MSGLOG_GEN TYYPE

MSGLOG SEQ NUM

MSGLOG_SPEC TYPE

MSGLOG_STRIN̄GI

MSGLOG DT

MSGLOG_ERROR_FLAG
PCSOMS CL“871690961 LLW_P CK 1 - TTP099"

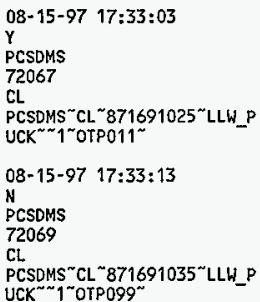

08-15-97 17:59:38

$Y$

PCSDMS

72140

POPD

PCSDMS $" P O P{ }^{\sim} 871692620 \%$ L.WW

_EXIT OTP099\%TEST DRUM

08-15-97 17:59:40

(4)

PCSDMS

72142

CLW

PCSDMS CLW 871692620 LLW. PUCK ${ }^{2}$ if $82^{\sim}$

\author{
08-15-97 19:08:23 \\ MSGLOG_STRIN̄G?
}

$N$

PCSDMS

72327

CL

PCSDMS“CL"871696744 LLW P UCK $^{\sim}$ 1 OTP099"

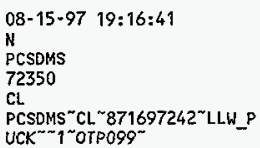

08-15-97 20:15:22

$\mathrm{Y}$

PCSDMS

72507

$\mathrm{CL}$.

PCSDMS CL $871700764^{*}$ LLW_E

XIT $\sim$ TEST DRUM

08-15-97 20:33:47

N

PCSDMS

72559

POPD

PCSDMS POPD $~ 871701868 \%$ LLW

EXIT OTPO02 TEST DRUM

08-15-97 20:33:48

(y)

PCSDMS

72560

CLW

PCSDMS CLW 871701868 LLW_ PUCK 1 fी $224^{\circ}$

08-15-97 20:40:37
MSGLOG GEN TYPE

MSGLOG'SEQ NUM

MSGLOG_SPEC

MSGLOG_STRINTGI

MSGLOGDT

MSGLOG_ERROR_FLAG

MSGLOG_GEN_TYYPE

MSGLOG SEQ NUM

MSGLOG_SPEC TYPE

MSGLOG_STRIN̄G1

MSGLOG DT

MSGLOG GEN TYYPE

MSGLOG SEQ NUM

MSGLOG_SPEC TYPE

MSGLOG_STRIN̄G1

MSGLOG DT

MSGLOG_ERROR_FLAG

MSGLOG GEN TYYPE

MSGLOG SEQ NUM

MSGLOG_SPEC TYPE

MSGLOG_STRINTGI

MSGLOG_DT

MSGLOG_ERROR_FLAG

MSGLOG_GEN_TYPE

MSGLOG SEQ NUM

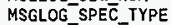

MSGLOG DT

MSGLOG_ERROR_FLAG

MSGLOG GEN TYPE

MSGLOG SEQ NUM

MSGLOG_SPEC̄ITYPE

MSGLOG_STRINTG1

MSGLOG DT

MSGLOG_ERROR_FLAG

MSGLOG_GEN TYYPE

MSGLOG SEQ NUM

MSGLOG_SPED IYPE

MSGLOG_STRIÑG1

MSGLOG DT

MSGLOG_ERROR_FLAG

MSGLOG GEN TYYPE

MSGLOG SEQ NUM

MSGLOG_SPEC_TYPE

MSGLOG_STRING!

MSGLOG DT

MSGLOG_ERROR_FLAG

MSGLOG_GEN_TYPE

MSGLOG SEQ NUM

MSGLOG_SPEC्C_TYPE

MSGLOG_STRING 1

MSGLOG OT

MSGLOG_ERROR_FLAG

MSGLOG GEN_TYPE

MSGLOG SEQ NUM

MSGLOG_SPEC

MSGLOG_STRING
MSGLOG_ERROR_FLAG
PCSOMS

72580

$\mathrm{Ct}$

PCSDMS CL $^{-871702278 \sim L L W E ~}$ XIT $\sim 1$ TEST ORUM

08-15-97 20:41:25

$\mathrm{Y}$

PCSDMS

72584

CL

PCSOMS“CL “871702326“LLW_E XIT $\sim$ ๆ TEST DRUM -

$08-15-97 \quad 20: 43: 32$

$Y$

PCSDMS

72592

$\mathrm{CL}$

PCSDMS $\approx$ CL 871702453 LLW_E XIT $\sim$ 1 TEST DRUM -

08-15-97 20:51:22

$N$

PCSDMS

72614

POPD

PCSDMS ${ }^{\sim P O P D}$ " 871702923 LLW _EXIT OTPOO1"TEST DRUM

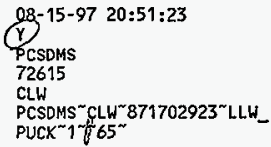

08-16-97 10:48:32

N

PCSOMS

73655

CL

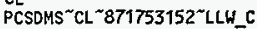
MPCT 1"OTP-LLW-WP-04"

08-16-97 11:06:43

$Y$

PCSDMS

73704

CL

PCSOMS “CL $\% 871754243$ LLW_P UCK ${ }^{\sim 1}$ \% OTP004"

08-16-97 11:19:07

N

PCSDMS.

73740

CL

PCSDMS CL" 87?754987“LLW_C $\mathrm{MPCT}^{\sim \sim 1 \text { 1 OTP-LLW-WP-04" }}$

$08-16-97 \quad 11: 24: 49$

N

PCSDMS

73755

CL

PCSDMS“CL ${ }^{-871755329 \% L L W C ~}$ MPCT ${ }^{\sim} 1$ OTP-LLW-WP-04\%

$08-16-97 \quad 11: 35: 35$

N

PCSDNS

73786

CL MPCT $\sim 1$ OTP-LLW-WP-04"- 
MSGLOG DT

MSGLOG_ERROR_FLAG

MSGLOG_GEN_TYPE

MSGLOG SEQ NUM

MSGLOG_SPEC_TYPE

MSGLOG_STRIN̄G1

MSGLOG DT

MSGLOG_ERROR_FLAG

MSGLOG GEN TYYPE

MSGLOG SEQ NUM

MSGLOG SPEC TYPE

MSGLOG_SIRIN̄G1

MSGLOG DT

MSGLOG_ERROR_FLAG

MSGLOG GEN TYYPE

MSGLOG SEQ NUM

MSGLOG SPEC TYPE

MSGLOG_STRIN̄G1

MSGLOG OT

MSGLOG_ERROR FLAG

MSGLOG GEN TYYPE

MSGLOG_SEQ-NUM

MSGLOG SPE $\vec{C}$ TYPE

MSGLOG_STRIN̄G1

MSGLOG DT

MSGLOG_ERROR_FLAG

MSGLOG GEN TYPE

MSGLOG SEQ NUM

MSGLOG SPE $\bar{C}$ TYPE

MSGLOG_STRIN̄GI

MSGLOG DT .

MSGLOG_ERROR_FLAG

MSGLOG GEN TYPE

MSGLOG_SEQ NUM

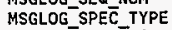

MSGLOG_STRINTGI

MSGLOG DT

MSGLOG_ERROR_FLAG

MSGLOG GEN TYYPE

MSGLOG-SEQ NUM

MSGLOG SPEC TYPE

MSGLOG_STRINTGI

MSGLOG DT

MSGLOG_ERROR_FLAG

MSGLOG GEN TYPE

MSGLOG SEQ NUM

MSGLOG_SPEC TYPE

MSGLOG STRING1

MSGLOG DT

MSGLOG_ERROR_FLAG

MSGLOG GEN TYYPE

MSGLOG SEQ NUM

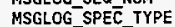

MSGLOG_STRINTG1

MSGLOG DT

MSGLOG_ERROR_FLAG

MSGLOG GEN TYYPE

MSGLOG_SEQ NUM

MSGLOG_SPEC_TYPE
N

PCSDMS

73793

$\mathrm{CL}$.

PCSDMS $\sim \mathrm{CL} \sim 871756135 \sim L L W \_C$

MPCT $\sim$ 1 OTP- $1 L W-W P-04^{\sim}$

$08-16-97 \quad 12: 23: 37$

$\mathrm{N}$

PCSDMS

73916

$\mathrm{CL}$

PCSDMS ${ }^{\sim}$ \% 871758857 LLW_C

MPCT $\sim 1 \sim 0 T P-L L W-W P-04 \approx-$

08-16-97 12:24:29

$\mathrm{N}$

PCSDNS

73919

$\mathrm{CL}$

PCSDMS ${ }^{\sim} \mathrm{CL}^{\sim 871758908}$ "LLH_C

MPCT $^{\sim} 1 \sim 0 T P-L L W-W P-04^{\sim}$

08-16-97 12:37:27 MSGLOG_STRING1

$\mathrm{N}$

PCSDMS

73954

$\mathrm{CL}$

PCSOMS $\sim 2 L 871759687$ LLW_C

MPCT $^{\sim-1 * O T P-L L W-W P-04 * ~}$

08-16-97 12:42:45

N

PCSDMS

73969

CL.

PCSDMS“CL"871760005“LLW_C

MPCT 1 "OTP-LLW-WP-04"

$08-16-97 \quad 13: 32: 46$

N

PCSDMS

74104

CL

PCSDMS $\approx \mathrm{CL}^{\sim} 871763006 \sim \mathrm{LLW} \mathrm{C}$ MPCT $\sim 1 \sim 0 T P-L L W-W P-04^{\sim}-$

08-16-97 13:35:57. ${ }_{\text {MSGLOG_STRINGT }}$

$N$

PCSDMS

74113

$\mathrm{CL}$

PCSDMS CL"871763197\%LLWC $\mathrm{MPCT}^{\sim} \sim 1 \sim \mathrm{OTP}-\mathrm{LLW}-\mathrm{WP}-04^{\sim}$

08-16-97 13:37:46 MSGLOG_STRING?

$\mathrm{N}$

PCSDMS

74120

CL

PCSDMS CL"871763305 LLW_C MPCT $^{\sim} 1^{\sim}$ OTP-LLW-WP-04

$08-16-97 \quad 14: 13: 58$.

$Y$

PCSDMS

74217

CL

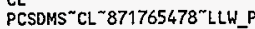

UCK $\sim 1$ "OTPOO3"

08-16-97 14:43:14

it

PCSDMS

74297

CL
MSGLOG_STRING1

MSGLOG_DT

MSGLOG ERROR FLAG

MSGLOG_GEN_TYYPE

MSGLOG SEQ NUM

MSGLOG SPEC TYPE

MSGLOG DT

MSGLOG_ERROR FLAG

MSGLOG_GEN_TYYPE

MSGLOG SEQ NUM

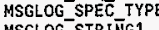

MSGLOG DT

MSGLOG_ERROR FLAG

MSGLOG GEN_TYPE

MSGLOG SEO NUM

\section{MSGLOG DT}

MSGLOG_ERROR_FLAG

MSGLOG_GEN_TYYPE

MSGLOG SEQ NUM

MSGLOG SPEC TYPE

MSGLOG_STRIN̄GI

\section{MSGLOG DT}

MSGLOG_ERROR FLA

MSGLOG SEQ NUM

MSGLOG STRINGI

\section{MSGLOG DT}

MSGLOG ERROR FLAG

MSGLOG GEN T TYPE

MSGLOG SEQ NUM

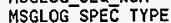

\section{MSGLOG DT}

MSGLOG ERROR FLAG MSGLOG GEN TYPE

MSGLOG SEQ NUM

MSGLOG SPE $\bar{C}$ TYPE

MSGLOG STRING?

MSGLOG DT

MSGLOG_ERROR FLAG

MSGLOG_GEN_TYYPE

MSGLOG SEQ NUM

MSGLOG_STRINGG1

\section{MSGLOG DT}

MSGLOG ERROR FLAG

MSGLOG_GEN_TYPE

MSGLOG SEQ NUM

MSGLOG_SPEC _TYPE

MSGLOG_SIRIN̄GI

MSGLOG DT

MSGLOG_ERROR_FLAG
MSGLOG SPEC TYPE

MSGLOG_SPE $\bar{C}$ TYPE
PCSDMS CL $871767233 \sim \mathrm{LLW} C$ MPCT $\sim 1 \sim 01 P-L L W-W P-04^{\sim}$

08-16-97 14:49:50

$N$

PCSDMS

74316

$\mathrm{CL}$

PCSDNS“CL“871767630 LLW C MPCT $\sim \sim 1 \% O T P-L L W-W P-04$ "

08-16-97 15:28:18

N

PCSDMS

74419

CL

PCSDMS ${ }^{*} \mathrm{CL}^{\circ 871769938 \sim L L W ~ C ~}$ MPCT $^{-m}$ 1 OTP-LLW-WP-04

08-16-97 15:50:16

N

PCSDMS

74476

CL

PCSDMS $\sim$ CL. $871771255 \%$ LLW E XIT $\sim$-OTP-LLW-OD-02

08-17-97 10:43:42

$Y$

PCSOMS

77499

$\mathrm{CL}$

PCSDMSCL $871839260^{\sim}$ TRU_S

ORT $\sim 1 \sim 202 A-T E S T-C A S E^{\sim}$

08-17-97 11:13:07

$y$

PCSDMS

77579

CL.

PCSDMS ${ }^{\circ}$-871841025 TRU_S ORT $\sim 1 \sim 202 A-T E S T$-CASE"

08-18-97 08:55:15

$N$

PCSDMS

81045

CL

PCSDMS $\mathrm{CL}^{2} 3080883314 \% \mathrm{LLH}$ CMPCT $\sim 1$ OTP-LLW-WP-O2

08-18-97 09:30:44

$N$

PCSDMS

81062

CL

PCSOMS“CL $\approx 71921280 \sim D I S C H$ CVR $^{\sim \sim}$ 1 RHZ-101-A13568

08-18-97 09:34:11

$Y$

PCSDMS

81073

$\mathrm{CL}$.

PCSDMS $\%$ CL"871921487 LLWS TORE1 1 OTPOO3

$08-18-97 \quad 09: 48: 24$

N

PCSDMS

81113

CL

PCSDMS ${ }^{\circ}$ \%871922340 LLH_P UCK $^{\sim 1 \text { 1 OTPOO2 }}$

08-18-97 09:48:29

N 
MSGLOG_GEN_TYPE

MSGLOG SEQ NUM

HSGLOG_SPEC TYPE

MSGLOG_STRIN̄GI

MSGLOG DT

MSGLOG_ERROR_FLAG

MSGLOG GEN TY YPE

MSGLOG_SEQ_NUM

MSGLOG SPE $\bar{C}$ TYPE

MSGLOG_STRING 1

MSGLOG DT

MSGLOG_ERROR FLAG

MSGLOG GEN TYYPE

MSGLOG_SEQ NUM

MSGLOG SPEC TYPE

MSGLOG_STRIN̄G1

MSGLOG DI

MSGLOG ERROR FLAG

MSGLOG GEN TYYPE.

MSGLOG SEQ NUM

MSGLOG SPE $\bar{C}$ TYPE

MSGLOG_STRING!

MSGLOG DT

MSGLOG ERROR FLAG

MSGLOG GEN TYYPE

MSGLOG SEO NUM

MSGLOG SPE $\bar{C}$ TYPE

MSGLOG_SIRINGG1

MSGLOG OT

MSGLOG ERROR_FLAG

MSGLOG GEN TYPPE

MSGLOG SEQ NUM

MSGLOG SPE $\bar{C}$ TYPE

MSGLOG_STRING1

MSGLOG OT

MSGLOG ERROR FLAG

MSGLOG GEN TYPE

MSGLOG SEQ NUM

MSGLOG_SPE $\bar{C}$ TYPE

MSGLOG_STRINGG1

MSGLOG DT

MSGLOG ERROR FLAG

MSGLOG GEN TYPE

MSGLOG SEQ MUM

MSGLOG_SPE $\bar{C}$ TYPE

MSGLOG_STRIN̄G1

MSGLOG DT

MSGLOG_ERROR_FLAG

MSGLOG_GEN TYPE

MSGLOG SEQ NUM

MSGLOG SPE $\bar{C}$ TYPE

MSGLOG_STRINNGI

\section{MSGLOG DT}

MSGLOG_ERROR_FLAG

MSGLOG GEN TYPE

MSGLOG SEQ NUM

MSGLOG SPEC̄_TYPE
PCSDMS

81114

$\mathrm{CL}$

PCSOMS“CL “871922342“LLW_P

UCK $\sim 1 \sim$ OTPOO2

08-18-97 10:07:08

N

PCSDMS

81165

$\mathrm{CL}$

PCSDMS $\mathrm{CL}^{2} 871923464$ LLW_P $\mathrm{UCK}^{\sim \sim 1}$ 1 OTPO02

08-18-97 10:12:15

$\mathrm{N}$

PCSDMS

81180

POPD

PCSDMS $\% O P D \sim 871923771 \% \mathrm{LLW}$

EXIT OTPOO4 OIP-LLW-OD-0

08-18-97 $10: 12: 17$
N
PCSDMS
81181
CLW
PCSDMS CLW $871923771^{\sim}$ LLW $_{-}$
PUCK $1 \%$ OTP004 32

08-18-97 11:29:25

PCSDMS

81388

$\mathrm{CL}$

PCSDMS “CL $871928401 \%$ LLW_S TORE2 ${ }^{\sim *} 1$ "OTPOO2

08-18-97 11:34:59

$\mathrm{N}$

PCSDMS

81395

$\mathrm{CL}$

PCSDMS CL $3080892898 \sim$ LLW CMPCT $^{\sim} 1 \sim$ OTP-LLW-WP-O3

08-18-97 11:39:49

$N$

PCSDMS

81406

CL.

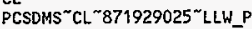
UCK 1 -OTP003

08-18-97 11:45:54

N

PCSDMS

81425

$\mathrm{CL}$

PCSDMS“CL 871929390〜LLWS

TORE1 " 1 OTP003

08-18-97 11:46:29

N

PCSDMS

81426

CL

PCSDMS ${ }^{\sim} \mathrm{CL}^{\sim} 871929425$ LLW_S

TORE 1 $\sim 1 \sim 0 T P O 03^{\sim}$

08-18-97 11:47:22

$\mathrm{N}$

PCSDMS

81431

CL
MSGLOG_STRINGI

MSGLOG OT

MSGLOG ERROR FLAG

MSGLOG_GEN_TŶPE

MSGLOG SEQ NUM

MSGLOG SPEC TYPE

MSGLOG_STRING

MSGLOG OT

MSGLOG_ERROR FLAG

MSGLOG GEN TYPPE

MSGLOG SEQ NUM

MSGLOG_SPE $\bar{C}$ TYPE

MSGLOG STRING

MSGLOG DT .

MSGLOG_ERROR_FLAG

MSGLOG GEN TYYPE

MSGLOG SEQ NUM

MSGLOG SPE $\bar{C}$ TYPE

MSGLOG_STRING 1

MSGLOG DT

MSGLOG_ERROR_FLAG

MSGLOG_GEN_TYPE

MSGLOG SEQ NUN

MSGLOG_SPE $\bar{C}$ TYPE

MSGLOG_STRIN̄G1

MSGLOG DT

MSGLOG ERROR FLAG

MSGLOG GEN TYPE

MSGLOG SEQ NUM

MSGLOG SPEC TYPE

MSGLOG_STRIÑG1

MSGLOG DT

MSGLOG ERROR FLAG

MSGLOG GEN TYYPE

MSGLOG SEQ NUM

MSGLOG SPEC TYPE

MSGLOG_STRIN̄G1

MSGLOG DT

MSGLOG ERROR FLAG

MSGLOG_GEN TYYPE

MSGLOG SEQ NUM

MSGLOG SPEC TYPE

MSGLOG_STRINTE1

MSGLOG_DT

MSGLOG ERROR FLAG

MSGLOG GEN_TYYPE

MSGLOG SEQ NUM

MSGLOG SPEC̄ TYPE

MSGLOG_STRINTG1

MSGLOG DT

MSGLOG ERROR FLAG

MSGLOG GEN TYYPE

MSGLOG SEQ NUM

MSGLOG SPEC TYPE

MSGLOG_STRIN̄G1

MSGLOG_OT
PCSDMS CL 871929476 LLW_S ORE1 $\sim$ 1 OTP0O3

08-18-97 11:48:49

$\mathrm{N}$

PCSDNS

81434

CL.

PCSDMS CL“871929565"LLW_S TORE1 ${ }^{\sim}$ ๆ OTPOOS

08-18-97 11:54:20

PCSDNS

81451

$\mathrm{Cl}$.

PCSDMS ${ }^{2}$ \%871929896 TRU S ORT $\sim 1$ "202A-TEST-CASE

08-18-97 11:55:41

$n$

PCSOMS

81457

CL

PCSDMS"CL“871929977"LLWS

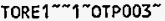

08-18-97 12:08:34

$\mathrm{N}$

PCSDMS

81492

POPD

PCSDMS POPD 871930750 LLW EXIT"OTPOO3"OTP-LLLH-OD-O

08-18-97 12:08:35

$N$

PCSOMS

81493

CLW

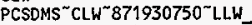
PUCK 1 OTP0O3 $1 \sim$

08-18-97 13:20:25

$Y$

PCSDMS

81686

$\mathrm{CL}$

PCSOMS“CL“871935061 TRU_S ORT $\sim 1 \sim 202 A$-TEST - CASE

08-18-97 13:37:32

N

PCSOMS

81732

$\mathrm{Cl}$

PCSDMS CL ${ }^{\sim} 871936088$ LLW_P UCK $^{\sim \sim}$ 1 OTPOO2

08-18-97 14:08:16

$\mathrm{N}$

PCSDMS

81815

$\mathrm{CL}$

PCSOMS"CL"871937932"LLWE NTRY" 1 OTP-LLW-OD-05

08- $-8-97$ 15:32:20

$\mathrm{N}$

PCSDMS

82040

POPD

PCSDMS"POPD"871942976"LLH

EXIT OTP-LLLW-OD-O

08-18-97 $15: 32: 21$ 


\begin{tabular}{|c|c|}
\hline $\begin{array}{l}\text { MSGLOG_ERROR FLAG } \\
\text { MSGLOG_GEN_TYYPE } \\
\text { MSGLOG_SEQ_NUM } \\
\text { MSGLOG_SPEC__TYPE } \\
\text { MSGLOG_STRINGG1 }\end{array}$ & $\begin{array}{l}Y \\
\text { PCSDMS } \\
82041 \\
\text { CLW } \\
\text { PCSDMS } \sim \text { CLW } 871942976^{\sim L L H} \\
\text { PUCK 11 } 31^{\sim}\end{array}$ \\
\hline $\begin{array}{l}\text { MSGLOG_DT } \\
\text { MSGLOG_ERROR FLAG } \\
\text { MSGLOG_GEN_TYPE } \\
\text { MSGLOG_SEQ_NUM } \\
\text { MSGLOG_SPEC̄_TYPE } \\
\text { MSGLOG_STRINTG1 }\end{array}$ & 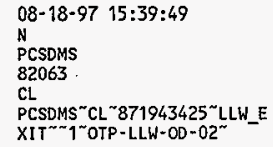 \\
\hline $\begin{array}{l}\text { MSGLOG_DT } \\
\text { MSGLOG_ERROR_FLAG } \\
\text { MSGLOG_GEN_TYPE } \\
\text { MSGLOG_SEQ_NUM } \\
\text { MSGLOG_SPEC TYPE } \\
\text { MSGLOG_STRINTG1 }\end{array}$ & $\begin{array}{l}08-18 \cdot 97 \quad 15: 41: 05 \\
N \\
\text { PCSDMS } \\
82068 \\
\text { POPD } \\
\text { PCSDMS POPD } \% 871943500 \sim L L W \\
\text { EXIT hickr! } 0 T P-L L W-0 D * 0\end{array}$ \\
\hline $\begin{array}{l}\text { MSGLOG_DT } \\
\text { MSGLOG_ERROR_FLAG } \\
\text { MSGLOG_GEN_TYPE } \\
\text { MSGLOG_SEQ_NUM } \\
\text { MSGLOG_SPEC TYPE } \\
\text { MSGLOG_STRINGT }\end{array}$ & 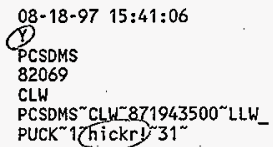 \\
\hline $\begin{array}{l}\text { MSGLOG_DT } \\
\text { MSGLOG_ERROR FLAG } \\
\text { MSGLOG_GEN_TYPE } \\
\text { MSGLOG_SEQ_NUM } \\
\text { MSGLOG_SPEC_TYPE } \\
\text { MSGLOG_STRINGI }\end{array}$ & $\begin{array}{l}\text { O8-18-97 } 15: 42: 58 \\
\text { N } \\
\text { PCSDMS } \\
82077 \\
\text { POPD } \\
\text { PCSDMS POPD } 871943614^{\sim} L L W \\
\text { EXIT OTP003 } O T P-L L W-O D-0\end{array}$ \\
\hline $\begin{array}{l}\text { MSGLOG_DT } \\
\text { MSGLOG_ERROR_FLAG } \\
\text { MSGLOG_GEN_TYPE } \\
\text { MSGLOG_SEQ_NUM } \\
\text { MSGLOG_SPEC TTYPE } \\
\text { MSGLOG_STRING1 }\end{array}$ & 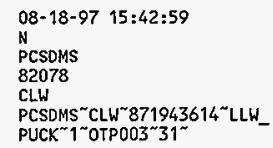 \\
\hline $\begin{array}{l}\text { MSGLOG_DT } \\
\text { MSGLOG_ERROR_FLAG } \\
\text { MSGLOG_GEN_TYPE } \\
\text { MSGLOG_SEQ_NUM } \\
\text { MSGLOG_SPEC̄TYPE } \\
\text { MSGLOG_STRING1 }\end{array}$ & $\begin{array}{l}\text { 08-18-97 } 15: 44: 37 \\
\text { N } \\
\text { PCSDMS } \\
82083 \\
\text { POPD } \\
\text { PCSDMS POPD } \% 871943712^{\sim L L W} \\
\text { EXIT OTP002 OTP-LLW-OD-0 }\end{array}$ \\
\hline $\begin{array}{l}\text { MSGLOG_DT } \\
\text { MSGLOG_ERROR_FLAG } \\
\text { MSGLOG_GEN_TYPE } \\
\text { MSGLOG_SEG_NUM } \\
\text { MSGLOG_SPEC̄_TYPE } \\
\text { MSGLOG_STRING }\end{array}$ & $\begin{array}{l}\text { 08-18-97 } 15: 44: 38 \\
\mathrm{~N} \\
\text { PCSOMS } \\
82084 \\
\text { CLW } \\
\text { PCSDMS"CLW"871943712 LLW_ } \\
\text { PUCK } 1 \text { "OTP002 31" }\end{array}$ \\
\hline $\begin{array}{l}\text { MSGLOG_DT } \\
\text { MSGLOG_ERROR_FLAG } \\
\text { MSGLOG_GEN_TYPE } \\
\text { MSGLOG_SEQ_NUM } \\
\text { MSGLOG_SPEC_TYPE } \\
\text { MSGLOG_STRINTG1 }\end{array}$ & $\begin{array}{l}\text { 08-18-97 } 16: 30: 59 \\
\text { N } \\
\text { PCSDMS } \\
82209 \\
\text { CL } \\
\text { PCSDMS CL } 871946495^{\sim L L W E} \\
\text { XIT } \sim 1 \sim O T P-L L W-00-01^{\sim}\end{array}$ \\
\hline $\begin{array}{l}\text { MSGLOG_DT } \\
\text { MSGLOG_ERROR_FLAG } \\
\text { MSGLOG_GEN TYYPE }\end{array}$ & $\begin{array}{l}08-18-97 \quad 16: 54: 00 \\
Y \\
\text { PCSDMS }\end{array}$ \\
\hline
\end{tabular}

MSGLOG_SEQ_NUM MSGLOG SPE $\vec{C}$ TYPE MSGLOG_STRINGG1

MSGLOG DT

MSGLOG_ERROR_FLAG

MSGLOG GEN TYYPE

MSGLOG SEQ MUM

MSGLOG SPEC TYPE

MSGLOG_STRIÑG?

MSGLOG DT

MSGLOG_ERROR_FLAG

MSGLOGGGEN TYPE

MSGLOG_SEQ -NUM

MSGLOG_SPEC

MSGLOG_STRIN̄G!.

MSGLOG DT

MSGLOG_ERROR_FLAG

MSGLOG GEN TYPE

MSGLOG SEQ NUM

MSGLOG_SPEC TYPE

MSGLOG_STRINGT

MSGLOG DT

MSGLOG_ERROR_FLAG

MSGLOG GEN TYYPE

MSGLOG_SEQ_MUM

MSGLOG_SPEC TYPE

MSGLOG_STRING 1

MSGLOG DT

MSGLOG_ERROR_FLAG

MSGLOG GEN TYPE

MSGLOG SEQ NUM

MSGLOG SPEC TYPE

MSGLOG_STRINGG1

MSGLOG DT

MSGLOG_ERROR FLAG

MSGLOG_GEN TYPPE

MSGLOG_SEO-NUM

MSGLOG SPEC TYPE

MSGLOG_STRINGG1

MSGLOG DT

MSGLOG_ERROR_FLAG

MSGLOG GEN TYYPE

MSGLOG_SEQ NUM

MSGLOG SPEC TYPE

MSGLOG_STRIN̄G1

MSGLOG_DT

MSGLOG ERROR FLAG

MSGLOG_GEN_TYYPE

MSGLOG SEQ NUM

MSGLOG_SPE $\bar{C}$ TYPE

MSGLOG_STRINGG1

MSGLOG_DT

MSGLOG_ERROR_FLAG

MSGLOG_GEN_TYYPE

MSGLOG_SEQ_NUM

MSGLOG_SPEC $\bar{C}_{-}$TYPE
82270

CL

PCSDMS "CL 871947876 LLW_P

UCK" 1 "OTPOO1"

08-18-97 17:09:18

$N$

PCSDMS

82314

POPD

PCSDMS"POPD 771948794 LLW EXIT OTPOO1 OTP-LLW-OD-O

98-18-97 17:09:19

(Y)

PCSDMS

82315

CLW

PCSDMS“CLW 871948794 LLH. PUCK 1 1 OTP001 $24^{\sim}$

08-18-97 17:22:04

N

PCSDMS

82351

CL

PCSDMS CL“871949559\%LLWE

XIT $\sim 1 \sim 0 T P-L L W-O D-01 \sim$

08-18-97 17:27:25

N

PCSDMS

82366

CL.

PCSDMS ${ }^{2}$ \%871949881 LLWE XIT $\sim$ १ OTP-LLW-OD-01

08-18-97 17:45:13

$Y$

PCSDMS

82415

$\mathrm{CL}$

PCSDMS"CL"871950949 LLW_P

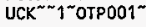

$08-18-97 \quad 17: 45: 15$

$\mathrm{Y}$

PCSDMS

82417

cl.

PCSDMS“CL.“871950949"LLW_P UCK $\sim 1 \sim 0 T P 001 \sim$

08-18-97 18:09:01

N

PCSDMS

82480

POPD

PCSDMS"POPD "871952376 "LW EXIT\%OTPOO1 OTP-LLW-OD-O

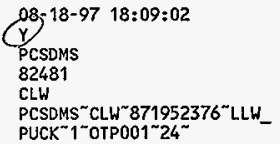

08-18-97 18:17:11
N
PCSDMS
82505
CL


MSGLOG_STRINGI

MSGLOG_OT

MSGLOG ERROR FLAG

MSGLOG_GEN_TY YPE

MSGLOG SEQ NUM

MSGLOG SPEC TYPE

MSGLOGSTRINGA1

MSGLOG DT

MSGLOG_ERROR_FLAG

MSGLOG_GEN TYYPE

MSGLOG SEQ NUM

MSGLOG SPEC TYPE

MSGLOG_STRINEG1

MSGLOG DT

MSGLOG_ERROR FLAG

MSGLOG GEN TYPE

MSGLOG SEQ NUM

MSGLOG_SPEC TYPE

MSGLOG_STRINGG?

MSGLOG OY

MSGLOG_ERROR_FLAG

MSGLOG GEN TVPPE

MSGLOG SEQ NUM

MSGLOG SPE $\bar{C}$ TYPE

MSGLOG STRIN̄G1

MSGLOG DT

MSGLOG_ERROR_FLAG

MSGLOG GEN TYPE

MSGLOG_SEQ_NUM

MSGLOG SPE $\bar{C}$ TYPE

MSGLOG_STRINTG1

MSGLOG DT

MSGLOG_ERROR FLAG

MSGLOG GEN TYPE

MSGLOG SEQ NUM

MSGLOG_SPE $\bar{C}$ TYPE

MSGLOG_STRINTG1

MSGLOG DT

MSGLOG_ERROR FLAG

MSGLOG GEN TYPE

MSGLOG SEO NUM

MSGLOG SPEC TYPE

MSGLOG_STRINGG?

MSGLOG DT

MSGLOG_ERROR_FLAG

MSGLOG GEN TYYPE

MSGLOGSSEQNUM

MSGLOG SPE $\bar{C}$ TYPE

MSGLOG_STRINGI

MSGLOG DT

MSGLOG ERROR FLAG

MSGLOG GEN TYYPE

MSGLOG_SEQ_NUM

MSGLOG SPE $\vec{C}$ TYPE

MSGLOG_STRINTG1

MSGLOG_DT

MSGLOG_ERROR_FLAG
PCSDMS CL 871952866 LLW_E

IT $^{\sim \sim 1}$ - OTP- $L L W-O D-01^{\sim}$

08-15-97 08:34:21

$Y$

PCSOMS

70534

CL

PCSDMS ${ }^{\circ L}$-871658704 TRU_S

ORT $\sim 1 \sim 202 A-T E S T$-CASE ${ }^{\sim}$

08-15-97 09:43:25

$\mathrm{Y}$

PCSOMS

70720

CL.

PCSOMS $\sim 2$ CL71662848 LLW_S

TORE1 “ 1“OTP010

08-15-97 11:22:14

$Y$

PCSDMS

70989

$\mathrm{CL}$

PCSOMS $\sim \mathrm{CL} \approx 871668776 \sim$ LLW_P

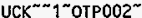

$\begin{array}{ll}08-15-97 \quad 11: 46: 51 & \text { MSGLOG_SPEC } \bar{C} \text { TYPE } \\ \text { MSGLOG_STRINGG1 }\end{array}$

$Y$

PCSOMS

71057

$\mathrm{CL}$

PCSDMS CL 871670253 LLW P

UCK $\sim 1 \sim 0 T P 001$

08-15-97 12:51:16

$Y$

PCSDMS

71233

$\mathrm{CL}$

PCSDMS ${ }^{*} \mathrm{CL}^{*} 871674118^{\sim}$ LLW_P

UCK" ${ }^{m \sim}{ }^{\sim}$ OTPOO1

08-15-97 12:51:55

$Y$

PCSDMS

71237

$\mathrm{CL}$

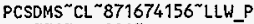

UCK ${ }^{N \sim} 1^{\sim}$ OTP0O1"

08-15-97 12:52:13

$Y$

PCSOMS

71239

CL

PCSDMS“CL 871674175 “LLWP UCK $\sim 1 \sim O T P 001 \sim$

08-15-97 12:54:08

$Y$

PCSDMS

71247

$\mathrm{CL}$

PCSDMS ${ }^{\sim} \mathrm{CL}^{\sim} 871674289 \%$ LLW P UCK ${ }^{\sim \sim} 1 \sim$ OTP001*

08-15-97 13:01:54

$Y$

PCSDMS

71269

$\mathrm{CL}$

PCSDMS“CL *871674756"LLW_S TORE 1 $~ 1 " O T P 001 "$

08-15-97 13:04:44

$Y$
MSGLOG_GEN_TYPE

MSGLOG SEQ NUM

MSGLOG SPE $\bar{C}$ TYPE

MSGLOG_STRIÑG1

MSGLOG_DT

MSGLOG ERROR FLAG

MSGLOG_GEN TYPE

MSGLOG SEQ NUM

MSGLOG SPE $\bar{C}$ TYPE

MSGLOG_STRIN̈G1

MSGLOG DT

MSGLOG ERROR FLAG

MSGLOG GEN TYPE

MSGLOG SEQ MUM

MSGLOG_SPE $\bar{C}$ TYPE

MSGLOG_STRINGG1

MSGLOG DT

MSGLOG_ERROR_FLAG

MSGLOG GEN TYPE

MSGLOG SEQ NUM

MSGLOG DT

MSGLOG_ERROR_FLAG

MSGLOG_GEN_TYYPE

MSGLOG SEQ NUM

MSGLOG_SPE $\bar{C}$ TYPE

MSGLOG_STRINTG1

MSGLOG OT

MSGLOG_ERROR_FLAG

MSGLOG GEN TYPE

MSGLOG_SEQ NUM

MSGLOG SPE $\vec{C}$ TYPE

MSGLOG STRIN̄G!

MSGLOG DT

MSGLOG_ERROR_FLAG

MSGLOG GEN TYYPE

MSGLOG SEQ NUM

MSGLQG SPE $\bar{C}$ TYPE

MSGLOG_STRINGG?

MSGLOG DT

MSGLOG_ERROR FLAG

MSGLOG GEN TYPE

MSGLOG_SEQ_NUM

MSGLOG_STRIN̄GI

MSGLOG DT

MSGLOG ERROR FLAG

MSGLOG GEN TYPE

MSGLOG SEQ NUM

MSGLOG_STRIN̄G1

MSGLOG DT

MSGLOG ERROR FLAG

MSGLOG GEN TŸPE

MSGLOG_SEQ_NUM

MSGLOG SPEC TYPE

MSGLOG_STRIN̄G1
MSGLOG SPEC TYPE

MSGLOG SPE $\vec{C}$ TYPE
PCSDMS

71277

c1.

PCSDMS"CL"871674926"LLW_S

TORE1 $\sim 1$ 1 OTPOO1

08-15-97 13:09:55

$Y$

PCSDMS

71293

CL

PCSOMS“CL“871675237"LLWS TORE1 q OTP001"

08-15-97 13:09:57

$Y$

PCSDMS

71295

CL.

PCSDMS “CL “871675239 LLW_S

TORE 1 \% 1 "OTP001"

08-15-97 13:10:02

$Y$

PCSDMS

71297

$\mathrm{CL}$

PCSDMS“CL 871675244\%LL.WS

TORE1 "1 1 OTPOO1

$08-15-97 \quad 13: 10: 14$

Y

PCSDMS

71301

$\mathrm{Cl}$

PCSDMS〜CL $\approx 71675255$ LLW_S

TORE1 $\sim 1$ "OTPOO1

08-15-97 13:10:32

$\mathrm{Y}$

PCSDMS

71303

$\mathrm{CL}$

PCSDMS“CL $871675274 \sim$ LLW_S TORE1 1 OTPOO1

08-15-97 13:11:36

$Y$

PCSDMS

71309

$\mathrm{CL}$.

PCSDMS“CL"871675336“LLW_S TORE1 $\sim 1 \% O T P O O 1^{\sim}$

$08-15-97 \quad 13: 12: 30$

$Y$

PCSDMS

71313

$\mathrm{CL}$

PCSOMS ${ }^{\sim} \mathrm{CL}^{\star} 871675392^{\sim}$ LLWS TORE 1 1"OTPOO1

08-15-97 13:26:58

$\gamma$

PCSDMS

71353

$\mathrm{CL}$

PCSDMS“CL"871676260 LLW_P UCK $^{\sim 1 \% \text { 1 }}$

08-15-97 13:27:00

$\mathrm{Y}$

PCSOMS

71355

$\mathrm{CL}$

PCSDMS“CL 871676261 LLW_P UCK $\sim$ 1 OTPOO2 
HSGLOG_DT

MSGLOG ERROR FLAG

MSGLOG GEN TYYPE

MSGLOG SEQ NUM

MSGLOG SPE $\bar{C}$ TYPE

MSGLOG_STRINTG1

MSGLOG OT

MSGLOG ERROR FLAG

MSGLOG_GEN_TȲPE

MSGLOG SEQ NUM

MSGLOG SPEC TYPE

MSGLOG_STRINTG1

MSGLOG DT

MSGLOG ERROR FLAO

MSGLOG_GEN_TYYPE

MSGLOG SEQ NUM

MSGLOG SPE C̃ TYPE

MSGLOG_STRIN̄G1

MSGLOG DT

MSGLOG ERROR FLAG

MSGLOG_GEN_TY YPE

MSGLOG SEQ NUM

MSGLOG SPEC TYPE

MSGLOG_STRINGG1

MSGLOG DI

MSGLOG ERROR FLAG

MSGLOG GEN IYPE

MSGLOG SEQ NUM

MSGLOG SPEC TYPE

MSGLOGSSIRIN̄GI

MSGLOG DT

MSGLOG_ERROR_FLAG

MSGLOG GEN TYYPE

MSGLOG SEQ NUM

MSGLOG SPEC TYPE

MSGLOG STRINTG?

MSGLOG DT

MSGLOG ERROR FLAG

MSGLOG GEN_TYYPE

MSGLOG SEQ NUM

MSGLOG SPE C TYPE

MSGLOG STRINTGI

MSGLOG DT

MSGLOG_ERROR FLAG

MSGLOG GEN TYPE

MSGLOG SEQ NUM

MSGLOG SPEC TYPE

MSGLOG STRINTG!

MSGLOG DT

MSGLOG_ERROR_FLAG

MSGLOG GEN TYYPE

MSGLOG SEQ NUM

MSGLOG SPEC TYPE

MSGLOG_STRINGG

MSGLOG DT

MSGLOG ERROR FLAG

MSGLOG GEN TYYPE

MSGLOG SEQ NUM

MSGLOG_SPE $\bar{C}_{-}$TYPE
08

PCSDMS

71358

$\mathrm{CL}$

PCSOMS CL 871676326 LLW_P

UCK $^{\sim \sim 1} \sim$

08-15-97 13:32:31

PCSDMS

71372

PCSDMS CL 871676593 LLW_P UCK $\sim \boldsymbol{1}^{\sim \sim}$

08-15-97 13:35:44 MSGLOG_STRIN̈G!

PCSDMS

71384

$\mathrm{CL}$

PCSOMS $\sim \mathrm{CL}^{2} 871676786 \sim$ LLW P UCK $\sim 1 \sim$ ORPOO2

08-15-97 13:35:46 MSGLOG_STRINTG1

PCSDMS

71386

$\mathrm{CL}$

PCSDMS“CL $871676787^{\sim}$ LLW_P UCK $\sim 1 \sim 0 T P 002 \sim$

$08-15-97 \quad 13: 35: 47$
$Y$
PCSDMS
71387
CL
PCSDMS CL $\approx 71676788 \sim L L H_{-P}$ UCK $^{\sim} \Upsilon^{\sim}$ OTP002

$08+15-97 \quad 13: 37: 32$

$Y$

PCSOMS

71392

$\mathrm{CL}$

PCSOMS“CL “871676894 "LLW_P

UCK $\sim 1 \sim 0 T P 002 \sim$

08-15-97 13:37:36

Y

PCSONS

71394

$\mathrm{CL}$

PCSDMS CL $^{2871676897 \% L L W ~ P ~}$ UCK" 1 "OTPOO2

08-15-97 14:35:14

$Y$

PCSDMS

71551

$\mathrm{CL}$

PCSOMS ${ }^{2}$ CL 871680356 TRU C OMPCT $^{\sim} \sqcap 96000001$

08-15-97 14:35:16

$Y$

PCSDMS

71553

$\mathrm{CL}$

PCSDMS"CL $\approx 71680356$ TRU_C OMPCT $^{\sim} \sim \mp 96000001$

08-15-97 14:35:18

Y

PCSDMS

71555

CL
MSGLOG_STRING1

MSGLOGDT

MSGLOG ERROR FLAG

MSGLOG GEN TŸPE

MSGLOG_SEQ NUM

MSGLOG SPE $\bar{C}$ TYPE

MSGLOG_STRIN̄GI

MSGLOG_DT

MSGLOG ERROR FLAG

MSGLOG GEN TYPE

MSGLOG SEQ NUM

MSGLOG SPE $\bar{C}$ TYPE

MSGLOG DT

MSGLOG ERROR FLAG

MSGLOG_GEN_T TPPE

MSGLOG SEO NUM

MSGLOG SPEC TYPE

MSGLOG DT

MSGLOG ERROR FLAG

MSGLOG GEN TYYPE

MSGLOG SEQ NUM

MSGLOG_SPE $\bar{C}$ TYPE

MSGLOG_STRINGG1

MSGLOG DT

MSGLOG_ERROR FLAG

MSGLOG GEN TYPPE

MSGLOG SEQ NUM

MSGLOG_SPEC_TYPE

MSGLOG STRIN̄G1

MSGLOG DT

MSGLOG_ERROR_FLAG

MSGLOG GEN TYPE

MSGLOG SEO NUM

MSGLOG SPE $\vec{C}$ TYPE

MSGLOGSTRINTG1

MSGLOG DT

MSGLOG_ERROR_FLAG

MSGLOG GEN TYPE

MSGLOG SEQ NUM

MSGLOG_SPE $\bar{C}$-TYPE

MSGLOG_STRING1

MSGLOG DT

MSGLOG_ERROR_FLAG

MSGLOG GEN TYPE

MSGLOG SEQ NUM

MSGLOG SPE $\bar{C}$ TYPE

MSGLOG_STRINGG1

MSGLOG OT

MSGLOG_ERROR FLAG

MSGLOG GEN TYYPE

MSGLOG SEQ NUM

MSGLOG_SPEC TYPE

MSGLOG_STRIN̄G1

MSGLOG DT

MSGLOG ERROR_FLAG
PCSDMS"CL“871680357"TRU_C OMPCT $^{\sim 1} \sim 96000001$

08-15-97 14:35:20

$Y$

PCSDMS

71557

$\mathrm{CL}$

PCSDMS"CL "871680357"TRU_C OMPCT $\sim 1 " 96000001$

08-15-97 14:37:20

PCSDMS

71565

CL.

PCSDMS“CL 871680481 TRU_C OMPCF $\sim 1 \sim 96000001$

08-15-97 14:37:22

$Y$

PCSDMS

71567

$\mathrm{CL}$

PCSDMS $\approx$ CL $871680482 \% T R U \_C$ OMPCT $\sim 1 \% 96000001$

08-15-97 14:37:24

PCSDMS

71569

CL

PCSDMS "Cl. "871680482 TRU_C OMPCT $^{\sim}$ P $^{*} 96000001$

08-15-97 14:37:55

PCSOMS

71573

CL

PCSDMS $\approx$ CL $871680517^{\sim}$ TRU_C OMPCT $^{\sim} 1 \sim 96000001$

08-15-97 14:37:57

$Y$

PCSDMS

71575

$\mathrm{CL}$

PCSDHS“CL "871680517"TRU_C OMPCT $\sim 1 \sim 96000001$

08-15-97 14:37:59

Y

PCSDMS

71577

$\mathrm{CL}$

PCSOMS“CL “871680518“TRU_C

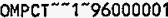

08-15-97 14:38:01

Y

PCSDMS

71579

CL

PCSDMS"CL $\approx 871680518 \% T R U$ C OMPCT $^{\sim} 1 \sim 96000001$

08-15-97 14:38:03

$Y$

PCSDMS

71581

$\mathrm{CL}$

PCSDMS $\sim \mathrm{CL}^{\sim} 871680518^{\sim}$ TRU_C OMPCT $^{\sim 1} \sim 96000001$

08-15-97 15:19:03

Y 
MSGLOG_GEN_TYPE MSGLOG_SEQ_NUM MSGLOG_SPEC TYPE MSGLOG_STRINTGI

\section{MSGLOG DT}

MSGLOG_ERROR_FLAG

MSGLOG GEN TTYPE

MSGLOG SEQ NUM

MSGLOG SPEC TYPE

MSGLOG STRINTG1

MSGLOG DT

MSGLOG_ERROR_FLAG

MSGLOG GEN TYYPE

MSGLOG_SEQ_NUM

MSGLOG SPEC TYPE

MSGLOG_STRING!

MSGLOG DT

NSGLOG_ERROR FLAG

MSGLOG GEN TŸPE

MSGLOG_SEQ_NUM

MSGLOG_SPE $\bar{C}$ TYPE

MSGLOG_STRIN̄G1

MSGLOG DT

MSGLOG_ERROR_FLAG

MSGLOG GEN TYPPE

MSGLOG_SEQ_NUN

MSGLOG SPE $\bar{C}$ TYPE

MSGLOG_STRIN̄G

MSGLOG_DT

MSGLOG_ERROR_FLAG

MSGLOG GEN TYPE

MSGLOG_SEQ_NUM

MSGLOG SPE $\bar{C}$ TYPE

MSGLOG_STRINGG1

MSGLOG_DT

MSGLOG ERROR FLAG

MSGLOG_GEN_TYPE

MSGLOG-SEQ NUN

MSGLOG SPEC TYPE

MSGLOG_STRING1

MSGLOG_DT

MSGLOG ERROR FLAG

MSGLOG_GEN TYPE

MSGLOG SEQ NUM

MSGLOG SPEC TYPE

MSGLOG_STRIN̄GI

MSGLOG DT

MSGLOG ERROR FLAG

MSGLOG_GEN_TYYPE

MSGLOG SEQ NUM

MSGLOG SPE $\bar{C}$ TYPE

MSGLOG_STRINTG1

MSGLOG DT

MSGLOG_ERROR FLAG

MSGLOG GEN TYYPE

MSGLOG SEO NUM

MSGLOG SPE $\bar{C}$ TYPE

MSGLOG_STRIN̄GI
PCSDMS

71691

CL

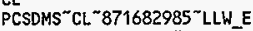
XIT $I^{\sim T E S T ~ D R U M ~ ~}$

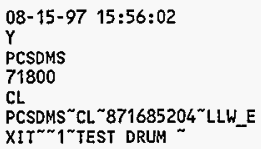

08-15-97 17:20:39

$Y$

PCSOMS

72026

$\mathrm{CL}$

PCSOHS ${ }^{2}$ CL 871690281 LLW_E XIT $\sim$ T TEST DRUM

08-15-97 17:33:03

$Y$

PCSDMS

72067

$\mathrm{CL}$

PCSDMS “CL“871691025 "LLW_P UCK 1 "OTP011

08-15-97 17:59:38

$Y$

PCSOMS

72140

POPD

PCSDMS ${ }^{\sim P O P D}$ \% $871692620^{\sim} \mathrm{LLW}$ EXIT OTP099 TEST DRUM

O8- $15-97 \quad 17: 59: 40$
PCSDMS
72142
CLW
PCSDMS CLW $871692620 \sim$ LLH $_{-}$
PUCK $1 \sim 82 \sim$

08-15-97 20:15:22

PCSOMS

72507

$\mathrm{CL}$

PCSDMS“CL"871700764"LLW_E XIT $\sim \sim$ 个TEST DRUM

08-15-97 20:33:48 Y

PCSDMS

72560

CLW

PCSDMS ${ }^{\circ} \mathrm{CLW}^{\sim} 871701868^{2}{ }^{\mathrm{LLW}}$ PUCK ${ }^{\sim}{ }^{\sim 224}$

08-15-97 20:40:37

$Y$

PCSDMS

72580

$\mathrm{Cl}$

PCSDMS ${ }^{\sim} \mathrm{CL}^{-871702278 \sim L L W E}$ XIT $\sim 1^{\sim}$ TEST DRUM -

08-15-97 20:41:25

$Y$

PCSDMS

72584

$\mathrm{CL}$

PCSDMS“CL "871702326“LLW_E

XIT $\sim 1 \sim T E S T$ DRUM
MSGLOG_DT

MSGLOG ERROR FLAG

MSGLOG GEN TYYPE

MSGLOG SEQ NUM

MSGLOG SPEC TYPE

MSGLOG_STRIN̈G1

MSGLÓg_DT

MSGLOG ERROR FLAG

MSGLOG_GEN TYYPE

MSGLOG SEQ NUM

MSGLOG SPEC̄ TYPE

MSGLOG_STRITIG1

MSGLOG DT

MSGLOG ERROR FLAG

MSGLOG_GEN_TYPE

MSGLOG SEQ MUM

MSGLOG SPE $\overline{\mathrm{C}}$ TYPE

MSGLOG_STRIÑG1

MSGLOG DT

MSGLOG_ERROR_FLAG

MSGLOG GEN TYYPE

MSGLOG SEQ NUM

MSGLOG_SPEC̄_TYPE

MSGLOG_STRINGG

MSGLOGDT

MSGLOG ERROR FLAG

MSGLOG GEN TYPPE

MSGLOG SEQ NUM

MSGLOG_SPE $\bar{C}$ TYPE

MSGLOG_STRINTGI

MSGLOG DT

MSGLOG_ERROR_FLAG

MSGLOG GEN TYPE

MSGLOG SEQ NUM

MSGLOG SPEC TYPE

MSGLOG STRIN̄G1

MSGLOG DT

MSGLOG_ERROR_FLAG

MSGLOG GEN TYYPE

MSGLOG SEO NUM

MSGLOG_SPE $\overline{\mathrm{C}}$ TYPE

MSGLOG_STRINGG1

MSGLOG OT

MSGLOG_ERROR_FLAG

MSGLOG GEN TYYPE

MSGLOG SEQ NUM

MSGLOG_SPEC

MSGLOG_STRIN̄G1

MSGLOG OT

MSGLOGERROR FLAG

MSGLOG GEN TYYPE

MSGLOG SEQ NUM

MSGLOG SPEC TYPE

MSGLOG_STRIN̄G1

MSGLOG OT

MSGLOG_ERROR_FLAG

MSGLOG GEN TYYPE

MSGLOG SEQ NUM

MSGLOG SPEC C TYPE
$08-15-9720: 43: 32$

$Y$

PCSDMS

72592

PCSDMS“CL"871702453"LLH_E

XIT $\sim 1$ TEST DRUM

$08-15-9720: 51: 23$

$Y$

PCSDMS

72615

CLW

PCSDHS CLN 871702923 LLW PUCK $1^{* *} 65^{n}$

$08-16-97 \quad 11: 06: 43$

PCSDMS

73704

$\mathrm{CL}$

PCSDMS“CL"871754243" LLW P UCK $^{\sim-1}$ 1 OTP004

08-16-97 14:13:58

PCSDMS

74217

CL

PCSDMS CL"871765478 LLW_P UCK $\sim 1$ 1 OTPOOS

$08-17-97 \quad 10: 43: 42$

$Y$

PCSDMS

77499

CL

PCSDMS“CL $\% 71839260^{\sim T R U S}$ ORT $^{\sim 1} 1^{\sim 202 A-T E S T}$-CASE

08-17-97 11:13:07

$Y$

PCSDMS

77579

CL

PCSDMS ${ }^{\sim} \mathrm{CL} \sim 871841025^{\sim}$ IRU_S ORT $^{\sim 1}$ 1 2O2A-TEST-CASE

08-18-97 09:34:11

$Y$

PCSDMS

81073

C1

PCSDMS ${ }^{\circ} \mathrm{CL}^{\circ} 871921487^{\sim}$ LLW S TORE $1^{\sim \sim 1}$ OTPOOZ

08-18-97 11:54:20

$\checkmark$

PCSDMS

81451

CL

PCSDMS $\approx C^{\sim} 871929896$ TRU S ORT $\sim^{\sim} \sim 202$ A-TEST-CASE

08-18-97 13:20:25

$Y$

PCSDMS

81686

CL

PCSDMS “CL $\approx 71935061 “ T R U S$ $\mathrm{ORT}^{\sim-1} 1$ 2O2A-TEST-CASE

08-18-97 15:32:21

Y

PCSDMS

82041

CLW 


\begin{tabular}{|c|c|}
\hline MSGLOG_STRING1 & $\begin{array}{l}\text { PCSDMS } \sim \text { CLW }^{\sim} 871942976^{\sim} \mathrm{LLW}_{2} \\
\text { PUCK }^{\sim} \sim 31^{\sim}\end{array}$ \\
\hline $\begin{array}{l}\text { MSGLOG_DT } \\
\text { MSGLOG_ERROR_FLAG } \\
\text { MSGLOG_GEN_TYPE } \\
\text { MSGLOG_SEQ_NUM } \\
\text { MSGLOG_SPE } \bar{C} \text { TYPE } \\
\text { MSGLOG_SIRINGG }\end{array}$ & $\begin{array}{l}08-18-97 \quad 15: 41: 06 \\
Y \\
\text { PCSDMS } \\
82069 \\
\text { CLW } \\
\text { PCSDMS CLW 871943500 LLW } \\
\text { PUCK }{ }^{\sim} \text { Hickr! } 31^{\sim}\end{array}$ \\
\hline $\begin{array}{l}\text { MSGLOG_DT } \\
\text { MSGLOG_ERROR_FLAG } \\
\text { MSGLOG_GEN_TYPE } \\
\text { MSGLOG_SEQ_NUM } \\
\text { MSGLOG_SPEC TYPE } \\
\text { MSGLOG_STRINGI }\end{array}$ & 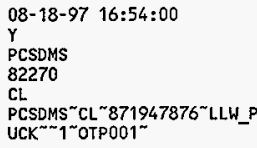 \\
\hline $\begin{array}{l}\text { MSGLOG_DT } \\
\text { MSGLOG_ERROR_FLAG } \\
\text { MSGLOG_GEN_TYYPE } \\
\text { MSGLOG_SEQ_NUM } \\
\text { MSGLOG_SPEC_TYYE } \\
\text { MSGLOG_SIRING1 }\end{array}$ & $\begin{array}{l}08-18-97 \quad 17: 09: 19 \\
Y \\
\text { PCSDMS } \\
82315 \\
\text { CLW } \\
\text { PCSDMS }{ }^{\sim} \text { CLW } 871948794^{\sim} \text { LLW }^{-} \\
\text {PUCK 1 OTP001 } 24^{\sim}\end{array}$ \\
\hline $\begin{array}{l}\text { MSGLOG_DT } \\
\text { MSGLOG_ERROR_FLAG } \\
\text { MSGLOG_GEN_TYYPE } \\
\text { MSGLOG_SEQ_NUM } \\
\text { MSGLOG_SPEד̄_TYPE } \\
\text { MSGLOG_STRINGI }\end{array}$ & 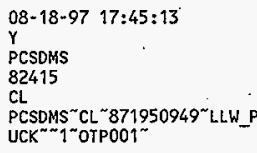 \\
\hline $\begin{array}{l}\text { MSGLOG_DT } \\
\text { MSGLOG_ERROR_FLAG } \\
\text { MSGLOG_GEN_TYYPE } \\
\text { MSGLOG_SEQ_NUM } \\
\text { MSGLOG_SPEC_TYPE } \\
\text { MSGLOG_STRINGI }\end{array}$ & 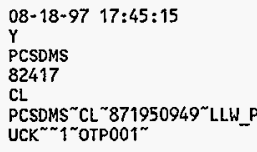 \\
\hline $\begin{array}{l}\text { MSGLOG_DT } \\
\text { MSGLOG_ERROR_FLAG } \\
\text { MSGLOG_GEN_TYYPE } \\
\text { MSGLOG_SEQ_NUM } \\
\text { MSGLOG_SPEC̄_TYPE } \\
\text { MSGLOG_STRING1 }\end{array}$ & $\begin{array}{l}\text { 08-18-97 18:09:02 } \\
\text { Y } \\
\text { PCSDMS } \\
82481 \\
\text { CLW } \\
\text { PCSDMS }{ }^{\sim} \text { CLW } 871952376^{\sim} \text { LLW } \\
\text { PUCK } 1^{\sim} \text { OTP001 } 24^{\sim}\end{array}$ \\
\hline
\end{tabular}




\section{WRAP 1 PLANT OPERATING PROCEDURE}

SYSTEM. PROCESS

\section{GLOVEBOX HOUSEKEEPING}

(HNF-SD-W026-OTP-015)

\section{SYSTEM DESCRIPTION}

This procedure provides instructions for cleanup of spil1s, debris and general housekeeping in WRAP-1 gloveboxes.

Glovebox housekeeping shall be performed after processing of each waste stream batch, and as needed during glovebox operations for spills or other debris bui 7 dup. This wi 11 ensure adequate, current, process knowledge to help determine possible sampling requirements \& environmental compliance when disposing of cleanup residue.

\section{REFERENCE DOCUMENTS / DOCUMENTED BASIS}

WRP1-OP-0704, G1ovebox Manipulator Operation WRP1-OP-0705, Bagless Transfer Manual Operation

\section{PRESTART CONDITIONS}

Al1 personnel performing this procedure sha11 be qualified in accordance with Waste Management Hanford Procedures Manua 1, Section 5.1, "Training and Qualification," and on-the-job training.

Verify daily radiological dose rates and contamination level for gloves have been established by Radiological Control.

\begin{tabular}{|c|c|c|c|c|c|}
\hline Release bate & Print 0.90 & Appr Des & Gocument No & Revimod & page \\
\hline
\end{tabular}




\section{WRAP 1 PLANT OPERATING PROCEDURE}

(HNF SB. W026 - OTP. 015)

IV. SAFETY

A11 potential hazards, such as lifting heavy containers or handling sharp objects present in waste containers, must be mitigated by protective equipment. procedures, and administrative controls to ensure acceptablerisk operating conditions.

A17 work must be performed per applicable Radiation work Permits (RWPS).

Clean up spil1s immediately. Use only facility approved chemical or cleaning agents. Use materials sparingly and in accordance with MSDS information to prevent unwanted chemical reactions or formulation of new chemical compositions.

\section{TOOLS AND SUPPLIES}

Approved cleaning agents and supplies as required.

\section{TABLE OF CONTENTS}

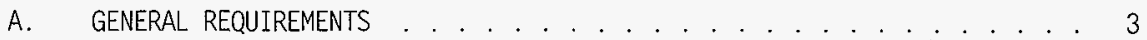

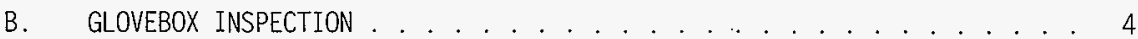

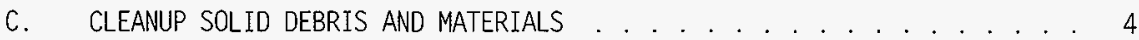

D. ClEANUP LIQUID AND SPILLS. .................... 5

E. WIPEDOWN GLOVEBOX ......................... 6

F. SAMPLING AND LOADOUT .................. . . 7

\section{ATTACHMENTS}

None 


\section{WRAP 1 PLANT OPERATING PROCEDURE}

(HNF SB. W026 - OTP 015 )

\section{PROCEDURE}

\section{CAUTION}

INTRODUCTION OF UNAPPROVED CLEANING AGENTS INTO GLOVEBOXES MAY RESULT IN ENVIRONMENTAL NON-COMPLIANCE, UNWANTED CHEMICAL REACTIONS OR OTHER UNDESIRABLE EFFECTS. ONLY FACILITY APPROVED CLEANING AGENTS MAY BE USED.

Note -

Administrative hold steps are identified by letters in parentheses at the left margin of the procedure step. Direction given in the procedure step must be satisfied before work continues.

(M) - Operations management sha71 approve operation.

(R) - Radiological Control (RC) shall complete surveys or agree to permit continued operation.

A. GENERAL REQUIREMENTS

1. Sections VII.C through VII.E of this procedure may be performed repeatedly and in any order necessary to facilitate work.

\section{WARNING}

WORK THROUGH GLOVEPORTS MAY INVOLVE PLACING HANDS IN CLOSE PROXIMITY TO MOVING EQUIPMENT. CARE MUST BE TAKEN TO PREVENT INJURY.

2. IF at any time during performance of this procedure, work is to be performed through gloveports,

THEN:

a. CHECK status and location of moving equipment in proximity of gloveport(s) to be used.

b. TAKE action, including any of the following, as deemed necessary to prevent injury:

- $\quad$ AVOID equipment when reaching through port(s)

- $\quad$ PRESS glovebox E-Stop to stop glovebox equipment

- CONSULT DOS when unsure 


\section{WRAP 1 PLANT OPERATING PROCEDURE}

(HNF. SD. W026.07P-015)

c. PERFORM a contamination survey of hands, arms and front of body upon exiting glove:

(1) IF contamination is detected.

THEN, CONTACT Radiological Control Technician.

\section{B. GLOVEBOX INSPECTION}

1. CHECK, visua7ly throughout glovebox for:

- Loose items, spills, droppings or tailings from waste handling process.

- Improperly stored glovebox tools and equipment.

- Leaks from glovebox hydraulic systems.

- Left-over agents introduced for cleaning or other purposes.

- Foreign matter on glovebox wa71s, windows and/or equipment.

2. CHECK glovebox equipment (manipulators, ports, lift tables, etc) for damage, leaks, etc.

3. PERFORM glovebox cleanup as required, per Sections VII.D through VII.F. of this procedure.

\section{CLEANUP SOLID DEBRIS AND MATERIALS}

NOTE 1 - Glovebox manipulators are operated per WRP1-OP-0704, Glovebox Manipulator Operation.

1. USING manipulators to the extent possible, PERFORM the following:

a. PICKUP and BAG smal1 waste and debris.

(1) USE (with manipulators) broom, dustpan, and shove] located inside glovebox, as needed to aid cleanup.

b. PLACE waste in desired area for removal or processing as directed by DOS.

c. MOVE glovebox tools and equipment to proper glovebox storage location. 


\section{WRAP 1 PLANT OPERATING PROCEDURE}

$(\mathrm{HN}=\mathrm{SD} \cdot \mathrm{W026} \cdot 0 \mathrm{IP} \cdot 015)$

2. IF any items cannot be accessed with manipulators,

THEN:

a. LOCATE gloveport(s) which provide best access to item(s),

b. PROCESS items through gloveports per Steps VII.C.1.a through VII.C.1.C.

3. IF other cleanup work is to be performed,

THEN, GO TO applicable section(s).

OTHERWISE, GO TO Section VII.F, Sampling and Loadout.

D. CLEANUP LIQUID AND SPILLS

NOTE - Spill kits will norma77y be pre-packaged in marked sample transfer canisters and stored in the Warm Maintenance Area. Room 108.

1. TRANSFER required Spi 17 Cleanup Kit into glovebox through bagless transfer port, sample transfer port or consumable materials entry port, as needed, per applicable operating procedure(s):

- WRP1-0P-0705, Bagless Transfer Manual Operation.

- WRP1-0P-0710, Waste Sampling.

2. WORKING with manipulators and/or through gloveport(s), as required. PERFORM the following:

NOTE - Cleanup is accomplished by best suited method. The following steps are used as a guideline.

a. PLACE dam, dike, or absorbent containment from spill kit around spil1.

b. ABSORB, using approved pads and absorbent, as required, all spilled material.

c. PLACE used pads and absorbent into plastic bag(s).

d. PLACE waste in desired area for removal or processing as directed by DOS. 


\section{WRAP 1 PLANT OPERATING PROCEDURE}

(BNS SD W026 - 0TP 015$)$

3. IF necessary,

THEN, WIPEDOWN spi11 area per Section VII.E.

4. IF other cleanup work is to be performed.

THEN, GO TO applicable section(s).

OTHERWISE, GO TO Section VII.F, Sampling and Loadout.

E. WIPEDOWN GLOVEBOX

1. TRANSFER required materials (cleaning agents, rags, pads) into glovebox through bagless transfer port, sample transfer port or consumable materials entry port, as needed, per applicable operating procedure(s):

- WRP1-0P-0705, Bagless Transfer Manual Operation.

- WRP1-OP-0710, Waste Sampling.

2. WORKING with manipulators and/or through gloveport(s), as required, PERFORM the following:

NOTE - Cleanup is accomplished by best suited method (i.e. cleaning agents may be poured directly on area or applied to rags or pads, then wiped over area). The following steps are used as a guideline.

a. APPLY cleaning agent to affected area(s).

b. SCRUB/BRUSH affected area(s).

c. WIPE, affected area(s) and equipment with clean, dry, absorbent pads or rags.

d. PLACE used pads, rags and other items to be disposed. into plastic bag(s).

e. PLACE waste in desired area for removal or processing as directed by DOS.

3. IF other cleanup work is to be performed,

THEN, GO TO applicable section(s),

OTHERWISE, GO TO Section VII.F. Sampling and Loadout. 


\section{WRAP 1 PLANT OPERATING PROCEDURE}

F. SAMPLING AND LOADOUT

NOTE 1 - Once cleaned up, items are treated as if processing ordinary waste for applicable glovebox.

NOTE 2 - Sampling requirements are determined by review of process history for waste stream preceding cleanup activities.

(M) 1. DETERMINE sorting, sampling and processing requirements for waste gathered or generated during cleanup activities.

2. PERFORM sorting, sampling and processing of waste gathered or generated during cleanup activities per applicable procedures. as directed. 


\title{
WRAP 1 PLANT OPERATING PROCEDURE
}

\author{
SWSTEM. PROGESS
}

\section{RESTART LLW GLOVEBOX OPERATION}

(HNF-SD-W026-OTP-015)

\section{SYSTEM DESCRIPTION}

This procedure provides instructions for restart of Low Level Waste (LLW) Gloveboxes after Emergency. Stop. Fire Alarm. Power Outage, Equipment Failure, Low Glovebox Pressure or other abnormal shutdown.

Emergency Stop or other abnormal shutdown of the gloveboxes may leave various glovebox equipment including entry/exit ports, transfer cart, sorting table, hoists, lift tables etc. in an abnormal state (i.e., partially open/closed, tilted, seals deflated or inflated at the wrong time, etc.). Prior to restart, the condition of al1 glovebox equipment must be analyzed, and as required, returned to normal initialization status. Because of numerous interlocks and other safety features return of most equipment to the proper position must be accomplished manually through use of the Maintenance Mode menus on the glovebox Operator Interface Units (OIUs), Lift Table Controllers and other operator control stations.

When an OIU is in maintenance mode, most interlocks and safety features are bypassed. This allows equipment to be manipulated to a desired state to satisfy operationa 7 needs. It is also possible to damage equipment, and/or bypass safety restrictions such as glovebox containment. Caution must be exercised to prevent unwanted violation of operational safety requirements or personnel injury.

\section{REFERENCE DOCUMENTS}

WRP1-0P-0719. LLW Glovebox Line Manual Operation

WRP1-OP-0713, LLW Entry Glovebox Operation.

WRP1-0P-0714, LLW Sorting Glovebox Operation.

WRP1-OP-0716. LLW Exit Glovebox Operation.

\section{PRESTART CONDITIONS}

A11 personnel performing this procedure shall be qualified in accordance with WHC-CM-5-34. Section 1.8, "Training Plan," and on-the-job training.

Process area Heating, Ventilation, Air Conditioning (HVAC) in OPERATION. Applicable light curtains in OPERATION.

Applicable portions of plant electrical system in OPERATION.

Compressed air system in OPERATION.

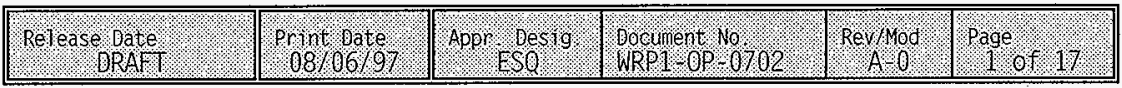




\section{WRAP 1 PLANT OPERATING PROCEDURE}

(HNF S1 1026 - OTP 015$)$

\section{SAFETY}

Potential hazards, such as lifting heavy containers or handling sharp objects present in waste containers, must be mitigated by protective equipment, procedures, and administrative controls to ensure acceptable-risk operating conditions.

Consult with Operations Management and Industrial Hygiene, and refer to the Job Hazards Analysis (JHA), Radiological Work Permit (RWP) and Material Safety Data Sheets (MSDS) to determine required. Personal Protective Equipment (PPE).

This procedure requires accessing gloveboxes through gloveports on a regular basis. Operators shall perform self survey upon exiting gloveport áfter each use. Radiological control Technician (RCT) shall be contacted anytime self survey limits are exceeded.

Warning - Use care to prevent injury. Possible safety hazards include:

- Tripping/STipping

- Injuries to the hands at glovebox ports

- Radiological Contamination

- Interference with the Automatic Guided Vehicle (AGV)

\section{TOOLS AND SUPPLIES}

None. 


\section{WRAP 1 PLANT OPERATING PROCEDURE}

\section{TABLE OF CONTENTS}

PAGE

A. DETERMINE GLOVEBOX EQUIPMENT STATUS . . . . . . . . . . . . . 4

B. RETURN ENTRY GLOVEBOX TO OPERATION ............. 5

C. RETURN SORTING GLOVEBOX TO OPERATION . . . . . . . . . . . . . . . . . 6

D. RETURN EXIT GLOVEBOX TO OPERATION . . . . . . . . . . . . . . 6 ATTACHMENTS

ATTACHMENT 1: ENTRY GLOVEBOX EQUIPMENT STATUS . . . . . . . . . . . . . . . . . 7 ATTACHMENT 2: SORTING GLOVEBOX EQUIPMENT STATUS . . . . . . . . . . . . . . 10 ATTACHMENT 3: EXIT GLOVEBOX EQUIPMENT STATUS $: \ldots \ldots \ldots$ 


\section{WRAP 1 PLANT OPERATING PROCEDURE}

(HNF SD .1026 . OJP O 015)

\section{PROCEDURE}

NOTE -

Equipment status is determined by visual observation of equipment and status indicating 7ights as applicable.

A. DETERMINE GLOVEBOX EQUIPMENT STATUS

1. CHECK each glovebox equipment/component listed on Attachment 1 , LLW Entry Glovebox Equipment Status, and RECORD AS FOUND status as follows:

a. DRAW a line through non-applicable positions for each component.

b. IF component is at other than a defined position, THEN:

(1) DRAW a line through all defined positions.

(2) DESCRIBE As Found state in Comments section.

c. RECORD a 17 other indications, conditions or supplemental information in Comments section.

2. CHECK each glovebox equipment/component listed on Attachment 2, LLW Sorting Glovebox Equipment Status, and RECORD AS FOUND status as follows:

a. DRAW a line through non-applicable position(s) for each component.

b. IF component is at other than a defined position, THEN:

(1) DRAW a line through all defined positions.

(2) DESCRIBE As Found state in Comments section.

c. RECORD al1 other indications, conditions or supplemental information in Comments section. 


\section{WRAP 1 PLANT OPERATING PROCEDURE}

(HN) SB -1026 - 0IP 015$)$

3. CHECK each glovebox equipment/component listed on Attachment 3, LLW Exit Glovebox Equipment Status, and RECORD AS FOUND status as follows:

a. DRAW a line through non-applicable positions for each component.

b. IF component is at other than a defined position. THEN:

(1) DRAW a line through all defined positions.

(2) DESCRIBE As Found state in Comments section.

c. RECORD a11 other indications, conditions or supplemental information in Comments section.

\section{B. RETURN ENTRY GLOVEBOX TO OPERATION}

1. ANALYZE As Found state of glovebox to determine which equipment must be manipulated to correct operational deficiencies, as follows:

a. COMPARE As Found status on Attachment 1, to the following:

- INITIALIZATION POSITION on Attachment 1.

- Interlock information in applicable section of WRP1-OP-0719, LLW Glovebox Line Manual Operation.

- $\quad$ Operational sequencing in WRPI-0P-0713, LLW Entry GTovebox Operation.

b. RECORD required corrections in Comments section of Attachment 1.

2. GO TO applicable section of WRP1-OP-0719 and PERFORM necessary actions to return equipment to desired status.

3. RETURN glovebox 0IU to automatic operation per WRP1-OP-0719.

\begin{tabular}{|c|c|c|c|}
\hline $\begin{array}{l}\text { Oocuinent } 110 \\
\text { WRPL Ge } 0702\end{array}$ & prnt bater & Rev/Mod & page \\
\hline
\end{tabular}




\section{WRAP 1 PLANT OPERATING PROCEDURE}

(UNF-SO-W626-0TP-015)

C. RETURN SORTING GLOVEBOX TO OPERATION

1. ANALYZE As Found state of glovebox to determine which equipment must be manipulated to correct operational deficiencies, as follows:

a. COMPARE As Found status on Attachment 2, to the following:

- $\quad$ inITIALIZATION POSITION on Attachment 2.

- Interlock information in applicable section of WRP1-0P-0719. LLW GTovebox Line Manual Operation.

- $\quad$ Operational sequencing in WRP1-0P-0714, LLW Sorting Glovebox Operation.

b. RECORD required corrections in Comments section of Attachment 2.

2. GO TO applicable section of WRP1-OP-0719 and PERFORM necessary actions to return equipment to desired status.

3. RETURN glovebox 0IU to automatic operation per WRP1-0P-0719.

D. RETURN EXIT GLOVEBOX TO OPERATION

1. ANALYZE As Found state of glovebox to determine which equipment must be manipulated to correct operational deficiencies, as follows:

a. COMPARE As Found status on Attachment 3, to the following:

- INITIALIZATION POSITION on Attachment 3.

- Interlock information in applicable section of WRP1-OP-0719. LLW Glovebox Line Manual Operation.

- Operational sequencing in WRP1-OP-0716. LLW Exit Glovebox Operation.

b. RECORD required corrections in Comments section of Attachment 3.

2. GO TO applicable section of WRP1-OP-0719 and PERFORM necessary actions to return equipment to desired status:

3. RETURN glovebox OIU to automatic operation per WRP1-0P-0719. 


\section{WRAP 1 PLANT OPERATING PROCEDURE}

(HNF SD WO26 - 0 TP 015 )

\section{ATTACHMENT 1: ENTRY GLOVEBOX EQUIPMENT STATUS}

\begin{tabular}{|c|c|c|}
\hline LQUPPMENT/COMPONENT: & AS FOUNB POSTMION & WOSTION \\
\hline \multicolumn{3}{|c|}{ 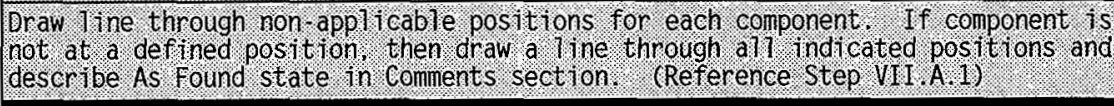 } \\
\hline \multicolumn{3}{|c|}{ 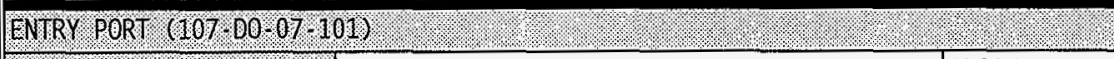 } \\
\hline PORT OOOR . & $(\mathrm{ZSO}-701 / \mathrm{ZSC} \cdot 701)$ & CLOSED \\
\hline 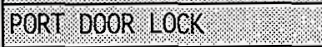 & LOCKED / UNLOCKED & LOCKED \\
\hline ORUM OOOR & $\begin{aligned} \text { OPEN / CLOSED / IN POSITION (1/2" up) } \\
\\
\text { (ZS-703C / ZS-703A / ZS-703B) }\end{aligned}$ & CLOSED \\
\hline IO AGAINST QRUM OOOR & YES / NO & NO \\
\hline BRUH CENI GRING OEVICE. & EXTENDED / RETRACTED & RETRACTED \\
\hline \multicolumn{3}{|c|}{ 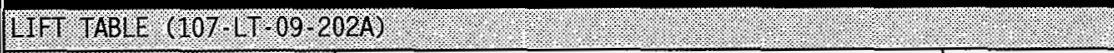 } \\
\hline 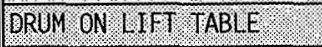 & YES / NO & YES \\
\hline OROM UNDER PORT WEO & ON / OFF & OFF \\
\hline QRUM IN POS T WON WEO & ON / OFF & OFF \\
\hline DRUM AT AGY $\mathrm{NNG}$ LEO & ON / OFF & ON \\
\hline AGY $\angle O A B$ UEIGH YGO & ON / OFF & ON \\
\hline GOWN QVERTRAVE LEO & ON / OFF & OFF \\
\hline \multicolumn{3}{|c|}{ 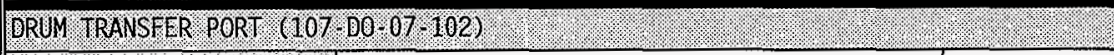 } \\
\hline 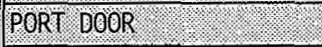 & (ZSO.705/ZSC.705) & CLOSED \\
\hline WAC SEAL & INFLATED / DEFLATED & INFLATED \\
\hline \multicolumn{3}{|c|}{ 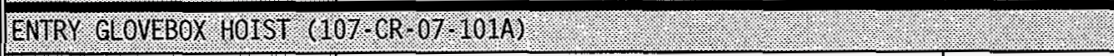 } \\
\hline GROM W U UIER . & YES / NO & NO \\
\hline TIUR JAWS & OPEN / CLOSED & OPEN \\
\hline HOUST IULY RATSED & YES / NO & YES \\
\hline HOIST TROUSY POST ITON & ENTRY PORT / TRANSFER PORT & ENTRY PORT. \\
\hline
\end{tabular}




\section{WRAP 1 PLANT OPERATING PROCEDURE}

(MNF SO W026 - 0TP -015)

\section{ATTACHMENT 1: ENTRY GLOVEBOX EQUIPMENT STATUS}

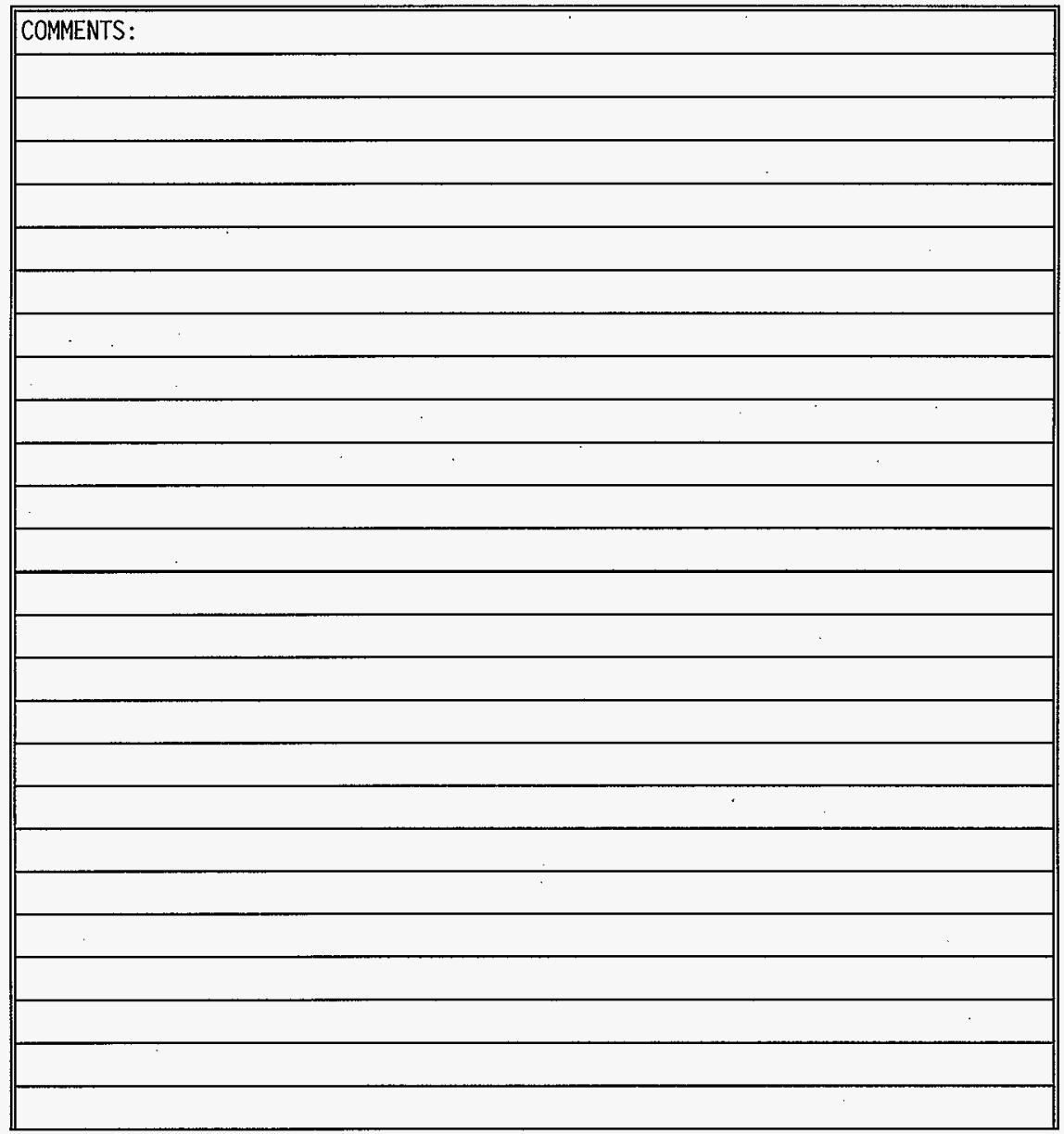


HNF-SD-W026-OTR-015, Rev. 0, Page OP-16

WRAP 1 PLANT OPERATING PROCEDURE

UNF SB WO26 OTP 015)

\section{ATTACHMENT 1: ENTRY GLOVEBOX EQUIPMENT STATUS}

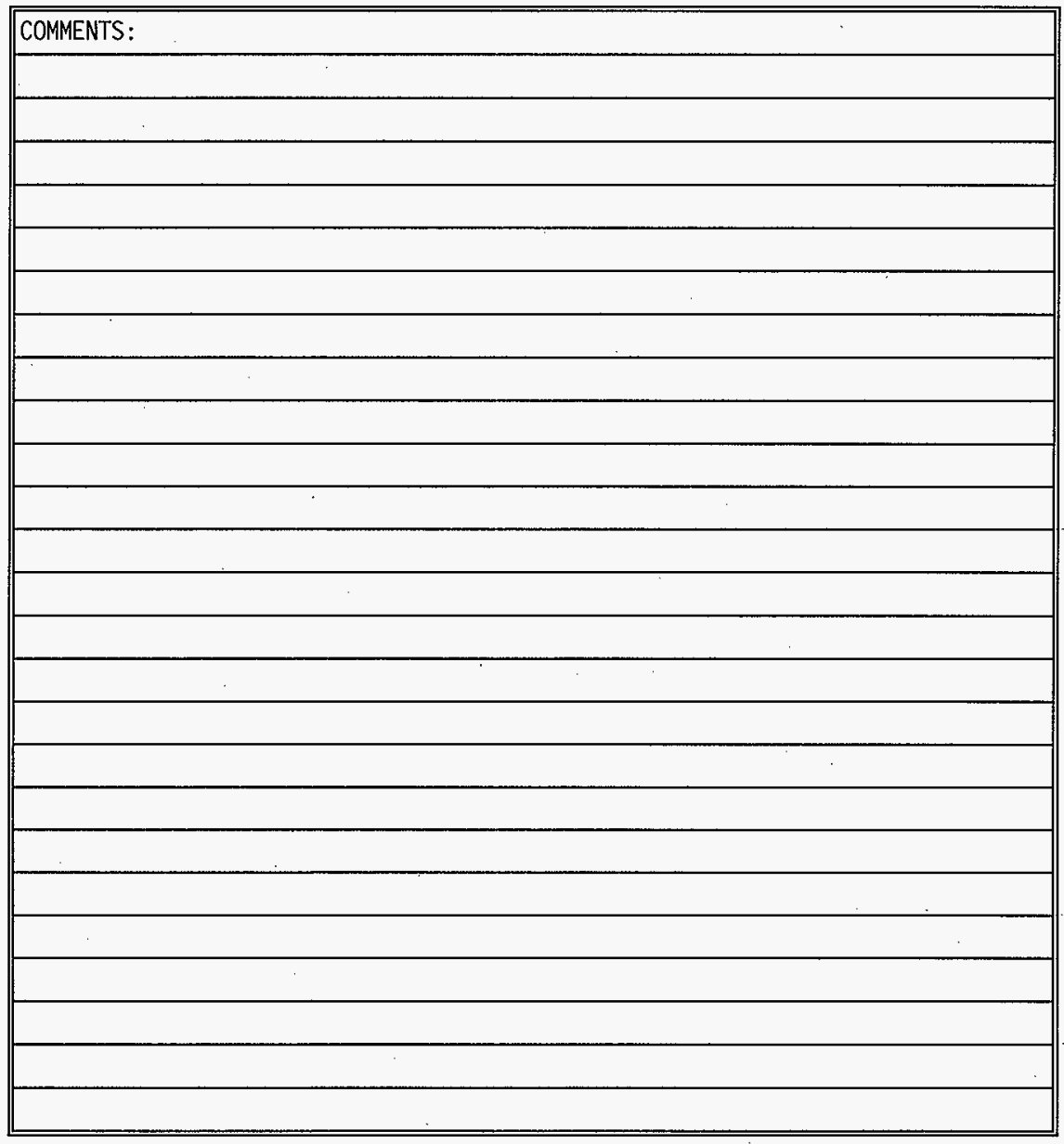




\section{WRAP 1 PLANT OPERATING PROCEDURE}

(UNF.SD . N026.0TP - 015)

\section{ATTACHMENT 2: SORTING GLOVEBOX EQUIPMENT STATUS}

\begin{tabular}{|c|c|c|c|}
\hline EQUIPUENT/GOMPONENT & \multicolumn{2}{|c|}{ AS FOUNA POSITION } & 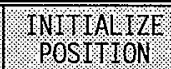 \\
\hline \multicolumn{4}{|c|}{ 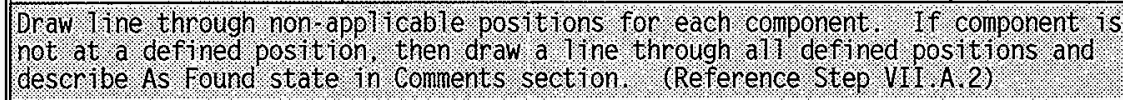 } \\
\hline \multicolumn{4}{|c|}{ DRUM TPPER (107 BH.07 103) } \\
\hline 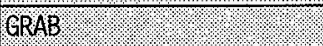 & OPEN / CLOSED & (ZS.720B / ZS.720A) & OPEN \\
\hline GRAB : : : : : : & RAISED / LOWERED & (ZS-332A / ZS-332B) & RAISED \\
\hline TIPPER GRAB VERTTCAL & YES / NO & $(25 \cdot 333)$ & YES \\
\hline TPPER GLUTOH TRIPPED & YES / NO & $(\mathrm{ZS}-335)$ & NO \\
\hline \multicolumn{4}{|c|}{ 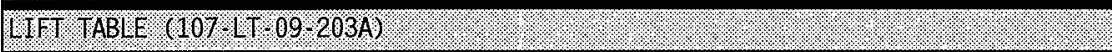 } \\
\hline DRUM GN LIIT TABLE: & YES / NO & (VISUAL) & YES \\
\hline BRUM UNBER PORT LEO & $\mathrm{ON} / \mathrm{OFF}$ & $(Z S-241 F)$ & OFF \\
\hline DRUM IN POSTITION IFE & ON / OFF & (ZS-241D) & OFF \\
\hline DRUM AT ACV ENB IED. & ON / OFF & (ZS-241E) & ON \\
\hline AGV LOAD IEIGHI YGD & ON / OFF & (ZS-241G) & ON \\
\hline DOWN OVER RAVEL LED & $\mathrm{ON} / \mathrm{OFF}$ & (ZS-241C) & OFF \\
\hline \multicolumn{4}{|c|}{ ENIRY PORT (107. DO 07 . 105 ) } \\
\hline PORT DOOR : & OPEN / CLOSED & (ZS-734B / ZS-734A) & CLOSED \\
\hline PORT OOOOR LOCK. & LOCKED / UNLOCKED & $(Z S-730 A \& B)$ & LOCKED \\
\hline MU LIUER: & LIFTED / LOWERED & (ZS-731A / ZS-731B) & CLOSED \\
\hline WD ATIACHED : & YES / NO & $(Z S-733 B / Z S-733 A)$ & NO \\
\hline \multicolumn{4}{|c|}{ SORTING GLOVEBOX HOIST (107. CR 07 T 10IC) } \\
\hline XFER STANG ATTACHED : & YES / NO & (VISUAL) & YES \\
\hline HOIST FUIY Y RATSED. & YES / NO & (ZS-730C) & YES \\
\hline HOIST TROLLEY POSTTION & TRAY LOAD OUT / TF & $(Z S-730 A \& B)$ & LOAD OUT \\
\hline
\end{tabular}

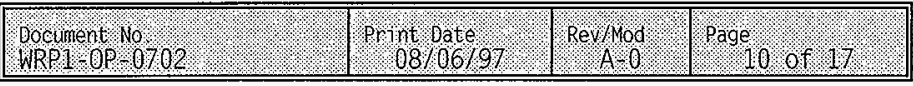




\section{WRAP 1 PLANT OPERATING PROCEDURE}

(HNF . SD - NO26 OIP 015 )

\section{ATTACHMENT 2: SORTING GLOVEBOX EQUIPMENT STATUS}

\begin{tabular}{|c|c|c|}
\hline EQUIPMEN/ COMPONENT. & AS FOUNO POSTITON & $\begin{array}{l}\text { INIJIALIZ } \\
\text { POSTTION }\end{array}$ \\
\hline \multicolumn{3}{|c|}{ 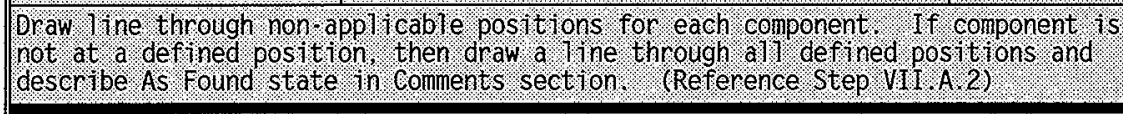 } \\
\hline \multicolumn{3}{|c|}{ 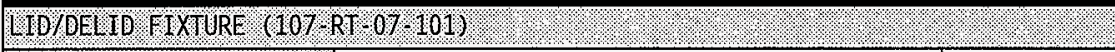 } \\
\hline M XTURE ELEVATON: : & RAISED / LOWERED & RAISED \\
\hline TXURE TRAVE & PARK-RELID / DELID & PARK \\
\hline FIXURE ROAATION & PARKED / OTHER INDEX & PARKED \\
\hline М1 ATACHEV. & (PS-716 \& VISUAL) & NO \\
\hline \multicolumn{3}{|l|}{ WD/OE TO I ITURE SAW } \\
\hline SAW ARM : $: 0.0$ & EXTENDED / RETRACTED & RETRACTED \\
\hline MERT GAS FIOH : & YES / NO & NO \\
\hline SAW MOTOR: & (FEV-378A \& VISUAL) & OFF \\
\hline \multicolumn{3}{|c|}{ MANIPU ATOR $(107$. EM:07 101$)$. } \\
\hline MANIPUIATOR PARKED & POS $1 /$ POS $2 /$ NO (ZS-715A / ZS-715B / NONE) & POS 1 OR 2 \\
\hline LEFI ARU PARKED & YES / NO & YES \\
\hline RTCHT ARM PARKED: & YES / NO & YES \\
\hline \multicolumn{3}{|l|}{ PAGKET X RAY : } \\
\hline X RAY DRAWER & $(\mathrm{ZS} \cdot 334 \mathrm{~A} / \mathrm{ZS} \cdot 334 \mathrm{~B})$ & CLOSED \\
\hline X RAY TRAY: & EXTENDED / RETRACTED & RETRACTED \\
\hline \multicolumn{3}{|c|}{ SORING TABEE (107 ST $07.10 \%$. } \\
\hline TABEE EXIENSION & EXTENDED / RETRACTED (ZS-717A / ZS-717C) & RETRACTED \\
\hline TABUE PIVYATION & (ZS-717B / ZS-716A \& B) & LOWERED \\
\hline LOCKM N PINS: & LOCKED / UNLOCKED & LOCKED \\
\hline
\end{tabular}




\section{WRAP 1 PLANT OPERATING PROCEDURE}

(HNF-SD $1026.0 T P$ P 015$)$

\section{ATTACHMENT 2: SORTING GLOVEBOX EQUIPMENT STATUS}

\begin{tabular}{|c|c|c|c|}
\hline EQGIPMENI SOMPONEN & AS POUNO POSI & 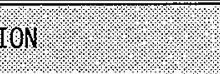 & 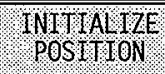 \\
\hline \multicolumn{4}{|c|}{ 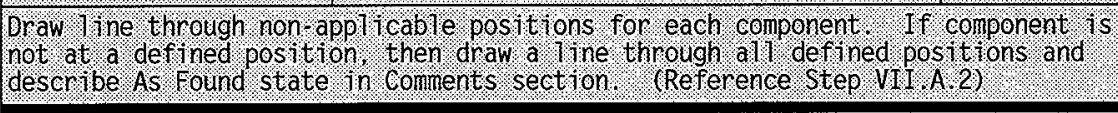 } \\
\hline \multicolumn{4}{|c|}{ BRUM TRANSIER CAR $(107 \%(07,101)$} \\
\hline BRUM ON GAR & YES / NO & (ZS-331) & NO \\
\hline CAR POSTIOT: & $\begin{array}{l}\text { ENTRY TRANSFER PORT } \\
\text { DELID POSITION } \\
\text { RELID POSITION } \\
\text { COMPACTOR TRANSFER PORT }\end{array}$ & $\begin{array}{l}(Z S-710) \\
(Z S-725 C) \\
(Z S-725 B) \\
(Z S-725 A)\end{array}$ & ENTRY \\
\hline 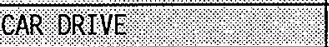 & ENGAGED / DISENGAGED & (ZS-718A / ZS-718B) & DISENGAGED \\
\hline
\end{tabular}


HNF-SD-W026-0TR-015, Rev. 0, Page OP-20

\section{WRAP 1 PLANT OPERATING PROCEDURE}

(HNF SB W026-01P - 015 )

\section{ATTACHMENT 2: SORTING GLOVEBOX EQUIPMENT STATUS}

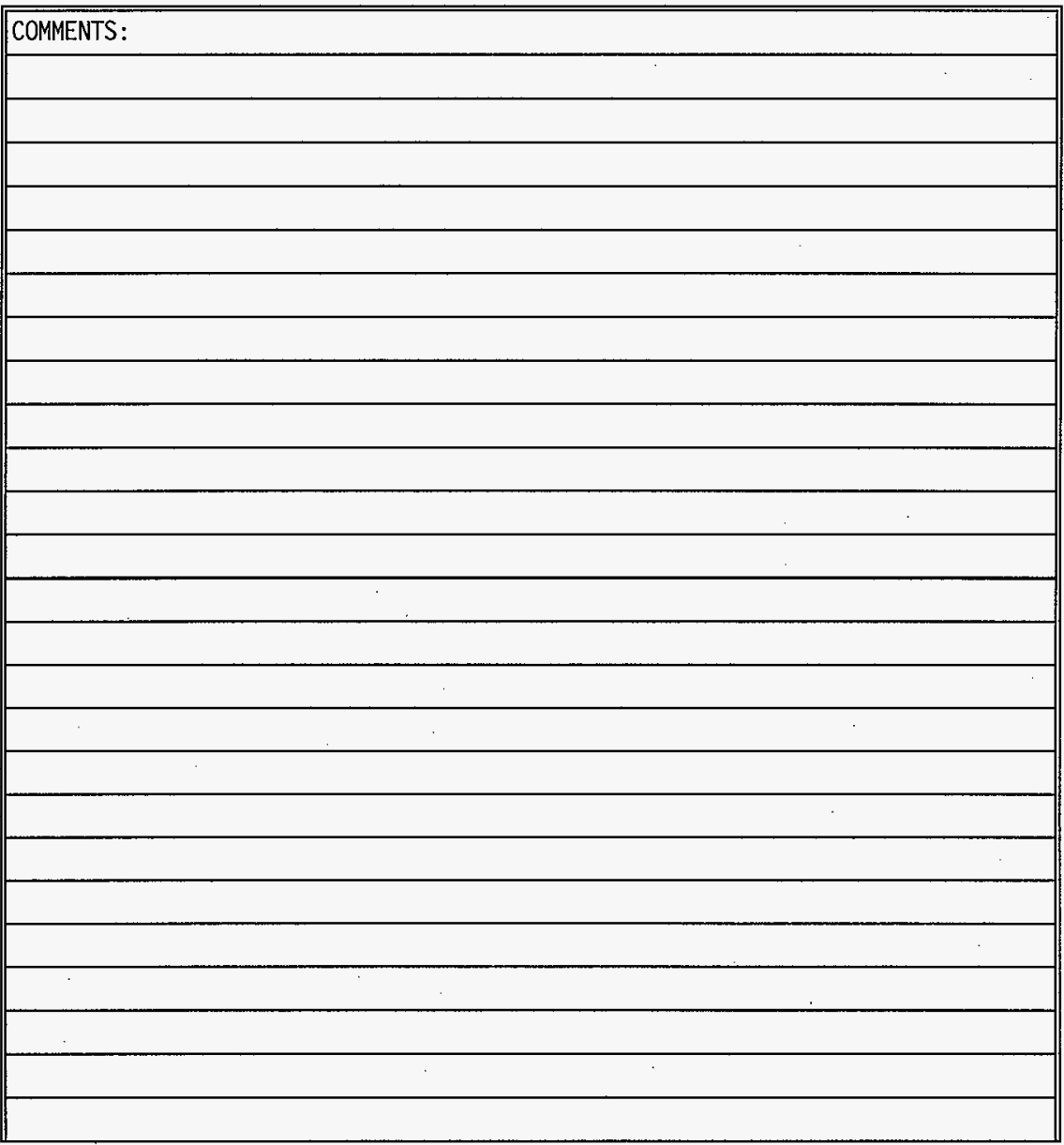




\section{WRAP 1 PLANT OPERATING PROCEDURE}

(HNF SB .026 - ONP 015 )

\section{ATTACHMENT 2: SORTING GLOVEBOX EQUIPMENT STATUS}

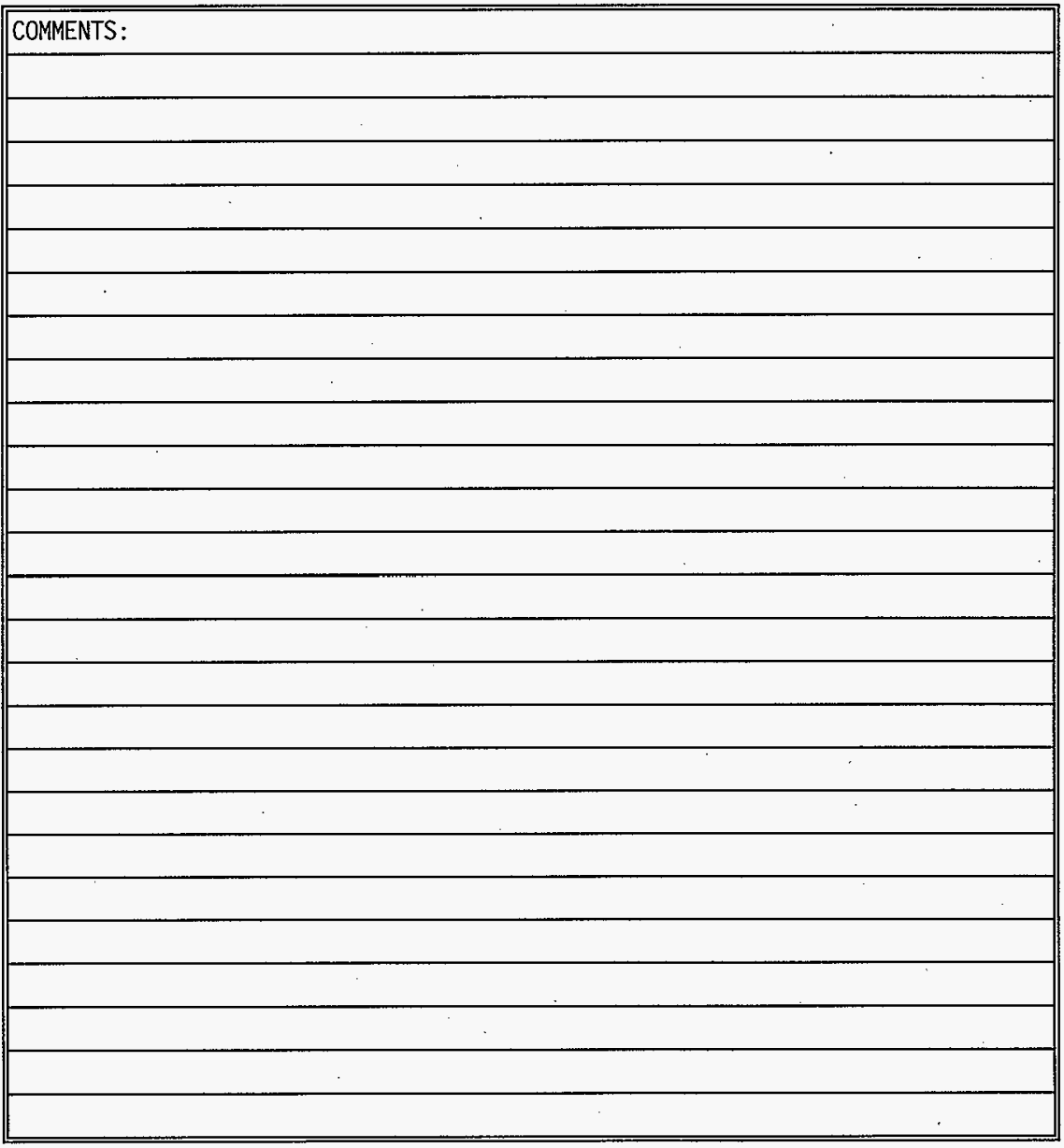




\section{WRAP 1 PLANT OPERATING PROCEDURE}

UNF SD W026 - OTP - 015)

\section{ATTACHMENT 3: EXIT GLOVEBOX EQUIPMENT STATUS}

\begin{tabular}{|c|c|c|}
\hline EOUTPMENI / COMPONENI & AS FOUND POSTIION & INIMIALTLE. \\
\hline \multicolumn{3}{|c|}{ 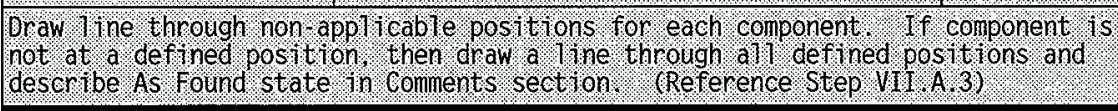 } \\
\hline \multicolumn{3}{|c|}{ EXIT PORT $10 \% .00 .07 .06 \%$ : } \\
\hline PORT OOOR : : : : $: 1:$ : & $(\mathrm{ZSO}-760 / \mathrm{ZSC} \cdot 760)$ & CLOSED \\
\hline PORT BOQR LOCK. & LOCKED / UNLOCKED & YES \\
\hline QRUM OOOOR & $\begin{aligned} \text { OPEN / CLOSED / IN POSITION (1/2" up) } & \\
& (\mathrm{ZS}-7061 \mathrm{C} / \mathrm{ZS}-761 \mathrm{~A} / \mathrm{ZS}-761 \mathrm{~B})\end{aligned}$ & CLOSED \\
\hline IB AGA NST ORUM BOOR & YES / NO & NO \\
\hline DRUM GENTERING DEVTCE & EXTENDED / RETRACTED & RETRACTED \\
\hline SEAL INFLATED. & YES / NO & NO \\
\hline \multicolumn{3}{|c|}{ 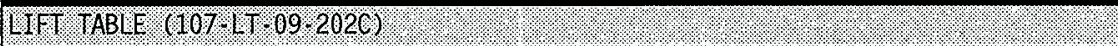 } \\
\hline BRUM ON LIFT TABLE. : & YES / NO (VISUAL) & YES \\
\hline QRUM UNEER PORT LED: & ON / OFF & OFF \\
\hline ERUM IN POS T ION WED & ON / OFF & OFF \\
\hline GRUM AT AGV ENO LED & ON / OFF & ON \\
\hline AGV LOAB HEIGHI LEO & ON / OFF & OFF \\
\hline BOWN OVERTRAVE IKE. & ON / OFF & OFF \\
\hline \multicolumn{3}{|c|}{ EXIT GOUVBOX HOIST ( 107 (R $07 \%$ 101D) } \\
\hline BRUM IN I I TTER: : & YES / NO & NO \\
\hline LITER JAWS : : & $(250.766 / Z S C-766)$ & OPEN \\
\hline HOIST FULY Y RATSEO & YES / NO & YES \\
\hline HOIST IROL EY POSTITION & STORE 1 / STORE 2 / RECEIPT (ZS-702B ,C \& D) & RECEIPT \\
\hline \multicolumn{3}{|c|}{ 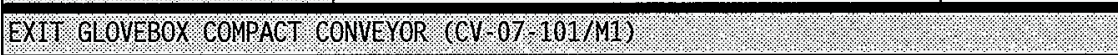 } \\
\hline PUGK ON CONVEYOR: : & YES / NO (ZS-762) & NO \\
\hline GONYEYOR ORIYL : & ENERGIZED / DE-ENERGIZED & DE-ENERGIZED \\
\hline
\end{tabular}

\begin{tabular}{|c|c|c|c|c|}
\hline bocument No 10.72 & Pront bate & Rev/10d & page 15.07 & 17 \\
\hline
\end{tabular}


HNF-SD-W026-0TR-015, Rev. 0, Page OP-23

WRAP 1 PLANT OPERATING PROCEDURE

(HNF. SB W026 OIP 015)

\section{ATTACHMENT 3: EXIT GLOVEBOX EQUIPMENT STATUS}

\begin{tabular}{||l||}
\hline COMMENTS: \\
\hline \\
\hline \\
\hline \\
\hline \\
\hline \\
\hline \\
\hline \\
\hline \\
\hline \\
\hline \\
\hline \\
\hline \\
\hline \\
\hline \\
\hline \\
\hline \\
\hline \\
\hline \\
\hline \\
\hline \\
\hline \\
\hline \\
\hline \\
\hline \\
\hline \\
\hline \\
\hline \\
\hline \\
\hline
\end{tabular}


HNF-SD-W026-0TR-015, Rev. O, Page OP-24

WRAP 1 PLANT OPERATING PROCEDURE

(HNF. SD WO26 010015$)$

\section{ATTACHMENT 3: EXIT GLOVEBOX EQUIPMENT STATUS}

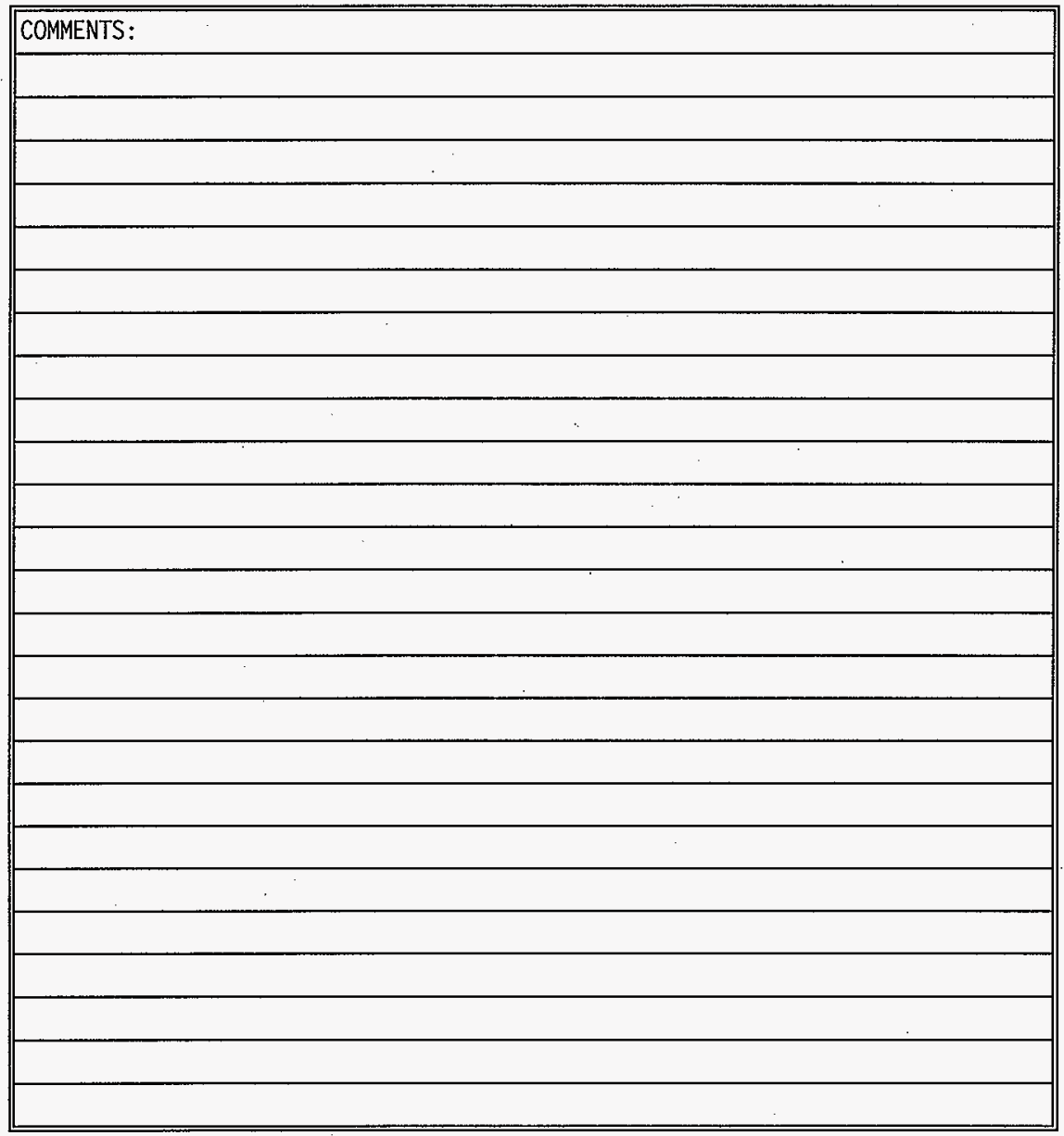




\section{WRAP 1 PLANT OPERATING PROCEDURE}

SYSTEM: PROCESS

\section{GLOVEBOX LIFT TABLE MANUAL OPERATION \\ (HNF-SD-W026-0TP-015)}

\section{SYSTEM DESCRIPTION}

This procedure provides instructions for manual operation of drum lift tables at various locations throughout the facility. Automatic operation of lift tables is described in applicable glovebox operating procedures.

\section{REFERENCE DOCUMENTS}

WRP1-0P-0704, Glovebox Manipulator Operation.

WRP1-OP-0713, LLW Entry Glovebox Operation.

WRP1-0P-0714, LLW Sorting Glovebox Operation.

WRP1-0P-0716, LLW Exit G1ovebox Operation.

WRP1-0P-0717, LLW/RWM Process Glovebox Operation.

\section{PRESTART CONDITIONS}

A11 personnel performing this procedure shall be qualified in accordance with Waste Management Hanford Procedures Manua1, Section 5.1, "Training and Qualification," and on-the-job training.

Verify daily radiological dose rates and contamination level for gloves have been established by Radiological control.

\section{SAFETY}

All potential hazards, such as lifting heavy containers or handling sharp objects present in waste containers, must be mitigated by protective equipment, procedures, and administrative controls to ensure acceptablerisk operating conditions.

Warning - In manual control (maintenance) mode there is no interlock to prevent drum over-travel. If the reverse switch is held in after the drum reaches the Automatic Guided Vehicle (AGV) end of the table and the AGV is not in place, the drum wil1 fal1 to the floor. There is also no interlock to stop up-travel based on weight setpoint and no indication that lift table is at drum centering height.

Caution - Lift tables LT-09-201C, LT-09-202D, and LT-09-202E, when lowered, interfere with AGV travel near the LLW and TRU RWM glovebox 1 ines. Before lowering these tables below AGV LOAD HEIGHT (27 [26-7/8 to 27-1/8] inches from floor to top of rollers), the operator must disable the AGV to ensure that it will not travel into the lift table area. 


\section{WRAP 1 PLANT OPERATING PROCEDURE}

V. TOOLS AND SUPPLIES

- $\quad$ Tape measure.

VI. TABLE OF CONTENTS

PAGE

A. GENERAL REQUIREMENTS .................. . . 3

B. LIFT TABLE MANUAL OPERATION . . . . . . . . . . . . . . . 4 Place Lift Table In Manual Control (Maintenance Mode) . . . . . . . . . . 4 Lower Drum From AGV Load Height . . . . . . . . . . . . . . . . . . . . 4

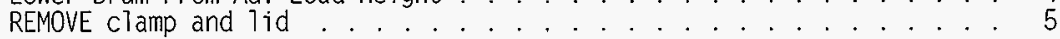

c. LIFT TABLE MANUAL OPERATION .................. 5

Position Drum Under Port . . . . . . . . . . . 5 Lift Drum To Port . . . . . . . . . . . . . . . . . . . . . . . . . . . 5 Lower Drum From Port . . . . . . . . . . . . . . . 7 MOVE drum to AGV end of iift tabie . . . . . . . . . . . . 8

D. RETURN LIFT TABLE TO NORMAL OPERATION . . . . . . . . . . . . . . . 8

E. REHOME LIFT TABLE . . . . . . . . . . . . . . . . . . . . . . . . . . . 9

FIGURE 1: LIFT TABLE LOCAL CONTROL PANEL . . . . . . . . . . . . . . 10

TABLE 1: LIFT TABLE/CONTROL PANEL DESCRIPTIONS . . . . . . . . . . . . . . . 10

TABLE 2: DRUM MATING PRESSURE/CENTERING HEIGHT . . . . . . . . . . . . . . . . . 11 


\section{WRAP 1 PLANT OPERATING PROCEDURE}

(HNF SB WO26-OTP 015 )

\section{PROCEDURE}

NOTE 1 - This procedure covers manual operation of all WRAP lift tables. therefore equipment tag numbers are not referenced in the individual steps. Tag numbers for referenced equipment are located in Table 1. When equipment is called out by name. reference to applicable equipment number is understood.

NOTE 2 - Unless otherwise stated. switches and indicators are located on the applicable Lift Table Control Pane7.

\section{CAUTION}

TABLE RESPONDS QUICKLY TO PRESSURE ON CONTROL SWITCHES. A "LIGHT TOUCH" MUST BE USED ON SWITCHES WHEN JOGGING DRUM INTO POSITION. OVER-DRIVING DRUM INTO PORT COULD RESULT IN EQUIPMENT DAMAGE, DO NOT EXCEED LIFT TABLE MATING PRESSURE BY MORE THAN 50 KILOGRAMS (Reference Table 2).

\section{WARNING}

IN MANUAL CONTROL (MAINTENANCE) MODE THERE IS NO INTERLOCK TO PREVENT DRUM OVER-TRAVEL. IF THE REVERSE SWITCH IS HELD AFTER THE DRUM REACHES THE AGV END OF THE TABLE AND THE AGV IS NOT IN PLACE, THE DRUM WILL FALL TO THE FLOOR. THERE IS ALSO NO INTERLOCK TO STOP UP-TRAVEL BASED ON WEIGHT SETPOINT AND NO INDICATION THAT LIFT TABLE IS AT DRUM CENTERING HEIGHT.

A. GENERAL REQUIREMENTS

1. After placing lift table in Manual Control (Maintenance) mode per Section VII.B, VII.C, other sections of this procedure may be performed repeatedly and in any order necessary to facilitate work. Section VII.E must always be performed before returning the lift table to automatic operation.

2. WHEN positioning lift tables, operator may "jog" UP/DOWN or FORWARD/REVERSE buttons as required. 
HNF-SD-W026-0TR-015, Rev. 0, Page OP-28

\section{WRAP 1 PLANT OPERATING PROCEDURE}

(HNF SO NO26 0TP 015)

\section{WARNING}

WORK THROUGH GLOVEPORTS MAY INVOLVE PLACING HANDS IN CLOSE PROXIMITY TO MOVING EQUIPMENT. CARE MUST BE TAKEN TO PREVENT INJURY.

3. IF at any time during performance of this procedure, work is to be performed through gloveports,

THEN:

a. CHECK status and location of moving equipment in proximity of gloveport(s) to be used.

b. TAKE action, including any of the following, as deemed necessary to prevent injury:

- $\quad$ AVOID equipment when reaching through port(s)

- $\quad$ PRESS glovebox E-Stop to stop glovebox equipment

- CONSULT DOS when unsure

c. PERFORM a contamination survey of hands, arms and front of body upon exiting glove:

(1) IF contamination is detected,

THEN, CONTACT Radiological Control Technician.

\section{B. LIFT TABLE MANUAL OPERATION}

1. Place Lift Table In Manual Control (Maintenance Mode)

a. ENSURE glovebox manipulator is PARKED (Reference WRP1-OP-0704, Glovebox Manipulator Operation).

b. PLACE "MODE" (MAINT/OFF/NORMAL) switch to "MAINT".

2. Lower Drum From AGV Load Height

NOTE - On7y those Lift Tables indicated by (*) in Table 1, need to be lowered from AGV Load Height for drum to clear port. Port clearance height is checked visually or with a tape measure when visual inspection is not possible.

a. LOWER lift table to DRUM CLEARANCE HEIGHT. (Reference Table 2).

b. IF "DOWN OVERTRAVEL" light comes ON, THEN, PRESS "UP" switch until light goes OFF. 


\section{WRAP 1 PLANT OPERATING PROCEDURE}

(TNF SB W026.0TP 0 015 )

3. REMOVE clamp and $1 \mathrm{id}$, as needed, from drum per applicable procedure:

- WRP1-0P-0713, LLW Entry Glovebox Operation.

- WRP1-0P-0714, LLW Sorting Glovebox Operation.

- WRP1-0P-0716, LLW Exit Glovebox Operation.

- WRP1-OP-0717, LLW/RWM Process Glovebox Operation.

c. LIFT TABLE MANUAL OPERATION

1. Position Drum Under Port

a. VERIFY heights of listed items, as applicable:

(1) 55 gallon drum with lid and clamp band attached:

- $34-5 / 8(34-1 / 2$ to $34-3 / 4)$ inches.

(2) 85 gallon overpack drum with lid and clamp band attached:

- $39-1 / 16(38-15 / 16$ to $39-3 / 16)$ inches.

NOTE - Only those lift tables indicated by (*) on Table 1. need 7owered from AGV LOAD HEIGHT for drum to clear port. Port clearance height is checked visually.

b. IF necessary,

THEN, ENSURE lift table is lowered to port clearance height.

c. FORWARD 1 ift table until drum is centered beneath port and DRUM UNDER PORT light is ON.

2. Lift Drum To Port

a. ENSURE drum is properiy positioned under port.

b. ENSURE drum port is CLOSED, per applicable procedure.

c. VERIFY the following for Entry/Exit ports:

- Centering devices: RETRACTED.

- HVAC seat, as applicable: DEFLATED

- Lid Detach rod: RETRACTED. 


\section{WRAP 1 PLANT OPERATING PROCEDURE}

d. VERIFY correct drum PIN, per applicable procedure:

- WRP1-OP-0713, LLW Entry GTovebox Operation.

- WRP1-0P-0714, LLW Sorting Glovebox Operation.

- WRP1-OP-0716, LLW Exit G1ovebox Operation.

- WRP1-0P-0717. LLW/RWM Process Glovebox Operation.

NOTE - Proper drum to port mating is determined by drum to port mating pressure or drum height, depending on port type.

e. DETERMINE correct drum to port MATING PRESSURE or DRUM CENTERING HEIGHT per Table 2.

f. RAISE drum to correct height, non-ENTRY/EXIT Port:

\section{CAUTION}

(1) ENSURE 7ift table scale (on top of table control pedesta 1) units are set to "kg" (kilograms).

(2) RAISE lift table until drum mates to port.

(3) JOG UP/DOWN to correct MATING PRESSURE.

g. RAISE drum to correct height, ENTRY/EXIT Port:

(1) RAISE lift table to DRUM CENTERING HEIGHT.

(2) VERIFY with tape measure, lift table at DRUM CENTERING HEIGHT (Reference Table 2).

(3) EXTEND centering clamps.

(4) VERIFY centering clamps extended.

NOTE - Entry/Exit port maintenance menus have a light that indicates port LID IN POSITION sensor is actuated by the drum lid.

(5) RAISE lift table to LID IN POSITION HEIGHT.

(6) TURN ON port vacuum.

(7) VERIFY vacuum generated. 


\section{WRAP 1 PLANT OPERATING PROCEDURE}

(UNF SD WO26 07 P 015$)$

(8) IF HVAC Seal Present:

(a) INFLATE HVAC seaT.

(b) VERIFY HVAC seal inflated.

(9) UNLOCK port.

(10) VERIFY port unlocked.

NOTE: Entry/Exit port maintenance menus have a jight that indicates when port DRUM IN POSITION sensor is activated by raising the port door $\sim 112$ inch above glovebox floor.

(11) RAISE lift table to DRUM IN POSITION height.

h. JOG lift table "UP/DOWN" switches to adjust position/mating pressure as required.

i. OPERATE drum port per applicable procedure.

3. Lower Drum From Port

a. ENSURE drum port is CLOSED, per applicable procedure.

b. PERFORM the following for Entry/Exit Ports:

(1) LOWER lift table to LID IN POSITION HEIGHT.

(2). VERIFY Port Door LOWERED.

(3) LOCK Port Door.

(4) VERIFY. Port Door Locked.

(5) EXTEND lid detach cylinder

(6). LOWER lift table to DRUM CENTERING HEIGHT.

(7) RETRACT lid detach cylinder.

(8) RETRACT centering clamps.

(9) IF port is equipped with HVAC sea? THEN:

(a) DEFLATE HVAC seal.

(b) VERIFY HVAC seal deflated. 


\section{WRAP 1 PLANT OPERATING PROCEDURE}

$(H N F$ SO WO26 OTP 015$)$

NOTE - Only those Lift Tables indicated by (*) in Tab7e 1. need lowered from AGV Load Height for drum to clear port. Port clearance height is checked visual7y.

c. LOWER lift table to AGV LOAD HEIGHT (AGV LOAD HEIGHT

light ON), or port clearance height, as applicable.

d. IF DOWN OVERTRAVEL light comes ON,

THEN, PRESS UP switch until light goes OFF.

e. REQUEST RCT survey drum.

4. MOVE drum to AGV end of lift table

NOTE - $\quad$ OnTy those Lift Tables indicated by (*) in Table 1. need lowered from AGV Load Height for drum to clear port. Port clearance height is checked visual7y.

a. IF necessary,

THEN, ENSURE lift table is lowered to port clearance height.

b. REVERSE lift table until DRUM AT AGV END light is ON.

c. IF drum extends past end of lift table,

THEN, FORWARD lift table until drum is at desired position.

D. RETURN LIFT TABLE TO NORMAL OPERATION

1. PLACE MODE switch to NORMAL.

NOTE - Only Lift Tables 107-LT-09-201A through 201F are equipped with Table Control switch.

2. IF equipped with TABLE CONTROL switch,

THEN, PLACE TABLE CONTROL switch to AGV. 


\section{WRAP 1 PLANT OPERATING PROCEDURE}

E. REHOME LIFT TABLE

1. IF any of the following have occured:

- Power outage affecting lift table,

- Lift table was used in manual mode,

- Lift table rope switch was activated,

THEN, REHOME affected lift table(s) as follows:

a. PLACE glovebox in Maintenance Mode.

b. VERIFY lift table is EMPTY.

c. POSITION lift table ABOVE AGV LOAD HEIGHT.

d. IF affected lift table is marked by an (*) on Table 1 , THEN, LOWER 7 ift table BELOW AGV LOAD HEIGHT.

e. SELECT MISCELLANEOUS EQUIPMENT MAINTENANCE MENU.

NOTE - $\quad X X$ refers to last two digits in lift table tag number.

f. PRESS REHOME LIFT TABLE LT-09-20XX button.

g. VERIFY lift table at AGV LOAD. HEIGHT:

- $\quad$ AGV LOAD HEIGHT indicator ON and/or,

- Lift table rollers at 27 (26-7/8 to 27-1/8) inches above floor. 
HNF-SD-W026-OTR-015, Rev. 0, Page OP-34

\section{WRAP 1 PLANT OPERATING PROCEDURE}

(HNF SB W026 OIP . 015)

FIGURE 1: LIFT TABLE LOCAL CONTROL PANEL

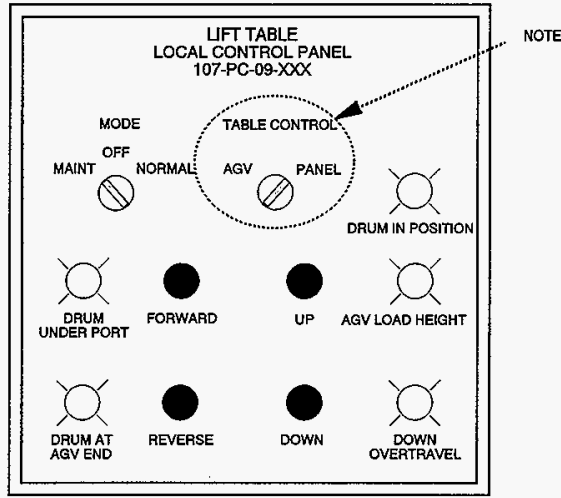

TABLC 1. LIT TABLE/CONTROL PANEL DESCRIPTLONS

\begin{tabular}{|c|c|c|}
\hline $\begin{array}{l}\text { LOCAL CONTROL } \\
\text { PANEL NUMER }\end{array}$ & IF TABLE NUMBER & DESCRTPTION \\
\hline $107.90 .09 \cdot 201 \mathrm{~A}$ & $107.1 .09 .201 \mathrm{~A}$ & TRU RWY TREATED WASTL LOABOUT PORT/LIST TABLE \\
\hline $107 . \mathrm{PC}, 09.201 \mathrm{~B}$ & $107.1 \mathrm{~T}, 09.201 \mathrm{~B}$ & TRU RWH COMPL IANT WASTE LOADQUT PORT /LIFT TABLE: \\
\hline 107.80 .092010 & 107.4 .092010 & TRU RWM TRANSEER DRUM PORT/LIT TABLE. \\
\hline 107.90 .09 .2010 & * 107.109 .2010 & LWH RWM TREATEO WASTE LOABOUT RORT MLIFI TABLE \\
\hline $107 \cdot 2 C 09.201 \mathrm{C}$ & $107 / 1709-201 \mathrm{E}$ & LIN RWM COMPL IANT WASTE LOADOUT PORT/L ITT TABLEE \\
\hline $107 . \mathrm{PC} 09.201 \mathrm{~F}$ & $107.1009 .201 \mathrm{~F}$ & LW RWY TRANSFER GRUM PORT/MT TABLE, \\
\hline $107 . \mathrm{PC} .09 .2024$ & $107.40 .09 .202 A$ & LUW ENTRY GLOVEBOX PORTIUIFT TABLE. \\
\hline $107 \cdot \mathrm{PC} 009.202 \mathrm{~B}$ & $107,109,2028$ & TRU ENIRY GLOVEBOX PORT/LIFT TABLE. \\
\hline $107.9 C 09-2026$ & * $107 / 1-09.2020$ & LWW EXIT GL GVEBOX LOABOUT POSITIONILITT TABLE. \\
\hline 107.90 .092020 & t. 107.11 .09 .2020 & TRU EXIT GLOVEBOX LOABOUT POSITION \#1/LIT T ABLG \\
\hline $107.96 .09 \cdot 2025$ & t. $107.09 .202 \mathrm{E}$ & GRU EXIT GLOVEBOX LOABOUT POSITION \#2/LIIT TABLC: \\
\hline 107 . PC. $09.203 \mathrm{~A}$ & $107.1109 .203 A$ & LIW RANSFER PORT/LIT TABLE IOR RWM TRANSFER DRUMS \\
\hline $107 \cdot \mathrm{PC} .09 \cdot 203 \mathrm{~B}$ & $107.1092,203 \mathrm{~B}$ & TRU TRANSFER PORT/L IFT TABLE FOR RWM TRANSFER DRUMS \\
\hline $107 \cdot \mathrm{PC} 00.2030$ & 107.1092030 & TRU EMPTY DRUM COMPACT ION LOABQUT PORT / LIFT TABLE. \\
\hline
\end{tabular}

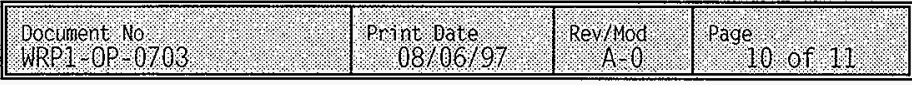




\section{WRAP 1 PLANT OPERATING PROCEDURE}

(HNF-SO $1026-0 \mathrm{TP} 015)$

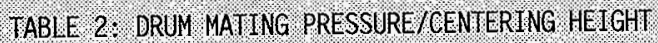

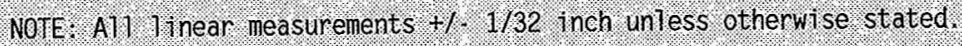

\begin{tabular}{|c|c|c|c|c|}
\hline IIIT TABIC & MATING PRESSURE & 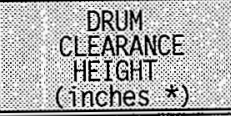 & 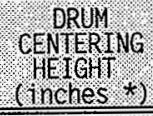 & 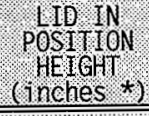 \\
\hline $107.4009201 \mathrm{~A}$ & $300(250$ to 350$)$ & & & \\
\hline $107.1 \mathrm{~T} 09-2018$ & $300 /(250$ to 350$)$ & & & \\
\hline 107 L 109,2010 & 600 (550 to 650$)$ & & & \\
\hline $107.7 .09,2019$ & & $(18.0 .80,19,0)$ & $5.1 \%$ & 8 \\
\hline $107-1709-201 \mathrm{E}$ & & & & \\
\hline $107 / 1.09201 \%$ & 600 ( 550 to 650$)$ & & & \\
\hline 107 LI $09.202 A$ & & & $38 \% 5 \% 10$ & $33-1 \%$ \\
\hline $107,4.09-2028$ & & & $37.1 / 16$ & $37 \% 116$ \\
\hline $107 / 4.09 \cdot 2020$ & & $1,(12,5,130,13.5)$ & 24,78 & $25.3 / 8$ \\
\hline $107,-T \cdot 09-2029$ & $300 \cdot(250,0.350)$ & $(1,18,5,40,19,5)$ & & \\
\hline $107 . T \cdot 09,202 E$ & $300,(250 \cdot \mathrm{to} \% 350$ & $(18.5 \%$ & & \\
\hline $107,57,09,203 \mathrm{~A}$ & & & & \\
\hline 107 LT $09 \% 203 \mathrm{~B}$ & $600 .(550, t 0.650)$ & & & \\
\hline $107409-2030$ & & & & \\
\hline
\end{tabular}




\section{WRAP 1 PLANT OPERATING PROCEDURE}

\section{SYSTEY. PROCESS}

\section{GLOVEBOX MANIPULATOR}

(HNF-SD-W026-0TP-015)

\section{SYSTEM DESCRIPTION}

This procedure provides instructions for operation of Schi17ing Robotic Systems, Glovebox Manipulators.

Glovebox Manipulators are used for moving materials and equipment, and performing tasks inside the gloveboxes.

\section{REFERENCE DOCUMENTS}

None.

\section{PRESTART CONDITIONS}

A11 personnel performing this procedure shal1 be qualified in accordance with Waste Management Hanford Procedures Manua 1. Section 5.1, "Training and - Qualification," and on-the-job training.

\section{SAFETY}

Potential hazards, such as lifting heavy containers or handling sharp objects present in waste containers, must be mitigated by protective equipment, procedures, and administrative controls to ensure acceptablerisk operating conditions.

\section{TOOLS AND SUPPLIES}

None. 


\section{WRAP 1 PLANT OPERATING PROCEDURE}

(GNF SO W026.0TP 015 )

VI. TABLE OF CONTENTS

PAGE

A. GENERAL REQUIREMENTS .................. . . 3

B. SYSTEM STARTUP ......................... 4

C. NORMAL SYSTEM OPERATION .................. 7

Master Arm Operation . . . . . . . . . . . . . . . . . . . . 999

Changing STave Arm To Master Arm Correspondence . . . . . . . . . . . . . . 9

Jaw Operation . . . . . . . . . . . . . . . . . . . . . 10

Controlling Wrist Rotation . . . . . . . . . . . 11

Stowing Slave Arms ...................... . . . 12

Moving Carriages . . . . . . . . . . . . . . . . . . . . 13

Vertical Carriage Obstructions

Horizontal Carriage Obstructions . . . . . . . . . . . . . . 14

Changing Individual System Operating Characteristics . . . . . . . 15

D. STARTUP WITH SYSTEM ERRORS . . . . . . . . . . . . . . . . . 15

E. SET SLAVE ARM CHARACTERISTICS ............... 18

Set Stow Sequence $\ldots$

Freezing Entire Slave Arm . . . . . . . . . . . . . . . . . . . . . . . 19

Freezing Individual Slave Arm Joints . . . . . . . . . . . . . . 20

Controlling Slave Arm Speed . . . . . . . . . . . . . . . . . . 21

Enabling And Disabling Slave Arm Hydraulics . . . . . . . 21

Set Slave Arm Movement Limits . . . . . . . . . . . . . . . . . . . . . 22

F. SET JAW OPERATING CHARACTERISTICS . . . . . . . . . . . . . . . 24

Set Jaw Closing Speed . . . . . . . . . . . . . . . . . . . . . . . 24

Set Jaw offset values $\ldots \ldots \ldots$

G. SET DISPLAY ATTRIBUTES . . . . . . . . . . . . . . . . . . 27

H. SYSTEM SHUTDOWN . . . . . . . . . . . . . . . . . . . . . 28

I. EMERGENCY SHUTDOWN ...................... 30

\section{ATTACHMENTS}

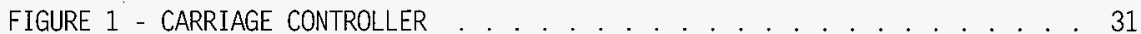

FIGURE 2 - MASTER CONTROL PANEL . . . . . . . . . . . . . . . . . . . 32

FIGURE 3 - MASTER ARM . . . . . . . . . . . . . . . . . . . . 


\section{WRAP 1 PLANT OPERATING PROCEDURE}

(1NF SB - N026 - $0 \mathrm{PP} 015$ )

\section{VII : $\quad$ PROCEDURE}

A. GENERAL REQUTREMENTS

1. Sections VII.B through VII.I of this procedure may be performed repeatedly and in any order necessary to facilitate work.

\section{WARNING}

WORK THROUGH GLOVEPORTS MAY INVOLVE PLACING HANDS IN CLOSE PROXIMITY TO MOVING EQUIPMENT. CARE MUST BE TAKEN TO PREVENT INJURY.

2. If at any time during performance of this procedure, work is to be performed through gloveports,

THEN:

a. CHECK status and location of moving equipment in proximity of gloveport(s) to be used.

b. TAKE action, including any of the following, as deemed necessary to prevent injury:

- $\quad$ AVOID equipment when reaching through port(s)

- $\quad$ PRESS glovebox E-Stop to stop glovebox equipment

- CONSULT DOS when unsure

c. PERFORM a contamination survey of hands, arms and front of body upon exiting glove:

(1) IF contamination is detected,

THEN, CONTACT Radiological Control Technician. 


\section{WRAP 1 PLANT OPERATING PROCEDURE}

(HNF SD 1026 - OTP 015$)$

\section{B. SYSTEM STARTUP}

\section{CAUTION}

ERRATIC, UNCONTROLLED MOVEMENT OF SLAVE ARMS MAY DAMAGE EQUIPMENT.

1. IF at any time during startup or operation, slave arms move erratically or uncontrolled,

THEN immediately:

a. PRESS EMERGENCY STOP button on either side of carriage controller (Figure 1) IN.

b. TURN master control panel (Figure 2) POWER switch OFF.

NOTE - Problem which caused erratic movement must be corrected prior to restart of system.

c. CONTACT Duty Operation Supervisor (DOS).

2. PERFORM pre-start checks:

a. INSPECT, visually, fasteners and fittings are properly connected and in good condition.

b. INSPECT, visually, hoses are free of cuts, nicks, kinks, and excessive sagging.

c. INSPECT, visually, slave arms are free of hydraulic leaks.

3. ENSURE disconnect switch on Control \& Instrumentation (C\&I) enclosure for desired manipulator is set to ONE.

4. ENSURE EMERGENCY STOP buttons on carriage controller are OUT.

5. TURN master control panel POWER switch ON.

NOTE - $\quad$ Screen briefly displays "Establishing communication with slave unit" when power is first applied.

6. IF screen displays no readable text,

THEN, TOGGLE master control POWER switch (OFF and back ON).

a. IF screen remains blank,

THEN, NOTIFY DOS. 


\section{WRAP 1 PLANT OPERATING PROCEDURE}

(HNF SO W026 OTP 015 )

B. SYSTEM STARTUP (Cont.)

7. IF STARTUP WITH ERRORS screen displays,

THEN, GO TO SEction VII.D, STARTUP WITH SYSTEM ERRORS.

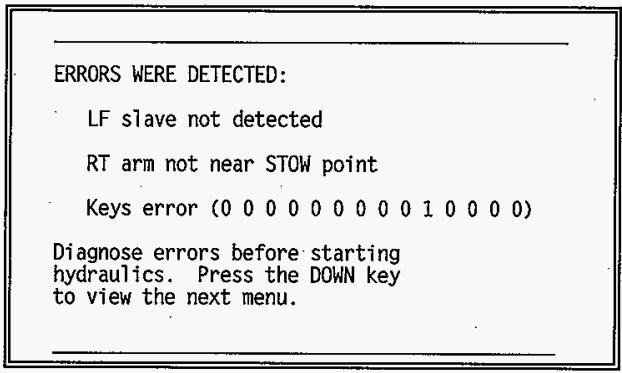

8. IF NORMAL STARTUP screen displays,

THEN, PROCEED as folTows:

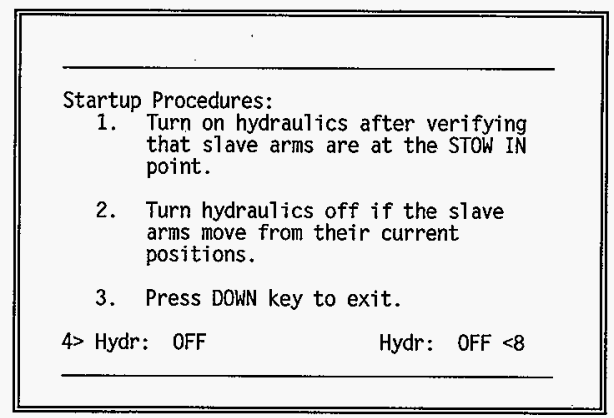

NOTE -

HPU 107-HU-07-302 is common to a 77 manipulators and will be running already if another manipulator is operating Another Start/Stop switch is located on the HPU Control Panel at HPU on overhead walkway.

a. IF Hydraulic Power Unit (HPU), 107-HU-07-302 is OFF, THEN, PRESS "START" button "I" beam behind Glovebox 102.

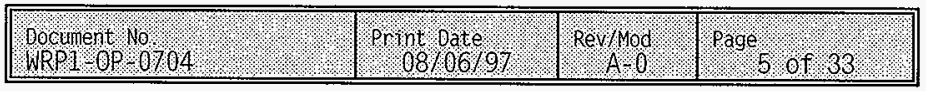




\section{WRAP 1 PLANT OPERATING PROCEDURE}

(HNF SB W.W26 OTP 015 )

B. SYSTEM STARTUP (Cont.)

NOTE - $\quad$ Pressing indicated function keys toggles hydraulic power to slave arms by opening and closing isolation valves. Corresponding screen flag will change to indicate state OFF/ON.

b. PRESS the following STARTUP screen keys to enable slave arm hydraulic power:

- $\quad$ "4> HYDR" - enable left slave arm

- $\quad$ "8> HYOR" - enable right slave arm

c. CHECK, HYDR indicators on display:

- 4> HYDR: ON

- 8> HYDR: ON

d. PRESS DN (down) function key on master control panel.

e. VERIFY STOW OUT menu displayed.

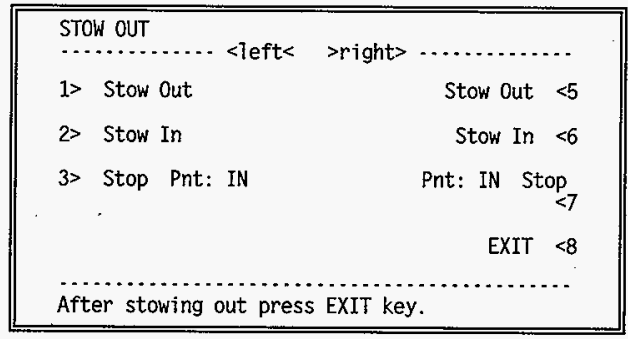

f. PRESS "1> STOW OUT" (1eft arm).

g. VERIFY left arm display (PNT), indicates OUT.

h. PRESS "5> STOW OUT" (right arm).

i. VERIFY right arm display (PNT), indicates OUT.

j. PRESS "8> EXIT" (to MAIN menu).

k. GO TO Section VII.C, NORMAL SYSTEM OPERATION. 


\section{WRAP 1 PLANT OPERATING PROCEDURE}

\section{NORMAL SYSTEM OPERATION}

NOTE - This section assumes dual-arm system operation. If one slave arm controller is disabled, menus and choices for only the active arm wil7 be available.

Normal operation of the manipulator, with exception of setting different system characteristics is gained primarily from on-the-job training and experience.

The user manipulates the system via master arms on the master control pane7, and arm carriages, and selects or changes desired operating characteristics as needed.

This section describes basic master arm operating functions, carriage movement, and OPERATE menu selections. The user is referenced to applicable sections for setting specific characteristics.

The master control panel display is normal7y 7 eft on the OPERATE menu. which provides easy access to the most common functions and allows for quick change between LEFT and RIGHT by use of LEFT/RIGHT function keys.

1. VERIFY MAIN menu displayed.

NOTE - User returns to MAIN menu as needed by pressing EXIT from other menus until MAIN menu displays.

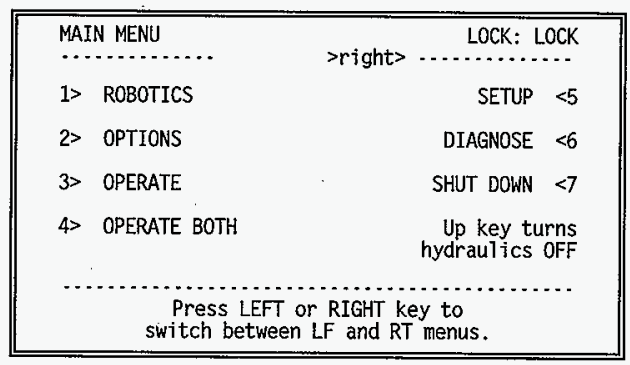

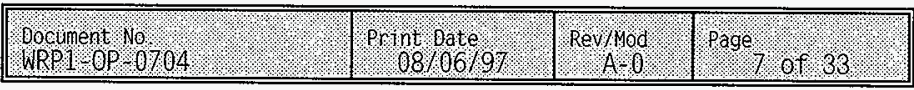




\section{WRAP 1 PLANT OPERATING PROCEDURE}

(HNF-SD W026- OTP. - 15 )

\section{NORMAL SYSTEM OPERATION (Cont.)}

NOTE - OPERATE selects left/right OPERATE menu depending on f7ag when selection is made. To select opposite OPERATE menu, operator must return to MAIN menu. OPERATE BOTH a 7 lows operator to toggle left/right OPERATE menus using LFIRT function keys, without returning to MAIN menu.

2. IF access to only one OPERATE menu is desired. THEN:

a. PRESS LF (left) OR RT (right) function key until desired choice is flagged on MAIN menu.

b. PRESS " $3>$ OPERATE".

3. IF access to both LEFT and RIGHT OPERATE menus is desired, THEN, PRESS "4> OPERATE BOTH".

4. VERIFY OPERATE menu displayed.

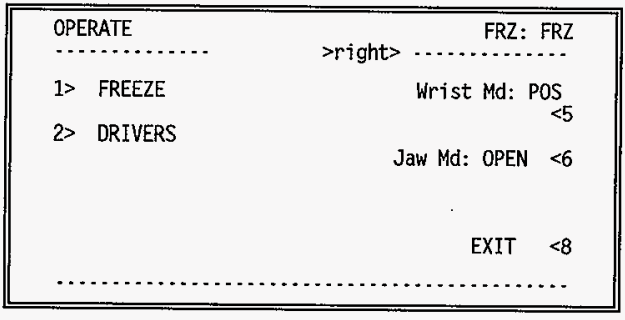

\begin{tabular}{|c|c|}
\hline & TABLE 1 . OPERATS MENU FUNC \\
\hline SELECTION & 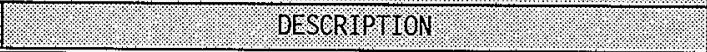 \\
\hline FREZZE & 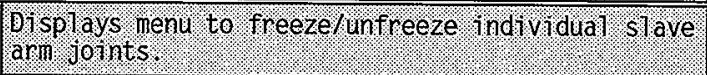 \\
\hline DRIVERS: & Controls slave arm hydraulies through hydraul ho \\
\hline WRTST MEDE & Toggles wrist operat ng node. \\
\hline JAW MQEE & Toggles jaw operatting mode. \\
\hline
\end{tabular}




\section{WRAP 1 PLANT OPERATING PROCEDURE}

(MNF. SO $1026-01 P-015)$

\section{NORMAL SYSTEM OPERATION (Cont.)}

5. Master Arm Operation

NOTE - Master arms (Figure 3) are miniature replicas of left and right slave arms, with the same relative range of motion. Each slave arm joint or function has a corresponding master arm action.

a. ACTUATE master arm action(s) (TABLE 2), as required, to manipulate corresponding slave arm:

\begin{tabular}{|c|c|}
\hline TABLE 2, MAS & TER ARI CONTROL ACT IONS \\
\hline BESTRED SLAVE ARI FUNCTION & OPERATOR ACTION ON MASTER ARM \\
\hline MOVE SLAVE ARM JOINT & $\begin{array}{l}\text { Move naster arm joint corresponding } \\
\text { to slave arm joint. }\end{array}$ \\
\hline $\begin{array}{l}\text { PREEZE SLAVE ARM IN } \\
\text { PRESENT POSTTION. }\end{array}$ & $\begin{array}{l}\text { PRESS FREE } 7 \text { b button on end of } \\
\text { master arn (toggles arn rreeze). }\end{array}$ \\
\hline ROMATE WRTST & ROTATE master arn wrist collar. \\
\hline OPEN G GOSE JAW & $\begin{array}{l}\text { SQUEEZE appl licable textured jaw } \\
\text { band on master arm. }\end{array}$ \\
\hline NOTE Ghanging from one & to another automat tically freezes \\
\hline
\end{tabular}

6. Changing Slave Arm To Master Arm Correspondence

NOTE - When a slave arm is frozen, its master arm can be repositioned to a more comfortable operating position without moving the slave arm. This is useful when a task results in an awkward position for the master arm.

a. FREEZE slave arm.

b. REPOSITION master arm as desired.

c. UNFREEZE slave arm.

d. RESUME normat operation.

\begin{tabular}{|c|c|c|c|}
\hline Oocunent. No & Prnt vate & Rev/10d & page, 907.03 \\
\hline
\end{tabular}




\section{WRAP 1 PLANT OPERATING PROCEDURE}

$(1 \mathrm{HF}$ SO $102601 \mathrm{TP} 015)$

C. NORMAL SYSTEM OPERATION (Cont.)

7.. Jaw Operation

NOTE - STave arm must be unfrozen to change jaw mode.

a. SET jaws to desired mode as follows:

(1) VERIFY OPERATE menu displayed.

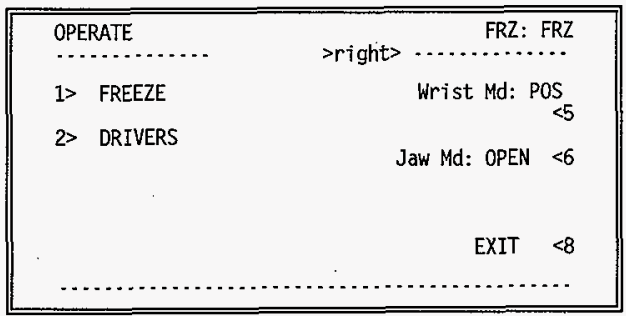

(2) PRESS "6> JAW MD" to toggle modes (TABLE 3).

\begin{tabular}{|c|c|}
\hline & TABLE $3 . J$ AW MOOES \\
\hline MODE. & JAW OPERATING CHARACTERIST ICS. \\
\hline QPEN & 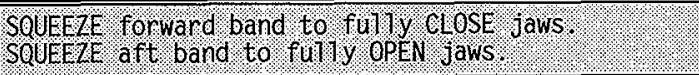 \\
\hline TogGle & $\begin{array}{l}\text { SQUEEZE forward band to toggle jaws between } \\
\text { OPENEE and CloSED (art band has no effect). }\end{array}$ \\
\hline Logk: & $\begin{array}{l}\text { IREFE jaws in current posttion unthl another } \\
\text { mode is selected. }\end{array}$ \\
\hline POSTIION & 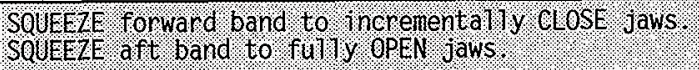 \\
\hline
\end{tabular}




\section{WRAP 1 PLANT OPERATING PROCEDURE}

(1HNF. SD W026-0TP 015$)$

C. NORMAL SYSTEM OPERATION (Cont.)

8. Controlling Wrist Rotation

NOTE - The wrist has two modes of operation: In POSITION mode the slave arm wrist follows (direction and speed) rotation of the master arm wrist collar. Rotation is limited by master arm wrist collar to $270^{\circ}$.

In RATE mode, rotating the master arm wrist collar in either direction from nul7 position causes the slave arm wrist to rotate continuously in the corresponding direction. Rate of rotation is proportional to degree of rotation of the master col7ar from nul7 position. Freezing the slave arm or rotating the wrist slightly in the opposite direction wi 77 stop rotation.

a. CHANGE wrist. mode as follows:

(1) ENSURE OPERATE menu displayed.

(2) ENSURE slave arm unfrozen.

(3) PRESS " $5>$ WRS MD" (toggles setting).

NOTE - $\quad$ Nu77 position applies to RATE mode on7y. NU77 position is the position of the master arm wrist collar at the point where either: (1) wrist mode was changed from POSITION to RATE, or (2) slave arm was unfrozen. This means the nul7 position could be close to one of the wrist col7ar mechanical stops.

b. ESTABLISH new wrist nul1 position as follows:

(1) FREEZE slave arm.

NOTE - NULL is norma77y set close to center of wrist collar mechanical range.

(2) MOVE wrist collar to desired null position.

(3) UNFREEZE slave arm. 


\section{WRAP 1 PLANT OPERATING PROCEDURE}

OHNF SD W026 OTP 015)

C. NORMAL SYSTEM OPERATION (Cont.)

9. Stowing Slave Arms

NOTE - STave arms are moved to their STOWED IN, (protected) position for transport by carriages inside glovebox and to their STOWED OUT (dep7oyed) position for working.

\section{CAUTION}

IF A SLAVE ARM IS NOT STOWED OUT WHEN A STOW COMMAND IS ISSUED, THE MESSAGE "ARM NOT NEAR STOW POINT" DISPLAYS. THE ARM THEN MOVES FIRST, TO THE STOW OUT POSITION, THEN TO THE COMMANDED POINT. BE PREPARED FOR ARM TO MOVE TO STOW OUT POSITION BEFORE IT MOVES THROUGH THE STOW/DEPLOY SEQUENCE.

ONCE STOW IN IS SELECTED. ARM MUST BE ALLOWED TO MOVE TO FULL STOW IN POSITION BEFORE EXITING APPLICABLE MENU.

a. ENSURE stow paths are clear of obstructions which could damage or be damaged by slave arms.

b. SELECT "Shutdown" from Main Menu.

c. PRESS function keys (TABLE 6), as required, to STOW slave $\operatorname{arm}(\mathrm{s})$.to desired position:

(1) PRESS STOP to halt slave arm at next point in sequence.

(2) IF slave arm is being STOWED IN,

THEN, PRESS STOW OUT at any point to reverse sequence.

(3) IF slave arm is being STOWED OUT,

THEN, PRESS STOW IN at any point to reverse sequence.

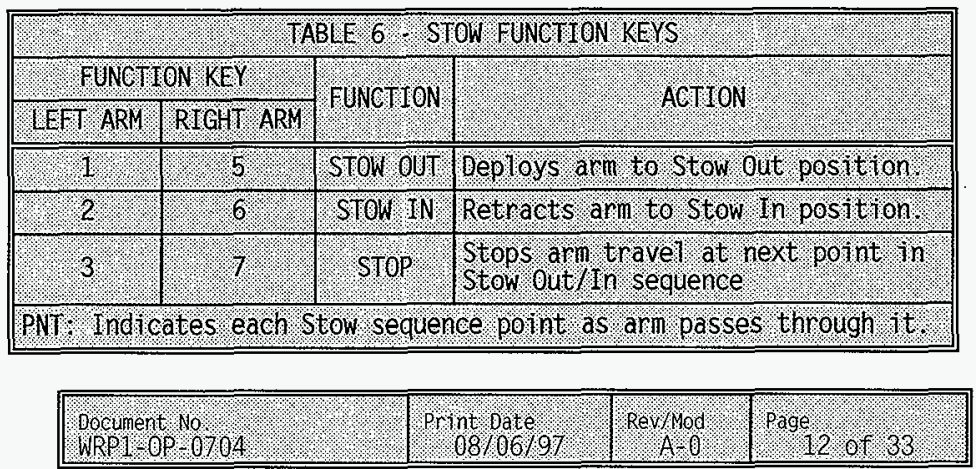




\section{WRAP 1 PLANT OPERATING PROCEDURE}

(HNF. SO W026 OIP 015)

c. NORMAL SYSTEM OPERATION (Cont.)

10. Moving Carriages

\section{CAUTION}

CARRIAGES MAY RUN INTO OBSTRUCTIONS (CABLES, HOSES, PROCESS ITEMS OR PROCESS EQUIPMENT) INSIDE GLOVEBOX.

CARRIAGE WILL STOP MOVING WHEN MECHANICAL STOP IS REACHED. DO NOT HOLD MOVEMENT BUTTONS AFTER CARRIAGE REACHES MECHANICAL STOP OR EQUIPMENT MAY BE DAMAGED.

CARRIAGE MUST COME TO A COMPLETE STOP BEFORE REVERSING DIRECTIONS.

OPERATING HORIZONTAL CARRIAGE WITH LOW HYDRÁULIC PRESSURE CAN DAMAGE EQUIPMENT.

a. PRESS and HOLD movement button(s) on carriage controller unti1 carriage reaches desired position,

THEN, RELEASE.

11. Vertical Carriage Obstructions

a. If vertical carriage encounters an obstruction too heavy to push aside,

THEN:

(1) RELEASE activated movement button.

(2) CHECK for obstruction(s).

(3) MOVE obstructions as necessary.

(4) RESUME normal operations. 


\section{WRAP 1 PLANT OPERATING PROCEDURE}

(HNF SD - W026 - 0TP - 015)

\section{NORMAL SYSTEM OPERATION (Cont.)}

12. Horizontal Carriage Obstructions

NOTE - Horizontal carriage is equipped with a torque limiter which may be tripped by any of the following: horizontal carriage hits obstacle too heavy to push aside, foreign obstacle in carriage pulley or sprocket, slave arm grasping fixed object during carriage movement, carriage at end of travel, carriage rail is bent or damaged.

HORIZ SLIP 7ight is ON only when torque 7imiter trips and movement button is pressed.

a. IF HORIZ SLIP Tight i 17uminates, THEN:

(1) RELEASE activated movement button.

(2) CHECK for obstructions or other cause.

NOTE - Torque limiter may self reset when fault is cleared.

(3) CORRECT obstructions or fault as necessary.

(4) RESUME normat operations.

b. IF cause of fault is not readily apparent,

$\underline{Q R}$

IF torque limiter remains tripped after fault is cleared. THEN, RESET torque limiter as follows:

NOTE - HORIZ SLIP light wi77 be ON when moving carriage.

(1) MOVE carriage in opposite direction of fault until mechanical stop is encountered.

(2) JOG (repeatedly press and release) button used to move carriage to mechanical stop, until torque limiter resets (listen for reset).

(3) VERIFY HORIZ SLIP Tight remains OFF when carriage is moved in either direction.

(4) REPEAT actions unti1 reset.

(5) RESUME norma1 operations.

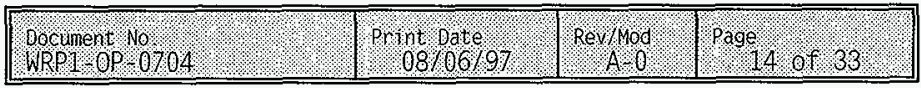




\section{WRAP 1 PLANT OPERATING PROCEDURE}

ANF :SD W026.0TP 015)

C. NORMAL SYSTEM OPERATION (Cont.)

13. Changing Individual System Operating Characteristics

a. CHANGE characteristics as follows:

(1) GO TO Section indicated in TABLE 4.

(2) MAKE changes as required.

(3) RESUME normal operation.

\begin{tabular}{|c|c|}
\hline \multicolumn{2}{|r|}{ TABLE 4 : OPERATING CHARACTERTSTILS ATTAGMENIS } \\
\hline SECTION & TILE I CHARACTERISTICS: \\
\hline VII. I & 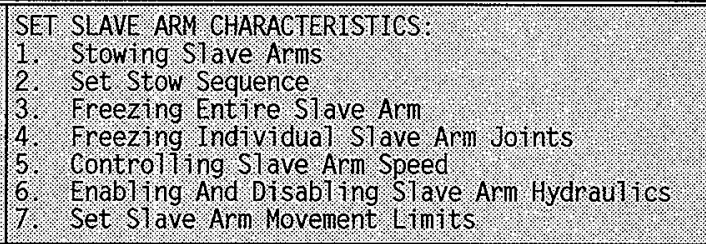 \\
\hline VITIF & 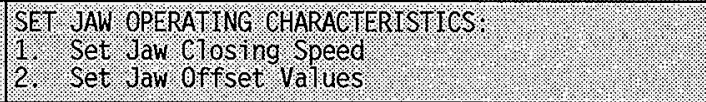 \\
\hline VII.G. & SET DISPLAY ATIRIBUTES. \\
\hline
\end{tabular}

D. STARTUP WITH SYSTEM ERRORS

1. DETERMINE cause of error(S) (TABLE 5).

2. TAKE applicable corrective action per TABLE 5 .

ERRORS WERE DETECTED:

LF slave not detected

RT arm not near STOW point

Keys error $\left(\begin{array}{llllllllllllll}0 & 0 & 0 & 0 & 0 & 0 & 0 & 0 & 0 & 1 & 0 & 0 & 0 & 0\end{array}\right)$

Diagnose errors before starting

hydraulics. Press the DOWN key

to view the next menu. 


\section{WRAP 1 PLANT OPERATING PROCEDURE}

(HNF SB W026-0TP 015)

D. STARTUP WITH SYSTEM ERRORS (Cont.)

\begin{tabular}{|c|c|c|}
\hline $4.6 .4 .4 \mathrm{AB}$ & 5 S & \\
\hline ERROR MESSAGE & CAUSS & ACTION \\
\hline$(x+\%)$ slave not detegted & $\begin{array}{l}\text { Master controver farled to } \\
\text { establish compunication with } \\
\text { one of the slave controllers }\end{array}$ & Nottify Supervis sion \\
\hline$(x)$ arm not near sToW point & left or r rght slave arm not. & 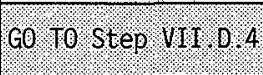 \\
\hline 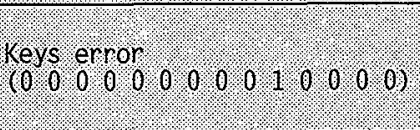 & 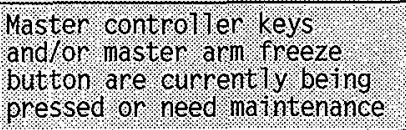 & Go 10.4 step VII. B. \\
\hline 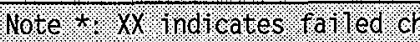 & net, l. $($ left) or RT $(r$ & \\
\hline
\end{tabular}

3. If a key error message is displayed,

THEN, PROCEED as fOl lows:

a. IF a key or FREEZE button is actually being pressed, THEN:

(1) REMOVE pressure from key or REPOSITION master arm so freeze button is released.

(2) SKIP to Step VII.D.4.

b. IF a key or freeze button is not being pressed, THEN:

(1) DO NOT enable hydraulics or deploy slave arms.

(2) PRESS DN function key on master control panel.

(3) VERIFY MAIN menu displayed.

(4) NOTIFY DOS.

4. PRESS DN function key on master control panel.

5. VERIFY MAIN menu for right slave arm displayed.

6. PRESS " $3>$ OPERATE". 


\section{WRAP 1 PLANT OPERATING PROCEDURE}

D. STARTUP WITH SYSTEM ERRORS (Cont.)

7. VERIFY OPERATE menu displayed.

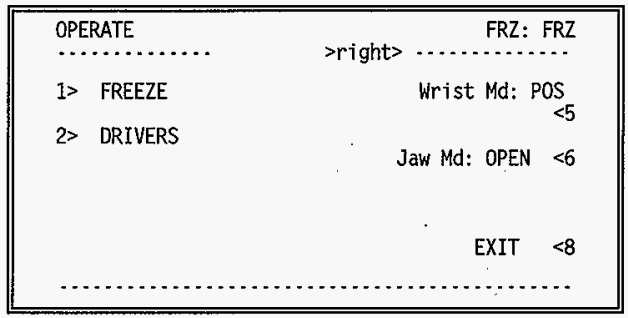

8. PRESS "2> DRIVERS".

9. VERIFY DRIVERS menu displayed.

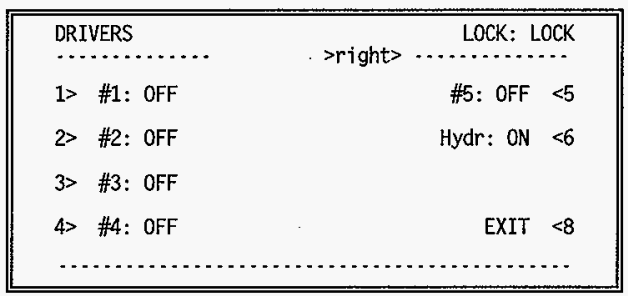

10. PRESS "6> HYDR" (enable right slave arm hydraulics).

11. EXIT to MAIN menu,

THEN, DISPLAY OPERATE menu for left slave arm.

12. REPEAT Steps VII.D.8 through VII.D.10 (enable left slave arm hydraulics).

13. GO TO Section VII.C, NORMAL SYSTEM OPERATION. 


\section{WRAP 1 PLANT OPERATING PROCEDURE}

(HNF SB: WO26 ONP 015)

\section{E. SET SLAVE ARM CHARACTERISTICS}

1. Set Stow Sequence

NOTE - Stow sequence is a series of arm movements that positions the slave arm between the protected (STOW IN) position and the deployed (STOW OUT) position. The sequence may consist of 2 to 16 slave arm positions (points).

a. PRESS "5> SETUP" from MAIN menu.

b. VERIFY SETUP menu displayed.

\begin{tabular}{|c|c|}
\hline SETUP & LOCK: LOCK \\
\hline 1> SET LIMITS & SET JAW' SPEED $<5$ \\
\hline 2> SET STOW & \\
\hline 3> DISPLAY & \\
\hline 4> SET JAW OFFSET & EXIT \\
\hline
\end{tabular}

c. PRESS "2> SET STOW".

d. VERIFY SET STOW menu displayed.

\begin{tabular}{|c|c|}
\hline $\begin{array}{l}\text { SET STOW } \\
\text {............. }\end{array}$ & >right> .... LOCK: FRZ \\
\hline Point: 01 & Last $<5$ \\
\hline & Next $<6$ \\
\hline & Teach $<7$ \\
\hline$<4$ Point: 16 & EXIT $<8$ \\
\hline
\end{tabular}

e. DETERMINE desired number of points in stow path sequence.

f. PRESS "4> POINT", until determined number is displayed.

g. UNFREEZE slave arm.

NOTE - Point 01 is first point in stow path. (STOW IN position).

h. VERIFY point counter indicates 01 . 


\section{WRAP 1 PLANT OPERATING PROCEDURE}

(1NF. SD - W026.0TP. 015)

\section{E. SET SLAVE ARM CHARACTERISTICS (Cont.)}

i. MOVE arm to desired position (point).

\section{CAUTION}

WHEN THE LAST POINT SPECIFIED IN SEQUENCE IS TAUGHT, POINT COUNTER DOES NOT INCREMENT. IF "7> TEACH" IS NOT PRESSED THE LAST POINT ACTUALLY TAUGHT PRIOR TO PRESSING "8> EXIT" BECOMES THE STOW OUT POINT. IT IS IMPORTANT TO TEACH DESIRED STOW OUT POINT BEFORE EXITING SET STOW MENU.

ALL POINTS MUST BE CLEAR OF GLOVEBOX WALLS \& EQUIPMENT OR DAMAGE MAY OCCUR.

NOTE - Pressing " $5>\angle A S T$ ", instead of "7> TEACH" returns to previous point. Pressing "6> NEXT", instead of "7> TEACH" skips point \& increments point counter.

j. PRESS "7> TEACH".

k. VERIFY point counter increments to next number.

1. REPEAT Steps VII.E.1. 1 through VII.E.1.k unti1 ạ11 desired points have been programmed.

m. PRESS "8> EXIT" (to SETUP menu).

2. Freezing Entire Slave Arm

NOTE - Changing from one menu to another automatically freezes both slave arms.

a. CHECK slave arm status in upper right corner of menu:

- REIN - normal unfrozen operation.

- $\quad$ FRZ - arm frozen (can be unfrozen with master arm freeze button).

- $\quad$ LOCK - arm frozen (cannot be unfrozen with freeze button from current menu).

b. PRESS freeze button (toggles FRZ/REIN) on master arm. 


\section{WRAP 1 PLANT OPERATING PROCEDURE}

E. SET SLAVE ARM CHARACTERISTICS (Cont.)

3. Freezing Individual Slave Arm Joints

NOTE - $\quad$ Freezing individua7 joints not required for a given task. provides a more stable platform for the task.

a. FREEZE slave arm.

b. PRESS "3> OPERATE" from MAIN menu.

c. VERIFY OPERATE menu displayed.

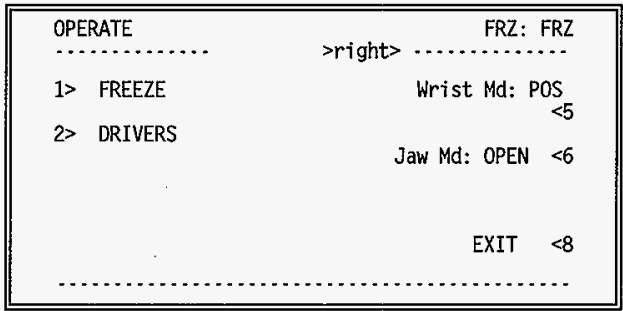

d. PRESS " $1>$ FREEZE".

e. VERIFY FREEZE menu displayed.

\begin{tabular}{|c|c|}
\hline $\begin{array}{l}\text { FREEZE } \\
\ldots \ldots \ldots\end{array}$ & $\begin{array}{l}\text { LOCK: LOCK } \\
>\text { right }>\ldots . . . . . . . . . .\end{array}$ \\
\hline 1> Rol: FRZ & Wrs: REIN $<5$ \\
\hline 2> Sho: REIN & . \\
\hline 3> Elb: FRZ & REIN a11 $<7$ \\
\hline 4> Yaw: REIN & EXIT $<8$ \\
\hline
\end{tabular}

f. CHECK status of individua? joints on menu.

g. PRESS indicated function to toggle status (FRZ/REIN) of each joint as desired ("7> REIN ALL", unfreezes a11).

h. PRESS " $8>$ EXIT" (to OPERATE menu). 


\section{WRAP 1 PLANT OPERATING PROCEDURE}

(HNF SO . WO26 - 07 P 015

E. SET SLAVE ARM CHARACTERISTICS (Cont.)

4. Controlling Slave Arm Speed

NOTE - . In genera7, speed of master arm movement controls speed of slave arm movement. System starts in FAST mode, however SLOW mode may be selected when more precise control is needed.

a. PRESS "2> OPTIONS", from MAIN menu.

b. VERIFY OPTIONS menu displayed.

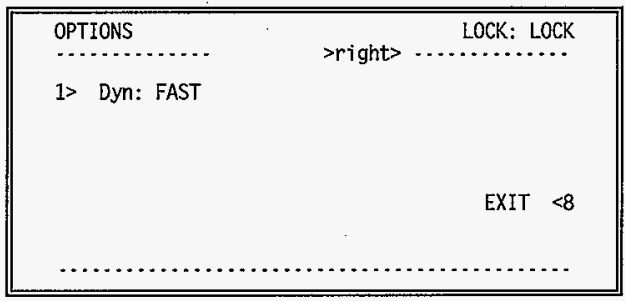

c. PRESS " $1>$ DYNAMICS" (togg TeS FAST/SLOW modes).

d. PRESS "8> EXIT" (to MAIN menu).

5. Enabling And Disabling Slave Arm Hydraulics

\section{CAUTION}

IF COMMANDED SLAVE ARM POSITION CHANGES WHILE HYDRAULICS ARE DISABLED, SLAVE ARM MAY ABRUPTLY MOVE TO THE NEW COMMANDED POSITION WHEN HYDRAUL ICS ARE RE-ENABLED. (This can happen if operator disables hydraulics, freezes a slave arm. moves the master arm, unfreezes the slave arm, and then re-enables hydraulics). IT IS IMPERATIVE THAT SLAVE ARM BE FROZEN BEFORE DISABL ING SLAVE ARM HYDRAULICS. HYDRAULICS MUST BE RE-ENABLED BEFORE UNFREEZING SLAVE ARM.

NOTE - Each slave arm has a hydraulic isolation valve that is either OPEN (enable hydraulic flow to slave arm actuators), or CLOSED (disable arm movement). Hydraulics are enabled as part of the startup process, but may be toggled for operation as desired.

a. IF hydraulics are to be immediately disabled, THEN:

(1) FREEZE slave arm.

(2) PRESS UP function key on master control panel.

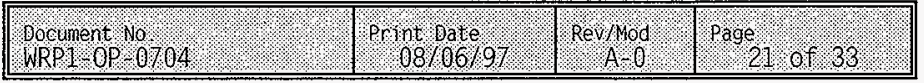


HNF-SD-W026-0TR-015, Rev. 0, Page OP-57

\section{WRAP 1 PLANT OPERATING PROCEDURE}

E. SET SLAVE. ARM CHARACTERISTICS (Cont.)

b. IF hydraulics are to be re-enabled or toggled, THEN:

(1) PRESS "2> DRIVERS" from OPERATE menu.

(2) VERIFY DRIVERS menu displayed.

\begin{tabular}{|c|c|c|c|}
\hline $\begin{array}{l}\text { DRIVERS } \\
\ldots \ldots \ldots \ldots \ldots\end{array}$ & >right> & $\begin{array}{r}\text { LOCK: L } \\
.\end{array}$ & \\
\hline 1> \#1: OFF & & \#5: OFF & $<5$ \\
\hline 2> \#2: OFF & & Hydr: ON & $<6$ \\
\hline $3>\quad \# 3:$ OFF & & & \\
\hline 4> \#4: OFF & & EXIT & $<8$ \\
\hline
\end{tabular}

(3) CHECK "6> HYDR" display (ON = hyd enabled, OFF $=$ disabled).

(4) PRESS " $6>$ HYDR", to toggle hydraulics as desired.

(5) PRESS "8> EXIT" (to OPERATE menu).

6. Set Slave Arm Movement Limits

NOTE - $\quad$ Range of motion of each slave arm joint (except wrist) can be limited by specifying left/right or up/down position limit.

a. PRESS "5> SETUP" from MAIN menu.

b. VERIFY SETUP menu displayed.

\begin{tabular}{|c|c|}
\hline $\begin{array}{l}\text { SETUP } \\
\end{array}$ & >right> .................. \\
\hline 1> SET LIMITS & SET JAW SPEED \\
\hline 2> SET STOW & \\
\hline 3> DISPLAY & \\
\hline $4>$ SET JAW OFFSET & EXIT \\
\hline
\end{tabular}

c. PRESS " $1>$ SET LIMITS".

\begin{tabular}{|c|c|c|c|}
\hline Bocument 10 . & 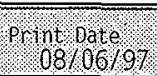 & Rev/Mod & page 2,0183 \\
\hline
\end{tabular}




\section{WRAP 1 PLANT OPERATING PROCEDURE}

(HNF SD - WO26-OTP 015 )

\section{E. SET SLAVE ARM CHARACTERISTICS (Cont.)}

NOTE - Limits are inactive when menu is displayed.

d. VERIFY SET LIMITS menu displayed.

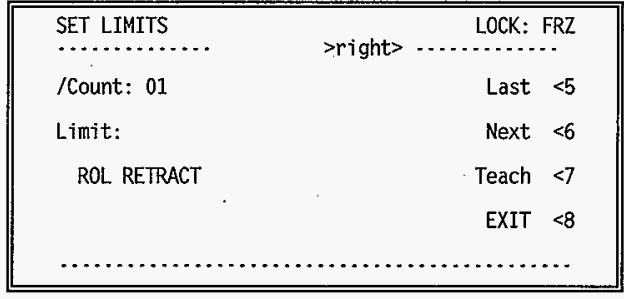

e. UNFREEZE slave arm.

f. VERIFY count indicates 01 .

g. PRESS "6> NEXT" OR "5> LAST" to scroll through 1ist, until desired 1imit (TABLE 7), indicates on display.

\begin{tabular}{|c|c|c|}
\hline \multicolumn{3}{|c|}{ TABLE 7. SLAVE ARM JOMNI IIMUTS } \\
\hline 1MTT NUMBER & JOMT: & AGTLATOR BIRECTION \\
\hline $01 \%$ & \multirow{2}{*}{ Shoulder Rol? } & RETRACT \\
\hline 02 & & EXIENO \\
\hline 03. & \multirow{2}{*}{ Shoulder P Ittch } & RETRAC \\
\hline . 04 & & EXTEND: \\
\hline .05 & \multirow[b]{2}{*}{ Eloow } & RETRACT \\
\hline 06 & & EXIENO \\
\hline (1. 07 & \multirow{2}{*}{ Yaw } & RETRACT \\
\hline 08. & & EXTLNO \\
\hline
\end{tabular}

h. MOVE joint indicated on display to desired limit.

i. PRESS "7> TEACH".

j. VERIFY counter increments to next position number.

k. REPEAT Steps VII.E.6.g through VII.E.6.j unti1 a11 desired limits have been set. 


\section{WRAP 1 PLANT OPERATING PROCEDURE}

(HNF SD 1026 - OTP-015)

\section{E. SET SLAVE ARM CHARACTERISTICS (Cont.)}

NOTE - $\quad$ Any joints remaining outside new 7 imits wi7l move to position inside limits when arm is unfrozen.

1. MOVE arm inside new set limits.

m. PRESS "8> EXIT" (to SETUP menu).

F. SET JAW OPERATING CHARACTERISTICS

1. Set Jaw Closing Speed

NOTE - Jaw speed increments jaw CLOSING speed when JAW MODE is set to POSITION. OPENING speed is not affected.

a. SET jaw mode to POSITION (Reference VII.C.7).

b. PRESS " $8>$ EXIT" (to MAIN menu).

c. PRESS "5> SETUP", from MAIN menu.

d. VERIFY SETUP menu displayed.

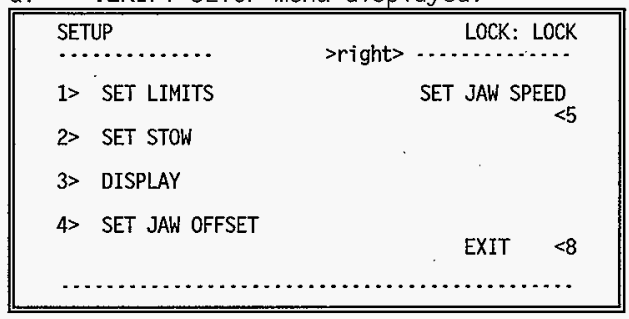

e. PRESS " $5>$ SET JAW SPEED".

f. VERIFY SET JAW SPEED menu displayed.

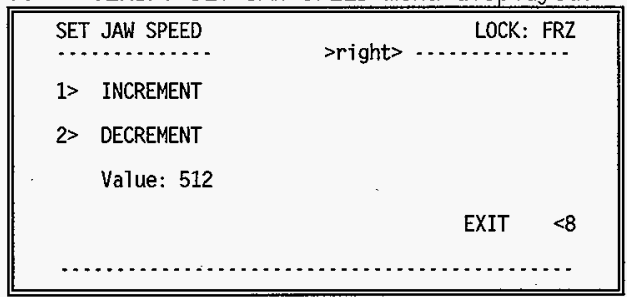




\section{WRAP 1 PLANT OPERATING PROCEDURE}

\section{F. SET JAW OPERATING CHARACTERISTICS (Cont.)}

NOTE - Jaw speed is incremented in arbitrary units

(counts), ranging from 128 to 2048 . Higher count setting equals faster jaw closure. Each switch press changes setting by 128 .

g. PRESS " $1>$ INCREMENT" OR "2> DECREMENT", unti1 desired setting is displayed.

h. PRESS " $8>$ EXIT" (to MAIN menu).

2. Set Jaw Offset values

NOTE - Jaw offset compensates for differences in servo valves to prevent jaws from drifting from a command position during operation. Once set it should not need to be changed, unless servo valve is replaced.

a. PRESS " $5>$ SETUP", from MAIN menu.

b. VERIFY SETUP menu displayed.

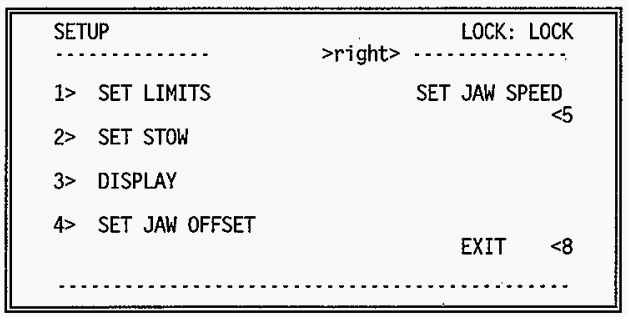

c. PRESS " $4>$ SET JAW OFFSET". 
HNF-SD-W026-OTR-015, Rev. 0, Page 0P-61

\section{WRAP 1 PLANT OPERATING PROCEDURE}

(HNF SB WO26 - OTP 015 )

d. VERIFY SET JAW OFFSET menu displayed.

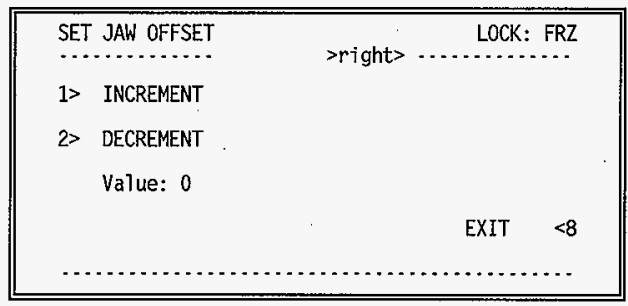




\section{WRAP 1 PLANT OPERATING PROCEDURE}

F. SET JAW OPERATING CHARACTERISTICS (Cont.)

NOTE - Jaw offset is incremented in arbitrary units (counts), ranging from -512 to +512 . Each switch press changes setting by 32 .

e. PRESS " $1>$ INCREMENT" $\underline{\text { OR }}$ " $2>$ DECREMENT", until desired setting is displayed.

f. PRESS "8> EXIT" (to OPERATE menu).

g. RESUME normal operation.

G. SET DISPLAY ATTRIBUTES

NOTE - Allows for change of screen viewing angle and/or reversing dark and light portions of the screen.

1. PRESS "5> SETUP", from MAIN menu.

2. VERIFY SETUP menu displayed.

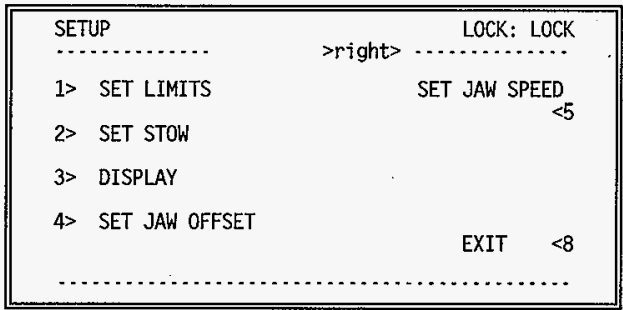

3. PRESS " $3>$ DISPLAY".

4. VERIFY DISPLAY menu displayed.

\begin{tabular}{|c|c|}
\hline DISPLAY & >right> ............. LOCK \\
\hline 1> View Angle - UP & Save to Default $<5$ \\
\hline 2> View Angle - DOWN & \\
\hline 3) Invert background & \\
\hline 4> Default & EXIT $<8$ \\
\hline
\end{tabular}

\begin{tabular}{|c|c|c|c|}
\hline bocunent lo lo & Print bate & Rev/110d & Page 27.0 of 33 \\
\hline
\end{tabular}




\section{WRAP 1 PLANT OPERATING PROCEDURE}

(HNF SO WO26 OTP 015)

G. SET DISPLAY ATTRIBUTES (Cont.)

5. PRESS function key(s) (TABLE 8), for desired screen attributes.

\begin{tabular}{|c|c|}
\hline Tit & SCR \\
\hline .. IUNCTION & SCREEN EFFECT \\
\hline 1) VEW ANGL UP & $\begin{array}{l}\text { Repeated pressing rotates viewing angl } \\
\text { up to destred position. }\end{array}$ \\
\hline 2?. YIEW ANGLE DOWN & $\begin{array}{l}\text { Repeated pressing rotates viewing an } \\
\text { down to desired position. }\end{array}$ \\
\hline 3> INVERT BACKGROUND & Reverses (toggle) screen contrast. \\
\hline $4>$ BEFAUIT & Revert to startup screen conf figuration. \\
\hline 5> SAVE IO DEFAAULT & $\begin{array}{l}\text { save current screen attributes to } \\
\text { defautl. }\end{array}$ \\
\hline
\end{tabular}

6. PRESS "8> EXIT" (to MAIN menu).

H. SYSTEM SHUTDOWN

\section{CAUTION}

SHUTTING DOWN MANIPULATOR WILL CAUSE SLAVE ARMS TO SAG UNTIL MECHANICAL STOPS ARE REACHED. HYDRAULIC OR CONTROL POWER SHOULD NOT BE TURNED OFF UNTIL ARMS ARE POSITIONED IN GLOVEBOX SO THEY WILL NOT HARM, OR BE HARMED BY OTHER OBJECTS WHEN THEY SAG.

1. PRESS "7> SHUTDOWN" from MAIN menu.

2. VERIFY SHUTDOWN menu displayed.

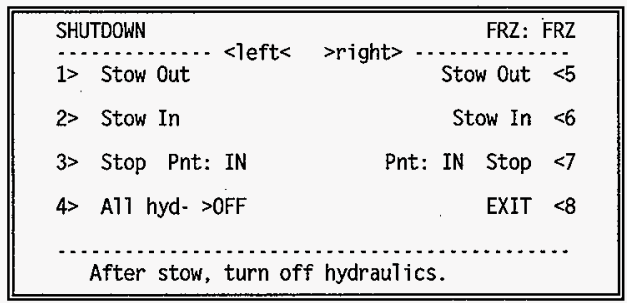

3. MOVE each slave arm to a position near its STOW OUT point.

4. PRESS "2> STOW IN" (left slave arm).

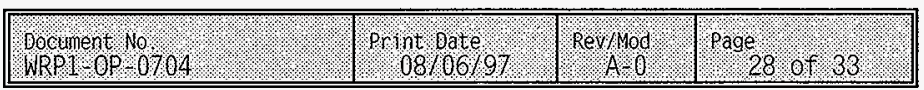




\section{WRAP 1 PLANT OPERATING PROCEDURE}

(HNF SB 1026 - 0IP - 015)

H. SYSTEM SHUTDOWN (Cont.)

5. VERIFY left slave arm STOWS IN.

6. PRESS "5> STOW IN" (right slave arm).

7. VERIFY right slave arm STOWS IN.

8. PRESS " $4>$ ALL HYDR OFF".

9. TURN master control power switch OFF.

10. PUSH EMERGENCY STOP button on either side of carriage controller IN.

11. IF manipulator is to be electricaliy isolated, THEN, SET disconnect switch on C\&I enclosure to ZERO.

12. IF HPU is to be SHUT DOWN, THEN:

a. REPEAT Steps VII.H.1 through VII.H.11 for each manipulator.

\section{CAUTION}

ALL MANIPULATORS MUST BE NEAR STOW OUT POINT BEFORE SHUTTING OFF HPU.

SHUTTING OFF HPU WILL DISABLE ALL MANIPULATORS.

NOTE -

HPU 107-HU-07-302 is common to a77 manipulators. Another Start/Stop switch is located on the HPU Control Panel at HPU on overhead walkway.

b. PRESS "STOP" button on "I" beam behind Glovebox 102. 


\section{WRAP 1 PLANT OPERATING PROCEDURE}

(HNF SD- W026-0IP -015)

\section{EMERGENCY SHUTDOWN}

NOTE - $\quad$ Emergency Stop immediately shuts down manipulator by turning off a 77 manipulator components that receive power from C\&I enclosure (master controller, carriage controller, slave controllers, slave arms, and horiz and vertical carriages).

Hydraulic power unit (HPU) remains ON but hydraulical7y powered manipulator components cannot be used without electrical power as hydraulic control valves are disabled.

\section{CAUTION}

SHUTTING DOWN MANIPULATOR WILL CAUSE SLAVE ARMS TO SAG UNTIL MECHANICAL STOPS ARE REACHED. HYDRAULIC OR CONTROL POWER SHOULD NOT BE TURNED OFF UNTIL ARMS ARE POSITIONED IN GLOVEBOX SO THEY WILL NOT HARM, OR BE HARMED BY OTHER OBJECTS WHEN THEY SAG.

1. PUSH EMERGENCY STOP button on either side of carriage controller IN. 


\section{WRAP 1 PLANT OPERATING PROCEDURE}

FIGURE 1 - CARRIAGE CONTROLLER

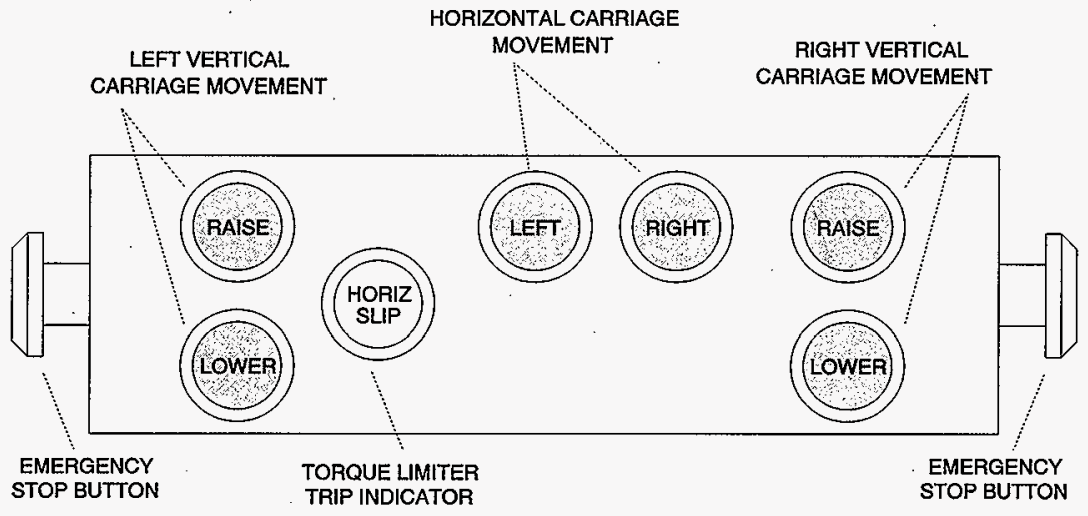




\section{WRAP 1 PLANT OPERATING PROCEDURE}

(HNF SD 1026 OTP $<015$ )

\section{FIGURE 2 - MASTER CONTROL PANEL}

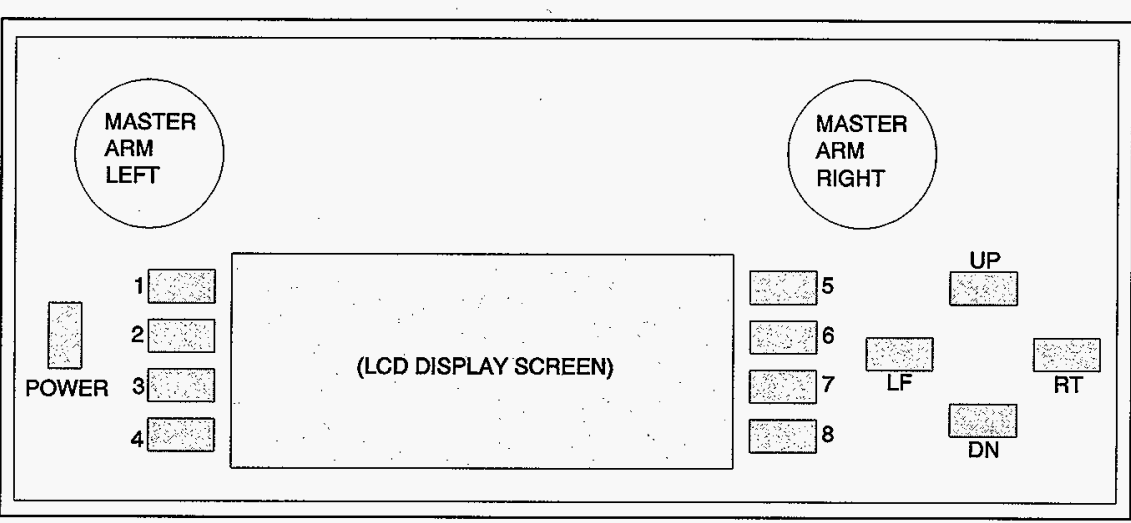

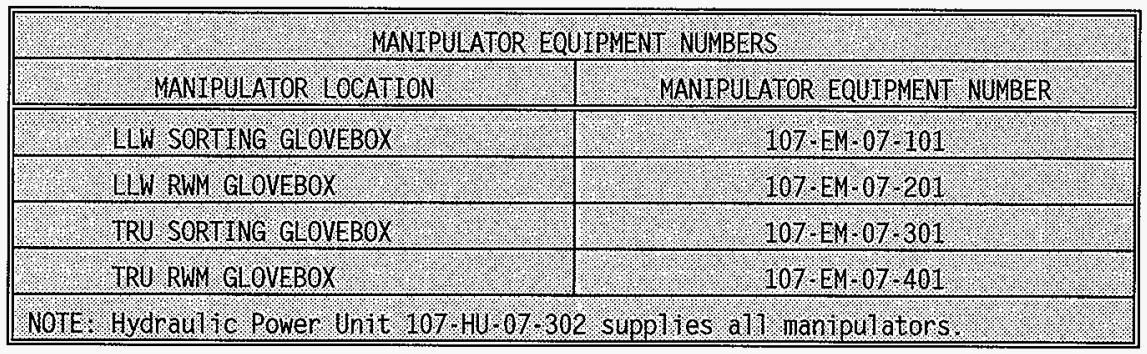

\begin{tabular}{|c|c|c|c|}
\hline 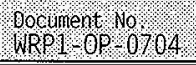 & prot $00 / 0 \mathrm{ate}, 97$ & Rev/ 1000 & p.ge 30.0 . 33 \\
\hline
\end{tabular}


HNF-SD-W026-0TR-015, Rev, 0, Page 0P-68

\section{WRAP 1 PLANT OPERATING PROCEDURE}

FIGURE 3 - MASTER ARM

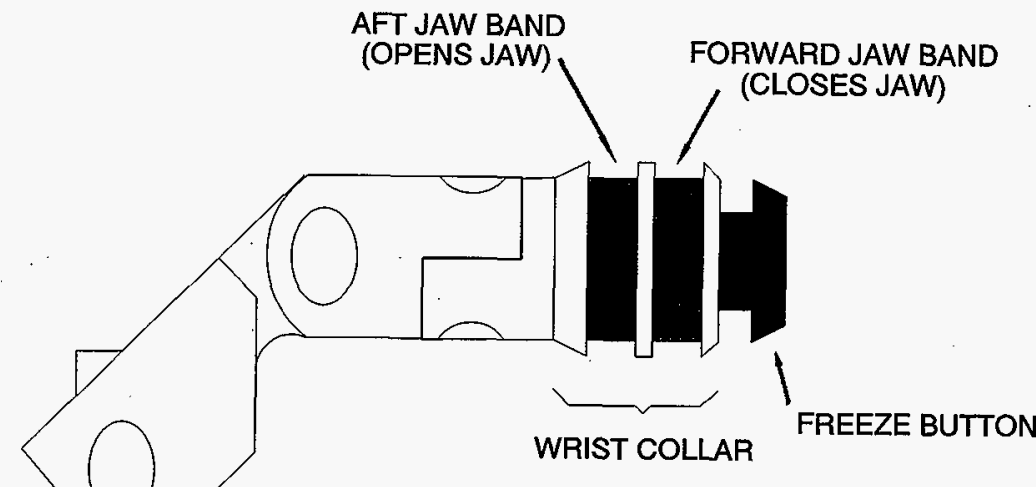




\section{WRAP 1 PLANT OPERATING PROCEDURE}

SYSTEY PROGESS

\section{BAGLESS TRANSFER SYSTEM MANUAL OPERATION}

(HNF-SD-W026-OTP-015)

\section{SYSTEM DESCRIPTION}

NOTE - The bag7ess transfer system may also be referred to as the Drath \& Schrader system in some plant documentation.

This procedure provides instructions for manual operation of the Deutsche Babcock, Bagless Transfer System (200/300 liter Drum Loading Ports).

The drum loading port is normally operated in automatic mode. However, if automatic operation is interrupted (inadvertently or otherwise), it may be necessary to enter manual mode to perform drum transfers or to return unit to proper sequencing. The drum loading port should be operated in manual only if absolutely necessary because the PLC ignores all limit switch signals. Major equipment damage is possible if improper operating sequence is used.

\section{REFERENCE DOCUMENTS}

None.

\section{PRESTART CONDITIONS}

A11 personnel performing this procedure shal1 be qualified in accordance with Waste Management Hanford Procedures Manual, Section 5.1, "Training and Qualification," and on-the-job training.

Verify daily radiological dose rates and contamination level for gloves have been established by Radiological Control.

\begin{tabular}{|c|c|c|c|c|c|}
\hline Retease bate & Prum Date & Appr. besig & Wocument No 10.705 & rev. & 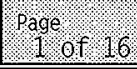 \\
\hline
\end{tabular}




\section{WRAP 1 PLANT OPERATING PROCEDURE}

\section{SAFETY}

All potential hazards, such as lifting heavy containers or handling sharp objects present in waste containers, must be mitigated by protective equipment, procedures, and administrative controls to ensure acceptablerisk operating conditions.

Al1 shall be performed per applicable Radiation Work Permits (RWPs).

Failure to follow proper port sequencing as described in this procedure may result in contamination of drum port and surrounding area.

\section{TOOLS AND SUPPLIES}

None.

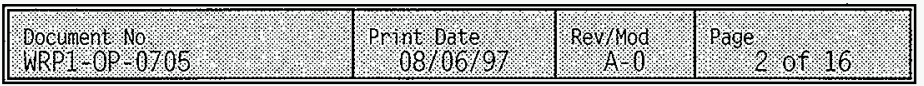




\section{WRAP 1 PLANT OPERATING PROCEDURE}

\section{TABLE OF CONTENTS}

A. GENERAL REQUIREMENTS . . . . . . . . . . . . . . . . . . . . . 4

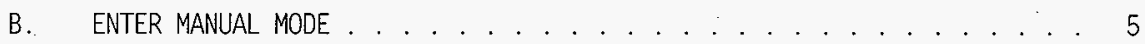

c. DETERMINE PORT STATUS . . . . . . . . . . . . . . . . . 7

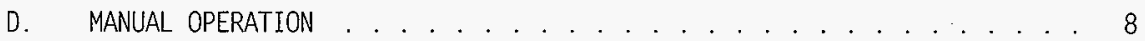

E. FAULT RECOVERY OPERATION . . . . . . . . . . . . . . . . . . . . . 13

F. FAULT ACKNOWLEDGEMENT SEQUENCE . . . . . . . . . . . . . . . 15

G. RESET PORT CONTROL PANEL EMERGENCY STOP . . . . . . . . . . . . . . 15 ATTACHMENTS

ATTACHMENT 1 - OPERATOR PANEL . . . . . . . . . . . . . . . . 16 


\section{WRAP 1 PLANT OPERATING PROCEDURE}

(HNF SO WO26 OTP . 015 )

\section{PROCEDURE}

\section{CAUTION}

THE DRUM LOADING PORT SHOULD BE OPERATED IN MANUAL, ONLY IF ABSOLUTELY

NECESSARY, BECAUSE THE PLC IGNORES ALL LIMIT SWITCH SIGNALS. MAJOR EQUIPMENT DAMAGE OR CONTAMINATION IS POSSIBLE IF IMPROPER OPERATING SEQUENCE IS USED.

A. GENERAL REQUIREMENTS

1. Once placed in Manual Mode per Section VII.B of this procedure, other sections of this procedure may be performed repeatedly and in any order necessary to facilitate work. However STEPS within each section MUST BE PERFORMED IN SEQUENCE.

\section{WARNING}

WORK THROUGH GLOVEPORTS MAY INVOLVE PLACING HANDS IN CLOSE PROXIMITY TO MOVING EQUIPMENT. CARE MUST BE TAKEN TO PREVENT INJURY.

2. IF at any time during performance of this procedure, work is to be performed through gloveports,

THEN:

a. CHECK status and location of moving equipment in proximity of gloveport(s) to be used.

b. TAKE action, including any of the following, as deemed necessary to prevent injury:

- $\quad$ AVOID equipment when reaching through port(s)

- $\quad$ PRESS glovebox E-Stop to stop glovebox equipment

- CONSULT DOS when unsure

c. PERFORM a contamination survey of hands, arms and front of body upon exiting glove:

(1) IF contamination is detected,

THEN, CONTACT Radiological Control Technician. 


\section{WRAP 1 PLANT OPERATING PROCEDURE}

B. ENTER MANUAL MODE

1. OBTAIN Duty Operations Supervisor (DOS) permission to enter MANUAL mode.

2. VERIFY the following equipment is positioned so as not to interfere with port operation:

3. VERIFY manipulator wil1 not interfere with port operation.

4. VERIFY other equipment wi 11 not interfere with port operation.

5. ACCESS, on operator panel keyboard (Attachment 1). MANUAL mode, as follows:

a. PRESS STOP button (not EMERGENCY STOP).

b. VERIFY $\mathrm{X} 5$ key pilot light ILLUMINATED.

C. PRESS K5 key.

d. VERIFY MAIN menu displayed.

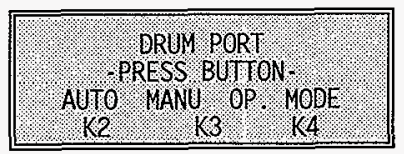

e. PRESS K3 key.

f. VERIFY display.

MANUAL SERVIGE

BE CAREFUL

K5 =ESC.

CONIINUE PRESS F 8 
HNF-SD-W026-0TR-015, Rev. 0, Page OP-74

\section{WRAP 1 PLANT OPERATING PROCEDURE}

(HNF SO . . 026 . OTP 015 )

(Step VII.B.5 Cont.)

g. PRESS F8 key.

h. VERIFY display.

NOTE - Pressing ESC key at this point will cancel entry to manua 7 .

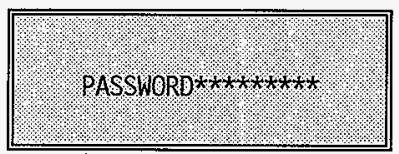

(DOS) i. KEY IN password.

j. PRESS ENTER kEY.

k. PRESS ESC key.

1. PRESS F8 key.

m. VERIFY display.

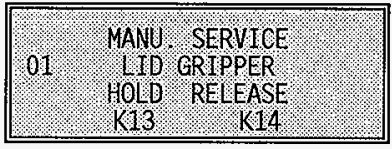

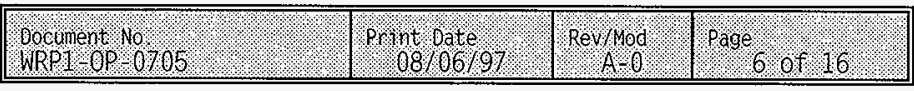




\section{WRAP 1 PLANT OPERATING PROCEDURE}

(HNF SO W026-0IP 015 )

\section{DETERMINE PORT STATUS}

1. DETERMINE port status as follows:

a. PRESS UP/DOWN arrow keys, as required, to select each function listed in Table 1.

NOTE - Either K13 or K14 wi7l be STEADY ON, while the other will be FLASHING. Option listed above FLASHING indicator is present position/status of selected function.

b. OBSERVE K13 / K14 key at each function to determine position of corresponding actuator.

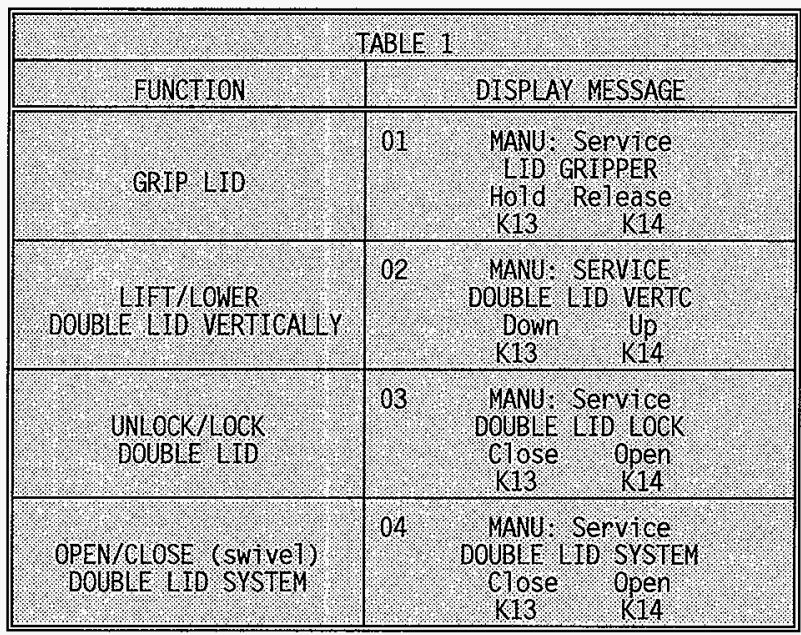




\section{WRAP 1 PLANT OPERATING PROCEDURE}

(1NF SD W026 OTP 015)

D. MANUAL OPERATION

\section{CAUTION}

FAILURE TO FOLLOW PROPER SEQUENCE MAY RESULT IN MAJOR EQUIPMENT DAMAGE OR CONTAMINATION OF EQUIPMENT AND SURROUNDING AREA.

1. VERIFY port travel path clear of obstructions.

2. VERIFY port closed and locked as per port close sequence below.

3. VERIFY Lid Gripper in "Release" position.

4. ENSURE drum properly positioned under port.

5. OPEN port as follows:

a. GRIP Tid:

(1) IF the following LID GRIPPER menu with title is displayed.

THEN, PRESS DOWN arrow key to display LID GRIPPER Menu without title.

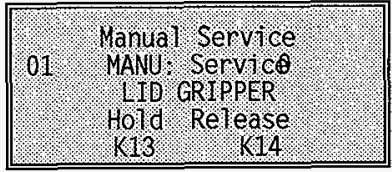

(2) PRESS UP/DOWN arrow keys, as required, to access LID GRIPPER menu:

\begin{tabular}{|c|c|}
\hline 01 & 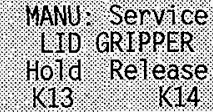 \\
\hline
\end{tabular}

(3) PRESS K13 key.

(4) VERIFY K13 FLASHING, K14 STEADY ON. 


\section{WRAP 1 PLANT OPERATING PROCEDURE}

(HNF SD W026. OTP - 015 )

(Step VII.D.5 Cont.)

b. LIFT double lid:

(1) PRESS DOWN arrow key.

(2) VERIFY DOUBLE LID VERTC menu displayed.

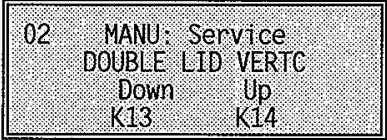

(3) PRESS K14 key.

(4) VERIFY K14 FLASHING, K13 STEADY ON.

(5) VERIFY lid retained against raised port door.

c. OPEN double lid lock:

(1) PRESS DOWN arrow key.

(2) VERIFY DOUBLE LID LOCK menu displayed.

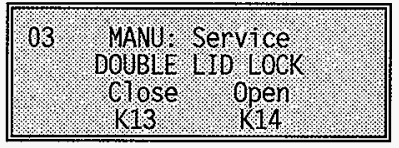

(3) PRESS K14 key.

(4) VERIFY K14 FLASHING, K13 STEADY ON.

\begin{tabular}{|c|c|c|c|}
\hline Uocument No & ontnt bater & Revmod & page 90016 \\
\hline
\end{tabular}




\section{WRAP 1 PLANT OPERATING PROCEDURE}

(HNF SB W026-0TP-015)

(Step VII.D.5 Cont.)

NOTE - Upon completion of the following step, port will be rotated open and drum completely exposed to glovebox.

d. OPEN double lid system:

(1) PRESS DOWN arrow key.

(2) VERIFY DOUBLE LID SYSTEM menu displayed.

\begin{tabular}{|c|}
\hline 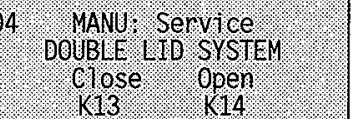 \\
\hline
\end{tabular}

(3) PRESS and HOLD K14 key.

NOTE - K14 key must be held until door reaches full OPEN position.

(4) VERIFY K14 FLASHING, K13 STEADY ON.

(5) RELEASE K14 key.

6. CLOSE port as follows:

a. VERIFY no debris present on port.

b. CLOSE double lid system:

(1) PRESS UP/DOWN arrow keys, as required, to access DOUBLE LID SYSTEM menu.

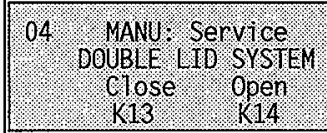

NOTE - Key must be held unti7 door is full CLOSED.

(2) PRESS and HOLD K13 key.

(3) VERIFY K13 FLASHING, K14 STEADY ON.

(4) RELEASE K13 key.

\begin{tabular}{|c|c|c|c|}
\hline 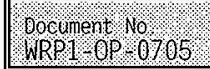 & p. 00106069 & Revorod & page 10.01 \\
\hline
\end{tabular}




\section{WRAP 1 PLANT OPERATING PROCEDURE}

(Step VII.D. 6 Cont.)

c. CLOSE double Tid lock:

(1) PRESS IJP arrow key.

(2) VERIFY DOUBLE LID LOCK menu displayed.

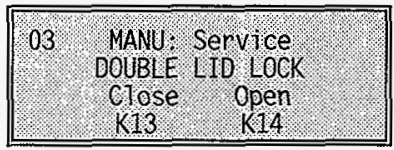

(3) PRESS K13 key.

(4) VERIFY K13 FLASHING, K14 STEADY ON.

d. LOWER double 1id:

(1) PRESS UP arrow key.

(2) VERIFY DOUBLE LID VERTC menu displayed.

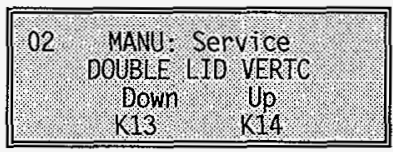

(3) PRESS K13 key.

(4) VERIFY K13 FLASHING, K14 STEADY ON.

e. RELEASE lid:

(1) PRESS UP arrow key.

(2). VERIFY LID GRIPPER menu displayed.

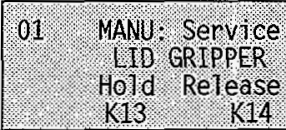

(3) PRESS KI4 key.

(4) VERIFY K14 FLASHING, K13 STEADY ON. 


\section{WRAP 1 PLANT OPERATING PROCEDURE}

7. WHEN desired.

THEN, RETURN to automatic operation as follows:

a. ENSURE port CLOSED per Step VII.D.6.

b. REQUEST RCT survey the closed port and immediate area to verify no contamination.

c. PRESS K5 key.

d. VERIFY MAIN menu displayed.

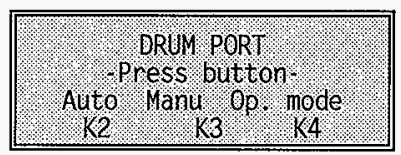

e. PRESS K2 key.

f. PRESS "START" when prompted by menu screen.

NOTE - Port wi77 not go into Automatic with low regulator pressure or with EMERGENCY STOP depressed.

8. IF AUTOMATIC mode cannot be accessed, THEN:

a. VERIFY regulator for port being operated is adjusted to greater than six (6) bar.

b. VERIFY glovebox emergency stops CLEARED.

\begin{tabular}{|c|c|c|c|}
\hline 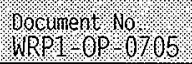 & Print Dote, & Revoldod & 92901201.16 \\
\hline
\end{tabular}




\section{WRAP 1 PLANT OPERATING PROCEDURE}

(HNF SD W026 OSP - 015)

E. FAULT RECOVERY OPERATION

NOTE - A typical fault may involve an emergency stop, a loss of power, or loss of air pressure, stopping the port during the OPENING/CLOSING sequence. In MANUAL, the operator determines where in the sequence the port stopped. manual7y operates the actuators to restore sequence, then CLOSES the port and returns to AUTOMATIC mode.

1. DETERMINE port status per Section VII.C.

2. EVALUATE port for fault condition relative to proper OPENING/CLOSING sequence per Tables 2 and 3.

NOTE - Manual operating sequence must follow automatic sequences shown in Tables 2 and 3 (sequences assume design drum proper7y positioned).

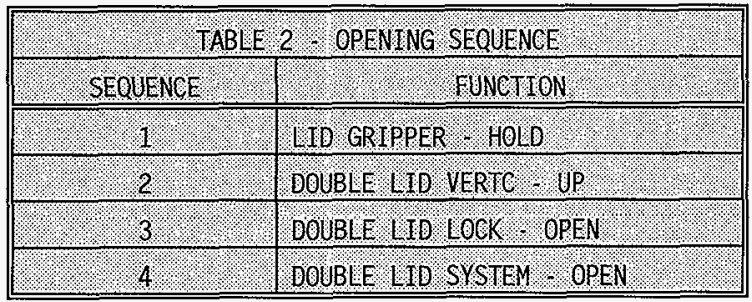

\begin{tabular}{|c|c|}
\hline 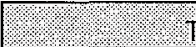 & 3. CLOSING SEQUENCE \\
\hline S.: SEQUENGE & (1:.4: : FUNCTION \\
\hline 1 & DOUBLE ITO SYSTEN - CLOSE \\
\hline 2 & DOUBLE LID LOCK CLOSE \\
\hline 3.3 & DOUBLE LIO VERTC DOWN \\
\hline .8 & LIO GRIPPER - RELEASE \\
\hline
\end{tabular}




\section{WRAP 1 PLANT OPERATING PROCEDURE}

(MNF SB W026 -0IP -015)

3. DETERMINE, based on observations, corrections required to return port to normal sequencing.

a. IF in doubt as to proper corrective actions, THEN:

(1) STOP work immediately.

(2) CONTACT DOS and COG Engineer for assistance.

4. CORRECT port sequencing fault using Section VII.D, as follows:

a. PRESS UP/DOWN arrow key, as applicable, to select desired function.

b. PRESS K13 / K14 key as applicable; to return corresponding actuator to proper position.

c. REPEAT Steps VII.E.4.a and VII.E.4.b, as required, to properly position each port actuator.

NOTE - Once port sequence has been interrupted, the port must be complete7y CLOSED before it can be returned to automatic.

5. WHEN port has been returned to correct sequencing, THEN :

a. DETERMINE port condition relative to CLOSING sequence per Step VII.D.6. AND continue sequence to fu17 CLOSED position.

b. RETURN to automatic mode per Step VII.D.7. 


\section{WRAP 1 PLANT OPERATING PROCEDURE}

F. FAULT ACKNOWLEDGEMENT SEQUENCE

NOTE 1 - $\quad$ Al7 faults must be acknowledged and cleared before operation can commence.

NOTE 2 - An unacknowledged fault exists when either the "ACK" button is i77uminated, or the Control Panel screen is flashing.

1. READ Fault message.

2. RECORD Fault message.

3. PRESS "ACK" button.

4. IF "ACK" button pilot light is ILLUMINATED,

AND screen is flashing.

THEN, REPEAT steps VII.F.1 through VII.F.3.

5. VERIFY "ACK" button pilot light is OFF.

G. RESET PORT CONTROL PANEL EMERGENCY STOP

1. TWIST EMERGENCY STOP button CLOCKWISE.

2. ACKNOWLEDGE all faults. 
HNF-SD-W026-0TR-015, Rev. 0, Page OP-84

\section{WRAP 1 PLANT OPERATING PROCEDURE}

(BNF SB W026 OIP - 015)

\section{ATTACHMENT 1 - OPERATOR PANEL}

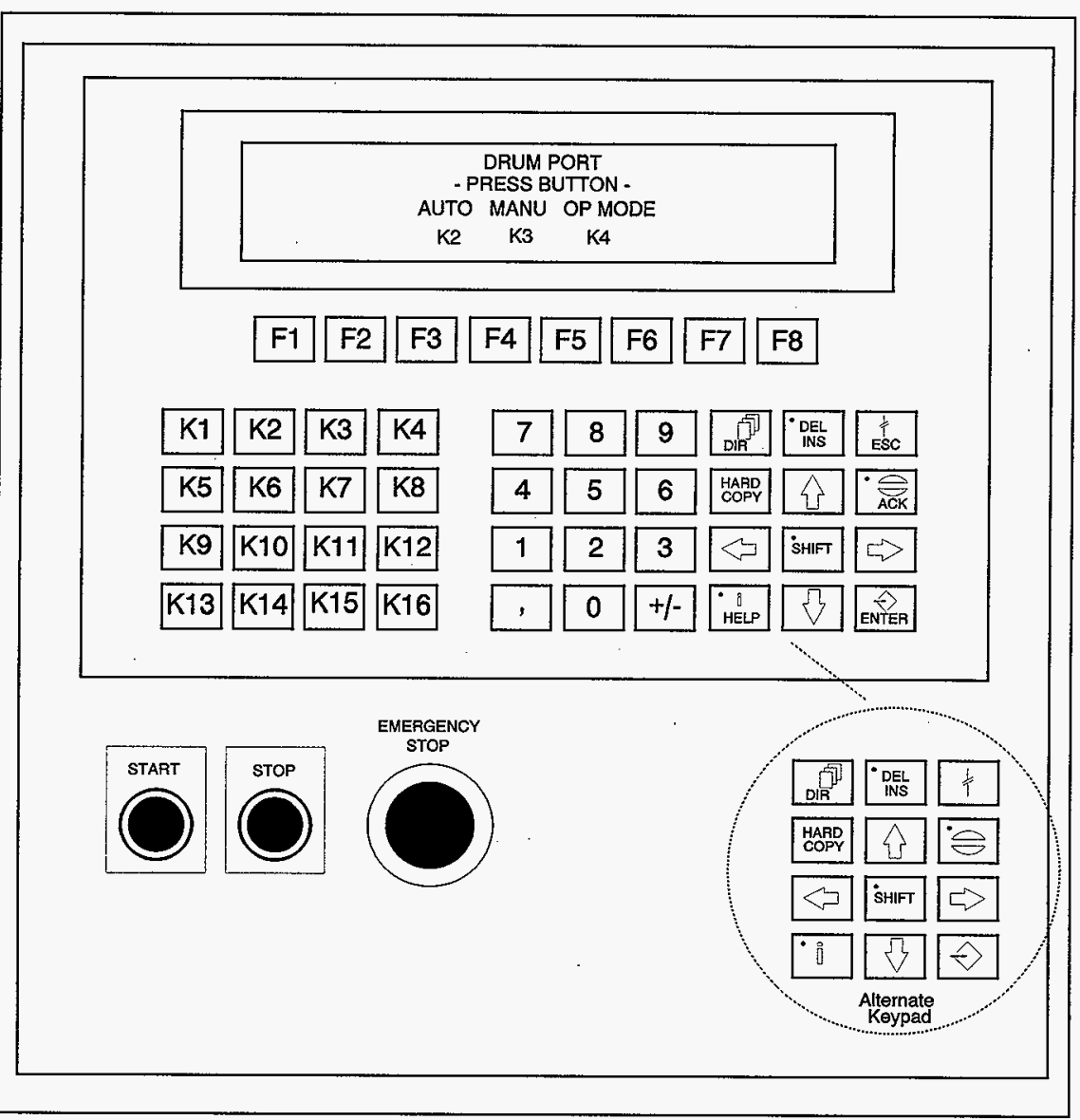

\begin{tabular}{|c|c|c|c|}
\hline oocument No 1 \% & P.10 0ate & Rev/Mod & page 6.6 .07 .16 \\
\hline
\end{tabular}




\section{WRAP 1 PLANT OPERATING PROCEDURE}

SYSTEM: PROCESS

\section{PACKET NDE OPERATION \\ (HNF-SD-W026-OTP-015)}

\section{SYSTEM DESCRIPTION}

This procedure provides instructions for operation of EG\&G Corp, TORR II D, $X$-Ray Inspection System.

$X$-Ray systems are used for packet non-destructive examination (NDE) in the Low Level Waste (LLW) and Transuranic Waste (TRU) sorting gloveboxes.

\section{REFERENCE DOCUMENTS}

None.

\section{PRESTART CONDITIONS

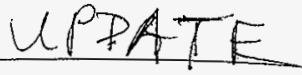

All personnel performing this procedure shall be qualified in accordance with WHC-CM-5-34, Section 1.8, "Training and Qualification, " and on-the-job training.

\section{SAFETY}

Radiological

$X$-ray leakage is certified $<0.5 \mathrm{mrem} / \mathrm{hr}$.

System is equipped with door interlock to prevent accidental exposure.

\section{TOOLS AND SUPPLIES}

$X$-ray Control Panel Power Key. 


\section{WRAP 1 PLANT OPERATING PROCEDURE}

\section{TABLE OF CONTENTS}

PAGE

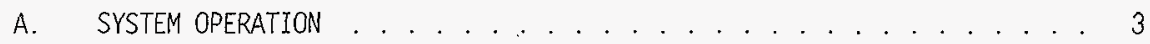

\section{ATTACHMENTS}

FIGURE 1: LLW, OIU-12-103B MAIN MENU . . . . . . . . . . . . . . . . 5

FIGURE 2: TRU, OIU-12-104B MAIN MENU $\ldots . . . . . . . . . . .6$

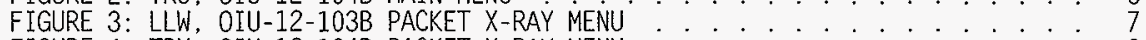

FIGURE 4: TRU, OIU-12-104B PACKET X-RAY MENU . . . . . . . . . . . . . . 8

FIGURE $5:$ X-RÁY CONTROL PANEL . . . . . . . . . . . . . . . . . 9 


\section{WRAP 1 PLANT OPERATING PROCEDURE}

$(\mathrm{HN} / \mathrm{SB}, \mathrm{NO} 260 \mathrm{PP} 015)$

VII. PROCEDURE NOJK-Packet $x$-Ray Cantrol Pawel
Glavebox isvlo7-ND-07-101
labeled

A. SYSTEM OPERATION

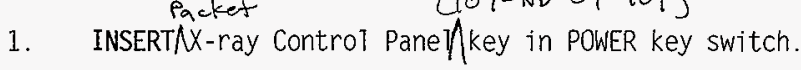

2. TURN POWER key switch ON.

3. SELECT packet to be X-Rayed.

4. SELECT "PACKET X-RAY MENU" on Operator Interface Unit

(0IU-12-103B) \{0IU-12-104B\} MAIN menu (Figures 1 \& 2).

5. VERIFY, PACKET X-RAY menu (Figures 3 \& 4) displayed.

6. SELECT "OPEN PACKET $X$-RÂ DOOR ON PACKET $X$-RAY menu.

Svarty VERIFY:

$8 \overrightarrow{P L A C}$ DOOR Sel "Retruct $X$ Ray Tray

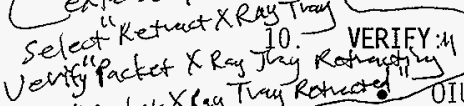

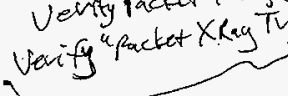

OIU message: "PACKET X-RAY CLOSING...".

OIU message: "PACKET X-RAYNOPENING...".

Packet $X$-Ray door OPENS.

Packet tray EXIENOS? Dook

OIU message: "PACKET X-RAYNOPEN".

PLACE selected packet in center of packet tray.

SELECT "CLOSE RACKET X-RAY D O ON PACKET X-RAY menu.

- Packet tray-RETRACTS.

- Packet X-Ray door CLOSES. Poor

- OIU message: "PACKET X-RAY ${ }_{1}$ CLOSED".

11. SELECT "ENABLE PACKET $X$-RAY" On PACKET X RAY menu. are locked out

12. VERIFY, OIU message: "PACKET X-RAY ENABLED".

Turn Brightness bicloce $=13$. DEPRESS-ANO HOLD X-Ray footswitch.

entrol to $20^{\text {ocloc }}=3$. PRESS "START" button on X-RAY CONTROL PANEL (FIGURE 5).

position-

15. VIEW image on $/$ ackef monitor $107-10 \%-10$

16. ADJUST "BRIGHTNESS" control on X-RAY CONTROL PANEL (FIGURE 5) as required, for clear picture of packet being $X$-Rayed.

\begin{tabular}{|c|c|c|c|}
\hline Gocunent $10 \%$ & rrant oate $.08106 / 97$ & Revimod & frge .0 .079 \\
\hline
\end{tabular}




\section{WRAP 1 PLANT OPERATING PROCEDURE}

(HNF SD. W026-0TP - 015 )

17. WHEN inspection is complete:

-a. RELEASE-X-Ray-footswitch

b. PRESS "STOP" on X-RAY CONTROL PANEL.

18. PRESS "OPEN PACKEF $X$-RAY Poor" on PACKET X-RAY menU.

19. VERIFY:

- OIU message: "PACKET X-RAYTOPENING...".

- Packet X-Ray door OPENS.

- Packet tray EXTENAS. - D soor

- OIU message: "PACKET X-RAYMOPEN".

20. REMOVE packet from $X$-Ray tray. relect "Closex-Ray zoor"

PROCESS packet (compliant or non-compliant) per applicable glovebox sorting procedure.

22. REPEAT Steps VII.A.3 through VII.A.21, as applicable, until all non-compliant items have been removed from waste.

NOTE - $\quad$ X-ray will typical7y be turned on at beginning of shift and turned off at end of shift.

23. IF X-ray activities are complete,

THEN, TURN POWER key switch OFF.

24. REMOVE X-ray Control Panel key. 


\section{WRAP 1 PLANT OPERATING PROCEDURE}

(UNF SO - 1026 - OTP 015 )

FIGURE 1: LLW, OIU-12-103B MAIN MENU

SORTING GLOVEBOX MAIN MENU

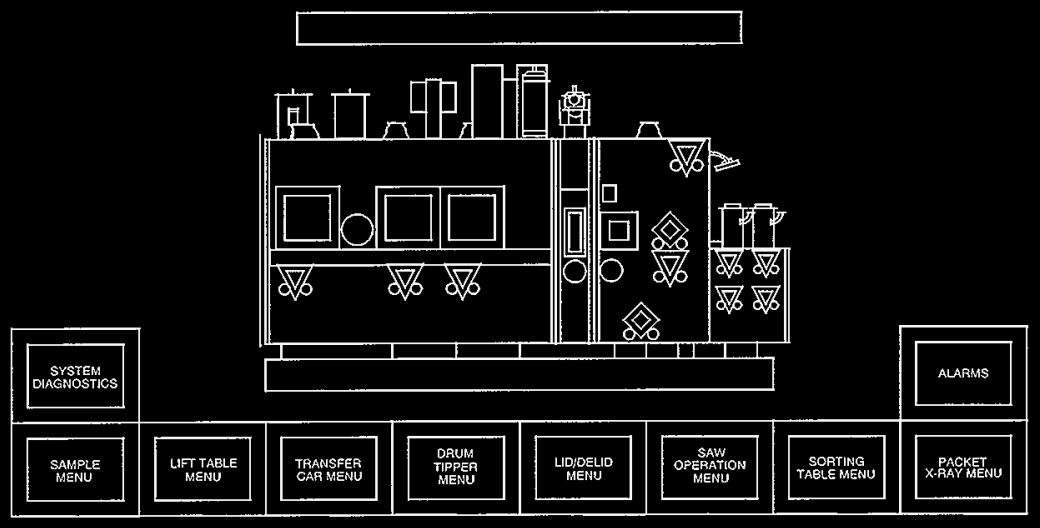


HNF-SD-W026-OTR-015, Rev. 0, Page 0P-90

\section{WRAP 1 PLANT OPERATING PROCEDURE}

(HNF SB W026 0 0IP 015)

\section{FIGURE 2: TRU, OIU-12-104B MAIN MENU}

\section{TRU SORTING GLOVEBOX MAIN MENU}

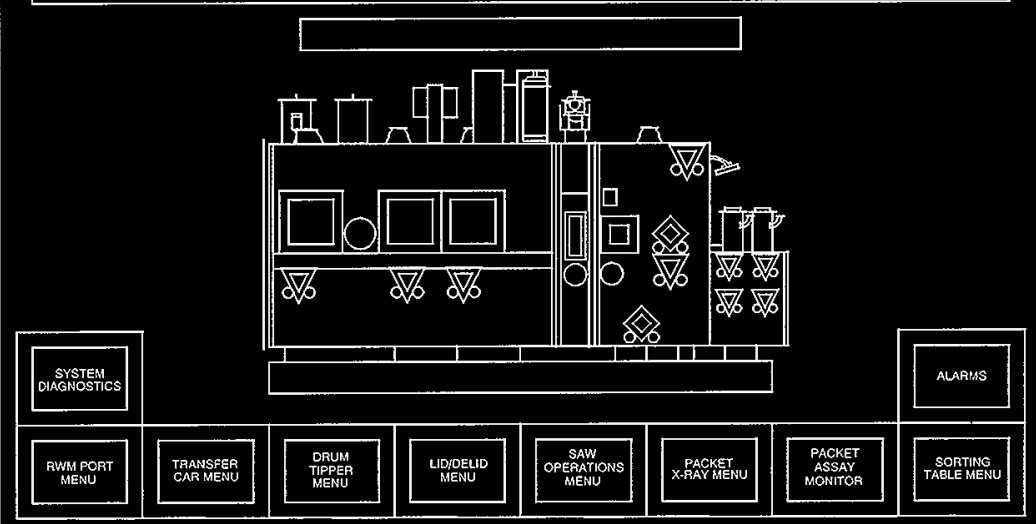




\section{WRAP 1 PLANT OPERATING PROCEDURE}

\section{(HNF SO 1026 - OTP 015 )}

FIGURE 3: LLW, OIU-12-103B-PACKET X-RAY MENU

\section{PACKET X-RAY MENU}

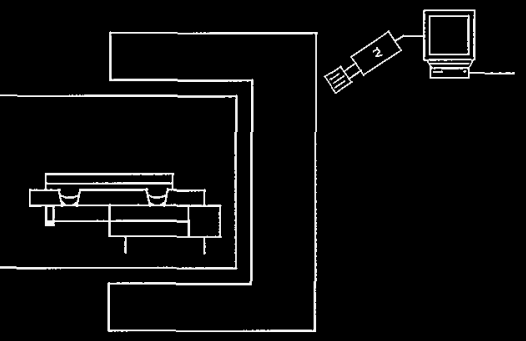




\section{WRAP 1 PLANT OPERATING PROCEDURE}

FIGURE 4: TRU, OIU-12-104B PACKET. X-RAY MENU

\section{PACKET X-RAY MENU}

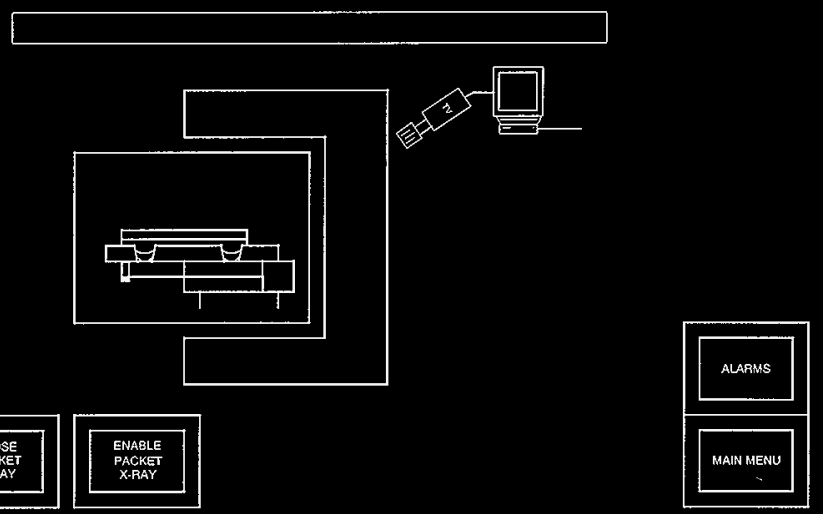




\section{WRAP 1 PLANT OPERATING PROCEDURE}

(HNF. SO W026 OTP - 015)

FIGURE 5: X-RAY CONTROLS PAXELL(107-nD-07 101

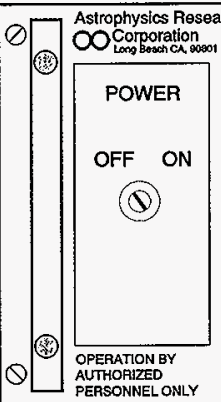

TORREX II X-RAY SCREENING SYSTEM
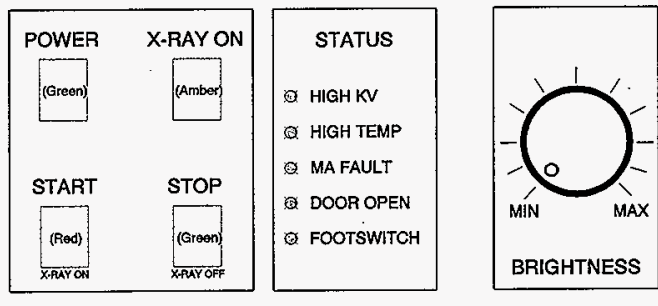

CAUTION: X-RAYS PRODUCED WHEN ENERGIZED

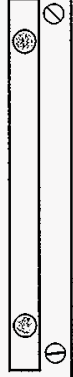

\section{Control panel switch/indicator functions:}

\begin{tabular}{|l|l|}
\hline POWER keylock switch & Power OFF/ON switch. \\
\hline POWER indicator & Green when power ON. \\
\hline X-RAY ON indicator & Amber when X-Ray ON. \\
\hline START button/indicator & Enables X-Ray, Red when X-Ray ON. \\
\hline STOP button/indicator & Stops X-Ray, Green when X-Ray OFF.
\end{tabular}

\section{Control panel STATUS LEDS:}

\begin{tabular}{l|l} 
HIGH KV: & Brightness setting exceeds rating of $X$-Ray generator.
\end{tabular} Turns $X$-Rays OFF. Decrease brightness setting.

HIGH TEMP: $\quad$ High temperature condition.

MA FAULT: $\quad$ Brightness too low to sustain required beam current. Turns X-Rays OFF. Increase brightness setting.

DOOR OPEN:

Door OPEN. Turns $X$-Rays OFF.

FOOTSWITCH:

Footswitch activated.

\begin{tabular}{|c|c|c|c|}
\hline Bocunent vo & pront $00 \mathrm{ate}$ & Rev/Mod & page 9.00 \\
\hline
\end{tabular}




\title{
WRAP 1 PLANT OPERATING PROCEDURE
}

\author{
SYSTENT: PROCESS
}

\section{GLOVEBOX WASTE SAMPLING \\ (HNF-SD-W026-OTP-015)}

\section{SYSTEM DESCRIPTION}

This procedure defines the approach used to sample WRAP 1 process area glovebox waste. Additional requirements will be provided if it becomes necessary to take RCRA protocol samples.

WRAP Operation's responsibility is to sample for laboratory analysis in accordance with this procedure. Sample data shall be recorded using either the Data Management System. DMS Screen DMS\$0501, which is the primary method of recording data, or on Attachment 2, Lab Sample Acquisition which is available as a backup method.

The Sample/Treatment Director shall provide recommendations on the specific method to be used, primarily via DMS Screen DMSS0504. Attachment 1, Laboratory Sample Analysis Request, shall be prepared for each sample as appropriate. The Sample/Treatment Director shall also be responsible for ensuring that the data collected by Operations personnel either in DMS or on Attachment 2, Lab Sample Acquisition. is transferred to the Facility Sample Log as required.

Additionally, this procedure may also be used to collect samples that may undergo field screening within the glovebox prior to sampling for laboratory analysis.

This procedure will also provide guidance for transferring samples and other various items in and out of the gloveboxes through the use of the Sample Transfer container (STC). This will include mounting and unmounting the container, item transfer, and al1 associated DMS interface requirements.

\section{REFERENCE DOCUMENTS}

WHC-CM-5-34, Solid Waste Division Operations Administration

WHC-CM-5-36, SWD Interna] Requirements, Chapter 7-5, Environmental Compliance

\begin{tabular}{|c|c|c|c|c|c|}
\hline Rellease pate & prnt bate & Apod besig & Gogument No 10 & rewmod & page \\
\hline
\end{tabular}




\section{WRAP 1 PLANT OPERATING PROCEDURE}

(HNF SB W026 OTP 015)

\section{PRESTART CONDITIONS}

Al1 personnel performing this procedure shal1 be qualified in accordance with Waste Management Hanford Procedures Manual, Section 5.1, "Training and Qualification," and on-the-job training.

\section{SAFETY}

Appropriate protective clothing will be worn depending on the sampling activity involved. The clothing required will be identified on the RWP and/or during the pre-job evaluation by the operations personnel, Operations Management, or Industrial Safety and Hygiene, as appropriate.

Anticipated radiological conditions shall be evaluated prior to starting work through the RWP and ALARA Management Worksheet (AMW), if applicable.

\section{TOOLS AND SUPPLIES (As Needed)}

Sampling equipment (i.e., glass tube or pipet sampler, scoop, hand auger. etc.)

Sample container(s)

Evidence tape

Tape

cooler

Plastic sheets

Plastic bags

Camera

Other tools, supplies, or protective clothing as defined by the pre-job evaluation.

Chain of Custody Form (Hard Copy) 


\section{WRAP 1 PLANT OPERATING PROCCEDURE}

(HNF. SD. W026-0TP-015)

\section{TABLE OF CONTENTS}

PAGE

A. FIELD SCREENING IN RWM GLOVEBOXES ............... 4

B. PREPARING TO SAMPLE .................. 6

c. LOAD EMPTY SAMPLE BOTTLES INTO GLOVEBOX . . . . . . . . . . . . . . 8

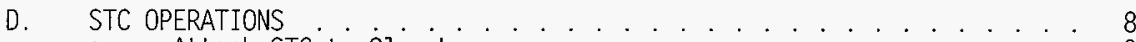

a. Attach STC to GTovebox . . . . . . . . . . . . . . . . 8

b. Open STC . . . . . . . . . . . . . 9

c. Unload STC . . . . . . . . . . . . . . . 10

d. Load Sample in STC . . . . . . . . . . . . . . . . . 10

E. SAMPLING LIQUIDS . . . . . . . . . . . . . . . . . . . . . 11

E. SAMPLING LIQUIDS (Cont.) . . . . . . . . . . . . . 12

F. SAMPLING SEMI-SOLID/SOLID WASTE MATRICES . . . . . . . . . . . 12

a. Scoop Sampler . . . . . . . . . . . . . . . . . 13

b. Hand Auger Sampler . . . . . . . . . . . 14

c. Snip and Wipe Method . . . . . . . . . . . . . . . . . . . . 15

G. FINAL SAMPLE PREPARATION AND LOAD OUT . . . . . ....... 15

H. SAMPLE ACTIVITY LOGBOOKS AND DMS ............... 20

I. SAMPLING ACTIVITIES PHOTO ALBUM . . . . . . . . . . . . 20

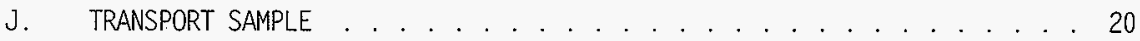

ATTACHMENT 1 - LABORATORY SAMPLE ANALYSIS REQUEST . . . . . . . . . . 21

ATTACHMENT 2 - LAB SAMPLE ACQUISITION . . . . . . . . . . . . . 22

ATTACHMENT 3 - SAMPLE AND TREATMENT LOOKUP TABLES . . . . . . . . . . . . 23 


\section{WRAP 1 PLANT OPERATING PROCEDURE

\section{PROCEDURE}

NOTE 1 - Administrative hold steps are identified by initials in parenthesis at the left margin of the procedure step. The direction given in the procedure step must be satisfied before work continues.

(M) - Operations management sha77 approve continued operation.

(R) - Radiological Control sha77 complete surveys or agree to permit continued operation.

NOTE 2 - The Sample/Treatment Director has the authority to decide whether any given quantity of waste should be Field Screened or Sampled.

\section{A. FIELD SCREENING IN RWM GLOVEBOXES}

NOTE 1 - A supply of bottles, dishes, and other supplies to be used in field screening wi7l be kept in, or loaded into the RWM Glovebox as needed.

NOTE 2 - DMS Screen DMSS0508 (Sample Labe7s) is accessed from Screen DMSS0504 (Laboratory Sample Analysis Request) which can be accessed from DMSS0503 (Field Screening).

NOTE 3 - DMS records the sample/packet relationship.

NOTE 4 - This Section is used when material from a packet or collection container located at an RWM Glovebox sorting location requires field screening.

1. VERIFY that all Prestart Conditions have been satisfied.

2. DISPLAY screen DMSS0503, Field Screening, by selecting the "Field Screening" button on the RWM Waste Repackaging DMS Screen. (DMSS0322 for LLW RWM and DMSS0342 for TRU RWM).

NOTE - $\quad$ Screen DMSS0503 displays a 7ist of all packets and collection containers located at the Rwil Sorting Location.

3. SELECT PIN of item to be sampled from the list.

4. SELECT "Next Sample":

NOTE - DMS, at this point assigns a Screening ID.

5. CONDUCT field screening in accordance with the information provided on DMS Screen DMSS0503 and any additional guidance provided by the Sample Treatment Director. 


\section{WRAP 1 PLANT OPERATTING PROCEDURE \\ (HNF SD - W026-0TP-015)}

HNF-SD-W026-OTR-015, Rev. 0, Page OP-98

\section{A. FIELD SCREENING IN RWM GLOVEBOXES (Cont.)}

6. ENTER results of field screening on screen DMSS0503, including the following parameters as a minimum:

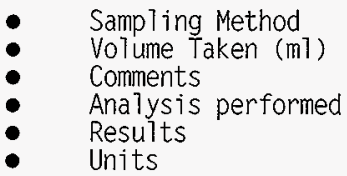

7. ENTER a signature password and commit the data.

8. IF Sample/Treatment Director determines a sample is required based on results of the field screening,

THEN Sampling/Treatment Director sha17 perform the following:

a. PREPARE a sample analysis request using screen DMSS0504, Laboratory Sample Analysis Request, or Attachment 1, Laboratory Sample Analysis Request.

b. PRINT (in Sample Management Office) the sample bottle labels using screen DMSS0508. Sample Bottle Labels.

9. IF another screening sample is required,

THEN SELECT NEXT SAMPLE button on DMSS0503 screen to re-display list of packets and collection containers at the RWM sampling location.

10. If no other screening sample is required,

THEN return to screen DMSS0322 or DMSS0342.

11. WHEN field screening analyses are complete,

PACKAGE field screened material with the original material if possible.

NOTE: If it is necessary to package a sample separately, a new packet wi 77 be created and labeled.

12. If sample cannot be combined with original material,

THEN perform the following:

a. SCAN LOCATION barcode.

b. SCAN original packet barcode.

c. LABEL new packet.

d. SCAN new packet(s) barcode.

e. SCAN END barcode.

f. SCAN YES barcode.

g. ENTER descriptions of new packets on DMSS0322 or DMSS0342 Screen. 


\section{WRAP 1 PLANT OPERATING PROCEDURE}

(UNF - SD 1026 - OTP 015 )

HNF-SD-W026-OTR-015, Rev. 0, Page OP-99

\section{B. PREPARING TO SAMPLE}

\section{WARNING}

APPROPRIATE EYE, HAND, AND PROTECTION CLOTHING AS SPECIFIED BY THE RWP, JHA OR PRE-JOB EVALUATION SHALL BE WORN DURING SAMPLING.

NOTE 1 - The appropriate methods for sample collection, required volumes, and sample containers are determined prior to sampling and recorded in DMS or on Attachment 1, Laboratory Sample Analysis Request. Sampling is performed on the following waste matrices:

- S7udges

- Soils, sand, silt, other particulates

- Free tiquids

- Sorbents (stained or saturated only).

Other matrices can be sampled if they fall under matrices identified in SW-846, specific sampling and analysis plan. waste analysis plan. or work package.

If discrepant articles or conditions are encountered that cannot be identified and resolved with resources available, contact the Operations Management for assistance.

NOTE 2 - Sampling documentation (including photographs) is collected throughout the sampling activity, as appropriate.

1. ENSURE Sample/Treatment Director has performed one of the following:

- PREPARE Attachment 1, Laboratory Sample Analysis Request.

- UPDATE DMS Screen DMSS0504 Laboratory Sample Analysis Request.

2. OBTAIN appropriate sampling equipment, sample container(s), camera, etc. 


\section{WRAP 1 PLANT OPERATING PROCEDURE}

(UNF. SB . 1026 - OTP - 015)

HNF-SD-W026-0TR-015, Rev. 0, Page OP-100

B. PREPARING TO SAMPLE (Cont.)

\section{CAUTION}

SAMPLE CONTAINERS MAY NOT BE OPENED PRIIOR TO THEIR INTENDED USE. DOING SO MAY NEGATE THE PEDIGREE OF THE CONTAINER. DO NOT USE PREVIOUSLY OPENED CONTAINERS FOR RCRA PROTOCOL SAMPLING ACTIVITIES.

3. ENSURE empty sample containers have been maintained in a compliant manner as follows:

- Kept under lock and key (e.g., locked storage cabinet, locked storeroom, etc.), with limited access. A custodian and alternate(s) sha11 be designated by Operations.

- The outside of each case must be legibly initialed and dated by the individual opening that case. Pedigree papers for each case should be checked against the contents and any anomalies noted at that time in the logbook. Pedigree papers are kept on file.

4. ENSURE the following information is recorded in the Empty Sample Container Logbook for each sample container:

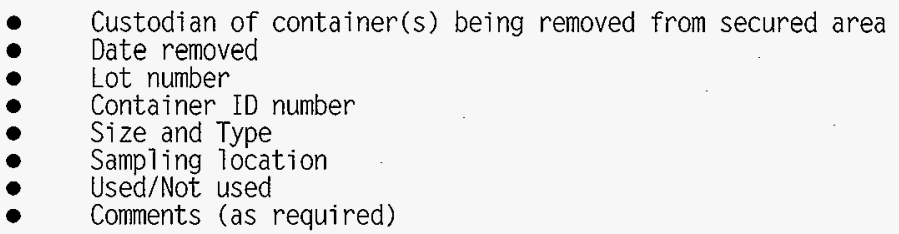

5. PRINT sample bottle barcode labels using screen DMSS0508.

6. LABEL empty sample bottle(s) with barcode label(s). 


\section{WRAP 1 PLANT OPERATING PROCEDURE}

HNF-SD-W026-0TR-015, Rev. 0, Page 0P-101

C. LOAD EMPTY SAMPLE BOTTLES INTO GLOVEBOX

1. ENSURE sample bottle is properly labeled.

2. OPEN door on Consumable Materials Port.

3. INSERT a properly sized bottle into the Consumable Materials Port, pushing the bottle already in the Port all the way through to the interior of the glovebox.

4. INSERT another properly sized bottle into the Consumable Materials Port, pushing desired sample bottle(s) into the interior of the glovebox.

D. STC OPERATIONS

a. Attach STC to GTovebox

(1) REMOVE STC from transfer pig (if applicable).

(2) ENSURE port door is CLOSED and LOCKED:

(a) Door latch CLOSED (down).

(b). Port door latch locking pin dropped into locking hole.

NOTE - During rotation, the STC is locked and sealed to the port flange, and the STC cover is unlocked from the STC and locked and sealed to the cell port.

(3) MATE STC flange to cell flange, with locking bayonets aligned to cell flange openings and ROTATE. STC clockwise, $30^{\circ}$.

(4) ENSURE STC fully engages port as follows:

(a) Visual1y CHECK mounting flanges are fully engaged.

(b) TIGHTEN STC with STC Tool as necessary.

(5) PERFORM a contamination survey of Port area. 


\section{WRAP 1 PLANT OPERATING PROCEDUURE}

$(1 N F$ - SB - W026-0TP-015)

HNF-SD-W026-0TR-015, Rev. 0, Page OP-102

(6) SCAN STC to glovebox location with the apeprate portable scanner-as follows (peference Table-1):

(a) LOCATION barcode on glovebox wall.

(b) STC barcode.

Cequ

(c) END barcode on glovebox wall.

(d) YES barcode on glovebox wa 11.

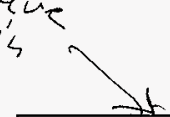
Table 1

\begin{tabular}{|c|c|c|}
\hline GLOVEBOX & PORYABLG SCANALR & IOEATION \\
\hline TRU SORTING & $12-\mathrm{NE}-300$ & TRU /RP \\
\hline TRU RWM & 12-AEF303 & TRURW RP \\
\hline LLW SORTNG & 12-) & LLW_PP \\
\hline LLW RWM & 12-NE-313 & LLWRW_PP \\
\hline
\end{tabular}

(7) VERIFY Operator Interface Unit (OIU) display on oca 1033 Sample Menu, "Purge Port Canister Present".

b. Open STC

This section assumes STC is properly mated to the Glovebox per Section VII.D...a.

(1) PULL port door latch locking pin and TURN counter-clockwise, $30^{\circ}$.

(2) MOVE port door latch to OPEN (UP).

(3) OPEN port door. 


\section{WRAP 1 PLANT OPERATING PROCEDURE}

(HNF SD N026 OIP O15)

HNF-SD-W026-0TR-015, Rev. 0, Page 0P-103

D. STC OPERATIONS (Cont.)

c. Unload STC

(1) INSPECT glovebox sampling area for objects or conditions that could puncture, rip, cut, or could otherwise injure or harm sampling personne?.

(2) REQUEST Radiological Control (RC) coverage to the extent indicated on Attachment 1, Laboratory Sample Analysis Request.

(3) UNLOAD sample container, or applicable item, into the glovebox, using gloves.

(a) IF sample containers needing treatment are being unloaded.

THEN REFER to WRP1-OP-0717, LLW/RWM GLOVEBOX OPERATION, Section titled, "REMOVE SAMPLE BOTTLES FROM STC FOR TREATMENT".

d. Load Sample in STC

(1) SELECT "Loadout Samples" on OIU.

(2) SCAN barcodes as follows (reference Table 2):

(a) LOCATION barcode on glovebox wall.

(b) Sample Bottle PIN barcode.

(c) END barcode on glovebox walT.

(d) YES barcode on glovebox wa11.

Table 2

\begin{tabular}{||c|c|c||}
\hline \multirow{2}{*}{ GLOVEBOX } & PORTABLE_SCANMER & IOCATION \\
\hline \hline TRU SORTING & $12-\mathrm{NE}-300$ & TRU_PP \\
\hline TRU RWM & $12-\mathrm{NE}-303$ & TRURW_PP \\
\hline LLW SORTING & $12-\mathrm{NE}-310$ & LLW_PP \\
\hline LLW RWM & $12-\mathrm{NE}-313$ & LLWR_PP \\
\hline
\end{tabular}


E. SAMPLING LIQUIDS

NOTE 1 - Liquids may be sampled using several methods - pipet, glass tube, etc. The determination of which method and sample containers to use is made prior to sampling and is defined on Attachment 2, Laboratory Sample Analysis Request OR in the DMS.

NOTE 2 - The sample container should be placed over the waste container to minimize potential contamination spread as much as practical.

1. SCAN barcodes as follows (reference Table 3):

a. LOCATION barcode on glovebox wä17.

b. Sample Bottle PIN barcode.

c. IF sampling in an RWM Glovebox, THEN SCAN packet or collection container being sampled.

d. END barcode on glovebox wal1.

e. YES barcode on glovebox wall.

Table 3

\begin{tabular}{|c|c|l||}
\hline GLOVEBOX & PORTABLE SCANMER & IOCATION \\
\hline TRU SORTING & $12-\mathrm{NE} \cdot 300$ & TRU_SORT \\
\hline TRU RWM & $12-\mathrm{NE}-303$ & TRURWSAMPL \\
\hline LLW SORTING & $12-\mathrm{NE}-310$ & LLW_SORT \\
\hline LLW RWM & $12-\mathrm{NE}-313$ & LLWRWSAMPL \\
\hline
\end{tabular}

2. OBTAIN sampling information from DMS Screen DMSS0501 or from Attachment 2. Lab Sample Acquisition.

3. ENTER Room Temperature, Sample Matrix, and Comments as appropriate per Attachment 2, Lab Sample Acquisition.

4. ENTER Signature Password.

5. SELECT Commit.

6. LOWER designated sampler into the selected waste container and obtain the sample. 


\section{WRAP 1 PLANT OPERATING PROCEDURE}

E. SAMPLING LIQUIDS (Cont.)

7. CAREFULLY WITHDRAW waste sample material and ADD required volume to sample container(s), as appropriate:

a. IF there are phases,

THEN FILL a sample container(s) for each phase.

b. NOTE the order of each phase and NUMBER the vials appropriately, (e.g., 1 of 3,2 of 3,3 of 3 ).

8. SECURE cap on each container(s)

9. SEAL around cap/container with tape.

10. RETURN unused waste sample material to original container.

11. CLOSE and SECURE lid on the waste container that was sampled.

12. GO TO Section VII.G.

F. SAMPLING SEMI-SOLID/SOLID WASTE MATRICES

1. SCAN barcodes as follows (reference Table 4):

a. LOCATION barcode on glovebox wa 17.

b. Sample Bottle PIN barcode.

c. IF sampling in an RWM Glovebox,

THEN SCAN packet or collection container being sampled.

d. END barcode on glovebox wall.

e. YES barcode on glovebox wa17.

Table 4

\begin{tabular}{|c|c|c|}
\hline GLOVEBOX & PORTABLE SCANNER I B & LOGATION \\
\hline TRU SORTING & $12-\mathrm{NE}-300$ & TRU SORT \\
\hline TRU RWM & $12-\mathrm{NE}-303$ & TRURWSAMPL \\
\hline LLW SORTING & $12-\mathrm{NE}-310$ & LLW SORT \\
\hline LLW RWM & $12-\mathrm{NE}-313$ & LLWRWSAMPL \\
\hline
\end{tabular}




\section{WRAP 1 PLANT OPERATING PROCEDURE}

(HNF SB N026 OTP 015)

HNF-SD-W026-0TR-015, Rev. 0, Page OP-106

\section{F. SAMPLING SEMI-SOLID/SOLID WASTE MATRICES (Cont.)}

2. OBTAIN sampling information from DMS Screen DMSS0501 or from Attachment 2, Lab Sample Acquisition.

3. ENTER Room Temperature, Sample Matrix, and Comments as appropriate per DMS screen or Attachment 2, Lab Sample Acquisition.

4. ENTER Signature Password.

5. SELECT Commit.

6. DIVIDE waste surface into a sampling grid, as appropriate.

NOTE - Each distinguishable matrix is sampled using one of the following methods:

a. Scoop Sampler

NOTE - This sampling technique should only be used when the material is homogenous throughout the container and a representative sample can be taken from a portion of the container.

(1) MIX (thoroughly) the material to be sampled using a scoop or shovel,

THEN SCOOP enough material for a representative sample.

(2) FILL the sample container(s).

(3) SECURE cap on each container(s).

(4) SEAL around cap/container with tape.

(5) RETURN unused sample material to original waste container, as appropriate.

(6) CLOSE and SECURE the lid on sampled waste container, as applicable.

(7) GO TO Section VII.G. 


\section{WRAP 1 PLANT OPERAT ING PROCEDURE}

(HNF SD-W026:0TP-015)

HNF-SD-W026-0TR-015, Rev. 0, Page OP-107

F. SAMPLING SEMI-SOLID/SOLID WASTE MATRICES (Cont.)

b. Hand Auger Sampler

NOTE - $\quad$ There are a wide variety of hand augers available in varying sizes for different materials. If the material wi 77 not stay in the auger, or if the sample hole wi 77 not stay open for reentry, an a7ternate sampling method should be chosen.

(1) PLACE auger end at selected sampling location.

(2) AUGER into the sample material.

(3) WHEN the auger is full of material. THEN REMOVE the auger by PULLING and TURNING counterclockwise. Power equipment may be used as appropriate.

(4) PLACE a bow1 underneath the auger.

(5) PUSH material from either the top or bottom of the auger into bowl using spoon or other suitable tool.

(a) TAP the auger with a hammer or other suitable tool, as needed to encourage material flow.

(6) REPEAT Steps VII.F.6.b. (1) through VII.F.6.b. (5) above, the appropriate number of times in the same sampling hole to obtain sample material from the entire depth of the matrix.

(7) IF more than one bowl is needed,

THEN SUB-SAMPLE from each bow1 into a single composite bowl and MIX thoroughly.

(8) MIX (thoroughiy) the material to be sampled using a scoop or shovel,

THEN SCOOP enough material for a representative sample.

(9) FILL sample container(s).

(10) SECURE cap on each container(s).

(11) SEAL around cap/container with tape.

(12) RETURN unused sample material to original waste container, if appropriate.

(13) CLOSE and SECURE Tid on sampled waste container, as applicable. 
F: SAMPLING SEMI-SOLID/SOLID WASTE MATRICES (Cont.)

(14) GO TO Section VII.G for instruction on completing sample preparation.

c. Snip and Wipe Method

(1) If the solid item being sampled is hard, THEN WIPE item with an approved filter paper or other media in such a way as to obtain a representative sample of the whole.

(2) IF the solid item being sampled is soft. THEN SNIP sections of the soft item with scisors in such a way as to abtain a representative sample of the whole.

(3) Prepare the sample per instructions obtained from the laboratory that will be performing the analysis and identified on Attachment 1, Laboratory Sample Analysis Request or DMS Screen DMSS0501.

(4) FILL the container as appropriate.

(5) SECURE cap on each container(s).

(6) SEAL around cap/container with tape.

(7) RETURN unused sample material to original waste container, as appropriate.

(8) CLOSE and SECURE the lid on sampled waste container, as applicable.

(9) GO To Section VII.G for information on completion of sample preparation.

\section{G. FINAL SAMPLE PREPARATION AND LOAD OUT}

1. Sample custodian must ENSURE sample containers are sealed with evidence tape and appropriately labeled as follows:

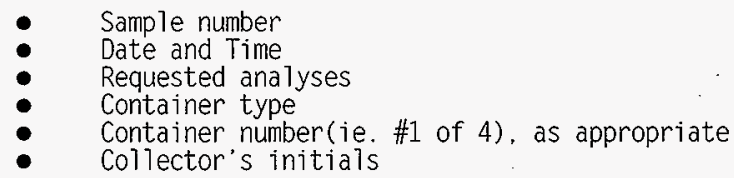

2. LOAD sample containers into STC for transfer.

\begin{tabular}{|c|c|c|c|}
\hline bocument 10.0 & Prot Bate, & Rev/Mod & page 15 of 24 \\
\hline
\end{tabular}




\section{WRAP 1 PLANT OPERATING PROCEDURE}

(HNF. SB W026-0TP 015)

G. FINAL SAMPLE PREPARATION AND LOAD OUT (Cont.)

HNF-SD-W026-0TR-015, Rev. 0, Page OP-109

NOTE - If "LOADOUT SAMPLES" is selected without a STC in place, OIU responds with "NO PURGE PORT PRESENT".

a. SELECT "LOADOUT SAMPLES" on OIU (reference Table 5). Table 5

\begin{tabular}{|c|c|}
\hline GGOUEBOX & OIU I D \\
\hline TRU SORTING & OIU-12-104B \\
\hline TRU RWM & $0 I U-12-106 \mathrm{~B}$ \\
\hline LLW SORTING & OIU-12:103B \\
\hline LLW RWM & OIU-12-105B \\
\hline
\end{tabular}

b. VERIFY display "SAMPLE LOADOUT IN PROGRESS" at OIU-12-105B.

c. SCAN sample container barcode with portable scanner as follows (reference Table 6):

(1) LOCATION barcode on glovebox wall.

(2) Sample Bottle PIN barcode.

(3) END barcode on glovebox wal1.

(4) YES barcode on glovebox wall. Table 6

\begin{tabular}{|c|c|c|}
\hline GLUVEBOX & $\begin{array}{l}\text { PORTABIE SCANMER } \\
\text { I }, \mathrm{B} \text {. }\end{array}$ & MOSATIION. \\
\hline TRU SORTING & $12-N E-300$ & TRU SORT \\
\hline TRU RWM & 12-NE-303 & TRURWSAMPL \\
\hline LLW SORTING & 12-NE-310 & LLW_SORT \\
\hline LLW RWM & $12-\mathrm{NE}-313$ & LLWRWSAMPL \\
\hline
\end{tabular}

d. LOAD sample container into sample transfer canister.

e. REPEAT Steps VII.G.2.c and VII.G.2.d for each sample container to be transferred.

f. ENSURE all sample bottles in the Sample Transfer Canister are displayed on DMSS0501. 


\section{WRAP 1 PLANT OPERATING PROCEDURE}

(HNF SD 1026 - OTP 015 )

HNF-SD-W026-0TR-015, Rev. 0, Page OP-110

G. FINAL SAMPLE PREPARATION AND LOAD OUT (Cont.)

g. SELECT "END SAMPLE LOADOUT" on OIU.

h. VERIFY display "SAMPLE LOADOUT COMPLETE" at OIU-12-105B.

3. CLOSE and DETACH STC from the glovebox.

a. CLOSE port door:

b. MOVE port door Tatch to CLOSED position (down).

c. TURN port door latch locking pin clockwise, $30^{\circ}$ and RELEASE.

d. ENSURE port door latch locking pin drops into locking hole.

NOTE - During rotation, the STC cover is locked and sealed to the STC.

\section{CAUTION}

FAILURE TO SURVEY STC AND CELL FLANGE MAY RESULT IN EQUIPMENT AND/OR PERSONNEL CONTAMINATION.

e. REQUEST Radiological Control Technician (RCT) coverage for STC removal.

f. ROTATE STC counter-clockwise, $60^{\circ}$ AND REMOVE from port flange. 


\section{WRAP 1 PLANT OPERATING PROCEDURE}

(HNF SD- W026- OTP 015)

HNF-SD-W026-OTR-015, Rev. 0, Page OP-111

G. FINAL SAMPLE PREPARATION AND LOAD OUT (Cont.)

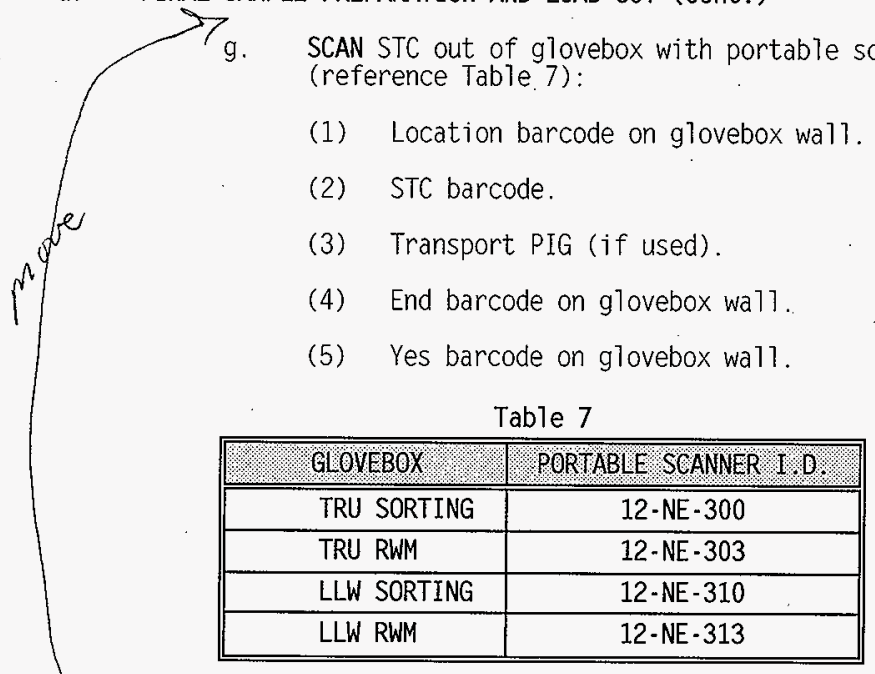

(R) 5. REQUEST RCT survey and appropriately release the sealed STC.I

6. IF surface contamination is found.

THEN DECONTAMINATE the container.

7. IF no surface contamination is detected,

THEN PLACE STC in transport PIG or cooler as required.

8. ENSURE COC/SAR information is entered into screen DMSS0502 and the hard copy is transferred with the STC.

9. IF COC for STC is transferred between operations personne] prior to removal from the Process Area,

THEN ENSURE COC/SAR, DMSS502 "Relinquished By" and

"Relinquished To" information is entered into screen DMSS0502 $\underline{\mathrm{OR}}$ an updated hard copy is printed. 


\section{WRAP 1 PLANT OPERATING PROCEDURE}

(UNF.SB - W026 - OTP. 015)

HNF-SD-W026-0TR-015, Rev. 0, Page OP-112

\section{G. FINAL SAMPLE PREPARATION AND LOAD OUT (Cont.)}

10. TRANSFER STC to Sample Management Area, OR

a. SECURE in a locked cabinet until such time that containers will be transported to Sample Management Area.

b. RECORD time, date, and person placing the sample into storage in the cabinet log sheet.

11. RELINQUISH sample(s) to the sample custodian

12. ENSURE the COC/SAR "Relinquished By" and the "Relinquished To" information is entered into Screen DMSS0502 and on the hard copy form or an updated hard copy is printed.

13. PLACE STC in appropriate shipping container, cushioned as appropriate.

14. MAKE a copy of the Chain of Custody Form and Laboratory Sample Analysis Request. STORE originals in ZipLoc bag and PLACE in shipping container. DELIVER copies to STD for sample file.

15. SEAL shipping container.

16. MARK and LABEL shipping container as directed by STD. 


\section{WRAP 1 PLANT OPERATING PROCEDURE}

(WNF SD W026 OTP 015)

HNF-SD-W026-0TR-015, Rev. 0, Page OP-113

H. SAMPLE ACTIVITY LOGBOOKS AND DMS

NOTE - DMS or Attachment 2, Lab Sample Acquisition, shall be used for data collection. The Sample/Treatment Director shal7 transfer the information to the Sample Logbook, in a timely manner as required for compliant record keeping.

1. Sample/Treatment Director ENSURE and/or RECORD the following information applicable to the samples taken, in the Sample Log book and in the DMS:

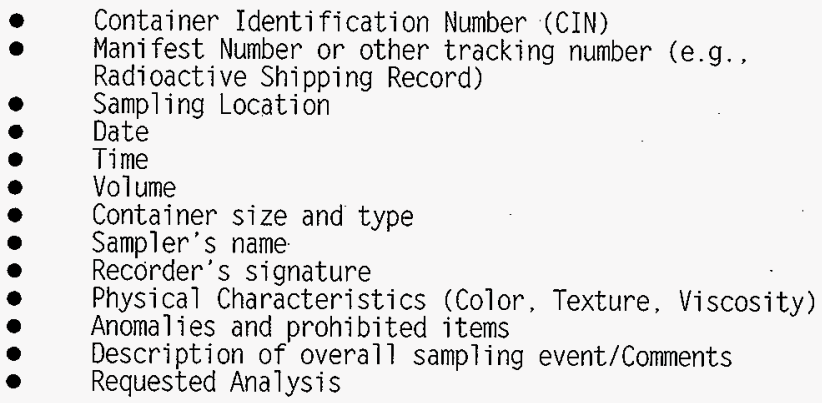

2. NOTE all discrepancies in COMMENTS section of Sample Log Book.

I. SAMPLING ACTIVITIES PHOTO ALBUM

1. RECORD the following information for each photo taken and ENTER in the Sampling Activities Photo Album:

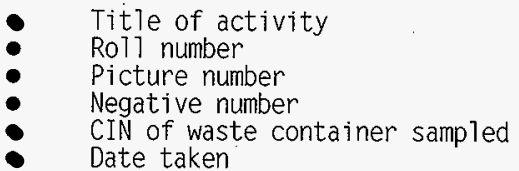

\section{J. TRANSPORT SAMPLE}

1. ENSURE Radioactive Shipment Record (RSR) is completed and signed by an authorized shipper.

2. TRANSPORT sample in vehicle specified on the RSR.

(R) 3. REQUEST RCT SURVEY and RELEASE transport vehicle after delivering sample to laboratory. 


\section{WRAP 1 PLANT OPERATING PROCEDURE (HNF. SD. W026- OTP - 015)}

HNF-SD-W026-0TR-015, Rev. 0, Page OP-114

\section{ATTACHMENT 1 - LABORATORY SAMPLE ANALYSIS REQUEST}

\section{NOTE}

The data recorded in this attachment corresponds to the data recorded on DMSS0504 - Laboratory Sample Analysis Request.

Requested by:

Date:

(Corresponds to Signature Password on DMSS0504)

Drum/Packet PIN

Sample Number:

Sample Location:

Analyses Selected: (Using codes from Attachment 3, Sample and Treatment Lookup Tables. each selected Laboratory Analysis wi 11 be associated with the following predetermined parameters: Description. Preservative, Container Type, No. of Containers, and required volume. Enter codes below for each requested analysis.)

Description of each Analysis:

Total number, type, and volume of sample containers required:

Preservative required:

Special Instructions/Graphics:

Additional sheet(s) attached: __ YES _ NO

\begin{tabular}{|c|c|c|c|}
\hline 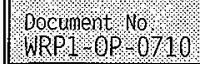 & Prnt onto & Rev/Mod & page \\
\hline
\end{tabular}




\section{ATTACHMENT 2 - LAB SAMPLE ACQUISITION}

\section{NOTE}

The data recorded in this attachment corresponds to the data recorded on DMSS0501 - Lab Sample Acquisition.

Requested by:

Date:

(Corresponds to Signature Password on DMSS0501)

\begin{tabular}{|l|l|l|l}
\hline Drum/Packet PIN: & \multicolumn{2}{l|}{ Location: } & STC PIN: \\
\hline Room Temperature: & $\left({ }^{\circ} \mathrm{C}\right)$ & Sample Matrix: . (from Attachment 3) \\
& & & \\
\hline
\end{tabular}

Bottles in STC by Bottle PIN and Analysis Description Code for each from Attachment 3 , Sample and Treatment Lookup Tab7es:

Bottle PIN Analysis Code

Bottle PIN Anatysis Code

$\frac{1}{\frac{1}{1}}$

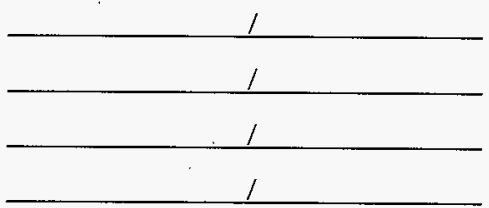

Comments:

Additional sheet(s) attached: __ YES _ NO 


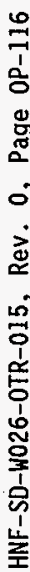

WRAP 1 PLANT OPERATING PROCEDURE

(HAF SO W026 0.P 015)

ATTACHMENT 3 - SAMPLE AND TREATMENT LOOKUP TABLES

(Page 1 of 2 )

\begin{tabular}{|c|c|c|c|c|c|}
\hline \multicolumn{6}{|c|}{ Laboratory Analysis Table (LABANAL) } \\
\hline $\begin{array}{l}* \text { LABANAL CD } \\
\operatorname{VCHAR2(8)}\end{array}$ & $\begin{array}{l}\text { LABANAL_CNTR_COUNT } \\
\text { NUMBER(1) }\end{array}$ & $\begin{array}{l}-\angle A B A N A L \text { CNTYP_CD } \\
\operatorname{VCHAR2(3)}\end{array}$ & $\begin{array}{l}\text { LABANAL_DESCR } \\
\text { VCHAR2(20) }\end{array}$ & $\begin{array}{l}\text { LABANAL_PRESERV } \\
\operatorname{VCHAR2(12)}\end{array}$ & $\begin{array}{l}\text { LABANAL_REQD_VOL } \\
\text { NUMBER(4). }\end{array}$ \\
\hline AQLIQ & 1 & $P$ & Aqueous liquid & None & $\dot{250}$ \\
\hline OILS & 1 & $\mathrm{P}$ & $0 \mathrm{ils}$ & None & 250 \\
\hline SOLVENTS & 1 & G & Solvents & None & 250 \\
\hline TCLP & 1 & $\mathrm{P}$ & TCLP & None & 250 \\
\hline SOLIDS & 1 & $M C$ & Solids & None & 250 \\
\hline COND & 1 & $\mathrm{P}$ & Conductivity & None & 250 \\
\hline
\end{tabular}

\begin{tabular}{||l|l||}
\hline \multicolumn{2}{||}{ Sample Container Type (SAMPCONTYP) DMSS0463 } \\
\hline \hline $\begin{array}{l}\text { *SAMP_CNTYPCD } \\
\text { VCHAR2(3) }\end{array}$ & $\begin{array}{l}\text { SAMP CNTR DESCR } \\
\text { VCHAR2 (30) }\end{array}$ \\
\hline \hline$P$ & Polyethylene Bottle \\
\hline$G$ & Glass \\
\hline AG & Amber glass \\
\hline AGS & Amber glass septa \\
\hline GS & Glass septa \\
\hline MC & Metal can \\
\hline
\end{tabular}




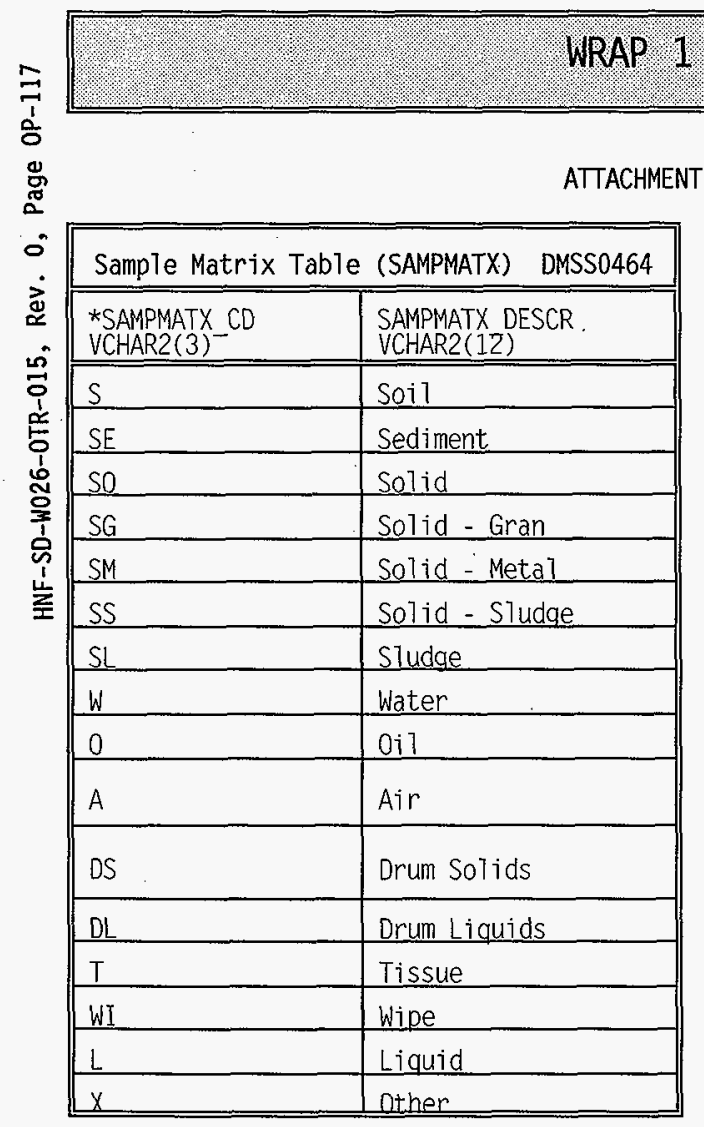

LANT OPERAT ING PROCEDURE

$(\mathrm{HNF}$ SB -1026 OTP 015$)$

(Page 2 of 2)
\begin{tabular}{|l|l|}
\hline \multicolumn{2}{|c|}{ Sampling Method (SAMPMETH) DMSSO465 } \\
\hline \hline \begin{tabular}{|l|} 
*SAMPLING_METHOD_CD \\
VCHAR2(4)-_
\end{tabular} & $\begin{array}{l}\text { SAMPLING METHOD_DESC } \\
\text { R_VCHAR2(30) }\end{array}$ \\
\hline \hline PIPE & Pipet sampler \\
\hline SCOP & Scoop sampler \\
\hline TRIR & Trier sampler \\
\hline AUGR & Hand auger sampler \\
\hline SNIP & Solid snip sample \\
\hline SWIP & Swipe sample \\
\hline CLWS & Colowasa sampler \\
\hline INS & Insitu \\
\hline
\end{tabular}

\begin{tabular}{||l|l|l||}
\hline \multicolumn{3}{|c|}{ Field Analysis Type Table (FLDANALTY) DMSS0459 } \\
\hline \hline $\begin{array}{l}\text { *FAT CD } \\
\text { VCHAR2(4) }\end{array}$ & $\begin{array}{l}\text { FAT DESCR } \\
\text { VCHAR2(70) }\end{array}$ & $\begin{array}{l}\text { FAT UNITS } \\
\text { VCHAR2(6) }\end{array}$ \\
\hline \hline LEAD & Lead Stick test & red/no \\
\hline PHDP & DH dip.Strip & $\mathrm{pH}$ \\
\hline PHMR & pH meter & $\mathrm{pH}$ \\
\hline PCBO & CLOR-N-OIL & $\mathrm{ppm}$ \\
\hline PCBS & CLOR-N-SOIL & $\mathrm{ppm}$ \\
\hline CLOR & CLOR-D-TECT & $\mathrm{ppm}$ \\
\hline
\end{tabular}

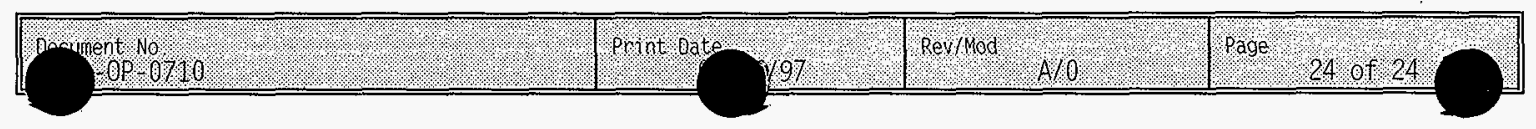




\title{
WRAP 1 PLANT OPERATING PROCEDURE
}

\author{
SYSTEM. PROCESS
}

\section{LLW ENTRY GLOVEBOX OPERATION}

(HNF-SD-W026-OTP-015)

\section{SYSTEM DESCRIPTION}

This procedure provides instructions for operation of the Low Level Waste (LLW) Entry Glovebox.

\section{REFERENCE DOCUMENTS}

WRP1-0P-0703, GTovebox Lift Table Operation.

WRP1-0P-0701, Glovebox Housekeeping.

\section{PRESTART CONDITIONS}

All personnel performing this procedure shall be qualified in accordance with WHC-CM-5-34, Section 1.8, "Training P7an," and on-the-job training.

Process area Heating, Ventilation. Air Conditioning (HVAC) in OPERATION.

\section{SAFETY}

Potential hazards, such as lifting heavy containers or handling sharp objects present in waste containers, must be mitigated by protective equipment, procedures, and administrative controls to ensure acceptable-risk operating conditions.

Consult with Operations Management and Industrial Hygiene, and refer to the Job Hazards Analysis (JHA); Radiological Work Permit (RWP) and Material Safety Data Sheets (MSDS) to determine required Personal Protective Equipment (PPE).

This procedure requires accessing gloveboxes through gloveports on a regular basis. Operators shall perform self survey upon exiting gloveport after each use. Radiological Control Technician (RCT) shall be contacted anytime self survey limits are exceeded.

Spills inside the glovebox sha11 be cleaned up per WRP1-0P-0701, Glovebox Housekeeping.

\begin{tabular}{|c|c|c|c|c|c|}
\hline Rerlease Bater & prog. 0ate & Aopr vesto & Wocumert vo 6 . & Rev/Mod & page \\
\hline
\end{tabular}




\section{WRAP 1 PLANT OPERATING PROCEDURE}

\section{SAFETY (Continued)}

Warning - Use care to prevent injury. Possible safety hazards include:

- Tripping/STipping

- Injuries to the hands at glovebox ports

- Radiological Contamination

- Interference with the Automatic Guided Vehicle (AGV)

\section{TOOLS AND SUPPLIES}

None.

VI. TABLE OF CONTENTS

PAGE

A. INITIALIZE SYSTEM . . . . . . . . . . . . . . . . . . . . 3

B. DRUM ENTRY TO LLW GLOVEBOX . . . . . . . . . . . . . . . . . . . . . . 4

C. CLOSE ENTRY PORT . . . . . . . . . . . . . . . . . . 6

D. MOVE DRUM TO DRUM TRANSFER CAR ............... 8

ATTACHMENTS

ATTACHMENT 1: HOIST CONTROL FUNCTIONS ............... 10 


\section{WRAP 1 PLANT OPERATING PROCEDURE (UNF SD. W026. 0IP - 015)}

\section{PROCEDURE}

NOTE 1 - Administrative hold steps are identified by letters in parentheses at the left margin of the procedure step. The direction given in the procedure step must be satisfied before work continues.

(M) - Operations management sha77 approve operation.

$(R)$ - Radiological Control (RC) shall complete surveys or agree to permit continued operation.

NOTE 2 - Un7ess otherwise specified Data Management System (DMS) actions are performed on DMS Terminal (107-TE-12-103).

NOTE 3 - Unless otherwise specified Operator Interface Unit (OIU) actions are performed on LLW Entry Glovebox OIU (OIU-12-103A).

NOTE 4 - A71 scanning is performed with portable scanner 12-NE-310-or assigned spare.

A. INITIALIZE SYSTEM
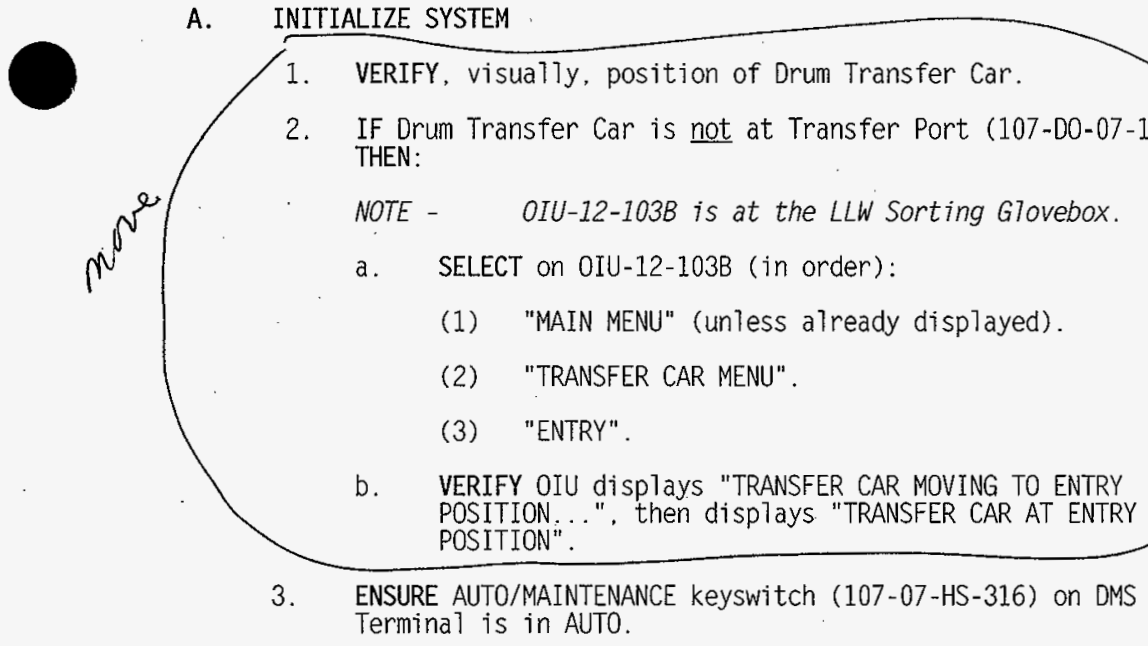

(PCS) When powered up, performs system initialization check and displays $r$ results on OIU Main Menu.

4. SELECT OIU-12-103A "MAIN MENU".

\begin{tabular}{|c|c|c|c|}
\hline Gocunent ro, & mont oate & gev Mod & Page, \\
\hline
\end{tabular}




\section{WRAP 1 PLANT OPERATING PROCEDURE}

5. SELECT "DIAGNOSTICS MENU".

6. VERIFY display, "DIAGNOSTICS MENU".

7. IF OIU displays "PASS",

THEN, GO TO Section VII.B.

8. IF OIU displays "FAIL"

PERFORM the following:

a. NOTIFY Duty Operations Supervisor (DOS).

b. PLACE AUTO/MAINTENANCE keyswitch (107-07-HS-316) on DMS Terminal to "MAINTENANCE".

c. ENTER MAINTENANCE access password on DMS terminal.

d. ACCESS MAINTENANCE MENU screens as necessary to correct indicated initialization faults.

e. WHEN a11 faults have been corrected, SELECT "MAIN MENU".

f. PLACE AUTO/MAINTENANCE keyswitch (107-07-HS-316) on DMS Terminal to "AUTO".

B. DRUM ENTRY TO LLW GLOVEBOX

1. SCAN location (2Y) bar code on side of Entry Glovebox.

2. SCAN drum (S) bar code.

(M) 3. IF scanner displays "PIN MIS-MATCH",

THEN:

a. RECORD PIN displayed on barcode scanner on PIN Change Form.

b. SCAN Cancellation barcode.

c. NOTIFY DOS.

d. CORRECT PIN information in PCS.

4. SCAN End $(Y)$ barcode.

5. SCAN Yes barcode. 


\section{WRAP 1 PLANT OPERATING PROCEDURE}

6. IF drum is overpacked.

THEN, REMOVE clamp band from overpack 85 gal (322 liter) drum as follows:

NOTE - Manual Lift table operation is performed per WRP1-0P-0703, Glovebox Lift Tab7e Operation.

a. LOWER Lift Table as required.

b. REMOVE clamp band.

c. RAISE Lift Table to AGV LOAD HEIGHT.

d. RETURN Lift Table to NORMAL operation.

7. If drum is overpacked

FHEN, SELECT "OVERPACK ENTRY MENU" on OIU.

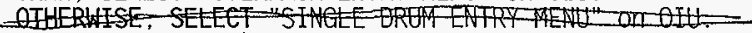

(M) 8. IF OIU displays "NO DRUM LOCATED ON LIFT TABLE",

THEN:

a. NOTIFY Duty Operations Supervisor (DOS).

b. CORRECT drum deficiency as directed.

9. SELECT "START DRUM ENTRY" on OIU.

10. VERIFY OIU displays "DRUM ENTRY IN PROGRESS...".

11. WAIT until OIU displays "DRUM ENTRY COMPLETE",

THEN, SELECT "OPEN PORT" on OIU.

12. VERIFY OIU displays "PORT OPENING...".

13. VERIFY OIU displays "PORT OPEN".

\section{WARNING}

Improper operation of the hoist may cause injury or equipment damage. Failure to properly position and close or open drum lifter could result in dropped drum. Caution must be exercised if reaching through gloveports to manually position drum lifter.

\begin{tabular}{|c|c|c|c|}
\hline bocument vo & $\begin{array}{l}\text { mat sate } \\
\text { mog/66/97. }\end{array}$ & $\mathrm{ReY} / \mathrm{AOd}$ & $\mathrm{Page}, 00$ \\
\hline
\end{tabular}


HNF-SD-W026-0TR-015, Rev. 0, Page 0P-123

\section{WRAP 1 PLANT OPERATING PROCEDURE \\ (HNF SD. W026.0TP. 015)}

NOTE - Unless otherwise specified, hoist control activities are performed on Hoist Control (107-PC-07-001). Controls must be activated unti7 associated indicator i77uminates. Reference Attachment 1: Hoist Control Functions.

14. PERFORM the following with Hoist Control:

a. ENSURE LIFTER jaWS FULLY RAISED.

b. ENSURE LIFTER jaWS FULL OPEN.

c. ENSURE HOIST properly positioned above 55 gal (208 Titer) drum.

d. LOWER HOIST over 55 gal drum.

e. CLOSE LIFTER on 55 gal drum.

f. RAISE HOIST (and 55 gal drum) unt $\uparrow 1$ "RAISED LIMIT" LED ILLUMINATES.

15. SELECT on OIU (in order):

a. "MAIN MENU" (unless a Tready displayed).

b. "TROLLEY MENU".

c. "SWAB POSITION".

16. VERIFY OIU displays "TROLLEY MOVING TO SWAB POSITION..." then displays "TROLLEY AT SWAB POSITION".

c. CLOSE ENTRY PORT

1. SELECT on OIU (in order):

a. "MAIN MENU" (unTess aTready displayed).

b. "OVERPACK ENTRY MENU".

2. COMPARE PIN displayed on OIU to PIN on drum inside glovebox. 


\section{WRAP 1 PLANT OPERATING PROCEDURE \\ (HNF. SD. W026. OTP. 015)}

3. SELECT the following at DMS terminat TE-12-103 from the DMS menu:
a. "Process Ops"
b. "LLW GTovebox"
c. "Drum Status at Entry".

4. VERIFY the correct outer drum PIN is displayed and the correct inner drum PIN is displayed, if overpacked.

5. NOTE whether sorting is required.

(M) 6. IF PINs do not match. THEN:
a. RECORD both PINs.
b. NOTIFY DOS.
c. ENTER PIN correction into DMS as directed.

7. SELECT "INNER DRUM ID VERIFIED" on OIU.

(R)

8. REQUEST RCT swab 55. gal drum for contamination.

9. VIEW inside of overpack drum for debris, on video monitor 107-TM-07-331B.

(M) $\quad$ 10. IF drum contains debris,

THEN, NOTIFY DOS.

11. SELECT "CLOSE PORT".

12. VERIFY OIU displays "PORT CLOSING...".

13. VERIFY OIU displays "PORT CLOSED".

14. SELECT "START DRUM EXIT".

15. VERIFY OIU displays, "DRUM EXIT IN PROGRESS".

16. VERIFY OIU displays, "DRUM EXIT COMPLETE". 


\section{WRAP 1 PLANT OPERATING PROCEDURE}

(UNF SB 1026 -0TP O 5 )

17. VERIFY 7ights on Lift Table Control Pedestal (107-PC-09-202A), ILLUMINATED:

a. AGV LOAD HEIGHT.

b. DRUM AT AGV END.

(R) 18. REQUEST RCT survey drum for contamination.

19. IF drum is contaminated,

a. THEN, decontaminate as directed.

b. RESURVEY drum.

20. REINSTALL clamp band on drum as follows:

NOTE - Manual Lift table operation is performed per WRP1-OP-0703. Glovebox Lift Tab7e Operation.

a. LOWER Lift Table as required.

b. REINSTALL clamp band.

c. RAISE Lift Table to AGV LOAD HEIGHT.

d. RETURN Lift Table to NORMAL operation.

21. IF drum is uncontaminated,

THEN, SELECT "CLEAN DRUM READY FOR PICKUP".

22. IF drum is contaminated;

THEN, SELECT "CONTAMIN. DRUM READY FOR PICKUP".

CON TAM M NATED

23. VERIFY OIU displays $\wedge$ "DRUM PICKUP IN PROGRESS...".

D. MOVE DRUM TO DRUM TRANSFER CAR/ $/ \mathrm{j}$.

1. SELECT on OIU (ingrder):

a. "MAIN MEAf" (unless already displayed).

b. "TROYEY/MENU".

c. "YRANSEER PORT".

2. VERIRY OIU/displays "TROLLEY MOVING TO TRANSFER PORT...".

3. WAIT until trolley STOPS. 


\section{WRAP 1 PLANT OPERATING PROCEDURE} (HNF SB W026 -0TP 015)

THEN, VERIPY OIU/displays "TROLIEY AT-TRAHSFER-PORF".

4. SELECT on OIV (in order)

a.

b. "MAIN MENU". "DRUM TRANSFER MENY".

5. VERIFY OIU displays "TRANSFER CAR IN POSTTION".

6. SELECT "OPEN TRANSFER PORT" on OIU.

7. VERIFY OIU displays "TRANSFER PORT OPENING...".

8. WAIT until OIU displays "TRANSFER PORT OPEN".

9. PERFORM the following with Hoist Control:

a. LOWER HOIST until drum is on transfer car AND hoist chain is slack.

b. OPEN LIFTER jawS.

c. RAISE HOIST untiT "RAISED LIMIT" LED ILLUMINATES.

10: SELECT "CLOSE TRANSFER PORT" on OIU.

11. VERIFY OIU displays "TRANSFER PORT CLOSING..." then displays "TRANSFER PORT CLOSED".

12. SELECT "MAIN MENU".

13. ENSURE "LLW ENTRY GLOVEBOX MAIN MENU" displayed on OIU. 


\section{WRAP 1 PLANT OPERATING PROCEDURE}

\section{ATTACHMENT 1: HOIST CONTROL FUNCTIONS}

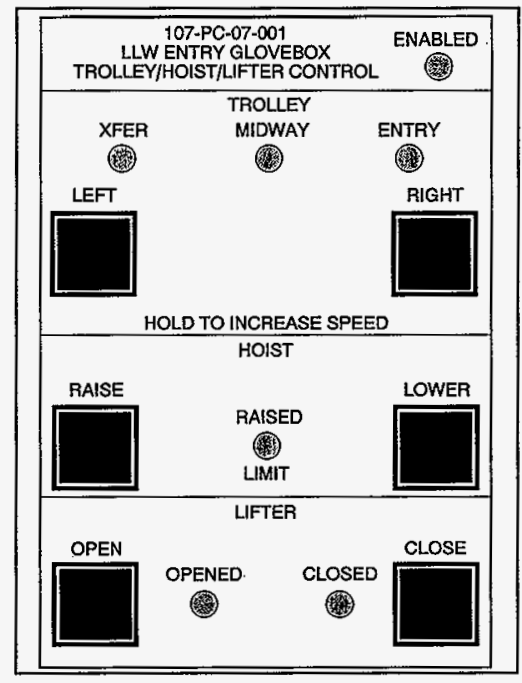

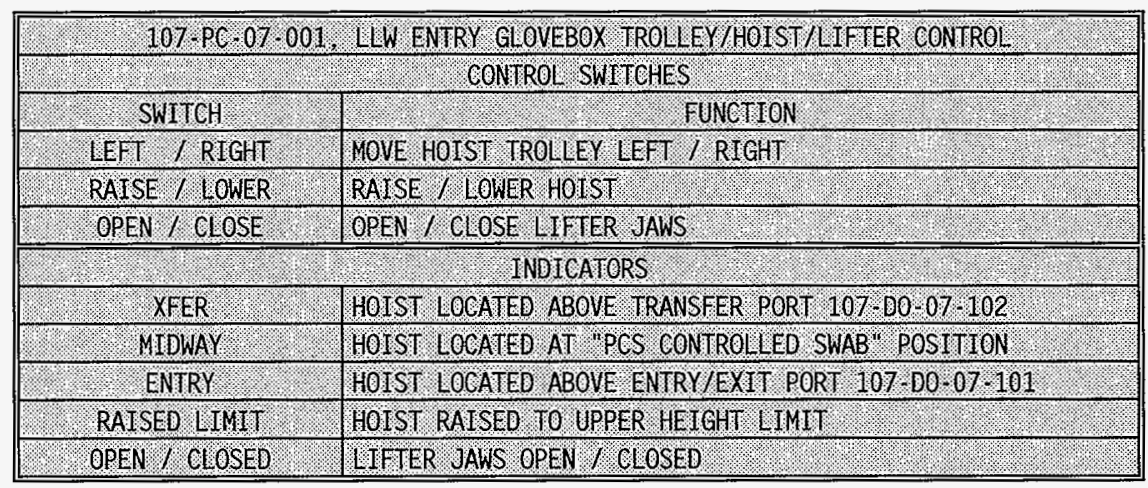

\begin{tabular}{|c|c|c|c|}
\hline bocument ro 1.73 & 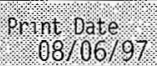 & Revolod & page 10.00 \\
\hline
\end{tabular}




\section{WASTE RECEIVING AND PROCESSING (WRAP) PLANT OPERATING PROCEDURE}

\section{LLW SORTING GLOVEBOX OPERATION \\ (HNF-SD-W026-OTP-015)}

I. SYSTEM DESCRIPTION

This procedure provides instructions for operation of the Low Level Waste (LLW) Sorting Glovebox (107-GB-07-102).

\section{REFERENCE DOCUMENTS}

HSRCM-1, Hanford Site Radiological Control Manual

WRP1-0P-0701, Glovebox Housekeeping

WRP1-0P-0702, Restart LLW Glovebox Operation

WRP1-0P-0704, Glovebox Manipulator Operation

WRP1-OP-0705, Bagless Transfer System Manua] Operation

WRP1-OP-0706, Packet NDE Operation

WRP1-0P-0710, Glovebox Waste Sampling

\section{PRESTART CONDITIONS}

Al1 personnel performing this procedure shall be qualified in accordance with WHC-CM-5-34, Section 1.8, "Training Plan," and on-the-job training.

Process area Heating, Ventilation. Air Conditioning (HVAC) in OPERATION.

Drum to be sorted is on the Transfer Car in the LLW Entry Glovebox, and ready to be transferred to the Sorting Glovebox.

RWM Transfer drum is located on Lift Table 107-LT-09-203A.

No faults or off normal conditions in the LLW Sorting glovebox as identified on the Diagnostics Menu screen of LLW Sorting Glovebox Operator Interface Unit 0IU-12-103B.

\begin{tabular}{|c|c|c|c|c|c|}
\hline Retease bate- & pront 6 ate & goprosig & 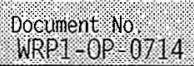 & Revord & page 901.20 \\
\hline
\end{tabular}




\section{WRAP 1 PLANT OPERATING PROCEDURE}

\section{SAFETY}

Potential hazards, such as lifting heavy containers or handling sharp objects present in waste containers, must be mitigated by protective equipment, procedures, and administrative controls to ensure acceptable-risk operating conditions.

Consult with Operations Management and Industrial Hygiene, and refer to the Job Hazards Analysis (JHA), Radiological Work Permit (RWP) and Material Safety Data Sheets (MSDS) to determine required Personal Protective Equipment (PPE).

This procedure requires accessing gloveboxes through gloveports on a regular basis. Operators shall perform self survey upon exiting gloveport after each use. Radiological Control Technician (RCT) shall be contacted anytime self survey limits are exceeded.

Spills inside the glovebox shall be cleaned up per WRPI-OP-0701. Glovebox Housekeeping.

Warning - Use care to prevent injury. Possible safety hazards include:

- Tripping/Slipping

- Injuries to the hands at glovebox ports

- Radiological Contamination

- Interference with the Automatic Guided Vehicle (AGV)

\section{TOOLS AND SUPPLIES}

None. 


\section{WRAP 1 PLANT OPERATING PROCEDURE}

\section{TABLE OF CONTENTS}

PAGE

A. INITIALIZE SYSTEM . . . . . . . . . . . . . . . . . . . . . . . . . . . 5

B. TRANSFER WASTE DRIM TO DELIO POSITION . . . . . . . . . . . . 6

c. GRIP WASTE DRUM WITH DRUM TIPPER $(107-\mathrm{DH}-07-103) \ldots \ldots$

D. SAW WASTE DRUM CLAMP BAND . . . . . . . . . . . . . . 7

E. DELID WASTE DRUM . . . . . . . . . . . . . . . . . . . . . . . . . 8

F. EMPTY WASTE DRUM ONTO SORTING TABLE . . . . . . . . . . . . . . . . . . . 8

G. REMOVE NON-COMPLIANT PACKETS FROM WASTE . . . . . . . . . . . . 10

H. RETURN COMPLIANT WASTE TO WASTE DRUM . . . . . . . . . . . . . . . . 12

I. MOVE WASTE DRUM TO RELID POSITION . . . . . . . . . . . . . . . . . . . 14

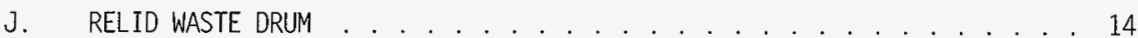

K. TRANSFER WASTE DRUM TO SUPERCOMPACTOR . . . . . . . . . . . 15

L. PLACE TRANSFER STAND IN RWM TRANSFER DRUM . . . . . . . . . . . 16

M. REMOVE DRUM FROM RWM TRANSFER PORT (107-D0-07-105) . . . . . . . . . . 17

N. MATE DRUM TO RWM TRANSFER PORT (107-D0-07-105) . . . . . . . . 18

0. REMOVE TRANSFER STAND FROM RWM TRANSFER DRUM . . . . . . . . . . . . . 19

ATTACHMENTS:

ATTACHMENT 1: SORTING GLOVEBOX HOIST CONTROL FUNCTTONS . . . . . . . . . 20 


\section{WRAP 1 PLANT OPERATING PROCEDURE} (HNF. SO W026 O OTP - 015 )

\section{PROCEDURE}

\section{WARNING}

IN THE EVENT OF A MAJOR PROBLEM, LLW GLOVEBOXES MAY BE SHUT DOWN BY OPERATION OF EMERGENCY STOP BUTTON ON. DMS CONSOLE TE-12-103. EMERGENCY STOP WILL SHUT DOWN ALL OPERATIONS IN THE GLOVEBOX LINE AND PLACE IT IN A SAFE CONDITION.

NOTE 1 - Administrative hold steps are identified by letters in parentheses at the left margin of the procedure step. The direction given in the procedure step must be satisfied before work continues.

(M) - Operations management sha77 approve operation.

$(R)$ - Radiological Conirol (RC) sha77 complete surveys or agree to permit continued operation.

NOTE 2 - UNLESS OTHERWISE SPECIFIED, selections and displays are on LLW Sorting Glovebox Operator Interface Unit (OIU-12-103B).

NOTE 3 - GToveports and manipulators are used as needed to perform tasks throughout this procedure. Manipulator operation is performed per WRP1-OP-0704, G10vebox Manipulator Operation.

NOTE 4 - Data Management System (OMS) actions, when specified, are performed on DMS Terminal (107-TE-12-103).

NOTE 5 - Lift Table 107-LT-09-203A controls and indicators are located on Lift Tab7e Control Pedesta7 107-PC-09-203A.

NOTE 6 - Hoist contro7 activities are performed on Hoist Contro7 (107-PC-07-003A or 003B) at operator discretion. Active contro77er is selected by switch on 107-PC-07-003A. Functions must be activated until associated indicator illuminates. Reference Attachment 1: Hoist Control Functions. 


\section{WRAP 1 PLANT OPERATING PROCEDURE}

\section{A. INITIALIZE SYSTEM}

1. ENSURE DMS AUTO/MAINTENANCE keyswitch (107-07-HS-317) set to AUTO.

2. ENSURE Lift Table MODE switch set to NORMAL.

3. REVIEW "MAIN MENU".

4. IF OIU displays "SYSTEM INITIALIZED", THEN, GO TO desired section(s). OTHERWISE, PERFORM the following:

a. NOTIFY Duty Operations Supervisor (DOS).

b. SELECT "DIAGNOSTICS MENU".

c. OBSERVE fault messages.

d. PLACE DMS AUTO/MAINTENANCE keyswitch to "MAINTENANCE".

e. ENTER Maintenance Access Password on MAINTENANCE ACCESS MENU.

f. ACCESS Maintenance Screens from SORTING GLOVEBOX MAINTENANCE MAIN MENU as necessary to correct indicated initialization faults.

g. WHEN all faults have been corrected:

(1) PLACE DMS AUTO/MAINTENANCE keyswitch to "AUTO".

(2) SELECT "MAIN MENU".

(3) VERIFY display "SYSTEM INITIALIZED".

(4) GO TO desired section(s). 


\section{WRAP 1 PLANT OPERATING PROCEDURE}

(HNF SD WO26 - 01 P 015 )

B. TRANSFER WASTE DRUM TO DELID POSITION

1. VERIFY (visual7y) waste drum on Transfer Car (107-TC-07-101) at Entry port (107-D0-07-102).

2. SELECT in order, as required:
a. "MAIN MENU"
b. "TRANSFER CAR MENU".
c. "DELID/TIPPER".

3. VERIFY display "TRANSFER CAR MOVING TO DELID POSITION...".

4. WAIT for display "TRANSFER CAR AT DELID POSITION".

C. GRIP WASTE DRUM WITH DRUM TIPPER (107-DH-07-103)

NOTE - Overbag must be lowered around-or removed from drum far enough down for unimpeded operation of drum grabber. Bag parts remaining lodged under drum wi 77 be removed later.

1. FF drum is enclosed in overbag, THEN:

a. REMOVE overbag-from-top-of drum.

-b. PLACE-7oose-overbag-materials-on sorting table.

2. ENSURE manipulator (107-EM-07-101) and arms PARKED.

3. SELECT in order, as required:

a. "MAIN MENU".

b. "DRUM TIPPER MENU" .

c. "LOWER AND CLOSE GRAB".

NOTE - OIU displays "LOWER AND CLOSE GRAB IN PROGRESS..." whi Te tipper lowers. "LOWER TIPPER" must be held until tipper reaches fun7 down and stops.

4. SELECT "LOWER TIPPER" until tipper down travel STOPS and display indicates "LOWER AND CLOSE GRAB COMPLETE". 


\section{WRAP 1 PLANT OPERATING PROCEDURE}

(HNF SO W026-0TP-015)

D. SAW WASTE DRUM CLAMP BAND

1. ENSURE manipulator and armS PARKED.

2. SELECT in order, as required:
a. "MAIN MENU".
b. "LAUTUELTO MENU".
c. "MOVE TO PELID POSITION".

3. WAIT unti? Delid/Relid fixture (107-RT-07-101) is at "DELID" position.

4. SELECT in order, as requrred:
a. "MAIN MENY". "SAW MENU".

NOTE -

- In Steps VII.D.4.C and VII.D.5. "CX" \& "CY" indicate desired position (C2 \& C5 or $(3$ \& 66$)$. Positions are selected as needed to saw
hitting clamp-band bolt.

c. "CX \& EY SAW ORIEAFATION".

5. VERIEY display "CX \&-EY SAW ORIENTATION REABY".

6. SELECT "START SAW SEQUENCE".

7. VERIFY display "SAWING IN PROGRESS...".

8. WAIT until saw STOPS and display indicates "OPERATOR: GRAB CLAMP BAND WITH MANIPULATOR...".

\section{CAUTION}

MANIPULATORS MUST BE CLEAR OF SAW OPERATING AREA.

9. GRIP clamp band on each side of cut with manipulators.

10. SELECT "CONFIRM CLAMP BAND HELD".

11. VERIFY display "SAWING IN PROGRESS...".

12. WAIT until saw STOPS and display indicates "OPERATOR: GRAB CLAMP BAND WITH MANIPULATOR...". 


\section{WRAP 1 PLANT OPERATING PROCEDURE}

(HNF SD-WO26-0TP 015 )

13. REMOVE clamp band pieces from drum and PLACE on sorting table.

14. PLACE manipulator and arms PARKED at POSITION 1.

15. VERIFY display "SAWING COMPLETE".

16. SELECT the following at DMS Screen DMSS0311:

a. "Process Ops"

b. "LLW Glovebox"

c. "Orum Status at Entry"

17. VERIFY the correct outer drum PIN is displayed and the correct inner drum PIN is displayed, if overpacked.

18. OBSERVE whether sorting is required.

19. IF drum is to be relidded without sorting,

THEN, gO TO Section VII.I, MOVE TRANSFER CAR TO RELID POSITION.

E. DELID WASTE DRUM

1. SELECT in order, as required:

a. "MAIN MENU".

b. "LID/DELID MENU".

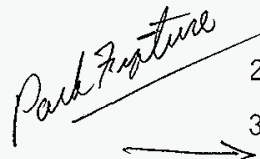

c. "START DELID SEQUENCE".

2. VERIFY display "OELID IN PROGRESS...".

3. WAIT for display "DELID COMPLETE".

F. EMPTY WASTE DRUM ONTO SORTING TABLE

1. ENSURE manipulator and arms are PARKED at POSITION 1.

2. SELECT in order, as required:

a. "MAIN MENU".

b. "DRUM TIPPER MENU".

c. "RAISE WITH GRAB CLOSED". 


\section{WRAP 1 PLANT OPERATING PROCEDURE}

NOTE .- $\quad$ OIU displays "RAISED WITH GRAB CLOSED IN PROGRESS..." while tipper raises. "RAISE TIPPER" must be held unti7 tipper reaches desired position and stops.

3. RAISE drum as follows:

a. IF overbag parts require removal, THEN:

(1) SELECT "RAISE TIPPER" until drum is at desired position.

(2) REMOVE overbag and place on sorting table, as applicable.

b. SELECT "RAISE TIPPER" unti1 tipper up trave1 STOPS and display indicates "RAISE WITH GRAB CLOSED COMPLETE ROTATE JOYSTICK".

NOTE - $\quad$ NDE X-Ray hard copies are used to prelocate non-compliant items. Drum contents should be dumped on sorting table in a controlled manner so packet position on table can be correlated with previous position in drum.

4. ROTATE drum tipper with joystick control until items begin emptying onto sorting table.

a. USE manipulators to aid movement of items, as required.

NOTE - Monitor (107-TM-07-331A) is focused on inside of drum.

5. VERIFY on video monitor (107-TM-07-331A), a11 waste has been emptied onto sorting table.

6. ENSURE manipulator and arms are PARKED.

7. ROTATE drum tipper upright to display "TIPPER AT VERTICAL".

8. SELECT "LOWER WITH GRAB CLOSED".

NOTE - OIU displays "LOWER AND CLOSE GRAB IN PROGRESS..." whi Te tipper lowers. "LOWER TIPPER" must be held until tipper reaches ful7 down and stops.

9. SELECT "LOWER TIPPER" until tipper down trave1 STOPS and display indicates "LOWER WITH. GRAB CLOSED COMPLETE".

\begin{tabular}{|c|c|c|c|}
\hline 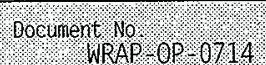 & Print bate $08 \% 06 \%$ & Rev/Mod & Page g or 20 \\
\hline
\end{tabular}




\section{WRAP 1 PLANT OPERATING PROCEDURE}

G. REMOVE NON-COMPLIANT PACKETS FROM WASTE

NOTE - Non-compliant packets are identified and located by review of drum NDE X-ray hard copies and association of packet location on sorting table to previous position in drum. Packet X-RAY is used if positive identification cannot be made visual7y.

1. SELECT the following at DMS terminal:

a. "Process Ops"

b. "LLW Glovebox"

c. "Sorting Table"

2. VERIFY the parent PIN (drum contents on sorting table).

3. REVIEW the Waste Description.

4. OBSERVE if "Sample" and/or "Compliant" are checked.

5. SELECT "NDE Comment" button on LLW Sorting Table screen to view radiographer comments on the NDE Comment screen.

6. SELECT "Return to Sorting Table Screen" when comment review complete.

7. REVIEW drum NDE hard copies as necessary to locate non-compliant packet/item.

8. IF necessary,

THEN, X-RAY packet per WRP1-OP-0706, Packet NDE Operation.

a. IF packet contains suspect article, THEN, GO TO Step VII.G.9.

b. IF NOT,

THEN, RETURN packet to sorting table and REPEAT step VII.G.8 for additional packets, as required.

9. ATTACH bar code to non-compliant packet.

10. SCAN attached bar code.

11. PLACE non-comptiant packet on Transfer stand.

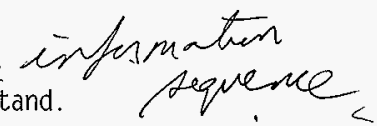




\section{WRAP 1 PLANT OPERATING PROCEDURE}

(HNF SD W026 OLP 015 )

12. SELECT the following at DMS.terminal:
a. "Process Ops"
b. "LLW Glovebox"
c. "Sorting Table"

13. SELECT "Non-Compliant" button on the LLW Sorting Table (DMSS0312) screen.

14. SELECT Material Group Code from the List of Values.

15. INPUT a brief Waste Description.

16. IF barcodes on additional packets are scanned while the LLW Non-Compliant screen is displayed

THEN SELECT "Refresh" button to display the new packet information.

17. REPEAT Steps VII.G.7 through VII.G.16, as required, until a11 non-compliant items have been removed.

18. IF Transfer Stand is full,

THEN. PERFORM Sections VII.L through VII.O

\section{AND}

RETURN to Step VII.G.17 OR Step VII.G.19, as applicable.

$\rightarrow$ 19. IF waste samples are required,

THEN, PERFORM sampling per WRP1-OP-0710, Glovebox Waste Sampling.

20. SELECT the following at DMS terminal:
a. "Process Ops"
b. "LLW Glovebox"
c. "Sorting Table"
d. . "Physcomp" on DMS Screen DMSS0312, LLW Sorting Table

NOTE - $\quad$ The total estimated volume \% of material must equa $7100 \%$ and the total estimated weight cannot exceed the original drum waste weight.

\begin{tabular}{|c|c|c|c|}
\hline Gocument vo. & print 0106 te & Rev/1900 & Page 10.0820 \\
\hline
\end{tabular}




\section{WRAP 1 PLANT OPERATING PROCEDURE}

21. UPDATE the following information as necessary to reflect the contents left on the Sorting table after the non-complaint packet(s) are removed:

- Material Description

- Estimated Volume \% of Material

- Estimated Weight of Material

22. WHEN data has been updated,

THEN perform the following:
a. SELECT "Return To Sorting Table Screen"
b. ENTER signature password.
c. COMMIT data.

23. SELECT "Chemcomp" button on Screen DMSS0312, LLW Sorting Table.

NOTE - Either the weight or weight \% can be entered. DMS wi77 then calculate the other value.

24. UPDATE the following information as necessary to reflect the contents left on the Sorting table after the non-complaint packet(s) are removed:

- Hazardous Chemical Component ID

- Hazardous Chemical Component Description

- $\quad$ Estimated weight of Hazardous Chemical Component

- Estimated weight \% of Hazardous Chemical Component

25. WHEN data has been updated,

THEN SELECT "Return To Sorting Table Screen".

26. ENTER signature password.

27. COMMIT data.

H. RETURN COMPLIANT WASTE TO WASTE DRUM

1. ENSURE all non-compliant items are removed from sorting table.

2. ENSURE DMS files have been updated, as required.

3. ENSURE manipuTator and arms are PARKED at POSITION 2.

\begin{tabular}{|c|c|c|c|}
\hline Document ro & Print oate $08 \% 06 / 97$ & Reverod & page, 12 of 20 \\
\hline
\end{tabular}




\section{WRAP 1 PLANT OPERATING PROCEDURE}

4. SELECT in order, as required:

a. "MAIN MENU".

b. "SORTING TABLE MENU".

c. "EXTEND TABLE".

5. VERIFY display "SORTING TABLE EXTENDING".

6. WAIT for display "EXTEND COMPLEFED".

7. SELECT "ENABLE RAISE/LOWER".

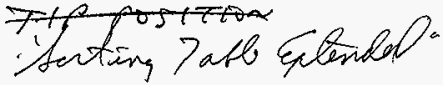

8. VERIFY display "RAISE/LOWER ENABLED".

\section{CAUTION}

TABLE SHOULD BE TIPPED ONLY ENOUGH TO START MATERIAL SLIDING. DUMPING MATERIAL TOO FAST COULD RESULT IN OVERFLOW OR BLOCKAGE.

NOTE - RAISE/LOWER buttons on OIU must be selected (touched) unti7 table reaches desired angle.

9. SELECT "RAISE SORTING TABLE" until tabTe reaches desired angle.

10. GUIDE waste into waste drum with manipulators, as necessary.

NOTE - A video camera is present which is focused on raised Sorting surface to aid in packet unloading.

11. ENSURE a 11 remaining packets have been returned to waste drum.

(M) 12. IF al1 waste cannot be placed in waste drum, THEN, NOTIFY DOS.

13. SELECT "LOWER SORTING TABLE" untiT table is FULL DOWN.

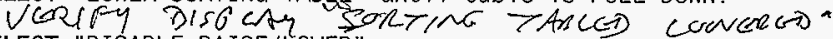

14. SELECT "DISABLE RAISE/LOWER".

15. VERIFY display "RAISE/LOWER DISABLED".

16. SELECT "RETRACT TABLE".

17. VERIFY display "SORTING TABLE RETRACTING...".

18. WAIT for display "SORTING TABLE RETRACTED". 


\section{WRAP 1 PLANT OPERATING PROCEDURE}

19. SELECT in order, as required:

a. "MAIN MENU".

b. "DRUM TIPPER MENU".

c. "OPEN GRAB AND PARK".

20. VERIFY display "OPEN GRAB AND PARK IN PROGRESS...".

21. WAIT for display "OPEN GRAB AND PARK COMPLETE".

I. MOVE WASTE DRUM TO RELID POSITION

1. SELECT in order, as required:
a. "MAIN MENU".
b. "TRANSFER CAR MENU".
c. "RELID".

2. VERIFY display "TRANSFER CAR MOVING TO RELID POSITION...".

3. WAIT for display "TRANSFER CAR AT RELID POSITION".

J. RELID WASTE DRUM

1. SELECT in order, as required:
a. "MAIN MENU".
b. "LID/DELID MENU".
c. "START RELID SEQUENCE".

2. VERIFY display "RELIO IN PROGRESS...".

3. MONITOR display during RELID sequence. 


\section{WRAP 1 PLANT OPERATING PROCEDURE}

$(\mathrm{HNF}$ SO 1026 -0TP 015$)$

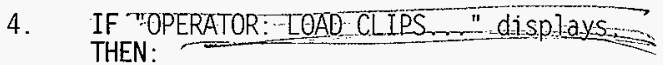

NOTE - Extra C-clips are stored on shelf inside glovebox, under Consumable Entry Port, on maintenance side of glovebox.

a. LOAD new supply of C-Clips in clip magazine.

(1) INSERT new clips through Consumable Entry Port, as required, when shelf supply is used.

-b.--_-SELECT "CONFIRM-E-CLIPS-LOABEO".

5. WAIT for disptay "REtTO COMPLEFE'.

6. VERIFY a11 C-Clips have been attached.

K. TRANSFER WASTE DRUM TO SUPERCOMPACTOR

1. SELECT in order, as required:

a. "MAIN MENU".

b. "TRANSFER CAR MENU".

2. SELECT "COMPACTOR TRANSFER".

3. VERIFY display "TRANSFER CAR MOVING TO COMPACT TRANSFER POSITION...".

4. WAIT for display "TRANSFER CAR AT COMPACT TRANSFER POSITION".

\section{CAUTION}

FAILURE TO PLACE SUPERCOMPACTOR IN MANUAL PRIOR SELECTING "COMPACTOR GLOVEBOX" ON OIU WILL RESULT IN AUTOMATIC COMPACTING OF DRUM.

5. IF the drum is to be moved through the Supercompactor without being compacted,

THEN GO TO WRP1-OP-0715, Section titled, "TRANSFERRING DRUM WITHOUT CRUSHING" .

AND PLACE SUpercompactor in MANUAL.

6. SELECT "COMPACTOR GLOVEBOX".

7. VERIFY display "TRANSFERRING DRUM TO COMPACTOR". 


\section{WRAP 1 PLANT OPERATING PROCEDURE}

8. IF OIU displays "COMPACTOR BUSY",

THEN, PERFORM one of the following:

a. (1) SELECT "COMPACTOR TRANSFER" (cancels transfer).

(2) CONFIGURE compactor to accept drum.

(3) REPEAT from Step VII.K.1.

$\underline{Q R}$

b. CONFIGURE compactor to accept drum. Orum wi11 transfer when compactor becomes available.

9. WAIT for display "DRUM TRANSFER TO COMPACTOR COMPLETE".

10. SELECT "ENTRY"

11. VERIFY display "TRANSFER CAR MOVING TO ENTRY POSITION..." .

12. WAIT for display "TRANSFER CAR AT ENTRY POSITION".

L. PLACE TRANSFER STAND IN RWM TRANSFER DRUM

1. SELECT in order, as required:
a. "MAIN MENU".
b. "RWM PORT MENU".
c. "OPEN PORT".

2. VERIFY display "PORT OPEN". 


\section{WRAP 1 PLANT OPERATING PROCEDURE}

\section{WARNING}

IMPROPER HOIST OPERATION MAY CAUSE INJURY. CAUTION MUST BE EXERCISED IF REACHING THROUGH GLOVEPORTS TO POSITION TRANSFER STAND.

\section{CAUTION}

ALLOWING TRANSFER STAND (on hoist) TO SWING INTO PORT SEAL ING SURFACES WHEN LOWERING INTO DRUM MAY DAMAGE PORT. USE OF ADDITIONAL OPERATOR MAY BE REQUIRED TO SAFELY GUIDE TRANSFER STAND INTO DRUM TO PREVENT DAMAGE.

3. PERFORM the following with Hoist Control:

a. RAISE HOIST (and puck) to "RAISED LIMIT".

b. MOVE TROLLEY to "PORT" position.

c. LOWER HOIST (and Transfer Stand) into drum.

d. RELEASE hook from Transfer Stand.

e. RAISE HOIST (and puck) to "RAISED LIMIT".

M. REMOVE DRUM FROM RWM TRANSFER PORT (107-D0-07-105)

1. ENSURE the following with hoist control:

a. Transfer Stand disengaged from Hoist.

b. HOIST at "RAISED LIMIT".

2. ENSURE port sealing surfaces are free of dirt and debris.

3. SELECT in order, as required:

a. "MAIN MENU".

b. "RWM PORT MENU".

c. "CLOSE PORT".

4. VERIFY display "PORT CLOSING...". 


\section{WRAP 1 PLANT OPERATING PROCEDURE}

5. IF port closing is disrupted,

THEN:

(M)

a. ACKNOWLEDGE OIU fault.

b. NOTIFY DOS.

C. CLOSE Bagless Transfer Port per WRP1-OP-0705, Bagless

Transfer System Manual Operation.

6. WAIT for display "PORT CLOSED".

7. SELECT "START DRUM EXIT".

8. VERIFY display "DRUM EXIT IN PROGRESS...".

9. WAIT for display "DRUM EXIT COMPLETE".

10. VERIFY Lift Table indicators ON:

- "AGV LOAD HEIGHT"

- "DRUM at agV END"

11. REQUEST RCT survey drum for contamination.

a. IF drum is contaminated,

THEN, decontaminate as directed.

b. RESURVEY drum.

12. SELECT "DRUM READY FOR PICKUP".

N. MATE DRUM TO RWM $\Lambda$ TRANSFER PORT (107-D0-07-105)

1. ENSURE with hoist control:

a. HOIST at "RAISED LIMIT"

b. TROLLEY at "PORF" m ID PO MY

2. ENSURE sorting tabTe FULCY RETRACFEO- aAd HORIZONTAL

3. ENSURE manipulator and arms PARKED.

4. SELECT in order, as required:

a. "MAIN MENU".

b. "RWM PORT MENU". 


\section{WRAP 1 PLANT OPERATING PROCEDURE}

5. VERIFY display "DRUM AT LIFT TABLE".

6. PERFORM the following:

a. SCAN Location (fí) bar code

b. SCAN Drum bar code.

(M)

c. IF scanner displays "PIN MIS-MATCH", THEN, NOTIFY DOS.

d. SCAN End $($ ) bar code.

7. SELECT "START DRUM ENTRY".

8. VERIFY display "DRUM ENTRY IN PROGRESS...".

9. WAIT for display "DRUM ENTRY COMPLETE".

- 10. VERIFY Lift-Table "ORUAM UMER PORT" indicaton-ON-.-

11. SELECT "OPEN PORT".

12. VERIFY display "PORT OPENING...".

13. WAIT for display "PORT OPEN".

0. REMOVE TRANSFER STAND FROM RWM TRANSFER DRUM

1. PERFORM the following with Hoist Control:
a. MOVE Trolley to "PORT".
b. LOWER Hoist.
c. ATTACH hoist hook to Transfer Stand.

NOTE - Transfer stand position and elevation may be changed, as needed, to enhance packet loading.

d. RAISE HOIST as needed.

e. MOVE TROLLEY to desired loading position.

f. LOWER HOIST to desired loading position. 


\section{WRAP 1 PLANT OPERATING PROCEDURE} (HNF - SD - W026-0TP-015)

ATTACHMENT 1: SORTING GLOVEBOX HOIST CONTROL FUNCTIONS

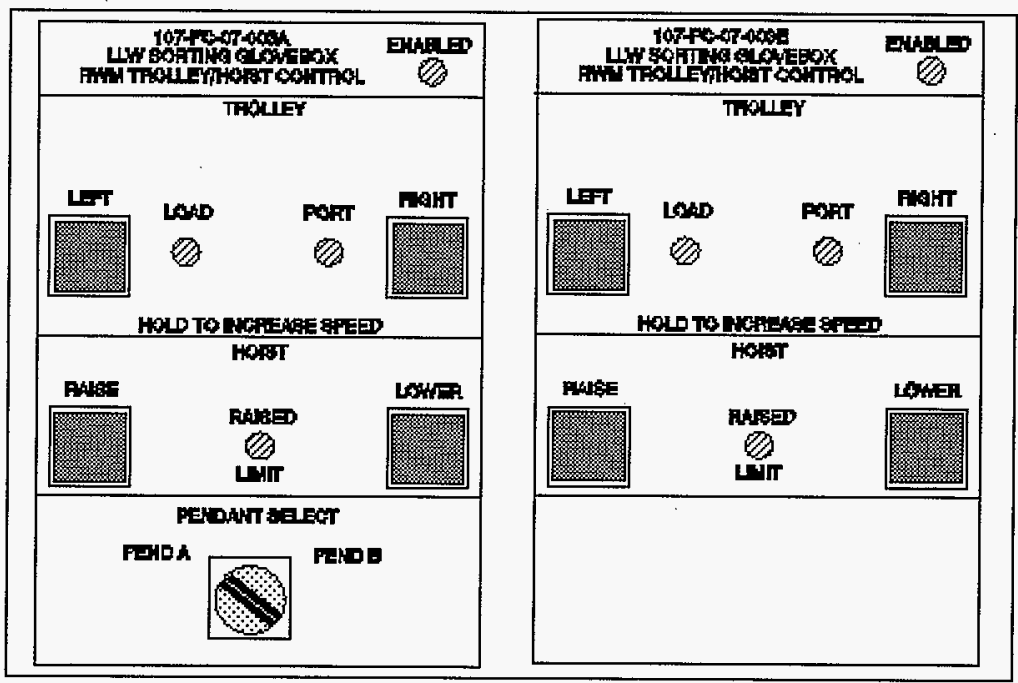

\begin{tabular}{|c|c|}
\hline$=107 \cdot 9 \mathrm{PC}: 07: 003 \mathrm{~A})$ & 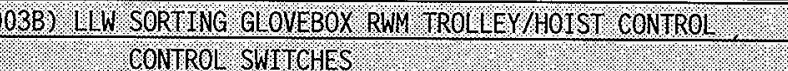 \\
\hline SWITCH & FUNCTION \\
\hline V. LEFT / RIGHT & MOVE LOIST ROLLEY LEFT I RTGH \\
\hline RAISE / L LOWER & RAISE / LOWER HOIST \\
\hline PENDANT SELECT ( $(\%)$ & SELECTS ACTIVE CONTROL (107 PC .07 003A or 003B) \\
\hline NOTE ( $($ ). Active cont & 07 is selected at Operater discretion. \\
\hline $2=2$ & INOTCATORS \\
\hline LOAB & HOIST AT TRANSFER STANB LOAD PESITLON \\
\hline D. PORT & HOLST LOCATED ABOVE RUM TRANSFER PORT ( 107 b0 07 105) \\
\hline RATSED LIMIT & HOTST RATSED TO UPPER HEIGHT LIMT \\
\hline
\end{tabular}

\begin{tabular}{|c|c|c|c|}
\hline 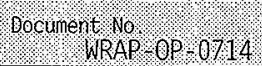 & Print 0 0ater & Rev/god & Prge, 20 or 20 \\
\hline
\end{tabular}




\section{WRAP 1 PLANT OPERATING PROCEDURE}

SYSTEY. PROCESS

\section{LLW SUPERCOMPACTOR OPERATION}

(HNF-SD-W026-0TP-015)

\section{SYSTEM DESCRIPTION}

This procedure provides instructions for operation of the Low Level Waste Drum Supercompactor.

The Supercompactor is used to reduce low level waste volume by compression.

\section{REFERENCE DOCUMENTS}

HSRCM-1, Hanford Site Radiological Control Manual

WHC-CM-5-34, Solid Waste Disposal Operations Administration

WHC-CM-5-36, SWD Internal Requirements

WHC-EP-0063, Hanford Site Solid Waste Acceptance Criteria

INET manua 1, MGT-SWV-429765

\section{PRESTART CONDITIONS}

Al1 personnel performing this procedure shall be qualified in accordance with WHC-CM-5-34, Section 1.8, "Training Plan," and on-the-job training.

Operator has Supercompactor Operator Key, stored in Controlled key lockbox.

\section{SAFETY}

All potential hazards, such as lifting heavy containers or handling sharp objects present in waste containers, must be mitigated by protective equipment, procedures, and administrative controls to ensure acceptable risk operating conditions.

Personnel sha11 not reach into gloveboxes when equipment is in operation.

\section{TOOLS AND SUPPLIES}

A11 tools necessary are stored in the Supercompactor glovebox.

Standard glovebox cleaning supplies.

One liter plastic bottle, for anvil liquids collection.

\begin{tabular}{|c|c|c|c|c|c|}
\hline 8etease Bate & prot adte & 4opr $\mathrm{Des} 19$ : & Wocument No & Revolod & page , of 16 \\
\hline
\end{tabular}




\section{WRAP 1 PLANT OPERATING PROCEDURE}

\section{TABLE OF CONTENTS}

PAGE

VII. PROCEDURE . . . . . . . . . . . . . . . . . . . . . 3

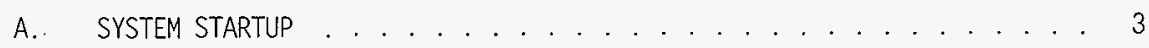

B. TRANSFERRING DRUM WITHOUT CRUSHING ............. . . 5

c. AUTOMATIC OPERATION ........................ . . . . 6

D. SEMI-AUTOMATIC OPERATION ................... . . . 6

E. MANUAL OPERATION ...................... 7

F. MANUAL MOLD STRIPPING . . . . . . . . . . . . . . . . . . 9

G. SYSTEM SHUTDOWN .......................... . . . 11

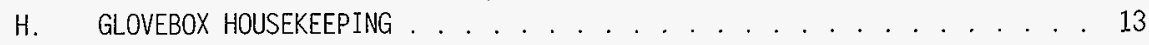

\begin{tabular}{|c|c|c|c|}
\hline bocument No & 00066197 & Rev/Mod & page $2.09 \% 16$ \\
\hline
\end{tabular}




\section{WRAP 1 PLANT OPERATING PROCEDURE}

\section{(HNF SD W026 - OTP - 015)}

\section{PROCEDURE}

A. SYSTEM STARTUP

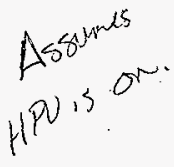

NOTE -

A77 control panel actions are performed at, and messages displayed on. Supercompactor Panelview panel, unless otherwise noted.

1. ENSURE conveyors, anvi1, and compactor glovebox are free of foreign objects.

2. ENSURE mold stripping blocks are down.

3. ENSURE any equipment, tools, and supplies, in glovebox are properly stored.

4. CLOSE disconnect (\#\#)

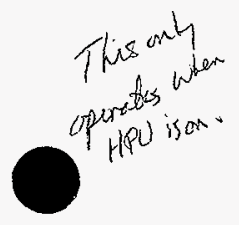

5. ENSURE Hydraulic Power Unit (HPU) cooling system is operating, as indicated on cooling system control panel.

6. INSERT Operator Control Key AND TURN ON. TO RUN

7. PRESS PanelView POWER ON button.

8. PRESS "F21-RESET".

9. PRESS "F7-HPU QEF". 15 ON"

10. CHECK message is "OFF MODE - AT HOME POSITION".

a. IF not in HOME position,

THEN PRESS "F21-GO HOME".

NOTE - $\quad$ Puck "spring back" needs to be considered when determining desired puck height.

Minimum puck height is 4.0 inches.

11. SET puck height:

a. PRESS "F17-CRUSHED PUCK HEIGHT"

b. ENTER desired puck height

c. PRESS ENTER

d. PRESS CANCEL. 


\section{WRAP 1 PLANT OPERATING PROCEDURE}

(HNF SD . 1026 - OTP - 015)

12. CHECK ram crushing pressure setting.

a. IF ram crushing pressure needs reset, THEN:

(1) PRESS "F18-CRUSHING PRESSURE"

(2) ENTER desired ram pressure

(3) PRESS ENTER

(4) PRESS CANCEL.

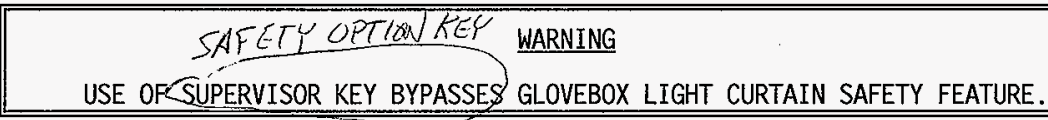

\section{CAUTION}

MAXIMUM RAM RELIEF PRESSURE SETTING IS 4200 psig.

13. CHECK ram relief pressure setting.

a. IF ram relief pressure needs reset, THEN:

(1) CONTACT Duty Operations Supervisor

(2) INSERT Supervisor Control Key AND TURN ON

(3) PRESS "F19-RELIEF PRESSURE".

(4) ENTER desired ram pressure

(5) PRESS ENTER

(6) PRESS CANCEL

(7) TURN Supervisor Control Key OFF AND REMOVE key. 


\section{WRAP 1 PLANT OPERATING PROCEDURE}

(HNF SD W026-01P - 015)

B. TRANSFERRING DRUM WITHOUT CRUSHING

NOTE - This section is intended for use when it is desired to pass a drum through the Supercompactor without crushing it.

NOTE - $\quad$ F10 may be used at any time to exit MANUAL mode.

\section{CAUTION}

FAILURE TO PLACE THE SUPERCOMPACTOR IN MANUAL PRIOR TO THE TRANSFER CAR IN THE SORTING GLOVEBOX DELIVERING THE DRUM TO THE ENTRY POSITION. WILL RESULT IN AUTOMATICALLY CRUSHING THE DRUM.

\section{CAUTION}

INTERLOCKS ARE BYPASSED IN MANUAL MODE.

In manual wock tide Fouttons, once momentarily pressed, will cause a step to pragress

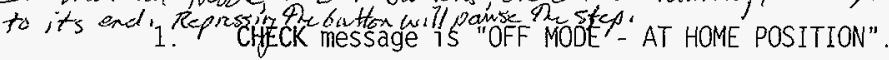

2. PRESS "F5-MANUAL".

3. ENSURE the desired drum is on input conveyor.

4. PRESS AND HOLD "F1-IN FWD" UNTIL drum loads into LUC.

5. PRESS AND HOKD "F3-LUC FWD" UNTIL puck is on output conveyor.

6. PRESS AND HOLD "F11-LUC REV" UNTIL LUC is ful7y retracted.

7. PRESS AND HOLD "F8-OUT FWD" UNTIL drum is at desired location.

8. PRESS "F10-OFF MODE".

9. CHECK message is "OFF MODE - AT HOME POSITION". 


\section{WRAP 1 PLANT OPERATING PROCEDURE}

\section{AUTOMATIC OPERATION}

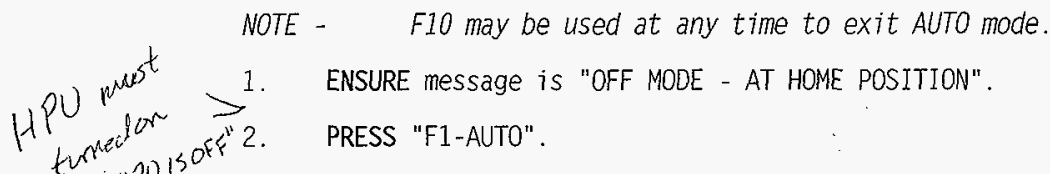

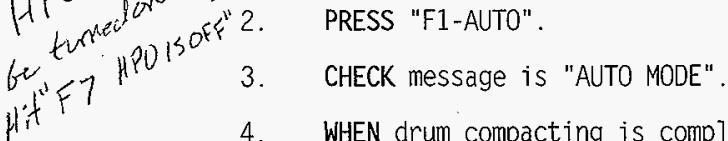

4. WHEN drum compacting is completed:

a. PRESS "F10-OFF MODE"

b. CHECK message is "OFF MODE - AT HOME POSITION"

c. SHUTDOWN supercompactor per section VII.G.

\section{SEMI-AUTOMATIC OPERATION}

NOTE - $\quad$ F10 may be used at any time to exit SEMI-AUTO mode.

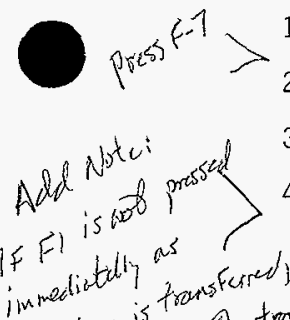

1. ENSURE message is "OFF MODE - AT HOME POSITION".

2. PRESS "F3-SEMI-AUTO".

3. CHECK message is "WAITING FOR LOAD COMMAND (F1)".

4. AFTER a drum is on input conveyor,

THEN :

qle drum is trang qhe transfer a. D. CHECK drum ioads into LUC.
it can $6 u^{2}$
c. PRESS "F3-CRUSH".
d. CHECK puck is stripped from mold.
e. PRESS "F5-PUSH".
f. CHECK puck moves onto outfeed conveyor.
5. REPEAT steps VII.D.3 to VII.D.4.f to crush additional drums. 


\section{WRAP 1 PLANT OPERATING PROCEDURE}

(HNF SD WO26 -0TP 015$)$

6. WHEN drum compacting is completed:

a. PRESS "F10-OFF MODE"

b. CHECK Panelview display is "OFF MODE - AT HOME POSITION"

c. SHUTDOWN supercompactor per section VII.G.

E. MANUAL OPERATION

NOTE - $\quad$ F10 may be used at any time to exit MANUAL mode.

\section{CAUTION}

INTERLOCKS ARE BYPASSED IN MANUAL MODE

1. CHECK message is "OFF MODE - AT HOME POSITION".

2. PRESS "F5-MANUAL".

3. AFTER a drum is on input conveyor.

a. PRESS AND HOLD "FI-IN FWD" UNTIL drum laads into LUC.

b. PRESS "F2-LASER IS OFF"

c. CHECK message is "LASER DETECTED".

NOTE - Pane7View wi77 display "LASER NOT DETECTED" when a drum breaks the beam. The drum is centered on the anvi 7 when Panelview display "LASER DETECTED" returns.

NOTE - Drum can be positioned using LUC jog keys (F4-F6 and F12-14).

d. PRESS AND HOLD "F3-LUC FWD". UNTIL drum is centered on anvil.

e. PRESS "F2-LASER IS ON".

f. CHECK "LASER DETECTED" is no longer displayed.

\section{CAUTION}

FAILURE TO FULLY RETRACT LUC CAN RESULT IN MOLD DAMAGING LUC.

g. PRESS AND HOLD "F11-LUC REV" UNTIL LUC is fu77y retracted. 


\section{WRAP 1 PLANT OPERATING PROCEDURE}

PRESS ANDO HOLD "F17-STOP MOLD" UNTIL mold is fully lowered.

PRESS ANDXWULD "F18-STOP PISTON" UNTIL ram reaches:

(1) crushing pressure

$\underline{\mathrm{OR}}$

(2) desired puck height.

j. PRESS "F19-STOP COMP".

k. WAIT five seconds,

THEN PRESS "F20-STOP_STRIP".

1. CHECK puck has stripped from mold.

m. WHEN mold reaches height of ram, THEN PRESS "F21-STOP RETURN".

n. CHECK mold and ram return HOME.

o. PRESS AND HOLD "F3-LUC FWD" UNTIL puck is on output conveyor.

p. PRESS AND HOLD "F11-LUC REV" UNTIL LUC is fully retracted.

NOTE - "F8-OUT FWD" and "F16-OUT REV" may be used to position puck.

q. PRESS AND HOLD "F8-OUT FWD" UNTIL puck is at desired location.

4. REPEAT steps VII.B.3, VII.E.3 to VII.B.7, VII.E.3.q to crush additional drums.

5. WHEN drum compacting is completed:

a. PRESS "F10-OFF MODE".

b. CHECK message is "OFF MODE - AT HOME POSITION".

c. SHUTDOWN supercompactor per section VII.G. 


\section{WRAP 1 PLANT OPERATING PROCEDURE}

F. MANUAL MOLD STRIPPING

NOTE - $\quad$ Flo may be used at any time to exit to the OFF mode.

1. REQUEST Duty Operations Supervisor authorization to perform manual mold stripping.

2. ENSURE mold and ram heights are greater than 12 inches.

a. IF mold and ram are less than 12 inches:

(1) PRESS "F21-RESET" to raise

(2) RELEASE "F21-RELEASE" WHEN mold and ram are greater than 12 inches.

3. PRESS "F10-OFF MODE".

4. PRESS "F7-HPU OFF".

5. TURN Operator Control Key OFF AND REMOVE key.

\section{WARNING}

PINCHING HAZARDS ARE PRESENT WHILE UPRIGHTING MOLD BLOCKS. CAUTION IS NECESSARY WHILE UPRIGHTING MOLD BLOCKS.

6. UPRIGHT mold stripping blocks.

7. INSERT Operator Control Key AND TURN ON.

8. PRESS PaneIView POWER ON button.

9. PRESS "F21-RESET".

10. PRESS "F19-STOP COMP"

11. PRESS "F7-HPU OFF".

12. CHECK message is "ON MODE - AT HOME POSITION".

\section{CAUTION}

INTERLOCKS ARE BYPASSED IN MANUAL MODE.

13. PRESS "F5-MANUAL". 


\section{WRAP 1 PLANT OPERATING PROCEDURE}

(HNF SB WO26 -0TP 015 )

14. PRESS "F17-STOP MOLD".

15. PRESS "F18-STOP PISTON".

16. PRESS "F20-STOP_STRIP":

17. WHEN mold reaches height of ram, THEN PRESS "F21-STOP RETURN".

18. CHECK puck has been stripped.

19. PRESS "F19-STOP COMP".

20. PRESS "F21-STOP-RETURN".

21. CHECK mold and ram return HOME.

22. PRESS "F10-OFF MODE".

23. PRESS "F7-HPU OFF".

24. TURN Operator Control Key OFF AND REMOVE key.

\section{WARNING}

PINCHING HAZARDS ARE PRESENT WHILE UPRIGHTING MOLD BLOCKS. CAUTION IS NECESSARY WHILE UPRIGHTING MOLD BLOCKS.

25. LOWER mold stripping blocks.

26. INSERT Operator Control Key AND TURN ON.

27. PRESS PaneIView POWER ON button.

28. PRESS "F21-RESET".

29. PRESS "F7-HPU OFF".

30. CHECK message is "OFF MODE - AT HOME POSITION".

\section{CAUTION}

UPRIGHTED MOLD STRIPPING BLOCKS CAN DAMAGE LUC.

31. PRESS AND HOLD "F3-LUC FWD" UNTIL puck is on output conveyor.

32. PRESS AND HOLD "F11-LUC REV" UNTIL LUC is ful7y retracted. 


\section{WRAP 1 PLANT OPERATING PROCEDURE}

$(1 N F$ SO 1026 - OTP 015$)$

NOTE - "F8-OUT FWD" and "F16-OUT REV" can be used to position puck.

33. PRESS AND HOLD "F8-OUT FWD" UNTIL puck is at desired Tocation.

34. PRESS "F10-OFF MODE".

G. SYSTEM SHUTDOWN

1. ENSURE anvil is clear of all objects.

2. CHECK message is "OFF MODE - AT HOME POSITION".

3. CHECK LUC is fully retracted.

\section{CAUTION}

INTERLOCKS ARE BYPASSED IN MANUAL MODE

a. IF LUC is not fully retracted,

THEN:

(1) PRESS "F5-MANUAL"

(2) PRESS AND HOLD "F11-LUC REV" UNTIL LUC is fu17y retracted

(3) PRESS "F10-OFF MOOE".

4. PRESS "F7-HPU OFF".

5. TURN Operator Control Key OFF AND REMOVE key.

\section{WARNING}

PINCHING HAZARDS ARE PRESENT WHILE UPRIGHTING MOLD BLOCKS. CAUTION IS NECESSARY WHILE UPRIGHTING MOLD BLOCKS.

6. UPRIGHT mold stripping blocks.

7. INSERT Operator Control Key AND TURN ON.

8. PRESS PanelVIEW POWER ON button.

9. PRESS "F21-RESET". 


\section{WRAP 1 PLANT OPERATING PROCEDURE}

(HNF SD WO26 - OTP 015$)$

10. SET puck height to four inches:
a. PRESS "F17-CRUSHED PUCK HEIGHT"
b. ENTER "4.0"
c. PRESS ENTER
d. PRESS CANCEL .

\section{CAUTION}

INTERLOCKS ARE BYPASSED IN MANUAL MODE.

11. PRESS "F5-MANUAL".

12. LOWER mold:

a. PRESS "F17-STOP MOLD"

b. CHECK mold is at 4.0 inches.

13. LOWER ram:

a. PRESS "F18-STOP PISTON"

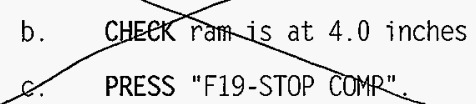

14. PRESS "F10-OFF MODE".

15. PRESS "F7-HPU OFF".

16. TURN Operator Control Key OFF AND REMOVE key.

17. RETURN Operator Control Key to controlled key lockbox.

18. PERFORM glovebox housekeeping, as required, per section VII.H. 


\section{WRAP 1 PLANT OPERATING PROCEDURE}

(GNF SD WO26 - OTP - 015 )

\section{H. GLOVEBOX HOUSEKEEPING}

\section{CAUTION}

INTRODUCTION OF UNAPPROVED CLEANING AGENTS INTO GLOVEBOXES MAY RESULT IN . ENVIRONMENTAL NON-COMPLIANCE, UNWANTED CHEMICAL REACTIONS OR UNDESIRABLE EFFECTS. USE ONLY FACILITY APPROVED CLEANING AGENTS.

\section{SUPERCOMPACTOR GLOVEBOX INSPECTION}

a. CHECK, visually throughout supercompactor glovebox for:

(1) Loose items, liquids, droppings or tailings from waste compaction

(2) Improperly stored glovebox tools and equipment

(3) Debris in the mold cleanout ports

(4) Debris in the anvil liquid collection ports

(5) Leaks from hydrautic systems

(6) Left-over agents introduced for cleaning or other purposes

(7) Material in Supercompactor base trough.

(8) Foreign matter on glovebox wal1s, windows and/or equipment.

b. CHECK supercompactor equipment for damage, leaks, etc 


\section{WRAP 1 PLANT OPERATING PROCEDURE}

(BNA . SO 6026 . 0 IP 015

c. CLEAN supercompactor as required, per following sections of this procedure:

(1) CLEANUP solids per Section VII.H.2.

(2) CLEANUP Tiquids per Section VII.H.3.

(3) WIPEDOWN glovebox per Section VII.H.4.

(4) LOADOUT of debris/cleanup materials per Section VII.H.5.

NOTE - Radiological survey is required upon exiting gloveports after each use.

(5) UPON exiting gloveports at any point in this procedure, PERFORM a self-survey.

(a) IF self-survey limits are exceeded,

THEN CONTACT Radiological Control Technician.

2. SOLID DEBRIS REMOVAL

a. ENSURE Supercompactor is SHUTOOWN.

b. DETERMINE gloveport(s) which provide best access.

c. PICKUP waste and debris items AND MOVE to glovebox sorting table.

(1) USE vacuum, broom, dustpan, or shovel, located inside glovebox, as necessary to aid cleanup.

NOTE - Tools may be stored in the press trough or under the conveyor.

d. STORE glovebox tools and equipment.

e. MOVE excess tools and equipment to glovebox sorting table. 


\section{WRAP 1 PLANT OPERATING PROCEDURE}

3. LIQUTDS CLEANUP

a. ENSURE Supercompactor is SHUTDOWN.

b. TRANSFER required materials into glovebox through bagless transfer port. sample transfer port or consumable entry port, as applicable.

NOTE - Cleanup is accomplished by best suited method. The following steps are a guideline.

c. PLACE dam, dike or absorbent containment from spi 11 kit around Tiquid.

d. ABSORB, using Rad-Pads and absorbent, a 11 liquids.

e. PLACE used absorbent and Rad-Pads into plastic bag(s).

f. PLACE bagged items on glovebox sorting table.

g. IF necessary,

THEN WIPEDOWN spil1 area per Section VII.H.4.

h. CHECK anvil liquids collection bottle liquid level.

i. IF anvil liquids collection bottle contains any liquid:

(a) INFORM Duty Operations Supervisor

(b) REMOVE AND CAP bottTe

(c) INSTALL empty bottle

(d) DISPOSE of fult bottle/liquid as directed. 


\section{WRAP 1 PLANT OPERATING PROCEDURE}

(HNF. SB W026 - OAP 015)

\section{GLOVEBOX WIPEDOWN}

a. ENSURE Supercompactor is SHUTDOWN.

b. TRANSFER required materials (approved cleaning agents, rags, Rad-Pads, etc.) into glovebox through bagless transfer port, sample transfer port, or consumable entry port, as applicable.

NOTE - Cleanup is accomplished by best suited method (i.e, cleaning agents may be poured directly on area or applied to rags or pads, then wiped over area). The following steps are a guideline.

c. APPLY cleaning agent to affected area(s).

d. SCRUB OR BRUSH affected area(s).

e. WIPE, affected area(s) and equipment with clean, dry, Rad-Pads or rags.

f. PLACE used Rad-Pads, rags and other items to be disposed, into plastic bag(s).

g. PLACE bagged items on glovebox sorting table.

\section{LOADOUT}

NOTE - Tools may be stored in the press trough or under the conveyor.

a. STORE Supercompactor operating/cleaning tools.

b. REMOVE excess tools or equipment per applicable glovebox operating and/or loadout procedure.

NOTE - Remaining cleanup items are treated as if processing waste typical of applicable glovebox.

c. PROCESS remaining cleanup items as directed, per applicable glovebox operating and loadout procedures. 


\section{WRAP 1 PLANT OPERATING PROCEDURE}

SYSTEM * PROCESS

\section{LLW EXIT GLOVEBOX OPERATION}

(HNF-SD-W026-OTP-015)

\section{SYSTEM DESCRIPTION}

This procedure provides instructions for operation of the Low Level Waste (LLW) Exit Glovebox.

\section{REFERENCE DOCUMENTS}

HSRCM-1, Hanford Site Radiological Control Manual

WHC-CM-5-34, Solid Waste Disposal Operations Administration

WHC-CM-5-36. SWD Internal Requirements

WHC-EP-0063, Hanford Site Solid Waste Acceptance Criteria

WRP1-OP-0703, Glovebox Lift Table Operation

WRP1-OP-0701, Glovebox Housekeeping

\section{PRESTART CONDITIONS}

Al1 personnel performing this procedure shall be qualified in accordance with WHC-CM-5-34, Section 1.8, "Training Plan," and on-the-job training.

Process area Heating, Ventilation, Air Conditioning (HVAC) in OPERATION.

\section{SAFETY}

Potential hazards, such as lifting heavy containers or handling sharp objects present in waste containers, must be mitigated by protective equipment, procedures, and administrative controls to ensure acceptable-risk operating conditions.

Consult with Operations Management and Industrial Hygiene, and refer to the Job Hazards Analysis (JHA). Radiological Work Permit (RWP) and Material Safety Data Sheets (MSDS) to determine required Persona? Protective Equipment (PPE). 


\section{WRAP 1 PLANT OPERATING PROCEDURE}

(HNF SD 1026 . OIP 015 )

\section{SAFETY (Continued)}

A light curtain is used to detect access at the gloveports above the conveyor between the Supercompactor and the LLW Exit Glovebox. If the light curtain is broken all automatic operations within the glovebox will be stopped to prevent possible injury to the operator.

This procedure requires accessing gloveboxes through gloveports on a regular basis. Operators shall perform self survey upon exiting gloveport after each use. Radiological Control Technician (RCT) shall be contacted anytime self survey limits are exceeded.

Spills inside the glovebox shall be cleaned up per WRP1-OP-0701, Glovebox Housekeeping.

Warning - Use care to prevent injury. Possible safety hazards include:

- Tripping/Slipping

- Injuries to the hands at glovebox ports

- Radiological Contamination

- Interference with the Automatic Guided Vehicle (AGV)

\section{TOOLS AND SUPPLIES}

Ruter for measuring puck height.

Indelible Black Marker Pens (e.g. Sharpie). 


\section{WRAP 1 PLANT OPERATING PROCEDURE}

\section{TABLE OF CONTENTS}

PAGE
A. INITIALIZE SYSTEM .
B. EXIT DRUM ACCESS TO LLW GLOVEBOX . . . . . . . . . . . . . . . 5
c. PUCK RECEIPT AND WEIGHT MEASUREMENT . . . . . . . . . . . . . . . . . . 6
D. MOVE PUCK FROM RECEIPT TO LOAD OUT . . . . . . . . . . . . . . . . . . 8
E. MOVE PUCK FROM RECEIPT TO STORAGE . . . . . . . . . . . . . . . . . . 9
F. MOVE PUCK FROM'STORAGE TO LOAD OUT . . . . . . . . . . . . . . . . . . 10
G. RECORD COMPACT DRUM (PUCK) DATA . . . . . . . . . . . . . . . . 11
H. REMOVE OVERPACK FROM GLOVEBOX . . . . . . . . . . . . . . . . . 12 ATTACHMENTS

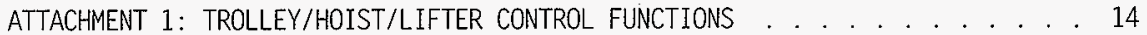




\section{WRAP 1 PLANT OPERATING PROCEDURE}

(BNF SD W026 OTP 015 )

\section{PROCEDURE}

\section{WARNING}

IN THE EVENT OF A MAJOR PROBLEM. THE LLW GLOVEBOX LINE MAY BE SHUT DOWN BY OPERATION OF LLW PROCESS GLOVEBOX EMERGENCY STOP BUTTON ON THE DMS CONSOLE. EMERGENCY STOP WILL SHUT DOWN ALL OPERATIONS IN THE GLOVEBOX LINE AND PLACE IT IN A SAFE CONDITION. RESTART FROM EMERGENCY STOP IS PERFORMED WITH PROCEDURE WRP1-0P-0702, RESTART GLOVEBOX OPERATION.

NOTE 1 - Administrative hold steps are identified by letters in parentheses at the left margin of the procedure step. The direction given in the procedure step must be satisfied before work continues.

(M) - Operations management shall approve operation.

$(R)$ - Radiological Control (RC) sha7l complete surveys or agree to permit continued operation.

NOTE 2 - Un7ess otherwise specified Data Management System (DMS) actions are performed on DMS Terminal (107-TE-12-103).

NOTE 3 - Unless otherwise specified Operator Interface Unit (OIU) actions are performed on OIU (OIU-12-103C).

\section{A. INITIALIZE SYSTEM}

1. ENSURE AUTO/MAINTENANCE keyswitch (107-07-HS-318) on DMS Termina 7 is in AUTO.

2. SELECT "DIAGNOSTICS MENU" on OIU MAIN MENU.

3. VERIFY OIU displays "DIAGNOSTICS MENU". 


\section{WRAP 1 PLANT OPERATING PROCEDURE}

4. IF DIAGNOSTIC MENU flashes "FAIL", THEN:

NOTE - Operator uses normally available OIU screens. If necessary. DOS may access maintenance mode on OIU.

a. IF possible,

THEN. CORRECT fault(s) indicated on OIU.

b. IF faults cannot be corrected,

THEN, NOTIFY Duty Operations Supervisor (DOS).

c. WHEN faults have been corrected,

THEN, SELECT "MAIN MENU".

d. REPEAT Section VII.A.

5. WHEN DIAGNOSTIC MENU f7ashes "PASS",

THEN, SELECT "MAIN MENU".

B. EXIT DRUM ACCESS TO LLW GLOVEBOX

1. SELECT "EXIT PORT MENU".

2. VERIFY display, "EXIT PORT MENU".

3. VERIFY display "DRUM AT LIFT TABLE". IF OIU displays "NO DRUM AT LIFT TABLE",

THEN:

a. NOTIFY Control Room and/or DOS.

b. CORRECT drum deficiency as directed.

4. SCAN the following in order with portable scanner 12-NE-310:

a. Location (2Y) barcode on side of glovebox.

b. Overpack Drum (S) barcode. 


\section{WRAP 1 PLANT OPERATING PROCEDURE}

(UNF SO W026-OIP 015)

(M)

5. IF Scanner displays "PIN MIS-MATCH", THEN, NOTIFY DOS.

6. SCAN "YEND" barcode on side of glovebox.

7. SCAN "YYES" barcode.

(M) 8. IF Scanner displays "PIN MIS-MATCH", THEN, NOTIFY DOS.

9. REMOVE clamp band from overpack drum.

10. SELECT "START DRUM ENTRY".

11. VERIFY display "DRUM ENTRY IN PROGRESS..."

12. WAIT unti] OIU displays "DRUM ENTRY COMPLETE", THEN, SELECT "OPEN PORT".

13. VERIFY display "PORT OPENING...".

14. VERIFY display "PORT OPEN".

15. SELECT the following in order at DMS Termina1:
a. "PROCESS OPS"
b. "LLW GLOVEBOX"
c. "EXIT GLOVEBOX"

16. VERIFY the Loadout Drum PIN.

17. VERIFY All other data fields on the screen are blank.

C. PUCK RECEIPT AND WEIGHT MEASUREMENT

1. SELECT in order:



2. VERIFY dISPTAY "COMPACTEQ RRIM RECETPI RAGESS".

3. WAIT for disPTay "COMPACTED DRUM RECEIPT COMPLETE".

4. PLACE barcode on compacted puck. 


\section{WRAP 1 PLANT OPERATING PROCEDURE}

5. SCAN puck barcode.

6. MEASURE puck height.

7. RECORD measured puck height on drum with black marker.

8. SELECT in order:

a. "MAIN MENU" (unless already displayed).

b. "TROLLEY MENU".

c. "COMPACT RECEIPT".

\section{WARNING}

IMPROPER HOIST OPERATION MAY CAUSE INJURY OR EQUIPMENT DAMAGE. FAILURE TO PROPERLY POSITION AND CLOSE OR OPEN DRUM LIFTER COULD RESULT IN DROPPED DRUM. CAUTION MUST BE EXERCISED IF REACHING THROUGH GLOVEPORTS TO MANUALLY POSITION DRUM LIFTER.

NOTE -

Unless otherwise specified hoist control activities are performed on Hoist Control (107-PC-07-004). Controls must be activated unt 77 associated indicator il7uminates. Reference Attachment 1: Hoist Control Functions.

9. PERFORM the following with Hoist Control:

a. POSITION HOIST above puck (unless al ready there).

b. OPEN LIFTER jaWS.

c. LOWER HOIST over puck.

d. CLOSE LIFTER on puck.

e. RAISE HOIST (and puck) unti 1 "RAISED LIMIT". LED illuminates.

10. SELECT in order:

a. "MAIN MENU" (unless already displayed).

b. "WEIGH MENU".

11. WAIT until OIU displays COMPACT DRUM WEIGHT value. 


\section{WRAP 1 PLANT OPERATING PROCEDURE}

NOTE - $\quad$ Pucks are norma7ly taken directly from receipt to load out, however at the discretion of the Operator or when overpack height. or weight limitations will be exceeded. the puck is placed in storage for later retrieval (i.e. When a new overpack drum is at the exit port.)

12. IF puck is to be moved to LOAD OUT, THEN, GO to Section VII.D, MOVE PUCK FROM RECEIPT TO LOAD OUT.

13. IF puck is to be placed in STORAGE, THEN:

a. RECORD puck weight on temporary data sheet.

b. GO to Section VII.E, MOVE PUCK FROM RECEIPT TO STORAGE.

D. MOVE PUCK FROM RECEIPT TO LOAD OUT

1. VERIFY measured puck height is within available drum space.

2. OBSERVE "COMPACT DRUM WEIGHT" and "CUMULATIVE WEIGHT".

3. IF either of the following are TRUE, THEN, GO to Section VII.E, MOVE PUCK FROM RECEIPT TO STORAGE.

a. Puck height exceeds available drum space.

NOTE - Documented calculation is not required. PCS gives warning message when ADO TO CUMULATIVE is selected, if weight exceeds maximum allowable.

b. "COMPACT DRUM WEIGHT" plus "CUMULATIVE WEIGHT" is greater than 1000 pounds.

4. SELECT "ADD TO CUMULATIVE".

5. IF OIU displays "CUMULATIVE WEIGHT EXCEEDED"

THEN, GO to Section VII.E, MOVE PUCK FROM RECEIPT TO STORAGE.

6. SELECT in order:

a. "MAIN MENU" (unless already displayed).

b. "TROLLEY MENU".

c. "LOAD OUT". 
HNF-SD-W026-OTR-015, Rev. 0, Page OP-172

\section{WRAP 1 PLANT OPERATING PROCEDURE}

(HNF SB 1026 - OTP 015 )

7. PERFORM the following with Hoist Control:

a. POSITION TROLLEY (puck) above overpack drum at LOAD OUT.

b. LOWER HOIST (puck) into overpack drum.

c. OPEN LIFTER jaws.

d. RAISE HOIST unti] "RAISED LIMIT" LED $i 17$ uminates.

8. GO TO Section VII.G, RECORD COMPACT DRUM (PUCK) DATA.

E. MOVE PUCK FROM RECEIPT TO STORAGE

NOTE - Storage Position 3 does not exist.

1. SELECT puck storage location on TROLLEY MENU screen:

- STORAGE POSITION 1

- STORAGE POSITION 2

2. PERFORM the following with Hoist Control:

a. POSITION TROLLEY (puck) above selected storage location.

b. LOWER HOIST (puck) onto storage table.

c. OPEN LIFTER jaWS.

d. RAISE HOIST unti7 "RAISED LIMIT" LED 117 uminates.

3. IF another puck is to be placed in overpack or storage, THEN, REPEAT Sections VII.C through VII.G as applicable. OTHERWISE, GO TO Section VII.H, REMOVE OVERPACK FROM GLOVEBOX. 


\section{WRAP 1 PLANT OPERATING PROCEDURE}

F. MOVE PUCK FROM STORAGE TO LOAD OUT

1. SELECT puck with height/weight compatible to available drum space.

2. SCAN the following:

a. Storage Location (2Y) barcode of selected puck.

b. Barcode on selected puck.

c. "YEND" barcode on side of glovebox.

d. "YYES" barcode.

(M) 3. IF OIU displays "PIN MIS-MATCH",

THEN, NOTIFY DOS.

4. SELECT in order:

a. "MAIN MENU" (unless a7ready displayed)

b. "TROLLEY MENU"

c. "STORAGE POSITION $X "$ ( $X$ is position of puck to be moved)

5. PERFORM the following with Hoist Control:

a. OPEN LIFTER jaws.

b. LOWER LIFTER onto puck.

c. CLOSE LIFTER jaWS.

d. RAISE HOIST unti 1 "RAISED LIMIT" LED i17uminates.

6. SELECT in order:

a. "MAIN MENU" (unless already displayed)

b. "WEIGH MENU"

7. WAIT for display "COMPACT DRUM WEIGHT" value.

8. SELECT "ADD TO CUMULATIVE". 


\section{WRAP 1 PLANT OPERATING PROCEDURE}

(HNF. SB WO26 OTP 015)

9. IF OIU displays "CUMULATIVE WEIGHT EXCEEDED".

THEN:

a. PERFORM the following with Hoist Control:

(1) LOWER HOIST (puck) back to storage tab7e.

(2) OPEN LIFTER jaws.

(3) RAISE HOIST unti1 "RAISED LIMIT" LED i 1 luminates.

b. SELECT another puck from storage or receipt, as applicable.

10. SELECT on in order:

a. $\quad$ "MAIN MENU" (unless already displayed).

b. "TROLLEY MENU".

c. "LOAD OUT".

11. VERIFY display "TROLLEY MOVING TO LOADOUT POSITION".

12. WAIT for display "TROLLEY AT LOADOUT POSITION".

13. PERFORM the following with Hoist Control:

a. POSITION TROLLEY (puck) above overpack drum at LOAD OUT.

b. LOWER HOIST (puck) into overpack drum.

c. OPEN LIFTER jaws.

d. RAISE HOIST unt 17 "RAISED LIMIT" LED $i 1$ luminates.

14. GO TO Section VII.G, RECORD COMPACT DRUM (PUCK) DATA.

G. RECORD COMPACT DRUM (PUCK) DATA

1. SELECT "Refresh" on LLW Exit Glovebox screen AND CONFIRM puck PIN is displayed.

2. ENTER the correct height if the puck height is different than the displayed default value.

3. IF another puck is to be placed in overpack or storage, THEN, REPEAT Sections VII.C through VII.G as applicable, OTHERWISE, GO TO Section VII.H, REMOVE OVERPACK FROM GLOVEBOX.

\begin{tabular}{|c|c|c|c|}
\hline Docuinent $10,0.60$ & ront bate $98.96 \%$ & Rev/Mod & $9 \mathrm{age} / 1.04$ \\
\hline
\end{tabular}




\section{WRAP 1 PLANT OPERATING PROCEDURE}

H. REMOVE OVERPACK FROM GLOVEBOX

1. RECORD information pertaining to the waste in the loadout drum using the LLW Exit Glovebox screen on DMS terminal TE-12-103:

a. ENTER signature password.

b. SELECT "Sum Waste Records" button.

c. ENTER Waste Description.

d. MODIFY primary waste type and hazardous waste storage category for the drum, as required.

e. ENTER void filler code and filler weight, as applicable.

f. ENTER signature password.

g. SELECT "Commit" button.

2. ENSURE area around port sealing surface is free of debris.

3. SELECT in order:

a. "MAIN MENU" (unless already displayed).

b. "EXIT PORT MENU".

c. "ClOSE \& RELT". ClOSÉ PORT

4. VERIFY OIU displays "PORT CLOSING...".

5. WAIT for display "PORT CLOSED".

6. SELECT "START DRUM EXIT",

7. VERIFY display "DRUM EXIT IN PROGRESS...".

8. WAIT for display "DRUM EXIT COMPLETE".

(R) 9. REQUEST RCT survey drum for contamination.

a. IF drum is contaminated.

THEN, decontaminate as directed.

b. RESURVEY drum.

10. ENTER surface beta-gamma dose rate on DMS screen DMSSO315: 
HNF-SD-W026-OTR-015, Rev. 0, Page OP-176

\section{WRAP 1 PLANT OPERATING PROCEDURE}

(ANF SD W026-0TP 015)

11. REINSTALL clamp band on overpack drum as follows:

NOTE - Manual Lift table operation is performed per WRPI-OP-0703. G7ovebox Lift Tab7e Operation.

a. LOWER Lift Table as required.

b. REINSTALL clamp band.

c. RAISE Lift Table to AGV LOAD HEIGHT.

d. RETURN Lift Table to NORMAL operation.

12. SELECT "DRUM READY FOR PICK-UP".

13. VERIFY display "DRUM PICKUP IN PROGRESS". 


\section{WRAP 1 PLANT OPERATING PROCEDURE}

(HNF SO W026 - OTP - 015)

\section{ATTACHMENT 1: TROLLEY/HOIST/LIFTER CONTROL FUNCTIONS}

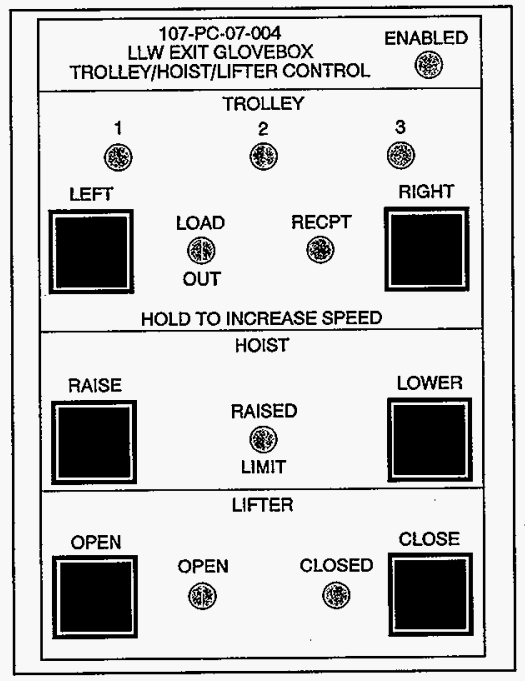

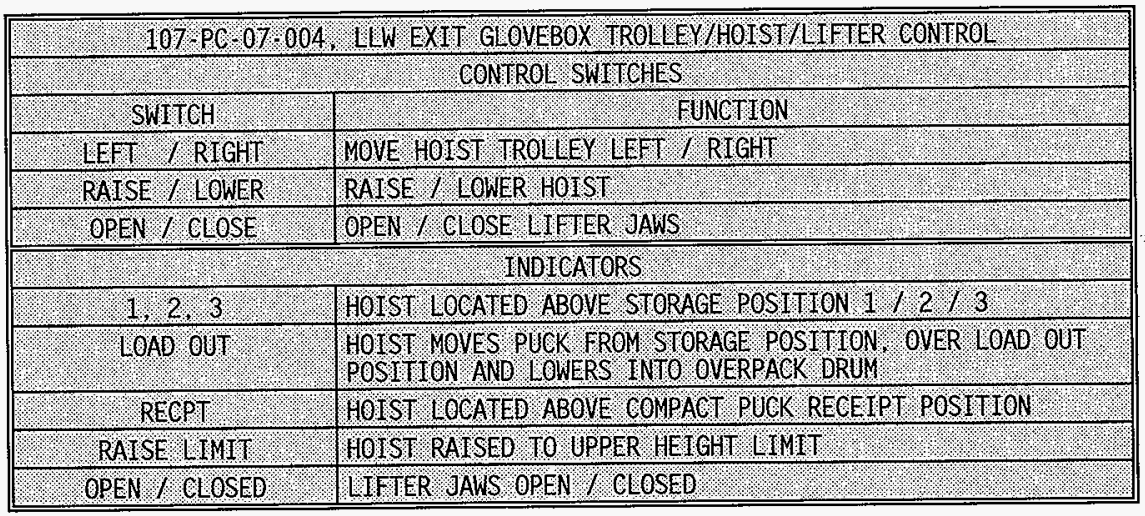

\begin{tabular}{|c|c|c|c|}
\hline Oocument No 1.76 & 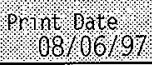 & Rev/Mod & Page 18 of 14 \\
\hline
\end{tabular}




\section{WRAP 1 PLANT OPERATING PROCEDURE}

SYSTEM: PROCESS

\section{LOW LEVEL WASTE GLOVEBOX LINE MANUAL OPERATION}

(HNF-SD-W026-OTP-015)

\section{SYSTEM DESCRIPTION}

This procedure provides instructions for manual (Operator Interface Unit (OIU) Maintenance Menu) operation of each glovebox (Entry, Sorting, Exit) in the Low Level Waste (LLW) Glovebox Line.

In Manual (Maintenance) mode automatic operations normally performed by the Plant Control System (PCS) may be individually actuated. This allows correction of interlock conflicts, time-outs or other out-of-sequence conditions which prevent the normal sequence of events from proceeding. once prohibiting conditions are corrected, automatic operation is resumed.

Maintenance Menus of the OIU are password protected and Duty Operation Supervisor (DOS) permission is required for activation. Depending on the situation, the DOS may simply correct a single prohibiting condition and return the system to normal, or in cases of numerous problems such as might occur after an Emergency Stop or power loss, the DOS may activate the maintenance mode and request additional assistance for the operator (i.e., Cognizant Engineer).

This procedure addresses each OIU function individual7y and is not intended to govern sequence of operations. Sequence of operations, hoid points and non-0IU related activities are governed by applicable glovebox operating procedures. Attachment 4, Tables 1 through 3, define inter lock and permissive signals in each glovebox and reference procedure sections or other procedures which manipulate the indicated source switch for the interlock or permissive. When a function will not actuate, applicable interlocks should be verified to the proper state, then the function rechecked. If a 17 interlocks are properly verified and a function will sti11 not actuate, maintenance is required.

\section{REFERENCE DOCUMENTS}

WRP1-OP-0703, Glovebox Lift Table Operation.

WRP1-OP-0701, Glovebox Housekeeping.

WRP1-OP-0704, Waste Manipu7ator Operation

WRP1-OP-0705, Bagless Transfer Manual Operation

WRP1-OP-0706. Packet NDE Operation

WRP1-OP-0713, LLW Entry Glovebox Operation.

WRP1-0P-0714, LLW Sorting Glovebox Operation.

WRP1-OP-0716, LLW Exit Giovebox Operation.

\begin{tabular}{|c|c|c|c|c|c|}
\hline Releast odte & prant oater & hes Ig & bocument No & $8 \mathrm{Rev} / \mathrm{Mrd}$ & page 1 of 44 \\
\hline
\end{tabular}




\section{WRAP 1 PLANT OPERATING PROCEDURE}

(HNF SD WO26 0TP 015$)$

\section{PRESTART CONDITIONS}

A11 personne 1 performing this procedure sha 11 be qualified in accordance with WHC-CM-5-34, Section 1.8, "Training Plan," and on-the-job training.

Process area Heating, Ventilation, Air Conditioning (HVAC) in OPERATION.

\section{SAFETY}

Potential hazards, such as lifting heavy containers or handling sharp objects present in waste containers, must be mitigated by protective equipment, procedures, and administrative controls to ensure acceptable-risk operating conditions.

Consult with Operations Management and Industrial Hygiene, and refer to the Job Hazards Analysis (JHA), Radiological Work Permit (RWP) and Material Safety Data Sheets (MSDS) to determine required Personal Protective Equipment (PPE).

This procedure requires accessing gloveboxes through gloveports on a regular basis. Operators shal1 perform self survey upon exiting gloveport after each use. Radiological Control Technician (RCT) shall be contacted anytime self survey limits are exceeded.

Spil1s inside gloveboxes shal1 be cleaned up per WRP1-OP-0701, Glovebox Housekeeping.

CAUTION - When an OIU is in maintenance mode, most interlocks and safety features are bypassed. It is possible to damage equipment, and/or bypass safety restrictions such as glovebox containment. Caution must be exercised to prevent unwanted violation of operational safety requirements or equipment damage.

WARNING - Use care to prevent injury. Possible safety hazards include:

- Tripping/Slipping

- Radiologicar Contamination

- Interference with Automatic Guided Vehicle (AGV)

WARNING - Improper operation of glovebox equipment including hoist, sorting table, manipulators, transfer car, etc. may cause injury. Caution must be exercised if reaching through gloveports while equipment is in operation.

\section{TOOLS AND SUPPLIES}

None.

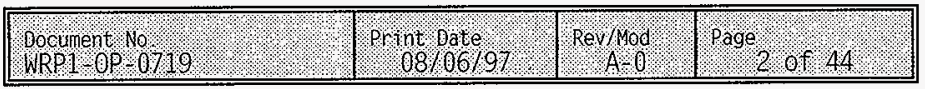




\section{WRAP 1 PLANT OPERATING PROCEDURE}

VI. TABLE OF CONTENTS

PAGE

VII. PROCEDURE (LLW ENTRY GLOVEBOX)

A. INITIALIZE ENTRY GLOVEBOX

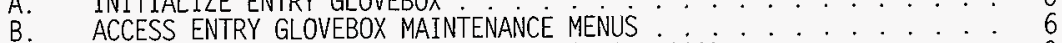

C. OPERATE ENTRY GLOVEBOX HOIST $(107-$ CR-07-101A) . . . . . . . . . . . . 8

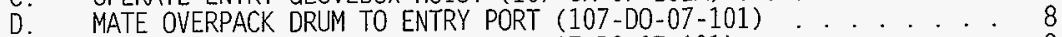

E. MATE SINGLE DRUM TO ENTRY PORT (107-D0-07-101) . . . . . . . . . . 8

F. OPEN ENTRY PORT $(107-00-07-101)$ (OVERPACK DRUM) . . . . . . . . . . . 9

G. OPEN ENTRY PORT (107-D0-07-101) (SINGLE DRUM) . . . . . . . . . . . . 10

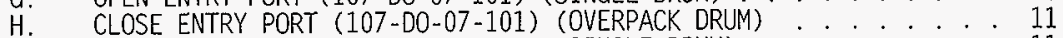

I. CLOSE ENTRY PORT (107-D0-07-101) (SINGLE DRUM) . . . . . . . . . . . . . 11

j. REMOVE OVERPACK DRUM FROM ENTRY PORT (107-D0-07-101) . . . . . . . 12

K. OPEN TRANSFER PORT (107-DO-07-102) .............. . . 12

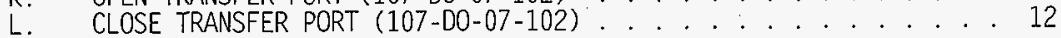

VIII. PROCEDURE (LLW SORTING GLOVEBOX) . . . . . . . . . . . . . . . . . . . 13

A. INITIALIZE SORTING GLOVEBOX . . . . . . . . . . . . . . . . . . . . 14

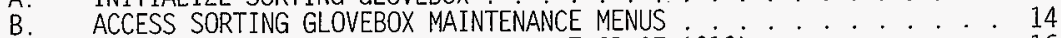

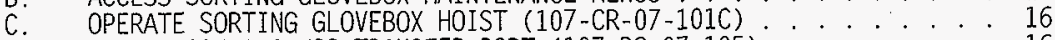

D. OPEN/CLOSE BAGLESS TRANSFER PORT (107-00-07-105) . . . . . . . . . . 16

E. MOVE DRUM TRANSFER CAR (107-TC-07-101) . . . ....... . 16

F. MOVE OR ROTATE DRUM LID/DELID FIXTURE $(107-R T-07-101)$. . . . . . . 17

G. OPERATE LID/DELID SAW. . . . . . . . . . . . . . . . . 18

H. DELID WASTE ORUM ....................... 19

I. RELID WASTE DRUM

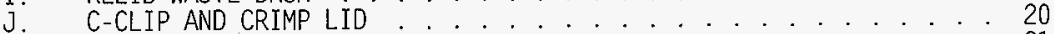

K. OPEN/CLOSE DRUM GRAB . . . . . . . . . . . . 21

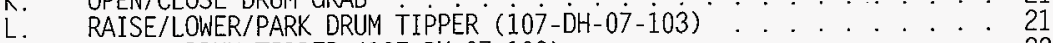

M. ROTATE DRUM TIPPER (107-DH-07-103) . . . . . . . . . . 22

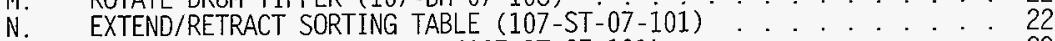

0 . RAISE/LOWER SORTING TABLE $(107-S T-07-101) \ldots \ldots . \ldots . \cdots$

IX. PROCEDURE (LLW EXIT GLOVEBOX) . . . . . . . . . . . . . . . . . . . . . . . 24

A. INITIALIZE EXIT GLOVEBOX

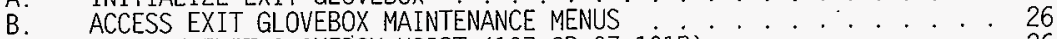

c. OPERATE EXIT GLOVEBOX HOIST (107-CR-07-1010) . . . . . . . . . . 26

D. MATE DRUM TO EXIT PORT (107-00-07-106) . . . . . . . . . . . . . 27

E. OPEN EXIT PORT (107-D0-07-106) . . . . . . . . . . . 28

F. OPERATE TRANSFER CAR CONVEYOR . . . . . . . . . . . . . . . . . . 29

G. RELID OVERPACK DRUM \& CLOSE EXIT PORT $(107-D \dot{0}-07-106) \cdots \cdots$

H. REMOVE OVERPACK DRUM FROM EXIT PORT (107-D0-07-106) . . . . . . . . 30

I. MATE DRUM TO EXIT PORT (107-DO-07-106) . . . . . . . . . . . . 31

\begin{tabular}{|c|c|c|c|}
\hline Document wo & printroate & Rev Mod & $\mathrm{page}, 3 \%$ \\
\hline
\end{tabular}




\section{TABLE OF CONTENTS (continued) PAGE}

ATTACHMENTS

ATTACHMENT 1: ENTRY GLOVEBOX HOIST CONTROL FUNCTIONS . . . . . . . . . . . . 32

ATTACHMENT 2: SORTING GLOVEBOX HOIST CONTROL FUNCTIONS $\ldots \ldots \ldots$

ATTACHMENT 3: EXIT GLOVEBOX HOIST CONTROL FUNCTIONS $\ldots \ldots \ldots$

ATTACHMENT 4: GLOVEBOX INTERLOCK SIGNALS . . . . . . . . . . . . . . 35 


\section{WRAP 1 PLANT OPERATING PROCEDURE}

(HNF-SD-W026-0TP. 015)

\section{PROCEDURE (LLW ENTRY GLOVEBOX)}

\section{WARNING}

IMPROPER OPERATION OF GLOVEBOX EQUIPMENT INCLUDING HOIST, SORTING TABLE, MANIPULATORS, TRANSFER CAR, ETC. MAY CAUSE INJURY. CAUTION MUST BE EXERCISED IF REACHING THROUGH GLOVEPORTS WHILE EQUIPMENT IS IN OPERATION.

\section{CAUTION}

WHEN AN OIU IS IN MAINTENANCE MODE, MOST INTERLOCKS AND SAFETY FEATURES ARE BYPASSED. IT IS POSSIBLE TO DAMAGE EQUIPMENT, AND/OR BYPASS SAFETY RESTRICTIONS SUCH AS GLOVEBOX CONTAINMENT. CAUTION MUST BE EXERCISED TO PREVENT VIOLATION OF OPERATIONAL SAFETY REQUIREMENTS OR EQUIPMENT DAMAGE.

NOTE 1 - In Maintenance mode, OIU selections must be selected (screen touched) until the actuated device is visually verified to have reached the desired state.

NOTE 2 - Gloveports and manipulators are used as needed to perform tasks throughout this procedure. Manipulator Operation is performed per WRP1-OP-0704, Glovebox Manipulator Operation.

NOTE 3 - Data Management System (DMS) actions throughout this procedure are performed on DMS Termina 7 (107-TE-12-103).

NOTE 4 - UNLESS OTHERWISE SPECIFIED, selections and displays in Section VII are on LLW Entry Operator Interface Unit (OIU-12-103A).

NOTE 5 - Lift Table 107-LT-09-202A contro7s and indicators in Section VII are located on Lift Table Contro7 Pedesta7 107-PC-09-202A. Manual Lift table operation is performed per WRP1-OP-0703. Glovebox Lift Table Operation.

NOTE 6 - Hoist activities in Section. VII are performed on Hoist Control (107-PC-07-001). Functions must be activated until associated indicator illuminates. Reference Attachment 1.

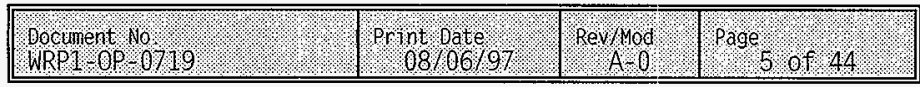




\section{WRAP 1 PLANT OPERATING PROCEDURE}

(HNF. SO W026-0TP-015)

A. INITIALIZE ENTRY GLOVEBOX

NOTE - Assumes Auto Initialization failed.

1. SELECT "DIAGNOSTICS MENU".

(PCS) Displays failed parameters in red, with negative messages and passed parameters in green with positive message.

2. OBSERVE diagnostic menu messages (Reference TabTe VII-1 on the following page).

3. ACCESS MAINTENANCE MENU screens per applicable sections of this procedure, to reset diagnostic flags as required.

4. VERIFY desired diagnostic flags are reset by performing the following:
a. RETURN to "MAINTENANCE MAIN MENU".
b. SELECT "DIAGNOSTICS MENU".
c. VERIFY display "PASS".
d. SELECT "MAIN MENU".
e. VERIFY display "SYSTEM INITIALIZED".
f. GO TO desired section(s).

NOTE - Sections VII.C through VII.L assume Maintenance Main Menu has been accessed per Section VII.B.

B. ACCESS ENTRY GLOVEBOX MAINTENANCE MENUS

$$
\text { LCW ENTrey GiLOUESOOD }
$$

1. PLACE DMAS keyswitch (107-07-HS-316) in "MAINTENANCE".

2. VERIFY display "MAINTENANCE ACCESS MENU".

3. KEY in maintenance password,

THEN, PRESS "ENTER".

4. VERIFY display "MAINTENANCE MAIN MENU".

5. SELECT desired maintenance submenu(s).

6. WHEN desired,

RETURN to Operating Menus as follows:
a. IF at any maintenance submenu, THEN, PRESS "MAINT MAIN MENU".
b. PLACE DMS keyswitch (107-07-HS-316) to "AUTO".

\begin{tabular}{|c|c|c|c|}
\hline $\begin{array}{l}\text { oocunent } 10, \\
\text { WRP }\end{array}$ & Print oate $08106 / 97$ & Rev/Mod & page 6.0144 \\
\hline
\end{tabular}




\section{WRAP 1 PLANT OPERATING PROCEDURE}

UNF SO W026-0IP 015)

TABLE VII-1: ENTRY GLOVEBOX INITIALIZATION INTERLOCKS

\begin{tabular}{|c|c|}
\hline : INTTIALITATION MESSAGE (faTled) & ST GNAL SOURCE \\
\hline D0-07-101 CENTERING CLAMPS NOT RETRACTED & 07-ZS-0712A/0712B \\
\hline LT-09-202A M/0/N NOT IN NORMAL & $07-H S-233 A$ \\
\hline LT-09-202A LIFT TABLE NOT AT AGV HEIGHT & $07-Z S-233 G$ \\
\hline DO-07-101 PORT DOOR NOT CLOSED AND LOCKED & 07-ZSC-700A/700B \\
\hline DO-07-101 VACUUM NOT RELEASED & 07-PS-0703 \\
\hline CR-07-101A HOIST NOT RAISED & $07-7 S-702 C$ \\
\hline DH-07-101 LIFTER NOT OPEN & $07 \cdot Z S-704$ \\
\hline CR-07-101A HOIST NOT AT TRANSFER PORT & $07-Z S-702 A / 702 B$ \\
\hline DO-07-102 PORT DOOR NOT CLOSED & $07-\mathrm{ZSC}-705$ \\
\hline D0-07-102 PORT DOOR SEAL NOT INFLATED & 07-PSL-700 \\
\hline
\end{tabular}




\section{WRAP 1 PLANT OPERATING PROCEDURE}

GNF SD 4026 OTP 015$)$

C. OPERATE ENTRY GLOVEBOX HOIST (107-CR-07-101A)

NOTE - Manual hoist operation is performed the same as in Automatic Mode, except hoist control is continuously enabled.

1. OPERATE hoist as required for glovebox tasks or maintenance.

D. MATE OVERPACK DRUM TO ENTRY PORT (107-D0-07-101)

1. REMOVE clamp band.

2. OPERATE Lift Table to position drum at "DRUM UNDER PORT".

3. SELECT in order, as required:

- $\quad$ "DRUM ENTRY/EXIT PORT MAINTENANCE MENU".

- "RETRACT CENTERING CLAMPS".

4. RAISE Lift Table to "DRUM CENTERING HEIGHT" as indicated by the local arrow Tabels.

5. SELECT "EXTEND CENTERING CLAMPS".

6. VERIFY display, "CENTERING CLAMPS EXTENDED".

7. RAISE Lift Table to "LID IN POSITION HEIGHT".

8. SELECT "MAINT MAIN MENU".

E. Mate single dRum to entry/h PORT (107-D0-07-101)

1. PQSiTION 55 gal drum centering spacer.

2. OPERATE Lift Table to position drum at "DRUM UNDER PORT".

3. SELECT in order, as required:

- "DRUM ENTRYYEXIT PORT MAINTENANCE MENU".

- "RETRACT CENTERING CLAMPS".

4. RAISE Lift Table to "LID IM POSITION HEIGHT".

5. SELECT "MAINT MAIN MENU".

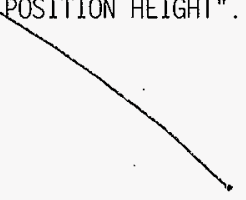




\section{WRAP 1 PLANT OPERATING PROCEDURE}

F. OPEN ENTRY PORT (107-D0-07-101) (OVERPACK DRUM)

1. SELECT in order as required:

- $\quad$ "DRUM ENTRY/EXIT PORT MAINTENANCE MENU".

- $\quad$ "TURN ON VACUUM".

- "UNLOCK PORT".

NOTE - Steps VII.F.2, VII.F.5, IX.E.8 and VII.F.4 are performed concurrent $7 y$.

2. PRESS and HOLD "OPEN VENTS" on OIU-12-103A.

3. VERIFY display "VENT SOLENOID ACTIVATED".

4. RAISE Lift Table until port door lifts $\sim 1 / 2$ inch.

5. RELEASE "OPEN VENTS" on OIU-12-103A.

6. SELECT "RAISE DOOR".

7. VERIFY display "DOOR RAISED".

8. SELECT "ROTATE PORT OPEN".

9. VERIFY display "PORT ROTATED OPEN".

10. SELECT "MAINT MAIN MENU". 


\section{WRAP 1 PLANT OPERATING PROCEDURE}

(HNF SB WO26 OTP 015 )

G. OPEN ENTRY PORT (107-D0-07-101) (SINGLE DRUM)

1. SELECT "DRUM ENTRY/EXIT PORT MAINTENANCE MENU".

2. PRESS and HOLD "OPEN VENTS" on OIU-12-103A.

3. VERIFY display "VENT SOLENOID ACTIVATED".

4. SELECT "UNLOEK PORT".

5. VERIFY displaY "PORT UNLOCKED".

6. RELEASE "OPEN VENTS" on 0IU-12-103A.

7. SELECT "RAISE DOOR"

8. VERIFY display "DOOR RAISED".

9. SELECT "ROTATE PORT OPEN".

10. VERIFY display "PORT ROTATED OPEN".

11. RAISE Lift Table until drum at desired height.

12. SELECT "MAINT MAIN MENU". 


\section{WRAP 1 PLANT OPERATING PROCEDURE}

H. CLOSE ENTRY PORT (107-D0-07-101) (OVERPACK DRUM)

1. SELECT "DRUM ENTRY/EXIT PORT MAINTENANCE MENU".

2. SELECT "ROTATE PORT CLOSED".

3. VERIFY display "PORT ROTATED CLOSED".

4 SELECT "LOWER DOOR".

5. VERIFY display, "DOOR LOWERED".

6. SELECT "TURN OFF VACUUM".

7. VERIFY display "TURN ON VACUUM" (e.g. , no VACUUM GENERATED display).

8. SELECT "EXTEND LID DETACH".

9. VERIFY display "EXTEND SOLENOID ACTIVATED".

10. SELECT "RETRACT LID DETACH".

11. VERIFY display "RETRACT SOLENOID ACTIVATED".

12. LOWER Lift Table to "DRUM CENTERING HEIGHT" as indicated by the local arrow labels.

13. SELECT "LOCK PORT".

14. VERIFY display "PORT LOCKED".

15. SELECT "MAINT MAIN MENU".

I. CLOSE ENTRY PORT (107-D0-07-101) (SINGLE DRUM)

1. SELECT "DRUMMENTRY/EXIT PORT MAINTENANCE MENU".

2. SELECT "ROTATE CLOSE PORT".

3. VERIFY display "PQRT ROTATED CLOSED".

4. SELECT "LOCK PORT".

5. VERIFY display "PORT LOLKED".

6. SELECT "MAINT MAIN MENU".

7. LOWER Lift Table to "AGV LOAD HEIGHT". 


\section{WRAP 1 PLANT OPERATING PROCEDURE}

J. REMOVE OVERPACK DRUM FROM ENTRY PORT (107-D0-07-101)

1. LOWER Lift Table to "AGV LOAD HEIGHT".

2. MOVE drum to "DRUM AT AGV END" position.

K. OPEN TRANSFER PORT (107-DO-07-102)

1. ENSURE Entry Port (107-D0-07-101) is CLOSED \& LOCKED.

2. SELECT "DRUM TRANSFER PORT MAINTENANCE MENU".

3. SELECT "OPEN ISOLATION VALVE".

4. VERIFY display "OPEN SOLEHOTD ACT YATEE".

5. SELECT "DEFLATE SEAL".

6. VERIFY display "SEAL DEFLATED".

7. SELECT "OPEN PORT".

8. VERIFY port OPEN.

9. SELECT "MAINT MAIN MENU".

L. CLOSE TRANSFER PORT (107-D0-07-102)

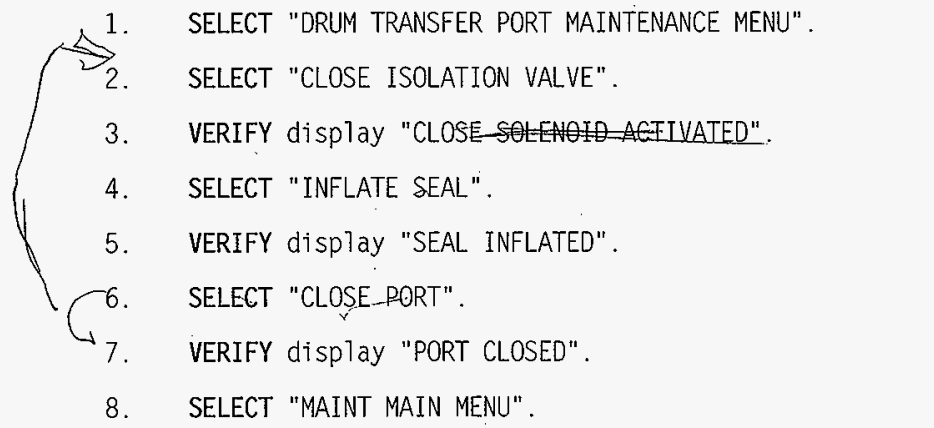


HNF-SD-W026-0TR-015, Rev. 0, Page OP-190

\section{WRAP 1 PLANT OPERATING PROCEDURE}

(1NF SB $1026-0 T P$ - 015$)$

VIII. PROCEDURE (LLW SORTING GLOVEBOX)

WARNING

IMPROPER OPERATION OF GLOVEBOX EQUIPMENT INCLUDING HOIST, SORTING TABLE, MANIPULATORS, TRANSFER CAR, ETC. MAY CAUSE INJURY. CAUTION MUST BE EXERCISED IF REACHING THROUGH GLOVEPORTS WHILE EQUIPMENT IS IN OPERATION.

\section{CAUTION}

WHEN AN OIU IS IN MAINTENANCE MODE, MOST INTERLOCKS AND SAFETY FEATURES ARE BYPASSED. IT IS POSSIBLE TO DAMAGE EQUIPMENT, AND/OR BYPASS SAFETY

RESTRICTIONS SUCH AS GLOVEBOX CONTAINMENT. CAUTION MUST BE EXERCISED TO PREVENT UNWANTED VIOLATION OF OPERATIONAL SAFETY REQUIREMENTS OR EQUIPMENT DAMAGE.

NOTE 1 - In Maintenance mode, OIU selections must be selected (screen touched) until the actuated device is visually verified to have reached the desired state.

NOTE 2 - Manual operation of Bagless Transfer Port 107-D0-07-105 is performed per WRP1-0P-0705, Bagless Transfer Manual Operation.

NOTE 3 - UNLESS OTHERWISE SPECIFIED, selections and displays in Section VIII are on LLW Sorting Operator Interface Unit (OIU-12-103B).

NOTE 4 - Lift Table 107-LT-09-203A controls and indicators in Section VIII are 10cated on Lift Table Control Pedesta 7 107-PC-09-203A. Manual Lift table operation is performed per WRP1-0P-0703. Glovebox Lift Table Operation.

NOTE 5 - Hoist activities in Section VIII are performed on Hoist Control (107-PC-07-003A or 003B). Active controller is selected by switch on 107-PC-07-003A. Functions must be activated unti7 associated indicator inluminates. Reference Attachment 2. 


\section{WRAP 1 PLANT OPERATING PROCEDURE}

(HNF. SD W026 - OTP . 015)

\section{A. INITIALIZE SORTING GLOVEBOX}

NOTE - Assumes Auto Initialization failed.

1. SELECT "INITIAL OIAGNOSTICS".

(PCS) Displays failed parameters in red, with negative messages and passed parameters in green with positive message.

2. OBSERVE fault messages (Reference Table VIII-1 on the following page) .

3. ACCESS MAINTENANCE MENU screens per applicable sections of this procedure, as necessary to correct indicated initialization faults.

4. WHEN all faults have been corrected:

a. RETURN to Operating Menus.

b. SELECT "MAIN MENU".

c. VERIFY display "SYSTEM INITIALIZED".

d. GO TO desired section(s).

NOTE - Sections VIII.C through VIII.O assume Maintenance Main Menu has been accessed per Section VIII.B.

B. ACCESS SORTING GLOVEBOX MAINTENANCE MENUS

1. PLACE DMS keyswitch (107-07-HS-317) to "MAINTENANCE".

2: VERIFY display "MAINTENANCE ACCESS MENU".

3. KEY in maintenance password, THEN, PRESS "ENTER".

4. VERIFY display "SORTING GLOVEBOX MAINTENANCE MAIN MENU".

5. SELECT desired maintenance submenu(s).

6. WHEN desired,

RETURN to Operating Menus as follows:

a. IF at any maintenance submenu.

THEN, PRESS "MAINT MAIN MENU".

b. PLACE DMS keyswitch (107-07-HS-317) to "AUTO". 


\section{WRAP 1 PLANT OPERATING PROCEDURE}

(HNF SD W026-0TP 015)

\begin{tabular}{|c|c|}
\hline 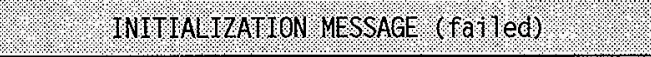 & . .1 .4 .5 SGNA. SOUREE. \\
\hline RT-07-101 FIXTURE ROTATION HEAD NOT PARKED & 07-ZS-727A \\
\hline D0-07-105 BAGLESS XFER PORT NOT CLOSED & $\begin{array}{l}\text { PORT CONTROL LOGIC } \\
\text { RELAY, DO-07-105/CS }\end{array}$ \\
\hline TC-07-101 TRAASFER CAR NOT AT ENTRY & $07-Z S-710$ \\
\hline ST-07-101 TABLE NOT IN LOWERED POSITION & $07-Z S-716 \mathrm{~A} / 716 \mathrm{~B}$ \\
\hline ST-07-101 TABLE NOT FULLY RETRACTED & $07-7 S-717 C$ \\
\hline DH-07-103 TIPPER NOT RAISED & $07-7 S-332 A$ \\
\hline DH-07-103 TIPPER NOT IN VERTICAL POSITION & $07 \cdot 7 S \cdot 333$ \\
\hline DH-07-103 DRUM GRAB NOT OPEN & $07-\mathrm{ZS}-720 \mathrm{~B}$ \\
\hline RT-07-101 FIXTURE NOT AT PARK/RELID POSITION & $07-7 S-728 \mathrm{~A}$ \\
\hline RT-07-101 FIXTURE NOT RAISED & $07 \cdot 7 S \cdot 726 \mathrm{~A}$ \\
\hline RT-07-101 SAW NOT RETRACTED & $07 \cdot 7 S \cdot 742 B$ \\
\hline RT-07-101 SAW IS RUNNING & 07-FEV-378A \\
\hline RT-07-101 CRIMP HEAD IS NOT BACK & 07-ZS-719A \\
\hline RT-07-101 CRIMP JAWS NOT OPEN & $07-7 S-721$ \\
\hline TC-07-101 TRANSFER CAR DRIVE NOT DE-ENERGIZED & $107-\mathrm{TC}-07-101 / \mathrm{M} 1$ \\
\hline ST-07-101 DRIVE ENGAGED & $07-7 S-718 \mathrm{~A}$ \\
\hline LT-09-203A M/0/N SWITCH NOT IN NORMAL & $07-H S-241 \mathrm{~A}$ \\
\hline CR-07-101C HOIST NOT AT LOADOUT & $07-Z S-730 \mathrm{~A}$ \\
\hline CR-07-101C HOIST NOT RAISED & $07-\mathrm{ZS}-730 \mathrm{C}$ \\
\hline EM-07-201 MANIPULATOR NOT PARKED & $07-2 S-715 A / 715 B$ \\
\hline EM-07-201 MANIPULATOR ARMS NOT PARKED & $07-2 S-715 C / 715 D$ \\
\hline
\end{tabular}

\begin{tabular}{|c|c|c|c|}
\hline bocument 10. & pront $00 \mathrm{ate}$ & Rey Mod & Page 15 of 44 \\
\hline
\end{tabular}




\section{WRAP 1 PLANT OPERATING PROCEDURE}

HNF SD W026-0TP 0:15)

C. OPERATE SORTING GLOVEBOX HOIST (107-CR-07-101C)

NOTE 1 - Manual hoist operation is performed the same as in Automatic Mode, except hoist control is continuously enabled.

NOTE 2 - Hoist 107-CR-07-101B is for maintenance and is not used during. normal operation. Its' controller functions the same as others.

1. OPERATE hoist as required for glovebox tasks or maintenance.

D. OPEN/CLOSE BAGLESS TRANSFER PORT (107-D0-07-105)

1. ENSURE hoist 107-CR-07-101C is at "RAISED LIMIT".

$E T R \rightarrow 2 . \quad$ ENSURE hoist 107-CR-07-101C TROLLEY is at "LOAD".

3. OPERATE port per WRP1-0P-0705. Bagless Transfer Manual Operation.

E. MOVE DRUM TRANSFER CAR (107-TC-07-101)

1. ENSURE manipulators and manipulator arms are PARKED.

2. ENSURE lidder/delidder is RAISED.

3. ENSURE drum tipper is RAISED.

4. SELECT "DRUM TRANSFER CAR AND MISCELLANEOUS EQUIPMENT MAINTENANCE MENU".

5. PERFORM on "DRUM TRANSFER CAR" submenu:

a. IF display "TRANSFER CAR STOP ENGAGED" is present, THEN SELECT "DISENGAGE TRANSFER CAR STOP".

b. SELECT one of the following until car nears destination:

- $\quad$ "MOVE LEFT" (move toward entry port).

- "MOVE RIGHT" (move toward transfer port).

c. WHEN the Transfer Car is in the desired position, THEN SELECT "ENGAGE TRANSFER CAR STOP".

6. SELECT "MAINT MAIN MENU". 


\section{WRAP 1 PLANT OPERATING PROCEDURE}

F. MOVE OR ROTATE DRUM LID/DELID FIXTURE (107-RT-07-1.01)

1. ENSURE manipulators and manipulator arms are PARKED.

2. SELECT "DRUM LID/DELID FIXTURE MAINTENANCE MENU".

NOTE - The displays shown in parentheses below are the alternate states for the indicated buttons.

3. SELECT on "DELID FIXTURE" submenu as required:

"RAISE FIXTURE"
"LOWER FIXTURE"
"MOVE LEFT"
"ROTATE RIGHT"
"ROTATE COUNTWWISE"

(FIXTURE RAISED)

(FIXTURE LOWERED)

(FIXTURE AT DELID POSITION)

(FIXTURE AT PARK/RELID POSITION)

(ROTATING CLOCKWISE)

(ROTATING COUNTER CLOCKWISE)

4. SELECT "MAINT MAIN MENU". 


\section{WRAP 1 PLANT OPERATING PROCEDURE}

G. OPERATE LID/DELID SAW

\section{CAUTION}

MANIPULATORS MUST BE CLEAR OF SAW OPERATING AREA.

1. ENSURE manipulators and manipulator arms are PARKED.

NOTE - Cut positions are selected as needed to saw through clamp band without hitting clamp band bolt.

2. ROTATE Tid/delid fixture to desired cut position.

3. LOWER lid/delid fixture onto drum.

\section{CAUTION}

BAND HALVES MUST BE HELD WITH MANIPULATORS AFTER FIRST GUT IS MADE.

4. IF second cut,

THEN, GRIP band halves with manipulators.

$\because$ 5. SELECT "DRUM LID/DELID FIXTURE MAINTENANCE MENU".

NOTE - $\quad$ Steps VIII.G.6 through VIII.G.17 below are accomplished using "LID/DELID SAW" submemr.

6. SELECT "TURN ON INERT GAS".

7. VERIFY display "INERT GAS ON".

8. SELECT "EXTEND SAW".

9. VERIFY display "SAW EXTENDED".

10. SELECT "TURN ON SAW".

11. VERIFY display "SAW ON".

12. WHEN clamp band is completely cut, SELECT "RETRACT SAW".

13. VERIFY display "SAW RETRACTED".

14. SELECT "TURN OFF SAW".

15. VERIFY display "SAW OFF". 


\section{WRAP 1 PLANT OPERATING PROCEDURE}

16. SELECT "TURN OFF INERT GAS"

17. VERIFY display "INERT GAS OFF".

18. RAISE lid/delid fixture.

19. REPEAT Steps VIII.G.2 through VIII.G.18 for each cut.

20. SELECT "MAINT MAIN MENU".

H. DELID WASTE DRUM

1. ENSURE clamp band is cut and removed.

2. ENSURE manipulators and manipulator arms are PARKED.

3. SELECT "DRUM LID/DELID FIXTURE MAINTENANCE MENU"

4. LOWER Tid/delid fixture onto drum.

5. SELECT on "LID LIFTER" submenu in order:

NOTE - Steps VIII.H.6 through VIII.H.9 below are accomplished using "LID/DELID SAW" submenu.

6. SELECT "OPEN ISOLATION VALVE",

7. VERIFY dTSPTAY "TSOLAFION VALVE OPEN".

8. SELECT "TURN ON VACUUM".

9. VERIFY display "VACUUM GENERATED".

10. RAISE lid/delid fixture.

11. MOVE Tid/delid fixture to relid/park position.

12. SELECT "MAINT MAIN MENU". 


\section{WRAP 1 PLANT OPERATING PROCEDURE}

I. RELID WASTE DRUM

1. ENSURE manipulators and manipulator arms are PARKED.

$\$ 2$. LOWER Tid/delid fixture onto drum.

SELECT "DRUM LID/DELID FIXTURE MAINTENANCE MENU".

NOTE - Steps VIII.I.4 through VIII.I.8 below are accomplished using "LID LIFTER" submenu.

4. SELECT "TURN OFF VACUUM".

5. VERIFY display "YALES

6. SELEGF "LLOSE ISOLADJUN VALVE .

7. WERIFY dispTaY "SOLAFIOWALVE-CLOSED".

8. RAISE lid/deTid fixture.

NOTE - Step VIII.I.9 may be skipped if lid is to be c-crimped.

9. SELECT "MAINT MAIN MENU".

J. C-CLIP AND CRIMP LID

1. ENSURE manipulators and manipulator arms are PARKED.

2. ENSURE drum lid is in place.

NOTE - Extra C-clips are stored on shelf inside glovebox, under Consumable Entry Port, on front side of glovebox.

3. MANUALLY PLACE C-clip in crimping device.

4. POSITION crimping device and C-clip on drum edge.

5. CRIMP $\mathrm{C}-\mathrm{C}$ - Tip by operating lever on side of crimping device.

6. - ROTAFE to next crimp position and repeat Steps VIII.J.3 through VIII.J.5 unti1 a11 desired C-clips are in place on drum lid. 


\section{WRAP 1 PLANT OPERATING PROCEDURE}

K. OPEN/CLOSE DRUM GRAB

1. ENSURE overbag is removed from drum, as applicable.

2. ENSURE manipulators PARKED in Position 1 and STOWED.

3. SELECT "SORTING TABLE/PACKET/DRUM TIPPER/DRUM GRAB MAINTENANCE MENU".

4. SELECT on "DRUM TIPPER" submenu, as required:

- $\quad$ "OPEN GRAB" to release drum.

- "ClOSE GRAB" to hold drum.

5. SELECT "MAINT MAIN MENU".

L. RAISE/LOWER/PARK DRUM TIPPER (107-DH-07-103)

1. ENSURE manipulators are in PARK POSITION \#1.

2. SELECT "SORTING TABLE/PACKET/QRUM TIPPER/DRUM GRAB MAINTENANCE MENU".

3. SELECT on "DRUM TIPPER" submenu, as required:

NOTE - Drum may be raised/lowered in intervals as necessary. Tipper PARK is FULL UP position.

- $\quad$ "RAISE TIPPER" until drum elevates to desired position.

- $\quad$ "LOWER TIPPER" until drum lowers to desired position.

4. SELECT "MAINT MAIN MENU". 


\section{WRAP 1 PLANT OPERATING PROCEDURE}

(INF SD . W026 -0TP - 0 15 )

M. ROTATE DRUM TIPPER (107-DH-07-103)

1. ENSURE manipulators are in PARK POSITION \#1.

2. ENSURE drum tipper is raised FULL UP.

3. SELECT "SORTING TABLE/PACKET/DRUM TIPPER/DRUM GRAB MAINTENANCE MENU".

NOTE - Steps VIII.M.4 through VIII.M.8 below are performed from the "DRUM TIPPER" submenu.

4. SELECT "ENABLE ROTATE JOYSTICK".

5. VERIFY display "ROTATE JOYSTICK ENABLED".

6. ROTATE grab right OR ROTATE grab left until tipper is at desired position.

7. SELECT ."DISABLE ROTATE JOYSTICK".

8. VERIFY "ROTATE JOYSTICK DISABLE".

9. SELECT "MAINT MAIN MENU".

N. EXTEND/RETRACT SORTING TABLE (107-ST-07-101)

1. ENSURE manipulators are PARKED above Lid/Delid Fixture.

2. SELECT "SORTING TABLE/PACKET/DRUM TIPPER/DRUM GRAB MAINTENANCE MENU".

NOTE - Steps VIII.N.3 through VIII.N.8 below are performed from the "SORTING TABLE" sub-menu.

3. SELECT "UNLOCK TABLE".

4. VERIFY display "TABLE UNLOCKED".

5. SELECT "EXTEND TABLE" OR "RETRACT TABLE" unti1 table is at desired position.

6. SELECT "LOCK TABLE".

7. VERIFY display "TABLE LOCKED".

8. SELECT "MAINT MAIN MENU". 
HNF-SD-W026-0TR-015, Rev, 0, Page OP-200

\section{WRAP 1 PLANT OPERATING PROCEDURE}

0. RAISE/LOWER SORTING TABLE (107-ST-07-101)

1. ENSURE manipulators are PARKED above Lid/Delid Fixture.

2. SELECT "SORTING TABLE/PACKET/DRUM-TIPPER/DRUM GRAB MAINTENANCE MENU".

NOTE - Steps through below are performed from the "SORTING TABLE" submenu.

3. SELECT "UNLOCK TABLE".

4. VERIFY display "TABLE UNLOCKED".

5. RAISE table OR LOWER table until table is at desired position.

6. SELECT "LOCK TABLE".

7. VERIFY display "TABLE LOCKED".

8. SELECT "MAINT MAIN MENU". 


\section{WRAP 1 PLANT OPERATING PROCEDURE}

(HNF SD - W026 - OTP 015)

\section{PROCEDURE (LLW EXIT GLOVEBOX)}

WARNING

IMPROPER OPERATION OF GLOVEBOX EQUIPMENT INCLUDING HOIST, SORTING TABLE, MANIPULATORS, TRANSFER CAR, ETC., MAY CAUSE INJURY. CAUTION MUST BE EXERCISED IF REACHING THROUGH GLOVEPORTS WHILE EQUIPMENT IS IN OPERATION.

\section{CAUTION}

WHEN AN OIU IS IN MAINTENANCE MODE, MOST INTERLOCKS AND SAFETY FEATURES ARE BYPASSED. IT IS POSSIBLE TO DAMAGE EQUIPMENT, AND/OR BYPASS SAFETY

RESTRICTIONS SUCH AS GLOVEBOX CONTAINMENT. CAUTION MUST BE EXERCISED TO PREVENT UNWANTED VIOLATION OF OPERATIONAL SAFETY REQUIREMENTS OR EQUIPMENT DAMAGE.

NOTE 1 - In Maintenance mode, OIU selections must be selected (screen touched) until the actuated device is visually verified to have reached the desired state.

NOTE 2 - UNLESS OTHERWISE SPECIFIED, selections \& displays in Section IX are on LLW Exit operator Interface Unit (OIU-12-103C).

NOTE 3 - Lift Tab7e 107-LT-09-202A contro7s and indicators in Section IX are located on Lift Table Control Pedestal 107-PC-09-202A. Manual Lift table operation is performed per WRP1-OP-0703. Glovebox Lift Table Operation.

NOTE 4 - Hoist activities in Section IX are performed on Hoist Control (107-PC-07-004). Functions must be activated unti7 associated indicator i77uminates. Reference Attachment 3. 


\section{WRAP 1 PLANT OPERATING PROCEDURE}

(HNF . SD . W026 - OTP . 015)

A. INITIALIZE EXIT GLOVEBOX

NOTE - Assumes Auto Initialization failed.

1. SELECT "DIAGNOSTICS MENU".

(PCS) Displays failed parameters in red, with negative messages and passed parameters in green with positive message.

2. OBSERVE fault messages (Reference Table IX-1.).

3. ACCESS MAINTENANCE MENU screens per applicable sections of this procedure, as necessary to correct indicated initialization faults.

4. WHEN all faults have been corrected:
a. RETURN to Operating Menus.
b. SELECT "MAIN MENU".
c. VERIFY display "SYSTEM INITIALIZED".
d. GO TO desired section(s).

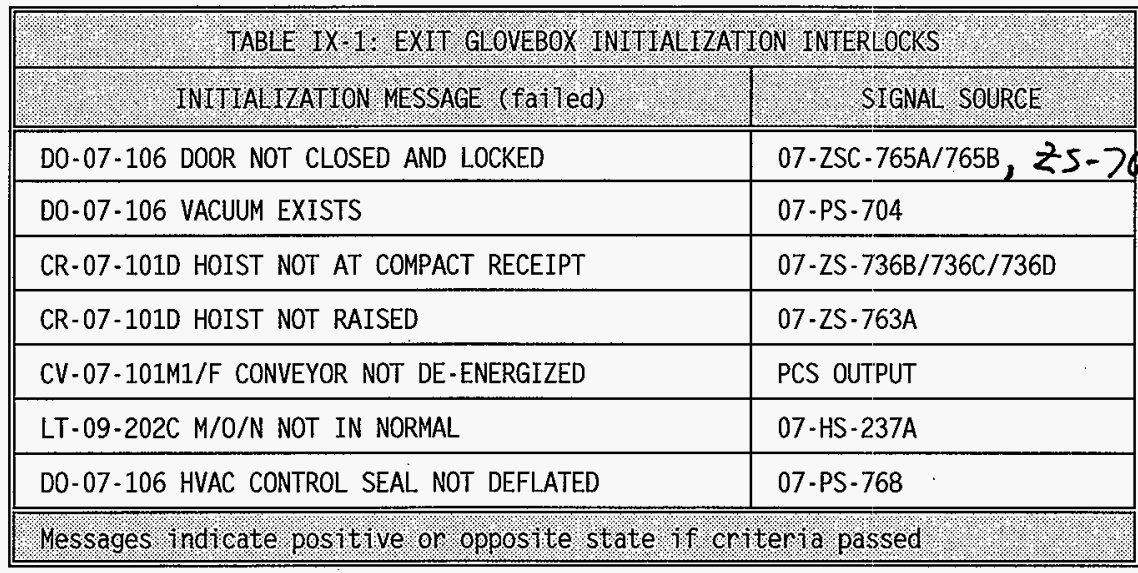




\section{WRAP 1 PLANT OPERATING PROCEDURE}

(MNF SO WO26 OTP - 015)

NOTE - $\quad$ Sections IX.C through IX.H assume Maintenance Main Menu has been accessed per Section IX.B.

B. ACCESS EXIT GLOVEBOX MAINTENANCE MENUS

1. PLACE DMS keyswitch (107-07-HS-318) to "MAINTENANCE".

2. VERIFY display "MAINTENANCE ACCESS MENU".

3. KEY in maintenance password, THEN, PRESS "ENTER".

4. VERIFY disp]ay "MAINTENANCE MAIN MENU".

5. SELECT desired maintenance submenu(s).

6. WHEN desired,

RETURN to Operating Menus as follows:

a. IF at any maintenance submenu. THEN, PRESS "MAINT MAIN MENU".

b. PLACE DMS keyswitch (107-07-HS-318) to "AUTO".

C. OPERATE EXIT GLOVEBOX HOIST (107-CR-07-101D)

NOTE - Manual hoist operation is performed the same as in Automatic Mode, except hoist control is continuously enabled.

1. OPERATE hoist as required for glovebox tasks or maintenance. 
HNF-SD-W026-0TR-015, Rev. 0, Page OP-204

WRAP 1 PLANT OPERATING PROCEDURE

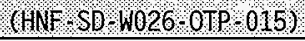

D. MATE DRUM TO EXIT PORT (107-D0-07-106)

SELECT MALT MADE

1. LOWER Lift Table as required.

2. REMOVE clamp band.

3. OPERATE Lift Table to position drum at "DRUM UNDER PORT" $\rightarrow$ SELECT "DRUM ENTRY/EXIT PORT MAINTENANCE MENU".

5. SELECT "RETRACT CENTERING CLAMPS".

6. VERIFY display "CENTERING CLAMPS RETRACTED".

7. RAISE Lift Table to "DRUM CENTERING HEIGHT". $-24 \%$

8. SELECT "EXTEND CENTERING CLAMPS".

9. VERIFY display "CENTERING CLAMPS EXTENDED".

10. RAISE Lift Table to "LID IN POSITION HEIGHT". -25 " 77 verify "COP IN PDSITION" or of pu

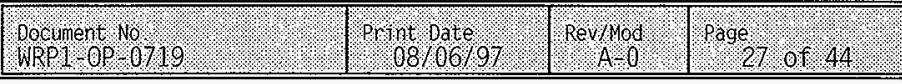




\section{WRAP 1 PLANT OPERATING PROCEDURE}

E. OPEN EXIT PORT (107-D0-07-106)

1. SELECT "DRUM EXIT/ENTRY PORT MAINTENANCE MENU"

2. SELECT "TURN ON VACUUM".

3. VERIFY display VACUUM GENERATED".

4. SELECT "UNLOCK PORT".

5. VERIFY display "PORT. UNLOCKED".

NOTE - Steps IX.E.6 and IX.E.7 are performed concurrent7y.

6. PRESS and HOLD "OPEN VENTS" on OIU.

7. RAISE Lift Table until port door lifts $\sim 1 / 2$ inch. VERIFY - "DOOR IN POSITION" ON OFu.

8. RELEASE "OPEN VENTS" on OIU.

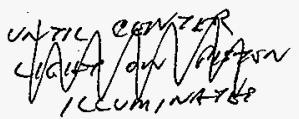

9. SELECT "RAISE DOOR".

10. VERIFY display "DOOR RAISED".

11. SELECT "ROTATE PORT OPEN".

12. VERIFY display "PORT ROTATED OPEN".

13. VERIFY port OPEN.

14. SELECT "MAINT MAIN MENU". 
HNF-SD-W026-0TR-015, Rev. 0, Page OP-206

\section{WRAP 1 PLANT OPERATING PROCEDURE}

(INF SB W026 - OTP 015)

F. OPERATE TRANSFER CAR CONVEYOR

1. SELECT "SORTING GLOVEBOX MAINTENANCE MAIN MENU".

2. SELECT "DRUM TRANSFER CAR AND MISCELLANEOUS EQUIPMENT MAINTENANCE MENU".

NOTE - StepS IX.F.3 through IX.F.10 are performed from the "TRANSFER CAR CONVEYOR" sUD-menu.

3. SELECT "ENGAGE CONVEYOR".

4. VERIFY display "CONVEYOR ENGAGED".

5. SELECT "TURN CONVEXOR ON" (until destination is reached).

6. VERIFY display "CONVEYOR ON".

7. SELECT "TURN CONVEYOR QFF".

8. VERIFY display "CONVEYOR QFF".

9. SELECT "DISENGAGE CONVEYOR"

10. VERIFY display "CONVEYOR DISENGAGED"

11. SELECT "MAINT MAIN MENU".

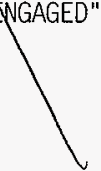




\section{WRAP 1 PLANT OPERATING PROCEDURE}

(HNF-SD W026 OTP 015$)$

G. RELID OVERPACK DRUM \& CLOSE EXIT PORT (107-D0-07-106)

1. ENSURE area around port sealing surface is free of debris.

2. SELECT "DRUM ENTRY/EXIT PORT MAINTENANCE MENU".

3. SELECT "ROTATE PORT CLOSED".

4. VERIFY display "PORT ROTATED CLOSED".

5. SELECT "LOWER DOOR".

6. VERIFY display "DOOR LOWERED" POSCT/ON" Or OIU. SELECT "TURN OFF VACUUM".

8. VERIFY display "TURN ON VACUUM" (i.e.. "VACUUM GENERATED" is o on not displayed.)

9. SELECT "EXTEND LID DETACH".

10. VERIFY display "EXTEND SOLENOID ACTIVATED".

11. SELECT "RETRACT LID DETACH".

12. VERIFY display "RETRACT SOLENOID ACTIVATED".

13. LOWER Lift Table to "DRUM CENTERING HEIGHT".

14. SELECT "LOCK PORT".

15. VERIFY display "PORT LOCKED".

16. VERIFY port CLOSED and LOCKED. UERIFY SEAC DEFLATQ

17. SELECT "MAINT MAIN MENU".

H. REMOVE OVERPACK DRUM FROM EXIT PORT (107-D0-07-106)

1. LOWER Lift Table to "DOWN OVERTRAVEL".

2. MOVE drum to "DRUM AT AGV END" position.

3. RAISE Lift Table to "AGV LOAD HEIGHT". 


\section{WRAP 1 PLANT OPERATING PROCEDURE}

I. MATE DRUM TO EXIT PORT (107-D0-07-106)

1. ENSURE drum clamp band is remover.

2. OPERATE Lift Table to position drum at "DRUM UNDER PORT".

3. SELECT "DRUM ENTRY/EXIT PORT MAINTENANCE MENU".

4. SELECT "RETRACT CENTERT NG CLAMPS".

5. VERIFY display "CENYERING CLAMPS RETRACTED".

6. RAISE Lift Table to "DRUM CENTERING HEIGHT".

7. SELECT "EXTEND CENTERING CLAMPS".

8. VERIFY display "CENTERING CLAMPS EXTENDED".

9. RAISE Ljft Table to "LID IN POSITION HEIGHT".

10. SELECT "MAINT MAIN MENU".

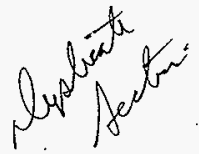


HNF-SD-W026-OTR-015, Rev. 0, Page OP-209

\section{WRAP 1 PLANT OPERATING PROCEDURE}

(HNF - SD W026- OIP 015 )

\section{ATTACHMENT 1: ENTRY GLOVEBOX HOIST CONTROL FUNCTIONS}

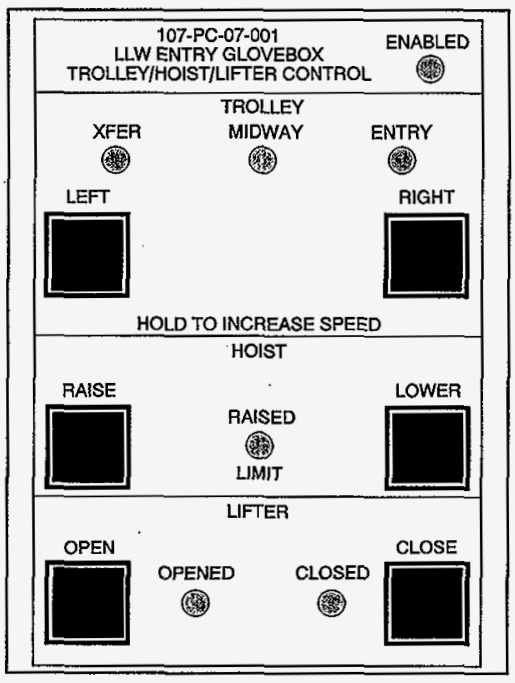

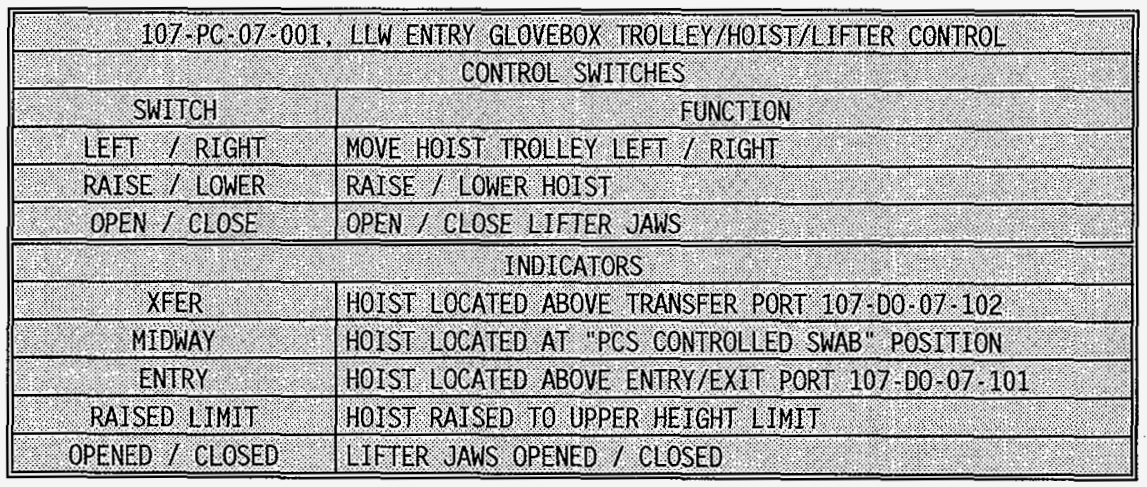

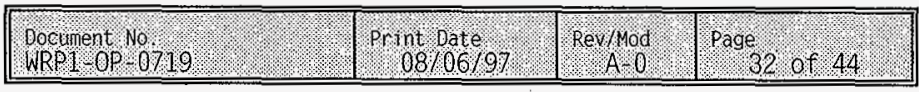




\section{WRAP 1 PLANT OPERATING PROCEDURE}

(HNF-SB 1026 - OTP 015$)$

ATTACHMENT 2: SORTING GLOVEBOX HOIST CONTROL FUNCTIONS

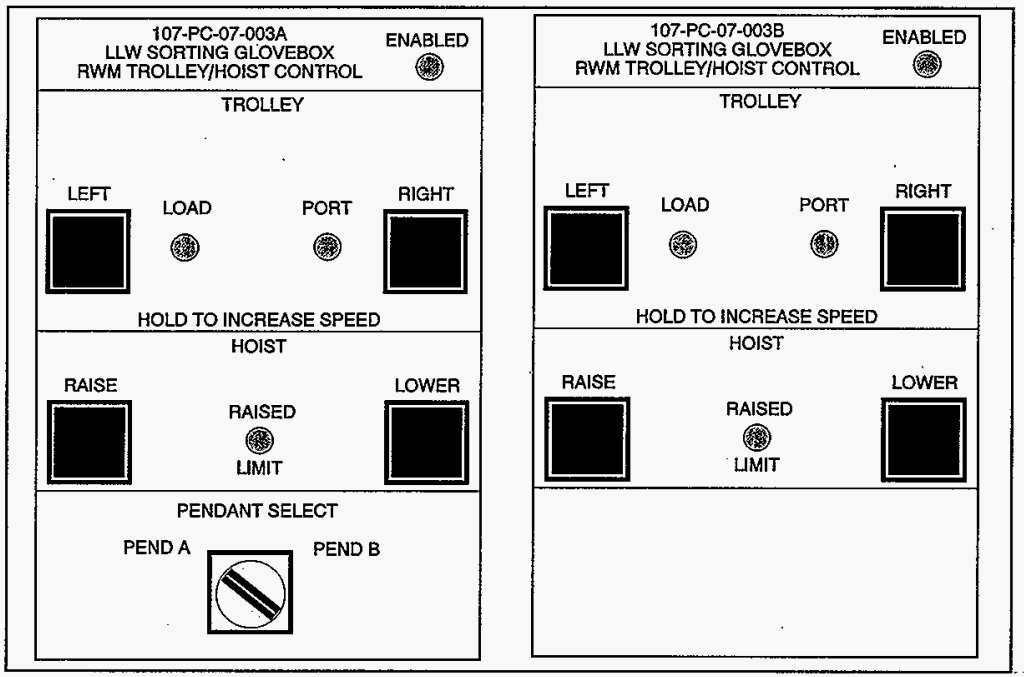

\begin{tabular}{|c|c|}
\hline \multicolumn{2}{|c|}{ 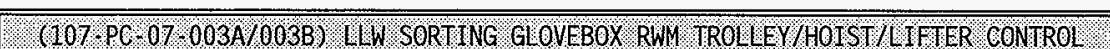 } \\
\hline (2.1.:. & 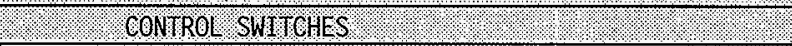 \\
\hline SWITH & . FUNCTION \\
\hline 2. LEFII I RIGHT & MOVE HOIST TROLLEY LEVI / RIGHT \\
\hline RAISE / LOWER & RAISE / IOWER IOIST: \\
\hline PENBANT SELECT $(\%)$ & SELECTS AC TVE CONTROL (107 PC 07 003A or $003 B)$ \\
\hline NOTE (t) : Act ive cont & 1) is selected at operator discretion. \\
\hline 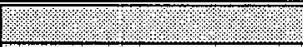 & 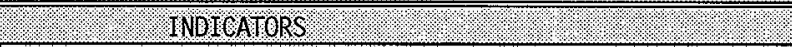 \\
\hline $10 \mathrm{AO}$ & HOIST AT TRANSFER STANO LOAO POSTMTON : $:$ : \\
\hline 1.:. PORT : & HOIST LOCALEO ABOVE RWI TRANSFER PORT ( 107 DO $0 \%$ 105) \\
\hline . RAISED IMTV & GOTST RAISEB TO UPPER HEIGAT I MMT : \\
\hline
\end{tabular}

\begin{tabular}{|c|c|c|c|}
\hline $\begin{array}{l}\text { gocument vo } \\
\text { wRP }\end{array}$ & pront 00 ave 1067 & Rev:yod & 48.80 .0844 \\
\hline
\end{tabular}




\section{WRAP 1 PLANT OPERATING PROCEDURE}

(HNF SD W026 0TP 015)

ATTACHMENT 3: EXIT GLOVEBOX HOIST CONTROL FUNCTIONS

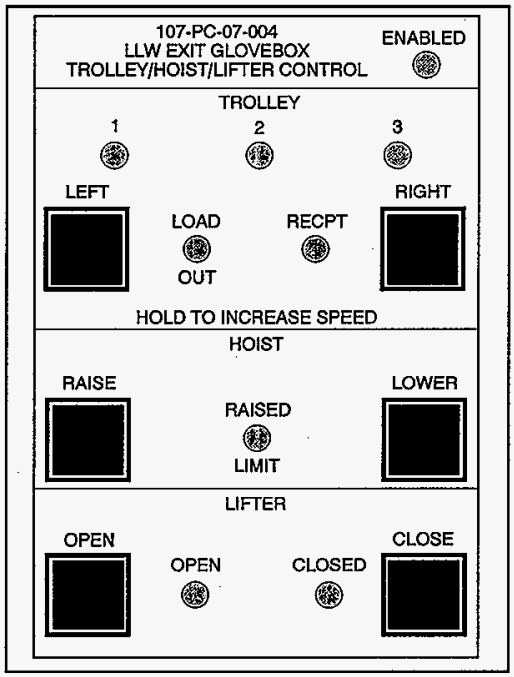

\begin{tabular}{|c|c|}
\hline.$\quad 107 \mathrm{PC} 07$ & 1) WW EXIT GLOVEBOX TROGLY/HOLST UITTER CONIROL \\
\hline 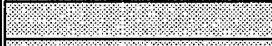 & CONTROL SWITCHES \\
\hline SWTCH: & 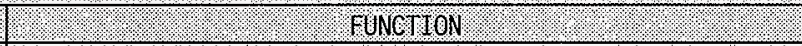 \\
\hline . LETI I RIGH & MOVE HOIST TROLGEY LETT I RIGHT: \\
\hline . RAISE / LOWER & 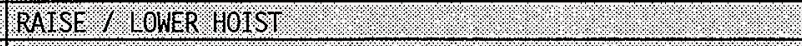 \\
\hline OPEN / CLOSE & OPEN / CLOSE LT LIER JANS \\
\hline 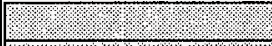 & TOLATORS \\
\hline 1.1 .2 .3 .3$. & 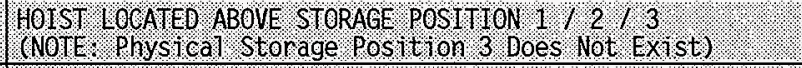 \\
\hline 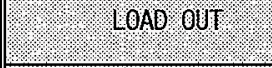 & $\begin{array}{l}\text { HOLST WOUES PUGK FRON STORAGE POST ION OVER LOAO OUT } \\
\text { POSI TON ANO LOKERS INTO OUERPACK DRUM }\end{array}$ \\
\hline : RECPT: $:$ : & MOLST LOCAIED ABOVE COMPACT PUCK RECETPT POSIIION \\
\hline 1. RAISED UMMT & HOIST RAISED TO UPPER HEIGHT LMIT: \\
\hline OPEN I CLOSED & GTTER JAWS OPEN / CLOSED \\
\hline
\end{tabular}

\begin{tabular}{|c|c|c|c|}
\hline Bocument 10, & Pr pre odte $0 \% 06$ & $\mathrm{Rey} / \mathrm{Mod}$ & Page 34 or 44 \\
\hline
\end{tabular}


HNF-SD-W026-OTR-015, Rev. 0, Page OP-212
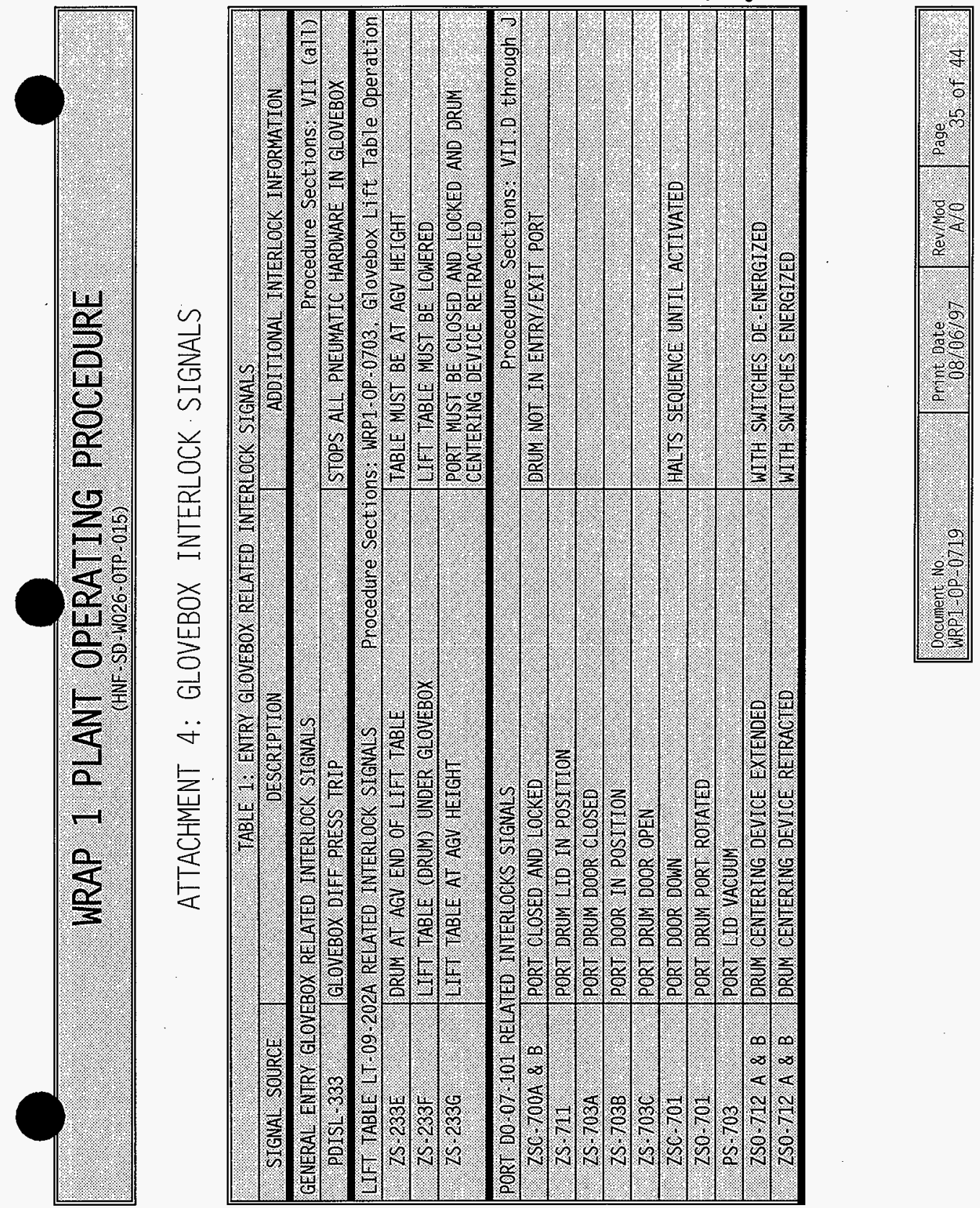


\section{WRAP 1 PLANT OPERATING PROCEDURE}

(INN SO WO26-OTP - 015)

\begin{tabular}{|c|c|c|}
\hline SIGNAL SOURCE & DESERIPTLON & ADOIIIONAL INIERLOCK INFORMATION \\
\hline 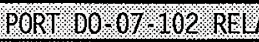 & LED INIERLOCK ST GNALS & Procedire Sections: \\
\hline 2SC 705 & PORT BO 07 T 102 CLOSEB & m. \\
\hline 250.705 & PORT BO 07 102 OPEN : & (1.1.1. \\
\hline PSL. 700 & PORT DO 07.102 SEAL INELATED: & BRUM IN SPHINGTER POSTTION : : : \\
\hline PSIL 701: & SEAL BEFATEB: : : & PORT MUST BE CLOSEB \& LOGKED : : \\
\hline \multicolumn{3}{|c|}{ HOIST REIATEB TNERLOCK STGNALS: Procedure Sections: UIT.C } \\
\hline $2 \mathrm{~S}, 702 \mathrm{~A} .2 .3$ & HOIST AT CENTER OF GLOVEBOX : : & DRUM LTITER MUST BE RATSEO . \\
\hline $2 \mathrm{~S} .102 \mathrm{~A} \& \mathrm{~B}$ & HOIST ABOVE IRANSFER PORT DO 07.102 & BRUM LIFTER MUST BE RATSEO \\
\hline $2 \mathrm{~S} .702 \mathrm{~B}$ & HOIST ABOVE ENTRY PORT DO OT $10 \mathrm{I}$. & DRUM LIF IER MUST BE RAISEB \\
\hline 25,7020 & 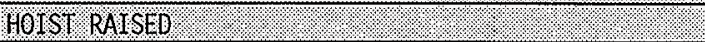 & (1.1. \\
\hline 250.704 & DRUM I IIIER OH 07 . 101 JAWS OPEN & DRUM NOT IN LIFTER \\
\hline $25 C 704$ & GRUM LIETER JAWS CLOSEB : : : & (1) \\
\hline
\end{tabular}


HNF-SD-W026-0TR-015, Rev, 0, Page OP-214

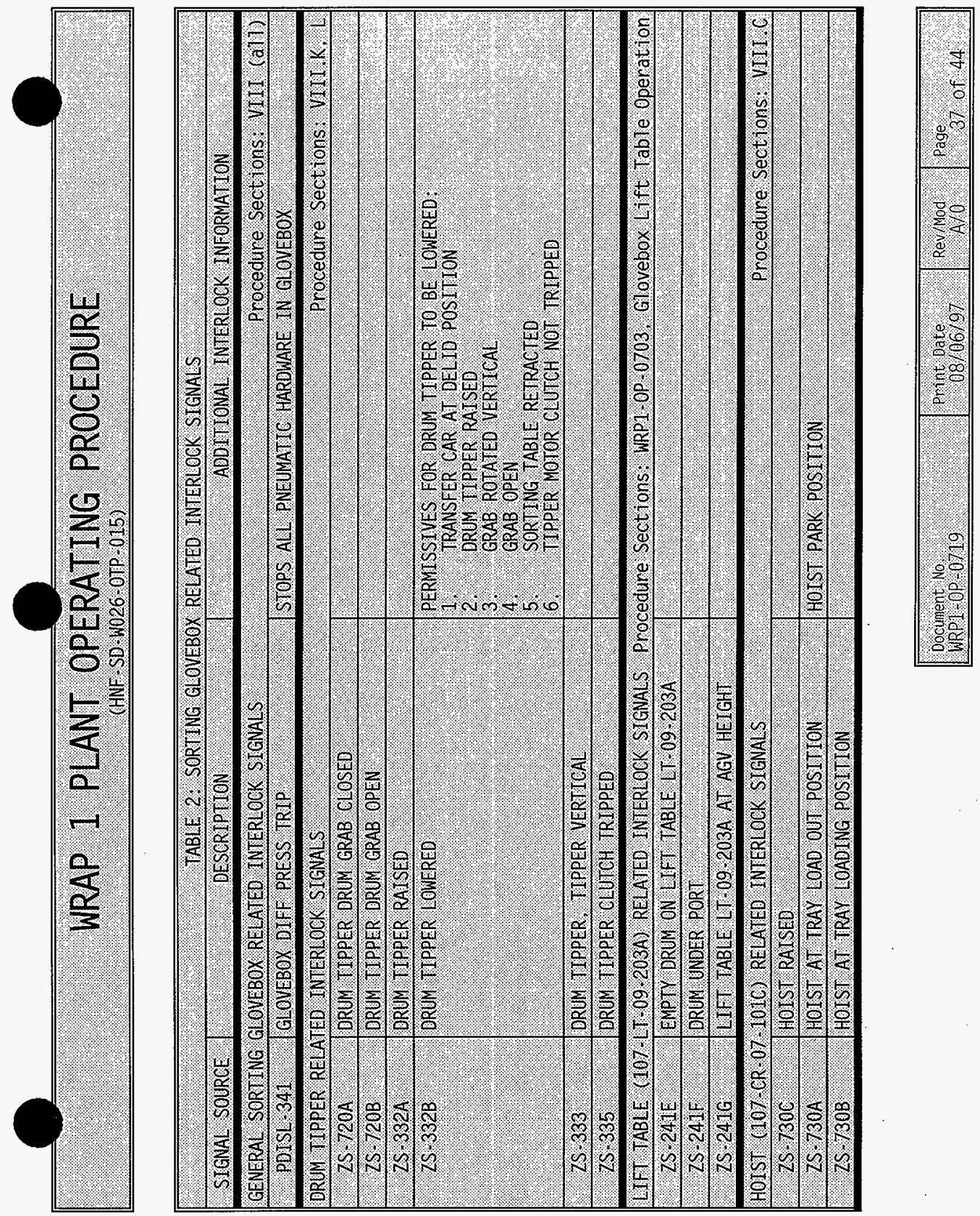


HNF-SD-W026-OTR-015, Rev. 0, Page OP-215
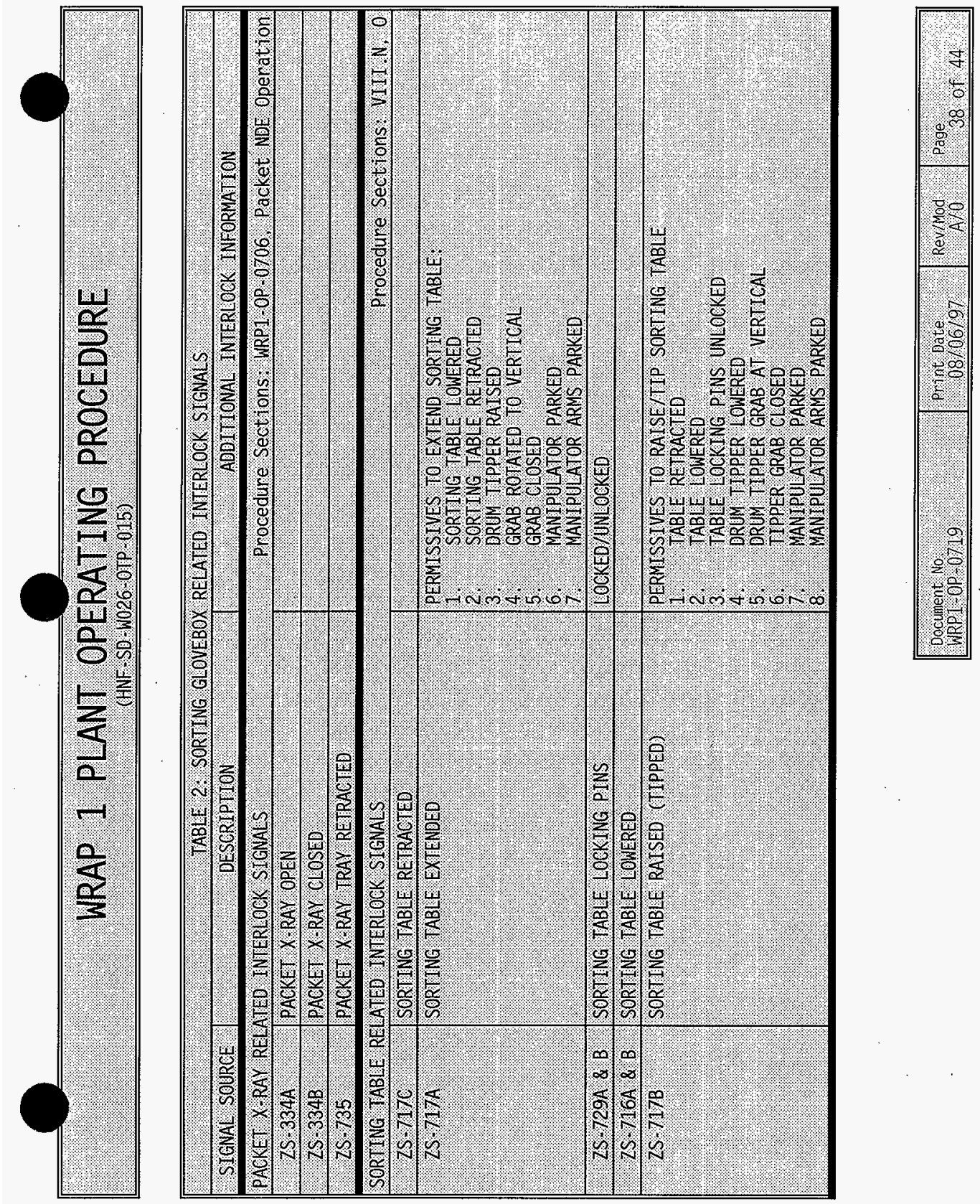


\section{WRAP 1 PLANT OPERATING PROCEDURE}

\begin{tabular}{|c|c|c|}
\hline SIGNAL SOIRCE & DESGRIPTION & ADDITHONAL INTERLOCK INFOR \\
\hline TO/BELL FTXI & RELATEO INTERLOQK STGNALS & Procedure Secttions: \\
\hline & LIB/GELID T TWUURE RAISED: & 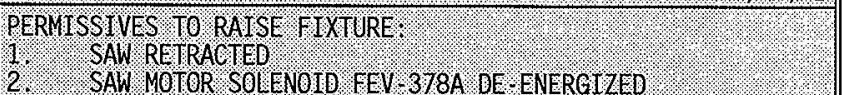 \\
\hline $2 S .726 \mathrm{~B}$ & LTO/OELIO ITYIURE LWERED & 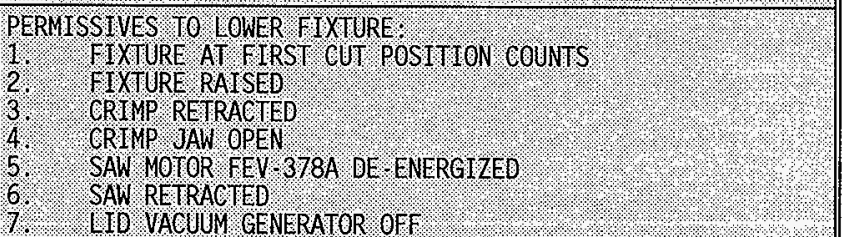 \\
\hline $25.727 \mathrm{~A}$ & ITO/BELIO FIXTURE ROTATION PARKED & 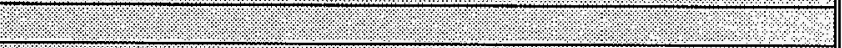 \\
\hline $2 S .727 B$ & LIO/OE IO FIXTURE ROTATTON INBEX. & SWITCHES REAB FLAGS ON FIXTURE AS IT YXTURE ROTATES \\
\hline $2 S .728 \mathrm{~A}$ & 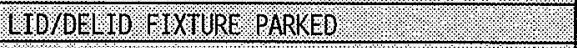 & REIID POSTTION : \\
\hline & IDVEELIO F XXIURE AT QELIO POSIIION & 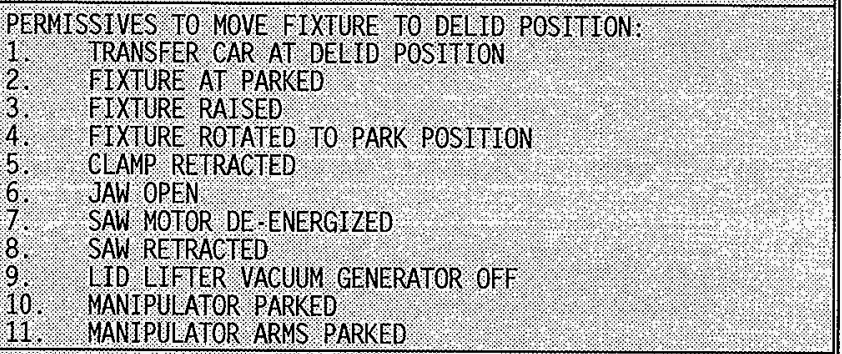 \\
\hline PS 17.6. & TDYOEL IO F TXTURE LID VACUUM & (1.9. \\
\hline
\end{tabular}

\begin{tabular}{|c|c|c|c|}
\hline 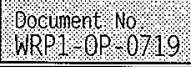 & Pront 0 ate $086 \%$ & Rev/Mod & page \\
\hline
\end{tabular}


HNF-SD-W026-OTR-015, Rev. 0, Page OP-219
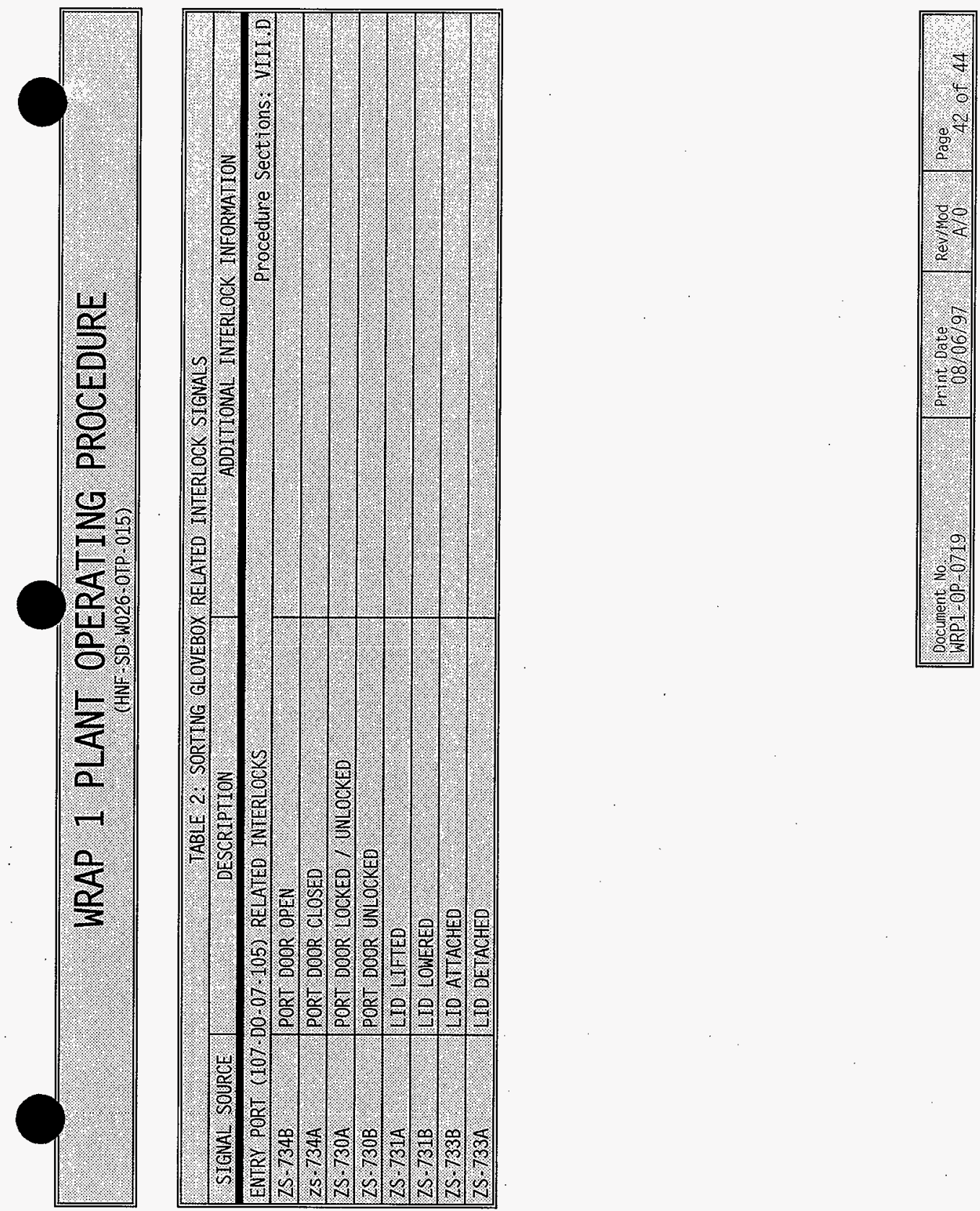


\section{WRAP 1 PLANT OPERATING PROCEDURE}

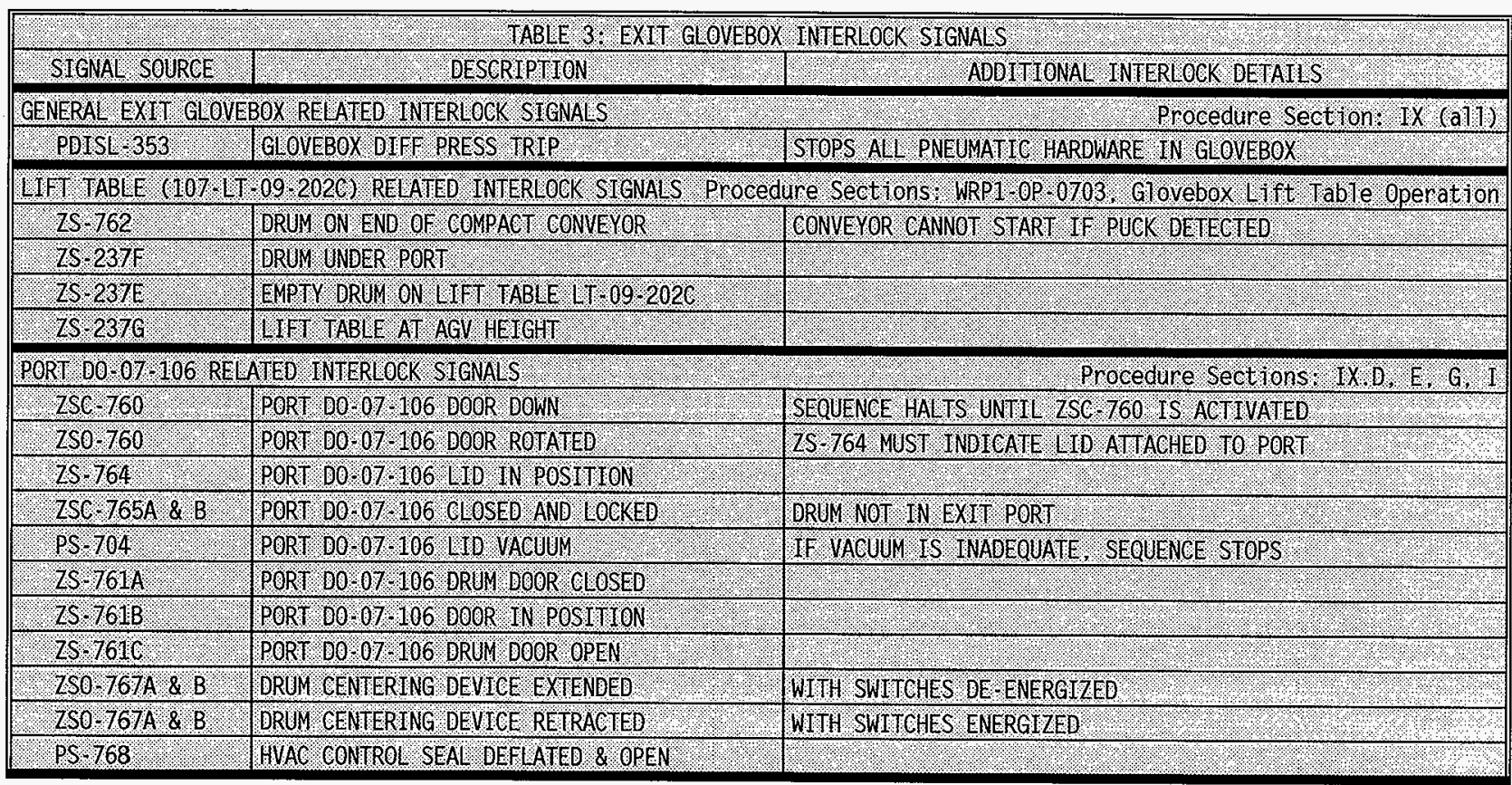

\begin{tabular}{|c|c|c|c|}
\hline 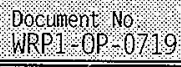 & 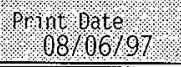 & Rev/Mod & page 43 of 44 \\
\hline
\end{tabular}




\section{WRAP 1 PLANT OPERATING PROCEDURE}

GHAF SD 1026 -0IP 015 )

\begin{tabular}{|c|c|c|}
\hline STGNAL SOURCE & DESOR PION & ABDTMLONAL MTERLOCK DETAILS. \\
\hline HOIST CR 07.1010 & ELATED INTERLOGK SIGNALS & Procedure Sections. IX.C \\
\hline $250-766$ & DRUM LITTER GRAB OPEN & l. \\
\hline $2 \mathrm{SC} 766$. & BREM LITER GRAB CLOSEB . & 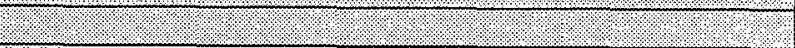 \\
\hline WS $763 \mathrm{~A}$ & HOIST CR $07.101 \mathrm{D}$ RA SED . & 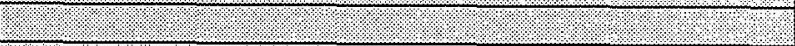 \\
\hline $2 S .763 \mathrm{~B} . \mathrm{C}$ & HOIST AT SLORAGE POSMTON 1.: : : & 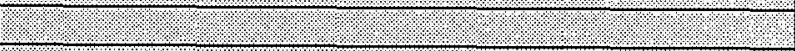 \\
\hline $2 S, 763 \mathrm{~B}, 0.8 .0$ & HOLST AT COMPACT RECETPT POSTIION. & BRUM IT ER MUST BE RAISEO : : : \\
\hline 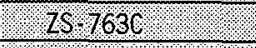 & HOIST AT STORAGE POSTMTON? & 1.:. \\
\hline 25.7630 .0 & HOIST AT LOAB OUT POSTTLN. & (1: \\
\hline
\end{tabular}

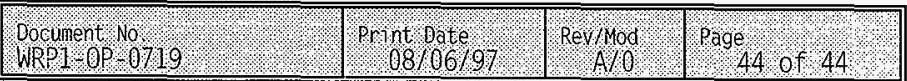


RJ Bottenus

WG Jasen

JK Kersten

RJ Koll

KJ Leist

WR Thackaberry

JR Weidert

Engineering Files
T4-51

$X$

T4-52 X

T4-52

$x$

T4-5I

$x$

T4-52

$x$

T4-52

$x$

T4-52

$x$

A3-88 $X$ 